DOE/EIA-0130(93/11)

Natur G

Month ,

\title{
November 19
}

$\because 11 / 6$

DEC O 8 SOB

c 21

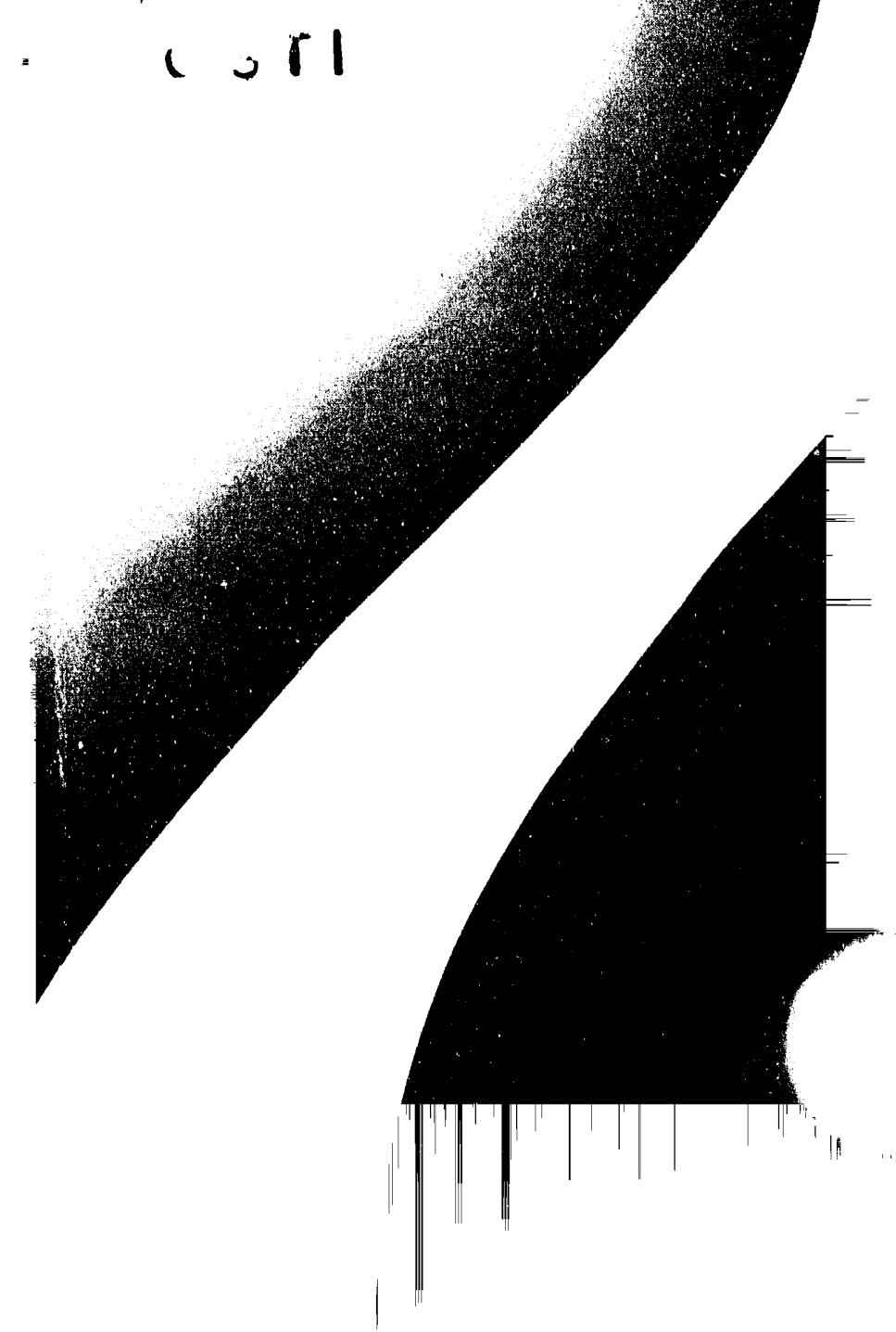


This publication and other Energy Information Administration (EIA) publications may be purchased from the Superintendent of Documents, U.S. Government Printing Office.

\title{
All telephone orders should be directed to:
}

U.S. Government Printing Office

McPherson Square Bookstore

1510 H Street, N.W.

Washington, DC 20005

(202)653-2050

FAX (202)376-5055

9 a.m. to 4:30 p.m., eastern time, M-F
Superintendent of Documents

U.S. Government Printing Office

Washington, DC 20402

(202)783-3238

FAX (202)512-2233

8 a.m. to 4 p.m., eastern time, M-F

\section{All mail orders should be directed to:}

\author{
U.S. Government Printing Office \\ P.O. Box 371954 \\ Piltsburgh, PA 15250-7954
}

Complimentary subscriptions and single issues are available to certain groups of subscribers, such as public and academic libraries, Federal, State, local and foreign governments, EIA survey respondents, and the media. For further information and for answers to questions on energy statistics, please contact EIA's National Energy Information Center. Address, telephone numbers, and hours are as follows:

National Energy Information Center, EI-231

Energy Information Administration

Forrestal Building, Room 1F-048

Washington, DC 20.585

(202) 586-8800

TTY: For people who are deaf or hard

of hearing: (202)586-1181

9 a.m. to 5 p.m., castern time, M-F

Released for Printing: November 29, 1993 by EIA, Office of Cil and Gas, Reserves and Natural Gas Division.

The Natural Gas Monthly (ISSN 0737-1713) is published monthly by the Eriergy Information Administration, 1000 Independence Avenue, SW, Washington, DC 20585, and sells for $\$ 85.00$ per year (price subject to change without advance notice). Second-class postage paid at Washington, DC 20066-9998, and additional mailing offices. POSTMASTER: Send address changes to Natural Gas Monthly, Energy Information Administration, EI-231, 1000 Independence Avenue, SW, Washington, DC 20585 
DOE/EIA-0130(93/11)

Distribution Category/UC-950

\title{
Natural Gas Monthly November 1993
}

\author{
Energy Information Administration \\ Office of Oil and Gas \\ U.S. Department of Energy \\ Washington, DC 20585
}




\section{Preface}

The Natural Gas Monthly (NGM) is prepared in the Data Operations Branch of the Reserves and Natural Gas Division, Office of Oil and Gas, Energy Information Administration (EIA), U.S. Department of Energy (DOE).

General questions and comments regarding the $N G M$ may be referred to Kendrick E. Brown, Jr. (202) 586-6077, Audrey E. J. Corley (202) 586-4804, or Eva M. Fleming (202) 586-6113. Specific technical questions may be referred to the appropriate persons listed in Appendix E.

The NGM highlights activities, events, and analyses of interest to public and private sector organizations associated with the natural gas industry. Volume and price data are presented each month for natural gas production, distribution, consumption, and interstate pipeline activities. Producer-related activities and underground storage data are also reported. From time to time, the NGM features articles designed to assist readers in using and interpreting natural gas information.
The data in this publication are collected on surveys conducted by the EIA to fulfill its responsibilities for gathering and reporting energy data. Some of the data are collected under the authority of the Federal Energy Regulatory Commission (FERC), an independent commission within the DOE, which has jurisdiction primarily in the regulation of electric utilities and the interstate natural gas industry. Geographic coverage is the 50 States and the District of Columbia.

Explanatory Notes supplement the information found in tables of the report. A description of the data collection surveys that support the $N G M$ is provided in the Data Sources section. A glossary of the terms used in this report is also provided to assist readers in understanding the data presented in this publication.

All natural gas volumes are reported at a pressure base of 14.73 pounds per square inch absolute (psia) and at 60 degrees Fahrenheit. 


\section{Common Abbreviations Used in the Natural Gas Monthly}

AGA

$\mathrm{Bbl}$

BLS

Bcf

BOM

Btu

DOE

DOI

EIA

FERC

\section{American Gas Association}

Barrels

Bureau of Labor Statistics, U.S.

Department of Labor

Billion Cubic Feet

Bureau of Mines, U.S. Department of the Interior

British Thermal Unit

U.S. Department of Energy

U.S. Department of the Interior

Energy Information Administration, U.S. Department of Energy

Federal Energy Regulatory Commission
IOGCC

LNG

Mcf

MMBtu

MMcf

MMS

NGL

NGPA

OCS

PGA

Tcf
Interstate Oil and Gas Compact Commission

Liquefied Natural Gas

Thousand Cubic Feet

Million British Thermal Units

Million Cubic Feet

United States Minerals Manage-

ment Service, U.S. Department of the Interior

Natural Gas Liquids

Natural Gas Policy Act of 1978

Outer Continental Shelf

Purchased Gas Adjustment

Trillion Cubic Feet 


\section{Contents}

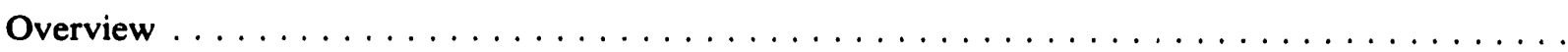

Appendices

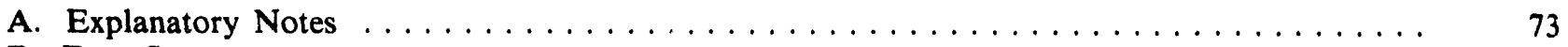

B. Data Sources $\ldots \ldots \ldots \ldots \ldots \ldots \ldots \ldots \ldots$

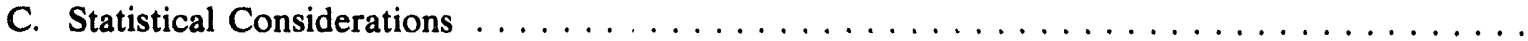

107

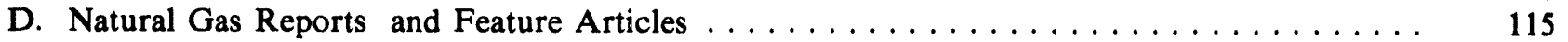

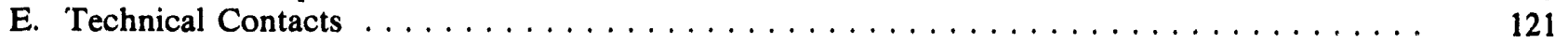

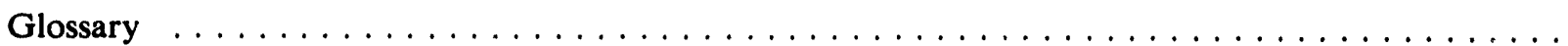

\section{Tables}

1. Summary of Natural Gas Production in the United States, 1987-1993 . . . . . . . . . . . . . .

2. Supply and Disposition of Dry Natural Gas in the United States, 1987-1993 . . . . . . . . .

3. Natural Gas Consumption in the United States, 1987-1993

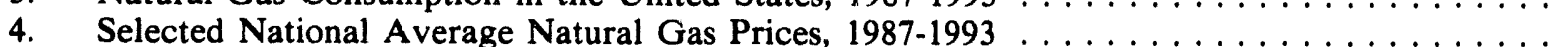

5. U.S. Natural Gas Imports by Country, $1987-1993 \ldots \ldots \ldots \ldots \ldots \ldots$

6. U.S. Natural Gas Exports by Country, $1987-1993 \ldots \ldots \ldots \ldots \ldots \ldots$

7. Marketed Production of Natural Gas by State, $1987-1993 \ldots \ldots \ldots$

8. Revenues, Expenses, and Income of Major Interstate Natural Gas Pipeline Companies,

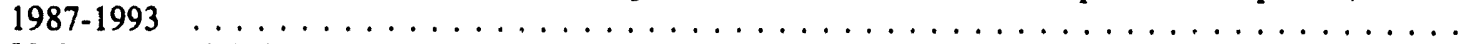

9. Volumes and Prices of Natural Gas Sold by Major Interstate Natural Gas Pipeline Companies, 1987-1993

10. Volumes and Prices of Natural Gas Sold by Major Interstate Natural Gas Pipeline Companies, by Company, August 1993

11. Natural and Other Gases Produced and Purchased by Major Interstate Natural Gas Pipeline Companies, $1987-1993 \ldots \ldots \ldots \ldots \ldots \ldots \ldots \ldots$

12. Natural and Other Gases Produced and Purchased by Major Interstate Natural Gas Pipeline Companies, August $1993 \ldots \ldots \ldots \ldots \ldots \ldots \ldots \ldots \ldots$

13. Underground Natural Gas Storage - All Operators, $1987-1993 \ldots \ldots \ldots$

14. Underground Natural Gas Storage - Interstate Operators of Storage Fields, 1987-1993 . . . .

15. Underground Natural Gas Storage - Intrastate Operators and Independent Producers, 1987-1993

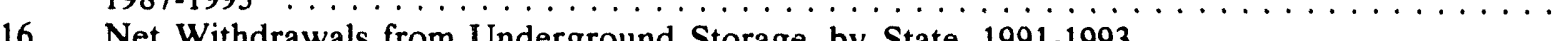

16. Net Withdrawals from Underground Storage, by State, 1991-1993 . . . . . . . . . . . . . .

17. Activities of Underground Natural Gas Storage Operators, by State, September 1993 . . .

18. Natural Gas Deliveries to Residential Consumers by State, 1992-1993 . . . . . . . . . . . . . .

19. Natural Gas Deliveries to Commercial Consumers by State, 1992-1993 . . . . . . . . . . . . .

20. Natural Gas Deliveries to Industrial Consumers by State, 1992-1993 . . . . . . . . . . . . . . .

21. Natural Gas Deliveries to Electric Utility Consumers by State, 1992-1993 . . . . . . . . . . . .

22. Natural Gas Deliveries to All Consumers by State, 1991-1993 . . . . . . . . . . . . . . . .

23. Average City Gate Price by State, $1992-1993 \ldots \ldots \ldots \ldots \ldots \ldots \ldots$

24. Average Price of Natural Gas Delivered to Residential Consumers by State, 1992-1993 . . .

25. Average Price of Natural Gas Sold to Commercial Consumers by State, 1992-1993 . . . . .

26. Average Price of Natural Gas Sold to Industrial Consumers by State, 1992-1993 . . . . . . .

27. Average Price of Natural Gas Delivered to Electric Utility Consumers by State, 1991-1993 .

28. Percentage of Total Deliveries Represented by Onsystem Sales by State, 1991-1993 . . . . . 
A1. Methodology for Reporting Initial Monthly Natural Gas Supply and Disposition Data . . . .

\section{Illustrations}

1. Production and Consumption of Natural Gas in the United States, 1990-1994 . . . . . . . .

2. Natural Gas Deliveries to Consumers in the United States, $1989-1993$. . . . . . . . . . . . .

3. Average Price of Natural Gas Delivered to Consumers in the United States, 1989-1993 . . . .

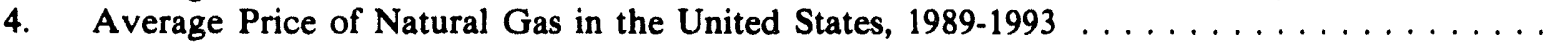

5. Underground Natural Gas Storage in the United States, $1989-1993 \ldots \ldots \ldots \ldots \ldots \ldots \ldots$

6. Percentage of Total Deliveries Represented by Onsystem Sales, 1989-1993 . . . . . . . . . . .

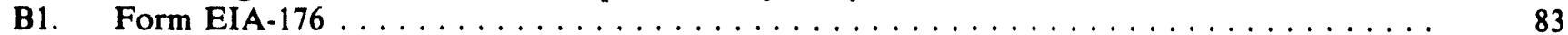

B2. Form EIA-176, Short Form $\ldots \ldots \ldots \ldots \ldots \ldots \ldots \ldots \ldots \ldots \ldots \ldots \ldots \ldots$

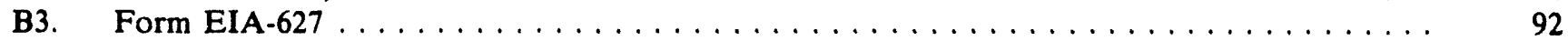

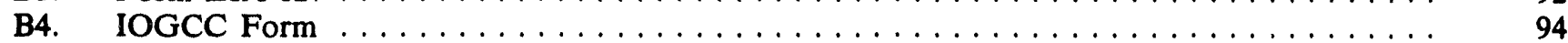

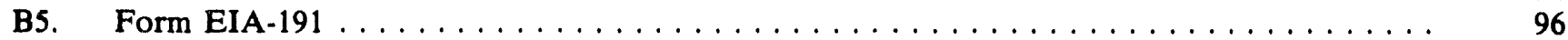

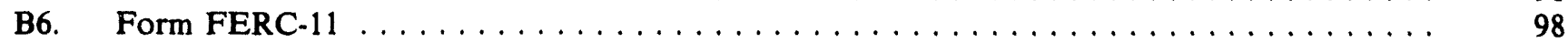

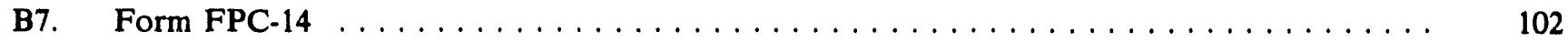

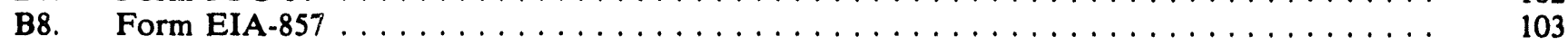




\section{Overview}

\section{Highlights}

The Energy Information Administration recently (November 1993) published its quarterly forecast of shortterm energy supply, demand, and prices in the ShortTerm Energy Outlook, Quarterly Projections, Fourth Quarter, 1993 (STEO). The STEO covers four major energy sources: petroleum and petroleum products, natural gas, coal, and electricity. The forecasts in this current STEO cover the period from the fourth quarter of 1993 through the fourth quarter of 1994

The projections made for natural gas are based on the following assumptions: real gross domestic product (GDP) growth will average 2.5 percent in 1993 and 3.2 percent in 1994 (after increasing by 0.4 percent in 1992); the price of oil will average about $\$ 17$ per barrel in 1993 and 1994; and weather temperatures will equal average temperatures over the last 15 years. Given these assumptions, the STEO forecasts the following for natural gas through the fourth quarter of 1994 :

\section{Consumption}

- 1993 gas demand continued to outpace production. Total consumption is up less than one percent in 1993 (to 19.8 trillion cubic feet) and is expected up 4 percent in 1994 (to 20.5 trillion cubic feet). Gas consumption was down for all sectors in the second quarter of 1993 due to an increase in gas wellhead prices.

- 1993 consumption in the industrial sector was down slightly. In response to higher prices, industrial consumption moderated during the first half of 1993. Continued industrial growth along with recluced upward pressures on prices will result in a gradual resumption of demand for this sector in 1994.

- 1993 residential and commercial consumption have both been rising by 4 percent due to normal weather conditions.

- Gas demand in 1994 is expected to grow across all sectors with normal weather and economic growth, particularly in the manufacturing production area. Normal colder winter weather drives consumption up in the residential and commercial sectors, while unseasonably warmer weather drives consumption down.

\section{Production}

- 1993 production was up by only 2 percent, to 18.1 trillion cubic feet. The low winter demand resulted in production cutbacks while the spring demand surge led to low storage levels.

- 1994 production is expected to go up by .3 trillion cubic feet or 2 percent over 1993.

\section{Imports}

- As demand continues to outpace domestic production, 1993 net imports are expected to rise to 2.1 trillion cubic feet, or over 10 percent. Net imports in 1994 are projected to rise to 2.5 trillion cubic feet, or 17 percent above 1993. The rapid growth in gas pipeline capacity has aided the steady rise in imports.

\section{Wellhead Prices}

- Wellhead prices increased sharply in 1993. The average wellhead price in 1993 of $\$ 2.15$ per thousand cubic feet is expected to be about 36 cents higher than the 1992 average price of \$1.79. During the first half of 1993 , however, prices were lower due to weak demand from warmer winter weather followed by a cold spring. The April price was 41 cents more than the January price which is usually the highest. Wellhead prices are normally lower in spring and summer months than they are in fall and winter months..

- Spot and wellhead prices should resume normal seasonal patterns, with normal weather conditions. Continued declines in production along with demand increases tend to push prices upward.

- 1994 wellhead prices are projected up slightly by 11 cents per thousand cubic feet over 1993 prices. Flat world crude oil prices will leelp offset price increases. 


\section{Supply and Disposition}

The Energy Information Administration estimates that marketed production (gross withdrawals less gas used for repressuring, quantities vented and flared, and nonhydrocarbon gases removed in treating or processing operations) totaled 1,531 billion cubic feet in September 1993 (Table 1). This is 2 percent higher than in September 1992.

The total gas supply available for disposition in September 1993 was an estimated 1,699 billion cubic feet, 4 percent greater than in September 1992 (Table 2). The September 1993 total includes 25 billion cubic feet withdrawn from storage, 9 billion cubic feet of supplemental fuel supplies, and 188 billion cubic feet of imported gas.

On the disposition side, the consumption of 1,311 billion cubic feet was 2 percent less than in August 1993 and 5 percent greater than in September 1992 (Table 2). Total disposition included 378 billion cubic feet of gas injected into underground storage reservoirs and exports of 11 billion cubic feet.

\section{Consumption}

Data for the four major end-use sectors indicate that the total amount of gas delivered to all consumers in August 1993 was 1,204 billion cubic feet, identical to July 1993 (Table 3). Consumption in the industrial sector decreased from 618 billion cubic feet in July 1993 to 612 billion cubic feet in August 1993, a decrease of 1 percent.
The electric utility sector consumed 357 billion cubic feet in August 1993, which is 7 percent greater than in July 1993 and an 18-percent increase from August 1992.

The residential sector consumed 120 billion cubic feet in August 1993, 8 percent lower than in August 1992. The commercial sector consumed 115 billion cubic feet in August 1993, 5 percent less than in August 1992.

\section{Prices}

In August 1993, major interstate pipeline companies paid an average of $\$ 2.35$ per thousand cubic feet for gas purchased from domestic producers, 16 percent greater than July's \$2.02 price and a 10-percent increase from the \$2.14 paid in August 1992 (Table 4). In August 1993 , these pipeline companies paid $\$ 2.25$ per thousand cubic feet for imported gas.

Distributors paid an average $\$ 3.35$ per thousand cubic feet for gas at the city gate in August 1993. This is almost identical to what these distributors paid in July 1993 and 5 percent greater than they paid in August 1992. Residential consumers paid $\$ 8.10$ per thousand cubic feet in August 1993, 9 percent higher than what they paid in August 1992. Commercial consumers paid \$5.26 per thousand cubic feet in August 1993, 5 percent greater than the $\$ 5.03$ paid in July 1993 and 11 percent higher than what they paid in August 1992.

Industrial consumers paid $\$ 2.86$ per thousand cubic feet in August 1993, a 6-percent increase from the July 1993 price of $\$ 2.71$ and 6 percent greater than in August 1992. Electric utilities paid an average of $\$ 2.46$ per thousand cubic feet in July 1993, slightly less than the $\$ 2.47$ per thousand cubic feet paid in June 1993. 
Table 1. Summary of Natural Gas Production in the United States, 1987-1993 (Billion Cubic Feet)

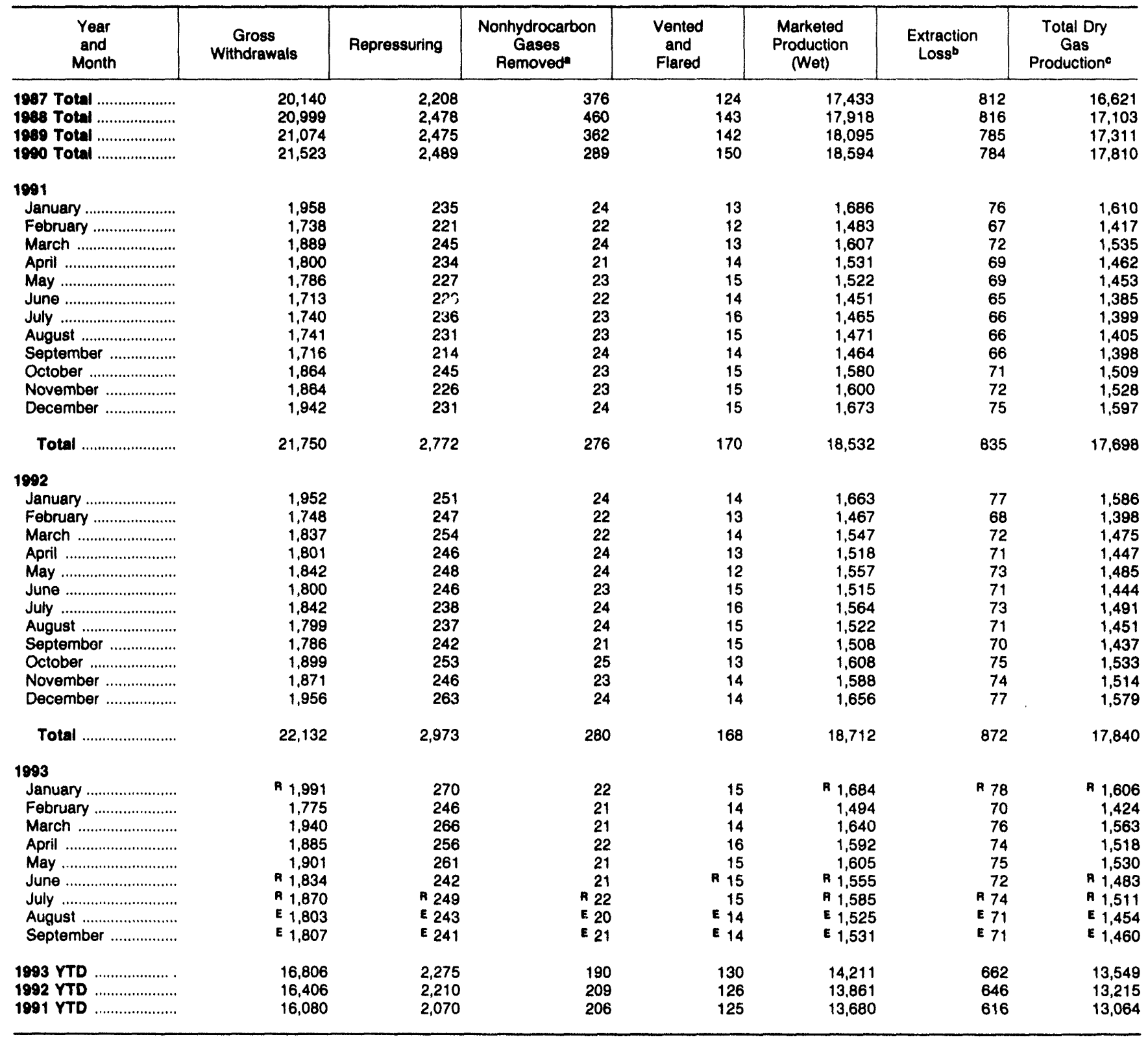

- See Appendix A, Explanatory Note 1 for a discussion of data on Nonhydrocarbon Gases Removed.

- Extraction loss is only collected on an annual basis. Annually it is between 4 and 5 percent of marketed production. Monthly extraction loss is estimated from monthly marketed production by assuming that the preceding annual percentage remains constant tor the next twelve months.

- Equal to marketed production (wet) minus extraction loss.

E Estimated Date.

A Revised Data.

Notes: Data for 1987 through 1992 are final. All other data are preliminary unless otherwise indicated. Geographic coverage is the 50 States and the District of Columbia. Totals may not equal sum of components because of independent rounding.

Sources: EIA, Natural Gas Annual 1992. Table 7 and ElA estimates, January 1993 through current month. See Appendix A, Explanatory Notes 1, 3, and 6 for discussion of computation, estimating procedures, and revision policy. 
Table 2. Supply and Disposition of Dry Natural Gas in the United States, 1987-1993 (Billion Cubic Feet)

\begin{tabular}{|c|c|c|c|c|c|c|c|c|c|}
\hline \multirow{2}{*}{$\begin{array}{l}\text { Year } \\
\text { and } \\
\text { Month }\end{array}$} & \multicolumn{5}{|c|}{ Supply } & \multirow{2}{*}{$\begin{array}{c}\text { Total } \\
\text { Supply' } \\
\text { Dispositiond }\end{array}$} & \multicolumn{3}{|c|}{ Disposition } \\
\hline & $\begin{array}{l}\text { Total Dry } \\
\text { Gas } \\
\text { Production }\end{array}$ & $\begin{array}{l}\text { Withorawals } \\
\text { from } \\
\text { Storage }\end{array}$ & $\begin{array}{l}\text { Supplemental } \\
\text { Gasoous } \\
\text { Fuela" }\end{array}$ & Imports & $\begin{array}{l}\text { Balancing } \\
\text { Item }\end{array}$ & & $\begin{array}{l}\text { Additions } \\
\text { to } \\
\text { Storage }\end{array}$ & Exports & Consumption \\
\hline $\begin{array}{l}1097 \text { Total } \\
1000 \text { Total } \\
1800 \text { Total } \\
1900 \text { Total }\end{array}$ & $\begin{array}{l}16,621 \\
17,103 \\
17,311 \\
17,810\end{array}$ & $\begin{array}{l}1,805 \\
2,270 \\
2,854 \\
1,886\end{array}$ & $\begin{array}{l}101 \\
101 \\
107 \\
123\end{array}$ & $\begin{array}{r}993 \\
1,294 \\
1,382 \\
1,532\end{array}$ & $\begin{array}{l}-444 \\
-453 \\
-218 \\
-148\end{array}$ & $\begin{array}{l}10,176 \\
20,315 \\
21,435 \\
21,302\end{array}$ & $\begin{array}{l}1,911 \\
2,211 \\
2,528 \\
2,499\end{array}$ & $\begin{array}{r}54 \\
74 \\
107 \\
86\end{array}$ & $\begin{array}{l}17,211 \\
18,030 \\
18,801 \\
18,716\end{array}$ \\
\hline $\begin{array}{l}1901 \\
\text { January } \\
\text { February } \\
\text { March } \\
\text { April } \\
\text { May } \\
\text { June } \\
\text { July } \\
\text { August } \\
\text { September } \\
\text { October } \\
\text { November } \\
\text { December ................ }\end{array}$ & $\begin{array}{l}1,610 \\
1,417 \\
1,535 \\
1,462 \\
1,453 \\
1,385 \\
1,399 \\
1,405 \\
1,398 \\
1,509 \\
1,528 \\
1,597\end{array}$ & 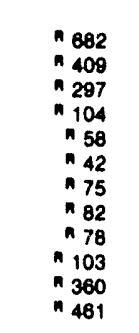 & $\begin{array}{r}12 \\
10 \\
11 \\
9 \\
9 \\
9 \\
8 \\
9 \\
9 \\
8 \\
0 \\
10 \\
9 \\
11\end{array}$ & $\begin{array}{l}163 \\
138 \\
151 \\
144 \\
141 \\
133 \\
135 \\
127 \\
134 \\
157 \\
169 \\
181\end{array}$ & $\begin{array}{l}n-44 \\
n 62 \\
n-15 \\
n-65 \\
n 13 \\
n-37 \\
n-28 \\
n-48 \\
-72 \\
n-88 \\
n-209 \\
n-88\end{array}$ & 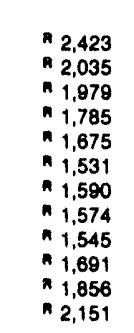 & 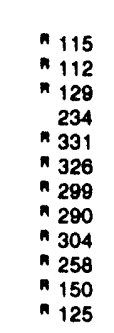 & $\begin{array}{r}10 \\
11 \\
10 \\
9 \\
8 \\
7 \\
7 \\
8 \\
10 \\
11 \\
14 \\
15 \\
18\end{array}$ & $\begin{array}{l}2,299 \\
1,912 \\
1,840 \\
1,542 \\
1,337 \\
1,198 \\
1,283 \\
1,274 \\
1,231 \\
1,419 \\
1,691 \\
2,009\end{array}$ \\
\hline Total & 17,698 & 2,752 & 113 & 1,773 & -500 & 21,836 & 2,672 & 129 & 19,035 \\
\hline 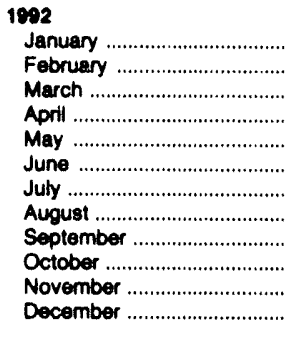 & $\begin{array}{l}1,586 \\
1,398 \\
1,475 \\
1,447 \\
1,485 \\
1,444 \\
1,491 \\
1,451 \\
1,437 \\
1,533 \\
1,514 \\
1,579\end{array}$ & $\begin{array}{r}624 \\
463 \\
397 \\
142 \\
44 \\
35 \\
42 \\
46 \\
40 \\
70 \\
282 \\
587\end{array}$ & $\begin{array}{r}12 \\
11 \\
11 \\
10 \\
9 \\
8 \\
8 \\
8 \\
8 \\
10 \\
11 \\
12\end{array}$ & $\begin{array}{l}165 \\
175 \\
180 \\
178 \\
174 \\
162 \\
167 \\
175 \\
166 \\
176 \\
210 \\
209 \\
\end{array}$ & $\begin{array}{r}-71 \\
42 \\
-42 \\
89 \\
68 \\
16 \\
-8 \\
-19 \\
-24 \\
-130 \\
-239 \\
-191\end{array}$ & $\begin{array}{l}2,315 \\
2,089 \\
2,022 \\
1,864 \\
1,780 \\
1,686 \\
1,700 \\
1,662 \\
1,629 \\
1,659 \\
1,778 \\
2,185\end{array}$ & $\begin{array}{r}60 \\
45 \\
74 \\
161 \\
344 \\
384 \\
373 \\
380 \\
362 \\
271 \\
88 \\
58\end{array}$ & $\begin{array}{l}16 \\
14 \\
23 \\
18 \\
18 \\
18 \\
18 \\
18 \\
18 \\
18 \\
19 \\
19 \\
18\end{array}$ & $\begin{array}{l}2,239 \\
2,031 \\
1,926 \\
1,685 \\
1,418 \\
1,264 \\
1,311 \\
1,264 \\
1,248 \\
1,368 \\
1,672 \\
2,119\end{array}$ \\
\hline Total & 17,840 & 2,772 & 118 & 2,138 & -508 & 22,360 & 2,599 & 216 & 19,544 \\
\hline 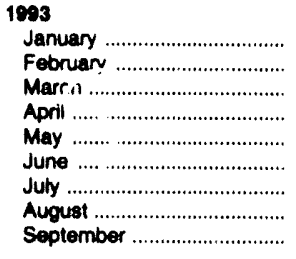 & $\begin{array}{l}1,606 \\
1,424 \\
1,563 \\
1,518 \\
1,530 \\
1,483 \\
1,511 \\
1,454 \\
1,460\end{array}$ & $\begin{array}{r}605 \\
578 \\
381 \\
111 \\
25 \\
43 \\
48 \\
98 \\
25\end{array}$ & $\begin{array}{r}13 \\
12 \\
12 \\
10 \\
8 \\
10 \\
9 \\
8 \\
8 \\
8\end{array}$ & $\begin{array}{r}198 \\
183 \\
199 \\
185 \\
160 \\
178 \\
180 \\
184 \\
184 \\
188\end{array}$ & $\begin{array}{l}-58 \\
n 17 \\
78 \\
78 \\
728 \\
n-8 \\
n-1 \\
n \\
n \\
n \\
n \\
18\end{array}$ & 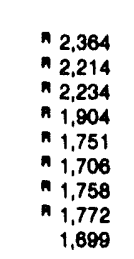 & $\begin{array}{r}50 \\
27 \\
78 \\
219 \\
447 \\
416 \\
388 \\
419 \\
378\end{array}$ & $\begin{array}{l}18 \\
13 \\
17 \\
12 \\
12 \\
11 \\
14 \\
14 \\
11 \\
11\end{array}$ & $\begin{array}{l}2,297 \\
2,174 \\
2,140 \\
1,673 \\
1,291 \\
1,280 \\
1,346 \\
1,342 \\
1,311\end{array}$ \\
\hline $\begin{array}{l}1903 \text { VTD } \\
1902 \text { VTD } \\
1901 \text { VTD }\end{array}$ & $\begin{array}{l}13,549 \\
13,215 \\
13,064\end{array}$ & $\begin{array}{l}1,815 \\
1,833 \\
1,827\end{array}$ & $\begin{array}{l}92 \\
85 \\
83\end{array}$ & $\begin{array}{l}1,668 \\
1,541 \\
1,267\end{array}$ & $\begin{array}{r}180 \\
53 \\
-104\end{array}$ & $\begin{array}{l}17,402 \\
16,728 \\
16,138\end{array}$ & $\begin{array}{l}2,430 \\
2,183 \\
2,139\end{array}$ & $\begin{array}{r}118 \\
160 \\
82\end{array}$ & $\begin{array}{l}14,854 \\
14,385 \\
13,817\end{array}$ \\
\hline
\end{tabular}

- Monthly and annual data for 1987 through 1992 include underground storage and liquefied natural gas storage. Data for January 1983 fonward include underground storage only. See Appendix A, Explanatory Note 7 for discussion of computation procedures.

- Supplemental gaseous fuels data are only collected on an annual basis except for the Dakota Gasification Inc. coal gasification facility where they are gathered each month. The ratio of annual supplemental tuels (excluding Dakota Gasification inc.) to the sum of dry gas production, net imports, and net withdrawals from storage is calculated. This ratio, which varies between .0028 and .0037, is applied to the monthly sum of these three elements. The Dakota Gasificution Inc. monthly value is added to the result to produce the monthily supplemental huels estimate.

- Represents quantities lost and imbalances in dats due to differences among data sources. See Appendix A, Explanatory Noto 10 for full discussion.

d "Total" data for 1987 through 1992 do not equal equivalent data in Table 1 of the Natural Gas Anmual 1992 due to the exclusion of intransit receipts and deliveries in the NGM

- Consiats of pipeline tuel use, lease and plant fuel use, and detiveries to consuming sectors as shown in Table 3.

- Estimated Date.

= Revised Date.

= Aovised Estimated Dats.

Notes: Data for 1997 through 1992 are final. All other data are preliminary unless othenwise indicated. Geographic coverage is the 50 States and the District of Columbia. To. tals may not equal sum of components because of independent rounding.

Sources: - Total Dry Gas Production: EIA Natural Gas Amual 1982, 1987 through 1992; IOGCC, MMS reporting, and ElA estimates, January 1993 through current month. See Appendix A. Explanatory Note 3 for extimation procedures and revision policy. WWithdrawals from and Additions to Storage: ElA Natural Gas Annual 1992, 1987 through 1982; Form EIA-191, January 1983 through current month. •Supplemental Gaseous Fuets: EIA Natural Gas Annual 1992 1987 through 1992; and EIA computations, January 1993 through current month. See Appendix A, Explanatory Note 2 for discuseion of computation procedures and revision policy. AImports and Exports: Form FPC-14, 1987 through 1992; and ElA estimates, January 1893 through the current month. See Appendix A. Explanatory Note 4 for diecusalon of procedures and revision policy. $\bullet$ Consumption and Balancing Itern: ElA Natural Gas Amnual 1892, 1987 through 1992; and ElA computations, January 1893 through current month. See Appendix A, Explanatory Notes 5 and 10 for discussion of computa. tion procedures and reviaion policy. 
Figure 1. Production and Consumption of Natural Gas in the United States, 1990-1994

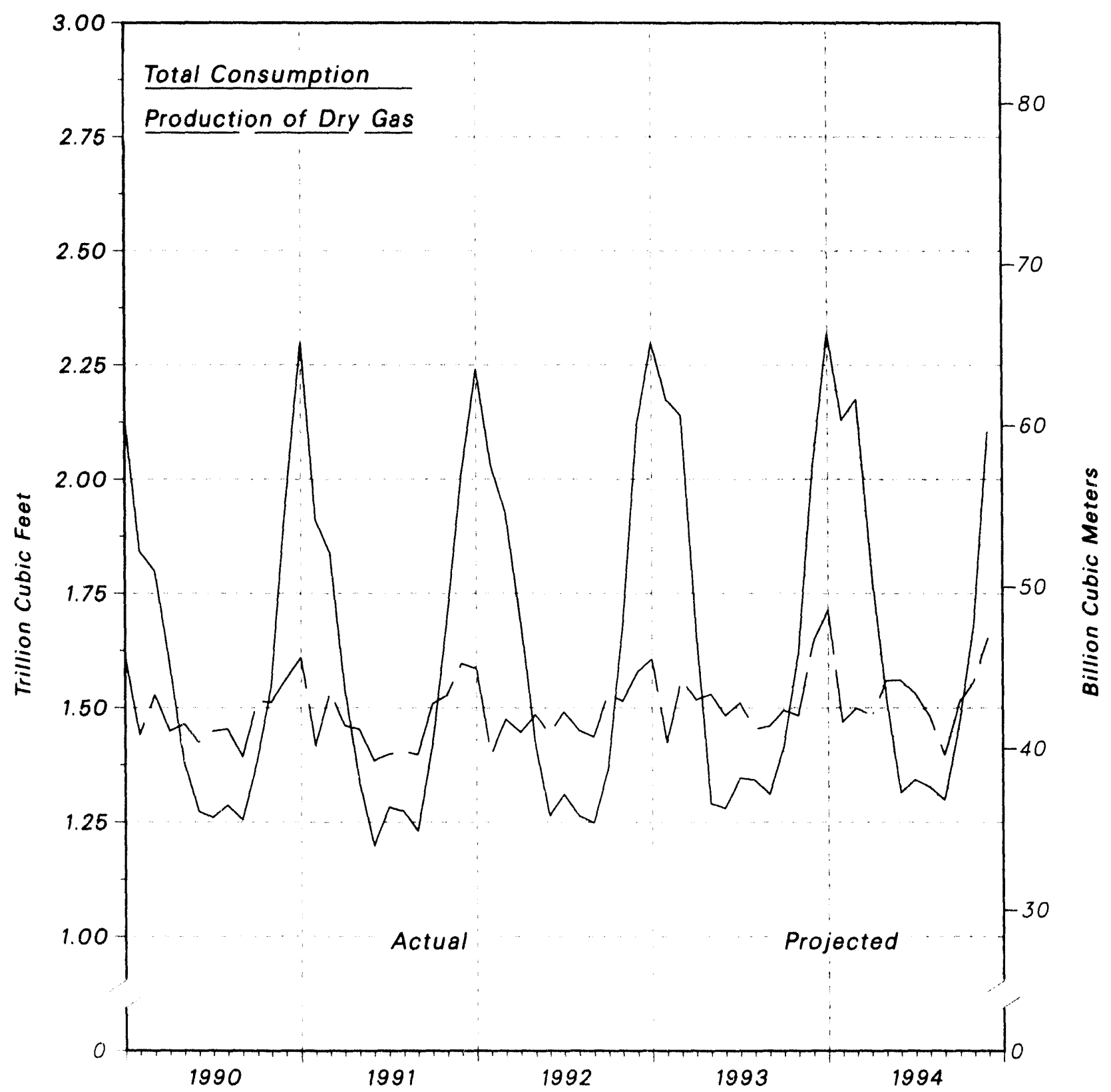

Source: Natural Gas Annual and the Short Term Energy Outlook. 
Table 3. Natural Gas Consumption in the United States, 1987-1993 (Billion Cubic Feet)

\begin{tabular}{|c|c|c|c|c|c|c|c|c|}
\hline \multirow{2}{*}{$\begin{array}{l}\text { Year } \\
\text { and } \\
\text { Month }\end{array}$} & \multirow{2}{*}{$\begin{array}{l}\text { Lease and } \\
\text { Plant } \\
\text { Fuel }\end{array}$} & \multirow{2}{*}{$\begin{array}{l}\text { Pipeline } \\
\text { Fue/t }\end{array}$} & \multicolumn{5}{|c|}{ Delivered to Consumers } & \multirow{2}{*}{$\begin{array}{c}\text { Total } \\
\text { Consumption }\end{array}$} \\
\hline & & & Residential & Commercial & Industrial & $\begin{array}{l}\text { Electric } \\
\text { Utilities }\end{array}$ & Totai & \\
\hline 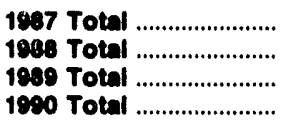 & $\begin{array}{l}1,149 \\
1,096 \\
1,070 \\
1,236\end{array}$ & $\begin{array}{l}519 \\
614 \\
629 \\
660\end{array}$ & $\begin{array}{l}4,315 \\
4,630 \\
4,781 \\
4,391\end{array}$ & $\begin{array}{l}2,430 \\
2,670 \\
2,718 \\
2,623\end{array}$ & $\begin{array}{l}5,953 \\
6,383 \\
6,816 \\
7,018\end{array}$ & $\begin{array}{l}2,844 \\
2,636 \\
2,787 \\
2,787\end{array}$ & $\begin{array}{l}15,542 \\
16,320 \\
17,102 \\
16,820\end{array}$ & $\begin{array}{l}17,211 \\
18,030 \\
18,801 \\
18,716\end{array}$ \\
\hline \multicolumn{9}{|l|}{1901} \\
\hline 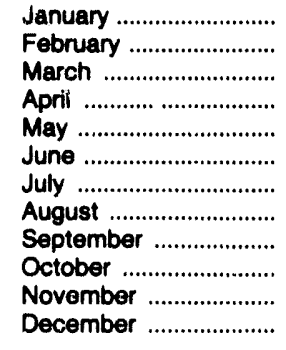 & $\begin{array}{r}102 \\
90 \\
98 \\
93 \\
93 \\
89 \\
90 \\
80 \\
89 \\
97 \\
97 \\
101\end{array}$ & $\begin{array}{l}74 \\
61 \\
58 \\
49 \\
42 \\
37 \\
40 \\
40 \\
38 \\
44 \\
54 \\
64\end{array}$ & $\begin{array}{l}844 \\
664 \\
573 \\
373 \\
229 \\
148 \\
126 \\
118 \\
138 \\
225 \\
459 \\
658\end{array}$ & $\begin{array}{l}434 \\
359 \\
310 \\
225 \\
154 \\
119 \\
125 \\
113 \\
121 \\
163 \\
256 \\
350\end{array}$ & $\begin{array}{l}672 \\
591 \\
607 \\
586 \\
571 \\
546 \\
572 \\
586 \\
582 \\
626 \\
627 \\
665\end{array}$ & $\begin{array}{l}173 \\
146 \\
193 \\
216 \\
249 \\
260 \\
330 \\
328 \\
263 \\
263 \\
198 \\
170\end{array}$ & $\begin{array}{l}2,123 \\
1,761 \\
1,683 \\
1,400 \\
1,202 \\
1,073 \\
1,153 \\
1,144 \\
1,103 \\
1,278 \\
1,540 \\
1,843\end{array}$ & $\begin{array}{l}2,299 \\
1,912 \\
1,840 \\
1,542 \\
1,337 \\
1,199 \\
1,283 \\
1,274 \\
1,231 \\
1,419 \\
1,691 \\
2,009\end{array}$ \\
\hline Total & 1,129 & 601 & 4,556 & 2,729 & 7,231 & 2,789 & 17,305 & 19,035 \\
\hline \multicolumn{9}{|l|}{$1 \% 02$} \\
\hline 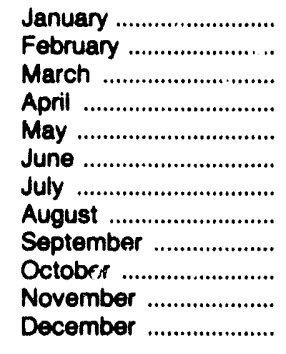 & $\begin{array}{r}104 \\
92 \\
97 \\
95 \\
97 \\
95 \\
98 \\
95 \\
94 \\
101 \\
99 \\
104\end{array}$ & $\begin{array}{l}68 \\
62 \\
58 \\
51 \\
42 \\
37 \\
39 \\
37 \\
37 \\
41 \\
50 \\
64\end{array}$ & $\begin{array}{l}786 \\
696 \\
574 \\
431 \\
251 \\
162 \\
132 \\
126 \\
137 \\
241 \\
437 \\
717\end{array}$ & $\begin{array}{l}410 \\
366 \\
315 \\
250 \\
170 \\
125 \\
122 \\
121 \\
121 \\
166 \\
256 \\
381\end{array}$ & $\begin{array}{l}701 \\
644 \\
674 \\
628 \\
620 \\
578 \\
587 \\
582 \\
586 \\
608 \\
641 \\
677\end{array}$ & $\begin{array}{l}169 \\
170 \\
208 \\
229 \\
236 \\
266 \\
334 \\
303 \\
274 \\
213 \\
189 \\
176\end{array}$ & $\begin{array}{l}2,067 \\
1,876 \\
1,770 \\
1,539 \\
1,278 \\
1,132 \\
1,175 \\
1,131 \\
1,117 \\
1,227 \\
1,523 \\
1,951\end{array}$ & $\begin{array}{l}2,239 \\
2,031 \\
1,926 \\
1,685 \\
1,418 \\
1,264 \\
1,311 \\
1,264 \\
1,249 \\
1,368 \\
1,672 \\
2,119\end{array}$ \\
\hline Total ............................. & 1,171 & 588 & 4,690 & 2,803 & 7,527 & 2,766 & 17,786 & 19,544 \\
\hline \multicolumn{9}{|l|}{1903} \\
\hline 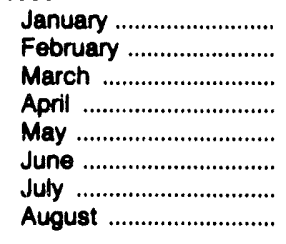 & $\begin{array}{r}105 \\
94 \\
103 \\
100 \\
100 \\
97 \\
99 \\
95\end{array}$ & $\begin{array}{l}\text { ค } 73 \\
\text { ค } 69 \\
\text { ค } 68 \\
\text { ค } 53 \\
\text { ค } 41 \\
\text { ค } 40 \\
\text { ค } 43 \\
42\end{array}$ & $\begin{array}{l}834 \\
770 \\
703 \\
450 \\
234 \\
164 \\
130 \\
120\end{array}$ & $\begin{array}{l}421 \\
408 \\
374 \\
257 \\
156 \\
127 \\
123 \\
115\end{array}$ & $\begin{array}{l}699 \\
672 \\
699 \\
639 \\
593 \\
597 \\
618 \\
612\end{array}$ & $\begin{array}{l}164 \\
162 \\
194 \\
174 \\
167 \\
255 \\
333 \\
357\end{array}$ & $\begin{array}{l}2,119 \\
2,012 \\
1,969 \\
1,521 \\
1,150 \\
1,142 \\
1,204 \\
1,204\end{array}$ & $\begin{array}{l}\text { ค } 2,297 \\
\text { ค } 2,174 \\
\text { ค } 2,140 \\
\text { ค } 1,673 \\
\text { ค } 1,291 \\
\text { ค } 1,280 \\
\text { ค } 1,346 \\
1,342\end{array}$ \\
\hline 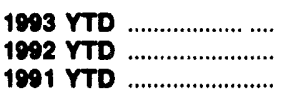 & $\begin{array}{l}794 \\
773 \\
745\end{array}$ & $\begin{array}{l}428 \\
395 \\
401\end{array}$ & $\begin{array}{l}3,405 \\
3,158 \\
3,075\end{array}$ & $\begin{array}{l}1,982 \\
1,879 \\
1,838\end{array}$ & $\begin{array}{l}5,129 \\
5,015 \\
4,731\end{array}$ & $\begin{array}{l}1,806 \\
1,914 \\
1,895\end{array}$ & $\begin{array}{l}12,322 \\
11,967 \\
11,540\end{array}$ & $\begin{array}{l}13,543 \\
13,136 \\
12,686\end{array}$ \\
\hline
\end{tabular}

- Plant fuel data are only collected on an annual basis and monthly lease fuel data are only collected annually. Lease and plant fuel estimates have been between 6 and 7 percent of marketed production annually. Monthly lease and plant fuel use is estimated from monthly marketed production by assuming that the preceding annual percentage remains constant for the next twelve months.

b Pipeline fuel use is only collected on an annual basis. Annually it is between 3 and 4 percent of total consumption. Monthly pipeline fuel data are estimated from monthly total consumption (excluding pipeline fuel) by assuming that the preceding annual percentage remains constant for the next twelve months.

R $=$ Revised Data.

Notes: Data for 1987 through 1992 are final. All other data are preliminary unless otherwise indicated. Geographic coverage is the 50 States and the District of Columbia. Totals may not equal sum of components because of independent rounding.

Sources: All data except electric utility: EIA Natura/ Gas Annual 1992, 1987 through 1992; and Form EIA-857 and computations January 1983 through the current month. See Appendix A, Explanatory Note 5 for computation procedures and revision policy. Electric utility data: Form ElA-759, "Monthly Power Plant Report" (formerty Form FPC-4). 
Trillion Cubic Feet

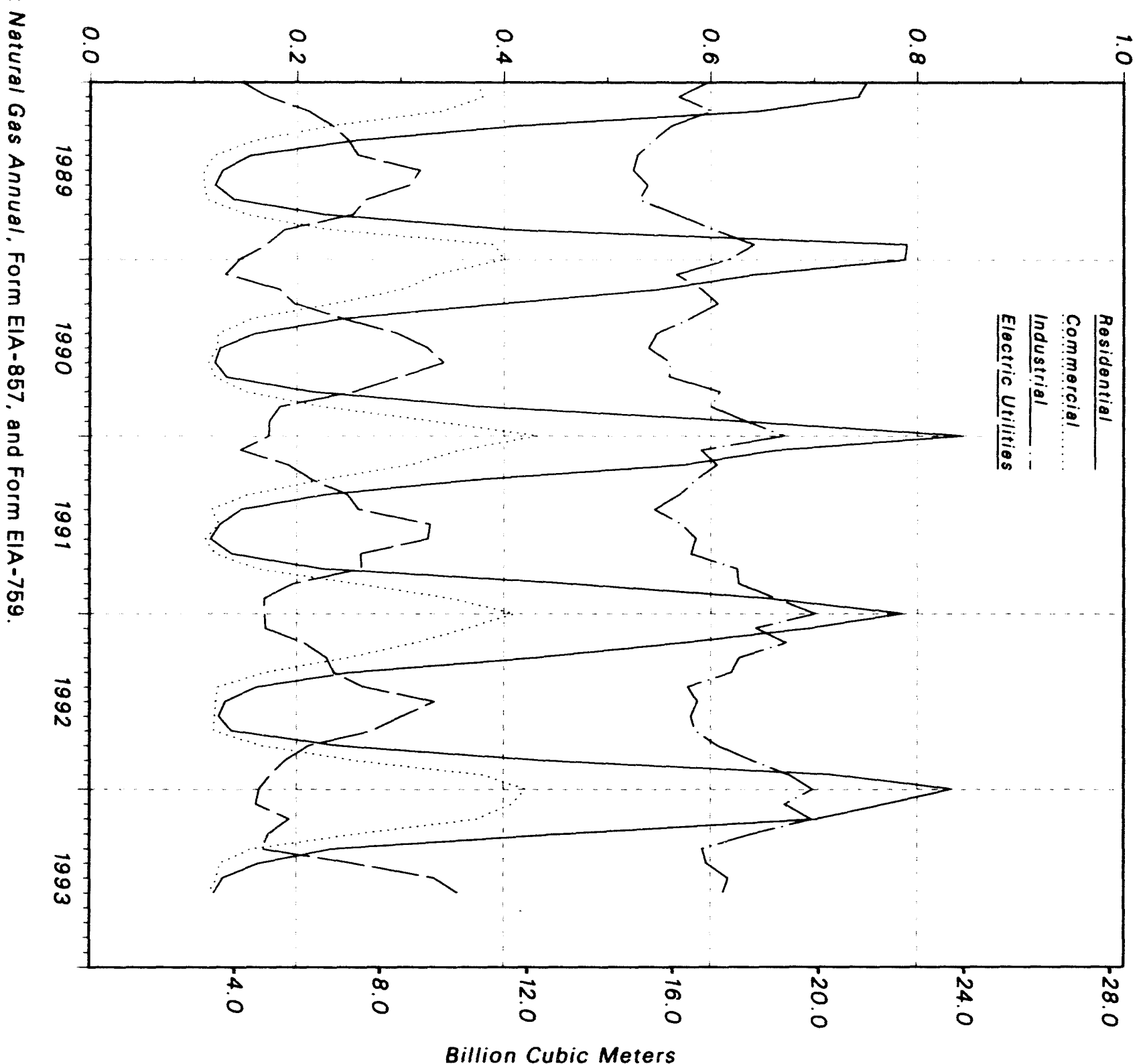

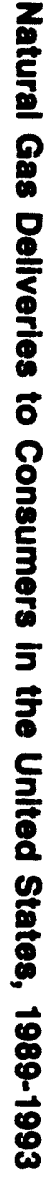


Table 4. Selected National Average Natural Gas Prices, 1987-1993 (Dollars per Thousand Cubic Feet)

\begin{tabular}{|c|c|c|c|c|c|c|c|c|}
\hline \multirow{2}{*}{$\begin{array}{l}\text { Year } \\
\text { and } \\
\text { Month }\end{array}$} & \multirow{2}{*}{$\begin{array}{l}\text { Wellhead } \\
\text { Price* }\end{array}$} & \multicolumn{2}{|c|}{$\begin{array}{c}\text { Major Interstate } \\
\text { Pipeline Companies }\end{array}$} & \multirow{2}{*}{$\begin{array}{l}\text { City } \\
\text { Gate }\end{array}$} & \multicolumn{4}{|c|}{ Delivered to Consumers } \\
\hline & & Imports & $\begin{array}{l}\text { Purchased from } \\
\text { Producers }\end{array}$ & & Residential & Commercial & Industrial & $\begin{array}{l}\text { Electric } \\
\text { Utilities }^{\circ}\end{array}$ \\
\hline 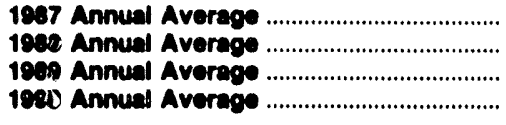 & $\begin{array}{l}1.67 \\
1.69 \\
1.69 \\
1.71\end{array}$ & $\begin{array}{l}2.17 \\
2.00 \\
2.04 \\
2.03\end{array}$ & $\begin{array}{l}2.10 \\
2.13 \\
2.18 \\
2.19\end{array}$ & $\begin{array}{l}2.87 \\
2.92 \\
3.01 \\
3.03\end{array}$ & $\begin{array}{l}5.54 \\
5.47 \\
5.64 \\
5.80\end{array}$ & $\begin{array}{l}4.77 \\
4.63 \\
4.74 \\
4.83\end{array}$ & $\begin{array}{l}2.94 \\
2.95 \\
2.96 \\
2.93\end{array}$ & $\begin{array}{l}2.32 \\
2.33 \\
2.43 \\
2.39\end{array}$ \\
\hline \multicolumn{9}{|l|}{1901} \\
\hline $\begin{array}{l}\text { January } \\
\text { February } \\
\text { March } \\
\text { April } \\
\text { May } \\
\text { June } \\
\text { July } \\
\text { August } \\
\text { September } \\
\text { October } \\
\text { November } \\
\text { December }\end{array}$ & $\begin{array}{l}1.86 \\
1.62 \\
1.49 \\
1.50 \\
1.48 \\
1.43 \\
1.34 \\
1.43 \\
1.59 \\
1.82 \\
1.89 \\
2.00\end{array}$ & $\begin{array}{l}2.20 \\
2.10 \\
1.92 \\
2.03 \\
1.99 \\
2.03 \\
2.11 \\
1.71 \\
1.84 \\
2.00 \\
2.20 \\
2.09\end{array}$ & $\begin{array}{l}2.19 \\
1.93 \\
2.02 \\
1.87 \\
1.96 \\
1.75 \\
1.79 \\
1.71 \\
1.76 \\
1.94 \\
2.02 \\
2.11\end{array}$ & $\begin{array}{l}3.08 \\
2.94 \\
2.78 \\
2.74 \\
2.76 \\
2.86 \\
2.74 \\
2.78 \\
2.91 \\
2.92 \\
2.92 \\
3.05\end{array}$ & $\begin{array}{l}5.54 \\
5.56 \\
5.60 \\
5.90 \\
6.28 \\
6.97 \\
7.23 \\
7.36 \\
6.92 \\
6.20 \\
5.51 \\
5.51\end{array}$ & $\begin{array}{l}4.94 \\
4.94 \\
4.89 \\
4.87 \\
4.65 \\
4.80 \\
4.50 \\
4.73 \\
4.57 \\
4.58 \\
4.71 \\
4.84\end{array}$ & $\begin{array}{l}3.25 \\
2.97 \\
2.75 \\
2.68 \\
2.40 \\
2.34 \\
2.23 \\
2.29 \\
2.40 \\
2.69 \\
2.84 \\
3.09\end{array}$ & $\begin{array}{l}2.70 \\
2.35 \\
2.21 \\
2.10 \\
2.01 \\
1.94 \\
1.88 \\
1.86 \\
2.19 \\
2.35 \\
2.43 \\
2.64\end{array}$ \\
\hline 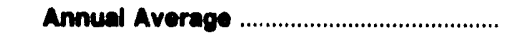 & 1.64 & 2.02 & 1.92 & 2.90 & 5.82 & 4.81 & 2.69 & 2.18 \\
\hline \multicolumn{9}{|l|}{1902} \\
\hline $\begin{array}{l}\text { January } \\
\text { February } \\
\text { April } \\
\text { May } \\
\text { June } \\
\text { July } \\
\text { August } \\
\text { September } \\
\text { October } \\
\text { November } \\
\text { December }\end{array}$ & $\begin{array}{ll}\text { A } & 1.74 \\
\text { A } & 1.26 \\
\text { R } & 1.35 \\
\text { R } & 1.42 \\
\text { R } & 1.51 \\
\text { R } & 1.62 \\
\text { R } & 1.55 \\
\text { R } & 1.84 \\
\text { ค } & 1.92 \\
\text { ค } 2.38 \\
\text { ค } 2.13 \\
\text { ค } 2.07\end{array}$ & $\begin{array}{r}2.20 \\
1.98 \\
1.45 \\
2.01 \\
1.79 \\
2.03 \\
1.89 \\
\mathrm{P} 1.85 \\
2.05 \\
2.13 \\
2.32 \\
1.92\end{array}$ & $\begin{array}{l}2.10 \\
1.70 \\
1.90 \\
1.73 \\
1.99 \\
2.16 \\
1.86 \\
2.14 \\
2.13 \\
2.69 \\
2.37 \\
2.40\end{array}$ & $\begin{array}{l}2.90 \\
2.70 \\
2.61 \\
2.74 \\
2.90 \\
3.00 \\
3.01 \\
3.18 \\
3.23 \\
3.50 \\
3.33 \\
3.17\end{array}$ & $\begin{array}{l}5.53 \\
5.54 \\
5.50 \\
5.62 \\
6.15 \\
6.84 \\
7.27 \\
7.45 \\
7.15 \\
6.52 \\
6.02 \\
5.74\end{array}$ & $\begin{array}{l}4.85 \\
5.03 \\
4.77 \\
4.77 \\
4.59 \\
4.72 \\
4.64 \\
4.73 \\
4.69 \\
4.90 \\
5.12 \\
5.11\end{array}$ & $\begin{array}{l}3.04 \\
2.78 \\
2.58 \\
2.54 \\
2.44 \\
2.53 \\
2.54 \\
2.71 \\
2.82 \\
3.21 \\
3.26 \\
3.38\end{array}$ & $\begin{array}{r}2.49 \\
2.03 \\
1.99 \\
\text { ค } 2.07 \\
2.11 \\
2.18 \\
2.13 \\
2.42 \\
2.51 \\
3.04 \\
2.87 \\
2.81\end{array}$ \\
\hline 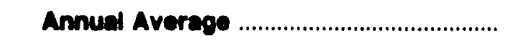 & ค 1.74 & 1.97 & 2.10 & 3.01 & 5.89 & 4.88 & 2.84 & 2.36 \\
\hline \multicolumn{9}{|l|}{1903} \\
\hline $\begin{array}{l}\text { January } \\
\text { February } \\
\text { March . } \\
\text { April } \\
\text { May } \\
\text { June } \\
\text { Juhy } \\
\text { August }\end{array}$ & $\begin{array}{l}\text { ค } 1.96 \\
\text { ค } 1.72 \\
\text { ค } 1.89 \\
\text { ค } 2.05 \\
\text { ค } 2.30 \\
\text { ค } 1.87 \\
\text { ค } 1.91 \\
\text { E } 2.07\end{array}$ & $\begin{array}{l}2.02 \\
1.91 \\
1.78 \\
2.15 \\
2.13 \\
1.95 \\
1.78 \\
2.02\end{array}$ & $\begin{array}{l}2.17 \\
1.94 \\
2.20 \\
2.34 \\
2.81 \\
2.03 \\
2.02 \\
2.35\end{array}$ & $\begin{array}{r}3.11 \\
2.94 \\
3.06 \\
3.24 \\
3.58 \\
3.44 \\
3.34 \\
3.35\end{array}$ & $\begin{array}{l}5.71 \\
5.71 \\
5.66 \\
5.99 \\
6.72 \\
7.32 \\
7.83 \\
8.10\end{array}$ & $\begin{array}{l}5.18 \\
5.08 \\
5.06 \\
5.13 \\
5.21 \\
5.31 \\
5.03 \\
5.26\end{array}$ & $\begin{array}{l}3.26 \\
3.12 \\
3.08 \\
3.13 \\
3.24 \\
2.95 \\
2.71 \\
2.86\end{array}$ & $\begin{array}{c}2.70 \\
2.55 \\
2.61 \\
2.75 \\
2.80 \\
2.47 \\
2.46 \\
\text { NA }\end{array}$ \\
\hline $\begin{array}{l}1993 \text { YTD } \\
1992 \text { YTD } \\
1901 \text { YTD }\end{array}$ & $\begin{array}{l}1.97 \\
1.54 \\
1.53\end{array}$ & $\begin{array}{l}2.00 \\
1.90 \\
2.01\end{array}$ & $\begin{array}{l}2.23 \\
1.95 \\
1.90\end{array}$ & $\begin{array}{l}3.19 \\
2.84 \\
2.87\end{array}$ & $\begin{array}{l}6.05 \\
5.80 \\
5.86\end{array}$ & $\begin{array}{l}5.13 \\
4.81 \\
4.85\end{array}$ & $\begin{array}{l}3.06 \\
2.66 \\
2.65\end{array}$ & $\begin{array}{l}2.60 \\
2.14 \\
2.12\end{array}$ \\
\hline
\end{tabular}

- See Appendix A, Explanatory Note 8 for discussion of wellhead price.

- See Appendix A, Explanatory Note $\theta$ for discussion of major interstate pipeline company data.

- Includes all steam electric utility generating plants with a combined capacity of $\mathbf{5 0}$ megawatts or greater.

$m=$ Not Available.

$n$ = Revised Data.

= Estimated Data.

Notes: Data for 1987 through 1992 are final. All other data are preliminary unless otherwise indicated. Geographic coverage is the 50 States and the District of Columbia. Prices for gas delivered to industrial consumers for 1987 include imputed averages for volumes of gas delivered for the account of others. From 1988 on, prices reflect on-8ystem sales prices onty. The change in series in 1988 affects the commercial and industrial sector prices.

Sources: -Average wollhead price: ElA Natural Gas Annual 1992, 1987 through 1992; and EIA estimates, January 1993 through current month. See Append'bx A, Explanatory Note 8 for estimation procedures and revision policy. -Imports and Interstate Pipeline Company Purchases: Form FERC-11. A Average City Gate, Residential, Commercial and Industrial average prices for 1987 through current month from Form ElA-857. See Appendix A, Explanatory Note 5 for discussion of revision policy. Earlier prices from EIA Natural Gas Annual 1992. Electric Utilities average prices from Form FERC-423, "Monthly Report of Cost and Quality of Fuels for Electric Plants." 
Figure 3. Average Price of Natural Gas Dellvered to Consumers in the United States, 1989-1993

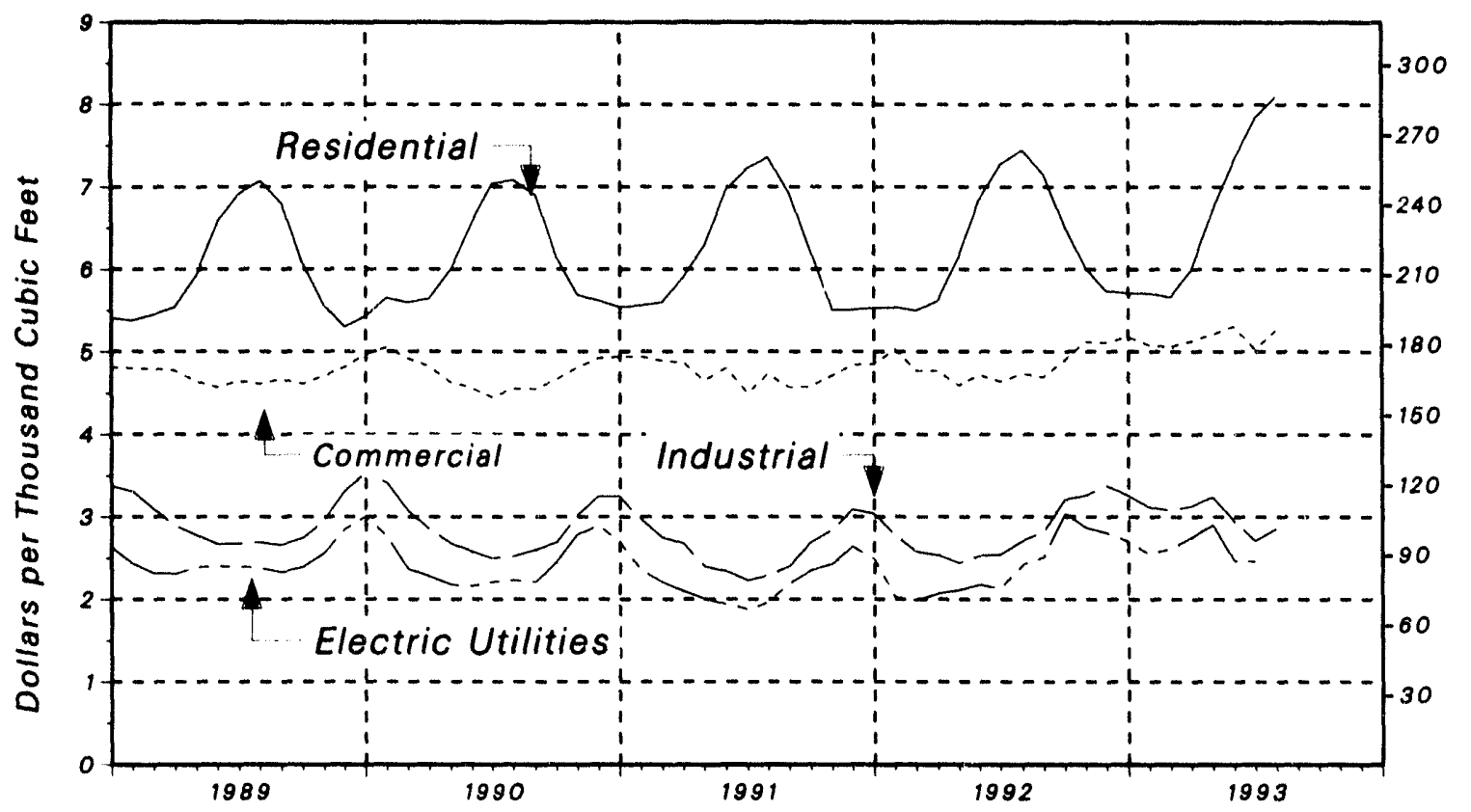

Source: Natural Gas Annual, Form ElA-857, and Form FERC-423.

Figure 4. Average Price of Natural Gas in the United States, 1989-1993

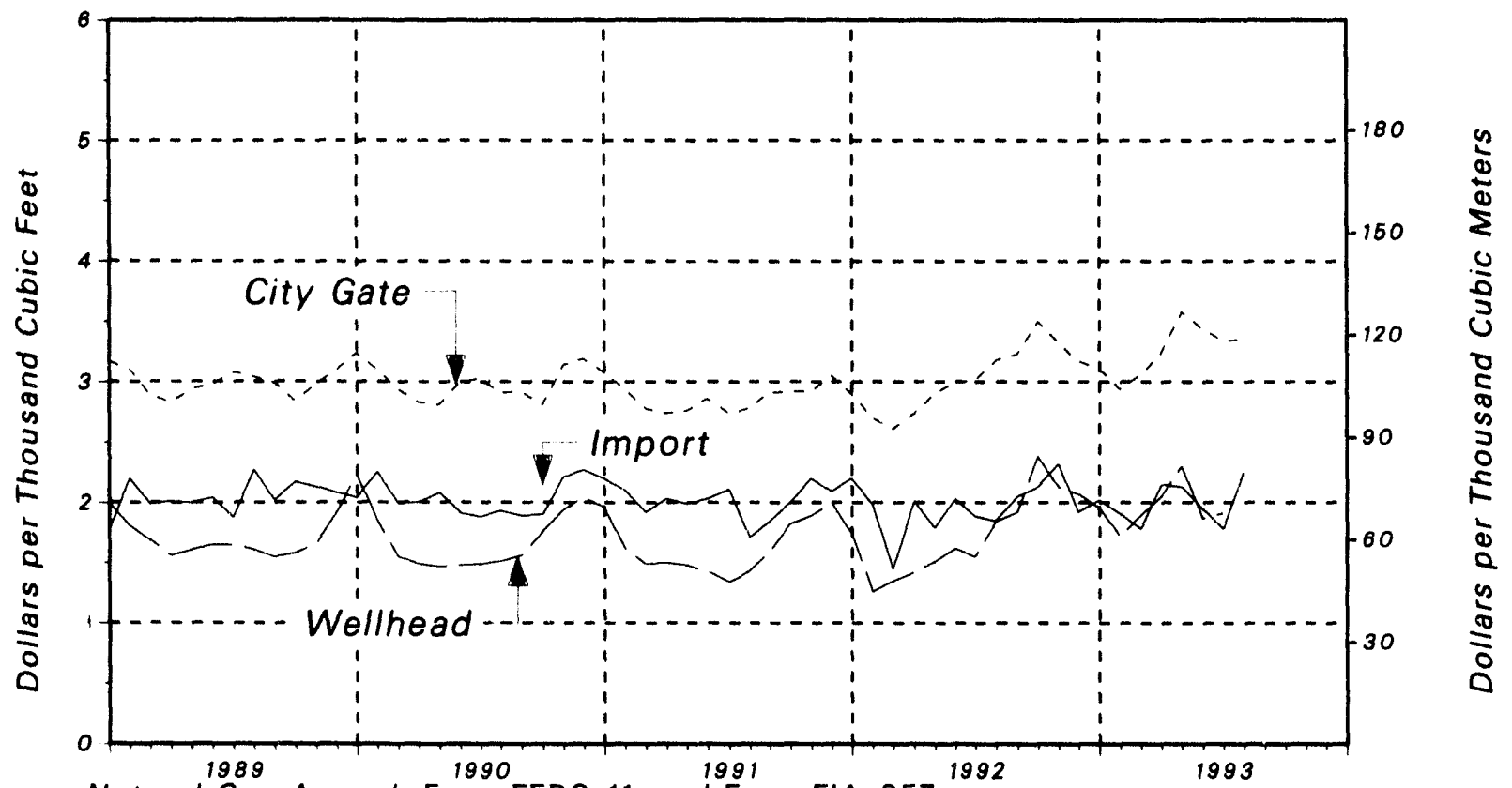

Source: Natural Gas Annual, Form FERC-11, and Form EIA-857. 
Table 5. U.S. Natural Gas Imports by Country, 1987-1993

(Volumes in Million Cubic Feet, Prices in Dollars per Thousand Cubic Fee.)

\begin{tabular}{|c|c|c|c|c|c|c|}
\hline \multirow{3}{*}{$\begin{array}{l}\text { Year } \\
\text { and } \\
\text { Month }\end{array}$} & \multirow{2}{*}{\multicolumn{2}{|c|}{ Pipeline }} & \multirow{2}{*}{\multicolumn{2}{|c|}{$\frac{\text { LNG }}{\text { Algeria }}$}} & \multicolumn{2}{|c|}{ Total } \\
\hline & & & & & \multirow[b]{2}{*}{ Volume } & \multirow{2}{*}{$\begin{array}{c}\text { Average } \\
\text { Price }\end{array}$} \\
\hline & Volume & $\begin{array}{l}\text { Average } \\
\text { Price }\end{array}$ & Volume & $\begin{array}{l}\text { Average } \\
\text { Price }\end{array}$ & & \\
\hline 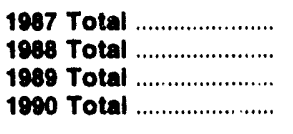 & $\begin{array}{r}992,532 \\
1,276,322 \\
1,339,357 \\
1,448,065\end{array}$ & $\begin{array}{l}1.95 \\
1.83 \\
1.81 \\
1.91\end{array}$ & $\begin{array}{r}0 \\
17,490 \\
42,163 \\
84,193\end{array}$ & $\begin{array}{l}2 . \overline{71} \\
2.22 \\
2.47\end{array}$ & $\begin{array}{r}992,532 \\
1,293,812 \\
1,381,520 \\
1,532,259\end{array}$ & $\begin{array}{l}1.95 \\
1.84 \\
1.82 \\
1.94\end{array}$ \\
\hline 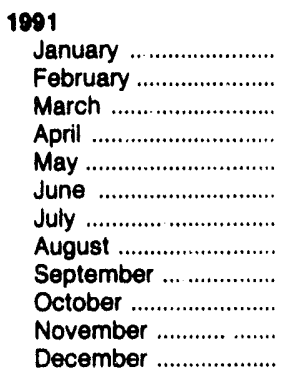 & $\begin{array}{l}155,805 \\
132,962 \\
146,383 \\
138,833 \\
136,202 \\
130,701 \\
129,768 \\
127,139 \\
131,345 \\
146,489 \\
163,605 \\
170,485\end{array}$ & $\begin{array}{l}2.06 \\
1.89 \\
1.83 \\
1.79 \\
1.74 \\
1.72 \\
1.67 \\
1.60 \\
1.67 \\
1.76 \\
1.89 \\
1.94\end{array}$ & $\begin{array}{r}7,643 \\
5,035 \\
5,105 \\
5,122 \\
5,091 \\
2,, 562 \\
5,091 \\
0 \\
2,589 \\
10,146 \\
5,113 \\
10,099\end{array}$ & $\begin{array}{l}2.94 \\
2.49 \\
2.91 \\
1.93 \\
1.66 \\
1.88 \\
1.86 \\
\overline{1.66} \\
2.41 \\
2.48 \\
2.63\end{array}$ & $\begin{array}{l}163,448 \\
137,998 \\
151,487 \\
143,955 \\
141,292 \\
133,264 \\
134,859 \\
127,139 \\
133,934 \\
156,635 \\
168,718 \\
180,585\end{array}$ & $\begin{array}{l}2.11 \\
1.92 \\
1.86 \\
1.79 \\
1.74 \\
1.72 \\
1.68 \\
1.60 \\
1.67 \\
1.80 \\
1.91 \\
1.98\end{array}$ \\
\hline 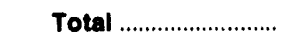 & $1,709,716$ & 1.81 & 63,596 & 2.36 & $1,773,313$ & 1.83 \\
\hline 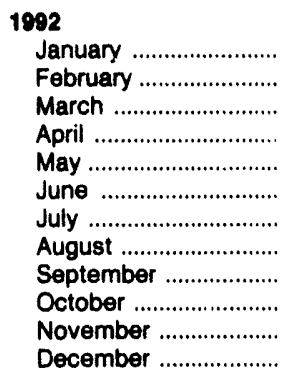 & $\begin{array}{l}157,026 \\
170,167 \\
177,978 \\
173,615 \\
174,312 \\
159,743 \\
167,216 \\
172,240 \\
163,584 \\
173,904 \\
202,846 \\
201,756\end{array}$ & $\begin{array}{l}1.95 \\
1.64 \\
1.63 \\
1.62 \\
1.66 \\
1.77 \\
1.70 \\
1.81 \\
1.96 \\
2.21 \\
1.94 \\
2.13\end{array}$ & $\begin{array}{r}7,610 \\
5,076 \\
2,503 \\
2,531 \\
0 \\
2,505 \\
0 \\
2,492 \\
2,533 \\
2,563 \\
7,633 \\
7,670\end{array}$ & $\begin{array}{r}2.49 \\
2.91 \\
3.32 \\
2.12 \\
2 . \overline{09} \\
-\overline{15} \\
1.83 \\
3.19 \\
2.57 \\
2.17\end{array}$ & $\begin{array}{l}164,636 \\
175,243 \\
180,482 \\
176,146 \\
174,312 \\
162,249 \\
167,216 \\
174,733 \\
166,117 \\
176,466 \\
210,479 \\
209,426\end{array}$ & $\begin{array}{l}1.98 \\
1.68 \\
1.65 \\
1.63 \\
1.66 \\
1.77 \\
1.70 \\
1.83 \\
1.96 \\
2.22 \\
1.96 \\
2.13\end{array}$ \\
\hline 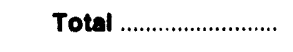 & $2,094,387$ & 1.84 & 43,116 & 2.54 & $2,137,504$ & 1.85 \\
\hline \multicolumn{7}{|l|}{1903} \\
\hline 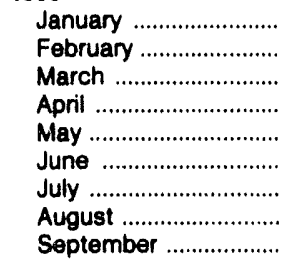 & $\begin{array}{r}192,899 \\
175,444 \\
193,830 \\
177,552 \\
154,640 \\
170,870 \\
\text { P } 182,550 \\
\text { RE } 179,384 \\
\text { E } 177,341\end{array}$ & $\begin{array}{l}2.06 \\
1.95 \\
1.98 \\
2.04 \\
2.08 \\
2.04 \\
\text { NA } \\
\text { NA } \\
\text { NA }\end{array}$ & $\begin{array}{r}5,141 \\
7,654 \\
5,146 \\
7,720 \\
5,236 \\
7,563 \\
7,642 \\
5,091 \\
10,252\end{array}$ & $\begin{array}{l}2.59 \\
2.93 \\
2.24 \\
2.12 \\
2.01 \\
1.94 \\
\text { NA } \\
\text { NA } \\
\text { NA }\end{array}$ & $\begin{array}{r}198,039 \\
183,097 \\
198,976 \\
185,272 \\
158,876 \\
178,433 \\
\text { ค } 190,192 \\
\text { คE } 184,475 \\
\varepsilon 187,593\end{array}$ & $\begin{array}{l}2.07 \\
1.99 \\
1.98 \\
2.04 \\
2.08 \\
2.03 \\
\text { NA } \\
\text { NA } \\
\text { NA }\end{array}$ \\
\hline 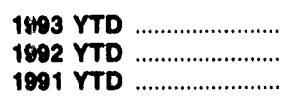 & $\begin{array}{l}1,604,510 \\
1,515,882 \\
1,229,137\end{array}$ & $\begin{array}{l}\text { NA } \\
1.75 \\
1.78\end{array}$ & $\begin{array}{l}61,444 \\
25,251 \\
38,238\end{array}$ & $\begin{array}{l}\text { NA } \\
2.58 \\
2.25\end{array}$ & $\begin{array}{l}1,665,954 \\
1,541,133 \\
1,267,376\end{array}$ & $\begin{array}{l}\text { NA } \\
1.76 \\
1.79\end{array}$ \\
\hline
\end{tabular}

$m=$ Not Available.

- - Not Applicable

R = Revised Data.

E = Estimated Data

HE = Revised Estimated data.

Source: 1987-1992: Energy Information Administration, Form FPC-14, "Annual Report for Importers and Exporters of Natural Gas." January 1983 through the current month: Office of Fossil Energy, U.S. Department of Energy. Natural Gas Imports and Exports. Monthly data (for the most current months), Pipeline Fuel: data shown with an E are taken from data from the National Energy Board of Canada plus EIA estimates. LNG: industry reports. 
Table 6. U.S. Natural Gas Exports by Country, 1987-1993

(Volumes in Million Cubic Feet, Prices in Dollars per Thousand Cubic Feet)

\begin{tabular}{|c|c|c|c|c|c|c|c|c|}
\hline \multirow{3}{*}{$\begin{array}{l}\text { Year } \\
\text { and } \\
\text { Month }\end{array}$} & \multicolumn{4}{|c|}{ Pipeline } & \multirow{2}{*}{\multicolumn{2}{|c|}{$\frac{\text { LNG }}{\text { Japan }}$}} & \multicolumn{2}{|c|}{ Total } \\
\hline & \multicolumn{2}{|c|}{ Canada } & \multicolumn{2}{|c|}{ Mexico } & & & \multirow[b]{2}{*}{ Volume } & \multirow{2}{*}{$\begin{array}{c}\text { Average } \\
\text { Price }\end{array}$} \\
\hline & Volume & $\begin{array}{c}\text { Average } \\
\text { Price }\end{array}$ & Volume & $\begin{array}{c}\text { Average } \\
\text { Price }\end{array}$ & Volume & $\begin{array}{c}\text { Average } \\
\text { Price }\end{array}$ & & \\
\hline 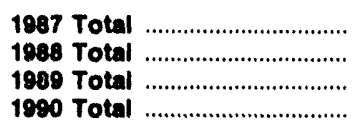 & $\begin{array}{r}3,297 \\
19,738 \\
38,443 \\
17,359\end{array}$ & $\begin{array}{l}1.81 \\
2.02 \\
2.00 \\
2.70\end{array}$ & $\begin{array}{r}2,125 \\
2,327 \\
17,004 \\
15,659\end{array}$ & $\begin{array}{l}3.18 \\
3.21 \\
2.14 \\
1.88\end{array}$ & $\begin{array}{l}48,599 \\
51,573 \\
51,424 \\
52,546\end{array}$ & $\begin{array}{l}3.15 \\
2.99 \\
3.01 \\
3.59\end{array}$ & $\begin{array}{r}54,020 \\
73,638 \\
106,871 \\
85,565\end{array}$ & $\begin{array}{l}3.07 \\
2.74 \\
2.51 \\
3.10\end{array}$ \\
\hline \multicolumn{9}{|l|}{1891} \\
\hline 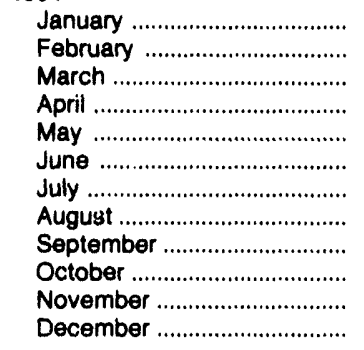 & $\begin{array}{r}1,710 \\
3,343 \\
1,228 \\
2 \\
36 \\
31 \\
195 \\
540 \\
446 \\
2,245 \\
2,385 \\
2,630\end{array}$ & $\begin{array}{l}2.47 \\
2.02 \\
2.04 \\
1.71 \\
1.73 \\
1.51 \\
1.24 \\
1.21 \\
1.30 \\
1.57 \\
1.87 \\
1.97\end{array}$ & $\begin{array}{l}3,477 \\
2,853 \\
4,404 \\
3,208 \\
4,762 \\
3,759 \\
3,235 \\
3,117 \\
5,921 \\
7,776 \\
8,349 \\
9,586\end{array}$ & $\begin{array}{l}2.19 \\
1.70 \\
1.61 \\
1.56 \\
1.46 \\
1.43 \\
1.28 \\
1.40 \\
1.68 \\
1.84 \\
2.00 \\
2.12\end{array}$ & $\begin{array}{l}4,375 \\
4,370 \\
4,369 \\
5,829 \\
2,947 \\
2,915 \\
4,400 \\
5,869 \\
4,370 \\
4,371 \\
4,371 \\
5,820\end{array}$ & $\begin{array}{l}5.19 \\
4.79 \\
4.13 \\
3.59 \\
3.23 \\
3.18 \\
3.20 \\
3.25 \\
3.31 \\
3.39 \\
3.50 \\
3.61\end{array}$ & $\begin{array}{r}9,562 \\
10,566 \\
10,001 \\
9,039 \\
7,744 \\
6,706 \\
7,830 \\
9,526 \\
10,737 \\
14,393 \\
15,105 \\
18,036\end{array}$ & $\begin{array}{l}3.61 \\
3.08 \\
2.76 \\
2.87 \\
2.14 \\
2.19 \\
2.36 \\
2.53 \\
2.32 \\
2.27 \\
2.41 \\
2.58\end{array}$ \\
\hline 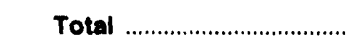 & 14,791 & 1.91 & 60,448 & 1.76 & 54,005 & 3.71 & 129,244 & 2.59 \\
\hline \multicolumn{9}{|l|}{1992} \\
\hline 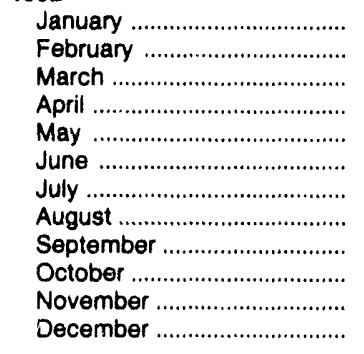 & $\begin{array}{r}2,377 \\
3,654 \\
11,437 \\
5,980 \\
5,919 \\
6,172 \\
5,230 \\
5,074 \\
5,547 \\
6,290 \\
3,487 \\
6,611\end{array}$ & $\begin{array}{l}1.87 \\
1.43 \\
1.95 \\
1.35 \\
1.56 \\
1.81 \\
1.67 \\
1.84 \\
1.91 \\
2.19 \\
2.04 \\
2.18\end{array}$ & $\begin{array}{r}9,687 \\
5,963 \\
6,707 \\
7,371 \\
7,285 \\
7,262 \\
6,258 \\
8,778 \\
8,181 \\
10,063 \\
10,674 \\
7,743\end{array}$ & $\begin{array}{l}1.83 \\
1.24 \\
1.37 \\
1.52 \\
1.69 \\
1.78 \\
1.68 \\
1.97 \\
2.00 \\
2.66 \\
2.26 \\
2.18\end{array}$ & $\begin{array}{l}4,368 \\
4,369 \\
4,367 \\
4,403 \\
5,827 \\
4,378 \\
4,379 \\
4,377 \\
4,408 \\
2,920 \\
4,366 \\
4,370\end{array}$ & $\begin{array}{l}3.58 \\
3.44 \\
3.27 \\
3.21 \\
3.23 \\
3.31 \\
3.44 \\
3.56 \\
3.61 \\
3.59 \\
3.56 \\
3.52\end{array}$ & $\begin{array}{l}16,432 \\
13,986 \\
22,511 \\
17,754 \\
19,030 \\
17,812 \\
15,868 \\
18,229 \\
18,136 \\
19,272 \\
18,527 \\
18,724\end{array}$ & $\begin{array}{l}2.30 \\
1.98 \\
2.03 \\
1.88 \\
2.12 \\
2.17 \\
2.16 \\
2.31 \\
2.36 \\
2.65 \\
2.52 \\
2.49\end{array}$ \\
\hline Total & 67.777 & 1.83 & 95,973 & 1.90 & 52,532 & 3.43 & 216,282 & 2.25 \\
\hline \multicolumn{9}{|l|}{1893} \\
\hline 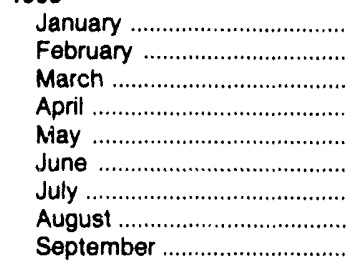 & $\begin{array}{r}5,577 \\
6,453 \\
7,583 \\
4,527 \\
3,941 \\
3,647 \\
\text { E } 5,000 \\
\text { E } 4,000 \\
\text { E } 3,500\end{array}$ & $\begin{array}{l}2.20 \\
2.06 \\
2.09 \\
1.99 \\
1.88 \\
1.88 \\
\text { NA } \\
\text { NA } \\
\text { NA }\end{array}$ & $\begin{array}{r}7,833 \\
2,121 \\
3,199 \\
2,875 \\
3,453 \\
4,050 \\
\text { ค } 3,805 \\
\text { ค } 2,582 \\
2,227\end{array}$ & $\begin{array}{l}2.03 \\
1.77 \\
1.96 \\
2.13 \\
2.44 \\
1.84 \\
\text { NA } \\
\text { NA } \\
\text { NA }\end{array}$ & $\begin{array}{l}4,367 \\
4,355 \\
5,812 \\
4,373 \\
4,381 \\
2,950 \\
5,162 \\
4,761 \\
5,156\end{array}$ & $\begin{array}{l}3.41 \\
3.29 \\
3.22 \\
3.21 \\
3.29 \\
3.22 \\
\text { NA } \\
\text { NA } \\
\text { NA }\end{array}$ & $\begin{array}{r}17,777 \\
12,929 \\
16,594 \\
11,775 \\
11,775 \\
10,647 \\
\text { A } 13,967 \\
\text { A } 11,343 \\
\text { E } 10,863\end{array}$ & $\begin{array}{l}2.42 \\
2.42 \\
2.43 \\
2.48 \\
2.57 \\
2.24 \\
\text { NA } \\
\text { NA } \\
\text { NA }\end{array}$ \\
\hline 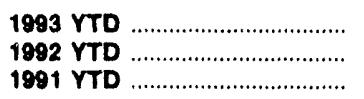 & $\begin{array}{r}44,228 \\
51,390 \\
7,531\end{array}$ & $\begin{array}{l}\text { NA } \\
1.73 \\
2.00\end{array}$ & $\begin{array}{l}32,145 \\
67,492 \\
34,737\end{array}$ & $\begin{array}{l}\text { NA } \\
1.70 \\
1.59\end{array}$ & $\begin{array}{l}41,298 \\
40,876 \\
39,442\end{array}$ & $\begin{array}{l}\text { NA } \\
3.40 \\
3.77\end{array}$ & $\begin{array}{r}117,671 \\
159,758 \\
81,711\end{array}$ & $\begin{array}{l}\text { NA } \\
2.15 \\
2.68\end{array}$ \\
\hline
\end{tabular}

MA $=$ Not Available.

R = Revised Data.

$\mathbf{E}=$ Estimated Data.

RE $=$ Revised Estimated Data.

Source: 1987-1982: Energy Information Administration, Form FPC-14, "Annual Report for Importers and Exporters of Natural Gas." January 1993 through the current month: Office of Fossil Energy, U.S. Department of Energy, Natural Gas Imports and Exports. Monthly data (for the most current months), Pipeline Fuel: data shown with an E are EIA estimates. LNG: industry reports. 
Table 7. Marketed Production of Natural Gas by State, 1987-1993 (Million Cubic Feet)

\begin{tabular}{|c|c|c|c|c|c|c|}
\hline Year and Month & Alabamab & Alaska & California & Colorado & Florida & Kansas \\
\hline 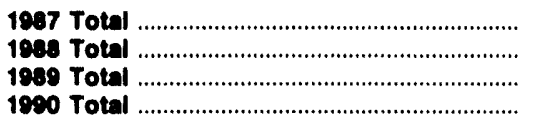 & $\begin{array}{l}117,241 \\
129,524 \\
128,411 \\
135,276\end{array}$ & $\begin{array}{l}359,837 \\
378,638 \\
393,729 \\
402,907\end{array}$ & $\begin{array}{l}424,621 \\
399,663 \\
362,860 \\
362,748\end{array}$ & $\begin{array}{l}164,557 \\
191,544 \\
216,737 \\
242,997\end{array}$ & $\begin{array}{l}8,281 \\
7,484 \\
7,534 \\
6,483\end{array}$ & $\begin{array}{l}472,752 \\
592,845 \\
601,196 \\
573,603\end{array}$ \\
\hline $\begin{array}{l}1991 \\
\text { January } \\
\text { February } \\
\text { March } \\
\text { April } \\
\text { May } \\
\text { June } \\
\text { July } \\
\text { August } \\
\text { September } \\
\text { October } \\
\text { November } \\
\text { December }\end{array}$ & $\begin{array}{l}13,267 \\
11,866 \\
13,131 \\
13,020 \\
13,906 \\
13,174 \\
14,400 \\
15,273 \\
11,850 \\
15,207 \\
15,502 \\
20,250\end{array}$ & $\begin{array}{l}40,203 \\
36,265 \\
38,847 \\
34,664 \\
32,434 \\
33,065 \\
33,840 \\
33,448 \\
41,609 \\
36,384 \\
38,363 \\
38,700\end{array}$ & $\begin{array}{l}30,159 \\
27,015 \\
32,268 \\
30,902 \\
31,072 \\
29,259 \\
32,169 \\
31,021 \\
31,944 \\
35,427 \\
34,194 \\
32,953\end{array}$ & $\begin{array}{l}20,110 \\
23,344 \\
23,648 \\
24,673 \\
22,724 \\
23,034 \\
23,088 \\
23,659 \\
22,546 \\
23,868 \\
24,349 \\
30,918\end{array}$ & $\begin{array}{r}290 \\
285 \\
325 \\
412 \\
301 \\
94 \\
381 \\
465 \\
543 \\
602 \\
591 \\
594\end{array}$ & $\begin{array}{l}63,891 \\
54,601 \\
57,863 \\
51,936 \\
50,732 \\
46,685 \\
44,913 \\
42,777 \\
43,491 \\
50,386 \\
57,959 \\
63,225\end{array}$ \\
\hline Total & 170,847 & 437,822 & 378,384 & 285,961 & 4,884 & 628,459 \\
\hline $\begin{array}{l}\text { Jeo2 } \\
\text { January } \\
\text { February } \\
\text { March } \\
\text { April } \\
\text { May } \\
\text { June } \\
\text { July } \\
\text { August } \\
\text { September } \\
\text { October } \\
\text { November } \\
\text { December }\end{array}$ & $\begin{array}{l}28,638 \\
28,093 \\
28,689 \\
28,690 \\
28,920 \\
29,312 \\
30,718 \\
29,338 \\
29,402 \\
31,435 \\
30,470 \\
31,386\end{array}$ & $\begin{array}{l}40,789 \\
37,311 \\
38,433 \\
37,128 \\
37,014 \\
34,222 \\
35,020 \\
32,832 \\
32,233 \\
39,237 \\
38,453 \\
40,924\end{array}$ & $\begin{array}{l}32,882 \\
30,127 \\
32,006 \\
31,789 \\
32,353 \\
29,550 \\
30,949 \\
30,046 \\
29,211 \\
30,386 \\
28,070 \\
28,262\end{array}$ & $\begin{array}{l}27,414 \\
25,451 \\
24,545 \\
25,138 \\
26,318 \\
26,270 \\
25,501 \\
27,256 \\
26,061 \\
27,474 \\
28,461 \\
33,152\end{array}$ & $\begin{array}{l}570 \\
536 \\
492 \\
488 \\
520 \\
572 \\
458 \\
574 \\
564 \\
654 \\
593 \\
635\end{array}$ & $\begin{array}{l}64,920 \\
56,375 \\
53,499 \\
47,445 \\
50,271 \\
48,589 \\
49,051 \\
48,567 \\
47,818 \\
56,304 \\
64,316 \\
70,853\end{array}$ \\
\hline Total & 355,099 & 443,597 & 365,632 & 323,041 & 6,657 & 658,007 \\
\hline $\begin{array}{l}1983 \\
\text { January } \\
\text { February } \\
\text { March } \\
\text { April } \\
\text { May } \\
\text { June }\end{array}$ & $\begin{array}{r}24,591 \\
22,157 \\
22,784 \\
22,235 \\
23,312 \\
22,847 \\
\text { E } 24,264\end{array}$ & $\begin{array}{r}41,375 \\
35,689 \\
41,674 \\
39,226 \\
35,702 \\
\text { E } 35,118 \\
\text { E } 36,023\end{array}$ & $\begin{array}{r}26,956 \\
24,024 \\
\text { E } 27,544 \\
25,320 \\
25,294 \\
27,398 \\
29,638\end{array}$ & $\begin{array}{l}\text { E } 28,356 \\
\text { E } 30,043 \\
\text { E } 29,757 \\
\text { E } 29,907 \\
\text { E } 29,932 \\
\text { E } 29,421 \\
\text { E } 29,521\end{array}$ & $\begin{array}{l}637 \\
573 \\
603 \\
601 \\
685 \\
577 \\
648\end{array}$ & $\begin{array}{r}64,279 \\
57,258 \\
61,084 \\
53,431 \\
54,054 \\
50,200 \\
\text { E } 50,972\end{array}$ \\
\hline 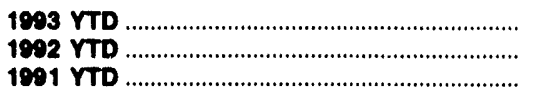 & $\begin{array}{r}162,190 \\
203,070 \\
92,765\end{array}$ & $\begin{array}{l}264,807 \\
259,917 \\
249,318\end{array}$ & $\begin{array}{l}186,174 \\
219,657 \\
212,845\end{array}$ & $\begin{array}{l}206,937 \\
180,637 \\
160,621\end{array}$ & $\begin{array}{l}4,324 \\
3,637 \\
2,090\end{array}$ & $\begin{array}{l}391,278 \\
370,149 \\
370,621\end{array}$ \\
\hline
\end{tabular}

See footnotes at end of table. 
Table 7. Marketed Production of Natural Gas by State, 1987-1993 (Continued) (Million Cubic Feet)

\begin{tabular}{|c|c|c|c|c|c|c|}
\hline Year and Month & Louisiana & Michigan & Mississippi & Montana & $\begin{array}{l}\text { New } \\
\text { Mexico }\end{array}$ & $\begin{array}{l}\text { North } \\
\text { Dakota }\end{array}$ \\
\hline $\begin{array}{l}1987 \text { Total } \\
1988 \text { Total } \\
1989 \text { Total } \\
1980 \text { Total }\end{array}$ & $\begin{array}{l}5,122,509 \\
5,180,267 \\
5,078,125 \\
5,241,989\end{array}$ & $\begin{array}{l}146,996 \\
146,145 \\
155,988 \\
172,151\end{array}$ & $\begin{array}{r}139,727 \\
124,053 \\
102,645 \\
94,616\end{array}$ & $\begin{array}{l}46,456 \\
51,654 \\
51,307 \\
50,429\end{array}$ & $\begin{array}{l}823,773 \\
791,819 \\
854,615 \\
965,104\end{array}$ & $\begin{array}{l}62,258 \\
57,747 \\
51,174 \\
52,169\end{array}$ \\
\hline \multicolumn{7}{|l|}{1901} \\
\hline $\begin{array}{l}\text { January } \\
\text { February } \\
\text { March } \\
\text { April } \\
\text { May } \\
\text { June } \\
\text { July } \\
\text { August } \\
\text { September } \\
\text { October } \\
\text { November } \\
\text { December }\end{array}$ & $\begin{array}{l}468,583 \\
408,977 \\
440,394 \\
421,649 \\
415,253 \\
388,442 \\
387,063 \\
392,982 \\
394,190 \\
430,728 \\
430,550 \\
455,550\end{array}$ & $\begin{array}{l}15,493 \\
13,850 \\
17,320 \\
18,019 \\
18,521 \\
15,994 \\
16,468 \\
15,697 \\
16,040 \\
15,055 \\
15,344 \\
16,948\end{array}$ & $\begin{array}{l}9,113 \\
7,601 \\
9,021 \\
9,346 \\
9,745 \\
9,499 \\
9,467 \\
8,866 \\
8,183 \\
8,615 \\
9,217 \\
9,359\end{array}$ & $\begin{array}{l}5,235 \\
4,433 \\
4,779 \\
4,786 \\
4,516 \\
3,467 \\
3,501 \\
4,081 \\
3,940 \\
4,393 \\
4,166 \\
4,702\end{array}$ & $\begin{array}{l}96,319 \\
79,943 \\
89,186 \\
86,221 \\
86,029 \\
81,862 \\
77,279 \\
83,204 \\
83,766 \\
91,254 \\
90,101 \\
93,120\end{array}$ & $\begin{array}{l}4,640 \\
4,162 \\
4,588 \\
4,351 \\
4,419 \\
4,481 \\
4,787 \\
4,625 \\
4,054 \\
4,253 \\
4,482 \\
4,638\end{array}$ \\
\hline Total & $5,034,361$ & 195,749 & 108,031 & 51,999 & $1,038,284$ & 53,479 \\
\hline \multicolumn{7}{|l|}{1002} \\
\hline $\begin{array}{l}\text { January } \\
\text { February } \\
\text { March } \\
\text { April } \\
\text { May } \\
\text { June } \\
\text { July } \\
\text { August } \\
\text { September } \\
\text { October } \\
\text { November } \\
\text { December }\end{array}$ & $\begin{array}{l}447,681 \\
397,242 \\
415,797 \\
406,614 \\
425,880 \\
411,275 \\
422,105 \\
377,693 \\
375,997 \\
409,724 \\
401,240 \\
423,050\end{array}$ & $\begin{array}{l}14,061 \\
12,580 \\
16,011 \\
15,126 \\
13,012 \\
20,668 \\
16,208 \\
17,200 \\
19,210 \\
17,614 \\
14,277 \\
18,848\end{array}$ & $\begin{array}{l}8,666 \\
7,632 \\
7,761 \\
7,549 \\
7,602 \\
7,447 \\
7,533 \\
7,553 \\
7,039 \\
7,618 \\
7,694 \\
7,601\end{array}$ & $\begin{array}{l}5,025 \\
4,831 \\
5,256 \\
4,583 \\
4,429 \\
3,645 \\
3,846 \\
3,746 \\
3,782 \\
4,503 \\
5,083 \\
5,138\end{array}$ & $\begin{array}{r}96,194 \\
86,756 \\
94,373 \\
102,157 \\
108,294 \\
101,770 \\
111,611 \\
114,145 \\
109,064 \\
111,646 \\
114,329 \\
118,523\end{array}$ & $\begin{array}{l}4,821 \\
4,469 \\
4,762 \\
4,389 \\
4,486 \\
4,436 \\
4,593 \\
4,609 \\
4,428 \\
4,772 \\
4,490 \\
4,628\end{array}$ \\
\hline Total & $4,914,300$ & 194,815 & 91,697 & 53,867 & $1,268,863$ & 54,883 \\
\hline \multicolumn{7}{|l|}{1093} \\
\hline 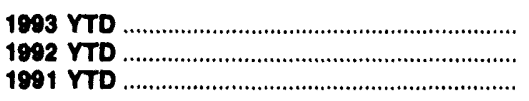 & $\begin{array}{l}2,976,262 \\
2,926,596 \\
2,930,361\end{array}$ & $\begin{array}{l}124,466 \\
107,666 \\
116,665\end{array}$ & $\begin{array}{l}49,662 \\
54,191 \\
63,792\end{array}$ & $\begin{array}{l}31,553 \\
31,615 \\
30,717\end{array}$ & $\begin{array}{l}803,702 \\
701,156 \\
596,840\end{array}$ & $\begin{array}{l}31,971 \\
31,956 \\
31,427\end{array}$ \\
\hline
\end{tabular}

See footnotes at end of table. 
Table 7. Marketed Production of Natural Gas by State, 1987-1993 (Continued) (Million Cubic Feet)

\begin{tabular}{|c|c|c|c|c|c|c|}
\hline Year and Month & Oklahoma & Texas & Utah & Wyoming & $\begin{array}{l}\text { Other* } \\
\text { States }\end{array}$ & $\begin{array}{l}\text { U.S. } \\
\text { Total }\end{array}$ \\
\hline $\begin{array}{l}1987 \text { Total } \\
1988 \text { Total } \\
1989 \text { Total } \\
1990 \text { Total }\end{array}$ & $\begin{array}{l}2,073,461 \\
2,167,050 \\
2,237,037 \\
2,258,471\end{array}$ & $\begin{array}{l}6,126,315 \\
6,286,029 \\
6,241,425 \\
6,343,146\end{array}$ & $\begin{array}{r}87,158 \\
101,372 \\
120,089 \\
145,875\end{array}$ & $\begin{array}{l}497,980 \\
509,058 \\
665,699 \\
735,728\end{array}$ & $\begin{array}{l}758,979 \\
803,573 \\
826,576 \\
810,100\end{array}$ & $\begin{array}{l}17,432,901 \\
17,918,465 \\
18,095,147 \\
18,593,792\end{array}$ \\
\hline $\begin{array}{l}1991 \\
\text { January } \\
\text { February } \\
\text { March } \\
\text { April } \\
\text { May } \\
\text { June } \\
\text { July } \\
\text { August } \\
\text { September } \\
\text { October } \\
\text { November } \\
\text { December }\end{array}$ & $\begin{array}{l}194,179 \\
176,577 \\
189,362 \\
182,592 \\
181,866 \\
168,292 \\
176,757 \\
171,545 \\
167,685 \\
180,504 \\
179,976 \\
184,516\end{array}$ & $\begin{array}{l}567,569 \\
496,899 \\
533,539 \\
506,976 \\
512,679 \\
500,166 \\
505,136 \\
503,988 \\
500,654 \\
539,982 \\
544,444 \\
568,621\end{array}$ & $\begin{array}{l}13,995 \\
11,573 \\
12,789 \\
11,468 \\
11,206 \\
10,567 \\
11,021 \\
12,009 \\
11,131 \\
13,869 \\
14,091 \\
11,098\end{array}$ & $\begin{array}{l}68,011 \\
60,587 \\
70,023 \\
66,126 \\
62,086 \\
61,837 \\
62,410 \\
65,429 \\
62,169 \\
63,623 \\
68,055 \\
66,172\end{array}$ & $\begin{array}{l}74,819 \\
65,447 \\
69,790 \\
64,125 \\
63,317 \\
60,626 \\
62,280 \\
61,788 \\
60,181 \\
66,247 \\
68,370 \\
71,338\end{array}$ & $\begin{array}{l}1,685,877 \\
1,483,427 \\
1,606,874 \\
1,531,264 \\
1,521,809 \\
1,450,544 \\
1,464,959 \\
1,470,857 \\
1,463,974 \\
1,580,397 \\
1,599,756 \\
1,672,701\end{array}$ \\
\hline Total & $2,153,852$ & $6,280,654$ & 144,817 & 776,528 & 788,328 & $18,532,439$ \\
\hline $\begin{array}{l}1992 \\
\text { January } \\
\text { February } \\
\text { March } \\
\text { April } \\
\text { May } \\
\text { June } \\
\text { July } \\
\text { August } \\
\text { September } \\
\text { October } \\
\text { November } \\
\text { December }\end{array}$ & $\begin{array}{l}184,091 \\
166,004 \\
165,802 \\
168,030 \\
165,080 \\
166,336 \\
160,800 \\
159,160 \\
160,231 \\
170,825 \\
172,795 \\
178,201\end{array}$ & $\begin{array}{l}551,786 \\
483,162 \\
506,941 \\
493,746 \\
510,273 \\
495,691 \\
508,414 \\
515,119 \\
509,836 \\
531,794 \\
513,103 \\
525,996\end{array}$ & $\begin{array}{l}11,797 \\
11,312 \\
12,727 \\
11,852 \\
13,378 \\
13,310 \\
13,930 \\
14,425 \\
15,250 \\
17,177 \\
16,718 \\
19,417\end{array}$ & $\begin{array}{l}74,325 \\
50,091 \\
71,930 \\
67,036 \\
62,977 \\
58,427 \\
77,202 \\
74,171 \\
73,315 \\
77,161 \\
78,509 \\
77,434\end{array}$ & $\begin{array}{l}69,450 \\
64,761 \\
68,252 \\
66,346 \\
66,344 \\
63,463 \\
65,761 \\
65,848 \\
64,218 \\
69,246 \\
68,952 \\
71,622\end{array}$ & $\begin{array}{l}1,663,111 \\
1,466,733 \\
1,547,288 \\
1,518,106 \\
1,557,151 \\
1,514,984 \\
1,563,702 \\
1,522,282 \\
1,507,660 \\
1,607,569 \\
1,587,552 \\
1,655,671\end{array}$ \\
\hline 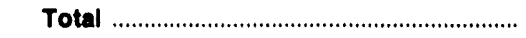 & $2,017,356$ & $6,145,862$ & 171,293 & 842,576 & 804,264 & $18,711,808$ \\
\hline \multicolumn{7}{|l|}{1993} \\
\hline $\begin{array}{l}\text { January } \\
\text { February } \\
\text { March } \\
\text { April } \\
\text { May } \\
\text { June } \\
\text { July }\end{array}$ & $\begin{array}{l}\text { E } 181,647 \\
\text { E } 162,327 \\
\text { E } 167,673 \\
\text { E } 165,335 \\
\text { E } 165,190 \\
\text { E } 165,784 \\
\text { E } 164,697\end{array}$ & $\begin{array}{l}553,029 \\
490,478 \\
554,949 \\
538,617 \\
546,915 \\
521,387 \\
528,063\end{array}$ & $\begin{array}{l}19,774 \\
19,304 \\
22,763 \\
18,986 \\
17,584 \\
18,487 \\
18,152\end{array}$ & $\begin{array}{r}69,028 \\
52,424 \\
57,523 \\
67,436 \\
55,759 \\
\text { R } 59,105 \\
55,843\end{array}$ & $\begin{array}{l}\text { E } 72,658 \\
\text { E } 65,382 \\
\text { E } 68,714 \\
65,504 \\
\text { E } 64,466 \\
63,598 \\
\text { E } 63,481\end{array}$ & $\begin{array}{r}1,684,354 \\
1,493,945 \\
1,639,591 \\
1,592,160 \\
1,604,852 \\
\text { R } 1,555,470 \\
1,585,016\end{array}$ \\
\hline 1983 YTD & $\begin{array}{l}1,172,653 \\
1,176,143 \\
1,269,625\end{array}$ & $\begin{array}{l}3,733,438 \\
3,550,014 \\
3,622,964\end{array}$ & $\begin{array}{r}135,050 \\
88,306 \\
82,619\end{array}$ & $\begin{array}{l}417,118 \\
461,987 \\
451,080\end{array}$ & $\begin{array}{l}463,803 \\
464,377 \\
460,404\end{array}$ & $\begin{array}{l}11,155,388 \\
10,831,075 \\
10,744,754\end{array}$ \\
\hline
\end{tabular}

- Includes Arizona, Arkansas, Illinois, Indiana, Kentucky, Maryland, Missouri, Nebraska, New York, Ohio, Oregon, Pennsylvania, South Dakota, Tennessee, Virginia and West Virginia. The 1992 and 1993 monthly values for these States are estimated.

b The 1992 monthly and annual values for Alabama include Federal Offshore production.

E = Estimated Data.

n Revised Data.

Notes: Data for 1987 through 1992 are final. All other data are preliminary unless otherwise indicated. Totals may not equal sum of components because of independent rounding. See Appendix A, Explanatory Notes 1 and 3 for discussion of computation procedures and revision policy.

Sources: - EIA Natural Gas Annual 1992, 1987 through 1992. •IOGCC, MMS reports, and EIA computations, January 1993 through current month. 
Table 8. Revenues, Expenses, and Income of Major Interstate Natural Gas Pipeline Companies, 1987-1993

(Million Dollars)

\begin{tabular}{|c|c|c|c|c|c|c|c|c|c|}
\hline \multirow{2}{*}{$\begin{array}{l}\text { Year } \\
\text { and } \\
\text { Month }\end{array}$} & \multirow{2}{*}{$\begin{array}{c}\text { Total Sales } \\
\text { Volume } \\
(B c f)\end{array}$} & \multirow{2}{*}{$\begin{array}{c}\text { Gas } \\
\text { Operating } \\
\text { Revenues }\end{array}$} & \multicolumn{4}{|c|}{ Gas Operating Expenses } & \multirow{2}{*}{$\begin{array}{l}\text { Total } \\
\text { Operating } \\
\text { Income }\end{array}$} & \multirow{2}{*}{$\begin{array}{l}\text { Total Income } \\
\text { Before Interest } \\
\text { Charges and } \\
\text { Extraordinary } \\
\text { Expenses }\end{array}$} & \multirow{2}{*}{$\begin{array}{c}\text { Net } \\
\text { Incomec }\end{array}$} \\
\hline & & & $\begin{array}{c}\text { Operation } \\
\text { and } \\
\text { Maintenance }\end{array}$ & $\begin{array}{l}\text { Depreciation, } \\
\text { Depletion, and } \\
\text { Amortization }\end{array}$ & $\operatorname{Taxes}^{b}$ & $\begin{array}{l}\text { Total Gas } \\
\text { Operating } \\
\text { Expenses }\end{array}$ & & & \\
\hline 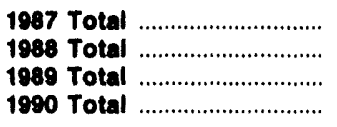 & $\begin{array}{l}6,758 \\
6,414 \\
5,652 \\
4,877\end{array}$ & $\begin{array}{l}27,469 \\
26,637 \\
26,703 \\
24,275\end{array}$ & $\begin{array}{l}21,871 \\
22,640 \\
21,522 \\
18,980\end{array}$ & $\begin{array}{l}1,520 \\
1,579 \\
1,451 \\
1,483\end{array}$ & $\begin{array}{l}552 \\
582 \\
596 \\
648\end{array}$ & $\begin{array}{l}25,151 \\
24,940 \\
24,168 \\
21,686\end{array}$ & $\begin{array}{l}2,333 \\
1,803 \\
2,575 \\
2,590\end{array}$ & $\begin{array}{l}3,493 \\
3,350 \\
4,595 \\
4,227\end{array}$ & $\begin{array}{l}1,414 \\
1,366 \\
2,311 \\
2,319\end{array}$ \\
\hline \multicolumn{10}{|l|}{1991} \\
\hline $\begin{array}{l}\text { January } \\
\text { February } \\
\text { March } \\
\text { April } \\
\text { May } \\
\text { June } \\
\text { July } \\
\text { August } \\
\text { September } \\
\text { October } \\
\text { November } \\
\text { December }\end{array}$ & $\begin{array}{l}686 \\
415 \\
384 \\
289 \\
227 \\
184 \\
212 \\
251 \\
229 \\
304 \\
439 \\
433\end{array}$ & $\begin{array}{l}3,126 \\
2,095 \\
1,960 \\
1,548 \\
1,386 \\
1,263 \\
1,295 \\
1,396 \\
1,387 \\
1,613 \\
2,127 \\
2,452\end{array}$ & $\begin{array}{r}2,395 \\
1,541 \\
1,445 \\
1,224 \\
1,045 \\
1,870 \\
989 \\
1,068 \\
1,268 \\
1,232 \\
1,615 \\
2,304\end{array}$ & $\begin{array}{r}116 \\
116 \\
117 \\
118 \\
118 \\
118 \\
117 \\
117 \\
118 \\
37 \\
116 \\
101\end{array}$ & $\begin{array}{l}61 \\
58 \\
53 \\
53 \\
56 \\
52 \\
53 \\
55 \\
46 \\
49 \\
50 \\
42\end{array}$ & $\begin{array}{l}2,729 \\
1,809 \\
1,699 \\
1,412 \\
1,229 \\
1,759 \\
1,133 \\
1,235 \\
1,364 \\
1,361 \\
1,863 \\
2,565\end{array}$ & $\begin{array}{r}397 \\
286 \\
261 \\
137 \\
157 \\
-496 \\
162 \\
162 \\
23 \\
252 \\
264 \\
-113\end{array}$ & $\begin{array}{r}448 \\
355 \\
273 \\
200 \\
215 \\
-422 \\
194 \\
128 \\
-398 \\
276 \\
312 \\
277\end{array}$ & $\begin{array}{r}303 \\
222 \\
151 \\
68 \\
74 \\
-540 \\
41 \\
-11 \\
-545 \\
120 \\
166 \\
130\end{array}$ \\
\hline 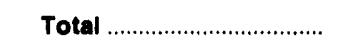 & 4,052 & 21,649 & 17,996 & 1,311 & 627 & 20,158 & 1,492 & 1,857 & 179 \\
\hline \multicolumn{10}{|l|}{1992} \\
\hline $\begin{array}{l}\text { January } \\
\text { February } \\
\text { March } \\
\text { April } \\
\text { May . } \\
\text { June } \\
\text { July } \\
\text { August } \\
\text { September } \\
\text { October } \\
\text { November } \\
\text { December }\end{array}$ & $\begin{array}{r}413 \\
347 \\
348 \\
276 \\
269 \\
224 \\
239 \\
279 \\
254 \\
268 \\
364 \\
435\end{array}$ & $\begin{array}{l}2,240 \\
1,800 \\
1,832 \\
1,526 \\
1,476 \\
1,438 \\
1,442 \\
1,564 \\
1,957 \\
1,739 \\
2,139 \\
2,430\end{array}$ & $\begin{array}{l}1,647 \\
1,281 \\
1,314 \\
1,112 \\
1,084 \\
1,056 \\
1,040 \\
1,157 \\
1,552 \\
1,363 \\
1,584 \\
1,892\end{array}$ & $\begin{array}{r}117 \\
42 \\
118 \\
119 \\
123 \\
113 \\
125 \\
131 \\
-24 \\
68 \\
126 \\
76\end{array}$ & $\begin{array}{l}64 \\
59 \\
56 \\
56 \\
58 \\
52 \\
56 \\
54 \\
49 \\
55 \\
54 \\
51\end{array}$ & $\begin{array}{l}1,954 \\
1,514 \\
1,586 \\
1,339 \\
1,311 \\
1,252 \\
1,259 \\
1,412 \\
1,628 \\
1,533 \\
1,895 \\
2,252\end{array}$ & $\begin{array}{l}286 \\
285 \\
246 \\
187 \\
165 \\
187 \\
183 \\
152 \\
329 \\
206 \\
244 \\
178\end{array}$ & $\begin{array}{l}382 \\
378 \\
380 \\
279 \\
368 \\
311 \\
301 \\
268 \\
378 \\
314 \\
359 \\
341\end{array}$ & $\begin{array}{l}241 \\
253 \\
246 \\
135 \\
232 \\
171 \\
147 \\
124 \\
252 \\
168 \\
220 \\
206\end{array}$ \\
\hline Total & R 3,716 & 21,583 & 16,083 & 1,133 & 665 & 18,935 & 2,648 & 4,058 & 2,395 \\
\hline \multicolumn{10}{|l|}{1893} \\
\hline 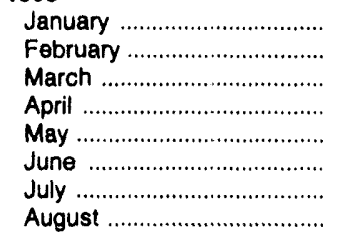 & $\begin{array}{l}366 \\
395 \\
401 \\
278 \\
185 \\
219 \\
225 \\
225\end{array}$ & $\begin{array}{l}2,070 \\
2,001 \\
2,013 \\
1,617 \\
1,420 \\
1,487 \\
1,451 \\
1,468\end{array}$ & $\begin{array}{r}1,434 \\
1,395 \\
1,396 \\
1,161 \\
1,062 \\
1,062 \\
980 \\
1,095\end{array}$ & $\begin{array}{l}121 \\
120 \\
118 \\
216 \\
105 \\
123 \\
116 \\
100\end{array}$ & $\begin{array}{l}57 \\
59 \\
55 \\
55 \\
54 \\
52 \\
51 \\
49\end{array}$ & $\begin{array}{l}1,703 \\
1,696 \\
1,705 \\
1,516 \\
1,251 \\
1,310 \\
1,212 \\
1,291\end{array}$ & $\begin{array}{l}367 \\
305 \\
308 \\
101 \\
168 \\
177 \\
239 \\
177\end{array}$ & $\begin{array}{l}388 \\
393 \\
404 \\
295 \\
108 \\
275 \\
319 \\
231\end{array}$ & $\begin{array}{l}267 \\
272 \\
268 \\
168 \\
-39 \\
144 \\
196 \\
106\end{array}$ \\
\hline
\end{tabular}

- Includes sales for resale and sales to ultimate consumers.

- Excludes income taxes.

c Total Income before Interest Charges and Extraordinary Expenses and Investment Tax Credits minus Income Taxes, Interest Charges, and Extraordinary Items.

$\mathbf{R}=$ Revised Data.

Notes: (1) Data up to the current month of the prior year are final. All other data are preliminary unless otherwise indicated. See Appendix A, Explanatory Note 9 for discussion of major interstate pipeline companies. Totals may not equal sum of components because of independent rounding. This table shows selected iterns only and therefore does not balance mathematically.

(2) As of January 1993, Arkla, Inc. does not meet the filing requirements for the FERC-11. Arkla Energy Resources Company (A.E.R. Co.) is a subsidiary of Arkla, Inc.. Data submitted on the FERC-11 for A.E.R. Co. will indicate less sales volumes and revenues than previous years reported by Arkla, Inc.

Source: Form FERC.11. 
Table 9. Volumes and Prices of Natural Gas Sold by Major Interstate Natural Gas Pipeline Companies, 1987-1993

(Volumes in Million Cubic Feet, Prices in Dollars per Thousand Cubic Feet)

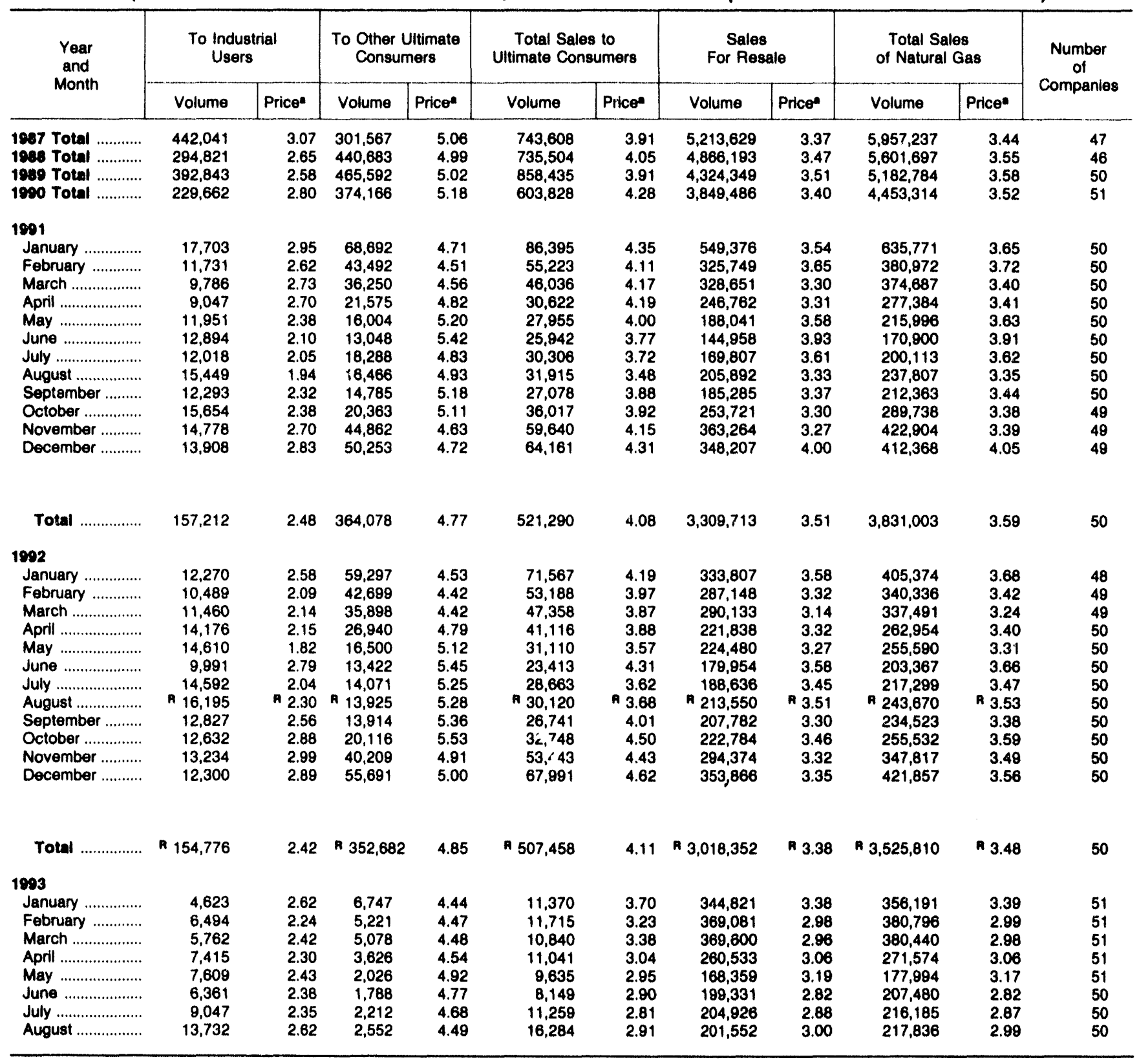

- All prices are weighted averages.

n = Revised Date.

Notes: (1) The summaries presented in this table are exclusive of transactions between major pipeline companies in the computation of total pipeline activities to eliminate double-counting. Data up to the current month of the prior year are final. All other data are preliminary unless otherwise indicated.

See Appendix A, Explanatory Note 9 for discussion of major interstate pipeline companies. Totals may not equal sum of components because of independent rounding.

(2) As of January 1993, Arkla, inc. does not meet the filing requirements for the FERC-11. Arkla Energy Resources Company (A.E.R. Co.) ls a subsidiary of Arkla, Inc.. Data submitted on the FERC-11 for A.E.R. Co. will indicate less sales volumes and revenues than previous years reported by Arkla, Inc.

Source: Form FERC-11. 
Table 10. Volumes and Prices of Natural Gas Sold by Major Interstate Natural Gas Pipeline Companies, by Company, August 1993 (Volumes in Million Cubic Feet, Prices in Dollars per Thousand Cubic Feet)

\begin{tabular}{|c|c|c|c|c|c|c|c|c|c|c|}
\hline \multirow{2}{*}{ Pipeline Company } & \multicolumn{2}{|c|}{$\begin{array}{l}\text { To Industrial } \\
\text { Users }\end{array}$} & \multicolumn{2}{|c|}{$\begin{array}{l}\text { To Other Ultimate } \\
\text { Consumers }\end{array}$} & \multicolumn{2}{|c|}{$\begin{array}{l}\text { Total Sales To } \\
\text { Uttimate Consumers }\end{array}$} & \multicolumn{2}{|c|}{$\begin{array}{c}\text { Sales } \\
\text { For Resale }\end{array}$} & \multicolumn{2}{|c|}{$\begin{array}{l}\text { Total Sele I } \\
\text { Of Natural G. is }\end{array}$} \\
\hline & Volume & Pricen & Volume & Price* & Volume & Pricen & Volume & Prices & Volume & Price" \\
\hline 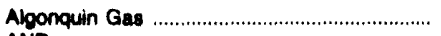 & 0 & - & 0 & - & 0 & - & 0 & - & 0 & - \\
\hline 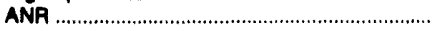 & 0 & - & 0 & - & 0 & - & 17,578 & 2.50 & 17,578 & 2.50 \\
\hline 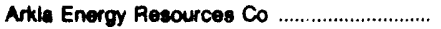 & 0 & - & 0 & - & 0 & - & 862 & 4.08 & 862 & 4.08 \\
\hline Bear Creok Storage & 0 & - & 0 & - & 0 & - & 0 & -- & 0 & - \\
\hline Colorado Interatate & 78 & 2.38 & 0 & - & 78 & 2.38 & 4,642 & 2.36 & 4,720 & 2.36 \\
\hline 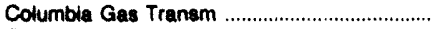 & 0 & - & 0 & - & 0 & - & 31,816 & 3.85 & 31,816 & 3.85 \\
\hline 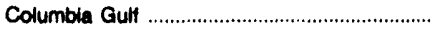 & 0 & - & 4 & 3.00 & 4 & 3.00 & 0 & - & 4 & 3.00 \\
\hline 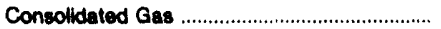 & 0 & - & 0 & - & 0 & - & 2,813 & 2.44 & 2,813 & 2.44 \\
\hline Esat Tennesser ……...................................... & 324 & 3.64 & 0 & - & 324 & 3.64 & 1,686 & 2.32 & 1,910 & 2.55 \\
\hline El Paso & 2,120 & 1.83 & 0 & - & 2,120 & 1.83 & 9,643 & 1.88 & 11,763 & 1.89 \\
\hline 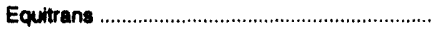 & 4,382 & 2.90 & 0 & - & 4,382 & 2.90 & 552 & 2.01 & 4,834 & 2.80 \\
\hline 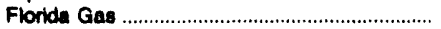 & 350 & 4.39 & 0 & - & 350 & 4.38 & 534 & 4.56 & 884 & 4.49 \\
\hline Great Lakes Gas & 0 & - & 0 & - & 0 & - & 0 & - & 0 & - \\
\hline High latand Otishore & 0 & -- & 0 & - & 0 & - & 0 & -- & 0 & - \\
\hline Iroquols Gas Transm. Sys. & 0 & - & 0 & - & 0 & - & 0 & - & 0 & - \\
\hline 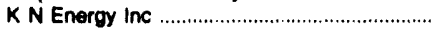 & 32 & 3.00 & 2,492 & 4.51 & 2,524 & 4.49 & 415 & 4.00 & 2,939 & 4.42 \\
\hline Kern River Gas Trans. & 0 & - & 0 & - & 0 & - & 0 & - & 0 & - \\
\hline Michigan Gas Storage & 0 & - & 0 & - & 0 & - & 84 & 2.61 & 84 & 2.61 \\
\hline 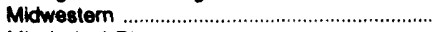 & 0 & - & 0 & - & 0 & - & 1,823 & 6.43 & 1,823 & 6.43 \\
\hline 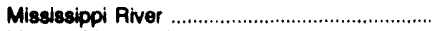 & 709 & 2.80 & 0 & -- & 709 & 2.80 & 2,440 & 4.95 & 3,148 & 4.47 \\
\hline 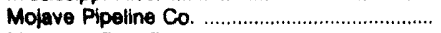 & u & - & 0 & - & 0 & - & 0 & - & 0 & - \\
\hline 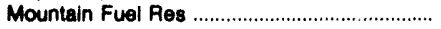 & 0 & -- & 0 & - & 0 & - & 0 & - & 0 & - \\
\hline 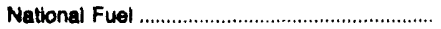 & 0 & - & 0 & - & 0 & -- & 0 & - & 0 & - \\
\hline 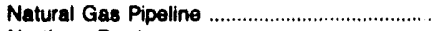 & 3 & 1.00 & 0 & - & 3 & 1.00 & 18,640 & 3.81 & 18,843 & 3.81 \\
\hline 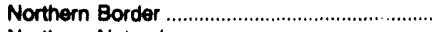 & 0 & - & 0 & - & 0 & - & 0 & - & 0 & - \\
\hline 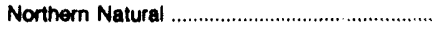 & 0 & - & 1 & 2.00 & 1 & 2.00 & 11,236 & 2.82 & 11,237 & 2.92 \\
\hline Northwest Alaskan & 0 & -. & 0 & - & 0 & - & 26,032 & 1.70 & 26,032 & 1.70 \\
\hline 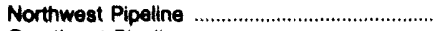 & 0 & -- & 0 & - & 0 & - & 2,181 & 2.44 & 2,181 & 2.44 \\
\hline 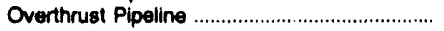 & 0 & - & 0 & -- & 0 & - & 0 & - & 0 & - \\
\hline Pactic Gas Transm & 0 & - & 0 & - & 0 & -- & 22,665 & 2.31 & $\mathbf{2 2 , 6 6 5}$ & 2.31 \\
\hline 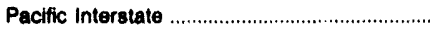 & 0 & - & 0 & -- & 0 & - & 5,878 & 2.66 & 5,878 & 2.66 \\
\hline 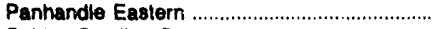 & 0 & - & 0 & - & 0 & - & 0 & - & 0 & - \\
\hline Sabine Pipeline Co & 0 & - & 0 & - & 0 & - & 0 & - & 0 & - \\
\hline Sea Robin Pipeline & 0 & -- & 0 & - & 0 & - & 0 & - & 0 & - \\
\hline 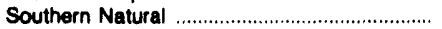 & 17 & 3.82 & 0 & -- & 17 & 3.82 & 1,559 & 3.02 & 1,578 & 3.03 \\
\hline 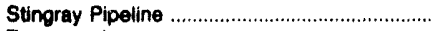 & 0 & - & 0 & - & 0 & - & 0 & - & 0 & - \\
\hline 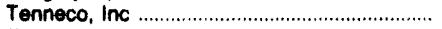 & 1,932 & 2.23 & 0 & - & 1,832 & 2.23 & 7,258 & 4.75 & 9,190 & 4.22 \\
\hline 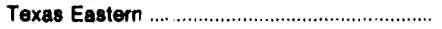 & 0 & -- & 0 & - & 0 & - & 2,000 & 3.84 & 2,000 & 3.94 \\
\hline 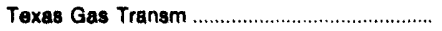 & 0 & - & 0 & -- & 0 & -- & 2,881 & 5.37 & 2,801 & 5.37 \\
\hline 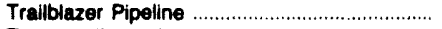 & 0 & - & 0 & - & 0 & - & 0 & - & 0 & - \\
\hline 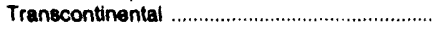 & 0 & - & 0 & - & 0 & - & 20,891 & 3.09 & 20,891 & 3.10 \\
\hline 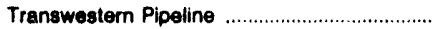 & 253 & 1.87 & 28 & 2.31 & 282 & 1.92 & 0 & - & 282 & 1.82 \\
\hline Trunkline Gas Co & 7 & 2.43 & 24 & 2.38 & 31 & 2.39 & 8,805 & 2.90 & 8,836 & 2.80 \\
\hline U-T Offehore & 0 & - & 0 & - & 0 & $\ldots$ & 0 & - & 0 & - \\
\hline United Gas Pipeline & 3,459 & 2.56 & 0 & - & 3,459 & 2.58 & 1,519 & 5.28 & 4,878 & 3.40 \\
\hline 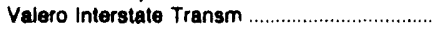 & 0 & - & 0 & -- & 0 & - & 1,241 & 2.62 & 1,241 & 2.62 \\
\hline 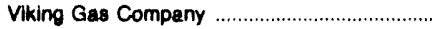 & 0 & - & 0 & - & 0 & - & 0 & - & 0 & - \\
\hline 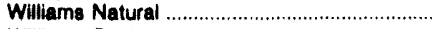 & 66 & 3.50 & 2 & 1.00 & 68 & 3.43 & 258 & 4.83 & 326 & 4.53 \\
\hline 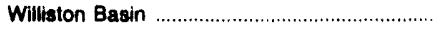 & 0 & - & 0 & - & 0 & -- & 189 & 2.59 & 189 & 2.59 \\
\hline 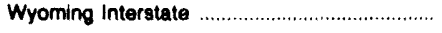 & 0 & - & 0 & -- & 0 & -- & 0 & - & 0 & - \\
\hline Total/Average Prices & 13,732 & 2.62 & 2,552 & 4.48 & 16,284 & 2.81 & 208.321 & 3.01 & 224,605 & 3.00 \\
\hline Sales to Other Major & & & & & & & & & & \\
\hline Companies & -- & -- & - & - & - & - & 6,769 & 3.49 & 0,780 & 3.49 \\
\hline Sales Excluding Sales to & & & & & & & & & & \\
\hline Major Companies & - & -- & - & - & - & -- & 201,552 & 3.00 & 217,836 & 2.89 \\
\hline
\end{tabular}

- All prices are computed weighted averages based on dollar and volume amounts reported, which may include or reflect out-ot-period dollar or volume adjustments, realatements or revisions, or account reclassifications or provisions for pending regulatory adjustments. See Appendix A, Explanatory Note 9 for discussion of apparent anomalies. - Not Applicable.

Notes: (1) Two lines have been added to this table to explicitly differentiate transactions between major and nonmajor pipeline companies. Totals may not equal sum of components due to independent rounding and provisions for pending regulatory adjustments.

(2) As of January 1993, Arkla, Inc. does not meet the filing requirements for the FERC.11. Arkla Energy Resources Company (A.E.R. Co.) is a subsidiary of Arkla, Inc.. Data submitted on the FERC-11 for A.E.R. Co. will indicate less sales volumes and revenues than previous years reported by Arkla, Inc..

Source: Form FERC-11. 
Table 11. Natural and Other Gases Produced and Purchased by Major Interstate Natural Gas Pipeline Companies, 1987-1993 (Million Cubic Feet)

\begin{tabular}{|c|c|c|c|c|c|c|c|c|}
\hline \multirow{2}{*}{$\begin{array}{l}\text { Year } \\
\text { and } \\
\text { Month }\end{array}$} & \multirow{2}{*}{$\begin{array}{c}\text { Transported } \\
\text { Gas" }\end{array}$} & \multirow{2}{*}{$\begin{array}{c}\text { Natural } \\
\text { Gas } \\
\text { Production }\end{array}$} & \multirow{2}{*}{\begin{tabular}{|} 
Manufactured Gas. \\
Liquefied \\
Natural Gas, \\
Gasified Coal, \\
and \\
Synthetic Gas \\
Production
\end{tabular}} & \multicolumn{5}{|c|}{ Purchased Natural Gas } \\
\hline & & & & $\begin{array}{c}\text { From } \\
\text { Producers }\end{array}$ & $\begin{array}{c}\text { Intracompany } \\
\text { Transfers }\end{array}$ & Imports & $\begin{array}{l}\text { From } \\
\text { Others }\end{array}$ & Total \\
\hline 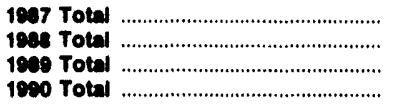 & $\begin{array}{l}13,479,989 \\
15,950,507 \\
18,696,398 \\
21,681,372\end{array}$ & $\begin{array}{r}152,039 \\
139,621 \\
108,036 \\
93,386\end{array}$ & $\begin{array}{l}22,905 \\
23,960 \\
24,609 \\
24,163\end{array}$ & $\begin{array}{l}4,848,577 \\
4,516,120 \\
3,532,588 \\
3,601,690\end{array}$ & $\begin{array}{r}124,572 \\
66,879 \\
74,058 \\
72,886\end{array}$ & $\begin{array}{l}759,345 \\
962,383 \\
903,488 \\
965,859\end{array}$ & $\begin{array}{l}400,134 \\
374,983 \\
411,897 \\
355,273\end{array}$ & $\begin{array}{l}6,132,628 \\
5,920,365 \\
4,922,131 \\
4,995,808\end{array}$ \\
\hline \multicolumn{9}{|l|}{1001} \\
\hline $\begin{array}{l}\text { January } \\
\text { February } \\
\text { March } \\
\text { April } \\
\text { May } \\
\text { June } \\
\text { July } \\
\text { August } \\
\text { September } \\
\text { October } \\
\text { November } \\
\text { December }\end{array}$ & $\begin{array}{l}2,098,858 \\
1,839,800 \\
1,865,092 \\
1,792,255 \\
1,723,696 \\
1,579,146 \\
1,610,413 \\
1,735,157 \\
1,691,806 \\
1,856,527 \\
2,103,022 \\
2,135,449\end{array}$ & $\begin{array}{l}8,065 \\
6,242 \\
5,837 \\
5,603 \\
5,534 \\
5,353 \\
5,571 \\
6,213 \\
5,898 \\
5,473 \\
6,378 \\
6,798\end{array}$ & $\begin{array}{l}1,588 \\
2,157 \\
2,120 \\
1,114 \\
2,202 \\
2,121 \\
2,285 \\
2,030 \\
1,880 \\
2,192 \\
1,708 \\
1,969\end{array}$ & $\begin{array}{l}372,884 \\
262,740 \\
222,064 \\
193,804 \\
174,738 \\
160,553 \\
155,126 \\
149,415 \\
169,800 \\
210,115 \\
261,011 \\
288,819\end{array}$ & $\begin{array}{r}7,194 \\
5,669 \\
5,388 \\
4,453 \\
4,808 \\
4,284 \\
5,186 \\
5,253 \\
5,586 \\
5,329 \\
11,226 \\
7,732 \\
\end{array}$ & $\begin{array}{l}79,076 \\
68,257 \\
78,343 \\
64,319 \\
61,258 \\
52,828 \\
49,545 \\
73,437 \\
60,109 \\
71,699 \\
73,042 \\
74,645\end{array}$ & $\begin{array}{l}40,958 \\
21,918 \\
19,280 \\
19,803 \\
14,697 \\
22,143 \\
15,099 \\
22,469 \\
14,208 \\
18,976 \\
33,149 \\
31,468\end{array}$ & $\begin{array}{l}500,112 \\
358,584 \\
325,075 \\
282,379 \\
255,501 \\
239,818 \\
224,956 \\
250,574 \\
249,703 \\
306,119 \\
378,428 \\
412,664\end{array}$ \\
\hline Total & $22,031,321$ & 73,065 & 23,376 & $2,631,069$ & 72,118 & 808,558 & 274,168 & $3,783,913$ \\
\hline \multicolumn{9}{|l|}{1902} \\
\hline $\begin{array}{l}\text { January } \\
\text { February } \\
\text { March } \\
\text { April } \\
\text { May } \\
\text { June } \\
\text { July } \\
\text { August } \\
\text { September } \\
\text { October } \\
\text { November } \\
\text { December }\end{array}$ & $\begin{array}{l}2,309,478 \\
2,159,190 \\
2,183,665 \\
2,107,984 \\
1,979,257 \\
1,912,627 \\
1,925,300 \\
1,888,726 \\
1,807,555 \\
1,985,790 \\
2,144,328 \\
2,194,128\end{array}$ & $\begin{array}{l}6,645 \\
6,079 \\
6,156 \\
6,796 \\
6,969 \\
5,243 \\
5,675 \\
6,146 \\
6,249 \\
6,352 \\
6,503 \\
6,604\end{array}$ & $\begin{array}{l}1,855 \\
2,070 \\
2,303 \\
2,208 \\
2,304 \\
2,076 \\
1,989 \\
2,045 \\
2,006 \\
2,209 \\
2,195 \\
2,633\end{array}$ & $\begin{array}{l}251,382 \\
205,682 \\
148,181 \\
164,991 \\
170,687 \\
155,643 \\
156,145 \\
166,766 \\
175,857 \\
192,808 \\
218,189 \\
247,161\end{array}$ & $\begin{array}{r}8,000 \\
7,942 \\
8,643 \\
5,813 \\
5,935 \\
4,803 \\
5,108 \\
5,429 \\
5,268 \\
5,380 \\
9,938 \\
10,494\end{array}$ & $\begin{array}{r}64,855 \\
63,868 \\
68,723 \\
59,849 \\
67,619 \\
56,782 \\
65,557 \\
\cap 72,672 \\
74,569 \\
71,677 \\
71,100 \\
79,706\end{array}$ & $\begin{array}{r}38,728 \\
24,695 \\
19,804 \\
21,858 \\
19,859 \\
17,899 \\
23,342 \\
18,715 \\
66,869 \\
20,536 \\
24,987 \\
45,325\end{array}$ & $\begin{array}{r}362,965 \\
302,187 \\
245,351 \\
252,511 \\
264,100 \\
235,227 \\
250,150 \\
263,582 \\
262,583 \\
290,401 \\
324,184 \\
382,686\end{array}$ \\
\hline 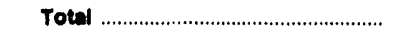 & $24,598,028$ & 75,417 & 25,893 & $2,253,492$ & 82,751 & " 816,977 & 282,697 & ค 3,435,817 \\
\hline \multicolumn{9}{|l|}{1003} \\
\hline $\begin{array}{l}\text { January } \\
\text { February . } \\
\text { March } \\
\text { April } \\
\text { May } \\
\text { June } \\
\text { July } \\
\text { August }\end{array}$ & $\begin{array}{l}2,334,905 \\
2,163,229 \\
2,214,880 \\
1,976,998 \\
1,934,569 \\
1,948,210 \\
1,958,562 \\
1,841,353\end{array}$ & $\begin{array}{l}6,430 \\
6,047 \\
6,310 \\
5,532 \\
5,578 \\
5,031 \\
5,343 \\
5,228\end{array}$ & $\begin{array}{r}2,335 \\
2,102 \\
2,460 \\
2,233 \\
894 \\
2,216 \\
2,140 \\
2,189\end{array}$ & $\begin{array}{l}203,273 \\
160,616 \\
171,111 \\
164,581 \\
162,910 \\
164,781 \\
172,148 \\
152,859\end{array}$ & $\begin{array}{r}5,475 \\
5,238 \\
5,563 \\
4,796 \\
5,037 \\
4,175 \\
4,500 \\
12,439\end{array}$ & $\begin{array}{l}76,801 \\
70,472 \\
71,018 \\
75,589 \\
63,140 \\
74,253 \\
76,334 \\
63,834\end{array}$ & $\begin{array}{r}7,912 \\
9,394 \\
11,065 \\
1,134 \\
5,158 \\
5,315 \\
2,357 \\
1,640\end{array}$ & $\begin{array}{l}293,461 \\
245,720 \\
258,757 \\
246,100 \\
236,245 \\
248,524 \\
255,339 \\
230,572\end{array}$ \\
\hline
\end{tabular}

- Gas transported for other companies through the production, transmission, or distribution lines or compressor stations of the reporting pipelines. - Includes out-of-period adjustments to correct data in prior month.

$n$ = Revised Data.

$m=$ Not Avallable.

Notes: (1) Previously published manufactured gas is now summartzed with liquefied natural gas, gasitied coal, and synthetic gas production. Also, the summaries presented in this table are exclusive of transactions between major pipeline companies in the computation of total pipeline activities to eliminate double counting. See Appendix A, Explana. tory Note $\theta$ for diecussion of major interstate pipeline compenies. Totals may not equal sum of components because of independent rounding.

(2) As of January 1993, Arkla, Inc. does not meet the filing requirements for the FERC-11. Arkla Energy Resources Company (A.E.R. Co.) is a subsidiary of Arkla, Inc. Data submitted on the FERC-11 for A.E.R. Co. will indicate less eales volurnes and revenues than previous years reported by Arkla, Inc.

Source: Form FERC-11. 
Table 12. Natural and Other Gases Produced and Purchased by Major Interstate Natural Gas Pipeline Companies, August 1993 (Million Cubic Feet)

\begin{tabular}{|c|c|c|c|c|c|c|c|c|}
\hline \multirow{2}{*}{ Pipeline Company } & \multirow{2}{*}{$\begin{array}{l}\text { Transported } \\
\text { Gas }\end{array}$} & \multirow{2}{*}{$\begin{array}{c}\text { Natural } \\
\text { Gas } \\
\text { Production }\end{array}$} & \multirow{2}{*}{$\begin{array}{c}\text { Manufactured } \\
\text { Gas, } \\
\text { Liquefied } \\
\text { Natural Gas, } \\
\text { Gasifled Coal } \\
\text { and } \\
\text { Synthetic Gas } \\
\text { Production }\end{array}$} & \multicolumn{5}{|c|}{ Purchased Natural Gas } \\
\hline & & & & $\begin{array}{l}\text { From } \\
\text { Producers }\end{array}$ & $\begin{array}{l}\text { Intracompany } \\
\text { Transfers }\end{array}$ & Imports & $\begin{array}{l}\text { From } \\
\text { Others }\end{array}$ & Total \\
\hline Algonquin Gas & 10,173 & 0 & 0 & 0 & 0 & 0 & 0 & 0 \\
\hline ANR & 77,599 & $\mathbf{0}$ & 1,109 & 12,230 & 0 & 9,289 & 0 & 21,518 \\
\hline 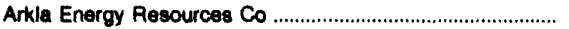 & 45,816 & 0 & 0 & 1,910 & 0 & 0 & 124 & 2,034 \\
\hline 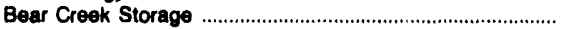 & & 0 & 0 & 0 & 0 & 0 & 0 & 0 \\
\hline Colorado Interstate & 41,160 & 4,327 & 0 & 4,749 & 4.327 & 0 & 0 & 8,076 \\
\hline Columbia Gas Transm & 55,227 & 0 & 0 & 28,467 & 0 & 0 & 520 & 29,987 \\
\hline Columbla Gult & 98,465 & 0 & 0 & 0 & 0 & 0 & $\cdot-326$ & -326 \\
\hline 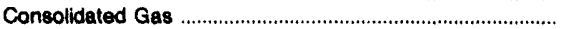 & 31,553 & 898 & 0 & 2,356 & 0 & 0 & $\cdot-37$ & 2,319 \\
\hline East Tennesser & 5,047 & 0 & 0 & 2,930 & 0 & 0 & 0 & 2,930 \\
\hline El Paso & 99,013 & 0 & 0 & 6,114 & 0 & 0 & 2,726 & 8,840 \\
\hline Equitrans & 4,175 & 0 & 0 & 614 & 0 & 0 & 0 & 614 \\
\hline 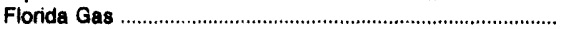 & 32,125 & 0 & 0 & 316 & 0 & 0 & 482 & 798 \\
\hline Great Lakes Gas & 71,342 & 0 & 0 & 0 & 0 & 0 & 0 & 0 \\
\hline High Island Otfshore & 24,739 & 0 & 0 & 0 & 0 & 0 & 0 & 0 \\
\hline 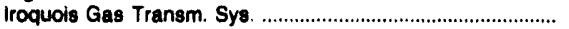 & 21,034 & 0 & 0 & 0 & 0 & 0 & 0 & 0 \\
\hline 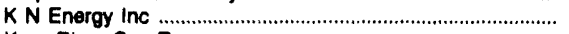 & 8,317 & 0 & 0 & 4,162 & 0 & 0 & 2,087 & 6,249 \\
\hline 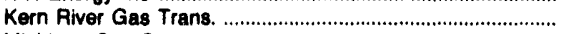 & 22,007 & 0 & 0 & 0 & 0 & 0 & 0 & 0 \\
\hline Michigan Gas Storage ............................................ & 24,444 & 0 & 0 & 0 & 0 & 0 & 8. & 84 \\
\hline Midwestern & 9,350 & 0 & 0 & 4,406 & 0 & 0 & 0 & 4,406 \\
\hline 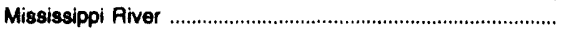 & 19,159 & 0 & 0 & 0,458 & 0 & 0 & 0 & 9,458 \\
\hline 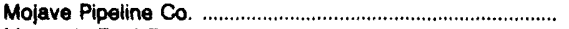 & 7,080 & 0 & 0 & 0 & 0 & 0 & 0 & 0 \\
\hline 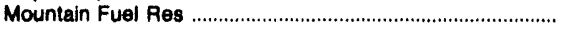 & 24,426 & 0 & 0 & 3,498 & 0 & 0 & -348 & 3,150 \\
\hline 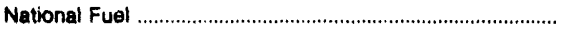 & 13,420 & 0 & 0 & 0 & 0 & 0 & 0 & 0 \\
\hline 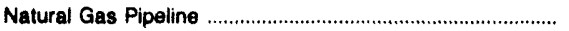 & 131,744 & 0 & 1,080 & 10,660 & 7,771 & 199 & 0 & 18,630 \\
\hline 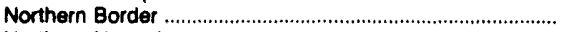 & 54,915 & 0 & 0 & 0 & 0 & 0 & 0 & 0 \\
\hline Northern Natural & 145,435 & 0 & 0 & 2,592 & 0 & 0 & 11,814 & 14,406 \\
\hline Northwest Alaskan & & 0 & 0 & 0 & 0 & 26,032 & 0 & 26,032 \\
\hline 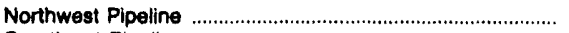 & 46,319 & 0 & 0 & 0 & 0 & 2,218 & 0 & 2,218 \\
\hline 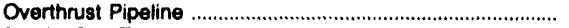 & 1,278 & 0 & 0 & 0 & 0 & 0 & 0 & 0 \\
\hline 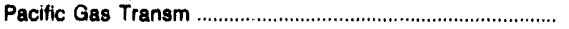 & 20,808 & 0 & 0 & 0 & 0 & 22,553 & 0 & 22,553 \\
\hline 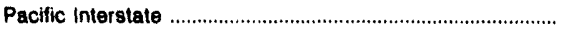 & & 0 & 0 & 0 & 0 & 0 & 5,968 & 5,869 \\
\hline 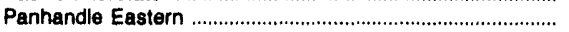 & 40,919 & 0 & 0 & $-8,758$ & 0 & 0 & 0 & $\cdot-8,758$ \\
\hline Sabine Pipeline Co. & 12,956 & 0 & 0 & 0 & 0 & 0 & 0 & 0 \\
\hline Sea Robin Pipeline & 22,628 & 0 & 0 & 0 & 0 & 0 & 0 & 0 \\
\hline Southern Natural ..................................................... & 35,277 & 0 & 0 & 8,509 & 0 & 0 & 1 & 8,510 \\
\hline 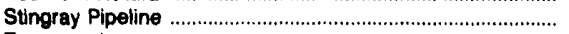 & 18,531 & 0 & 0 & 0 & 0 & 0 & 0 & 0 \\
\hline 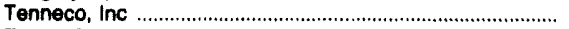 & 120,865 & 0 & 0 & 1,965 & 0 & 3,543 & $-5,761$ & $\cdot-253$ \\
\hline 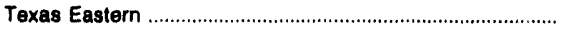 & 81,756 & 3 & 0 & 2,346 & 0 & 0 & -1 & 2,345 \\
\hline Texas Gas Transm & 53,314 & 0 & 0 & 3,637 & 0 & 0 & 5 & 3,642 \\
\hline 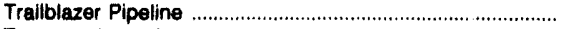 & 14,187 & 0 & 0 & 0 & 0 & 0 & 0 & 0 \\
\hline 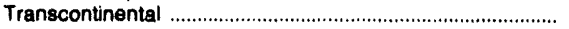 & 170,768 & 0 & 0 & 28,540 & 0 & 0 & 1,056 & 29,596 \\
\hline 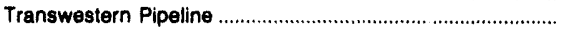 & 56,842 & 0 & 0 & 134 & 0 & 0 & 0 & 134 \\
\hline 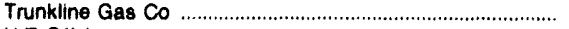 & 38,423 & 0 & 0 & 6,807 & 0 & 0 & 0 & 6,907 \\
\hline U-T OHshore & 15,043 & 0 & 0 & 0 & 0 & 0 & 0 & 0 \\
\hline United Gas Pipeline & 56.613 & 0 & 0 & 12,747 & 0 & 0 & 0 & 12,747 \\
\hline 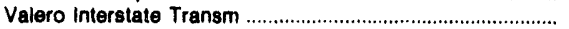 & 3,529 & 0 & 0 & 0 & 0 & 0 & 1,241 & 1,241 \\
\hline Viking Gas Company & 10,856 & 0 & 0 & 0 & 0 & 0 & 0 & 0 \\
\hline Williams Natural & 25,137 & 0 & 0 & 568 & 0 & 0 & 98 & 667 \\
\hline Williston Basin & 3,000 & 0 & 0 & 602 & 341 & 0 & 0 & 943 \\
\hline 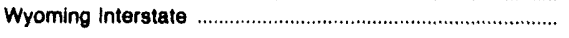 & 13,530 & 0 & 0 & 0 & 0 & 0 & 0 & 0 \\
\hline Total & $1,941,353$ & 5,228 & 2,189 & 152,659 & 12,439 & 63,834 & 19,735 & 248,667 \\
\hline \multicolumn{9}{|l|}{ Purchases from Other Major } \\
\hline Companies . & - & - & - & 0 & 0 & 0 & 18,095 & 18,085 \\
\hline \multicolumn{9}{|l|}{$\begin{array}{l}\text { Purchases Excluding } \\
\text { Purchases from Other }\end{array}$} \\
\hline Major Companies & - & - & - & 152,658 & 12,439 & 63,834 & 1,640 & 230,572 \\
\hline
\end{tabular}

- Includes out-ot-period adjustments to correct data in prior month.

- Not Applicable.

Notes: (1) Previously published manufactured gas is now summarized with liquefied natural gas, gasfited coal, und synthetlc gas production. Two lines have been added to this table that explicitly differentiate transactions between major and nonmajor pipeline companies. See Appendix $A$, Explanatory Note 9 for discussion of major interstate pipeline companies. Totals may not equal sum of components because of independent rounding.

(2) As of January 1993, Arkla, Inc. does not meet the filing requirements tor the FERC-11. Arkla Energy Resources Company (A.E.A. CO.) is a subsidiary of Arkla,

inc.. Data submitted on the FERC-11 for A.E.R. Co. will indicate less sales volumes and revenues than previous years reported by Arkla, Inc. 
Table 13. Underground Natural Gas Storage - All Operators, 1987-1993

(Volumes in Billion Cubic Feet)

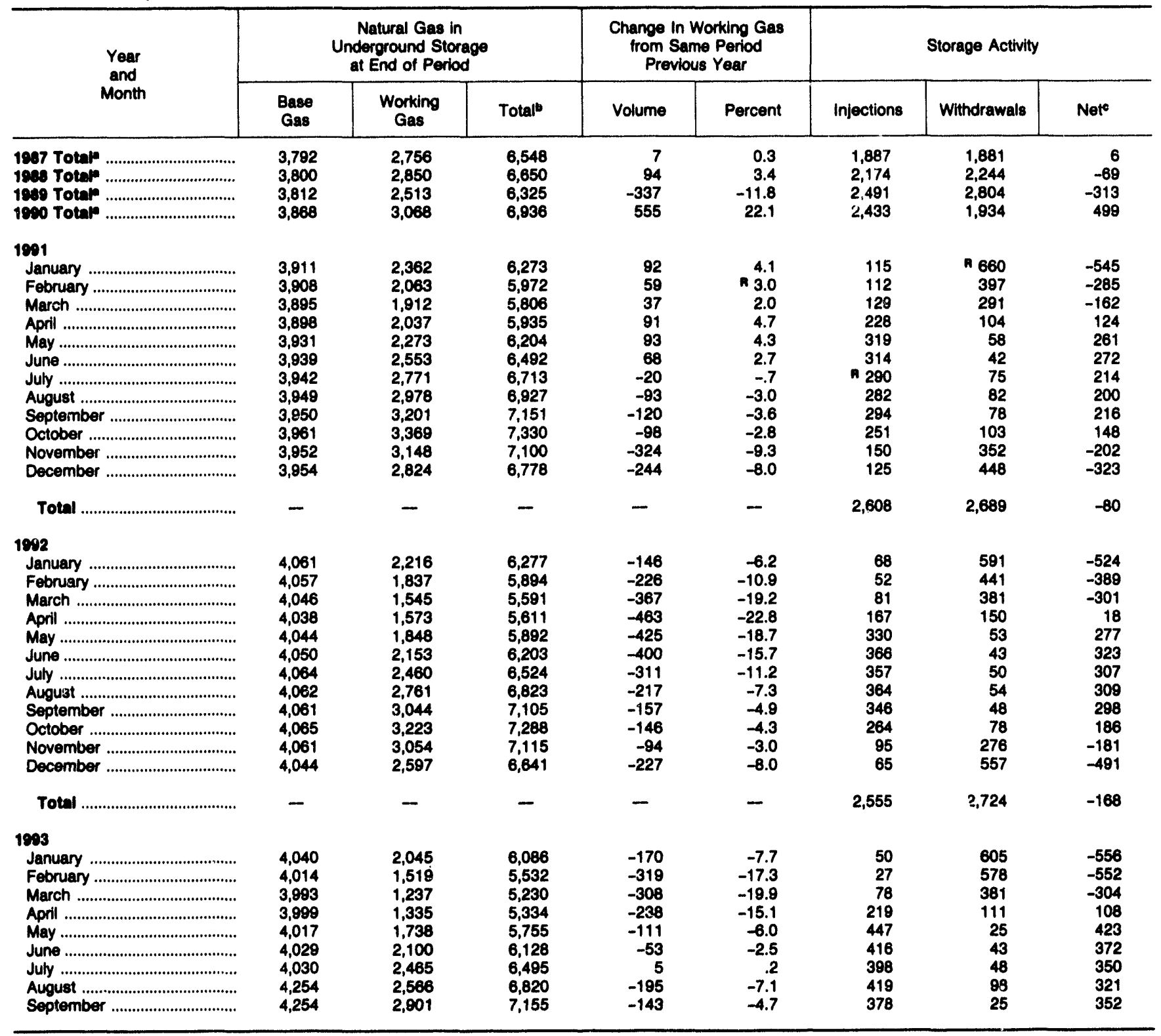

- Total as of December 31 .

b Total underground storage capacity at the end of each calendar year (in billion cubic feet): $1987,1988,8$ and $1989 \cdot 8,124 ; 1090-8,125 ; 1991 \cdot 7,993 ;$ and $1992-7,932$.

- Positive numbers indicate the volume of injections in excess of withdrawals. Negative numbers indicate the volume of withdrawals in excess of injections.

n = Revised Data.

- = Not Applicable.

Notes: Data for 1887 through 1992 are final. All other data are preliminary unless othenwise noted. See Appendix A, Explanatory Note 7 for discussion of revision policy. Gas in storage at the end of a reporting period may not equal the quantity derived by adding or subtracting net injections or withdrawals during the period to the quantity of gas in storage at the beginning of the period. This is due to changes in the quantties of native gas included in base gas and/or loseses in base gas due to migration from storage reservoirs. Totals may not equal sum of components because of independent rounding. Geographic coverage is the 50 States and the District of Columbia.

Sources: Form EIA-191, Form FERC-8, and Form EIA-176. 

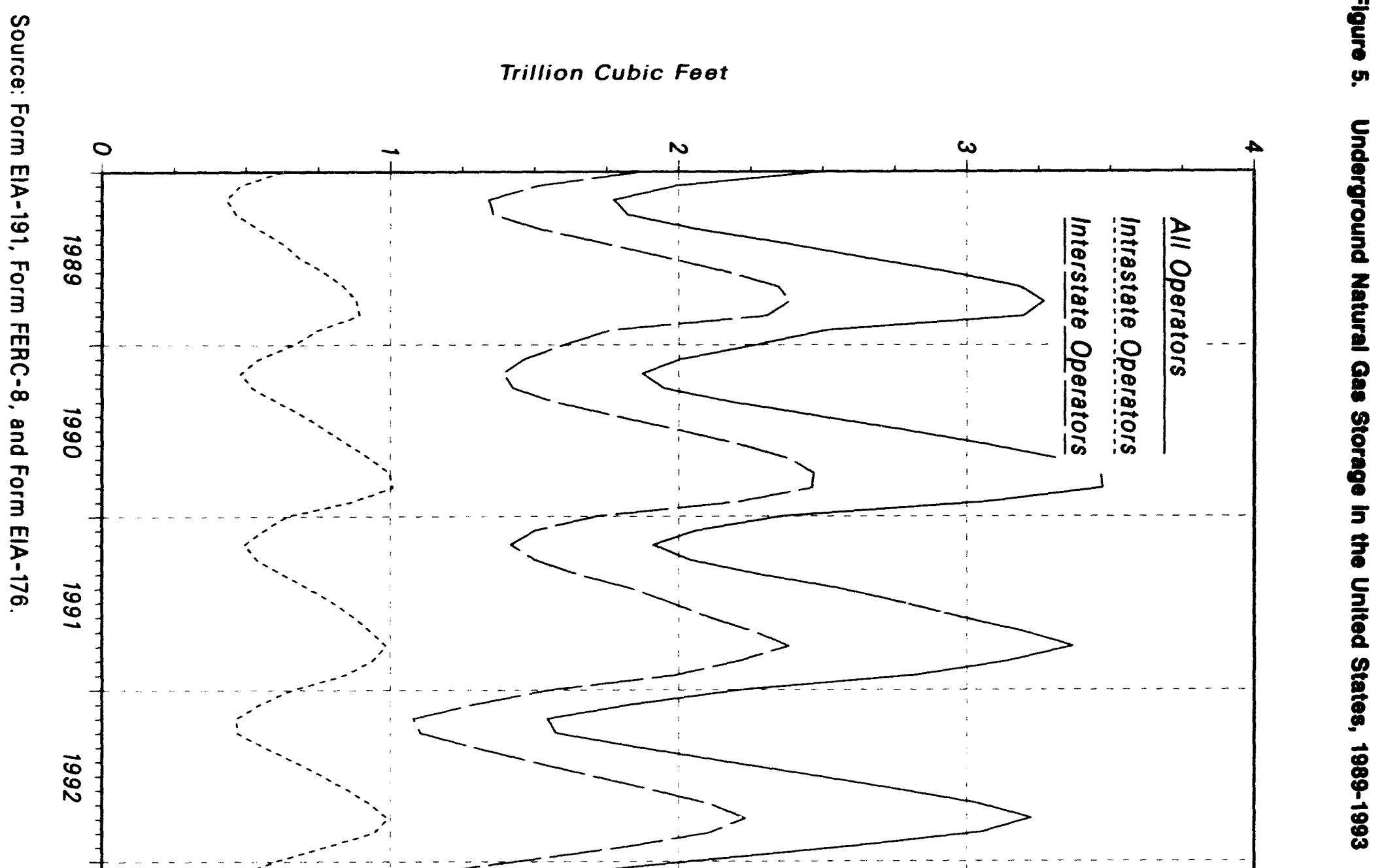

$\vec{\infty}$

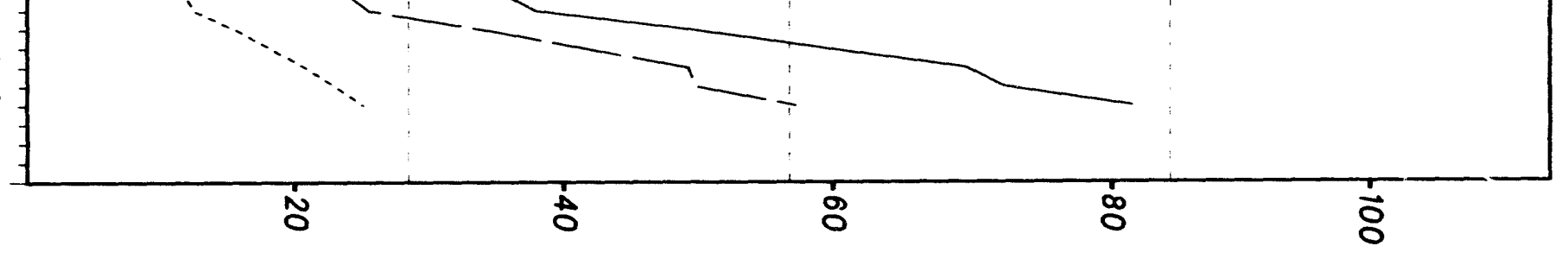

Billion Cubic Meters 
Table 14. Underground Natural Gas Storage - Interstate Operators of Storage Fields, 1987-1993 (Volumes in Billion Cubic Feet)

\begin{tabular}{|c|c|c|c|c|c|c|c|c|}
\hline \multirow{2}{*}{$\begin{array}{l}\text { Year } \\
\text { and } \\
\text { Month }\end{array}$} & \multicolumn{3}{|c|}{$\begin{array}{l}\text { Natural Gas in } \\
\text { Underground Storage } \\
\text { at End of Period }\end{array}$} & \multicolumn{2}{|c|}{$\begin{array}{c}\text { Change in Working Gas } \\
\text { from Same Period } \\
\text { Previous Year }\end{array}$} & \multicolumn{3}{|c|}{ Storage Activity } \\
\hline & $\begin{array}{c}\text { Base } \\
\text { Gas }\end{array}$ & $\begin{array}{c}\text { Working } \\
\text { Gas }\end{array}$ & Totalb & Volume & Percent & Injections & Withdrawals & Netc \\
\hline 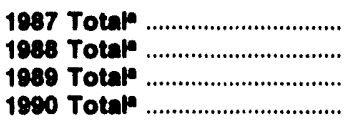 & $\begin{array}{l}2,461 \\
2,469 \\
2,478 \\
2,496\end{array}$ & $\begin{array}{l}2,024 \\
2,092 \\
1,764 \\
2,203\end{array}$ & $\begin{array}{l}4,485 \\
4,561 \\
4,242 \\
4,699\end{array}$ & $\begin{array}{r}14 \\
68 \\
-328 \\
439\end{array}$ & $\begin{array}{r}0.7 \\
3.3 \\
-15.7 \\
24.9\end{array}$ & $\begin{array}{l}1,365 \\
1,587 \\
1,788 \\
1,705\end{array}$ & $\begin{array}{l}1,348 \\
1,532 \\
2,072 \\
1,284\end{array}$ & $\begin{array}{r}17 \\
55 \\
-285 \\
421\end{array}$ \\
\hline $\begin{array}{l}1991 \\
\text { January } \\
\text { February ............. } \\
\text { March } \\
\text { April } \\
\text { May } \\
\text { June } \\
\text { July } \\
\text { August } \\
\text { September } \\
\text { October } \\
\text { November } \\
\text { December }\end{array}$ & $\begin{array}{l}2,542 \\
2,546 \\
2,535 \\
2,537 \\
2,569 \\
2,575 \\
2,576 \\
2,577 \\
2,571 \\
2,571 \\
2,572 \\
2,571\end{array}$ & $\begin{array}{l}1,718 \\
1,502 \\
1,418 \\
1,500 \\
1,647 \\
1,834 \\
1,970 \\
2,105 \\
2,265 \\
2,382 \\
2,210 \\
1,985\end{array}$ & $\begin{array}{l}4,261 \\
4,048 \\
3,952 \\
4,037 \\
4,216 \\
4,409 \\
4,546 \\
4,682 \\
4,836 \\
4,953 \\
4,782 \\
4,556\end{array}$ & $\begin{array}{r}114 \\
40 \\
26 \\
76 \\
78 \\
51 \\
-39 \\
-109 \\
-122 \\
-86 \\
-253 \\
-218\end{array}$ & $\begin{array}{r}7.1 \\
2.7 \\
\text { ค } 1.8 \\
5.3 \\
5.0 \\
2.9 \\
\text { ค }-2.0 \\
-4.9 \\
-5.1 \\
-3.5 \\
-10.3 \\
-9.9\end{array}$ & $\begin{array}{r}105 \\
93 \\
109 \\
165 \\
218 \\
213 \\
\text { A } 193 \\
186 \\
211 \\
181 \\
117 \\
112\end{array}$ & $\begin{array}{r}\text { ค } 482 \\
292 \\
200 \\
82 \\
\text { ค } 48 \\
35 \\
\text { R } 63 \\
\text { ค } 64 \\
64 \\
83 \\
274 \\
\text { ค } 327\end{array}$ & $\begin{array}{r}\text { R }-377 \\
-199 \\
-91 \\
83 \\
\text { A } 170 \\
178 \\
130 \\
130 \\
\text { A } 122 \\
148 \\
98 \\
-157 \\
\text { A }-215\end{array}$ \\
\hline Total & - & - & - & - & - & R 1,904 & A 2,015 & -111 \\
\hline 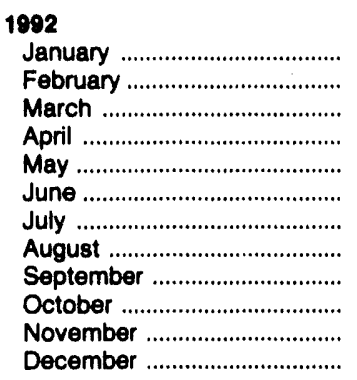 & $\begin{array}{l}2,675 \\
2,671 \\
2,661 \\
2,653 \\
2,659 \\
2,665 \\
2,679 \\
2,676 \\
2,675 \\
2,677 \\
2,673 \\
2,652\end{array}$ & $\begin{array}{l}1,554 \\
1,289 \\
1,080 \\
1,104 \\
1,285 \\
1,486 \\
1,695 \\
1,908 \\
2,111 \\
2,231 \\
2,110 \\
1,819\end{array}$ & $\begin{array}{l}4,230 \\
3,960 \\
3,741 \\
3,757 \\
3,945 \\
4,151 \\
4,375 \\
4,584 \\
4,785 \\
4,908 \\
4,784 \\
4,471\end{array}$ & $\begin{array}{l}-164 \\
-213 \\
-338 \\
-396 \\
-362 \\
-348 \\
-274 \\
-197 \\
-155 \\
-151 \\
-100 \\
-166\end{array}$ & $\begin{array}{r}-9.5 \\
-14.2 \\
-23.8 \\
-26.4 \\
-22.0 \\
-19.0 \\
-13.9 \\
-9.4 \\
-6.8 \\
-6.3 \\
-4.5 \\
-8.4\end{array}$ & $\begin{array}{r}60 \\
37 \\
65 \\
121 \\
237 \\
253 \\
254 \\
260 \\
240 \\
184 \\
74 \\
54\end{array}$ & $\begin{array}{r}410 \\
317 \\
284 \\
108 \\
42 \\
32 \\
34 \\
36 \\
27 \\
56 \\
209 \\
386\end{array}$ & $\begin{array}{r}-350 \\
-280 \\
-219 \\
12 \\
195 \\
221 \\
220 \\
224 \\
213 \\
128 \\
-135 \\
-332\end{array}$ \\
\hline 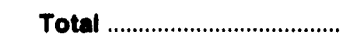 & - & - & - & - & -- & 1,838 & 1,940 & -102 \\
\hline $\begin{array}{l}1993 \\
\text { January } \\
\text { February } \\
\text { March } \\
\text { April } \\
\text { May } \\
\text { June } \\
\text { July } \\
\text { August } \\
\text { September }\end{array}$ & $\begin{array}{l}2,649 \\
2,632 \\
2,615 \\
2,621 \\
2,637 \\
2,648 \\
2,650 \\
2,872 \\
2,874\end{array}$ & $\begin{array}{r}1,443 \\
1,050 \\
829 \\
897 \\
1,191 \\
1,462 \\
1,734 \\
1,756 \\
2,018\end{array}$ & $\begin{array}{l}4,091 \\
3,683 \\
3,444 \\
3,518 \\
3,828 \\
4,110 \\
4,384 \\
4,628 \\
4,892\end{array}$ & $\begin{array}{r}-112 \\
-239 \\
-251 \\
-207 \\
-94 \\
-24 \\
38 \\
-152 \\
-93\end{array}$ & $\begin{array}{r}-7.2 \\
-18.5 \\
-23.3 \\
-18.7 \\
-7.3 \\
-1.6 \\
2.3 \\
-8.0 \\
-4.4\end{array}$ & $\begin{array}{r}42 \\
17 \\
46 \\
146 \\
328 \\
307 \\
286 \\
318 \\
274\end{array}$ & $\begin{array}{r}421 \\
425 \\
282 \\
78 \\
16 \\
26 \\
28 \\
77 \\
13\end{array}$ & $\begin{array}{r}-379 \\
-409 \\
-235 \\
68 \\
312 \\
281 \\
257 \\
241 \\
261\end{array}$ \\
\hline
\end{tabular}

- Total as of December 31 .

- Total underground storage capacity at the end of each calendar year (in billion cubic feet): 1987, 1988,1989, and 1990 - 5,622; 1991 - 5,512; and $1992-5,524$.

c Positive numbers indicate the volume of injections in excess of withdrawals. Negative numbers indicate the volume of withdrawals in excess of injections.

R = Revised Data.

- Not Applicable.

Notes: Data for 1987 through 1992 are final. All other data are preliminary unless othenwise noted. See Appendix A, Explanatory Note 7 for discussion of revision policy. Gas in storage at the end of a reporting period may not equal the quantity derived by adding or subtracting net injections or withdrawals during the period to the quantity of gas in storage at the beginning of the period. This is due to changes in the quantities of native gas included in base gas and/or losses in base gas due to migration from storage reservoirs. Totals may not $f$ Jal sum of components because of independent rounding. Geographic coverage is the $\mathbf{5 0}$ States and the District of Columbia.

Sources: Form EIA-191, Form FERC-8, and EIA-176. 
Table 15. Underground Natural Gas Storage - Intrastate Operators and Independent Producers, 1987-1993 (Volumes in Billion Cubic Feet)

\begin{tabular}{|c|c|c|c|c|c|c|c|c|}
\hline \multirow{2}{*}{$\begin{array}{l}\text { Year } \\
\text { and } \\
\text { Month }\end{array}$} & \multicolumn{3}{|c|}{$\begin{array}{l}\text { Natural Gas in } \\
\text { Underground Storage } \\
\text { at End of Period }\end{array}$} & \multicolumn{2}{|c|}{$\begin{array}{l}\text { Change in Working Gas } \\
\text { trom Same Period } \\
\text { Previous Year }\end{array}$} & \multicolumn{3}{|c|}{ Storage Activity } \\
\hline & $\begin{array}{c}\text { Base } \\
\text { Gas }\end{array}$ & $\begin{array}{c}\text { Working } \\
\text { Gas }\end{array}$ & Totalb & Volume & Percent & Injections & Withdrawals & Nete \\
\hline 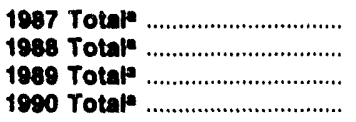 & $\begin{array}{l}1,331 \\
1,331 \\
1,335 \\
1,372\end{array}$ & $\begin{array}{l}732 \\
758 \\
749 \\
864\end{array}$ & $\begin{array}{l}2,063 \\
2,089 \\
2,083 \\
2,236\end{array}$ & $\begin{array}{r}-7 \\
26 \\
-9 \\
115\end{array}$ & $\begin{array}{r}0.1 \\
3.8 \\
-1.2 \\
15.4\end{array}$ & $\begin{array}{l}522 \\
587 \\
705 \\
728\end{array}$ & $\begin{array}{l}533 \\
711 \\
732 \\
650\end{array}$ & $\begin{array}{r}-11 \\
-124 \\
-26 \\
78\end{array}$ \\
\hline \multicolumn{9}{|l|}{1981} \\
\hline 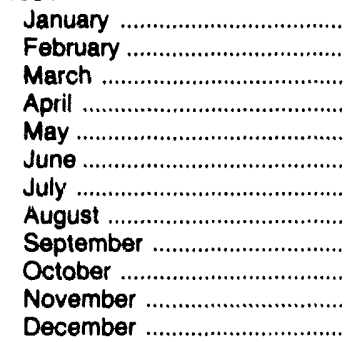 & $\begin{array}{l}1,369 \\
1,362 \\
1,360 \\
1,361 \\
1,362 \\
1,364 \\
1,366 \\
1,372 \\
1,379 \\
1,390 \\
1,380 \\
1,383\end{array}$ & $\begin{array}{l}644 \\
562 \\
494 \\
537 \\
626 \\
719 \\
802 \\
873 \\
936 \\
987 \\
937 \\
839\end{array}$ & $\begin{array}{l}2,012 \\
1,924 \\
1,854 \\
1,898 \\
1,988 \\
2,083 \\
2,168 \\
2,245 \\
2,315 \\
2,377 \\
2,317 \\
2,221\end{array}$ & $\begin{array}{r}-21 \\
21 \\
13 \\
16 \\
16 \\
18 \\
21 \\
17 \\
3 \\
-11 \\
-70 \\
-25\end{array}$ & $\begin{array}{r}-3.2 \\
\text { ค } 3.8 \\
2.7 \\
\text { ค } 3.0 \\
2.6 \\
\text { R } 2.5 \\
\text { ค } 2.6 \\
2.0 \\
.3 \\
\text { ค } \\
\text { ค } \\
-6.2 \\
-6 \\
-2.9\end{array}$ & $\begin{array}{r}10 \\
19 \\
20 \\
63 \\
101 \\
101 \\
97 \\
96 \\
83 \\
70 \\
33 \\
13\end{array}$ & $\begin{array}{r}178 \\
105 \\
\text { R } 90 \\
22 \\
11 \\
7 \\
13 \\
17 \\
14 \\
20 \\
78 \\
120\end{array}$ & $\begin{array}{r}-168 \\
-86 \\
\text { ค }-70 \\
41 \\
\text { ค } 91 \\
94 \\
84 \\
\text { ค } 78 \\
69 \\
\text { ค } 49 \\
-45 \\
-108\end{array}$ \\
\hline Total & - & - & -- & - & - & ค 705 & R 674 & R 31 \\
\hline \multicolumn{9}{|l|}{1992} \\
\hline 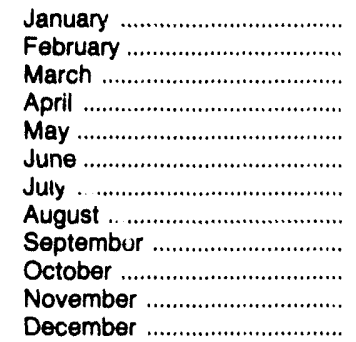 & $\begin{array}{l}1,386 \\
1,386 \\
1,385 \\
1,385 \\
1,385 \\
1,385 \\
1,385 \\
1,386 \\
1,386 \\
1,388 \\
1,388 \\
1,392\end{array}$ & $\begin{array}{l}661 \\
548 \\
465 \\
470 \\
563 \\
666 \\
764 \\
853 \\
933 \\
992 \\
943 \\
778\end{array}$ & $\begin{array}{l}2,047 \\
1,934 \\
1,850 \\
1,854 \\
1,948 \\
2,051 \\
2,150 \\
2,239 \\
2,320 \\
2,380 \\
2,331 \\
2,170\end{array}$ & $\begin{array}{r}18 \\
-13 \\
-29 \\
-67 \\
-63 \\
-52 \\
-37 \\
-20 \\
-3 \\
6 \\
6 \\
-61\end{array}$ & $\begin{array}{r}2.7 \\
-2.4 \\
-5.9 \\
-12.5 \\
-10.1 \\
-7.3 \\
-4.6 \\
-2.3 \\
-.3 \\
.6 \\
.6 \\
-7.3\end{array}$ & $\begin{array}{r}8 \\
15 \\
16 \\
47 \\
93 \\
113 \\
103 \\
103 \\
106 \\
80 \\
21 \\
11\end{array}$ & $\begin{array}{r}182 \\
124 \\
98 \\
42 \\
11 \\
11 \\
16 \\
18 \\
21 \\
22 \\
67 \\
171\end{array}$ & $\begin{array}{r}-174 \\
-109 \\
-82 \\
5 \\
82 \\
102 \\
87 \\
85 \\
85 \\
57 \\
-46 \\
-160\end{array}$ \\
\hline \multicolumn{9}{|l|}{1993} \\
\hline 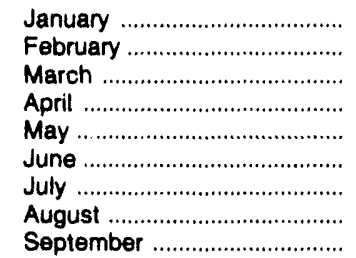 & $\begin{array}{l}1,392 \\
1,381 \\
1,377 \\
1,378 \\
1,381 \\
1,381 \\
1,380 \\
1,382 \\
1,380\end{array}$ & $\begin{array}{l}603 \\
468 \\
408 \\
439 \\
547 \\
638 \\
731 \\
810 \\
883\end{array}$ & $\begin{array}{l}1,994 \\
1,850 \\
1,786 \\
1,816 \\
1,927 \\
2,018 \\
2,111 \\
2,192 \\
2,263\end{array}$ & $\begin{array}{l}-59 \\
-80 \\
-57 \\
-31 \\
-16 \\
-29 \\
-33 \\
-43 \\
-50\end{array}$ & $\begin{array}{r}-8.9 \\
-14.6 \\
-12.2 \\
-6.6 \\
-2.9 \\
-4.3 \\
-4.3 \\
-5.0 \\
-5.4\end{array}$ & $\begin{array}{r}7 \\
10 \\
31 \\
72 \\
120 \\
108 \\
112 \\
101 \\
104\end{array}$ & $\begin{array}{r}184 \\
153 \\
100 \\
33 \\
9 \\
17 \\
19 \\
21 \\
13\end{array}$ & $\begin{array}{r}-177 \\
-143 \\
-69 \\
39 \\
110 \\
91 \\
93 \\
81 \\
91\end{array}$ \\
\hline
\end{tabular}

- Total as of December 31 .

b Total underground storage capacity at the end of each calendar year (in billion cubic feet): 1987, 1988, and 1989 - 2,502; 1990 - 2,503; 1991 - 2,481; and $1992 \cdot 2,407$.

- Positive numbers indicate the volume of injections in excess of withdrawals. Negative numbers indicate the volume of withdrawals in excess of injections.

A $=$ Revised Data.

- $=$ Not Applicable.

Notes: Data for 1987 through 1992 are final. All other data are preliminary unless otherwise noted. See Appendix A, Explanatory Note 7 for discussion of revision policy. Gas in storage at the end of a reporting period may not equal the quantity derived by adding or subtracting net injections or withdrawals during the period to the quantity of gas in storage at the beginning of the period. This is due to changes in the quantities of native gas included in base gas and/or losses in base gas due to migration from storage reservoirs. Totals may not equal sum of components because of independent rounding. Geographic coverage is the 50 States and the District of Columbia.

Sources: Form EIA-191, Form FERC-8, and EIA-176. 
Table 16. Net Withdrawals from Underground Storage, by State, 1991-1993 (Volume in Million Cubic Feet)

\begin{tabular}{|c|c|c|c|c|c|c|c|}
\hline States & $\begin{array}{c}\text { September } \\
1983\end{array}$ & $\begin{array}{c}\text { August } \\
1993\end{array}$ & $\begin{array}{l}\text { July } \\
1993\end{array}$ & $\begin{array}{l}\text { June } \\
1893\end{array}$ & $\begin{array}{l}\text { May } \\
1093\end{array}$ & $\begin{array}{l}\text { April } \\
1983\end{array}$ & $\begin{array}{c}\text { March } \\
1893\end{array}$ \\
\hline 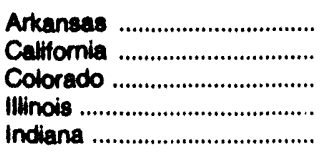 & $\begin{array}{r}0 \\
-14,928 \\
-4,187 \\
-36,219 \\
-4,192\end{array}$ & $\begin{array}{r}0 \\
-10,526 \\
-4,540 \\
-33,793 \\
-4,509\end{array}$ & $\begin{array}{r}10 \\
-23,040 \\
-3,880 \\
-30,687 \\
-4,420\end{array}$ & $\begin{array}{r}-14 \\
-13,993 \\
-4,646 \\
-32,862 \\
-2,089\end{array}$ & $\begin{array}{r}824 \\
-28,521 \\
-2,713 \\
-33,685 \\
-1,257\end{array}$ & $\begin{array}{r}121 \\
-15,646 \\
3,405 \\
-2,881 \\
-400\end{array}$ & $\begin{array}{r}478 \\
-6,478 \\
2,509 \\
25,052 \\
2,558\end{array}$ \\
\hline 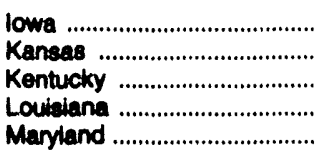 & $\begin{array}{r}-12,617 \\
-14,328 \\
-6,357 \\
-35,450 \\
-2,547\end{array}$ & $\begin{array}{r}-10,344 \\
-8,785 \\
-4,815 \\
-27,651 \\
-1,718\end{array}$ & $\begin{array}{r}-10,896 \\
-5,456 \\
-7,557 \\
-27,479 \\
-1,126\end{array}$ & $\begin{array}{r}-8,100 \\
-11,747 \\
-7,759 \\
-33,849 \\
-1,175\end{array}$ & $\begin{array}{r}-6,824 \\
-24,818 \\
-6,426 \\
-28,839 \\
-2,217\end{array}$ & $\begin{array}{r}-5,491 \\
-3,308 \\
726 \\
592 \\
456\end{array}$ & $\begin{array}{r}2,650 \\
4,688 \\
9,515 \\
23,512 \\
1,859\end{array}$ \\
\hline 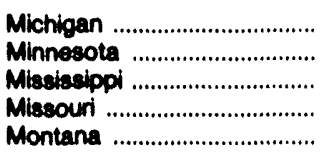 & $\begin{array}{r}-66,961 \\
-238 \\
-5,481 \\
-206 \\
-453\end{array}$ & $\begin{array}{r}-82,034 \\
-128 \\
-3,438 \\
-317 \\
-302\end{array}$ & $\begin{array}{r}-84,803 \\
-409 \\
-5,007 \\
-102 \\
-1,169\end{array}$ & $\begin{array}{r}-85,218 \\
-344 \\
-4,556 \\
-451 \\
685\end{array}$ & $\begin{array}{r}-75,304 \\
-318 \\
-7,012 \\
-1,049 \\
1,157\end{array}$ & $\begin{array}{r}-20,444 \\
49 \\
-2,489 \\
-1,551 \\
1,710\end{array}$ & $\begin{array}{r}65,891 \\
418 \\
6,098 \\
1,085 \\
2,759\end{array}$ \\
\hline 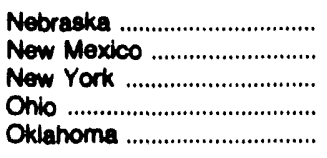 & $\begin{array}{r}-1,539 \\
-612 \\
-7,364 \\
-25,635 \\
-15,942\end{array}$ & $\begin{array}{r}-1,857 \\
2,318 \\
-8,269 \\
-26,949 \\
-13,432\end{array}$ & $\begin{array}{r}-2,561 \\
-269 \\
-10,095 \\
-29,068 \\
-14,917\end{array}$ & $\begin{array}{r}-4,278 \\
-1,221 \\
-10,768 \\
-31,671 \\
-17,825\end{array}$ & $\begin{array}{r}-2,958 \\
-2,705 \\
-10,921 \\
-29,544 \\
-20,586\end{array}$ & $\begin{array}{r}-975 \\
2,927 \\
-3,964 \\
-3,384 \\
-12,860\end{array}$ & $\begin{array}{r}1,010 \\
792 \\
10,303 \\
38,459 \\
8,856\end{array}$ \\
\hline 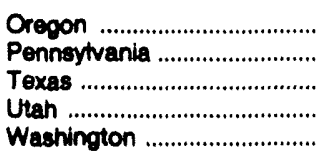 & $\begin{array}{r}-578 \\
-49,805 \\
-18,525 \\
-4,836 \\
-1,979\end{array}$ & $\begin{array}{r}-1,042 \\
-40,777 \\
-11,964 \\
-5,153 \\
-620\end{array}$ & $\begin{array}{r}-1,181 \\
-46,928 \\
-9,730 \\
-5,170 \\
-2,148\end{array}$ & $\begin{array}{r}-1,298 \\
-53,535 \\
-8,376 \\
-6,636 \\
-886\end{array}$ & $\begin{array}{r}-1,645 \\
-57,881 \\
-27,775 \\
-6,685 \\
-4,528\end{array}$ & $\begin{array}{r}426 \\
-18,784 \\
-19,952 \\
1,016 \\
-1,002\end{array}$ & $\begin{array}{r}728 \\
56,156 \\
12,328 \\
3,663 \\
-1,231\end{array}$ \\
\hline 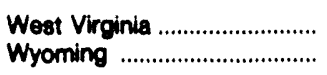 & $\begin{array}{r}-20,458 \\
-939\end{array}$ & $\begin{array}{r}-18,781 \\
-1,625\end{array}$ & $\begin{array}{r}-20,172 \\
-2,051\end{array}$ & $\begin{array}{r}-27,572 \\
-2,069\end{array}$ & $\begin{array}{r}-39,358 \\
-983\end{array}$ & $\begin{array}{r}-5,496 \\
-500\end{array}$ & $\begin{array}{r}29,813 \\
371\end{array}$ \\
\hline 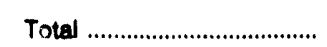 & $-352,488$ & $-321,050$ & $-350,311$ & $-372,334$ & $-422,670$ & $-107,702$ & 303,841 \\
\hline
\end{tabular}

See tootnotes at end of table. 
Table 16. Net Withdrawals from Underground Storage, by State, 1991-1993 (Volume in Million Cubic Feet)-Continued

\begin{tabular}{|c|c|c|c|c|c|c|c|}
\hline States & $\begin{array}{c}\text { Fobruary } \\
1993\end{array}$ & $\begin{array}{c}\text { January } \\
1993\end{array}$ & $\begin{array}{l}\text { Total } \\
1992\end{array}$ & $\begin{array}{l}\text { December } \\
1992\end{array}$ & $\begin{array}{c}\text { November } \\
1992\end{array}$ & $\begin{array}{l}\text { October } \\
1992\end{array}$ & $\begin{array}{c}\text { September } \\
1992\end{array}$ \\
\hline 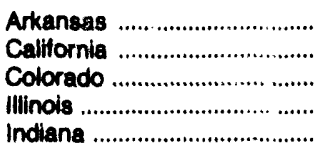 & $\begin{array}{r}872 \\
17,346 \\
5,116 \\
49,451 \\
5,026\end{array}$ & $\begin{array}{r}633 \\
37,972 \\
3,207 \\
59,730 \\
5,572\end{array}$ & $\begin{array}{r}514 \\
28,120 \\
4,860 \\
8,608 \\
840\end{array}$ & $\begin{array}{r}624 \\
54,629 \\
2,582 \\
42,048 \\
3,610\end{array}$ & $\begin{array}{r}366 \\
9,135 \\
3,085 \\
14,263 \\
2,364\end{array}$ & $\begin{array}{r}196 \\
-8,186 \\
-2,169 \\
-26,155 \\
-2,936\end{array}$ & $\begin{array}{r}-434 \\
-17,264 \\
-5,922 \\
-32,462 \\
-5,203\end{array}$ \\
\hline 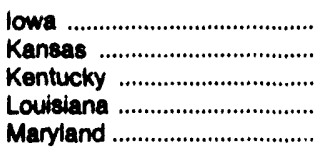 & $\begin{array}{r}10,789 \\
15,609 \\
10,958 \\
43,797 \\
4,290\end{array}$ & $\begin{array}{r}18,971 \\
18,609 \\
5,114 \\
47,855 \\
3,272\end{array}$ & $\begin{array}{r}4,259 \\
18,486 \\
-6,573 \\
13,958 \\
-333\end{array}$ & $\begin{array}{r}18,942 \\
12,181 \\
2,982 \\
31,771 \\
2,249\end{array}$ & $\begin{array}{l}7,997 \\
9,027 \\
1,514 \\
5,495 \\
-544\end{array}$ & $\begin{array}{r}-8,683 \\
-3,672 \\
-10,704 \\
-18,911 \\
-1,333\end{array}$ & $\begin{array}{r}-10,088 \\
-10,142 \\
-4,201 \\
-16,545 \\
-2,204\end{array}$ \\
\hline 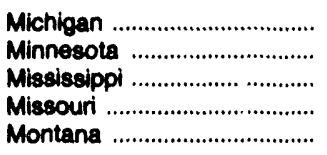 & $\begin{array}{r}103,072 \\
418 \\
8,450 \\
1,811 \\
4,420\end{array}$ & $\begin{array}{r}97,623 \\
396 \\
5,863 \\
1,091 \\
5,100\end{array}$ & $\begin{array}{r}2,251 \\
-43 \\
-1,732 \\
15 \\
12,602\end{array}$ & $\begin{array}{r}84,911 \\
193 \\
6,940 \\
7 \\
5,601\end{array}$ & $\begin{array}{r}47,094 \\
7 \\
998 \\
8 \\
3,347\end{array}$ & $\begin{array}{r}-28,345 \\
4 \\
-267 \\
-191 \\
1,357\end{array}$ & $\begin{array}{r}-67,401 \\
-126 \\
-6,250 \\
-208 \\
280\end{array}$ \\
\hline 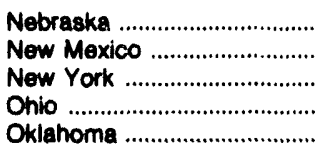 & $\begin{array}{r}2,009 \\
4,164 \\
16,122 \\
45,111 \\
21,531\end{array}$ & $\begin{array}{r}3,344 \\
2,613 \\
13,106 \\
38,493 \\
23,866\end{array}$ & $\begin{array}{r}-407 \\
2,458 \\
-1,572 \\
15,672 \\
10,058\end{array}$ & $\begin{array}{r}3,451 \\
-417 \\
10,534 \\
31,661 \\
31,363\end{array}$ & $\begin{array}{r}2,461 \\
1,036 \\
3,023 \\
5,352 \\
16,578\end{array}$ & $\begin{array}{r}316 \\
-2,559 \\
-3,455 \\
-11,598 \\
-7,728\end{array}$ & $\begin{array}{r}-1,167 \\
-1,172 \\
-6,488 \\
-15,846 \\
-12,709\end{array}$ \\
\hline 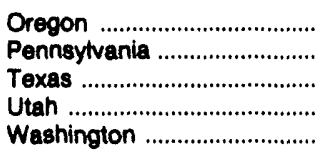 & $\begin{array}{r}1,397 \\
100,856 \\
24,856 \\
5,057 \\
3,347\end{array}$ & $\begin{array}{r}1,918 \\
71,301 \\
45,359 \\
5,873 \\
3,439\end{array}$ & $\begin{array}{r}872 \\
-5,873 \\
43,441 \\
-4,482 \\
5,667\end{array}$ & $\begin{array}{r}579 \\
57,665 \\
55,358 \\
6,756 \\
3,797\end{array}$ & $\begin{array}{r}1,100 \\
-2,844 \\
35,326 \\
5,453 \\
947\end{array}$ & $\begin{array}{r}-173 \\
-28,736 \\
-9,677 \\
-1,284 \\
760\end{array}$ & $\begin{array}{r}-453 \\
-47,631 \\
-15,735 \\
-3,128 \\
-1,196\end{array}$ \\
\hline 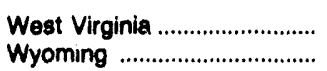 & $\begin{array}{r}43,205 \\
2,388\end{array}$ & $\begin{array}{r}31,863 \\
3,361\end{array}$ & $\begin{array}{l}8,180 \\
8,536\end{array}$ & $\begin{array}{r}17,912 \\
3,440\end{array}$ & $\begin{array}{l}6,875 \\
1,735\end{array}$ & $\begin{array}{r}-12,723 \\
1,034\end{array}$ & $\begin{array}{r}-13,281 \\
-814\end{array}$ \\
\hline 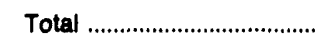 & 551,578 & 555,745 & 168,381 & 491,369 & 181,199 & $-185,819$ & $-297,793$ \\
\hline
\end{tabular}


Table 16. Net Withdrawals from Underground Storage, by State, 1991-1993

(Volume in Million Cubic Feet)-Continued

\begin{tabular}{|c|c|c|c|c|c|c|c|}
\hline States & $\begin{array}{l}\text { August } \\
1992\end{array}$ & $\begin{array}{l}\text { July } \\
1992\end{array}$ & $\begin{array}{l}\text { June } \\
1992\end{array}$ & $\begin{array}{l}\text { May } \\
1992\end{array}$ & $\begin{array}{l}\text { April } \\
1992\end{array}$ & $\begin{array}{l}\text { March } \\
1992\end{array}$ & $\begin{array}{c}\text { February } \\
1992\end{array}$ \\
\hline 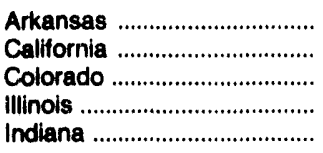 & $\begin{array}{r}-664 \\
-10,887 \\
-6,286 \\
-35,313 \\
-4,107\end{array}$ & $\begin{array}{r}-852 \\
-15,281 \\
-4,177 \\
-28,140 \\
-5,123\end{array}$ & $\begin{array}{r}-509 \\
-23,985 \\
-2,019 \\
-29,861 \\
-2,563\end{array}$ & $\begin{array}{r}16 \\
-15,170 \\
368 \\
-31,163 \\
-1,206\end{array}$ & $\begin{array}{r}159 \\
-15,220 \\
4,146 \\
8,578 \\
500\end{array}$ & $\begin{array}{r}443 \\
14,214 \\
6,690 \\
25,247 \\
4,104\end{array}$ & $\begin{array}{r}519 \\
15,305 \\
2,758 \\
43,448 \\
4,606\end{array}$ \\
\hline 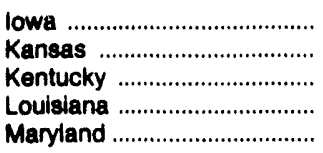 & $\begin{array}{r}-10,162 \\
-11,527 \\
-1,610 \\
-13,386 \\
-2,546\end{array}$ & $\begin{array}{r}-10,141 \\
-4,255 \\
-3,544 \\
-13,811 \\
-1,942\end{array}$ & $\begin{array}{r}-7,264 \\
-2,319 \\
-1,546 \\
-18,063 \\
-2,867\end{array}$ & $\begin{array}{r}-7,583 \\
-2,161 \\
1,236 \\
-19,021 \\
-3,527\end{array}$ & $\begin{array}{r}-2,061 \\
4,821 \\
1,675 \\
-719 \\
958\end{array}$ & $\begin{array}{r}3,325 \\
9,031 \\
4,848 \\
10,774 \\
2,840\end{array}$ & $\begin{array}{r}11,535 \\
13,082 \\
1,211 \\
24,031 \\
5,269\end{array}$ \\
\hline 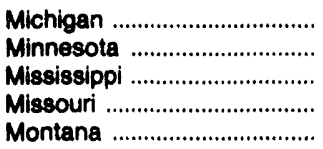 & $\begin{array}{r}-67,079 \\
-184 \\
-297 \\
-218 \\
-766\end{array}$ & $\begin{array}{r}-73,161 \\
-289 \\
-4,165 \\
-266 \\
-1,026\end{array}$ & $\begin{array}{r}-66,808 \\
-307 \\
-7,931 \\
-146 \\
-714\end{array}$ & $\begin{array}{r}-43,850 \\
-391 \\
-3,896 \\
-427 \\
-1,517\end{array}$ & $\begin{array}{r}-507 \\
198 \\
-106 \\
-1,570 \\
-63\end{array}$ & $\begin{array}{r}62,627 \\
311 \\
2,086 \\
330 \\
484\end{array}$ & $\begin{array}{r}72,236 \\
123 \\
1,362 \\
1,199 \\
2,207\end{array}$ \\
\hline 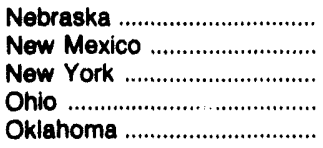 & $\begin{array}{r}-1,187 \\
-1,949 \\
-9,745 \\
-23,106 \\
-5,444\end{array}$ & $\begin{array}{r}-2,083 \\
2,103 \\
-10,849 \\
-28,770 \\
-16,356\end{array}$ & $\begin{array}{r}-2,611 \\
-542 \\
-9,127 \\
-29,912 \\
-11,329\end{array}$ & $\begin{array}{r}-1,609 \\
95 \\
-9,430 \\
-26,466 \\
-8,448\end{array}$ & $\begin{array}{r}-604 \\
2,400 \\
-2,328 \\
-2,246 \\
-5,813\end{array}$ & $\begin{array}{r}46 \\
734 \\
11,629 \\
36,501 \\
3,738\end{array}$ & $\begin{array}{r}1,041 \\
710 \\
13,614 \\
35,632 \\
8,339\end{array}$ \\
\hline 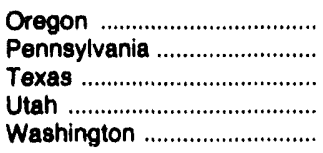 & $\begin{array}{r}-1,010 \\
-48,824 \\
-28,309 \\
-4,680 \\
-1,458\end{array}$ & $\begin{array}{r}-1,194 \\
-41,339 \\
-17,447 \\
-3,526 \\
-1,024\end{array}$ & $\begin{array}{r}-1,540 \\
-58,946 \\
-16,212 \\
-2,776 \\
-1,175\end{array}$ & $\begin{array}{r}-1,572 \\
-45,507 \\
-26,278 \\
-3,514 \\
-1,351\end{array}$ & $\begin{array}{r}443 \\
-6,017 \\
-2,096 \\
-1,845 \\
877\end{array}$ & $\begin{array}{r}1,586 \\
61,022 \\
4,693 \\
-252 \\
581\end{array}$ & $\begin{array}{r}1,540 \\
73,709 \\
20,462 \\
415 \\
2,979\end{array}$ \\
\hline 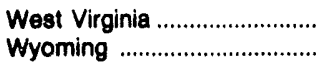 & $\begin{array}{r}-18,576 \\
-101\end{array}$ & $\begin{array}{r}-19,023 \\
-1,069\end{array}$ & $\begin{array}{r}-21,294 \\
-568\end{array}$ & $\begin{array}{r}-24,410 \\
-343\end{array}$ & $\begin{array}{r}-1,169 \\
84\end{array}$ & $\begin{array}{r}32,072 \\
866\end{array}$ & $\begin{array}{r}29,522 \\
2,005\end{array}$ \\
\hline Total & $-309,424$ & $-306,761$ & $-322,935$ & $-277,127$ & $-17,528$ & 300,570 & 388,858 \\
\hline
\end{tabular}


Table 16. Net Withdrawals from Underground Storage, by State, 1991-1993 (Volume in Million Cubic Feet)-Continued

\begin{tabular}{|c|c|c|c|c|c|c|}
\hline States & $\begin{array}{l}\text { January } \\
1892\end{array}$ & $\begin{array}{l}\text { Total } \\
1991\end{array}$ & $\begin{array}{l}\text { December } \\
1991\end{array}$ & $\begin{array}{c}\text { November } \\
1991\end{array}$ & $\begin{array}{l}\text { October } \\
1991\end{array}$ & $\begin{array}{l}\text { Septernber } \\
1991\end{array}$ \\
\hline 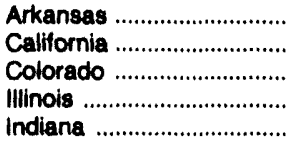 & $\begin{array}{r}652 \\
40,831 \\
5,804 \\
58,118 \\
6,794\end{array}$ & $\begin{array}{r}1,866 \\
-12,276 \\
-7,370 \\
878 \\
926\end{array}$ & $\begin{array}{r}684 \\
24,681 \\
588 \\
34,113 \\
4,626\end{array}$ & $\begin{array}{r}629 \\
6,113 \\
-1,468 \\
17,558 \\
-802\end{array}$ & $\begin{array}{r}0 \\
879 \\
-1,759 \\
-26,390 \\
-4,105\end{array}$ & $\begin{array}{r}0 \\
1,455 \\
-5,275 \\
-32,897 \\
-4,467\end{array}$ \\
\hline 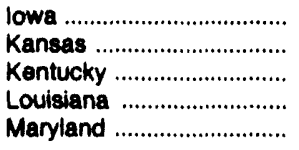 & $\begin{array}{r}18,442 \\
4,420 \\
1,568 \\
42,343 \\
3,314\end{array}$ & $\begin{array}{r}5,207 \\
-10,822 \\
1,397 \\
24,889 \\
-1,852\end{array}$ & $\begin{array}{r}13,739 \\
-2,317 \\
2,686 \\
30,577 \\
1,070\end{array}$ & $\begin{array}{r}4,997 \\
8,913 \\
6,590 \\
12,743 \\
-334\end{array}$ & $\begin{array}{r}-10,757 \\
-10,235 \\
460 \\
-23,315 \\
-800\end{array}$ & $\begin{array}{r}-10,675 \\
-10,203 \\
-3,437 \\
-18,632 \\
-2,084\end{array}$ \\
\hline 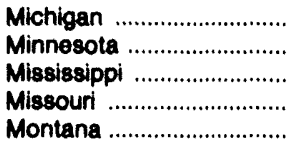 & $\begin{array}{r}82,535 \\
426 \\
9,794 \\
1,498 \\
3,412\end{array}$ & $\begin{array}{r}39,200 \\
-267 \\
5,884 \\
-1,117 \\
9,737\end{array}$ & $\begin{array}{r}74,384 \\
53 \\
6,130 \\
12 \\
3,548\end{array}$ & $\begin{array}{r}56,578 \\
77 \\
7,864 \\
-199 \\
2,875\end{array}$ & $\begin{array}{r}-32,872 \\
32 \\
-3,107 \\
-218 \\
1,379\end{array}$ & $\begin{array}{r}-27.703 \\
-93 \\
-5.101 \\
-212 \\
-120\end{array}$ \\
\hline 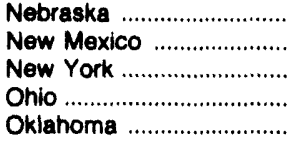 & $\begin{array}{r}1,540 \\
2,019 \\
11,051 \\
44,471 \\
17,866\end{array}$ & $\begin{array}{r}-229 \\
-3,694 \\
-11,619 \\
-431 \\
12,139\end{array}$ & $\begin{array}{r}2,362 \\
-1,002 \\
7,125 \\
27,810 \\
11,502\end{array}$ & $\begin{array}{r}402 \\
147 \\
3,662 \\
12,644 \\
26,655\end{array}$ & $\begin{array}{r}154 \\
9 \\
-5,237 \\
-9,113 \\
-4,702\end{array}$ & $\begin{array}{r}-901 \\
-2,642 \\
-8,681 \\
-16,055 \\
-10,249\end{array}$ \\
\hline 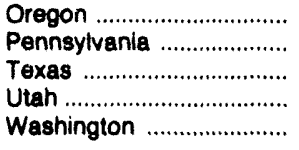 & $\begin{array}{r}1,568 \\
81,577 \\
43,357 \\
3,899 \\
1,930\end{array}$ & $\begin{array}{r}-566 \\
11,335 \\
-6,398 \\
9,976 \\
-3,200\end{array}$ & $\begin{array}{r}487 \\
37,591 \\
9,801 \\
7,418 \\
458\end{array}$ & $\begin{array}{r}-23 \\
10,030 \\
8,300 \\
5,792 \\
-1,040\end{array}$ & $\begin{array}{r}-339 \\
-13,835 \\
-856 \\
-560 \\
1,040\end{array}$ & $\begin{array}{r}-674 \\
-34,526 \\
-10,004 \\
-3,940 \\
5\end{array}$ \\
\hline $\begin{array}{l}\text { West Virginia } \\
\text { Wyoming }\end{array}$ & $\begin{array}{r}32,275 \\
2,269\end{array}$ & $\begin{array}{r}16,888 \\
-426\end{array}$ & $\begin{array}{r}22,834 \\
1,941\end{array}$ & $\begin{array}{r}11,077 \\
1,809\end{array}$ & $\begin{array}{r}-3,606 \\
-54\end{array}$ & $\begin{array}{r}-8,516 \\
-761\end{array}$ \\
\hline 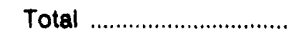 & 523,770 & 80,355 & 322,991 & 201,590 & $-147,808$ & $-216,387$ \\
\hline
\end{tabular}

Notes: This table contains total net withdrawals for each State with natural gas storage facilities. Positive numbers indicate the volume of withdrawals in excess of injections. Negative values indicate the volume of injections in excess of withdrawals. Data for "1991 and 1982" are final. All other data are preliminary at this time and are not considered final until publication of the Natural Gas Annual for that year.

Source: Form EIA-191. 
Table 17. Activities of Underground Natural Gas Storage Operators, by State, September 1993

(Volumes in Million Cubic Feet)

\begin{tabular}{|c|c|c|c|c|c|c|c|c|}
\hline \multirow[t]{2}{*}{ State } & \multirow{2}{*}{$\begin{array}{l}\text { Total } \\
\text { Storage } \\
\text { Capacity }\end{array}$} & \multicolumn{3}{|c|}{$\begin{array}{l}\text { Natural Gas in } \\
\text { Underground Storage } \\
\text { at End of Period }\end{array}$} & \multicolumn{2}{|c|}{$\begin{array}{l}\text { Change in Working Gas } \\
\text { from Same Period } \\
\text { Previous Year }\end{array}$} & \multicolumn{2}{|c|}{ Storage Activity } \\
\hline & & $\begin{array}{c}\text { Base } \\
\text { Gas }\end{array}$ & $\begin{array}{l}\text { Working } \\
\text { Gas }\end{array}$ & Total & Volume & Percent & Injections & Withdrawals \\
\hline 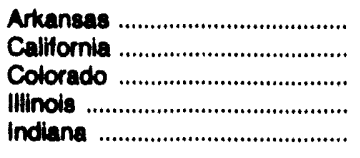 & $\begin{array}{r}31,278 \\
472,109 \\
105,780 \\
950,732 \\
108,677\end{array}$ & $\begin{array}{r}17,888 \\
248,944 \\
45,210 \\
623,466 \\
71,407\end{array}$ & $\begin{array}{r}4,969 \\
199,629 \\
36,057 \\
261,204 \\
33,303\end{array}$ & $\begin{array}{r}22,856 \\
448,573 \\
81,266 \\
884,669 \\
104,709\end{array}$ & $\begin{array}{r}-2,890 \\
2,580 \\
-713 \\
-40,897 \\
876\end{array}$ & $\begin{array}{r}-37.6 \\
1.3 \\
-1.9 \\
-13.5 \\
2.7\end{array}$ & $\begin{array}{r}0 \\
17,604 \\
4,288 \\
38,446 \\
4,412\end{array}$ & $\begin{array}{r}0 \\
2,676 \\
101 \\
2,227 \\
220\end{array}$ \\
\hline 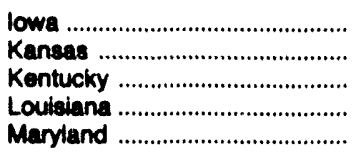 & $\begin{array}{r}279,700 \\
291,058 \\
210,242 \\
539,200 \\
62,400\end{array}$ & $\begin{array}{r}200,700 \\
186,188 \\
105,481 \\
252,953 \\
46,677\end{array}$ & $\begin{array}{r}58,949 \\
89,934 \\
98,956 \\
210,143 \\
11,746\end{array}$ & $\begin{array}{r}259,649 \\
276,121 \\
204,437 \\
463,097 \\
58,423\end{array}$ & $\begin{array}{r}-44,848 \\
10,177 \\
12,748 \\
35,589 \\
-1,700\end{array}$ & $\begin{array}{r}-43.2 \\
12.8 \\
14.8 \\
20.4 \\
-12.6\end{array}$ & $\begin{array}{r}12,617 \\
15,748 \\
6,901 \\
36,447 \\
2,547\end{array}$ & $\begin{array}{r}0 \\
1,420 \\
544 \\
997 \\
0\end{array}$ \\
\hline 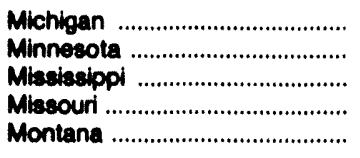 & $\begin{array}{r}1,043,782 \\
7,000 \\
114,627 \\
30,564 \\
375,010\end{array}$ & $\begin{array}{r}420,562 \\
4,655 \\
48,049 \\
21,600 \\
169,892\end{array}$ & $\begin{array}{r}547,612 \\
2,260 \\
61,026 \\
8,627 \\
88,012\end{array}$ & $\begin{array}{r}968,174 \\
6,916 \\
109,075 \\
30,227 \\
257,904\end{array}$ & $\begin{array}{r}43,300 \\
-58 \\
2,671 \\
-136 \\
-24,213\end{array}$ & $\begin{array}{r}8.6 \\
-2.5 \\
4.6 \\
-1.6 \\
-21.6\end{array}$ & $\begin{array}{r}68,548 \\
238 \\
7,280 \\
211 \\
1,608\end{array}$ & $\begin{array}{r}1,587 \\
0 \\
1,789 \\
5 \\
1,154\end{array}$ \\
\hline 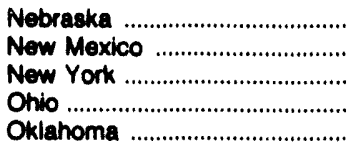 & $\begin{array}{r}93,312 \\
94,600 \\
167,835 \\
594,644 \\
359,616\end{array}$ & $\begin{array}{r}27,312 \\
30,426 \\
108,690 \\
358,924 \\
229,765\end{array}$ & $\begin{array}{r}61,281 \\
8,356 \\
63,952 \\
157,070 \\
117,788\end{array}$ & $\begin{array}{r}88,593 \\
38,782 \\
172,642 \\
515,994 \\
347,554\end{array}$ & $\begin{array}{r}439 \\
-5,056 \\
-8,772 \\
-1,489 \\
-48,848\end{array}$ & $\begin{array}{r}.7 \\
-37.7 \\
-12.1 \\
-.9 \\
-29.3\end{array}$ & $\begin{array}{r}1,614 \\
1,358 \\
8,546 \\
26,057 \\
16,427\end{array}$ & $\begin{array}{r}75 \\
746 \\
1,183 \\
423 \\
485\end{array}$ \\
\hline 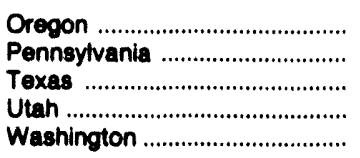 & $\begin{array}{r}11,445 \\
664,684 \\
598,783 \\
114,980 \\
34,100\end{array}$ & $\begin{array}{r}3,291 \\
359,530 \\
234,210 \\
54,873 \\
18,800\end{array}$ & $\begin{array}{r}7,750 \\
320,921 \\
214,597 \\
24,983 \\
15,508\end{array}$ & $\begin{array}{r}11,041 \\
680,452 \\
448,807 \\
79,956 \\
34,308\end{array}$ & $\begin{array}{r}-231 \\
14,808 \\
-126,560 \\
2,435 \\
975\end{array}$ & $\begin{array}{r}-2.9 \\
4.8 \\
-37.1 \\
10.8 \\
6.7\end{array}$ & $\begin{array}{r}578 \\
52,435 \\
24,800 \\
4,842 \\
2,383\end{array}$ & $\begin{array}{r}0 \\
2,530 \\
6,275 \\
5 \\
404\end{array}$ \\
\hline 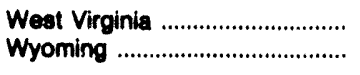 & $\begin{array}{l}519,286 \\
105,669\end{array}$ & $\begin{array}{r}304,701 \\
59,427\end{array}$ & $\begin{array}{r}170,597 \\
25,868\end{array}$ & $\begin{array}{r}475,297 \\
85,295\end{array}$ & $\begin{array}{r}41,628 \\
-4,687\end{array}$ & $\begin{array}{r}32.3 \\
-15.3\end{array}$ & $\begin{array}{r}20,858 \\
1,020\end{array}$ & $\begin{array}{r}400 \\
81\end{array}$ \\
\hline Total & $7,979,132$ & $4,253,720$ & $2,901,098$ & $7,154,818$ & $-142,972$ & -4.7 & 377,814 & 25,326 \\
\hline
\end{tabular}

Notes: Gas in storage at the end of a reporting period may not equal the quantity derived by adding or subtracting net injections or withdrawals during the period to the quantity of gas in storage at the beginning of the period. This is due to changes in the quantities of native gas included in base gas and/or losses in base gas due to migration from storage reservoirs. Totals may not equal sum of components because of independent rounding. Geographic coverage is the 50 States and the District of Columbia.

Source: Form EIA-181. 
Table 18. Natural Gas Deliveries to Residential Consumers by State, 1992-1993 (Million Cubic Feet)

\begin{tabular}{|c|c|c|c|c|c|c|}
\hline State & $\begin{array}{c}\text { YTD } \\
1993\end{array}$ & $\begin{array}{l}\text { YTD } \\
1992 \\
\end{array}$ & $\begin{array}{l}\text { YTD } \\
1991\end{array}$ & $\begin{array}{c}\text { August } \\
1893\end{array}$ & $\begin{array}{c}\text { July } \\
1893\end{array}$ & $\begin{array}{l}\text { June } \\
1993\end{array}$ \\
\hline 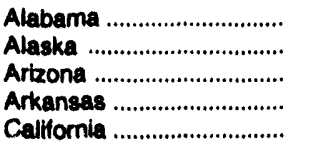 & $\begin{array}{r}37,824 \\
9,268 \\
19,918 \\
32,057 \\
341,266\end{array}$ & $\begin{array}{r}35,880 \\
9,373 \\
19,822 \\
27,550 \\
320,577\end{array}$ & $\begin{array}{r}32,685 \\
8,980 \\
22,818 \\
28,570 \\
352,829\end{array}$ & $\begin{array}{r}1,326 \\
402 \\
802 \\
1,017 \\
22,743\end{array}$ & $\begin{array}{r}1,426 \\
423 \\
833 \\
1,109 \\
23,641\end{array}$ & $\begin{array}{r}1,636 \\
577 \\
1,097 \\
1,309 \\
25,109\end{array}$ \\
\hline $\begin{array}{l}\text { Colorado .......................... } \\
\text { Connecticut ........................ } \\
\text { Delaware .......................... } \\
\text { Diatrict of Columbia .......... } \\
\text { Florida ................................. }\end{array}$ & $\begin{array}{r}74,992 \\
29,269 \\
6,222 \\
12,200 \\
9,864\end{array}$ & $\begin{array}{r}65,101 \\
29,237 \\
6,032 \\
11,971 \\
10,337\end{array}$ & $\begin{array}{r}69,448 \\
25,510 \\
5,315 \\
11,014 \\
8,796\end{array}$ & $\begin{array}{r}2,384 \\
859 \\
167 \\
414 \\
611\end{array}$ & $\begin{array}{r}2,843 \\
1,026 \\
184 \\
432 \\
671\end{array}$ & $\begin{array}{r}3,985 \\
1,257 \\
242 \\
540 \\
764\end{array}$ \\
\hline 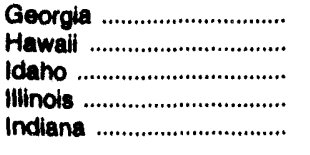 & $\begin{array}{r}74,549 \\
380 \\
8,519 \\
321,863 \\
108,837\end{array}$ & $\begin{array}{r}68,230 \\
374 \\
5,987 \\
300,279 \\
100,158\end{array}$ & $\begin{array}{r}60,649 \\
368 \\
7,045 \\
291,432 \\
95,426\end{array}$ & $\begin{array}{r}2,922 \\
41 \\
250 \\
9,736 \\
3,080\end{array}$ & $\begin{array}{r}3,013 \\
46 \\
301 \\
10,380 \\
3,140\end{array}$ & $\begin{array}{r}3,111 \\
47 \\
333 \\
12,724 \\
4,346\end{array}$ \\
\hline 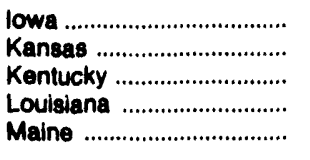 & $\begin{array}{r}56,412 \\
62,179 \\
42,822 \\
39,625 \\
586\end{array}$ & $\begin{array}{r}48,091 \\
48,468 \\
39,510 \\
38,389 \\
634\end{array}$ & $\begin{array}{r}51,679 \\
52,345 \\
37,500 \\
37,585 \\
502\end{array}$ & $\begin{array}{r}1,527 \\
1,684 \\
1,145 \\
1,837 \\
22\end{array}$ & $\begin{array}{r}1,566 \\
1,874 \\
1,299 \\
2,112 \\
22\end{array}$ & $\begin{array}{r}2,387 \\
2,459 \\
1,722 \\
2,217 \\
30\end{array}$ \\
\hline 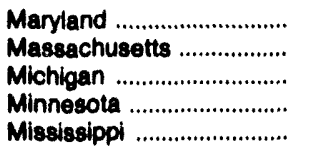 & $\begin{array}{r}53,040 \\
87,746 \\
249,499 \\
81,892 \\
20,275\end{array}$ & $\begin{array}{r}51,635 \\
86,715 \\
243,142 \\
72,691 \\
18,527\end{array}$ & $\begin{array}{r}46,797 \\
74,504 \\
226,847 \\
74,628 \\
17,925\end{array}$ & $\begin{array}{r}1,898 \\
3,090 \\
7,120 \\
2,389 \\
789\end{array}$ & $\begin{array}{r}1,943 \\
3,505 \\
8,494 \\
2,565 \\
831\end{array}$ & $\begin{array}{r}2,442 \\
4,472 \\
11,337 \\
3,564 \\
949\end{array}$ \\
\hline 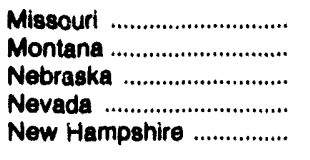 & $\begin{array}{r}98,616 \\
13,152 \\
37,042 \\
14,475 \\
4,693\end{array}$ & $\begin{array}{r}79,310 \\
9,984 \\
26,642 \\
12,321 \\
4,734\end{array}$ & $\begin{array}{r}83,881 \\
12,033 \\
29,755 \\
13,652 \\
4,062\end{array}$ & $\begin{array}{r}2,486 \\
526 \\
954 \\
612 \\
124\end{array}$ & $\begin{array}{r}2,870 \\
604 \\
1,068 \\
600 \\
149\end{array}$ & $\begin{array}{r}3,675 \\
639 \\
1,427 \\
910 \\
211\end{array}$ \\
\hline 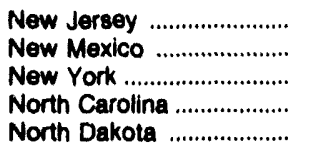 & $\begin{array}{r}141,260 \\
22,318 \\
274,944 \\
34,217 \\
7,521\end{array}$ & $\begin{array}{r}137,250 \\
22,326 \\
269,578 \\
29,736 \\
6,489\end{array}$ & $\begin{array}{r}121,751 \\
21,580 \\
237,115 \\
26,310 \\
6,997\end{array}$ & $\begin{array}{r}4,617 \\
828 \\
9,195 \\
762 \\
210\end{array}$ & $\begin{array}{r}5,104 \\
856 \\
10,140 \\
830 \\
268\end{array}$ & $\begin{array}{r}5,972 \\
1,062 \\
13,837 \\
1,040 \\
350\end{array}$ \\
\hline 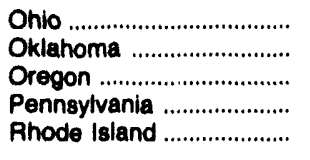 & $\begin{array}{r}237,531 \\
57,012 \\
20,893 \\
184,547 \\
14,727\end{array}$ & $\begin{array}{r}227,857 \\
45,443 \\
15,115 \\
182,310 \\
14,440\end{array}$ & $\begin{array}{r}212,827 \\
48,149 \\
19,371 \\
164,757 \\
12,383\end{array}$ & $\begin{array}{r}6,501 \\
1,616 \\
672 \\
5,668 \\
472\end{array}$ & $\begin{array}{r}7,220 \\
1,837 \\
768 \\
\text {. } 595 \\
558\end{array}$ & $\begin{array}{r}11,797 \\
2,338 \\
945 \\
8,528 \\
671\end{array}$ \\
\hline 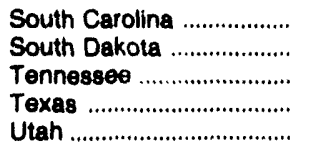 & $\begin{array}{r}17,458 \\
8,399 \\
40,454 \\
154,417 \\
35,713\end{array}$ & $\begin{array}{r}15,658 \\
6,810 \\
35,637 \\
143,709 \\
29,778\end{array}$ & $\begin{array}{r}13,485 \\
7,294 \\
33,689 \\
147,591 \\
35,288\end{array}$ & $\begin{array}{r}373 \\
236 \\
1,007 \\
7,393 \\
1,353\end{array}$ & $\begin{array}{r}402 \\
281 \\
1,102 \\
7,750 \\
1,533\end{array}$ & $\begin{array}{r}485 \\
370 \\
1,353 \\
8,441 \\
1,845\end{array}$ \\
\hline 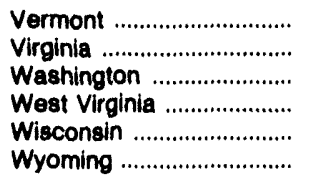 & $\begin{array}{r}1,879 \\
45,089 \\
37,554 \\
24,052 \\
86,182 \\
8,741\end{array}$ & $\begin{array}{r}1,826 \\
42,707 \\
27,335 \\
25,307 \\
80,234 \\
7,111\end{array}$ & $\begin{array}{r}1,544 \\
37,010 \\
32,662 \\
22,152 \\
79,800 \\
8,306\end{array}$ & $\begin{array}{r}45 \\
1,391 \\
1,270 \\
616 \\
2,570 \\
282\end{array}$ & $\begin{array}{r}50 \\
1,420 \\
1,455 \\
529 \\
2,487 \\
366\end{array}$ & $\begin{array}{r}84 \\
1,608 \\
2,676 \\
955 \\
3,726 \\
506\end{array}$ \\
\hline 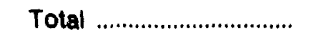 & $3,404,584$ & $3,158,355$ & $3,074,653$ & 120,146 & 129,524 & 164,118 \\
\hline
\end{tabular}

See footnote at end of table. 
Table 18. Natural Gas Dellveries to Residential Consumers by State, 1992-1993 (Continued)

(Million Cubic Feet)

\begin{tabular}{|c|c|c|c|c|c|c|}
\hline State & $\begin{array}{l}\text { May } \\
1993\end{array}$ & $\begin{array}{l}\text { April } \\
1993\end{array}$ & $\begin{array}{c}\text { March } \\
1993\end{array}$ & $\begin{array}{c}\text { February } \\
1993\end{array}$ & $\begin{array}{c}\text { January } \\
1993\end{array}$ & $\begin{array}{l}\text { Total } \\
1992\end{array}$ \\
\hline 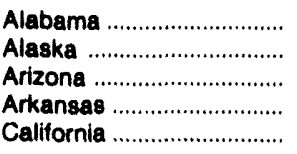 & $\begin{array}{r}2,840 \\
891 \\
1,298 \\
2,256 \\
29,404\end{array}$ & $\begin{array}{r}5,652 \\
1,288 \\
2,179 \\
4,970 \\
36,242\end{array}$ & $\begin{array}{r}8,691 \\
1,471 \\
4,080 \\
6,735 \\
50,580\end{array}$ & $\begin{array}{r}8,165 \\
2,138 \\
4,122 \\
7,123 \\
65,997\end{array}$ & $\begin{array}{r}8,087 \\
2,079 \\
5,507 \\
8,139 \\
87,540\end{array}$ & $\begin{array}{r}49,644 \\
14,350 \\
28,386 \\
39,474 \\
479,537\end{array}$ \\
\hline 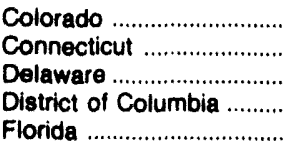 & $\begin{array}{r}6,487 \\
1,921 \\
447 \\
817 \\
952\end{array}$ & $\begin{array}{l}9,870 \\
3,989 \\
1,016 \\
1,624 \\
1,463\end{array}$ & $\begin{array}{r}14,984 \\
6,171 \\
1,483 \\
2,838 \\
1,903\end{array}$ & $\begin{array}{r}15,937 \\
7,302 \\
1,382 \\
2,795 \\
1,849\end{array}$ & $\begin{array}{r}18,500 \\
6,635 \\
1,301 \\
2,740 \\
1,650\end{array}$ & $\begin{array}{r}94,614 \\
42,394 \\
8,194 \\
16,587 \\
14,380\end{array}$ \\
\hline 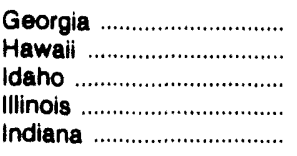 & $\begin{array}{r}4,449 \\
44 \\
689 \\
18,265 \\
6,398\end{array}$ & $\begin{array}{r}9,292 \\
48 \\
1,040 \\
42,438 \\
14,327\end{array}$ & $\begin{array}{r}15,685 \\
52 \\
1,699 \\
68,000 \\
23,924\end{array}$ & $\begin{array}{r}18,101 \\
51 \\
1,951 \\
74,465 \\
25,852\end{array}$ & $\begin{array}{r}17,977 \\
51 \\
2,256 \\
85,855 \\
27,770\end{array}$ & $\begin{array}{r}108,214 \\
551 \\
9,659 \\
475,360 \\
152,692\end{array}$ \\
\hline 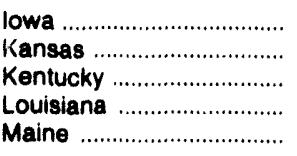 & $\begin{array}{r}3,458 \\
4,727 \\
1,921 \\
2,946 \\
38\end{array}$ & $\begin{array}{r}7,613 \\
9,162 \\
5,024 \\
5,580 \\
71\end{array}$ & $\begin{array}{r}11,612 \\
13,049 \\
9,264 \\
7,940 \\
115\end{array}$ & $\begin{array}{r}13,078 \\
13,450 \\
11,225 \\
8,409 \\
153\end{array}$ & $\begin{array}{r}15,171 \\
15,775 \\
11,222 \\
8,584 \\
144\end{array}$ & $\begin{array}{r}74,879 \\
71,522 \\
61,911 \\
55,221 \\
872\end{array}$ \\
\hline 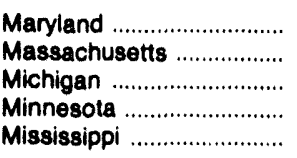 & $\begin{array}{r}3,419 \\
7,277 \\
18,067 \\
5,382 \\
1,467\end{array}$ & $\begin{array}{r}6,596 \\
13,203 \\
34,718 \\
10,745 \\
2,965\end{array}$ & $\begin{array}{r}12,217 \\
18,308 \\
54,009 \\
16,317 \\
4,402\end{array}$ & $\begin{array}{r}12,767 \\
19,634 \\
56,815 \\
18,621 \\
4,393\end{array}$ & $\begin{array}{r}11,757 \\
18,257 \\
58,940 \\
22,308 \\
4,480\end{array}$ & $\begin{array}{r}75,122 \\
119,670 \\
358,088 \\
113,560 \\
26,487\end{array}$ \\
\hline 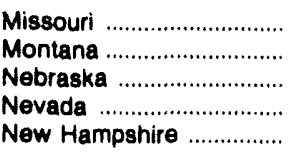 & $\begin{array}{r}7,478 \\
997 \\
2,252 \\
1,087 \\
349\end{array}$ & $\begin{array}{r}13,434 \\
1,674 \\
4,864 \\
1,503 \\
721\end{array}$ & $\begin{array}{r}21,556 \\
2,591 \\
7,316 \\
2,715 \\
1,009\end{array}$ & $\begin{array}{r}21,884 \\
2,490 \\
7,759 \\
3,157 \\
1,081\end{array}$ & $\begin{array}{r}25,234 \\
3,631 \\
11,404 \\
3,892 \\
1,048\end{array}$ & $\begin{array}{r}116,655 \\
16,673 \\
41,414 \\
18,184 \\
6,449\end{array}$ \\
\hline 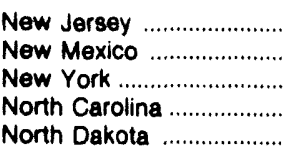 & $\begin{array}{r}8,744 \\
1,699 \\
20,317 \\
1,879 \\
568\end{array}$ & $\begin{array}{r}19,991 \\
2,798 \\
39,136 \\
5,036 \\
1,082\end{array}$ & $\begin{array}{r}31,606 \\
4,026 \\
60,720 \\
8,033 \\
1,462\end{array}$ & $\begin{array}{r}34,599 \\
4,736 \\
62,212 \\
8,149 \\
1,522\end{array}$ & $\begin{array}{r}30,626 \\
6,313 \\
58,387 \\
8,389 \\
2,059\end{array}$ & $\begin{array}{r}198,462 \\
31,433 \\
378,689 \\
42,588 \\
9,693\end{array}$ \\
\hline 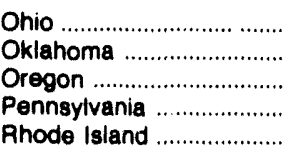 & $\begin{array}{r}13,734 \\
3,917 \\
1,769 \\
10,967 \\
1,117\end{array}$ & $\begin{array}{r}32,024 \\
8,042 \\
2,533 \\
24,899 \\
2,200\end{array}$ & $\begin{array}{r}52,333 \\
12,165 \\
3,844 \\
42,203 \\
3,291\end{array}$ & $\begin{array}{r}58,337 \\
12,493 \\
4,570 \\
44,864 \\
3,326\end{array}$ & $\begin{array}{r}55,585 \\
14,604 \\
5,790 \\
41,824 \\
3,091\end{array}$ & $\begin{array}{r}340,628 \\
65,811 \\
23,109 \\
266,528 \\
20,000\end{array}$ \\
\hline 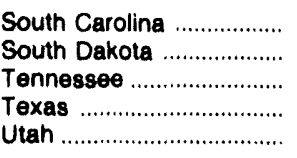 & $\begin{array}{r}947 \\
\text { An6 } \\
2,288 \\
10,676 \\
3,055\end{array}$ & $\begin{array}{r}2,532 \\
1,153 \\
5,647 \\
17,317 \\
4,375\end{array}$ & $\begin{array}{r}4,230 \\
1,678 \\
9,840 \\
27,195 \\
6,589\end{array}$ & $\begin{array}{r}4,247 \\
1,784 \\
9,089 \\
34,330 \\
8,045\end{array}$ & $\begin{array}{r}4,232 \\
2,291 \\
10,127 \\
41,315 \\
8,918\end{array}$ & $\begin{array}{r}22,392 \\
10,791 \\
52,220 \\
214,682 \\
44,701\end{array}$ \\
\hline 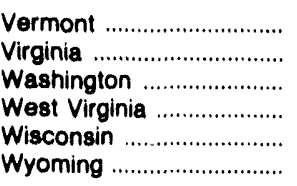 & $\begin{array}{r}138 \\
2,402 \\
3,191 \\
1,596 \\
5,548 \\
811\end{array}$ & $\begin{array}{r}271 \\
5,781 \\
4,766 \\
3,534 \\
12,504 \\
1,166\end{array}$ & $\begin{array}{r}433 \\
10,351 \\
6,815 \\
5,724 \\
18,048 \\
1,855\end{array}$ & $\begin{array}{r}446 \\
11,461 \\
7,491 \\
5,916 \\
18,316 \\
1,799\end{array}$ & $\begin{array}{r}412 \\
10,675 \\
9,891 \\
5,182 \\
22,983 \\
2,145\end{array}$ & $\begin{array}{r}2,520 \\
62,431 \\
43,048 \\
35,291 \\
123,405 \\
10,895\end{array}$ \\
\hline 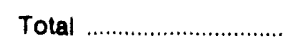 & 234,089 & 450,088 & 702,955 & 769,882 & 833,773 & $4,690,065$ \\
\hline
\end{tabular}

See footnote at end of table. 
Table 18. Natural Gas Deliveries to Residential Consumers by State, 1992-1993 (Continued) (Million Cubic Feet)

\begin{tabular}{|c|c|c|c|c|c|c|}
\hline State & $\begin{array}{c}\text { December } \\
1992\end{array}$ & $\begin{array}{c}\text { November } \\
1992\end{array}$ & $\begin{array}{c}\text { October } \\
1992\end{array}$ & $\begin{array}{c}\text { September } \\
1992\end{array}$ & $\begin{array}{c}\text { August } \\
1992\end{array}$ & $\begin{array}{l}\text { July } \\
1992\end{array}$ \\
\hline 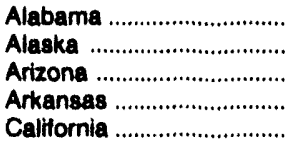 & $\begin{array}{r}7,261 \\
1,682 \\
5,118 \\
6,185 \\
75,266\end{array}$ & $\begin{array}{r}3,402 \\
1,442 \\
1,576 \\
3,132 \\
37,291\end{array}$ & $\begin{array}{r}1,728 \\
1,209 \\
1,000 \\
1,511 \\
24,434\end{array}$ & $\begin{array}{r}1,373 \\
643 \\
870 \\
1,096 \\
21,969\end{array}$ & $\begin{array}{r}1,311 \\
438 \\
815 \\
1,124 \\
22,039\end{array}$ & $\begin{array}{r}1,423 \\
474 \\
947 \\
1,194 \\
22,397\end{array}$ \\
\hline 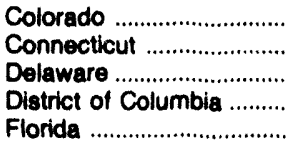 & $\begin{array}{r}15,715 \\
5,855 \\
1,044 \\
2,213 \\
1,766\end{array}$ & $\begin{array}{r}7,607 \\
3,876 \\
628 \\
1,314 \\
956\end{array}$ & $\begin{array}{r}3,547 \\
2,271 \\
307 \\
642 \\
685\end{array}$ & $\begin{array}{r}2,644 \\
1,155 \\
183 \\
446 \\
636\end{array}$ & $\begin{array}{r}2,438 \\
996 \\
179 \\
420 \\
627\end{array}$ & $\begin{array}{r}2,809 \\
1,125 \\
197 \\
469 \\
674\end{array}$ \\
\hline 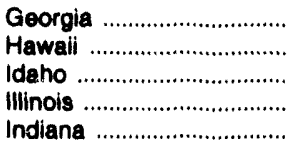 & $\begin{array}{r}18,555 \\
50 \\
1,946 \\
77,632 \\
24,261\end{array}$ & $\begin{array}{r}12,934 \\
42 \\
1,024 \\
54,950 \\
15,953\end{array}$ & $\begin{array}{r}5,284 \\
42 \\
428 \\
28,742 \\
8,361\end{array}$ & $\begin{array}{r}3,211 \\
43 \\
274 \\
13,758 \\
3,960\end{array}$ & $\begin{array}{r}2,981 \\
42 \\
183 \\
11,171 \\
3,312\end{array}$ & $\begin{array}{r}3,170 \\
45 \\
233 \\
10,391 \\
3,484\end{array}$ \\
\hline 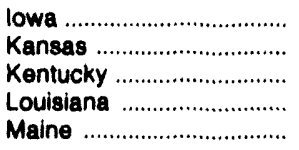 & $\begin{array}{r}12,827 \\
12,732 \\
10,617 \\
8,411 \\
111\end{array}$ & $\begin{array}{r}8,350 \\
6,102 \\
6,997 \\
4,185 \\
70\end{array}$ & $\begin{array}{r}3,725 \\
2,421 \\
3,376 \\
2,222 \\
41\end{array}$ & $\begin{array}{r}1,885 \\
1,798 \\
1,413 \\
2,014 \\
17\end{array}$ & $\begin{array}{r}1,668 \\
1,740 \\
1,416 \\
1,960 \\
22\end{array}$ & $\begin{array}{r}1,546 \\
1,932 \\
1,326 \\
2,067 \\
24\end{array}$ \\
\hline 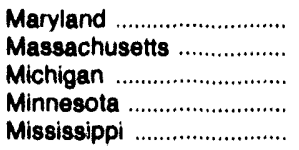 & $\begin{array}{r}10,567 \\
14,189 \\
50,376 \\
18,543 \\
4,198\end{array}$ & $\begin{array}{r}6,855 \\
10,056 \\
34,278 \\
12,857 \\
1,927\end{array}$ & $\begin{array}{r}4,038 \\
5,342 \\
20,856 \\
6,449 \\
979\end{array}$ & $\begin{array}{r}2,027 \\
3,369 \\
9,435 \\
3,021 \\
856\end{array}$ & $\begin{array}{r}2,068 \\
3,203 \\
8,655 \\
2,525 \\
817\end{array}$ & $\begin{array}{r}2,153 \\
3,453 \\
8,773 \\
2,702 \\
896\end{array}$ \\
\hline 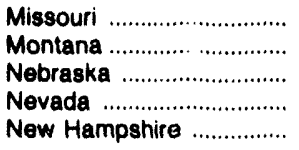 & $\begin{array}{r}19,801 \\
3,065 \\
7,085 \\
3,302 \\
750\end{array}$ & $\begin{array}{r}10,344 \\
1,792 \\
4,561 \\
1,226 \\
526\end{array}$ & $\begin{array}{r}4,259 \\
1,106 \\
2,030 \\
727 \\
288\end{array}$ & $\begin{array}{r}2,942 \\
727 \\
1,096 \\
608 \\
151\end{array}$ & $\begin{array}{r}2,681 \\
421 \\
983 \\
529 \\
139\end{array}$ & $\begin{array}{r}2,931 \\
485 \\
1,047 \\
671 \\
159\end{array}$ \\
\hline 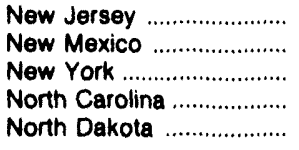 & $\begin{array}{r}27,553 \\
5,194 \\
49,715 \\
6,819 \\
1,472\end{array}$ & $\begin{array}{r}17,953 \\
1,992 \\
30,758 \\
3,446 \\
990\end{array}$ & $\begin{array}{r}10,368 \\
1,033 \\
18,633 \\
1,781 \\
458\end{array}$ & $\begin{array}{r}5,338 \\
889 \\
10,006 \\
806 \\
285\end{array}$ & $\begin{array}{r}4,918 \\
851 \\
9,649 \\
734 \\
214\end{array}$ & $\begin{array}{r}5,239 \\
929 \\
10,508 \\
837 \\
260\end{array}$ \\
\hline 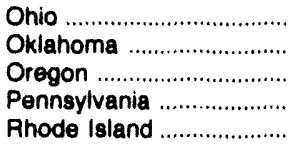 & $\begin{array}{r}49,551 \\
11,635 \\
4,337 \\
36,609 \\
2,403\end{array}$ & $\begin{array}{r}34,181 \\
4,722 \\
1,993 \\
24,806 \\
1,720\end{array}$ & $\begin{array}{r}20,929 \\
2,236 \\
1,017 \\
15,853 \\
890\end{array}$ & $\begin{array}{r}8,111 \\
1,775 \\
648 \\
6,950 \\
547\end{array}$ & $\begin{array}{r}7,238 \\
1,712 \\
526 \\
5,902 \\
423\end{array}$ & $\begin{array}{r}8,098 \\
1,948 \\
610 \\
6,134 \\
638\end{array}$ \\
\hline $\begin{array}{l}\text { South Carolina } \\
\text { South Dakota } \\
\text { Tennessee } \\
\text { Texas } \\
\text { Utah }\end{array}$ & $\begin{array}{r}3,784 \\
1,797 \\
9,125 \\
35,434 \\
7,957\end{array}$ & $\begin{array}{r}1,750 \\
1,267 \\
4,473 \\
19,205 \\
3,844\end{array}$ & $\begin{array}{r}792 \\
602 \\
1,885 \\
8,584 \\
1,802\end{array}$ & $\begin{array}{r}408 \\
315 \\
1,100 \\
7,750 \\
1,321\end{array}$ & $\begin{array}{r}386 \\
254 \\
1,051 \\
7,553 \\
1,255\end{array}$ & $\begin{array}{r}424 \\
265 \\
1,120 \\
8,118 \\
1,406\end{array}$ \\
\hline 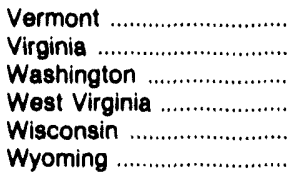 & $\begin{array}{r}308 \\
9,396 \\
7,930 \\
4,146 \\
19,080 \\
1,803\end{array}$ & $\begin{array}{r}205 \\
5,553 \\
4,236 \\
3,120 \\
13,233 \\
1,071\end{array}$ & $\begin{array}{r}129 \\
3,307 \\
2,236 \\
1,899 \\
7,630 \\
551\end{array}$ & $\begin{array}{r}53 \\
1,467 \\
1,310 \\
819 \\
3,229 \\
360\end{array}$ & $\begin{array}{r}49 \\
1,406 \\
956 \\
661 \\
2,662 \\
278\end{array}$ & $\begin{array}{r}53 \\
1,495 \\
910 \\
683 \\
2,604 \\
323\end{array}$ \\
\hline Total & 717,170 & 436,771 & 240,662 & 137,106 & 125,580 & 131,801 \\
\hline
\end{tabular}

See footnote at end of table. 
Table 18. Natural Gas Deliveries to Residential Consumers by State, 1992-1993 (Continued) (Million Cubic Feet)

\begin{tabular}{|c|c|c|c|c|c|c|}
\hline State & $\begin{array}{l}\text { June } \\
1992\end{array}$ & $\begin{array}{c}\text { May } \\
1992\end{array}$ & $\begin{array}{l}\text { April } \\
1992\end{array}$ & $\begin{array}{c}\text { March } \\
1992\end{array}$ & $\begin{array}{c}\text { Fobruary } \\
1992\end{array}$ & $\begin{array}{c}\text { January } \\
1982\end{array}$ \\
\hline 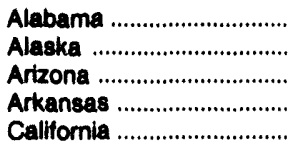 & $\begin{array}{r}1,772 \\
628 \\
1,132 \\
1,371 \\
24,100\end{array}$ & $\begin{array}{r}2,714 \\
1,012 \\
1,207 \\
1,854 \\
28,422\end{array}$ & $\begin{array}{r}5,012 \\
1,346 \\
2,313 \\
3,828 \\
37,311\end{array}$ & $\begin{array}{r}5,605 \\
1,764 \\
3,589 \\
4,305 \\
46,860\end{array}$ & $\begin{array}{r}9,267 \\
1,933 \\
4,220 \\
6,724 \\
61,574\end{array}$ & $\begin{array}{r}8,777 \\
1,777 \\
5,598 \\
7,150 \\
77,875\end{array}$ \\
\hline 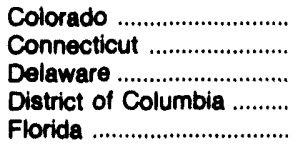 & $\begin{array}{r}3,963 \\
1,394 \\
301 \\
630 \\
733\end{array}$ & $\begin{array}{r}5,005 \\
2,417 \\
528 \\
948 \\
936\end{array}$ & $\begin{array}{r}8,789 \\
4,284 \\
948 \\
1,820 \\
1,340\end{array}$ & $\begin{array}{r}11,499 \\
5,777 \\
1,158 \\
2,172 \\
1,478\end{array}$ & $\begin{array}{r}14,400 \\
6,571 \\
1,417 \\
2,829 \\
2,345\end{array}$ & $\begin{array}{r}16,198 \\
6,675 \\
1,303 \\
2,683 \\
2,206\end{array}$ \\
\hline 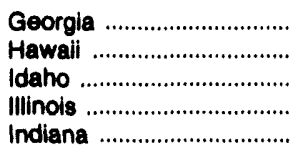 & $\begin{array}{r}3,536 \\
46 \\
247 \\
12,963 \\
4,627\end{array}$ & $\begin{array}{r}4,718 \\
47 \\
445 \\
22,488 \\
7,584\end{array}$ & $\begin{array}{r}7,360 \\
46 \\
730 \\
40,752 \\
14,093\end{array}$ & $\begin{array}{r}12,768 \\
48 \\
844 \\
59,187 \\
18,963\end{array}$ & $\begin{array}{r}14,531 \\
50 \\
1,361 \\
63,013 \\
22,018\end{array}$ & $\begin{array}{r}19,167 \\
51 \\
1,843 \\
80,315 \\
26,077\end{array}$ \\
\hline 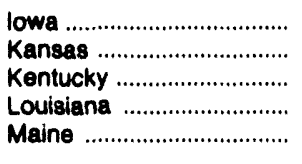 & $\begin{array}{r}2,186 \\
2,609 \\
1,835 \\
2,288 \\
37\end{array}$ & $\begin{array}{r}3,335 \\
3,788 \\
2,626 \\
2,799 \\
66\end{array}$ & $\begin{array}{r}6,857 \\
6,917 \\
4,179 \\
4,544 \\
96\end{array}$ & $\begin{array}{r}8,482 \\
7,890 \\
7,813 \\
5,650 \\
122\end{array}$ & $\begin{array}{r}11,637 \\
10,939 \\
8,511 \\
9,220 \\
139\end{array}$ & $\begin{array}{r}12,381 \\
12,653 \\
11,805 \\
9,861 \\
128\end{array}$ \\
\hline 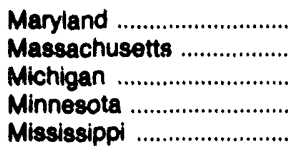 & $\begin{array}{r}2,577 \\
4,822 \\
11,927 \\
3,130 \\
963\end{array}$ & $\begin{array}{r}4,007 \\
8,475 \\
21,545 \\
5,136 \\
1,287\end{array}$ & $\begin{array}{r}7,087 \\
13,384 \\
36,421 \\
10,391 \\
2,440\end{array}$ & $\begin{array}{r}9,717 \\
16,712 \\
46,640 \\
14,009 \\
2,850\end{array}$ & $\begin{array}{r}11,583 \\
19,067 \\
52,688 \\
15,904 \\
4,551\end{array}$ & $\begin{array}{r}12,442 \\
17,599 \\
56,513 \\
18,895 \\
4,724\end{array}$ \\
\hline 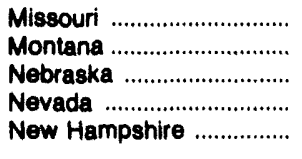 & $\begin{array}{r}3,838 \\
541 \\
1,311 \\
695 \\
219\end{array}$ & $\begin{array}{r}5,858 \\
837 \\
2,020 \\
803 \\
461\end{array}$ & $\begin{array}{r}11,044 \\
1,371 \\
3,760 \\
1,394 \\
766\end{array}$ & $\begin{array}{r}13,548 \\
1,620 \\
4,706 \\
2,094 \\
869\end{array}$ & $\begin{array}{r}18,433 \\
2,180 \\
5,956 \\
2,611 \\
1,089\end{array}$ & $\begin{array}{r}20,977 \\
2,529 \\
6,858 \\
3,525 \\
1,032\end{array}$ \\
\hline 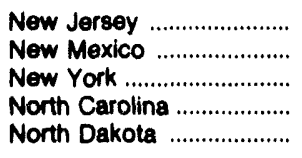 & $\begin{array}{r}6,166 \\
1,122 \\
13,470 \\
1,301 \\
311\end{array}$ & $\begin{array}{r}11,680 \\
1,514 \\
23,835 \\
2,222 \\
616\end{array}$ & $\begin{array}{r}20,212 \\
2,723 \\
41,548 \\
4,509 \\
1,002\end{array}$ & $\begin{array}{r}26,880 \\
3,842 \\
53,492 \\
5,194 \\
1,128\end{array}$ & $\begin{array}{r}31,006 \\
5,376 \\
59,000 \\
7,366 \\
1,456\end{array}$ & $\begin{array}{r}31,150 \\
5,968 \\
58,076 \\
7,573 \\
1,500\end{array}$ \\
\hline 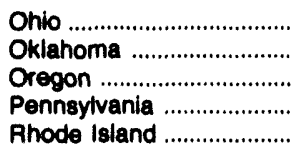 & $\begin{array}{r}10,336 \\
2,549 \\
772 \\
8,047 \\
771\end{array}$ & $\begin{array}{r}17,276 \\
3,219 \\
1,248 \\
13,989 \\
1,446\end{array}$ & $\begin{array}{r}31,344 \\
5,961 \\
1,891 \\
26,243 \\
2,271\end{array}$ & $\begin{array}{r}45,783 \\
7,235 \\
2,477 \\
36,068 \\
2,802\end{array}$ & $\begin{array}{r}50,254 \\
10,656 \\
3,223 \\
41,812 \\
3,092\end{array}$ & $\begin{array}{r}57,528 \\
12,164 \\
4,367 \\
44,113 \\
2,997\end{array}$ \\
\hline 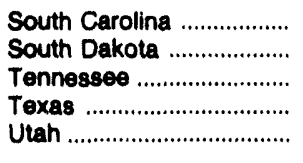 & $\begin{array}{r}597 \\
321 \\
1,437 \\
8,776 \\
1,524\end{array}$ & $\begin{array}{r}1,033 \\
638 \\
2,186 \\
10,307 \\
1,986\end{array}$ & $\begin{array}{r}2,295 \\
996 \\
4,859 \\
14,934 \\
3,184\end{array}$ & $\begin{array}{r}2,650 \\
1,202 \\
6,002 \\
21,265 \\
4,869\end{array}$ & $\begin{array}{r}4,071 \\
1,497 \\
8,849 \\
33,008 \\
7,132\end{array}$ & $\begin{array}{r}4,203 \\
1,639 \\
10,132 \\
39,747 \\
8,422\end{array}$ \\
\hline 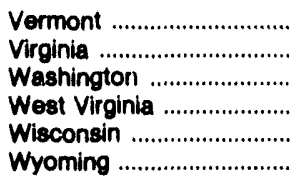 & $\begin{array}{r}72 \\
1,788 \\
1,384 \\
1,105 \\
3,681 \\
431\end{array}$ & $\begin{array}{r}145 \\
3,269 \\
2,377 \\
2,076 \\
6,373 \\
613\end{array}$ & $\begin{array}{r}284 \\
5,884 \\
3,736 \\
4,038 \\
11,914 \\
951\end{array}$ & $\begin{array}{r}367 \\
7,853 \\
4,719 \\
4,328 \\
15,740 \\
1,146\end{array}$ & $\begin{array}{r}426 \\
10,106 \\
5,966 \\
6,231 \\
17,320 \\
1,492\end{array}$ & $\begin{array}{r}431 \\
10,805 \\
7,286 \\
6,183 \\
19,941 \\
1,876\end{array}$ \\
\hline Total & 162,378 & 251,416 & 431,401 & 573,979 & 696,082 & 785,718 \\
\hline
\end{tabular}

$n=$ Revised Data.

Notes: Geographic coverage is the 50 States and the District of Columbia. See Appendbx A, Explanatory Note 5 for discussion of computations and revision policy.

Source: Form ElA-857. 
Table 19. Natural Gas Dellveries to Commercial Consumers by State, 1992-1993 (Million Cubic Feet)

\begin{tabular}{|c|c|c|c|c|c|c|}
\hline State & $\begin{array}{l}\text { YTD } \\
1993\end{array}$ & $\begin{array}{c}\text { YTD } \\
1992\end{array}$ & $\begin{array}{l}\text { YTD } \\
1991\end{array}$ & $\begin{array}{c}\text { August } \\
1993\end{array}$ & $\begin{array}{c}\text { July } \\
1993\end{array}$ & $\begin{array}{l}\text { June } \\
1993\end{array}$ \\
\hline 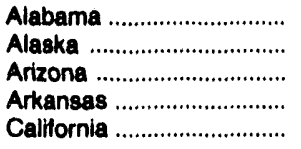 & $\begin{array}{r}18,271 \\
13,249 \\
19,079 \\
20,469 \\
178,204\end{array}$ & $\begin{array}{r}17,455 \\
13,939 \\
18,477 \\
17,392 \\
196,912\end{array}$ & $\begin{array}{r}16,220 \\
13,901 \\
19,595 \\
17,963 \\
190,304\end{array}$ & $\begin{array}{r}1,096 \\
958 \\
1,611 \\
1,044 \\
15,595\end{array}$ & $\begin{array}{r}1,105 \\
918 \\
1,665 \\
1,010 \\
19,856\end{array}$ & $\begin{array}{r}1,199 \\
1,055 \\
2,046 \\
1,075 \\
16,987\end{array}$ \\
\hline 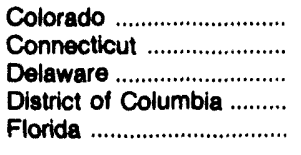 & $\begin{array}{r}50,732 \\
20,690 \\
3,698 \\
11,638 \\
28,098\end{array}$ & $\begin{array}{r}45,117 \\
20,708 \\
3,546 \\
11,674 \\
28,692\end{array}$ & $\begin{array}{r}48,243 \\
18,739 \\
3,010 \\
11,368 \\
25,995\end{array}$ & $\begin{array}{r}2,393 \\
809 \\
154 \\
697 \\
2,835\end{array}$ & $\begin{array}{r}2,482 \\
973 \\
163 \\
658 \\
2,923\end{array}$ & $\begin{array}{r}3,162 \\
1,012 \\
139 \\
724 \\
3,075\end{array}$ \\
\hline 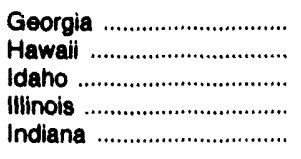 & $\begin{array}{r}38,831 \\
1,427 \\
7,342 \\
132,539 \\
50,885\end{array}$ & $\begin{array}{r}35,915 \\
1,436 \\
5,651 \\
123,619 \\
46,104\end{array}$ & $\begin{array}{r}33,784 \\
1,431 \\
6,566 \\
122,630 \\
44,356\end{array}$ & $\begin{array}{r}2,514 \\
170 \\
362 \\
5,640 \\
1,793\end{array}$ & $\begin{array}{r}2,600 \\
174 \\
385 \\
5,877 \\
1,800\end{array}$ & $\begin{array}{r}2,559 \\
183 \\
389 \\
5,099 \\
2,057\end{array}$ \\
\hline 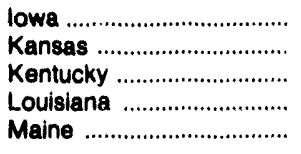 & $\begin{array}{r}33,414 \\
41,056 \\
24,118 \\
17,697 \\
1,529\end{array}$ & $\begin{array}{r}28,347 \\
35,270 \\
22,390 \\
19,492 \\
1,553\end{array}$ & $\begin{array}{r}30,370 \\
41,673 \\
21,141 \\
17,594 \\
1,267\end{array}$ & $\begin{array}{r}1,208 \\
3,420 \\
1,005 \\
1,280 \\
67\end{array}$ & $\begin{array}{r}1,215 \\
3,504 \\
1,029 \\
1,269 \\
71\end{array}$ & $\begin{array}{r}1,481 \\
2,248 \\
1,443 \\
1,456 \\
80\end{array}$ \\
\hline 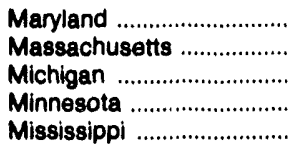 & $\begin{array}{r}29,650 \\
45,121 \\
122,222 \\
56,354 \\
13,986\end{array}$ & $\begin{array}{r}28,898 \\
45,360 \\
118,102 \\
52,337 \\
12,278\end{array}$ & $\begin{array}{r}25,628 \\
38,510 \\
114,501 \\
54,573 \\
11,960\end{array}$ & $\begin{array}{r}1,555 \\
2,513 \\
4,133 \\
2,183 \\
824\end{array}$ & $\begin{array}{r}1,496 \\
2,614 \\
5,039 \\
2,176 \\
970\end{array}$ & $\begin{array}{r}1,846 \\
3,121 \\
5,968 \\
2,533 \\
958\end{array}$ \\
\hline 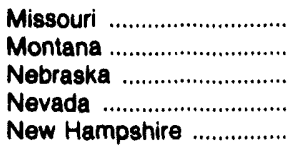 & $\begin{array}{r}48,472 \\
9,065 \\
25,832 \\
11,994 \\
4,338\end{array}$ & $\begin{array}{r}41,230 \\
6,859 \\
21,344 \\
11,056 \\
4,163\end{array}$ & $\begin{array}{r}43,111 \\
8,335 \\
28,769 \\
12,023 \\
3,537\end{array}$ & $\begin{array}{r}1,988 \\
418 \\
2,343 \\
940 \\
171\end{array}$ & $\begin{array}{r}2,093 \\
439 \\
1,289 \\
921 \\
177\end{array}$ & $\begin{array}{r}2,293 \\
436 \\
1,351 \\
1,054 \\
204\end{array}$ \\
\hline 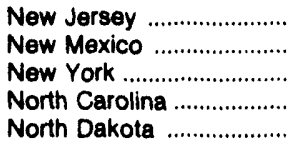 & $\begin{array}{r}93,002 \\
18,857 \\
150,853 \\
24,903 \\
7,088\end{array}$ & $\begin{array}{r}90,768 \\
18,236 \\
152,160 \\
24,649 \\
6,256\end{array}$ & $\begin{array}{r}82,786 \\
17,577 \\
136,677 \\
23,111 \\
6,976\end{array}$ & $\begin{array}{r}3,901 \\
1,408 \\
10,413 \\
1,415 \\
294\end{array}$ & $\begin{array}{r}5,985 \\
1,416 \\
10,369 \\
1,314 \\
317\end{array}$ & $\begin{array}{r}5,256 \\
1,464 \\
11,087 \\
1,421 \\
347\end{array}$ \\
\hline 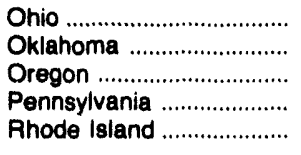 & $\begin{array}{r}111,082 \\
29,346 \\
16,523 \\
89,275 \\
6,129\end{array}$ & $\begin{array}{r}108,271 \\
24,122 \\
12,784 \\
90,068 \\
6,455\end{array}$ & $\begin{array}{r}100,650 \\
27,072 \\
15,889 \\
83,826 \\
5,695\end{array}$ & $\begin{array}{r}3,843 \\
1,442 \\
839 \\
3,897 \\
364\end{array}$ & $\begin{array}{r}3,918 \\
1,343 \\
914 \\
\mathbf{9 , 5 0 5} \\
273\end{array}$ & $\begin{array}{r}5,194 \\
1,458 \\
1,009 \\
4,462 \\
330\end{array}$ \\
\hline 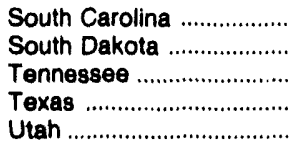 & $\begin{array}{r}11,484 \\
7,170 \\
35,279 \\
144,523 \\
13,662\end{array}$ & $\begin{array}{r}11,328 \\
5,661 \\
31,422 \\
122,166 \\
11,184\end{array}$ & $\begin{array}{r}10,560 \\
6,115 \\
31,199 \\
123,173 \\
13,980\end{array}$ & $\begin{array}{r}834 \\
292 \\
1,839 \\
15,027 \\
414\end{array}$ & $\begin{array}{r}826 \\
315 \\
1,912 \\
16,324 \\
471\end{array}$ & $\begin{array}{r}881 \\
349 \\
2,023 \\
15,471 \\
560\end{array}$ \\
\hline 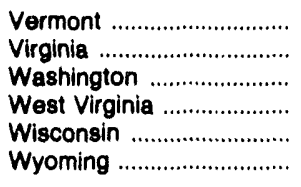 & $\begin{array}{r}1,598 \\
36,298 \\
30,151 \\
16,531 \\
52,047 \\
6,518\end{array}$ & $\begin{array}{r}1,618 \\
33,970 \\
24,700 \\
16,539 \\
46,247 \\
5,251\end{array}$ & $\begin{array}{r}1,371 \\
30,036 \\
29,092 \\
13,828 \\
45,450 \\
6,276\end{array}$ & $\begin{array}{r}67 \\
2,052 \\
1,765 \\
1,012 \\
1,896 \\
361\end{array}$ & $\begin{array}{r}62 \\
1,986 \\
1,816 \\
906 \\
2,016 \\
336\end{array}$ & $\begin{array}{r}80 \\
2,171 \\
1,821 \\
1,088 \\
2,594 \\
369\end{array}$ \\
\hline 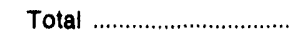 & $1,982,317$ & $1,879,171$ & $1,838,436$ & 115,091 & 123,216 & 126,742 \\
\hline
\end{tabular}

See footnote at end of table. 
Table 19. Natural Gas Deliveries to Commercial Consumers by State, 1992-1993 (Continued) (Million Cubic Feet)

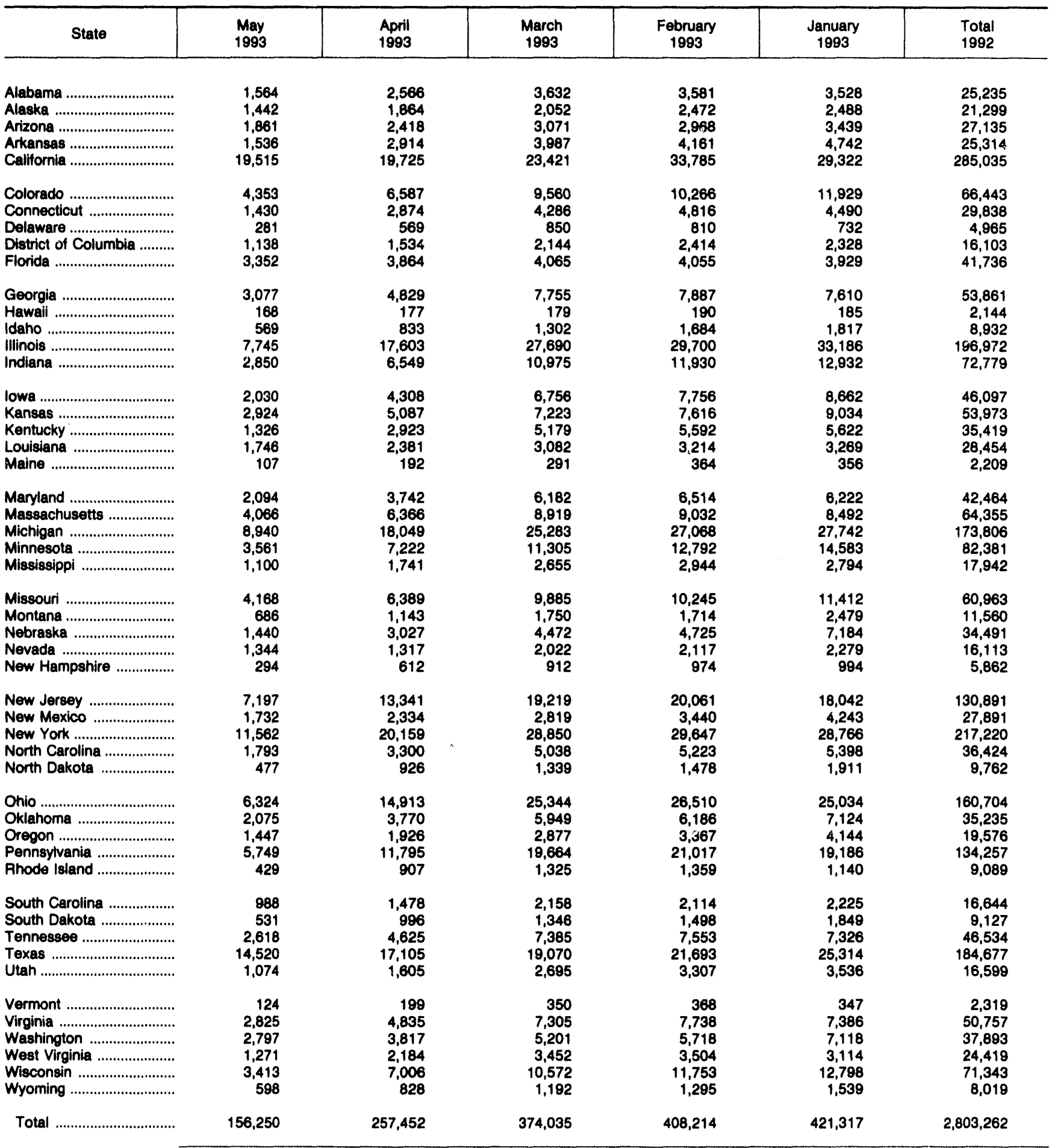

See footnote at end of table. 
Table 19. Natural Gas Deliveries to Commerclal Consumers by State, 1992-1993 (Continued)

(Million Cubic Feet)

\begin{tabular}{|c|c|c|c|c|c|c|}
\hline State & $\begin{array}{c}\text { December } \\
1992\end{array}$ & $\begin{array}{c}\text { November } \\
1992\end{array}$ & $\begin{array}{c}\text { October } \\
1992\end{array}$ & $\begin{array}{c}\text { September } \\
1992\end{array}$ & $\begin{array}{c}\text { August } \\
1992\end{array}$ & $\begin{array}{l}\text { July } \\
1992\end{array}$ \\
\hline 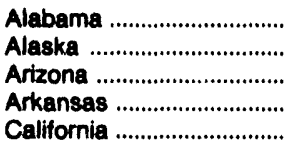 & $\begin{array}{r}3,338 \\
2,390 \\
3,573 \\
3,596 \\
28,738\end{array}$ & $\begin{array}{r}1,983 \\
1,828 \\
1,858 \\
2,078 \\
20,999\end{array}$ & $\begin{array}{r}1,320 \\
1,790 \\
1,580 \\
1,219 \\
19,112\end{array}$ & $\begin{array}{r}1,136 \\
1,252 \\
1,601 \\
1,029 \\
19,247\end{array}$ & $\begin{array}{r}1,078 \\
1,013 \\
1,505 \\
1,022 \\
21,633\end{array}$ & $\begin{array}{r}1,108 \\
1,084 \\
1,690 \\
1,062 \\
21,150\end{array}$ \\
\hline 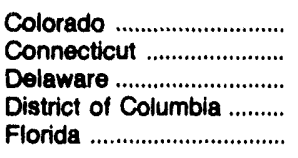 & $\begin{array}{r}10,319 \\
3,871 \\
622 \\
1,921 \\
3,914\end{array}$ & $\begin{array}{r}5,594 \\
2,623 \\
395 \\
1,103 \\
3,317\end{array}$ & $\begin{array}{r}3,078 \\
1,562 \\
240 \\
753 \\
2,954\end{array}$ & $\begin{array}{r}2,312 \\
1,075 \\
162 \\
653 \\
2,849\end{array}$ & $\begin{array}{r}2,183 \\
1,061 \\
152 \\
673 \\
2,881\end{array}$ & $\begin{array}{r}2,430 \\
1,046 \\
166 \\
718 \\
2,976\end{array}$ \\
\hline 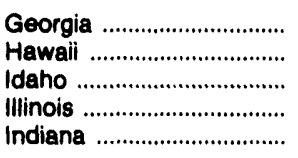 & $\begin{array}{r}7,642 \\
184 \\
1,557 \\
31,575 \\
12,097\end{array}$ & $\begin{array}{r}4,640 \\
178 \\
889 \\
22,757 \\
8,135\end{array}$ & $\begin{array}{r}3,201 \\
168 \\
465 \\
12,394 \\
4,253\end{array}$ & $\begin{array}{r}2,462 \\
178 \\
369 \\
6,618 \\
2,131\end{array}$ & $\begin{array}{r}2,503 \\
173 \\
330 \\
6,025 \\
1,955\end{array}$ & $\begin{array}{r}2,536 \\
174 \\
345 \\
5,811 \\
1,811\end{array}$ \\
\hline 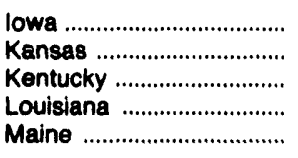 & $\begin{array}{r}7,827 \\
7,543 \\
5,519 \\
3,574 \\
285\end{array}$ & $\begin{array}{r}5,843 \\
4,867 \\
4,064 \\
2,295 \\
188\end{array}$ & $\begin{array}{r}2,645 \\
3,162 \\
2,237 \\
1,577 \\
116\end{array}$ & $\begin{array}{r}1,433 \\
3,132 \\
1,209 \\
1,508 \\
67\end{array}$ & $\begin{array}{r}1,257 \\
3,462 \\
1,158 \\
1,431 \\
52\end{array}$ & $\begin{array}{r}1,100 \\
3,609 \\
1,066 \\
1,556 \\
79\end{array}$ \\
\hline 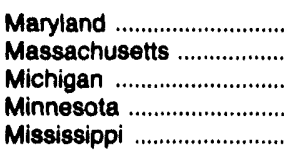 & $\begin{array}{r}5,565 \\
6,933 \\
24,211 \\
12,795 \\
2,387\end{array}$ & $\begin{array}{r}3,895 \\
5,530 \\
16,298 \\
9,526 \\
1,489\end{array}$ & $\begin{array}{r}2,491 \\
3,776 \\
9,760 \\
5,092 \\
942\end{array}$ & $\begin{array}{r}1,615 \\
2,753 \\
5,431 \\
2,631 \\
847\end{array}$ & $\begin{array}{r}1,586 \\
2,884 \\
4,964 \\
2,296 \\
867\end{array}$ & $\begin{array}{r}1,553 \\
3,072 \\
4,981 \\
2,244 \\
885\end{array}$ \\
\hline 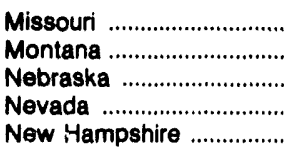 & $\begin{array}{r}9,646 \\
2,095 \\
4,941 \\
2,170 \\
715\end{array}$ & $\begin{array}{r}5,335 \\
1,271 \\
3,988 \\
1,022 \\
515\end{array}$ & $\begin{array}{r}2,728 \\
810 \\
2,508 \\
982 \\
295\end{array}$ & $\begin{array}{r}2,024 \\
523 \\
1,710 \\
872 \\
174\end{array}$ & $\begin{array}{r}1,993 \\
331 \\
2,393 \\
824 \\
169\end{array}$ & $\begin{array}{r}1,849 \\
365 \\
2,366 \\
1,015 \\
179\end{array}$ \\
\hline 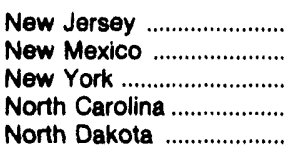 & $\begin{array}{r}16,470 \\
4,165 \\
25,808 \\
4,941 \\
1,549\end{array}$ & $\begin{array}{r}11,027 \\
2,269 \\
16,804 \\
3,010 \\
1,015\end{array}$ & $\begin{array}{r}7,164 \\
1,673 \\
12,860 \\
2,138 \\
576\end{array}$ & $\begin{array}{r}5,462 \\
1,541 \\
9,582 \\
1,679 \\
363\end{array}$ & $\begin{array}{r}4,981 \\
1,513 \\
9,456 \\
1,607 \\
262\end{array}$ & $\begin{array}{r}5,240 \\
1,200 \\
9,327 \\
1,591 \\
279\end{array}$ \\
\hline 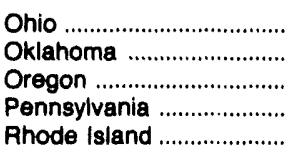 & $\begin{array}{r}23,770 \\
5,771 \\
3,166 \\
18,385 \\
951\end{array}$ & $\begin{array}{r}15,094 \\
2,608 \\
1,652 \\
12,784 \\
654\end{array}$ & $\begin{array}{r}9,092 \\
1,449 \\
1,105 \\
8,439 \\
609\end{array}$ & $\begin{array}{r}4,418 \\
1,240 \\
863 \\
4,579 \\
411\end{array}$ & $\begin{array}{r}4,260 \\
1,292 \\
782 \\
4,132 \\
479\end{array}$ & $\begin{array}{r}4,291 \\
1,407 \\
784 \\
4,046 \\
448\end{array}$ \\
\hline 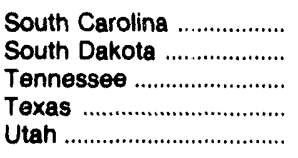 & $\begin{array}{r}2,089 \\
1,476 \\
6,846 \\
22,093 \\
3,120\end{array}$ & $\begin{array}{r}1,345 \\
1,104 \\
3,963 \\
17,386 \\
1,329\end{array}$ & $\begin{array}{r}1,006 \\
558 \\
2,368 \\
11,672 \\
545\end{array}$ & $\begin{array}{r}875 \\
324 \\
1,933 \\
11,356 \\
405\end{array}$ & $\begin{array}{r}882 \\
284 \\
1,681 \\
12,966 \\
372\end{array}$ & $\begin{array}{r}820 \\
291 \\
1,838 \\
13,768 \\
410\end{array}$ \\
\hline 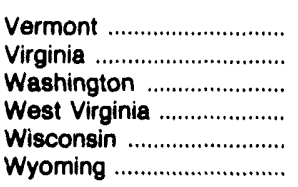 & $\begin{array}{r}269 \\
6,895 \\
5,709 \\
3,396 \\
11,087 \\
1,271\end{array}$ & $\begin{array}{r}211 \\
4,714 \\
3,418 \\
2,092 \\
7,918 \\
760\end{array}$ & $\begin{array}{r}144 \\
3,275 \\
2,240 \\
1,482 \\
4,108 \\
439\end{array}$ & $\begin{array}{r}77 \\
1,903 \\
1,734 \\
910 \\
1,954 \\
287\end{array}$ & $\begin{array}{r}75 \\
2,055 \\
1,514 \\
855 \\
1,615 \\
269\end{array}$ & $\begin{array}{r}79 \\
2,085 \\
1,575 \\
812 \\
1,759 \\
264\end{array}$ \\
\hline 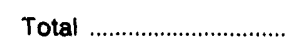 & 380,772 & 255,758 & 166,151 & 120,899 & 121,186 & 122,339 \\
\hline
\end{tabular}

See footnote at end of table. 
Table 19. Natural Gas Dellveries to Commercial Consumers by State, 1992-1993 (Continued)

(Million Cubic Feet)

\begin{tabular}{|c|c|c|c|c|c|c|}
\hline State & $\begin{array}{l}\text { June } \\
1992\end{array}$ & $\begin{array}{l}\text { May } \\
1902\end{array}$ & $\begin{array}{l}\text { April } \\
1992\end{array}$ & $\begin{array}{c}\text { March } \\
1992\end{array}$ & $\begin{array}{c}\text { February } \\
1992\end{array}$ & $\begin{array}{l}\text { Jaingry } \\
1992\end{array}$ \\
\hline 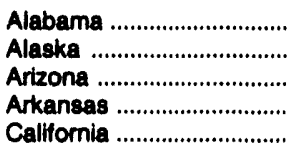 & $\begin{array}{r}1,225 \\
1,206 \\
1,754 \\
1,108 \\
22,150\end{array}$ & $\begin{array}{r}1,513 \\
1,597 \\
1,620 \\
1,358 \\
27,118\end{array}$ & $\begin{array}{r}2,287 \\
1,935 \\
2,352 \\
2,114 \\
18,379\end{array}$ & $\begin{array}{r}2,671 \\
2,319 \\
2,898 \\
2,708 \\
24,413\end{array}$ & $\begin{array}{r}3,833 \\
2,372 \\
3,011 \\
3,861 \\
23,834\end{array}$ & $\begin{array}{r}3,739 \\
2,414 \\
3,647 \\
4,159 \\
38,234\end{array}$ \\
\hline 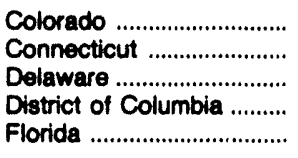 & $\begin{array}{r}3,055 \\
1,149 \\
198 \\
764 \\
3,085\end{array}$ & $\begin{array}{r}3,631 \\
1,779 \\
308 \\
1,056 \\
3,382\end{array}$ & $\begin{array}{r}5,965 \\
2,941 \\
537 \\
1,774 \\
3,741\end{array}$ & $\begin{array}{r}7,609 \\
3,940 \\
686 \\
2,132 \\
3,856\end{array}$ & $\begin{array}{r}9,450 \\
4,374 \\
755 \\
2,256 \\
4,364\end{array}$ & $\begin{array}{r}10,794 \\
4,417 \\
744 \\
2,300 \\
4,408\end{array}$ \\
\hline 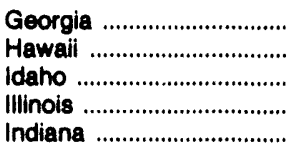 & $\begin{array}{r}2,596 \\
184 \\
343 \\
4,007 \\
2,004\end{array}$ & $\begin{array}{r}3,103 \\
177 \\
447 \\
8,872 \\
3,400\end{array}$ & $\begin{array}{r}4,381 \\
183 \\
643 \\
17,598 \\
6,172\end{array}$ & $\begin{array}{r}5,778 \\
174 \\
834 \\
23,400 \\
8,437\end{array}$ & $\begin{array}{r}6,952 \\
180 \\
1,167 \\
26,501 \\
10,265\end{array}$ & $\begin{array}{r}8,066 \\
190 \\
1,543 \\
30,506 \\
12,060\end{array}$ \\
\hline 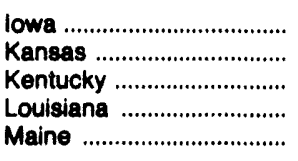 & $\begin{array}{r}1,427 \\
2,814 \\
1,132 \\
1,564 \\
80\end{array}$ & $\begin{array}{r}1,877 \\
3,736 \\
1,600 \\
1,769 \\
157\end{array}$ & $\begin{array}{r}3,883 \\
4,571 \\
2,612 \\
2,367 \\
229\end{array}$ & $\begin{array}{r}4,949 \\
4,372 \\
3,874 \\
2,743 \\
315\end{array}$ & $\begin{array}{r}6,709 \\
5,862 \\
4,758 \\
4,003 \\
318\end{array}$ & $\begin{array}{r}7,145 \\
6,844 \\
6,191 \\
4,060 \\
322\end{array}$ \\
\hline 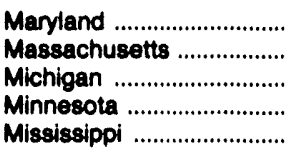 & $\begin{array}{r}1,784 \\
2,768 \\
5,883 \\
2,467 \\
888\end{array}$ & $\begin{array}{r}2,391 \\
4,450 \\
10,406 \\
3,602 \\
994\end{array}$ & $\begin{array}{r}3,807 \\
7,049 \\
17,769 \\
7,517 \\
1,473\end{array}$ & $\begin{array}{r}5,073 \\
8,217 \\
22,443 \\
10,134 \\
1,758\end{array}$ & $\begin{array}{r}6,382 \\
8,736 \\
24,979 \\
11,075 \\
2,595\end{array}$ & $\begin{array}{r}6,323 \\
8,184 \\
26,677 \\
13,000 \\
2,817\end{array}$ \\
\hline $\begin{array}{l}\text { Missouri } \\
\text { Montana ........................... } \\
\text { Nebraska ........................ } \\
\text { Nevada ........................ } \\
\text { New Hampshire .............. }\end{array}$ & $\begin{array}{r}2,547 \\
377 \\
1,330 \\
945 \\
208\end{array}$ & $\begin{array}{r}3,144 \\
568 \\
1,781 \\
1,012 \\
376\end{array}$ & $\begin{array}{r}5,601 \\
930 \\
2,411 \\
1,335 \\
629\end{array}$ & $\begin{array}{r}6,635 \\
1,099 \\
2,970 \\
1,725 \\
762\end{array}$ & $\begin{array}{r}9,060 \\
1,464 \\
3,760 \\
1,873 \\
931\end{array}$ & $\begin{array}{r}10,201 \\
1,726 \\
4,332 \\
2,327 \\
911\end{array}$ \\
\hline 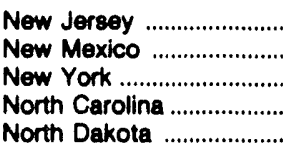 & $\begin{array}{r}5,294 \\
1,171 \\
9,915 \\
1,733 \\
298\end{array}$ & $\begin{array}{r}8,392 \\
1,687 \\
13,518 \\
2,205 \\
529\end{array}$ & $\begin{array}{r}14,066 \\
2,372 \\
23,039 \\
3,296 \\
937\end{array}$ & $\begin{array}{r}16,759 \\
2,730 \\
27,748 \\
3,883 \\
1,082\end{array}$ & $\begin{array}{r}18,398 \\
3,445 \\
29,912 \\
5,066 \\
1,380\end{array}$ & $\begin{array}{r}17,638 \\
4,118 \\
29,246 \\
5,169 \\
1,489\end{array}$ \\
\hline 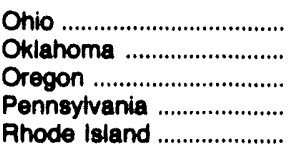 & $\begin{array}{r}5,016 \\
1,540 \\
884 \\
4,520 \\
498\end{array}$ & $\begin{array}{r}7,455 \\
1,785 \\
1,171 \\
6,793 \\
676\end{array}$ & $\begin{array}{r}15,893 \\
2,956 \\
1,542 \\
12,210 \\
1,047\end{array}$ & $\begin{array}{r}20,215 \\
3,653 \\
1,925 \\
17,679 \\
1,103\end{array}$ & $\begin{array}{r}24,820 \\
5,310 \\
2,465 \\
19,482 \\
1,128\end{array}$ & $\begin{array}{r}26,321 \\
6,180 \\
3,231 \\
21,204 \\
1,076\end{array}$ \\
\hline 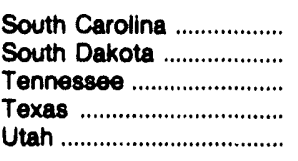 & $\begin{array}{r}944 \\
303 \\
2,016 \\
10,879 \\
457\end{array}$ & $\begin{array}{r}1,068 \\
460 \\
2,436 \\
12,557 \\
642\end{array}$ & $\begin{array}{r}1,436 \\
828 \\
4,205 \\
14,174 \\
1,155\end{array}$ & $\begin{array}{r}1,745 \\
1,007 \\
5,069 \\
15,866 \\
1,866\end{array}$ & $\begin{array}{r}2,158 \\
1,174 \\
6,834 \\
19,049 \\
2,952\end{array}$ & $\begin{array}{r}2,276 \\
1,314 \\
7,343 \\
22,907 \\
3,330\end{array}$ \\
\hline 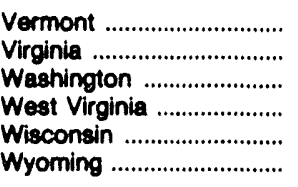 & $\begin{array}{r}75 \\
2,271 \\
1,830 \\
989 \\
1,886 \\
309\end{array}$ & $\begin{array}{r}118 \\
3,289 \\
2,343 \\
1,341 \\
3,632 \\
438\end{array}$ & $\begin{array}{r}231 \\
4,523 \\
3,179 \\
2,322 \\
6,870 \\
668\end{array}$ & $\begin{array}{r}320 \\
5,985 \\
3,855 \\
2,986 \\
9,113 \\
821\end{array}$ & $\begin{array}{r}354 \\
6,690 \\
4,840 \\
3,565 \\
10,037 \\
1,078\end{array}$ & $\begin{array}{r}366 \\
7,072 \\
5,564 \\
3,650 \\
11,235 \\
1,404\end{array}$ \\
\hline Total & 124,881 & 169,759 & 250,136 & 314,629 & 365,815 & 410,424 \\
\hline
\end{tabular}

n = Revised Data.

= Not Available.

Notes: Geographic coverage is the 50 States and the District of Columbia. Deliveries for total year 1982 may not equal the sum of the twelve months. Gas volumes delivered for use as vehicle tuel are included in the annual total but not in the monthly components. See Appendix A, Explanatory Note 5 for discussion of computations and revision policy.

Source: Form EIA-857. 
Table 20. Natural Gas Deliveries to Industrial Consumers by State, 1992-1993

(Million Cubic Feet)

\begin{tabular}{|c|c|c|c|c|c|c|}
\hline State & $\begin{array}{l}\text { YTD } \\
1893\end{array}$ & $\begin{array}{l}\text { YTD } \\
1992\end{array}$ & $\begin{array}{l}\text { YTD } \\
1991\end{array}$ & $\begin{array}{c}\text { August } \\
1993\end{array}$ & $\begin{array}{c}\text { July } \\
1993\end{array}$ & $\begin{array}{l}\text { June } \\
1993\end{array}$ \\
\hline 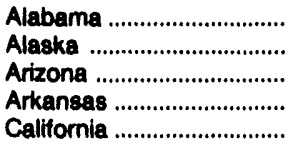 & $\begin{array}{r}116,984 \\
49,809 \\
13,389 \\
74,132 \\
371,059\end{array}$ & $\begin{array}{r}111,826 \\
53,063 \\
13,048 \\
78,695 \\
402,893\end{array}$ & $\begin{array}{r}102,001 \\
51,434 \\
12,826 \\
68,843 \\
398,725\end{array}$ & $\begin{array}{r}13,419 \\
4,919 \\
1,676 \\
7,887 \\
46,480\end{array}$ & $\begin{array}{r}14,029 \\
6,934 \\
1,598 \\
8,207 \\
52,134\end{array}$ & $\begin{array}{r}13,539 \\
5,517 \\
1,316 \\
8,515 \\
44,010\end{array}$ \\
\hline $\begin{array}{l}\text { Colorado ........................ } \\
\text { Connecticut ..................... } \\
\text { Delaware .......................... } \\
\text { District of Columbia .......... } \\
\text { Florida ................................ }\end{array}$ & $\begin{array}{r}58,126 \\
24,389 \\
13,196 \\
0 \\
64,867\end{array}$ & $\begin{array}{r}35,804 \\
24,194 \\
11,185 \\
0 \\
57,020\end{array}$ & $\begin{array}{r}37,768 \\
20,927 \\
10,905 \\
0 \\
55,731\end{array}$ & $\begin{array}{r}6,431 \\
1,817 \\
1,658 \\
0 \\
8,166\end{array}$ & $\begin{array}{r}6,778 \\
2,504 \\
1,604 \\
0 \\
8,164\end{array}$ & $\begin{array}{r}7,245 \\
2,619 \\
1,516 \\
0 \\
8,044\end{array}$ \\
\hline 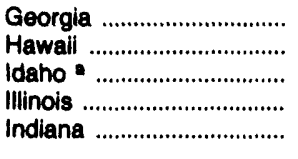 & $\begin{array}{r}111,774 \\
0 \\
19,421 \\
200,093 \\
175,105\end{array}$ & $\begin{array}{r}116,005 \\
0 \\
17,600 \\
186,834 \\
159,264\end{array}$ & $\begin{array}{r}110,686 \\
0 \\
17,552 \\
195,646 \\
149,126\end{array}$ & $\begin{array}{r}14,733 \\
0 \\
2,101 \\
17,810 \\
18,983\end{array}$ & $\begin{array}{r}14,267 \\
0 \\
2,154 \\
19,314 \\
18,558\end{array}$ & $\begin{array}{r}13,515 \\
0 \\
2,307 \\
19,630 \\
19,517\end{array}$ \\
\hline 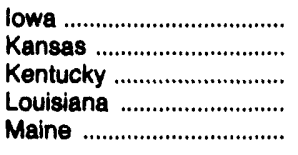 & $\begin{array}{r}66,223 \\
91,296 \\
49,311 \\
523,925 \\
957\end{array}$ & $\begin{array}{r}65,374 \\
82,606 \\
47,927 \\
651,155 \\
1,360\end{array}$ & $\begin{array}{r}62,357 \\
82,603 \\
45,603 \\
618,077 \\
1,408\end{array}$ & $\begin{array}{r}8,174 \\
10,933 \\
5,190 \\
69,247 \\
141\end{array}$ & $\begin{array}{r}7,164 \\
12,117 \\
4,909 \\
70,055 \\
112\end{array}$ & $\begin{array}{r}7,690 \\
10,374 \\
5,146 \\
63,367 \\
69\end{array}$ \\
\hline 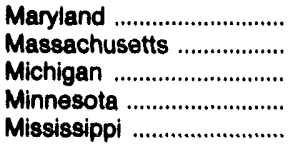 & $\begin{array}{r}31,645 \\
46,745 \\
214,646 \\
59,787 \\
76,944\end{array}$ & $\begin{array}{r}33,267 \\
46,053 \\
204,699 \\
61,910 \\
68,769\end{array}$ & $\begin{array}{r}31,535 \\
38,222 \\
182,380 \\
60,919 \\
67,937\end{array}$ & $\begin{array}{r}3,892 \\
6,310 \\
23,408 \\
6,152 \\
10,343\end{array}$ & $\begin{array}{r}J, 374 \\
5,428 \\
22,728 \\
6,189 \\
9,573\end{array}$ & $\begin{array}{r}3,742 \\
6,288 \\
24,666 \\
7,192 \\
9,031\end{array}$ \\
\hline 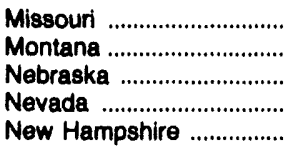 & $\begin{array}{r}38,324 \\
7,807 \\
23,457 \\
23,348 \\
2,587\end{array}$ & $\begin{array}{r}38,768 \\
7,385 \\
16,569 \\
6,168 \\
2,625\end{array}$ & $\begin{array}{r}37,518 \\
5,839 \\
16,628 \\
4,442 \\
2,260\end{array}$ & $\begin{array}{r}3,992 \\
921 \\
3,464 \\
3,209 \\
290\end{array}$ & $\begin{array}{r}3,727 \\
954 \\
2,624 \\
3,322 \\
311\end{array}$ & $\begin{array}{r}4,098 \\
780 \\
2,276 \\
3,240 \\
297\end{array}$ \\
\hline 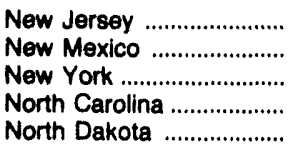 & $\begin{array}{r}124,028 \\
11,693 \\
102,193 \\
62,620 \\
4,088\end{array}$ & $\begin{array}{r}111,438 \\
11,417 \\
96,865 \\
60,190 \\
3,953\end{array}$ & $\begin{array}{r}65,104 \\
12,409 \\
77,449 \\
55,127 \\
2,999\end{array}$ & $\begin{array}{r}14,051 \\
1,743 \\
10,394 \\
7,905 \\
411\end{array}$ & $\begin{array}{r}14,046 \\
1,414 \\
9,984 \\
6,829 \\
421\end{array}$ & $\begin{array}{r}14,198 \\
1,335 \\
10,889 \\
7,828 \\
482\end{array}$ \\
\hline 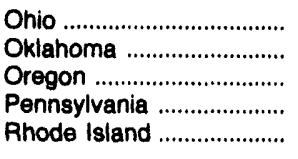 & $\begin{array}{r}196,031 \\
117,489 \\
40,906 \\
166,890 \\
30,941\end{array}$ & $\begin{array}{r}193,801 \\
118,254 \\
37,527 \\
157,673 \\
32,511\end{array}$ & $\begin{array}{r}181,793 \\
120,497 \\
36,263 \\
153,070 \\
14,962\end{array}$ & $\begin{array}{r}21,157 \\
15,069 \\
5,054 \\
17,884 \\
4,421\end{array}$ & $\begin{array}{r}14,829 \\
13,959 \\
4,889 \\
\text { A } 17,868 \\
3,803\end{array}$ & $\begin{array}{r}22,180 \\
13,198 \\
4,725 \\
19,245 \\
3,799\end{array}$ \\
\hline 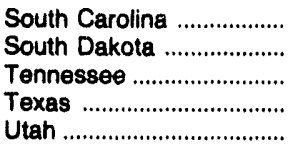 & $\begin{array}{r}62,830 \\
3,105 \\
78,549 \\
1,263,003 \\
30,323\end{array}$ & $\begin{array}{r}63,604 \\
2,942 \\
83,906 \\
1,151,999 \\
25,824\end{array}$ & $\begin{array}{r}56,246 \\
3,125 \\
77,633 \\
1,133,447 \\
26,343\end{array}$ & $\begin{array}{r}8,188 \\
285 \\
9,463 \\
157,355 \\
3,167\end{array}$ & $\begin{array}{r}7,449 \\
334 \\
8,827 \\
167,329 \\
2,826\end{array}$ & $\begin{array}{r}7,884 \\
340 \\
7,665 \\
154,284 \\
3,254\end{array}$ \\
\hline 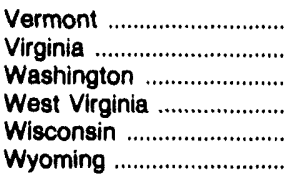 & $\begin{array}{r}1,337 \\
66,956 \\
61,427 \\
32,341 \\
88,253 \\
34,009\end{array}$ & $\begin{array}{r}1,281 \\
46,887 \\
52,817 \\
28,389 \\
84,744 \\
37,192\end{array}$ & $\begin{array}{r}1,078 \\
40,744 \\
51,594 \\
26,617 \\
84,015 \\
20,910\end{array}$ & $\begin{array}{r}139 \\
11,058 \\
7,438 \\
3,730 \\
8,083 \\
3,650\end{array}$ & $\begin{array}{r}137 \\
10,951 \\
7,134 \\
3,815 \\
7,378 \\
4,475\end{array}$ & $\begin{array}{r}136 \\
7,470 \\
6,335 \\
3,777 \\
8,031 \\
4,123\end{array}$ \\
\hline Total & $5,129,362$ & $5,015,381$ & $4,731,323$ & 612,391 & 617,831 & 596,727 \\
\hline
\end{tabular}

See footnotes at end of table. 
Table 20. Natural Gas Deliverles to Industrial Consumers by State, 1992-1993 (Continued)

(Million Cubic Feet)

\begin{tabular}{|c|c|c|c|c|c|c|}
\hline State & $\begin{array}{l}\text { May } \\
1983\end{array}$ & $\begin{array}{l}\text { April } \\
1993\end{array}$ & $\begin{array}{c}\text { March } \\
1993\end{array}$ & $\begin{array}{c}\text { February } \\
1993\end{array}$ & $\begin{array}{c}\text { January } \\
1993\end{array}$ & $\begin{array}{l}\text { Total } \\
1992\end{array}$ \\
\hline 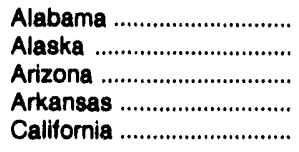 & $\begin{array}{r}13,800 \\
5,906 \\
1,679 \\
8,939 \\
46,173\end{array}$ & $\begin{array}{r}14,999 \\
6,220 \\
1,867 \\
9,393 \\
43,228\end{array}$ & $\begin{array}{r}15,943 \\
6,258 \\
1,979 \\
10,786 \\
48,371\end{array}$ & $\begin{array}{r}15,268 \\
6,298 \\
1,561 \\
9,765 \\
49,424\end{array}$ & $\begin{array}{r}15,987 \\
7,757 \\
1,713 \\
10,639 \\
41,239\end{array}$ & $\begin{array}{r}169,049 \\
80,938 \\
19,774 \\
118,850 \\
594,569\end{array}$ \\
\hline 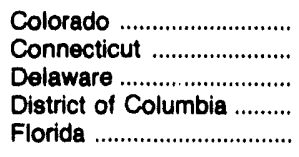 & $\begin{array}{r}7,322 \\
2,737 \\
1,632 \\
0 \\
8,210\end{array}$ & $\begin{array}{r}7,372 \\
3,176 \\
1,726 \\
0 \\
7,917\end{array}$ & $\begin{array}{r}7,220 \\
3,903 \\
1,776 \\
0 \\
8,362\end{array}$ & $\begin{array}{r}7,683 \\
4,043 \\
1,571 \\
0 \\
7,983\end{array}$ & $\begin{array}{r}8,075 \\
3,590 \\
1,713 \\
0 \\
8,022\end{array}$ & $\begin{array}{r}57,579 \\
36,383 \\
18,060 \\
0 \\
84,829\end{array}$ \\
\hline 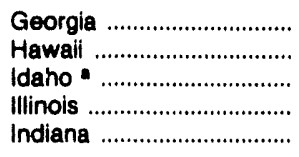 & $\begin{array}{r}13,488 \\
0 \\
2,272 \\
21,681 \\
19,866\end{array}$ & $\begin{array}{r}13,685 \\
0 \\
2,383 \\
24,113 \\
21,485\end{array}$ & $\begin{array}{r}14,155 \\
0 \\
2,759 \\
31,811 \\
25,813\end{array}$ & $\begin{array}{r}13,736 \\
0 \\
2,718 \\
33,445 \\
25,146\end{array}$ & $\begin{array}{r}14,196 \\
0 \\
2,726 \\
32,289 \\
25,736\end{array}$ & $\begin{array}{r}172,227 \\
0 \\
27,044 \\
300,366 \\
245,523\end{array}$ \\
\hline 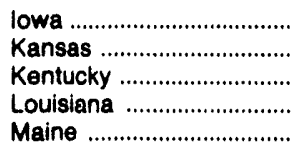 & $\begin{array}{r}7,765 \\
10,749 \\
5,351 \\
60,391 \\
80\end{array}$ & $\begin{array}{r}7,847 \\
10,619 \\
6,128 \\
64,569 \\
142\end{array}$ & $\begin{array}{r}9,406 \\
11,489 \\
7,429 \\
65,052 \\
136\end{array}$ & $\begin{array}{r}9,036 \\
12,998 \\
7,801 \\
63,341 \\
126\end{array}$ & $\begin{array}{r}9,143 \\
12,017 \\
7,356 \\
67,902 \\
152\end{array}$ & $\begin{array}{r}100,752 \\
130,807 \\
73,619 \\
932,467 \\
2,048\end{array}$ \\
\hline 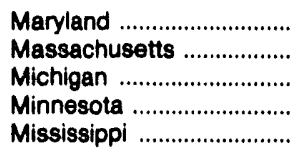 & $\begin{array}{r}3,868 \\
5,846 \\
24,531 \\
6,251 \\
9,194\end{array}$ & $\begin{array}{r}3,635 \\
5,305 \\
27,239 \\
7,093 \\
9,837\end{array}$ & $\begin{array}{r}4,634 \\
5,911 \\
31,681 \\
8,841 \\
9,854\end{array}$ & $\begin{array}{r}4,283 \\
5,694 \\
30,798 \\
8,690 \\
8,840\end{array}$ & $\begin{array}{r}4,217 \\
5,963 \\
29,595 \\
9,380 \\
10,271\end{array}$ & $\begin{array}{r}49,720 \\
70,872 \\
305,416 \\
93,025 \\
102,612\end{array}$ \\
\hline 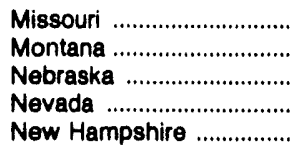 & $\begin{array}{r}4,188 \\
755 \\
2,294 \\
2,995 \\
375\end{array}$ & $\begin{array}{r}4,947 \\
931 \\
3,653 \\
4,051 \\
366\end{array}$ & $\begin{array}{r}6,015 \\
1,192 \\
2,747 \\
2,181 \\
325\end{array}$ & $\begin{array}{r}5,789 \\
1,067 \\
2,663 \\
1,679 \\
304\end{array}$ & $\begin{array}{r}6,568 \\
1,206 \\
3,737 \\
2,672 \\
318\end{array}$ & $\begin{array}{r}58,466 \\
12,218 \\
26,451 \\
9,259 \\
3,828\end{array}$ \\
\hline 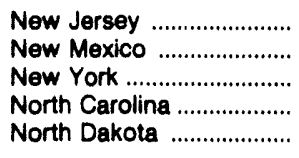 & $\begin{array}{r}14,283 \\
1,315 \\
11,505 \\
7,584 \\
467\end{array}$ & $\begin{array}{r}16,500 \\
1,533 \\
13,610 \\
8,054 \\
504\end{array}$ & $\begin{array}{r}16,846 \\
1,416 \\
15,669 \\
8,604 \\
582\end{array}$ & $\begin{array}{r}16,874 \\
1,458 \\
14,948 \\
7,620 \\
586\end{array}$ & $\begin{array}{r}17,230 \\
1,480 \\
15,192 \\
8,195 \\
635\end{array}$ & $\begin{array}{r}174,569 \\
17,070 \\
147,520 \\
90,984 \\
5,940\end{array}$ \\
\hline 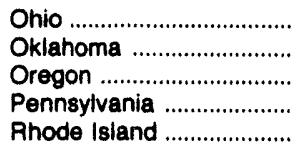 & $\begin{array}{r}22,139 \\
14,390 \\
5,009 \\
18,966 \\
3,941\end{array}$ & $\begin{array}{r}26,126 \\
14,510 \\
5,329 \\
20,885 \\
3,726\end{array}$ & $\begin{array}{r}30,726 \\
15,270 \\
5,457 \\
24,765 \\
3,771\end{array}$ & $\begin{array}{r}29,475 \\
14,773 \\
4,878 \\
23,606 \\
3,695\end{array}$ & $\begin{array}{r}29,299 \\
16,320 \\
5,565 \\
23,571 \\
3,786\end{array}$ & $\begin{array}{r}294,805 \\
175,168 \\
58,519 \\
236,708 \\
47,917\end{array}$ \\
\hline 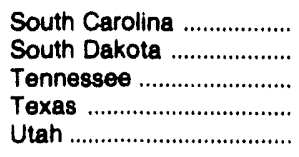 & $\begin{array}{r}7,301 \\
346 \\
10,212 \\
143,652 \\
3,164\end{array}$ & $\begin{array}{r}7,967 \\
364 \\
9,456 \\
157,790 \\
3,527\end{array}$ & $\begin{array}{r}8,723 \\
458 \\
10,368 \\
166,799 \\
4,062\end{array}$ & $\begin{array}{r}7,408 \\
462 \\
10,444 \\
149,368 \\
4,733\end{array}$ & $\begin{array}{r}7,909 \\
515 \\
12,014 \\
166,428 \\
5,590\end{array}$ & $\begin{array}{r}94,327 \\
4,488 \\
126,230 \\
1,734,001 \\
40,878\end{array}$ \\
\hline 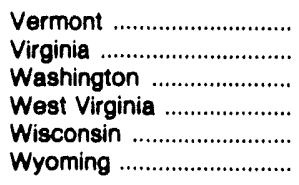 & $\begin{array}{r}159 \\
4,907 \\
7,582 \\
3,924 \\
8,792 \\
4,737\end{array}$ & $\begin{array}{r}173 \\
7,099 \\
7,503 \\
4,116 \\
12,166 \\
4,461\end{array}$ & $\begin{array}{r}191 \\
7,839 \\
8,979 \\
4,588 \\
14,082 \\
3,404\end{array}$ & $\begin{array}{r}187 \\
9,108 \\
8,143 \\
4,083 \\
14,459 \\
4,306\end{array}$ & $\begin{array}{r}215 \\
8,523 \\
8,313 \\
4,310 \\
15,263 \\
4,852\end{array}$ & $\begin{array}{r}1,958 \\
68,808 \\
79,766 \\
44,178 \\
130,267 \\
56,046\end{array}$ \\
\hline Total & 582,712 & 639,321 & 698,633 & 672,366 & 699,383 & $7,526,898$ \\
\hline
\end{tabular}

See footnotes at end of table. 
Table 20. Natural Gas Deliveries to Industrial Consumers by State, 1992-1993 (Continued)

(Million Cubic Feet)

\begin{tabular}{|c|c|c|c|c|c|c|}
\hline State & $\begin{array}{c}\text { December } \\
1992\end{array}$ & $\begin{array}{c}\text { November } \\
1992\end{array}$ & $\begin{array}{c}\text { October } \\
1992\end{array}$ & $\begin{array}{c}\text { September } \\
1992\end{array}$ & $\begin{array}{l}\text { August } \\
1992\end{array}$ & $\begin{array}{l}\text { July } \\
1992\end{array}$ \\
\hline $\begin{array}{l}\text { Alabama } \\
\text { Alaska } \\
\text { Arizona } \\
\text { Arkansas } \\
\text { California }\end{array}$ & $\begin{array}{r}14,906 \\
7,887 \\
1,974 \\
10,701 \\
48,770\end{array}$ & $\begin{array}{r}14,853 \\
7,337 \\
1,533 \\
9,916 \\
50,165\end{array}$ & $\begin{array}{r}13,968 \\
7,810 \\
1,657 \\
9,760 \\
40,509\end{array}$ & $\begin{array}{r}13,496 \\
4,841 \\
1,562 \\
9,778 \\
52,231\end{array}$ & $\begin{array}{r}13,309 \\
5,355 \\
1,581 \\
9,314 \\
49,895\end{array}$ & $\begin{array}{r}13,659 \\
6,968 \\
1,627 \\
9,129 \\
43,635\end{array}$ \\
\hline 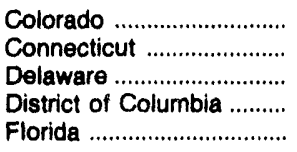 & $\begin{array}{r}6,583 \\
3,490 \\
1,760 \\
0 \\
7,792\end{array}$ & $\begin{array}{r}5,439 \\
3,102 \\
1,892 \\
0 \\
7,151\end{array}$ & $\begin{array}{r}5,093 \\
2,867 \\
1,790 \\
0 \\
6,330\end{array}$ & $\begin{array}{r}4,660 \\
2,732 \\
1,433 \\
0 \\
6,536\end{array}$ & $\begin{array}{r}4,044 \\
2,643 \\
1,354 \\
0 \\
6,618\end{array}$ & $\begin{array}{r}4,197 \\
2,522 \\
1,238 \\
0 \\
6,606\end{array}$ \\
\hline $\begin{array}{l}\text { Georgia } \\
\text { Hawaii } \\
\text { Idaho } \\
\text { Illinois } \\
\text { Indiana }\end{array}$ & $\begin{array}{r}14,203 \\
0 \\
2,615 \\
31,294 \\
24,807\end{array}$ & $\begin{array}{r}13,951 \\
0 \\
2,502 \\
26,739 \\
22,453\end{array}$ & $\begin{array}{r}14,085 \\
0 \\
2,351 \\
24,506 \\
20,770\end{array}$ & $\begin{array}{r}13,982 \\
0 \\
1,895 \\
20,992 \\
18,229\end{array}$ & $\begin{array}{r}13,862 \\
0 \\
1,657 \\
18,500 \\
16,896\end{array}$ & $\begin{array}{r}14,270 \\
0 \\
1,975 \\
19,944 \\
17,145\end{array}$ \\
\hline $\begin{array}{l}\text { lowa } \\
\text { Kansas } \\
\text { Kentucky } \\
\text { Louisiana } \\
\text { Maine }\end{array}$ & $\begin{array}{r}9,493 \\
12,952 \\
7,162 \\
72,036 \\
165\end{array}$ & $\begin{array}{r}9,725 \\
12,023 \\
6,649 \\
71,738 \\
207\end{array}$ & $\begin{array}{r}8,384 \\
13,293 \\
6,574 \\
72,644 \\
152\end{array}$ & $\begin{array}{r}7,775 \\
9,933 \\
5,308 \\
64,894 \\
164\end{array}$ & $\begin{array}{r}7,636 \\
9,956 \\
5,401 \\
73,865 \\
162\end{array}$ & $\begin{array}{r}8,064 \\
10,029 \\
4,797 \\
80,422 \\
161\end{array}$ \\
\hline 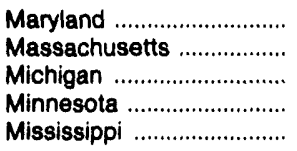 & $\begin{array}{r}4,346 \\
6,530 \\
27,672 \\
8,994 \\
8,476\end{array}$ & $\begin{array}{r}4,674 \\
6,385 \\
28,339 \\
8,883 \\
8,480\end{array}$ & $\begin{array}{r}3,916 \\
5,899 \\
23,810 \\
6,997 \\
8,514\end{array}$ & $\begin{array}{r}3,517 \\
6,004 \\
20,895 \\
6,241 \\
8,372\end{array}$ & $\begin{array}{r}3,615 \\
5,895 \\
21,680 \\
6,348 \\
7,936\end{array}$ & $\begin{array}{r}3,825 \\
4,698 \\
20,153 \\
6,436 \\
8,302\end{array}$ \\
\hline 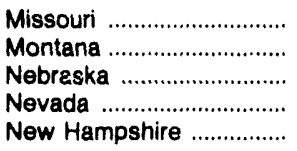 & $\begin{array}{r}5,823 \\
1,280 \\
3,015 \\
754 \\
292\end{array}$ & $\begin{array}{r}5,291 \\
1,345 \\
2,529 \\
777 \\
305\end{array}$ & $\begin{array}{r}4,626 \\
1,138 \\
2,226 \\
903 \\
270\end{array}$ & $\begin{array}{r}3,958 \\
1,071 \\
2,111 \\
658 \\
336\end{array}$ & $\begin{array}{r}4,132 \\
904 \\
1,966 \\
732 \\
333\end{array}$ & $\begin{array}{r}3,808 \\
883 \\
1,917 \\
718 \\
302\end{array}$ \\
\hline 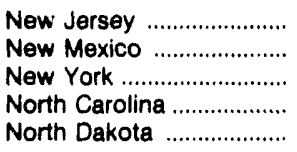 & $\begin{array}{r}16,237 \\
1,533 \\
14,944 \\
7,854 \\
570\end{array}$ & $\begin{array}{r}17,046 \\
1,389 \\
12,875 \\
7,862 \\
525\end{array}$ & $\begin{array}{r}15,572 \\
1,493 \\
11,595 \\
7,852 \\
484\end{array}$ & $\begin{array}{r}14,276 \\
1,238 \\
11,241 \\
7,226 \\
408\end{array}$ & $\begin{array}{r}14,687 \\
1,260 \\
10,137 \\
7,160 \\
390\end{array}$ & $\begin{array}{r}14,037 \\
1,038 \\
10,465 \\
6,663 \\
438\end{array}$ \\
\hline $\begin{array}{l}\text { Ohio } \\
\text { Oklahoma } \\
\text { Oregon } \\
\text { Pennsylvania } \\
\text { Rhode Island }\end{array}$ & $\begin{array}{r}29,294 \\
15,365 \\
4,908 \\
21,697 \\
3,803\end{array}$ & $\begin{array}{r}26,573 \\
14,394 \\
5,103 \\
21,164 \\
3,734\end{array}$ & $\begin{array}{r}23,979 \\
13,263 \\
5,544 \\
18,784 \\
4,140\end{array}$ & $\begin{array}{r}21,158 \\
13,290 \\
5,437 \\
17,389 \\
3,730\end{array}$ & $\begin{array}{r}19,914 \\
14,236 \\
5,229 \\
16,876 \\
3,688\end{array}$ & $\begin{array}{r}19,985 \\
14,509 \\
4,923 \\
17,173 \\
4,209\end{array}$ \\
\hline $\begin{array}{l}\text { South Carolina } \\
\text { South Dakota } \\
\text { Tennessee } \\
\text { Texas } \\
\text { Utah }\end{array}$ & $\begin{array}{r}7,032 \\
526 \\
11,079 \\
155,355 \\
4,741\end{array}$ & $\begin{array}{r}8,337 \\
412 \\
10,956 \\
137,499 \\
4,615\end{array}$ & $\begin{array}{r}7,589 \\
330 \\
10,577 \\
142,094 \\
3,175\end{array}$ & $\begin{array}{r}7,766 \\
278 \\
9,712 \\
147,054 \\
2,522\end{array}$ & $\begin{array}{r}7,702 \\
246 \\
9,933 \\
143,664 \\
2,512\end{array}$ & $\begin{array}{r}7,890 \\
358 \\
10,197 \\
146,392 \\
2,538\end{array}$ \\
\hline 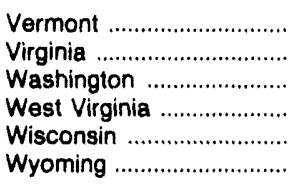 & $\begin{array}{r}196 \\
5,590 \\
7,179 \\
4,630 \\
14,008 \\
4,677\end{array}$ & $\begin{array}{r}169 \\
5,951 \\
6,962 \\
3,973 \\
12,610 \\
4,419\end{array}$ & $\begin{array}{r}167 \\
4,648 \\
6,744 \\
3,751 \\
10,269 \\
4,894\end{array}$ & $\begin{array}{r}145 \\
5,732 \\
5,964 \\
3,435 \\
8,636 \\
4,864\end{array}$ & $\begin{array}{r}130 \\
6,018 \\
6,214 \\
3,426 \\
8,127 \\
4,551\end{array}$ & $\begin{array}{r}119 \\
7,366 \\
5,717 \\
3,209 \\
7,610 \\
4,841\end{array}$ \\
\hline Total & 676,992 & 640,954 & 607,784 & 585,706 & 581,772 & 587,203 \\
\hline
\end{tabular}

See footnotes at end of table. 
Table 20. Natural Gas Deliveries to Industrial Consumers by State, 1992-1993 (Continued)

(Million Cubic Feet)

\begin{tabular}{|c|c|c|c|c|c|c|}
\hline State & $\begin{array}{l}\text { June } \\
1092 \\
\end{array}$ & $\begin{array}{c}\text { May } \\
1992 \\
\end{array}$ & $\begin{array}{l}\text { April } \\
1992\end{array}$ & $\begin{array}{c}\text { March } \\
1992\end{array}$ & $\begin{array}{c}\text { February } \\
1992\end{array}$ & $\begin{array}{c}\text { January } \\
1992\end{array}$ \\
\hline 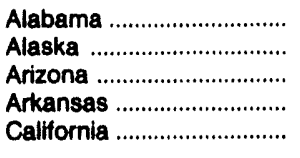 & $\begin{array}{r}13,739 \\
6,317 \\
1,613 \\
9,098 \\
47,916\end{array}$ & $\begin{array}{r}13,894 \\
6,902 \\
1,543 \\
9,868 \\
60,503\end{array}$ & $\begin{array}{r}13,624 \\
6,696 \\
1,642 \\
9,864 \\
32,593\end{array}$ & $\begin{array}{r}15,147 \\
6,561 \\
1,723 \\
10,513 \\
49,236\end{array}$ & $\begin{array}{r}13,986 \\
6,910 \\
1,588 \\
9,941 \\
55,101\end{array}$ & $\begin{array}{r}14,469 \\
7,354 \\
1,731 \\
10,967 \\
64,014\end{array}$ \\
\hline 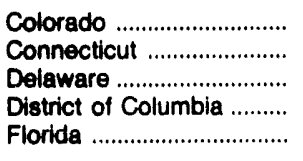 & $\begin{array}{r}4,173 \\
2,621 \\
1,343 \\
0 \\
6,891\end{array}$ & $\begin{array}{r}3,949 \\
2,775 \\
1,501 \\
0 \\
7,389\end{array}$ & $\begin{array}{r}4,555 \\
3,241 \\
1,548 \\
0 \\
7,303\end{array}$ & $\begin{array}{r}4,597 \\
3,672 \\
1,430 \\
0 \\
7,293\end{array}$ & $\begin{array}{r}5,035 \\
3,376 \\
1,409 \\
0 \\
6,908\end{array}$ & $\begin{array}{r}5,254 \\
3,343 \\
1,362 \\
0 \\
7,911\end{array}$ \\
\hline $\begin{array}{l}\text { Georgia } \\
\text { Hawaii } \\
\text { Idaho } \\
\text { Illinolis } \\
\text { Indiana }\end{array}$ & $\begin{array}{r}13,854 \\
0 \\
2,241 \\
18,988 \\
17,745\end{array}$ & $\begin{array}{r}14,505 \\
0 \\
2,286 \\
22,957 \\
18,817\end{array}$ & $\begin{array}{r}14,863 \\
0 \\
2,243 \\
25,873 \\
19,954\end{array}$ & $\begin{array}{r}15,505 \\
0 \\
2,464 \\
29,274 \\
22,941\end{array}$ & $\begin{array}{r}14,390 \\
0 \\
2,244 \\
29,992 \\
22,081\end{array}$ & $\begin{array}{r}14,755 \\
0 \\
2,490 \\
31,306 \\
23,685\end{array}$ \\
\hline 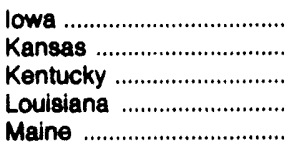 & $\begin{array}{r}7,792 \\
9,699 \\
5,273 \\
74,285 \\
157\end{array}$ & $\begin{array}{r}7,771 \\
9,303 \\
5,449 \\
78,686 \\
172\end{array}$ & $\begin{array}{r}8,161 \\
10,097 \\
6,023 \\
87,445 \\
219\end{array}$ & $\begin{array}{r}8,801 \\
11,009 \\
6,715 \\
87,311 \\
203\end{array}$ & $\begin{array}{r}8,350 \\
10,345 \\
6,823 \\
81,119 \\
159\end{array}$ & $\begin{array}{r}8,798 \\
12,169 \\
7,446 \\
88,023 \\
127\end{array}$ \\
\hline 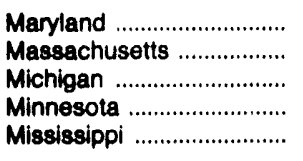 & $\begin{array}{r}3,603 \\
6,041 \\
22,387 \\
6,144 \\
8,251\end{array}$ & $\begin{array}{r}3,785 \\
6,524 \\
24,900 \\
6,951 \\
8,453\end{array}$ & $\begin{array}{r}4,419 \\
7,528 \\
28,501 \\
7,863 \\
8,936\end{array}$ & $\begin{array}{r}5,128 \\
4,978 \\
30,508 \\
8,665 \\
9,453\end{array}$ & $\begin{array}{r}4,435 \\
5,545 \\
29,056 \\
9,635 \\
7,916\end{array}$ & $\begin{array}{r}4,457 \\
4,746 \\
27,534 \\
9,868 \\
9,524\end{array}$ \\
\hline 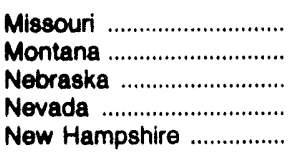 & $\begin{array}{r}4,084 \\
782 \\
2,197 \\
783 \\
300\end{array}$ & $\begin{array}{r}4,268 \\
841 \\
1,710 \\
821 \\
312\end{array}$ & $\begin{array}{r}5,027 \\
870 \\
2,172 \\
814 \\
422\end{array}$ & $\begin{array}{r}5,533 \\
861 \\
2,015 \\
821 \\
315\end{array}$ & $\begin{array}{r}5,483 \\
1,096 \\
2,350 \\
704 \\
331\end{array}$ & $\begin{array}{r}6,332 \\
1,147 \\
2,243 \\
775 \\
308\end{array}$ \\
\hline 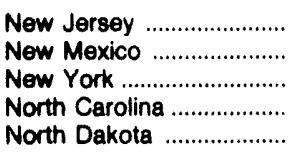 & $\begin{array}{r}14,027 \\
1,004 \\
10,805 \\
7,088 \\
420\end{array}$ & $\begin{array}{r}13,014 \\
1,323 \\
11,273 \\
7,410 \\
469\end{array}$ & $\begin{array}{r}14,133 \\
1,563 \\
12,441 \\
7,565 \\
472\end{array}$ & $\begin{array}{r}13,905 \\
1,468 \\
14,028 \\
8,652 \\
608\end{array}$ & $\begin{array}{r}13,898 \\
1,819 \\
13,968 \\
7,935 \\
528\end{array}$ & $\begin{array}{r}13,736 \\
1,942 \\
13,748 \\
7,716 \\
627\end{array}$ \\
\hline 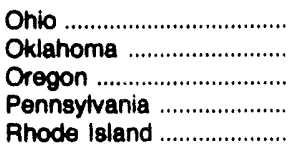 & $\begin{array}{r}21,123 \\
13,932 \\
4,296 \\
16,470 \\
4,241\end{array}$ & $\begin{array}{r}22,201 \\
14,326 \\
3,796 \\
18,523 \\
4,017\end{array}$ & $\begin{array}{r}26,498 \\
14,259 \\
3,926 \\
20,700 \\
4,321\end{array}$ & $\begin{array}{r}26,339 \\
15,620 \\
4,352 \\
22,389 \\
3,999\end{array}$ & $\begin{array}{r}28,592 \\
15,250 \\
5,507 \\
22,868 \\
4,323\end{array}$ & $\begin{array}{r}29,149 \\
16,123 \\
5,497 \\
22,674 \\
3,713\end{array}$ \\
\hline 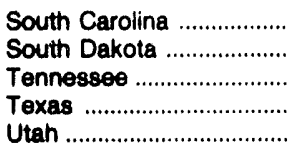 & $\begin{array}{r}8,110 \\
323 \\
9,366 \\
138,927 \\
2,771\end{array}$ & $\begin{array}{r}8,183 \\
346 \\
9,693 \\
147,828 \\
2,632\end{array}$ & $\begin{array}{r}8,128 \\
418 \\
11,278 \\
145,134 \\
2,997\end{array}$ & $\begin{array}{r}8,921 \\
435 \\
10,643 \\
150,805 \\
3,701\end{array}$ & $\begin{array}{r}7,635 \\
439 \\
11,027 \\
125,335 \\
3,867\end{array}$ & $\begin{array}{r}7,035 \\
380 \\
11,769 \\
153,914 \\
4,808\end{array}$ \\
\hline 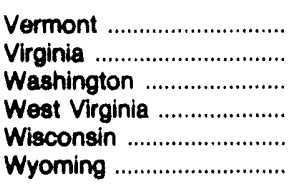 & $\begin{array}{r}128 \\
4,886 \\
5,900 \\
3,403 \\
7,867 \\
4,976\end{array}$ & $\begin{array}{r}149 \\
5,300 \\
6,222 \\
3,431 \\
8,838 \\
4,667\end{array}$ & $\begin{array}{r}141 \\
5,618 \\
6,616 \\
3,697 \\
11,327 \\
4,366\end{array}$ & $\begin{array}{r}223 \\
6,205 \\
7,073 \\
3,943 \\
13,296 \\
4,605\end{array}$ & $\begin{array}{r}200 \\
5,916 \\
7,401 \\
3,574 \\
13,093 \\
4,618\end{array}$ & $\begin{array}{r}190 \\
5,578 \\
7,774 \\
3,705 \\
14,585 \\
4,569\end{array}$ \\
\hline Total & 578,480 & 620,479 & 628,092 & 673,883 & 644,100 & 701,371 \\
\hline
\end{tabular}

- Small volumes of natural gas representing onsystem sales to industrial consumers in Idaho are included in the annual total but not in monthly components. Deliveries for total year 1992 do not equal the sum of the twelve months.

$\mathbf{n}=$ Revised Data.

$M=$ Not Available.

Notes: Geographic coverage is the 50 States and the District of Columbia. See Appendix A, Explanatory Note 5 for discussion of computations and revision policy.

Source: Form EIA-857. 
Table 21. Natural Gas Deliveries to Electric Utility" Consumers by State, 1992-1993 (Million Cubic Feet)

\begin{tabular}{|c|c|c|c|c|c|c|}
\hline State & $\begin{array}{l}\text { YTD } \\
1993\end{array}$ & $\begin{array}{l}\text { YTD } \\
1992\end{array}$ & $\begin{array}{c}\text { YTD } \\
1991\end{array}$ & $\begin{array}{c}\text { August } \\
1993\end{array}$ & $\begin{array}{c}\text { July } \\
1993\end{array}$ & $\begin{array}{l}\text { June } \\
1993\end{array}$ \\
\hline 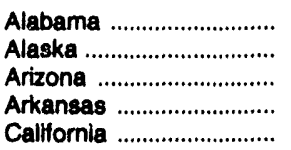 & $\begin{array}{r}3,645 \\
17,670 \\
14,377 \\
11,522 \\
278,298\end{array}$ & $\begin{array}{r}2,277 \\
18,650 \\
22,776 \\
23,503 \\
360,245\end{array}$ & $\begin{array}{r}2,829 \\
21,950 \\
14,731 \\
23,328 \\
261,055\end{array}$ & $\begin{array}{r}603 \\
1,794 \\
4,184 \\
4,587 \\
44,724\end{array}$ & $\begin{array}{r}1,444 \\
1,790 \\
2,580 \\
3,846 \\
31,247\end{array}$ & $\begin{array}{r}418 \\
2,027 \\
1,397 \\
1,646 \\
31,453\end{array}$ \\
\hline $\begin{array}{l}\text { Colorado ....................... } \\
\text { Connecticut ....................... } \\
\text { Delaware ........................ } \\
\text { District of Columbia ........ } \\
\text { Florida .............................. }\end{array}$ & $\begin{array}{r}3,278 \\
482 \\
4,346 \\
0 \\
121,895\end{array}$ & $\begin{array}{r}3,135 \\
2,021 \\
5,786 \\
0 \\
146,235\end{array}$ & $\begin{array}{r}3,374 \\
3,170 \\
10,410 \\
0 \\
136,620\end{array}$ & $\begin{array}{r}338 \\
152 \\
1,095 \\
0 \\
20,015\end{array}$ & $\begin{array}{r}470 \\
184 \\
1,231 \\
0 \\
19,776\end{array}$ & $\begin{array}{r}398 \\
13 \\
953 \\
0 \\
18,980\end{array}$ \\
\hline 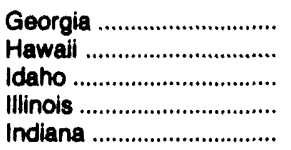 & $\begin{array}{r}2,811 \\
0 \\
0 \\
8,618 \\
3,919\end{array}$ & $\begin{array}{r}1,006 \\
0 \\
0 \\
7,789 \\
5,962\end{array}$ & $\begin{array}{r}449 \\
0 \\
0 \\
9,466 \\
5,833\end{array}$ & $\begin{array}{r}670 \\
0 \\
0 \\
3,369 \\
788\end{array}$ & $\begin{array}{r}1,554 \\
0 \\
0 \\
1,951 \\
611\end{array}$ & $\begin{array}{r}398 \\
0 \\
0 \\
1,089 \\
245\end{array}$ \\
\hline 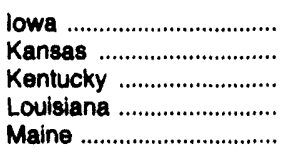 & $\begin{array}{r}3,582 \\
16,426 \\
153 \\
161,498 \\
0\end{array}$ & $\begin{array}{r}1,532 \\
10,698 \\
184 \\
187,470 \\
0\end{array}$ & $\begin{array}{r}2,492 \\
26,439 \\
159 \\
178,173 \\
0\end{array}$ & $\begin{array}{r}1,589 \\
5,391 \\
26 \\
39,699 \\
0\end{array}$ & $\begin{array}{r}835 \\
5,219 \\
24 \\
36,385 \\
0\end{array}$ & $\begin{array}{r}176 \\
2,037 \\
22 \\
26,473 \\
0\end{array}$ \\
\hline 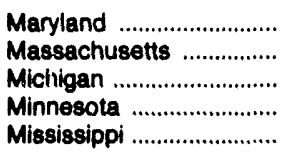 & $\begin{array}{r}6,017 \\
21,413 \\
13,864 \\
2,339 \\
27,248\end{array}$ & $\begin{array}{r}8,782 \\
25,864 \\
17,774 \\
3,690 \\
43,677\end{array}$ & $\begin{array}{r}12,806 \\
28,772 \\
15,560 \\
4,328 \\
47,862\end{array}$ & $\begin{array}{r}1,563 \\
4,857 \\
2,691 \\
804 \\
6,671\end{array}$ & $\begin{array}{r}2,097 \\
4,400 \\
2,153 \\
287 \\
7,334\end{array}$ & $\begin{array}{r}647 \\
2,606 \\
1,685 \\
282 \\
3,987\end{array}$ \\
\hline 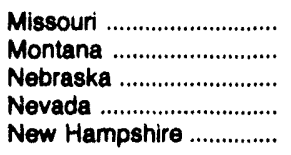 & $\begin{array}{r}4,098 \\
151 \\
1,515 \\
14,752 \\
136\end{array}$ & $\begin{array}{r}2,047 \\
123 \\
1,086 \\
18,381 \\
118\end{array}$ & $\begin{array}{r}10,453 \\
181 \\
1,995 \\
14,788 \\
0\end{array}$ & $\begin{array}{r}2,204 \\
52 \\
399 \\
2,657 \\
0\end{array}$ & $\begin{array}{r}1,305 \\
34 \\
454 \\
2,613 \\
3\end{array}$ & $\begin{array}{r}224 \\
13 \\
114 \\
1,852 \\
36\end{array}$ \\
\hline $\begin{array}{l}\text { New Jersey ..................... } \\
\text { New Mexico .................... } \\
\text { New York ....................... } \\
\text { North Carolina ................. } \\
\text { North Dakota ................... }\end{array}$ & $\begin{array}{r}28,459 \\
19,789 \\
125,944 \\
2,320 \\
1\end{array}$ & $\begin{array}{r}31,460 \\
15,653 \\
151,456 \\
2,362 \\
1\end{array}$ & $\begin{array}{r}51,319 \\
19,615 \\
146,770 \\
2,125 \\
0\end{array}$ & $\begin{array}{r}7,797 \\
3,097 \\
21,138 \\
564 \\
0\end{array}$ & $\begin{array}{r}11,574 \\
3,149 \\
25,652 \\
720 \\
0\end{array}$ & $\begin{array}{r}4,252 \\
2,710 \\
19,450 \\
323 \\
0\end{array}$ \\
\hline 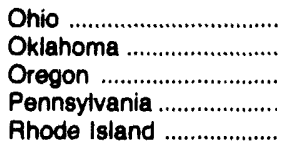 & $\begin{array}{r}2,456 \\
107,214 \\
7,426 \\
6,545 \\
221\end{array}$ & $\begin{array}{r}2,353 \\
105,240 \\
8,081 \\
2,154 \\
458\end{array}$ & $\begin{array}{r}2,099 \\
117,593 \\
3,268 \\
1,485 \\
1,318\end{array}$ & $\begin{array}{r}469 \\
22,607 \\
2,234 \\
932 \\
87\end{array}$ & $\begin{array}{r}399 \\
20,634 \\
0 \\
1,206 \\
52\end{array}$ & $\begin{array}{r}149 \\
13,882 \\
15 \\
814 \\
82\end{array}$ \\
\hline 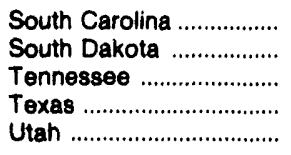 & $\begin{array}{r}1,685 \\
95 \\
1,508 \\
733,243 \\
4,234\end{array}$ & $\begin{array}{r}1,643 \\
11 \\
222 \\
651,324 \\
4,690\end{array}$ & $\begin{array}{r}9,542 \\
162 \\
108 \\
689,463 \\
2,956\end{array}$ & $\begin{array}{r}417 \\
78 \\
386 \\
142,579 \\
390\end{array}$ & $\begin{array}{r}808 \\
1 \\
904 \\
135,348 \\
368\end{array}$ & $\begin{array}{r}311 \\
1 \\
132 \\
111,153 \\
422\end{array}$ \\
\hline 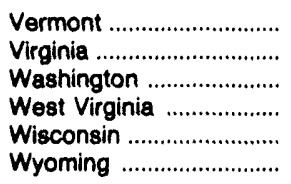 & $\begin{array}{r}245 \\
13,091 \\
4,571 \\
97 \\
2,258 \\
72\end{array}$ & $\begin{array}{r}647 \\
9,347 \\
4,423 \\
159 \\
1,849 \\
54\end{array}$ & $\begin{array}{r}929 \\
6,766 \\
54 \\
89 \\
2,022 \\
43\end{array}$ & $\begin{array}{r}0 \\
2,404 \\
5 \\
27 \\
559 \\
5\end{array}$ & $\begin{array}{r}129 \\
2,350 \\
4 \\
7 \\
245 \\
6\end{array}$ & $\begin{array}{r}79 \\
1,229 \\
0 \\
13 \\
297 \\
13\end{array}$ \\
\hline 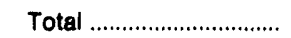 & $1,805,528$ & $1,914,394$ & $1,895,051$ & 356,695 & 333,405 & 254,601 \\
\hline
\end{tabular}

See footnotes at end of table. 
Table 21. Natural Gas Deliveries to Electric Utilitya Consumers by State, 1992-1993 (Continued)

(Million Cubic Feet)

\begin{tabular}{|c|c|c|c|c|c|c|}
\hline State & $\begin{array}{l}\text { May } \\
1993\end{array}$ & $\begin{array}{l}\text { April } \\
1993\end{array}$ & $\begin{array}{c}\text { March } \\
1993\end{array}$ & $\begin{array}{c}\text { February } \\
1993\end{array}$ & $\begin{array}{c}\text { January } \\
1993\end{array}$ & $\begin{array}{l}\text { Total } \\
1992\end{array}$ \\
\hline 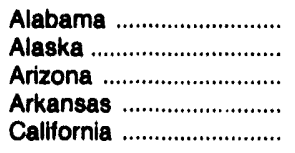 & $\begin{array}{r}266 \\
2,105 \\
1,765 \\
267 \\
17,816\end{array}$ & $\begin{array}{r}144 \\
2,155 \\
1,402 \\
311 \\
32,390\end{array}$ & $\begin{array}{r}215 \\
2,482 \\
1,921 \\
280 \\
38,720\end{array}$ & $\begin{array}{r}306 \\
2,535 \\
588 \\
263 \\
40,118\end{array}$ & $\begin{array}{r}249 \\
2,781 \\
530 \\
322 \\
41,829\end{array}$ & $\begin{array}{r}3,368 \\
28,953 \\
30,939 \\
27,015 \\
564,432\end{array}$ \\
\hline 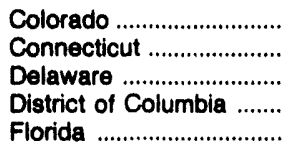 & $\begin{array}{r}278 \\
8 \\
272 \\
0 \\
16,262\end{array}$ & $\begin{array}{r}354 \\
10 \\
322 \\
0 \\
12,549\end{array}$ & $\begin{array}{r}514 \\
20 \\
253 \\
0 \\
12,232\end{array}$ & $\begin{array}{r}394 \\
62 \\
55 \\
0 \\
11,459\end{array}$ & $\begin{array}{r}531 \\
25 \\
164 \\
0 \\
10,621\end{array}$ & $\begin{array}{r}5,019 \\
2,100 \\
8,384 \\
0 \\
202,576\end{array}$ \\
\hline 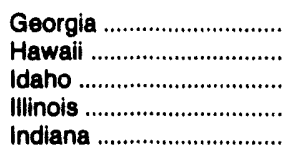 & $\begin{array}{r}74 \\
0 \\
0 \\
377 \\
273\end{array}$ & $\begin{array}{r}33 \\
0 \\
0 \\
445 \\
437\end{array}$ & $\begin{array}{r}22 \\
0 \\
0 \\
482 \\
457\end{array}$ & $\begin{array}{r}49 \\
0 \\
0 \\
396 \\
450\end{array}$ & $\begin{array}{r}12 \\
0 \\
0 \\
509 \\
658\end{array}$ & $\begin{array}{r}1,162 \\
0 \\
0 \\
9,293 \\
7,772\end{array}$ \\
\hline 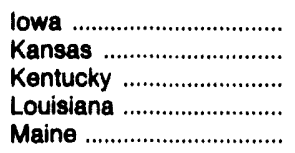 & $\begin{array}{r}233 \\
846 \\
19 \\
13,920 \\
0\end{array}$ & $\begin{array}{r}238 \\
649 \\
27 \\
10,869 \\
0\end{array}$ & $\begin{array}{r}169 \\
920 \\
11 \\
14,087 \\
0\end{array}$ & $\begin{array}{r}106 \\
670 \\
12 \\
9,246 \\
0\end{array}$ & $\begin{array}{r}236 \\
694 \\
12 \\
10,820 \\
0\end{array}$ & $\begin{array}{r}2,265 \\
13,981 \\
269 \\
254,922 \\
0\end{array}$ \\
\hline 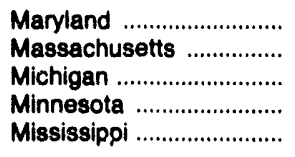 & $\begin{array}{r}385 \\
2,513 \\
1,345 \\
297 \\
2,968\end{array}$ & $\begin{array}{r}187 \\
3,815 \\
925 \\
185 \\
1,335\end{array}$ & $\begin{array}{r}560 \\
2,955 \\
1,380 \\
164 \\
2,114\end{array}$ & $\begin{array}{r}349 \\
128 \\
1,904 \\
144 \\
1,248\end{array}$ & $\begin{array}{r}229 \\
138 \\
1,781 \\
176 \\
1,591\end{array}$ & $\begin{array}{r}11,575 \\
38,341 \\
24,908 \\
4,906 \\
54,180\end{array}$ \\
\hline 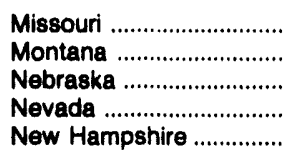 & $\begin{array}{r}54 \\
13 \\
137 \\
2,157 \\
91\end{array}$ & $\begin{array}{r}63 \\
4 \\
148 \\
1,695 \\
6\end{array}$ & $\begin{array}{r}113 \\
15 \\
111 \\
2,305 \\
0\end{array}$ & $\begin{array}{r}75 \\
16 \\
48 \\
926 \\
0\end{array}$ & $\begin{array}{r}59 \\
4 \\
104 \\
547 \\
0\end{array}$ & $\begin{array}{r}2,351 \\
220 \\
1,903 \\
24,355 \\
633\end{array}$ \\
\hline 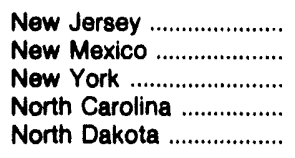 & $\begin{array}{r}1,278 \\
2,067 \\
12,276 \\
100 \\
0\end{array}$ & $\begin{array}{r}329 \\
2,811 \\
14,335 \\
99 \\
0\end{array}$ & $\begin{array}{r}1,508 \\
2,793 \\
12,983 \\
141 \\
0\end{array}$ & $\begin{array}{r}1,383 \\
1,699 \\
9,569 \\
205 \\
0\end{array}$ & $\begin{array}{r}338 \\
1,463 \\
10,543 \\
167 \\
0\end{array}$ & $\begin{array}{r}38,772 \\
22,486 \\
208,731 \\
3,159 \\
1\end{array}$ \\
\hline 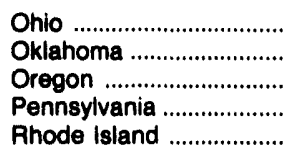 & $\begin{array}{r}135 \\
10,542 \\
0 \\
211 \\
0\end{array}$ & $\begin{array}{r}102 \\
10,485 \\
0 \\
337 \\
0\end{array}$ & $\begin{array}{r}417 \\
10,268 \\
1,990 \\
1,133 \\
0\end{array}$ & $\begin{array}{r}432 \\
9,249 \\
2,074 \\
1,156 \\
0\end{array}$ & $\begin{array}{r}352 \\
9,447 \\
1,113 \\
757 \\
0\end{array}$ & $\begin{array}{r}2,956 \\
148,980 \\
14,264 \\
3,100 \\
469\end{array}$ \\
\hline 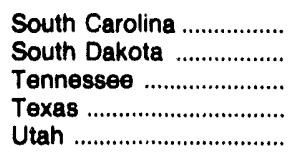 & $\begin{array}{r}24 \\
2 \\
15 \\
74,821 \\
507\end{array}$ & $\begin{array}{r}21 \\
0 \\
14 \\
71,510 \\
592\end{array}$ & $\begin{array}{r}69 \\
10 \\
15 \\
75,318 \\
697\end{array}$ & $\begin{array}{r}20 \\
2 \\
21 \\
59,828 \\
557\end{array}$ & $\begin{array}{r}17 \\
0 \\
20 \\
62,686 \\
700\end{array}$ & $\begin{array}{r}1,795 \\
48 \\
291 \\
968,165 \\
6,576\end{array}$ \\
\hline 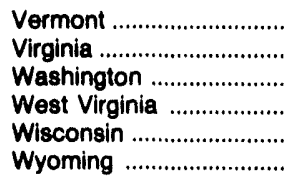 & $\begin{array}{r}18 \\
174 \\
0 \\
4 \\
222 \\
7\end{array}$ & $\begin{array}{r}15 \\
1,317 \\
683 \\
11 \\
343 \\
7\end{array}$ & $\begin{array}{r}1 \\
1,776 \\
1,836 \\
6 \\
318 \\
15\end{array}$ & $\begin{array}{r}1 \\
1,978 \\
1,896 \\
19 \\
130 \\
8\end{array}$ & $\begin{array}{r}1 \\
1,862 \\
147 \\
10 \\
143 \\
10\end{array}$ & $\begin{array}{r}801 \\
10,936 \\
5,385 \\
201 \\
2,584 \\
83\end{array}$ \\
\hline 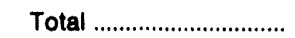 & 167,146 & 173,709 & $19 ?, 795$ & 161,778 & 164,400 & $2,765,608$ \\
\hline
\end{tabular}

See footnotes at end of table. 
Table 21. Natural Gas Deliveries to Electric Utilitya Consumers by State, 1992-1993 (Continued)

(Million Cubic Feet)

\begin{tabular}{|c|c|c|c|c|c|c|}
\hline State & $\begin{array}{c}\text { December } \\
1992\end{array}$ & $\begin{array}{c}\text { November } \\
1892\end{array}$ & $\begin{array}{c}\text { October } \\
1992\end{array}$ & $\begin{array}{c}\text { September } \\
1992\end{array}$ & $\begin{array}{c}\text { August } \\
1992\end{array}$ & $\begin{array}{c}\text { July } \\
1892\end{array}$ \\
\hline 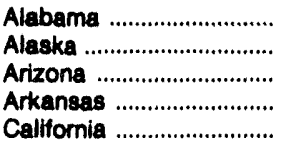 & $\begin{array}{r}171 \\
2,721 \\
328 \\
246 \\
47,146\end{array}$ & $\begin{array}{r}382 \\
2,756 \\
597 \\
209 \\
50,027\end{array}$ & $\begin{array}{r}234 \\
2,514 \\
2,631 \\
733 \\
53,026\end{array}$ & $\begin{array}{r}305 \\
2,311 \\
4,607 \\
2,324 \\
53,989\end{array}$ & $\begin{array}{r}286 \\
2,374 \\
5,277 \\
2,828 \\
66,474\end{array}$ & $\begin{array}{r}322 \\
2,342 \\
3,999 \\
4,145 \\
51,011\end{array}$ \\
\hline 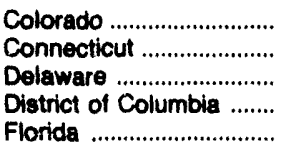 & $\begin{array}{r}526 \\
28 \\
580 \\
0 \\
10,390\end{array}$ & $\begin{array}{r}396 \\
18 \\
586 \\
0 \\
12,389\end{array}$ & $\begin{array}{r}493 \\
19 \\
581 \\
0 \\
12,525\end{array}$ & $\begin{array}{r}469 \\
14 \\
851 \\
0 \\
21,038\end{array}$ & $\begin{array}{r}479 \\
281 \\
1,140 \\
0 \\
20,829\end{array}$ & $\begin{array}{r}570 \\
407 \\
971 \\
0 \\
21,946\end{array}$ \\
\hline 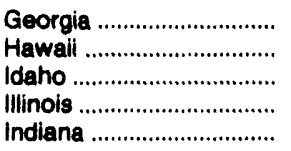 & $\begin{array}{r}13 \\
0 \\
0 \\
370 \\
824\end{array}$ & $\begin{array}{r}12 \\
0 \\
0 \\
279 \\
525\end{array}$ & $\begin{array}{r}65 \\
0 \\
0 \\
301 \\
158\end{array}$ & $\begin{array}{r}66 \\
0 \\
0 \\
554 \\
301\end{array}$ & $\begin{array}{r}120 \\
0 \\
0 \\
588 \\
370\end{array}$ & $\begin{array}{r}654 \\
0 \\
0 \\
854 \\
488\end{array}$ \\
\hline 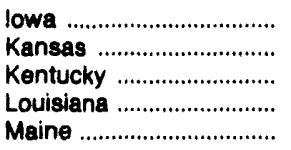 & $\begin{array}{r}193 \\
809 \\
20 \\
11,705 \\
0\end{array}$ & $\begin{array}{r}207 \\
534 \\
23 \\
13,510 \\
0\end{array}$ & $\begin{array}{r}200 \\
546 \\
19 \\
18,382 \\
0\end{array}$ & $\begin{array}{r}135 \\
1,395 \\
23 \\
23,855 \\
0\end{array}$ & $\begin{array}{r}277 \\
2,036 \\
28 \\
26,733 \\
0\end{array}$ & $\begin{array}{r}262 \\
2,833 \\
23 \\
33,952 \\
0\end{array}$ \\
\hline 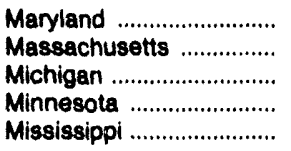 & $\begin{array}{r}430 \\
227 \\
1,727 \\
231 \\
1,625\end{array}$ & $\begin{array}{r}469 \\
3,903 \\
1,994 \\
336 \\
1,843\end{array}$ & $\begin{array}{r}665 \\
3,683 \\
1,675 \\
210 \\
1,616\end{array}$ & $\begin{array}{r}1,228 \\
4,664 \\
1,738 \\
440 \\
5,419\end{array}$ & $\begin{array}{r}1,457 \\
4,803 \\
2,421 \\
473 \\
5,359\end{array}$ & $\begin{array}{r}3,004 \\
7,643 \\
1,449 \\
509 \\
7,189\end{array}$ \\
\hline 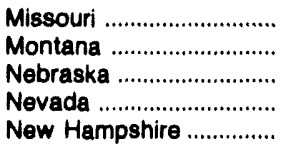 & $\begin{array}{r}67 \\
8 \\
136 \\
436 \\
0\end{array}$ & $\begin{array}{r}85 \\
47 \\
181 \\
808 \\
0\end{array}$ & $\begin{array}{r}67 \\
32 \\
210 \\
1,968 \\
16\end{array}$ & $\begin{array}{r}86 \\
10 \\
280 \\
2,761 \\
499\end{array}$ & $\begin{array}{r}200 \\
20 \\
85 \\
3,629 \\
118\end{array}$ & $\begin{array}{r}1,025 \\
29 \\
153 \\
3,491 \\
0\end{array}$ \\
\hline $\begin{array}{l}\text { New Jersey } \\
\text { New Mexico ......................... } \\
\text { New York ......................... } \\
\text { North Carolina ................ } \\
\text { North Dakota ................... }\end{array}$ & $\begin{array}{r}681 \\
1,408 \\
10,640 \\
169 \\
0\end{array}$ & $\begin{array}{r}1,209 \\
1,440 \\
11,688 \\
165 \\
0\end{array}$ & $\begin{array}{r}1,562 \\
1,803 \\
13,307 \\
127 \\
0\end{array}$ & $\begin{array}{r}3,860 \\
2,183 \\
21,641 \\
336 \\
0\end{array}$ & $\begin{array}{r}6,351 \\
2,594 \\
22,006 \\
361 \\
0\end{array}$ & $\begin{array}{r}12,179 \\
2,532 \\
25,124 \\
614 \\
0\end{array}$ \\
\hline 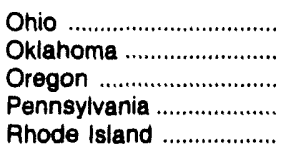 & $\begin{array}{r}53 \\
10,120 \\
977 \\
262 \\
0\end{array}$ & $\begin{array}{r}90 \\
8,514 \\
1,373 \\
148 \\
0\end{array}$ & $\begin{array}{r}217 \\
9,300 \\
1,789 \\
262 \\
0\end{array}$ & $\begin{array}{r}243 \\
15,806 \\
2,045 \\
274 \\
11\end{array}$ & $\begin{array}{r}288 \\
15,714 \\
1,854 \\
320 \\
78\end{array}$ & $\begin{array}{r}264 \\
17,018 \\
1,437 \\
824 \\
80\end{array}$ \\
\hline 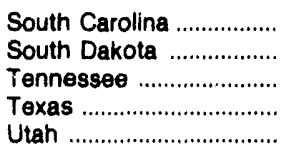 & $\begin{array}{r}5 \\
9 \\
25 \\
68,751 \\
542\end{array}$ & $\begin{array}{r}82 \\
27 \\
10 \\
72,018 \\
138\end{array}$ & $\begin{array}{r}22 \\
2 \\
18 \\
80,276 \\
665\end{array}$ & $\begin{array}{r}42 \\
1 \\
15 \\
95,796 \\
542\end{array}$ & $\begin{array}{r}82 \\
5 \\
7 \\
99,748 \\
550\end{array}$ & $\begin{array}{r}525 \\
0 \\
40 \\
120,114 \\
517\end{array}$ \\
\hline 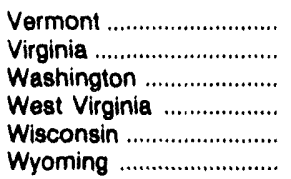 & $\begin{array}{r}1 \\
839 \\
9 \\
8 \\
146 \\
7\end{array}$ & $\begin{array}{r}28 \\
11 \\
4 \\
9 \\
207 \\
8\end{array}$ & $\begin{array}{r}122 \\
25 \\
426 \\
6 \\
134 \\
8\end{array}$ & $\begin{array}{r}2 \\
714 \\
523 \\
18 \\
249 \\
6\end{array}$ & $\begin{array}{r}34 \\
945 \\
2,698 \\
24 \\
178 \\
5\end{array}$ & $\begin{array}{r}186 \\
2,408 \\
185 \\
26 \\
219 \\
5\end{array}$ \\
\hline 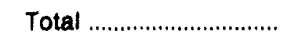 & 175,608 & 189,296 & 212,840 & 273,670 & 302,544 & 333,567 \\
\hline
\end{tabular}

See footnotes at end of table. 
Table 21. Natural Gas Dellverles to Electric Utility" Consumers by State, 1992-1993 (Continued)

(Million Cubic Feet)

\begin{tabular}{|c|c|c|c|c|c|c|}
\hline State & $\begin{array}{l}\text { June } \\
1992\end{array}$ & $\begin{array}{c}\text { May } \\
1992\end{array}$ & $\begin{array}{l}\text { April } \\
1992\end{array}$ & $\begin{array}{c}\text { March } \\
1992\end{array}$ & $\begin{array}{c}\text { February } \\
1992\end{array}$ & $\begin{array}{c}\text { January } \\
1992\end{array}$ \\
\hline 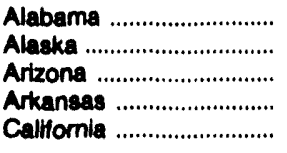 & $\begin{array}{r}307 \\
2,204 \\
2,778 \\
3,788 \\
36,692\end{array}$ & $\begin{array}{r}350 \\
2,116 \\
3,300 \\
3,881 \\
41,687\end{array}$ & $\begin{array}{r}216 \\
2,410 \\
2,300 \\
4,068 \\
43,478\end{array}$ & $\begin{array}{r}315 \\
2,449 \\
1,953 \\
2,403 \\
42,382\end{array}$ & $\begin{array}{r}204 \\
2,258 \\
1,719 \\
2,080 \\
39,134\end{array}$ & $\begin{array}{r}287 \\
2,498 \\
1,451 \\
311 \\
39,387\end{array}$ \\
\hline 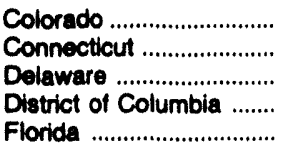 & $\begin{array}{r}355 \\
316 \\
1,034 \\
0 \\
21,623\end{array}$ & $\begin{array}{r}357 \\
131 \\
598 \\
0 \\
19,942\end{array}$ & $\begin{array}{r}243 \\
391 \\
593 \\
0 \\
18,239\end{array}$ & $\begin{array}{r}494 \\
424 \\
526 \\
0 \\
17,249\end{array}$ & $\begin{array}{r}309 \\
28 \\
467 \\
0 \\
14,042\end{array}$ & $\begin{array}{r}328 \\
34 \\
456 \\
0 \\
12,365\end{array}$ \\
\hline 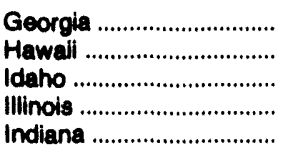 & $\begin{array}{r}75 \\
0 \\
0 \\
998 \\
760\end{array}$ & $\begin{array}{r}24 \\
0 \\
0 \\
955 \\
1,056\end{array}$ & $\begin{array}{r}106 \\
0 \\
0 \\
943 \\
605\end{array}$ & $\begin{array}{r}14 \\
0 \\
0 \\
1,720 \\
535\end{array}$ & $\begin{array}{r}10 \\
0 \\
0 \\
860 \\
1,030\end{array}$ & $\begin{array}{r}3 \\
0 \\
0 \\
869 \\
1,119\end{array}$ \\
\hline 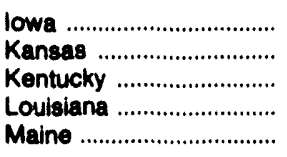 & $\begin{array}{r}208 \\
1,388 \\
28 \\
30,520 \\
0\end{array}$ & $\begin{array}{r}155 \\
1,385 \\
16 \\
26,127 \\
0\end{array}$ & $\begin{array}{r}195 \\
910 \\
23 \\
22,298 \\
0\end{array}$ & $\begin{array}{r}157 \\
717 \\
16 \\
20,481 \\
0\end{array}$ & $\begin{array}{r}182 \\
694 \\
14 \\
13,176 \\
0\end{array}$ & $\begin{array}{r}96 \\
735 \\
35 \\
14,182 \\
0\end{array}$ \\
\hline 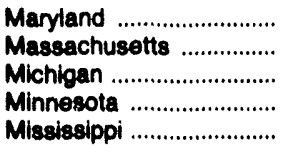 & $\begin{array}{r}1,367 \\
5,418 \\
1,473 \\
491 \\
5,227\end{array}$ & $\begin{array}{r}974 \\
3,630 \\
2,126 \\
352 \\
6,629\end{array}$ & $\begin{array}{r}772 \\
3,795 \\
2,516 \\
316 \\
5,878\end{array}$ & $\begin{array}{r}694 \\
463 \\
3,171 \\
508 \\
5,152\end{array}$ & $\begin{array}{r}244 \\
54 \\
2,574 \\
703 \\
5,439\end{array}$ & $\begin{array}{r}270 \\
59 \\
2,045 \\
337 \\
2,804\end{array}$ \\
\hline 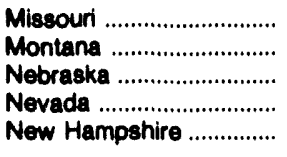 & $\begin{array}{r}131 \\
20 \\
139 \\
3,091 \\
0\end{array}$ & $\begin{array}{r}176 \\
26 \\
111 \\
2,681 \\
0\end{array}$ & $\begin{array}{r}79 \\
11 \\
211 \\
2,152 \\
0\end{array}$ & $\begin{array}{r}186 \\
7 \\
213 \\
1,499 \\
0\end{array}$ & $\begin{array}{r}143 \\
4 \\
76 \\
1,093 \\
0\end{array}$ & $\begin{array}{r}107 \\
6 \\
97 \\
744 \\
0\end{array}$ \\
\hline 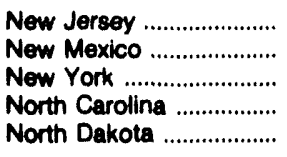 & $\begin{array}{r}3,330 \\
1,911 \\
23,875 \\
341 \\
0\end{array}$ & $\begin{array}{r}1,794 \\
2,444 \\
20,936 \\
180 \\
0\end{array}$ & $\begin{array}{r}3,878 \\
1,981 \\
18,809 \\
159 \\
0\end{array}$ & $\begin{array}{r}2,975 \\
1,288 \\
17,363 \\
206 \\
0\end{array}$ & $\begin{array}{r}487 \\
1,306 \\
13,500 \\
233 \\
1\end{array}$ & $\begin{array}{r}466 \\
1,597 \\
9,843 \\
269 \\
0\end{array}$ \\
\hline 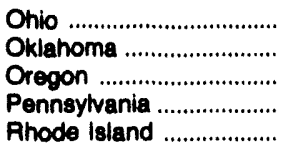 & $\begin{array}{r}173 \\
14,312 \\
661 \\
345 \\
90\end{array}$ & $\begin{array}{r}339 \\
13,004 \\
0 \\
129 \\
78\end{array}$ & $\begin{array}{r}409 \\
13,652 \\
0 \\
138 \\
90\end{array}$ & $\begin{array}{r}309 \\
12,412 \\
0 \\
178 \\
43\end{array}$ & $\begin{array}{r}259 \\
9,258 \\
2,005 \\
126 \\
0\end{array}$ & $\begin{array}{r}312 \\
9,870 \\
2,124 \\
94 \\
0\end{array}$ \\
\hline 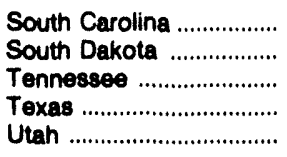 & $\begin{array}{r}202 \\
1 \\
35 \\
97,150 \\
509\end{array}$ & $\begin{array}{r}10 \\
1 \\
33 \\
76,427 \\
627\end{array}$ & $\begin{array}{r}13 \\
0 \\
37 \\
74,644 \\
642\end{array}$ & $\begin{array}{r}793 \\
1 \\
48 \\
66,472 \\
647\end{array}$ & $\begin{array}{r}3 \\
0 \\
5 \\
54,754 \\
573\end{array}$ & $\begin{array}{r}15 \\
2 \\
17 \\
62,015 \\
626\end{array}$ \\
\hline 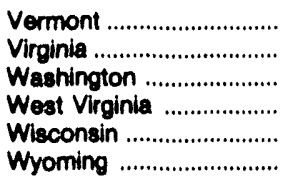 & $\begin{array}{r}160 \\
1,014 \\
1,028 \\
47 \\
259 \\
8\end{array}$ & $\begin{array}{r}122 \\
809 \\
195 \\
28 \\
269 \\
4\end{array}$ & $\begin{array}{r}122 \\
1,036 \\
293 \\
7 \\
345 \\
12\end{array}$ & $\begin{array}{r}20 \\
805 \\
6 \\
5 \\
351 \\
6\end{array}$ & $\begin{array}{r}1 \\
1,111 \\
7 \\
5 \\
116 \\
5\end{array}$ & $\begin{array}{r}2 \\
1,118 \\
11 \\
16 \\
113 \\
9\end{array}$ \\
\hline Total & 265,882 & 236,316 & 229,012 & 207,656 & 170,293 & 169,125 \\
\hline
\end{tabular}

- Includes all steam electric utility generating plants with a combined capacity of 50 megawatts or greater.

Notes: Geographic coverage is the 50 States and the District of Columbia. See Appendix A, Explanatory Note 5 for discussion of computations and revision policy.

Source: Form EIA-759. 
Table 22. Natural Gas Deliverles to All Consumers by State, 1992-1993 (Million Cubic Feet)

\begin{tabular}{|c|c|c|c|c|c|c|}
\hline State & $\begin{array}{c}\text { YTD } \\
1993\end{array}$ & $\begin{array}{c}\text { YTD } \\
1992\end{array}$ & $\begin{array}{l}\text { YrD } \\
1991\end{array}$ & $\begin{array}{c}\text { August } \\
1993\end{array}$ & $\begin{array}{c}\text { July } \\
1993\end{array}$ & $\begin{array}{l}\text { June } \\
1993\end{array}$ \\
\hline 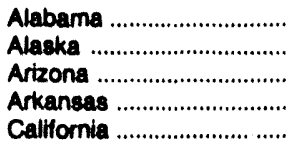 & $\begin{array}{r}176,724 \\
88,998 \\
66,763 \\
138,781 \\
1,168,818\end{array}$ & $\begin{array}{r}167,439 \\
95,025 \\
74,122 \\
147,141 \\
1,280,627\end{array}$ & $\begin{array}{r}153,734 \\
86,275 \\
69,970 \\
138,705 \\
1,202,914\end{array}$ & $\begin{array}{r}16,445 \\
8,073 \\
8,272 \\
14,534 \\
129,541\end{array}$ & $\begin{array}{r}18,003 \\
10,065 \\
6,686 \\
14,172 \\
126,878\end{array}$ & $\begin{array}{r}16,792 \\
9,176 \\
5,857 \\
12,546 \\
117,559\end{array}$ \\
\hline 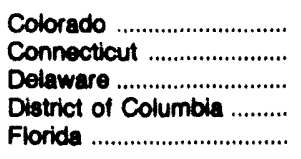 & $\begin{array}{r}187,128 \\
74,821 \\
27,461 \\
23,838 \\
224,724\end{array}$ & $\begin{array}{r}149,157 \\
76,160 \\
28,549 \\
23,645 \\
242,283\end{array}$ & $\begin{array}{r}158,834 \\
68,346 \\
29,640 \\
22,383 \\
227,142\end{array}$ & $\begin{array}{r}11,547 \\
3,736 \\
3,073 \\
1,111 \\
31,627\end{array}$ & $\begin{array}{r}12,575 \\
4,698 \\
3,182 \\
1,090 \\
31,533\end{array}$ & $\begin{array}{r}14,780 \\
4,801 \\
2,850 \\
1,265 \\
30,864\end{array}$ \\
\hline 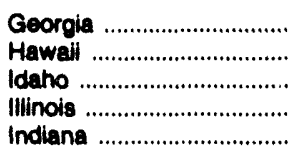 & $\begin{array}{r}227,866 \\
1,807 \\
35,282 \\
683,114 \\
338,747\end{array}$ & $\begin{array}{r}221,156 \\
1,810 \\
29,238 \\
628,521 \\
311,488\end{array}$ & $\begin{array}{r}205,568 \\
1,800 \\
31,163 \\
619,175 \\
294,741\end{array}$ & $\begin{array}{r}20,839 \\
211 \\
2,714 \\
36,555 \\
24,644\end{array}$ & $\begin{array}{r}21,434 \\
220 \\
2,840 \\
37,522 \\
24,110\end{array}$ & $\begin{array}{r}19,583 \\
231 \\
3,029 \\
38,542 \\
26,165\end{array}$ \\
\hline 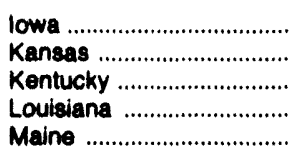 & $\begin{array}{r}159,632 \\
210,957 \\
116,404 \\
742,745 \\
3,082\end{array}$ & $\begin{array}{r}143,344 \\
177,042 \\
110,010 \\
896,506 \\
3,546\end{array}$ & $\begin{array}{r}146,898 \\
203,061 \\
104,403 \\
851,430 \\
3,178\end{array}$ & $\begin{array}{r}12,498 \\
21,427 \\
7,365 \\
112,063 \\
230\end{array}$ & $\begin{array}{r}10,780 \\
22,714 \\
7,260 \\
109,821 \\
205\end{array}$ & $\begin{array}{r}11,735 \\
17,117 \\
8,333 \\
93,514 \\
179\end{array}$ \\
\hline 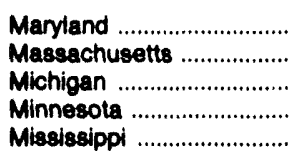 & $\begin{array}{l}120,352 \\
201,025 \\
600,231 \\
200,373 \\
138,453\end{array}$ & $\begin{array}{l}122,582 \\
203,992 \\
583,717 \\
190,628 \\
143,251\end{array}$ & $\begin{array}{l}116,765 \\
180,007 \\
539,289 \\
194,449 \\
145,784\end{array}$ & $\begin{array}{r}8,809 \\
16,770 \\
37,352 \\
11,528 \\
18,627\end{array}$ & $\begin{array}{r}8,909 \\
15,948 \\
38,414 \\
11,217 \\
18,709\end{array}$ & $\begin{array}{r}8,676 \\
16,486 \\
43,655 \\
13,572 \\
14,925\end{array}$ \\
\hline 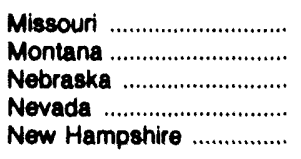 & $\begin{array}{r}180,510 \\
30,175 \\
87,846 \\
64,569 \\
11,753\end{array}$ & $\begin{array}{r}161,354 \\
24,351 \\
65,640 \\
47,826 \\
11,640\end{array}$ & $\begin{array}{r}174,862 \\
26,388 \\
77,147 \\
44,907 \\
9,858\end{array}$ & $\begin{array}{r}10,669 \\
1,818 \\
7,158 \\
7,418 \\
585\end{array}$ & $\begin{array}{r}8,995 \\
2,030 \\
5,435 \\
7,455 \\
639\end{array}$ & $\begin{array}{r}10,290 \\
1,869 \\
5,167 \\
7,057 \\
749\end{array}$ \\
\hline 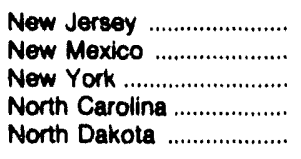 & $\begin{array}{r}386,749 \\
72,657 \\
653,834 \\
124,060 \\
18,698\end{array}$ & $\begin{array}{r}370,917 \\
67,631 \\
670,050 \\
116,937 \\
16,698\end{array}$ & $\begin{array}{r}320,960 \\
71,180 \\
598,010 \\
106,672 \\
16,971\end{array}$ & $\begin{array}{r}30,366 \\
7,076 \\
51,140 \\
10,646 \\
915\end{array}$ & $\begin{array}{r}36,709 \\
6,836 \\
56,145 \\
9,694 \\
1,006\end{array}$ & $\begin{array}{r}29,678 \\
6,571 \\
55,263 \\
10,613 \\
1,179\end{array}$ \\
\hline 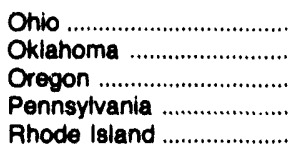 & $\begin{array}{r}547,100 \\
311,061 \\
85,748 \\
447,257 \\
52,017\end{array}$ & $\begin{array}{r}532,282 \\
293,059 \\
73,506 \\
432,205 \\
53,864\end{array}$ & $\begin{array}{r}497,368 \\
313,311 \\
74,791 \\
403,138 \\
34,358\end{array}$ & $\begin{array}{r}31,971 \\
40,734 \\
8,799 \\
28,381 \\
5,344\end{array}$ & $\begin{array}{r}26,466 \\
37,774 \\
6,571 \\
\text { ค } 28,273 \\
4,685\end{array}$ & $\begin{array}{r}39,320 \\
30,976 \\
6,694 \\
33,049 \\
4,882\end{array}$ \\
\hline 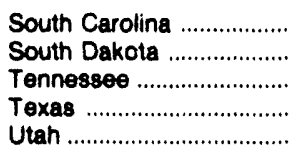 & $\begin{array}{r}93,457 \\
18,769 \\
155,791 \\
2,295,187 \\
83,931\end{array}$ & $\begin{array}{r}92,233 \\
15,423 \\
151,186 \\
2,089,198 \\
71,476\end{array}$ & $\begin{array}{r}89,833 \\
16,696 \\
142,629 \\
2,093,674 \\
78,568\end{array}$ & $\begin{array}{r}9,812 \\
892 \\
12,695 \\
322,354 \\
5,324\end{array}$ & $\begin{array}{r}9,483 \\
933 \\
12,845 \\
326,750 \\
5,199\end{array}$ & $\begin{array}{r}9,552 \\
1,054 \\
11,173 \\
289,348 \\
6,081\end{array}$ \\
\hline 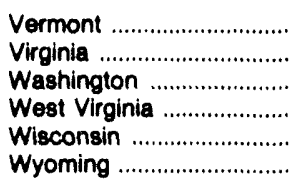 & $\begin{array}{r}5,059 \\
161,434 \\
133,703 \\
73,021 \\
228,741 \\
49,340\end{array}$ & $\begin{array}{r}5,372 \\
132,912 \\
109,374 \\
70,395 \\
213,073 \\
49,609\end{array}$ & $\begin{array}{r}4,922 \\
114,555 \\
113,402 \\
62,686 \\
211,287 \\
35,535\end{array}$ & $\begin{array}{r}251 \\
16,804 \\
10,477 \\
5,384 \\
13,108 \\
4,309\end{array}$ & $\begin{array}{r}379 \\
16,708 \\
10,410 \\
5,257 \\
12,127 \\
5,184\end{array}$ & $\begin{array}{r}380 \\
12,478 \\
10,932 \\
5,833 \\
14,647 \\
5,012\end{array}$ \\
\hline 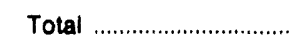 & $12,321,791$ & $11,967,301$ & $11,539,462$ & $1,204,323$ & $1,203,876$ & $1,142,188$ \\
\hline
\end{tabular}

See footnotes at end of tables. 
Table 22. Natural Gas Deliverles to All Consumers by State, 1992-1993 (Continued) (Million Cubic Feet)

\begin{tabular}{|c|c|c|c|c|c|c|}
\hline State & $\begin{array}{c}\text { May } \\
1993\end{array}$ & $\begin{array}{l}\text { April } \\
1993\end{array}$ & $\begin{array}{c}\text { March } \\
1993\end{array}$ & $\begin{array}{c}\text { February } \\
1993\end{array}$ & $\begin{array}{c}\text { January } \\
1993\end{array}$ & $\begin{array}{l}\text { Total } \\
1992\end{array}$ \\
\hline 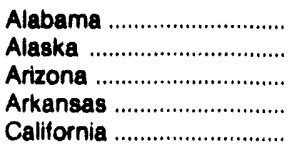 & $\begin{array}{r}18,470 \\
10,344 \\
6,603 \\
12,998 \\
112,908\end{array}$ & $\begin{array}{r}23,361 \\
11,527 \\
7,668 \\
17,589 \\
131,584\end{array}$ & $\begin{array}{r}28,481 \\
12,263 \\
11,052 \\
21,788 \\
161,093\end{array}$ & $\begin{array}{r}27,321 \\
13,443 \\
9,239 \\
21,311 \\
189,324\end{array}$ & $\begin{array}{r}27,851 \\
15,106 \\
11,189 \\
23,843 \\
189,930\end{array}$ & $\begin{array}{r}247,295 \\
145,541 \\
106,234 \\
210,653 \\
1,923,573\end{array}$ \\
\hline 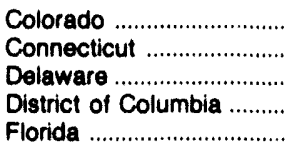 & $\begin{array}{r}18,440 \\
6,095 \\
2,632 \\
1,955 \\
28,777\end{array}$ & $\begin{array}{r}24,183 \\
10,048 \\
3,633 \\
3,158 \\
25,794\end{array}$ & $\begin{array}{r}32,278 \\
14,381 \\
4,363 \\
4,982 \\
26,562\end{array}$ & $\begin{array}{r}34,279 \\
16,223 \\
3,818 \\
5,209 \\
25,346\end{array}$ & $\begin{array}{r}39,036 \\
14,740 \\
3,910 \\
5,068 \\
24,221\end{array}$ & $\begin{array}{r}223,656 \\
110,715 \\
39,604 \\
32,690 \\
343,521\end{array}$ \\
\hline 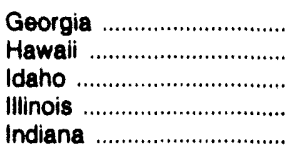 & $\begin{array}{r}21,088 \\
213 \\
3,530 \\
48,068 \\
29,387\end{array}$ & $\begin{array}{r}27,838 \\
225 \\
4,256 \\
84,599 \\
42,797\end{array}$ & $\begin{array}{r}37,618 \\
230 \\
5,760 \\
127,983 \\
61,168\end{array}$ & $\begin{array}{r}39,772 \\
242 \\
6,353 \\
138,006 \\
63,379\end{array}$ & $\begin{array}{r}39,794 \\
236 \\
6,799 \\
151,839 \\
67,096\end{array}$ & $\begin{array}{r}335,464 \\
2,695 \\
45,635 \\
981,991 \\
478,765\end{array}$ \\
\hline 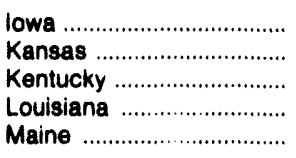 & $\begin{array}{r}13,485 \\
19,246 \\
8,617 \\
79,003 \\
226\end{array}$ & $\begin{array}{r}20,005 \\
25,517 \\
14,102 \\
83,399 \\
405\end{array}$ & $\begin{array}{r}27,943 \\
32,681 \\
21,883 \\
90,160 \\
542\end{array}$ & $\begin{array}{r}29,876 \\
34,734 \\
24,630 \\
84,210 \\
643\end{array}$ & $\begin{array}{r}33,211 \\
37,520 \\
24,212 \\
90,574 \\
652\end{array}$ & $\begin{array}{r}223,892 \\
270,284 \\
171,218 \\
1,271,064 \\
5,129\end{array}$ \\
\hline 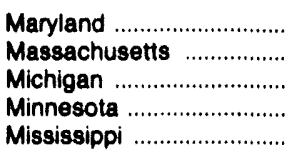 & $\begin{array}{r}9,765 \\
19,701 \\
52,883 \\
15,490 \\
14,729\end{array}$ & $\begin{array}{l}14,160 \\
28,688 \\
80,931 \\
25,245 \\
15,877\end{array}$ & $\begin{array}{r}23,593 \\
36,093 \\
112,353 \\
36,627 \\
19,025\end{array}$ & $\begin{array}{r}23,913 \\
34,489 \\
116,586 \\
40,247 \\
17,426\end{array}$ & $\begin{array}{r}22,426 \\
32,850 \\
118,057 \\
46,446 \\
19,136\end{array}$ & $\begin{array}{l}178,881 \\
293,238 \\
862,217 \\
293,873 \\
201,221\end{array}$ \\
\hline 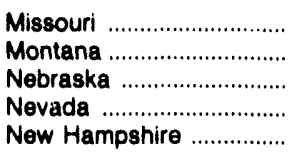 & $\begin{array}{r}15,888 \\
2,451 \\
6,122 \\
7,583 \\
1,111\end{array}$ & $\begin{array}{r}24,833 \\
3,752 \\
11,692 \\
8,566 \\
1,705\end{array}$ & $\begin{array}{r}37,569 \\
5,549 \\
14,646 \\
9,222 \\
2,245\end{array}$ & $\begin{array}{r}37,894 \\
5,287 \\
15,195 \\
7,878 \\
2,359\end{array}$ & $\begin{array}{r}43,272 \\
7,319 \\
22,429 \\
9,390 \\
2,361\end{array}$ & $\begin{array}{r}238,435 \\
40,671 \\
104,258 \\
67,912 \\
16,771\end{array}$ \\
\hline 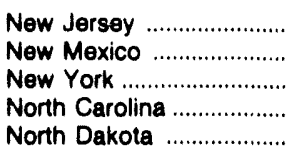 & $\begin{array}{r}31,501 \\
6,812 \\
55,661 \\
11,456 \\
1,512\end{array}$ & $\begin{array}{r}50,161 \\
9,476 \\
87,239 \\
16,490 \\
2,511\end{array}$ & $\begin{array}{r}69,180 \\
11,053 \\
118,222 \\
21,815 \\
3,383\end{array}$ & $\begin{array}{r}72,918 \\
11,333 \\
116,377 \\
21,197 \\
3,586\end{array}$ & $\begin{array}{r}66,236 \\
13,499 \\
113,887 \\
22,149 \\
4,606\end{array}$ & $\begin{array}{r}542,695 \\
98,881 \\
952,161 \\
173,156 \\
25,397\end{array}$ \\
\hline 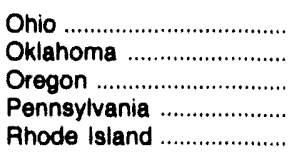 & $\begin{array}{r}42,332 \\
30,924 \\
8,225 \\
35,893 \\
5,487\end{array}$ & $\begin{array}{r}73,165 \\
36,806 \\
9,787 \\
57,915 \\
6,833\end{array}$ & $\begin{array}{r}108,821 \\
43,651 \\
14,168 \\
87,765 \\
8,388\end{array}$ & $\begin{array}{r}114,755 \\
42,702 \\
14,890 \\
90,643 \\
8,381\end{array}$ & $\begin{array}{r}110,270 \\
47,495 \\
16,613 \\
85,337 \\
8,017\end{array}$ & $\begin{array}{r}799,093 \\
425,185 \\
115,469 \\
640,593 \\
77,476\end{array}$ \\
\hline 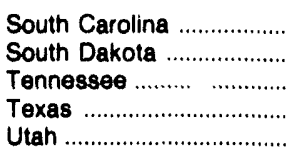 & $\begin{array}{r}9,259 \\
1,485 \\
15,132 \\
243,669 \\
7,800\end{array}$ & $\begin{array}{r}12,000 \\
2,513 \\
19,743 \\
263,722 \\
10,099\end{array}$ & $\begin{array}{r}15,180 \\
3,492 \\
27,608 \\
288,382 \\
14,043\end{array}$ & $\begin{array}{r}13,789 \\
3,746 \\
27,107 \\
265,219 \\
16,641\end{array}$ & $\begin{array}{r}14,382 \\
4,656 \\
29,487 \\
295,743 \\
18,744\end{array}$ & $\begin{array}{r}135,157 \\
24,454 \\
225,275 \\
3,101,526 \\
108,755\end{array}$ \\
\hline 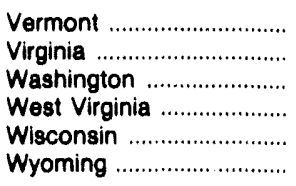 & $\begin{array}{r}438 \\
10,309 \\
13,570 \\
6,795 \\
17,976 \\
6,152\end{array}$ & $\begin{array}{r}658 \\
19,033 \\
16,769 \\
9,844 \\
32,018 \\
6,462\end{array}$ & $\begin{array}{r}976 \\
27,271 \\
22,830 \\
13,771 \\
43,020 \\
6,266\end{array}$ & $\begin{array}{r}1,003 \\
30,286 \\
23,248 \\
13,521 \\
44,657 \\
7,409\end{array}$ & $\begin{array}{r}975 \\
28,446 \\
25,468 \\
12,615 \\
51,188 \\
8,547\end{array}$ & $\begin{array}{r}7,598 \\
192,932 \\
166,092 \\
104,089 \\
327,599 \\
75,044\end{array}$ \\
\hline 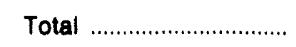 & $1,150,197$ & $1,520,579$ & $1,869,418$ & $2,012,238$ & $2,118,873$ & $17,785,833$ \\
\hline
\end{tabular}

See footnotes at end of tables. 
Table 22. Natural Gas Deliveries to All Consumers by State, 1992-1993 (Continued) (Million Cubic Feet)

\begin{tabular}{|c|c|c|c|c|c|c|}
\hline State & $\begin{array}{c}\text { December } \\
1992\end{array}$ & $\begin{array}{c}\text { November } \\
1992\end{array}$ & $\begin{array}{c}\text { October } \\
1992\end{array}$ & $\begin{array}{c}\text { September } \\
1992\end{array}$ & $\begin{array}{c}\text { August } \\
1992\end{array}$ & $\begin{array}{c}\text { July } \\
1992\end{array}$ \\
\hline 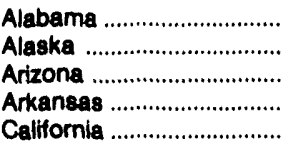 & $\begin{array}{r}25,675 \\
14,680 \\
10,994 \\
20,728 \\
199,920\end{array}$ & $\begin{array}{r}20,620 \\
13,463 \\
5,564 \\
15,335 \\
158,481\end{array}$ & $\begin{array}{r}17,250 \\
13,323 \\
6,868 \\
13,222 \\
137,081\end{array}$ & $\begin{array}{r}16,309 \\
9,049 \\
8,640 \\
14,227 \\
147,435\end{array}$ & $\begin{array}{r}15,994 \\
9,181 \\
9,178 \\
14,288 \\
160,041\end{array}$ & $\begin{array}{r}16,511 \\
10,869 \\
8,263 \\
15,530 \\
138,193\end{array}$ \\
\hline 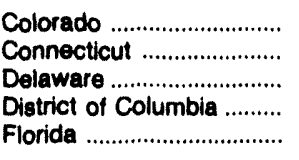 & $\begin{array}{r}33,143 \\
13,243 \\
4,005 \\
4,135 \\
23,862\end{array}$ & $\begin{array}{r}19,036 \\
9,619 \\
3,502 \\
2,416 \\
23,814\end{array}$ & $\begin{array}{r}12,212 \\
6,718 \\
2,919 \\
1,395 \\
22,494\end{array}$ & $\begin{array}{r}10,084 \\
4,975 \\
2,629 \\
1,100 \\
31,059\end{array}$ & $\begin{array}{r}9,145 \\
4,991 \\
2,825 \\
1,093 \\
30,956\end{array}$ & $\begin{array}{r}10,006 \\
5,100 \\
2,571 \\
1,187 \\
32,201\end{array}$ \\
\hline 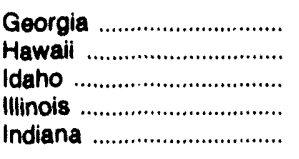 & $\begin{array}{r}40,414 \\
234 \\
6,119 \\
140,871 \\
61,989\end{array}$ & $\begin{array}{r}31,537 \\
221 \\
4,415 \\
104,726 \\
47,066\end{array}$ & $\begin{array}{r}22,635 \\
210 \\
3,244 \\
65,944 \\
33,542\end{array}$ & $\begin{array}{r}19,721 \\
221 \\
2,538 \\
41,922 \\
24,622\end{array}$ & $\begin{array}{r}19,466 \\
215 \\
2,171 \\
36,285 \\
22,532\end{array}$ & $\begin{array}{r}20,629 \\
218 \\
2,553 \\
37,000 \\
22,927\end{array}$ \\
\hline 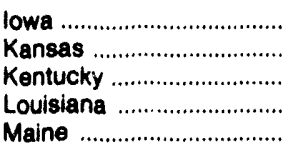 & $\begin{array}{r}30,340 \\
34,037 \\
23,317 \\
95,726 \\
561\end{array}$ & $\begin{array}{r}24,125 \\
23,526 \\
17,733 \\
91,729 \\
464\end{array}$ & $\begin{array}{r}14,953 \\
19,422 \\
12,206 \\
94,825 \\
309\end{array}$ & $\begin{array}{r}11,228 \\
16,258 \\
7,952 \\
92,270 \\
249\end{array}$ & $\begin{array}{r}10,838 \\
17,192 \\
8,003 \\
103,989 \\
236\end{array}$ & $\begin{array}{r}10,972 \\
18,403 \\
7,213 \\
117,997 \\
264\end{array}$ \\
\hline 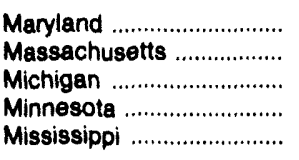 & $\begin{array}{r}20,908 \\
27,879 \\
103,987 \\
40,563 \\
16,686\end{array}$ & $\begin{array}{l}15,894 \\
25,875 \\
80,909 \\
31,602 \\
13,740\end{array}$ & $\begin{array}{l}11,110 \\
18,700 \\
56,101 \\
18,748 \\
12,051\end{array}$ & $\begin{array}{r}8,387 \\
16,790 \\
37,499 \\
12,333 \\
15,494\end{array}$ & $\begin{array}{r}8,727 \\
16,884 \\
37,701 \\
11,642 \\
14,978\end{array}$ & $\begin{array}{l}10,535 \\
18,866 \\
35,356 \\
11,892 \\
17,272\end{array}$ \\
\hline 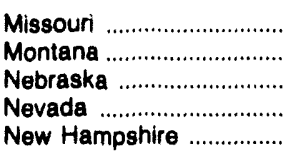 & $\begin{array}{r}35,337 \\
6,448 \\
15,177 \\
6,663 \\
1,756\end{array}$ & $\begin{array}{r}21,055 \\
4,455 \\
11,269 \\
3,833 \\
1,346\end{array}$ & $\begin{array}{r}11,679 \\
3,085 \\
6,974 \\
4,579 \\
868\end{array}$ & $\begin{array}{l}9,009 \\
2,330 \\
5,197 \\
4,899 \\
1,161\end{array}$ & $\begin{array}{r}9,007 \\
1,675 \\
5,428 \\
5,714 \\
759\end{array}$ & $\begin{array}{r}9,713 \\
1,761 \\
5,483 \\
5,896 \\
640\end{array}$ \\
\hline 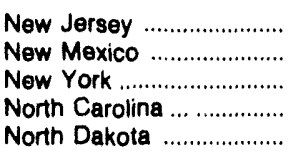 & $\begin{array}{r}60,941 \\
12,300 \\
101,107 \\
19,783 \\
3,592\end{array}$ & $\begin{array}{r}47,235 \\
7,091 \\
72,124 \\
14,484 \\
2,529\end{array}$ & $\begin{array}{r}34,665 \\
6,002 \\
56,395 \\
11,898 \\
1,518\end{array}$ & $\begin{array}{r}28,937 \\
5,851 \\
52,470 \\
10,047 \\
1,057\end{array}$ & $\begin{array}{r}30,937 \\
6,218 \\
51,248 \\
9,863 \\
866\end{array}$ & $\begin{array}{r}36,695 \\
5,700 \\
55,424 \\
9,704 \\
977\end{array}$ \\
\hline 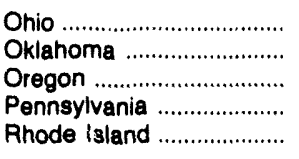 & $\begin{array}{r}102,668 \\
42,892 \\
13,388 \\
76,952 \\
7,158\end{array}$ & $\begin{array}{r}75,938 \\
30,238 \\
10,121 \\
58,902 \\
6,108\end{array}$ & $\begin{array}{r}54,217 \\
26,249 \\
9,455 \\
43,339 \\
5,639\end{array}$ & $\begin{array}{r}33,930 \\
32,711 \\
8,993 \\
29,191 \\
4,698\end{array}$ & $\begin{array}{r}31,699 \\
32,953 \\
8,391 \\
27,230 \\
4,667\end{array}$ & $\begin{array}{r}32,638 \\
34,883 \\
7,755 \\
28,178 \\
5,376\end{array}$ \\
\hline 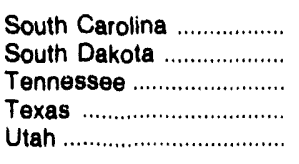 & $\begin{array}{r}12,911 \\
3,807 \\
27,075 \\
281,632 \\
16,360\end{array}$ & $\begin{array}{r}11,513 \\
2,810 \\
19,403 \\
246,108 \\
9,926\end{array}$ & $\begin{array}{r}9,408 \\
1,490 \\
14,849 \\
242,627 \\
6,188\end{array}$ & $\begin{array}{r}9,092 \\
918 \\
12,760 \\
261,956 \\
4,790\end{array}$ & $\begin{array}{r}9,051 \\
789 \\
12,672 \\
263,931 \\
4,689\end{array}$ & $\begin{array}{r}9,659 \\
912 \\
13,194 \\
288,393 \\
4,871\end{array}$ \\
\hline 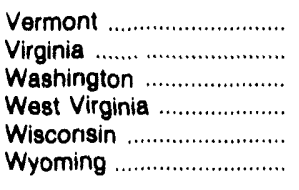 & $\begin{array}{r}775 \\
22,720 \\
20,827 \\
12,180 \\
44,320 \\
7,758\end{array}$ & $\begin{array}{r}613 \\
16,229 \\
14,620 \\
9,194 \\
33,967 \\
6,257\end{array}$ & $\begin{array}{r}561 \\
11,255 \\
11,646 \\
7,138 \\
22,141 \\
5,892\end{array}$ & $\begin{array}{r}277 \\
9,816 \\
9,531 \\
5,182 \\
14,068 \\
5,517\end{array}$ & $\begin{array}{r}288 \\
10,424 \\
11,383 \\
4,967 \\
12,581 \\
5,103\end{array}$ & $\begin{array}{r}436 \\
13,355 \\
8,387 \\
4,730 \\
12,191 \\
5,432\end{array}$ \\
\hline Total .............................. & $1,950,542$ & $1,522,779$ & $1,227,238$ & $1,117,381$ & $1,131,083$ & $1,174,910$ \\
\hline
\end{tabular}

See footnotes at end of tables. 
Table 22. Natural Gas Dellveries to All Consumers by State, 1992-1993 (Continued) (Million Cubic Feet)

\begin{tabular}{|c|c|c|c|c|c|c|}
\hline State & $\begin{array}{l}\text { June } \\
1992\end{array}$ & $\begin{array}{l}\text { May } \\
1892\end{array}$ & $\begin{array}{l}\text { April } \\
1992\end{array}$ & $\begin{array}{c}\text { March } \\
1992\end{array}$ & $\begin{array}{c}\text { February } \\
1992\end{array}$ & $\begin{array}{c}\text { January } \\
1992\end{array}$ \\
\hline $\begin{array}{l}\text { Alabama } \\
\text { Alaska } \\
\text { Arizona } \\
\text { Arkansas } \\
\text { California }\end{array}$ & $\begin{array}{r}17,043 \\
10,355 \\
7,277 \\
15,364 \\
130,858\end{array}$ & $\begin{array}{r}18,471 \\
11,627 \\
7,670 \\
16,961 \\
157,730\end{array}$ & $\begin{array}{r}21,138 \\
12,386 \\
8,607 \\
19,874 \\
131,762\end{array}$ & $\begin{array}{r}23,738 \\
13,093 \\
10,163 \\
18,929 \\
162,890\end{array}$ & $\begin{array}{r}27,290 \\
13,473 \\
10,538 \\
22,606 \\
179,643\end{array}$ & $\begin{array}{r}27,252 \\
14,042 \\
12,427 \\
22,588 \\
219,510\end{array}$ \\
\hline 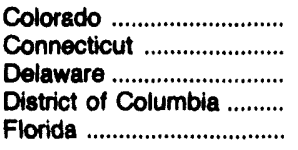 & $\begin{array}{r}11,545 \\
5,480 \\
2,877 \\
1,394 \\
32,431\end{array}$ & $\begin{array}{r}12,943 \\
7,102 \\
2,935 \\
2,005 \\
31,649\end{array}$ & $\begin{array}{r}19,552 \\
10,857 \\
3,626 \\
3,594 \\
30,622\end{array}$ & $\begin{array}{r}24,198 \\
13,813 \\
3,801 \\
4,304 \\
29,876\end{array}$ & $\begin{array}{r}29,194 \\
14,349 \\
4,049 \\
5,085 \\
27,659\end{array}$ & $\begin{array}{r}32,574 \\
14,468 \\
3,866 \\
4,984 \\
26,889\end{array}$ \\
\hline $\begin{array}{l}\text { Gsorgia } \\
\text { Hawail } \\
\text { Idaho } \\
\text { Illinois } \\
\text { Indiana }\end{array}$ & $\begin{array}{r}20,062 \\
230 \\
2,830 \\
37,856 \\
25,135\end{array}$ & $\begin{array}{r}22,350 \\
224 \\
3,178 \\
55,272 \\
30,857\end{array}$ & $\begin{array}{r}26,709 \\
229 \\
3,616 \\
85,166 \\
40,824\end{array}$ & $\begin{array}{r}34,065 \\
222 \\
4,242 \\
113,581 \\
50,877\end{array}$ & $\begin{array}{r}35,884 \\
231 \\
4,772 \\
120,366 \\
55,395\end{array}$ & $\begin{array}{r}41,990 \\
241 \\
5,877 \\
142,995 \\
62,941\end{array}$ \\
\hline $\begin{array}{l}\text { lowa } \\
\text { Kansas } \\
\text { Kentucky } \\
\text { Louisiana } \\
\text { Maine }\end{array}$ & $\begin{array}{r}11,613 \\
16,510 \\
8,268 \\
108,657 \\
274\end{array}$ & $\begin{array}{r}13,137 \\
18,212 \\
9,691 \\
109,381 \\
395\end{array}$ & $\begin{array}{r}19,096 \\
22,495 \\
12,836 \\
116,654 \\
545\end{array}$ & $\begin{array}{r}22,389 \\
23,989 \\
18,418 \\
116,184 \\
640\end{array}$ & $\begin{array}{r}26,879 \\
27,840 \\
20,106 \\
107,518 \\
617\end{array}$ & $\begin{array}{r}28,419 \\
32,400 \\
25,476 \\
116,126 \\
577\end{array}$ \\
\hline 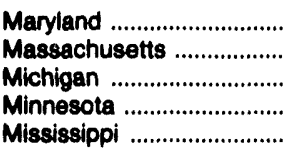 & $\begin{array}{r}9,331 \\
19,048 \\
41,669 \\
12,233 \\
15,329\end{array}$ & $\begin{array}{l}11,158 \\
23,079 \\
58,977 \\
16,041 \\
17,363\end{array}$ & $\begin{array}{l}16,084 \\
31,756 \\
85,208 \\
26,087 \\
18,727\end{array}$ & $\begin{array}{r}30,612 \\
30,370 \\
102,761 \\
33,315 \\
19,214\end{array}$ & $\begin{array}{r}22,644 \\
33,402 \\
109,277 \\
37,318 \\
20,500\end{array}$ & $\begin{array}{r}23,491 \\
30,588 \\
112,769 \\
42,100 \\
19,869\end{array}$ \\
\hline 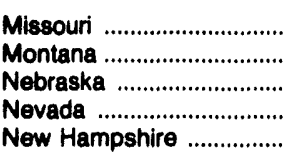 & $\begin{array}{r}10,600 \\
1,720 \\
4,977 \\
5,514 \\
727\end{array}$ & $\begin{array}{r}13,446 \\
2,273 \\
5,622 \\
5,317 \\
1,149\end{array}$ & $\begin{array}{r}21,751 \\
3,182 \\
8,555 \\
5,695 \\
1,817\end{array}$ & $\begin{array}{r}26,101 \\
3,587 \\
9,903 \\
6,139 \\
1,946\end{array}$ & $\begin{array}{r}33,119 \\
4,745 \\
12,142 \\
6,281 \\
2,350\end{array}$ & $\begin{array}{r}37,617 \\
5,408 \\
13,529 \\
7,371 \\
2,251\end{array}$ \\
\hline 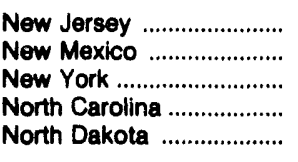 & $\begin{array}{r}28,817 \\
5,208 \\
58,065 \\
10,463 \\
1,029\end{array}$ & $\begin{array}{r}34,881 \\
6,969 \\
69,561 \\
12,017 \\
1,615\end{array}$ & $\begin{array}{r}52,289 \\
8,638 \\
95,837 \\
15,528 \\
2,411\end{array}$ & $\begin{array}{r}60,519 \\
9,327 \\
112,631 \\
18,034 \\
2,818\end{array}$ & $\begin{array}{r}63,790 \\
11,946 \\
116,379 \\
20,600 \\
3,364\end{array}$ & $\begin{array}{r}62,991 \\
13,625 \\
110,914 \\
20,728 \\
3,617\end{array}$ \\
\hline 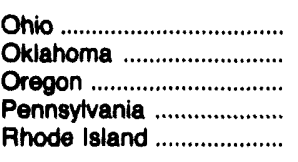 & $\begin{array}{r}36,649 \\
32,332 \\
6,614 \\
29,383 \\
5,600\end{array}$ & $\begin{array}{r}47,271 \\
32,334 \\
6,215 \\
39,434 \\
6,217\end{array}$ & $\begin{array}{r}74,144 \\
36,829 \\
7,359 \\
59,281 \\
7,729\end{array}$ & $\begin{array}{r}92,646 \\
38,920 \\
8,754 \\
76,315 \\
\quad 7,948\end{array}$ & $\begin{array}{r}103,925 \\
40,472 \\
13,200 \\
84,289 \\
8,543\end{array}$ & $\begin{array}{r}113,310 \\
44,337 \\
15,220 \\
88,085 \\
7,785\end{array}$ \\
\hline 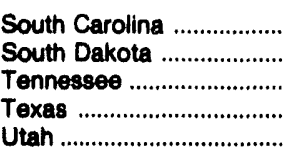 & $\begin{array}{r}9,853 \\
947 \\
12,854 \\
255,732 \\
5,260\end{array}$ & $\begin{array}{r}10,294 \\
1,444 \\
14,348 \\
247,119 \\
5,887\end{array}$ & $\begin{array}{r}11,871 \\
2,242 \\
20,379 \\
248,887 \\
7,978\end{array}$ & $\begin{array}{r}14,109 \\
2,644 \\
21,762 \\
254,408 \\
11,082\end{array}$ & $\begin{array}{r}13,866 \\
3,109 \\
26,715 \\
232,147 \\
14,524\end{array}$ & $\begin{array}{r}13,529 \\
3,335 \\
29,261 \\
278,583 \\
17,186\end{array}$ \\
\hline 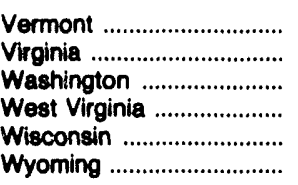 & $\begin{array}{r}435 \\
9,960 \\
10,143 \\
5,554 \\
13,793 \\
5,723\end{array}$ & $\begin{array}{r}534 \\
12,766 \\
11,137 \\
6,876 \\
19,112 \\
5,723\end{array}$ & $\begin{array}{r}778 \\
17,060 \\
13,824 \\
10,065 \\
30,455 \\
5,997\end{array}$ & $\begin{array}{r}930 \\
20,949 \\
15,652 \\
11,262 \\
38,500 \\
6,579\end{array}$ & $\begin{array}{r}981 \\
23,823 \\
18,213 \\
13,376 \\
40,566 \\
7,194\end{array}$ & $\begin{array}{r}989 \\
24,574 \\
20,635 \\
13,564 \\
45,875 \\
7,858\end{array}$ \\
\hline 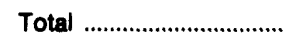 & $1,131,621$ & $1,277,970$ & $1,538,641$ & $1,770,147$ & $1,876,291$ & $2,066,637$ \\
\hline
\end{tabular}

n = Revised Data.

$m=$ Not Available.

Notes: Geographic coverage is the 50 States and the District of Columbia. Deliveries for total year 1982 may not equal the sum of the twelve months. Gas volumes delivered for use as vehicle fuol are included in the annual total for commercial deliveries but not in the monthly components. See Appendix A. Explanatory Note 5 for discussion of computations and revision policy.

Sources: Form EIA-857 and Form EIA-759. 
Table 23. Average City Gate Price by State, 1992-1993 (Dollars per Thousand Cubic Feet)

\begin{tabular}{|c|c|c|c|c|c|c|c|c|}
\hline State & $\begin{array}{l}\text { YTD } \\
1993\end{array}$ & $\begin{array}{l}\text { YTD } \\
1992\end{array}$ & $\begin{array}{l}\text { YTD } \\
1891\end{array}$ & $\begin{array}{c}\text { August } \\
1993\end{array}$ & $\begin{array}{c}\text { July } \\
1993\end{array}$ & $\begin{array}{l}\text { June } \\
1993\end{array}$ & $\begin{array}{l}\text { May } \\
1093\end{array}$ & $\begin{array}{l}\text { April } \\
1993\end{array}$ \\
\hline 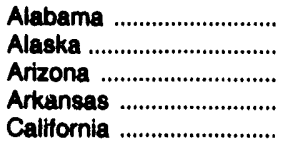 & $\begin{array}{r}3.47 \\
.34 \\
2.58 \\
2.57 \\
2.82\end{array}$ & $\begin{array}{r}3.05 \\
.34 \\
2.17 \\
2.60 \\
2.66\end{array}$ & $\begin{array}{r}3.11 \\
.33 \\
2.47 \\
2.50 \\
2.75\end{array}$ & $\begin{array}{r}3.96 \\
.41 \\
3.07 \\
2.71 \\
2.78\end{array}$ & $\begin{array}{r}3.83 \\
.33 \\
3.03 \\
2.49 \\
2.81\end{array}$ & $\begin{array}{r}3.98 \\
.33 \\
2.92 \\
2.44 \\
2.84\end{array}$ & $\begin{array}{r}3.88 \\
.33 \\
3.23 \\
2.65 \\
2.97\end{array}$ & $\begin{array}{r}3.38 \\
.33 \\
2.00 \\
2.60 \\
2.86\end{array}$ \\
\hline 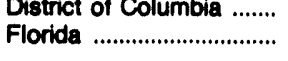 & $2 . \overline{76}$ & $2 . \overline{41}$ & $2 . \overline{46}$ & $2 . \overline{76}$ & $2 . \overline{57}$ & $2 . \overline{81}$ & $2 . \overline{89}$ & $2 . \overline{80}$ \\
\hline 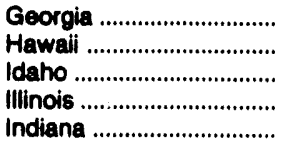 & $\begin{array}{l}3.70 \\
5.86 \\
2.12 \\
3.38 \\
3.17\end{array}$ & $\begin{array}{l}3.14 \\
7.65 \\
2.23 \\
2.99 \\
2.92\end{array}$ & $\begin{array}{l}3.44 \\
8.68 \\
2.18 \\
2.86 \\
3.06\end{array}$ & $\begin{array}{l}4.17 \\
5.18 \\
2.54 \\
3.25 \\
3.55\end{array}$ & $\begin{array}{l}4.06 \\
5.46 \\
2.92 \\
3.28 \\
3.40\end{array}$ & $\begin{array}{l}4.25 \\
5.62 \\
2.39 \\
3.18 \\
3.42\end{array}$ & $\begin{array}{l}4.62 \\
5.72 \\
2.24 \\
3.40 \\
3.74\end{array}$ & $\begin{array}{l}3.44 \\
5.69 \\
1.89 \\
3.43 \\
3.14\end{array}$ \\
\hline 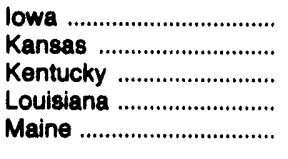 & $\begin{array}{l}3.21 \\
2.79 \\
3.21 \\
2.68 \\
3.79\end{array}$ & $\begin{array}{l}2.98 \\
2.48 \\
2.86 \\
2.25 \\
3.00\end{array}$ & $\begin{array}{l}2.66 \\
2.56 \\
2.89 \\
2.60 \\
2.96\end{array}$ & $\begin{array}{l}3.44 \\
2.44 \\
3.05 \\
2.74 \\
4.20\end{array}$ & $\begin{array}{l}3.52 \\
2.74 \\
3.00 \\
2.69 \\
4.77\end{array}$ & $\begin{array}{l}3.60 \\
2.37 \\
3.43 \\
2.77 \\
4.67\end{array}$ & $\begin{array}{l}4.28 \\
2.48 \\
3.94 \\
3.23 \\
3.67\end{array}$ & $\begin{array}{l}3.55 \\
2.54 \\
3.26 \\
2.78 \\
3.31\end{array}$ \\
\hline 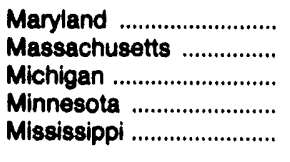 & $\begin{array}{l}3.53 \\
4.05 \\
2.90 \\
3.08 \\
2.77\end{array}$ & $\begin{array}{l}2.97 \\
3.35 \\
2.96 \\
2.65 \\
2.36\end{array}$ & $\begin{array}{l}2.95 \\
3.30 \\
3.06 \\
2.55 \\
2.54\end{array}$ & $\begin{array}{l}5.27 \\
4.72 \\
2.79 \\
3.39 \\
3.00\end{array}$ & $\begin{array}{l}5.41 \\
4.77 \\
2.60 \\
3.48 \\
2.89\end{array}$ & $\begin{array}{l}5.85 \\
4.81 \\
2.77 \\
3.46 \\
3.00\end{array}$ & $\begin{array}{l}4.98 \\
4.42 \\
3.17 \\
3.82 \\
3.41\end{array}$ & $\begin{array}{l}3.80 \\
3.92 \\
2.82 \\
2.98 \\
2.89\end{array}$ \\
\hline 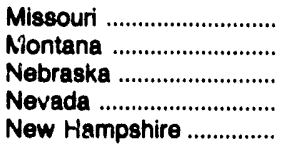 & $\begin{array}{l}3.22 \\
3.26 \\
3.03 \\
2.97 \\
3.74\end{array}$ & $\begin{array}{l}2.73 \\
3.76 \\
2.70 \\
2.33 \\
3.48\end{array}$ & $\begin{array}{l}2.86 \\
3.77 \\
2.72 \\
2.38 \\
3.38\end{array}$ & $\begin{array}{l}3.79 \\
4.53 \\
3.51 \\
4.75 \\
4.40\end{array}$ & $\begin{array}{l}3.63 \\
4.44 \\
3.37 \\
3.74 \\
4.55\end{array}$ & $\begin{array}{l}3.81 \\
4.47 \\
3.49 \\
3.55 \\
4.46\end{array}$ & $\begin{array}{l}3.89 \\
4.11 \\
3.94 \\
3.73 \\
3.86\end{array}$ & $\begin{array}{l}2.91 \\
3.16 \\
3.19 \\
3.29 \\
3.42\end{array}$ \\
\hline 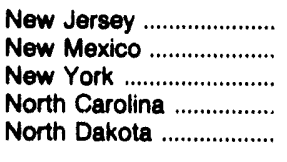 & $\begin{array}{l}3.55 \\
2.45 \\
3.29 \\
3.20 \\
3.32\end{array}$ & $\begin{array}{l}3.00 \\
2.04 \\
2.77 \\
2.64 \\
3.40\end{array}$ & $\begin{array}{l}3.07 \\
2.56 \\
2.86 \\
2.67 \\
3.51\end{array}$ & $\begin{array}{l}4.30 \\
2.10 \\
3.25 \\
3.53 \\
4.45\end{array}$ & $\begin{array}{l}4.69 \\
1.86 \\
3.49 \\
3.74 \\
4.57\end{array}$ & $\begin{array}{l}4.18 \\
2.36 \\
3.73 \\
3.57 \\
4.21\end{array}$ & $\begin{array}{l}4.26 \\
2.71 \\
4.08 \\
3.65 \\
4.05\end{array}$ & $\begin{array}{l}3.44 \\
3.04 \\
3.64 \\
3.24 \\
3.24\end{array}$ \\
\hline 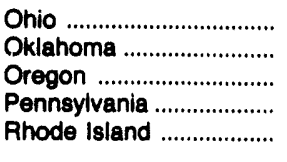 & $\begin{array}{l}3.62 \\
2.37 \\
2.34 \\
3.42 \\
4.25\end{array}$ & $\begin{array}{l}3.04 \\
2.11 \\
2.33 \\
3.10 \\
3.63\end{array}$ & $\begin{array}{l}3.09 \\
1.95 \\
2.37 \\
3.28 \\
3.63\end{array}$ & $\begin{array}{l}3.85 \\
2.00 \\
2.61 \\
4.43 \\
6.64\end{array}$ & $\begin{array}{r}3.82 \\
1.80 \\
2.51 \\
\text { ค } 3.97 \\
7.73\end{array}$ & $\begin{array}{l}4.23 \\
2.10 \\
2.83 \\
4.70 \\
6.17\end{array}$ & $\begin{array}{l}4.27 \\
2.54 \\
2.74 \\
4.19 \\
6.59\end{array}$ & $\begin{array}{l}4.28 \\
2.26 \\
2.18 \\
3.57 \\
4.09\end{array}$ \\
\hline 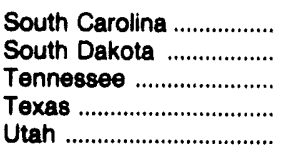 & $\begin{array}{l}3.54 \\
3.38 \\
3.13 \\
3.23 \\
3.07\end{array}$ & $\begin{array}{l}3.02 \\
3.05 \\
2.62 \\
2.85 \\
4.30\end{array}$ & $\begin{array}{l}2.83 \\
3.14 \\
2.63 \\
2.84 \\
4.05\end{array}$ & $\begin{array}{l}4.18 \\
4.69 \\
3.38 \\
3.16 \\
2.30\end{array}$ & $\begin{array}{l}4.25 \\
4.42 \\
3.34 \\
3.04 \\
2.89\end{array}$ & $\begin{array}{r}3.93 \\
4.31 \\
3.39 \\
3.14 \\
2.71\end{array}$ & $\begin{array}{l}4.20 \\
4.31 \\
3.80 \\
3.36 \\
5.61\end{array}$ & $\begin{array}{l}3.48 \\
3.36 \\
3.29 \\
3.09 \\
3.74\end{array}$ \\
\hline 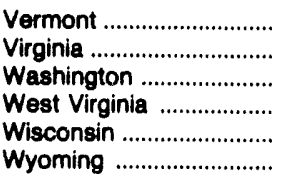 & $\begin{array}{l}3.00 \\
3.28 \\
2.19 \\
3.12 \\
3.71 \\
2.88\end{array}$ & $\begin{array}{l}2.92 \\
2.60 \\
1.79 \\
2.91 \\
3.25 \\
3.07\end{array}$ & $\begin{array}{l}2.82 \\
2.68 \\
1.86 \\
3.54 \\
3.14 \\
3.21\end{array}$ & $\begin{array}{l}4.38 \\
3.41 \\
2.35 \\
6.82 \\
6.22 \\
2.86\end{array}$ & $\begin{array}{l}3.78 \\
3.29 \\
2.37 \\
5.73 \\
5.71 \\
3.05\end{array}$ & $\begin{array}{l}3.98 \\
3.43 \\
2.56 \\
4.77 \\
5.65 \\
3.04\end{array}$ & $\begin{array}{l}3.85 \\
3.73 \\
2.52 \\
1.94 \\
5.43 \\
3.77\end{array}$ & $\begin{array}{l}3.03 \\
3.36 \\
1.85 \\
3.32 \\
3.86 \\
3.07\end{array}$ \\
\hline 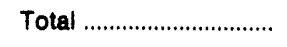 & 3.19 & 2.84 & 2.87 & 3.35 & 3.34 & ค 3.44 & 3.58 & 3.24 \\
\hline
\end{tabular}

See footnotes at end of table. 
Table 23. Average City Gate Price by State, 1992-1993 (Continued) (Dollars per Thousand Cubic Feet)

\begin{tabular}{|c|c|c|c|c|c|c|c|c|}
\hline State & $\begin{array}{c}\text { March } \\
1893\end{array}$ & $\begin{array}{l}\text { February } \\
1993\end{array}$ & $\begin{array}{l}\text { January } \\
1993\end{array}$ & $\begin{array}{l}\text { Total } \\
1992\end{array}$ & $\begin{array}{l}\text { December } \\
1992\end{array}$ & $\begin{array}{c}\text { November } \\
1992\end{array}$ & $\begin{array}{l}\text { October } \\
1992\end{array}$ & $\begin{array}{l}\text { September } \\
1992\end{array}$ \\
\hline 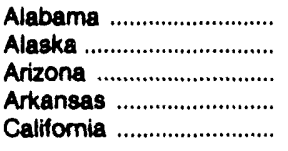 & $\begin{array}{r}3.16 \\
.33 \\
2.50 \\
2.63 \\
2.80\end{array}$ & $\begin{array}{r}3.37 \\
.33 \\
2.28 \\
2.45 \\
2.69\end{array}$ & $\begin{array}{r}3.33 \\
.33 \\
2.63 \\
2.62 \\
2.74\end{array}$ & $\begin{array}{r}3.21 \\
.34 \\
2.33 \\
2.60 \\
2.72\end{array}$ & $\begin{array}{r}3.43 \\
.33 \\
2.55 \\
2.64 \\
2.75\end{array}$ & $\begin{array}{r}3.44 \\
.33 \\
2.69 \\
2.71 \\
2.91\end{array}$ & $\begin{array}{r}3.91 \\
.33 \\
2.64 \\
2.49 \\
2.92\end{array}$ & $\begin{array}{r}3.67 \\
.33 \\
2.45 \\
2.41 \\
2.80\end{array}$ \\
\hline 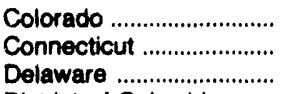 & $\begin{array}{l}2.57 \\
3.31 \\
3.22\end{array}$ & $\begin{array}{l}2.67 \\
3.27 \\
3.03\end{array}$ & $\begin{array}{l}2.80 \\
3.70 \\
3.13\end{array}$ & $\begin{array}{l}2.86 \\
3.73 \\
2.83\end{array}$ & $\begin{array}{l}2.81 \\
3.59 \\
3.53\end{array}$ & $\begin{array}{l}2.74 \\
3.80 \\
3.93\end{array}$ & $\begin{array}{l}2.98 \\
4.02 \\
3.74\end{array}$ & $\begin{array}{l}3.67 \\
4.13 \\
2.74\end{array}$ \\
\hline $\begin{array}{l}\text { District of Columbia } \\
\text { Florida }\end{array}$ & $2 . \overline{64}$ & $2 . \overline{63}$ & $2 . \overline{90}$ & $2 . \overline{61}$ & $3 . \overline{07}$ & $3 . \overline{19}$ & $3 . \overline{16}$ & $2 . \overline{64}$ \\
\hline 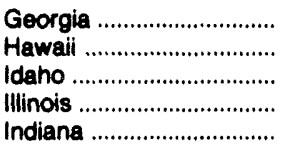 & $\begin{array}{l}3.25 \\
5.46 \\
2.12 \\
3.26 \\
2.91\end{array}$ & $\begin{array}{l}3.48 \\
7.01 \\
1.97 \\
3.48 \\
2.83\end{array}$ & $\begin{array}{l}3.79 \\
6.49 \\
2.03 \\
3.75 \\
3.04\end{array}$ & $\begin{array}{l}3.28 \\
7.72 \\
2.18 \\
3.20 \\
3.08\end{array}$ & $\begin{array}{l}3.51 \\
7.59 \\
2.06 \\
3.53 \\
3.26\end{array}$ & $\begin{array}{l}3.27 \\
7.81 \\
1.99 \\
3.47 \\
3.33\end{array}$ & $\begin{array}{l}3.93 \\
8.17 \\
2.37 \\
3.60 \\
3.69\end{array}$ & $\begin{array}{l}3.70 \\
7.87 \\
2.47 \\
3.36 \\
3.03\end{array}$ \\
\hline 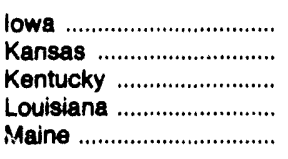 & $\begin{array}{l}2.94 \\
3.18 \\
3.24 \\
2.59 \\
3.66\end{array}$ & $\begin{array}{l}2.81 \\
2.66 \\
3.10 \\
2.38 \\
3.53\end{array}$ & $\begin{array}{l}3.02 \\
3.06 \\
3.13 \\
2.59 \\
3.69\end{array}$ & $\begin{array}{l}3.20 \\
2.50 \\
3.02 \\
2.48 \\
3.17\end{array}$ & $\begin{array}{l}3.21 \\
2.48 \\
3.26 \\
2.82 \\
4.00\end{array}$ & $\begin{array}{l}3.44 \\
2.79 \\
3.28 \\
2.83 \\
3.41\end{array}$ & $\begin{array}{l}3.97 \\
2.33 \\
3.53 \\
3.24 \\
3.45\end{array}$ & $\begin{array}{l}3.89 \\
2.31 \\
2.76 \\
2.70 \\
2.80\end{array}$ \\
\hline 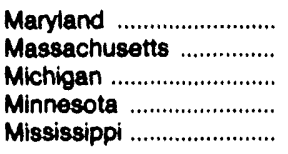 & $\begin{array}{l}2.90 \\
3.94 \\
2.84 \\
2.94 \\
2.61\end{array}$ & $\begin{array}{l}2.89 \\
3.53 \\
2.90 \\
2.78 \\
2.44\end{array}$ & $\begin{array}{l}3.02 \\
3.81 \\
3.43 \\
2.97 \\
2.62\end{array}$ & $\begin{array}{l}3.20 \\
3.52 \\
3.04 \\
2.92 \\
2.62\end{array}$ & $\begin{array}{l}3.17 \\
3.89 \\
3.16 \\
3.32 \\
2.97\end{array}$ & $\begin{array}{l}3.66 \\
3.95 \\
3.33 \\
3.31 \\
3.07\end{array}$ & $\begin{array}{l}3.91 \\
3.88 \\
3.31 \\
3.57 \\
3.59\end{array}$ & $\begin{array}{l}4.58 \\
3.40 \\
3.02 \\
3.28 \\
2.99\end{array}$ \\
\hline 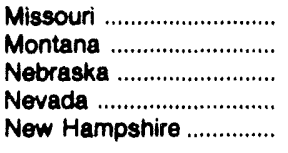 & $\begin{array}{l}2.96 \\
2.92 \\
2.78 \\
2.66 \\
3.68\end{array}$ & $\begin{array}{l}2.87 \\
2.96 \\
2.78 \\
2.36 \\
3.30\end{array}$ & $\begin{array}{l}3.37 \\
2.98 \\
2.92 \\
2.57 \\
3.78\end{array}$ & $\begin{array}{l}2.86 \\
3.45 \\
2.91 \\
2.37 \\
3.58\end{array}$ & $\begin{array}{l}2.78 \\
2.81 \\
3.09 \\
2.40 \\
3.96\end{array}$ & $\begin{array}{l}3.16 \\
3.01 \\
3.15 \\
2.43 \\
4.02\end{array}$ & $\begin{array}{l}3.56 \\
3.19 \\
3.55 \\
2.45 \\
3.70\end{array}$ & $\begin{array}{l}3.52 \\
3.85 \\
3.47 \\
2.67 \\
3.16\end{array}$ \\
\hline $\begin{array}{l}\text { New Jersey .................... } \\
\text { New Mexico } \\
\text { New York ............................. } \\
\text { North Carolina .................. } \\
\text { North Dakota ................... }\end{array}$ & $\begin{array}{l}3.27 \\
2.54 \\
3.11 \\
3.01 \\
3.01\end{array}$ & $\begin{array}{l}2.82 \\
2.34 \\
2.91 \\
2.83 \\
2.97\end{array}$ & $\begin{array}{l}3.32 \\
2.45 \\
3.10 \\
3.09 \\
3.11\end{array}$ & $\begin{array}{l}3.29 \\
2.25 \\
3.01 \\
2.88 \\
3.28\end{array}$ & $\begin{array}{l}3.32 \\
2.55 \\
3.34 \\
3.22 \\
3.06\end{array}$ & $\begin{array}{l}4.32 \\
2.66 \\
3.47 \\
3.32 \\
3.09\end{array}$ & $\begin{array}{l}3.95 \\
2.99 \\
3.75 \\
3.38 \\
3.16\end{array}$ & $\begin{array}{l}3.97 \\
2.30 \\
3.37 \\
3.26 \\
3.38\end{array}$ \\
\hline 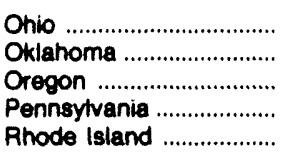 & $\begin{array}{l}3.44 \\
2.44 \\
2.16 \\
3.15 \\
3.34\end{array}$ & $\begin{array}{l}3.43 \\
2.40 \\
2.26 \\
2.93 \\
3.12\end{array}$ & $\begin{array}{l}3.40 \\
2.50 \\
2.22 \\
3.02 \\
3.57\end{array}$ & $\begin{array}{l}3.26 \\
2.22 \\
2.34 \\
3.29 \\
3.82\end{array}$ & $\begin{array}{l}3.69 \\
2.38 \\
2.39 \\
3.37 \\
3.67\end{array}$ & $\begin{array}{l}3.51 \\
2.52 \\
2.36 \\
3.61 \\
4.49\end{array}$ & $\begin{array}{l}3.84 \\
2.44 \\
2.35 \\
3.82 \\
4.45\end{array}$ & $\begin{array}{l}3.60 \\
1.84 \\
2.27 \\
4.20 \\
4.58\end{array}$ \\
\hline 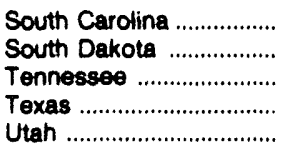 & $\begin{array}{l}3.09 \\
3.12 \\
3.07 \\
3.16 \\
3.49\end{array}$ & $\begin{array}{l}3.27 \\
2.98 \\
2.81 \\
3.21 \\
2.89\end{array}$ & $\begin{array}{l}3.39 \\
3.07 \\
2.85 \\
3.44 \\
2.94\end{array}$ & $\begin{array}{l}3.23 \\
3.10 \\
2.89 \\
3.06 \\
4.09\end{array}$ & $\begin{array}{l}3.56 \\
3.29 \\
3.20 \\
3.47 \\
3.00\end{array}$ & $\begin{array}{l}3.56 \\
3.18 \\
3.40 \\
3.47 \\
3.95\end{array}$ & $\begin{array}{l}3.74 \\
2.83 \\
3.66 \\
3.61 \\
4.71\end{array}$ & $\begin{array}{l}3.48 \\
3.25 \\
3.41 \\
3.01 \\
8.36\end{array}$ \\
\hline 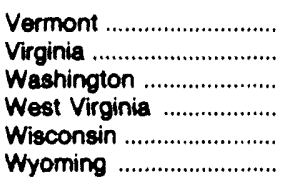 & $\begin{array}{l}2.57 \\
3.14 \\
2.06 \\
2.94 \\
3.15 \\
2.89\end{array}$ & $\begin{array}{l}2.53 \\
3.18 \\
2.11 \\
2.94 \\
2.95 \\
2.63\end{array}$ & $\begin{array}{l}2.52 \\
3.24 \\
2.18 \\
2.86 \\
3.06 \\
2.71\end{array}$ & $\begin{array}{l}2.93 \\
2.91 \\
1.90 \\
3.24 \\
3.36 \\
2.90\end{array}$ & $\begin{array}{l}2.60 \\
3.32 \\
2.16 \\
3.36 \\
3.17 \\
2.69\end{array}$ & $\begin{array}{l}2.86 \\
3.83 \\
1.98 \\
3.89 \\
3.48 \\
2.55\end{array}$ & $\begin{array}{l}2.95 \\
3.70 \\
2.00 \\
4.59 \\
3.97 \\
2.66\end{array}$ & $\begin{array}{l}4.06 \\
3.26 \\
1.99 \\
6.20 \\
4.47 \\
3.01\end{array}$ \\
\hline 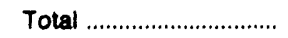 & 3.06 & 2.84 & 3.11 & 3.01 & 3.17 & 3.33 & 3.50 & 3.23 \\
\hline
\end{tabular}

See footnotes at end of table. 
Table 23. Average Clty Gate Price by State, 1992-1993 (Continued) (Dollars per Thousand Cubic Feet)

\begin{tabular}{|c|c|c|c|c|c|c|c|c|}
\hline State & $\begin{array}{l}\text { August } \\
1992\end{array}$ & $\begin{array}{l}\text { July } \\
1992\end{array}$ & $\begin{array}{l}\text { June } \\
1992\end{array}$ & $\begin{array}{l}\text { May } \\
1982\end{array}$ & $\begin{array}{l}\text { April } \\
1992\end{array}$ & $\begin{array}{l}\text { March } \\
1992\end{array}$ & $\begin{array}{c}\text { February } \\
1992\end{array}$ & $\begin{array}{c}\text { January } \\
1992\end{array}$ \\
\hline 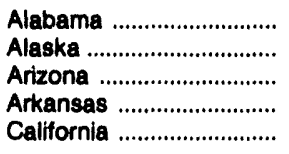 & $\begin{array}{r}3.65 \\
.34 \\
2.43 \\
2.38 \\
2.82\end{array}$ & $\begin{array}{r}3.25 \\
.33 \\
2.70 \\
2.41 \\
2.73\end{array}$ & $\begin{array}{r}3.28 \\
.33 \\
2.31 \\
2.53 \\
2.59\end{array}$ & $\begin{array}{r}3.01 \\
.33 \\
1.57 \\
2.43 \\
2.47\end{array}$ & $\begin{array}{r}2.80 \\
.33 \\
1.98 \\
2.38 \\
2.88\end{array}$ & $\begin{array}{r}2.68 \\
.34 \\
2.12 \\
2.62 \\
2.37\end{array}$ & $\begin{array}{r}3.06 \\
.36 \\
2.08 \\
2.61 \\
2.56\end{array}$ & $\begin{array}{r}3.16 \\
.36 \\
2.34 \\
2.87 \\
2.87\end{array}$ \\
\hline 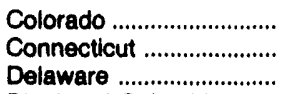 & $\begin{array}{l}3.61 \\
4.23 \\
1.78\end{array}$ & $\begin{array}{l}3.32 \\
4.12 \\
2.72\end{array}$ & $\begin{array}{l}3.23 \\
4.96 \\
2.90\end{array}$ & $\begin{array}{l}3.45 \\
4.76 \\
2.59\end{array}$ & $\begin{array}{l}2.75 \\
3.75 \\
2.55\end{array}$ & $\begin{array}{l}2.67 \\
3.18 \\
2.62\end{array}$ & $\begin{array}{l}2.60 \\
3.37 \\
1.81\end{array}$ & $\begin{array}{l}2.67 \\
3.39 \\
3.01\end{array}$ \\
\hline $\begin{array}{l}\text { District of Columbia } \\
\text { Florida }\end{array}$ & $2 . \overline{58}$ & $2 . \overline{41}$ & $2 . \overline{37}$ & $2 . \overline{29}$ & $2 . \overline{39}$ & $2 . \overline{34}$ & $2 . \overline{31}$ & $2 . \overline{56}$ \\
\hline 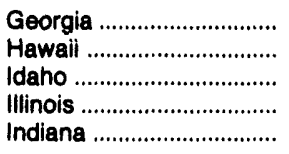 & $\begin{array}{l}3.55 \\
8.27 \\
3.61 \\
3.19 \\
\mathbf{3 . 5 2}\end{array}$ & $\begin{array}{l}3.10 \\
8.08 \\
2.52 \\
3.08 \\
2.97\end{array}$ & $\begin{array}{l}3.21 \\
7.55 \\
2.57 \\
2.89 \\
3.16\end{array}$ & $\begin{array}{l}2.90 \\
7.60 \\
2.55 \\
2.72 \\
2.71\end{array}$ & $\begin{array}{l}2.57 \\
7.64 \\
1.94 \\
2.97 \\
2.57\end{array}$ & $\begin{array}{l}2.61 \\
7.40 \\
2.28 \\
2.71 \\
2.75\end{array}$ & $\begin{array}{l}3.79 \\
7.22 \\
2.17 \\
2.99 \\
2.79\end{array}$ & $\begin{array}{l}3.27 \\
7.53 \\
1.96 \\
3.52 \\
3.07\end{array}$ \\
\hline 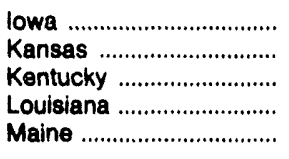 & $\begin{array}{l}3.86 \\
2.49 \\
2.74 \\
2.60 \\
4.19\end{array}$ & $\begin{array}{l}4.16 \\
2.30 \\
2.57 \\
2.26 \\
2.72\end{array}$ & $\begin{array}{l}4.12 \\
2.07 \\
2.98 \\
2.30 \\
2.82\end{array}$ & $\begin{array}{l}3.77 \\
2.32 \\
2.89 \\
2.42 \\
2.66\end{array}$ & $\begin{array}{l}2.48 \\
2.28 \\
2.88 \\
2.25 \\
2.44\end{array}$ & $\begin{array}{l}2.72 \\
2.09 \\
2.85 \\
2.06 \\
2.59\end{array}$ & $\begin{array}{l}2.58 \\
2.74 \\
2.89 \\
1.93 \\
3.39\end{array}$ & $\begin{array}{l}2.64 \\
2.73 \\
2.95 \\
2.34 \\
3.45\end{array}$ \\
\hline 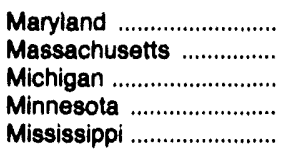 & $\begin{array}{l}4.83 \\
3.24 \\
2.95 \\
3.34 \\
2.81\end{array}$ & $\begin{array}{l}5.04 \\
3.35 \\
2.78 \\
3.12 \\
2.39\end{array}$ & $\begin{array}{l}4.46 \\
3.77 \\
2.82 \\
3.25 \\
2.59\end{array}$ & $\begin{array}{l}3.71 \\
3.66 \\
2.89 \\
3.02 \\
2.50\end{array}$ & $\begin{array}{l}2.88 \\
2.91 \\
2.93 \\
2.26 \\
2.32\end{array}$ & $\begin{array}{l}2.45 \\
2.97 \\
3.19 \\
2.47 \\
2.14\end{array}$ & $\begin{array}{l}2.41 \\
3.47 \\
3.11 \\
2.30 \\
2.06\end{array}$ & $\begin{array}{l}2.74 \\
3.67 \\
3.21 \\
2.68 \\
2.41\end{array}$ \\
\hline 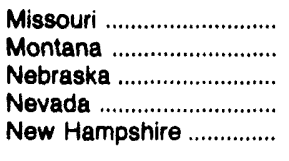 & $\begin{array}{l}3.39 \\
4.38 \\
3.23 \\
2.92 \\
3.83\end{array}$ & $\begin{array}{l}2.74 \\
4.80 \\
3.18 \\
2.72 \\
3.27\end{array}$ & $\begin{array}{l}3.20 \\
4.51 \\
3.28 \\
2.68 \\
3.62\end{array}$ & $\begin{array}{l}2.85 \\
4.03 \\
3.07 \\
2.65 \\
3.31\end{array}$ & $\begin{array}{l}2.60 \\
3.63 \\
2.47 \\
2.09 \\
2.92\end{array}$ & $\begin{array}{l}2.59 \\
3.58 \\
2.46 \\
2.20 \\
2.93\end{array}$ & $\begin{array}{l}2.46 \\
3.57 \\
2.36 \\
2.13 \\
4.00\end{array}$ & $\begin{array}{l}2.88 \\
3.57 \\
2.71 \\
2.20 \\
3.88\end{array}$ \\
\hline $\begin{array}{l}\text { New Jersey .................... } \\
\text { New Mexico ...................... } \\
\text { New York ....................... } \\
\text { North Carolina .................. } \\
\text { North Dakota ................... }\end{array}$ & $\begin{array}{l}3.38 \\
2.13 \\
3.15 \\
3.04 \\
3.79\end{array}$ & $\begin{array}{l}3.40 \\
2.09 \\
2.92 \\
3.13 \\
4.16\end{array}$ & $\begin{array}{l}3.17 \\
2.48 \\
3.11 \\
3.13 \\
3.55\end{array}$ & $\begin{array}{l}3.19 \\
2.09 \\
2.91 \\
2.85 \\
3.36\end{array}$ & $\begin{array}{l}2.66 \\
2.40 \\
2.67 \\
2.56 \\
3.21\end{array}$ & $\begin{array}{l}2.58 \\
1.82 \\
2.57 \\
2.18 \\
3.20\end{array}$ & $\begin{array}{l}2.81 \\
1.92 \\
2.49 \\
2.37 \\
3.34\end{array}$ & $\begin{array}{l}3.15 \\
2.05 \\
2.88 \\
2.77 \\
3.44\end{array}$ \\
\hline 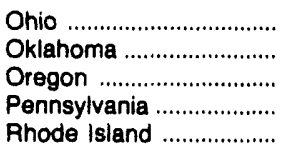 & $\begin{array}{l}3.71 \\
1.79 \\
2.41 \\
4.32 \\
4.87\end{array}$ & $\begin{array}{l}3.72 \\
1.82 \\
2.25 \\
3.92 \\
4.26\end{array}$ & $\begin{array}{l}3.43 \\
1.88 \\
2.14 \\
4.01 \\
4.21\end{array}$ & $\begin{array}{l}3.35 \\
2.97 \\
2.59 \\
3.62 \\
3.99\end{array}$ & $\begin{array}{l}2.96 \\
2.11 \\
2.22 \\
3.06 \\
3.38\end{array}$ & $\begin{array}{l}2.87 \\
2.06 \\
2.35 \\
2.77 \\
3.06\end{array}$ & $\begin{array}{l}2.98 \\
2.07 \\
2.27 \\
2.72 \\
3.40\end{array}$ & $\begin{array}{l}2.96 \\
2.17 \\
2.40 \\
2.86 \\
3.52\end{array}$ \\
\hline 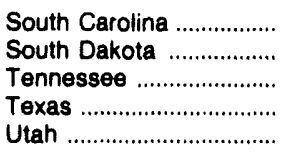 & $\begin{array}{l}3.38 \\
4.09 \\
3.41 \\
2.94 \\
7.45\end{array}$ & $\begin{array}{l}3.22 \\
3.91 \\
2.94 \\
2.71 \\
7.72\end{array}$ & $\begin{array}{l}3.10 \\
3.68 \\
3.09 \\
2.78 \\
7.48\end{array}$ & $\begin{array}{l}2.83 \\
3.42 \\
3.01 \\
2.85 \\
8.05\end{array}$ & $\begin{array}{l}2.75 \\
2.42 \\
2.68 \\
2.83 \\
5.26\end{array}$ & $\begin{array}{l}2.70 \\
2.98 \\
2.36 \\
2.77 \\
4.17\end{array}$ & $\begin{array}{l}2.99 \\
2.86 \\
2.26 \\
2.78 \\
3.54\end{array}$ & $\begin{array}{l}3.24 \\
2.85 \\
2.47 \\
2.98 \\
3.26\end{array}$ \\
\hline 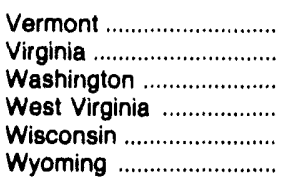 & $\begin{array}{l}4.17 \\
3.63 \\
1.93 \\
5.74 \\
4.94 \\
3.32\end{array}$ & $\begin{array}{l}3.25 \\
2.87 \\
1.96 \\
7.91 \\
4.76 \\
3.36\end{array}$ & $\begin{array}{l}3.25 \\
2.69 \\
1.87 \\
4.36 \\
3.84 \\
3.63\end{array}$ & $\begin{array}{l}3.04 \\
2.80 \\
1.84 \\
4.05 \\
3.45 \\
3.43\end{array}$ & $\begin{array}{l}2.76 \\
2.33 \\
1.63 \\
2.29 \\
3.12 \\
3.23\end{array}$ & $\begin{array}{l}2.65 \\
2.14 \\
1.80 \\
1.93 \\
3.03 \\
2.83\end{array}$ & $\begin{array}{l}2.76 \\
2.34 \\
1.77 \\
2.80 \\
2.88 \\
2.95\end{array}$ & $\begin{array}{l}2.76 \\
2.93 \\
1.76 \\
3.14 \\
3.02 \\
2.95\end{array}$ \\
\hline 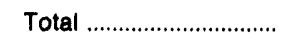 & 3.18 & 3.01 & 3.00 & 2.90 & 2.74 & 2.61 & 2.70 & 2.90 \\
\hline
\end{tabular}

MA $=$ Not Available

A $=$ Revised Data.

-- = Not Applicable.

Notes: Geographic coverage is the 50 States and the District of Columbia. Prices in this table represent the average price of natural gas by State at the point where the gas transferred from a pipeline to a local distribution company within the State. See Appendix A, Explanatory Note 5 for discussion of computations and revision policy.

Source: Form EIA-857. 
Table 24. Average Price of Natural Gas Dellvered to Residential Consumers by State, 1992-1993

(Dollars per Thousand Cubic Feet)

\begin{tabular}{|c|c|c|c|c|c|c|c|c|}
\hline State & $\begin{array}{l}\text { YTD } \\
1983\end{array}$ & $\begin{array}{l}\text { YTD } \\
1992\end{array}$ & $\begin{array}{c}\text { YTD } \\
1991\end{array}$ & $\begin{array}{c}\text { August } \\
1993\end{array}$ & $\begin{array}{c}\text { July } \\
1993\end{array}$ & $\begin{array}{l}\text { June } \\
1993\end{array}$ & $\begin{array}{l}\text { May } \\
1993\end{array}$ & $\begin{array}{l}\text { April } \\
1993\end{array}$ \\
\hline 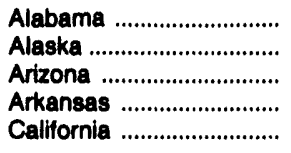 & $\begin{array}{l}6.80 \\
3.86 \\
7.10 \\
5.24 \\
6.14\end{array}$ & $\begin{array}{l}6.59 \\
3.80 \\
7.19 \\
5.02 \\
6.00\end{array}$ & $\begin{array}{l}7.09 \\
4.29 \\
6.86 \\
4.90 \\
6.29\end{array}$ & $\begin{array}{l}9.90 \\
4.50 \\
9.94 \\
7.29 \\
6.61\end{array}$ & $\begin{array}{l}9.81 \\
4.47 \\
9.50 \\
7.14 \\
6.61\end{array}$ & $\begin{array}{l}9.36 \\
4.25 \\
8.92 \\
6.65 \\
6.58\end{array}$ & $\begin{array}{l}7.74 \\
4.05 \\
8.23 \\
5.76 \\
6.10\end{array}$ & $\begin{array}{l}6.59 \\
3.93 \\
7.31 \\
5.12 \\
5.71\end{array}$ \\
\hline 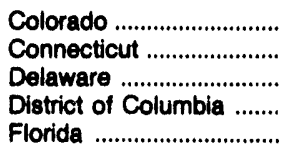 & $\begin{array}{r}4.39 \\
9.37 \\
6.39 \\
8.35 \\
10.21\end{array}$ & $\begin{array}{l}4.57 \\
9.34 \\
5.98 \\
7.23 \\
8.75\end{array}$ & $\begin{array}{l}4.54 \\
8.70 \\
5.80 \\
6.99 \\
8.80\end{array}$ & $\begin{array}{r}6.01 \\
11.59 \\
8.80 \\
8.68 \\
12.81\end{array}$ & $\begin{array}{r}5.62 \\
11.37 \\
8.66 \\
8.17 \\
12.62\end{array}$ & $\begin{array}{r}5.06 \\
10.89 \\
8.25 \\
8.28 \\
12.39\end{array}$ & $\begin{array}{r}4.56 \\
10.35 \\
7.09 \\
8.70 \\
11.42\end{array}$ & $\begin{array}{l}4.36 \\
9.59 \\
6.25 \\
8.27 \\
9.83\end{array}$ \\
\hline 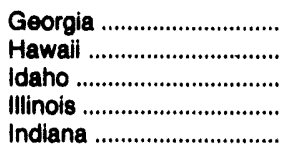 & $\begin{array}{r}6.76 \\
17.57 \\
5.19 \\
5.48 \\
5.87\end{array}$ & $\begin{array}{r}6.58 \\
17.88 \\
5.28 \\
4.91 \\
5.39\end{array}$ & $\begin{array}{r}6.83 \\
22.96 \\
5.17 \\
5.13 \\
5.69\end{array}$ & $\begin{array}{r}9.43 \\
18.43 \\
6.81 \\
8.26 \\
8.39\end{array}$ & $\begin{array}{r}9.06 \\
17.97 \\
6.30 \\
7.88 \\
8.49\end{array}$ & $\begin{array}{r}8.91 \\
17.57 \\
5.99 \\
7.64 \\
7.81\end{array}$ & $\begin{array}{r}8.08 \\
17.86 \\
5.49 \\
6.98 \\
6.81\end{array}$ & $\begin{array}{r}6.49 \\
17.70 \\
5.30 \\
5.63 \\
5.94\end{array}$ \\
\hline 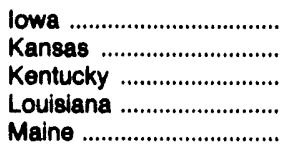 & $\begin{array}{l}5.69 \\
4.68 \\
5.24 \\
5.73 \\
7.28\end{array}$ & $\begin{array}{l}4.93 \\
4.69 \\
4.79 \\
5.24 \\
6.93\end{array}$ & $\begin{array}{l}4.70 \\
4.33 \\
4.93 \\
5.62 \\
6.80\end{array}$ & $\begin{array}{l}8.69 \\
6.49 \\
7.68 \\
8.06 \\
9.05\end{array}$ & $\begin{array}{l}9.13 \\
6.12 \\
7.44 \\
7.26 \\
8.88\end{array}$ & $\begin{array}{l}7.27 \\
6.28 \\
6.78 \\
7.29 \\
8.29\end{array}$ & $\begin{array}{l}7.04 \\
5.26 \\
6.72 \\
6.98 \\
8.11\end{array}$ & $\begin{array}{l}5.92 \\
4.58 \\
5.28 \\
5.67 \\
7.51\end{array}$ \\
\hline 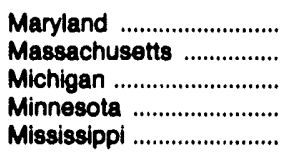 & $\begin{array}{l}7.12 \\
8.17 \\
4.77 \\
5.31 \\
4.77\end{array}$ & $\begin{array}{l}6.10 \\
7.83 \\
5.06 \\
4.52 \\
4.75\end{array}$ & $\begin{array}{l}6.15 \\
8.05 \\
5.11 \\
4.38 \\
5.30\end{array}$ & $\begin{array}{l}9.65 \\
8.56 \\
7.21 \\
7.36 \\
5.58\end{array}$ & $\begin{array}{l}9.65 \\
8.01 \\
6.69 \\
6.29 \\
5.55\end{array}$ & $\begin{array}{l}9.09 \\
7.52 \\
6.10 \\
6.13 \\
5.49\end{array}$ & $\begin{array}{l}8.05 \\
6.85 \\
5.37 \\
6.10 \\
5.76\end{array}$ & $\begin{array}{l}7.02 \\
8.54 \\
4.67 \\
5.35 \\
4.66\end{array}$ \\
\hline 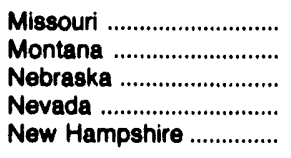 & $\begin{array}{l}5.08 \\
4.85 \\
4.85 \\
5.46 \\
7.38\end{array}$ & $\begin{array}{l}4.93 \\
4.79 \\
4.61 \\
5.59 \\
7.22\end{array}$ & $\begin{array}{l}5.14 \\
4.48 \\
4.55 \\
5.56 \\
7.08\end{array}$ & $\begin{array}{l}8.42 \\
5.95 \\
6.71 \\
7.32 \\
9.29\end{array}$ & $\begin{array}{l}7.96 \\
5.64 \\
6.45 \\
7.07 \\
8.60\end{array}$ & $\begin{array}{l}7.42 \\
5.65 \\
5.99 \\
6.50 \\
7.53\end{array}$ & $\begin{array}{l}5.82 \\
5.16 \\
5.51 \\
6.01 \\
6.59\end{array}$ & $\begin{array}{l}4.91 \\
4.85 \\
4.77 \\
5.54 \\
5.91\end{array}$ \\
\hline 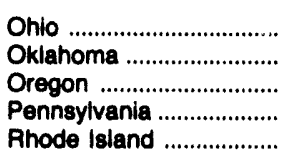 & $\begin{array}{l}5.60 \\
4.91 \\
6.22 \\
6.71 \\
7.89\end{array}$ & $\begin{array}{l}5.02 \\
4.94 \\
6.16 \\
6.63 \\
7.58\end{array}$ & $\begin{array}{l}5.34 \\
4.67 \\
6.09 \\
6.79 \\
7.54\end{array}$ & $\begin{array}{r}7.79 \\
7.51 \\
8.02 \\
9.54 \\
10.09\end{array}$ & $\begin{array}{r}7.39 \\
7.15 \\
7.78 \\
9.47 \\
9.72\end{array}$ & $\begin{array}{l}6.50 \\
6.55 \\
7.44 \\
8.20 \\
8.47\end{array}$ & $\begin{array}{l}5.97 \\
5.64 \\
6.55 \\
7.64 \\
8.49\end{array}$ & $\begin{array}{l}5.49 \\
4.76 \\
6.14 \\
6.69 \\
7.85\end{array}$ \\
\hline 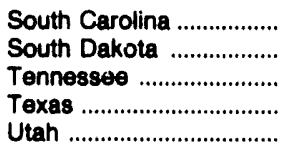 & $\begin{array}{l}7.28 \\
5.37 \\
5.68 \\
5.83 \\
5.13\end{array}$ & $\begin{array}{l}6.99 \\
4.92 \\
5.40 \\
5.71 \\
5.52\end{array}$ & $\begin{array}{l}7.00 \\
4.85 \\
5.14 \\
5.72 \\
5.53\end{array}$ & $\begin{array}{l}9.15 \\
7.09 \\
7.60 \\
7.78 \\
5.84\end{array}$ & $\begin{array}{l}8.93 \\
6.65 \\
7.38 \\
7.54 \\
5.60\end{array}$ & $\begin{array}{l}8.47 \\
6.13 \\
7.01 \\
7.20 \\
5.40\end{array}$ & $\begin{array}{l}7.34 \\
5.89 \\
6.23 \\
6.76 \\
4.82\end{array}$ & $\begin{array}{l}6.56 \\
5.38 \\
5.47 \\
5.78 \\
4.89\end{array}$ \\
\hline 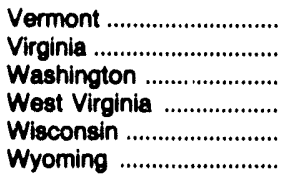 & $\begin{array}{l}6.06 \\
7.60 \\
4.90 \\
6.27 \\
6.22 \\
4.58\end{array}$ & $\begin{array}{l}6.68 \\
6.35 \\
5.01 \\
6.06 \\
5.67 \\
4.73\end{array}$ & $\begin{array}{l}6.11 \\
6.79 \\
4.62 \\
6.55 \\
5.61 \\
4.70\end{array}$ & $\begin{array}{r}7.81 \\
11.04 \\
7.24 \\
8.75 \\
7.73 \\
5.96\end{array}$ & $\begin{array}{r}7.60 \\
11.17 \\
6.76 \\
9.35 \\
7.61 \\
5.58\end{array}$ & $\begin{array}{r}6.91 \\
10.71 \\
3.75 \\
7.63 \\
7.29 \\
4.91\end{array}$ & $\begin{array}{l}6.46 \\
9.30 \\
5.37 \\
6.88 \\
7.36 \\
4.69\end{array}$ & $\begin{array}{l}6.08 \\
7.49 \\
5.03 \\
6.18 \\
6.15 \\
4.54\end{array}$ \\
\hline 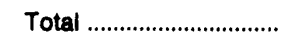 & 6.05 & 5.80 & 5.86 & 8.10 & 7.83 & 7.32 & 6.72 & 5.99 \\
\hline
\end{tabular}

See footnotes at end of table. 
Table 24. Average Price of Natural Gas Delivered to Residential Consumers by State, 1992-1993 (Continued)

(Dollars per Thousand Cubic Feet)

\begin{tabular}{|c|c|c|c|c|c|c|c|c|}
\hline State & $\begin{array}{c}\text { March } \\
1893\end{array}$ & $\begin{array}{c}\text { February } \\
1993\end{array}$ & $\begin{array}{c}\text { January } \\
1893\end{array}$ & $\begin{array}{l}\text { Total } \\
1992\end{array}$ & $\begin{array}{c}\text { December } \\
1992\end{array}$ & $\begin{array}{c}\text { November } \\
1992\end{array}$ & $\begin{array}{c}\text { October } \\
1992\end{array}$ & $\begin{array}{c}\text { September } \\
1992\end{array}$ \\
\hline 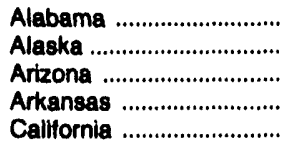 & $\begin{array}{l}6.15 \\
3.90 \\
6.68 \\
5.04 \\
5.87\end{array}$ & $\begin{array}{l}6.49 \\
3.82 \\
6.50 \\
5.02 \\
6.02\end{array}$ & $\begin{array}{l}6.53 \\
3.83 \\
6.36 \\
4.77 \\
6.20\end{array}$ & $\begin{array}{l}6.74 \\
3.79 \\
7.24 \\
5.10 \\
5.97\end{array}$ & $\begin{array}{l}6.42 \\
3.70 \\
6.55 \\
4.73 \\
5.80\end{array}$ & $\begin{array}{l}7.36 \\
3.74 \\
7.59 \\
5.30 \\
5.60\end{array}$ & $\begin{array}{l}8.36 \\
3.79 \\
9.19 \\
6.39 \\
6.35\end{array}$ & $\begin{array}{l}8.94 \\
4.02 \\
9.64 \\
6.86 \\
6.29\end{array}$ \\
\hline 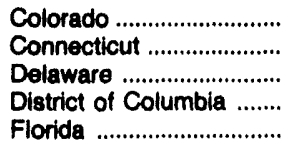 & $\begin{array}{l}4.21 \\
9.03 \\
6.01 \\
6.22 \\
9.27\end{array}$ & $\begin{array}{l}4.17 \\
8.79 \\
6.05 \\
8.33 \\
9.23\end{array}$ & $\begin{array}{l}4.13 \\
8.99 \\
6.09 \\
8.45 \\
9.07\end{array}$ & $\begin{array}{l}4.56 \\
8.96 \\
6.13 \\
7.61 \\
9.08\end{array}$ & $\begin{array}{l}4.17 \\
7.68 \\
6.22 \\
8.30 \\
8.63\end{array}$ & $\begin{array}{r}4.50 \\
7.84 \\
6.44 \\
8.58 \\
10.20\end{array}$ & $\begin{array}{r}5.37 \\
8.41 \\
7.20 \\
9.13 \\
11.36\end{array}$ & $\begin{array}{r}5.89 \\
10.66 \\
8.05 \\
9.18 \\
11.48\end{array}$ \\
\hline 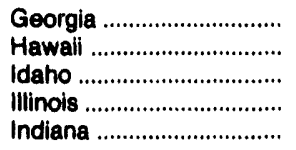 & $\begin{array}{r}6.13 \\
17.20 \\
5.02 \\
4.88 \\
5.36\end{array}$ & $\begin{array}{r}6.18 \\
16.50 \\
4.98 \\
5.02 \\
5.43\end{array}$ & $\begin{array}{r}6.51 \\
17.62 \\
4.93 \\
5.08 \\
5.58\end{array}$ & $\begin{array}{r}6.44 \\
18.03 \\
5.23 \\
5.09 \\
5.43\end{array}$ & $\begin{array}{r}6.11 \\
18.05 \\
4.89 \\
5.14 \\
5.54\end{array}$ & $\begin{array}{r}6.33 \\
18.42 \\
5.24 \\
5.42 \\
5.17\end{array}$ & $\begin{array}{r}7.64 \\
18.60 \\
5.62 \\
5.60 \\
5.44\end{array}$ & $\begin{array}{r}3.87 \\
18.46 \\
6.00 \\
6.37 \\
6.85\end{array}$ \\
\hline 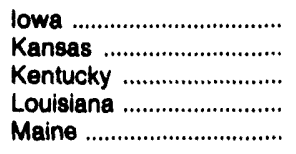 & $\begin{array}{l}5.27 \\
4.43 \\
4.83 \\
5.30 \\
7.11\end{array}$ & $\begin{array}{l}4.94 \\
4.41 \\
4.72 \\
4.71 \\
6.89\end{array}$ & $\begin{array}{l}5.34 \\
4.37 \\
5.08 \\
5.44 \\
6.75\end{array}$ & $\begin{array}{l}5.23 \\
4.70 \\
5.01 \\
5.60 \\
6.95\end{array}$ & $\begin{array}{l}5.29 \\
4.45 \\
5.04 \\
5.73 \\
7.12\end{array}$ & $\begin{array}{l}5.62 \\
4.71 \\
5.14 \\
6.61 \\
6.89\end{array}$ & $\begin{array}{l}6.90 \\
5.32 \\
6.34 \\
7.87 \\
6.77\end{array}$ & $\begin{array}{l}7.66 \\
5.81 \\
6.96 \\
7.34 \\
7.12\end{array}$ \\
\hline 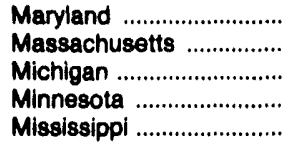 & $\begin{array}{l}6.43 \\
8.33 \\
4.46 \\
5.03 \\
4.36\end{array}$ & $\begin{array}{l}6.66 \\
8.28 \\
4.42 \\
4.95 \\
4.44\end{array}$ & $\begin{array}{l}6.88 \\
8.27 \\
4.43 \\
5.13 \\
4.80\end{array}$ & $\begin{array}{l}6.43 \\
7.92 \\
5.06 \\
4.86 \\
4.95\end{array}$ & $\begin{array}{l}6.99 \\
8.55 \\
4.73 \\
5.19 \\
5.05\end{array}$ & $\begin{array}{l}6.81 \\
8.61 \\
4.98 \\
5.43 \\
5.70\end{array}$ & $\begin{array}{l}7.43 \\
6.74 \\
5.39 \\
6.04 \\
6.25\end{array}$ & $\begin{array}{l}8.80 \\
7.70 \\
6.45 \\
5.93 \\
5.75\end{array}$ \\
\hline 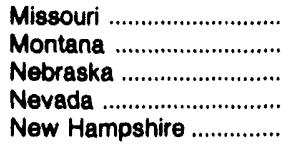 & $\begin{array}{l}4.62 \\
4.68 \\
4.51 \\
5.23 \\
7.66\end{array}$ & $\begin{array}{l}4.67 \\
4.73 \\
4.55 \\
5.06 \\
7.48\end{array}$ & $\begin{array}{l}4.72 \\
4.55 \\
4.72 \\
4.96 \\
7.86\end{array}$ & $\begin{array}{l}5.11 \\
4.80 \\
4.82 \\
5.59 \\
7.55\end{array}$ & $\begin{array}{l}4.93 \\
4.61 \\
4.90 \\
5.03 \\
8.00\end{array}$ & $\begin{array}{l}5.57 \\
4.77 \\
5.15 \\
5.68 \\
9.10\end{array}$ & $\begin{array}{l}6.59 \\
5.14 \\
5.78 \\
6.76 \\
8.09\end{array}$ & $\begin{array}{l}7.23 \\
5.45 \\
6.16 \\
7.06 \\
8.97\end{array}$ \\
\hline $\begin{array}{l}\text { New Jersey .................... } \\
\text { New Mexico .................... } \\
\text { New York ......................... } \\
\text { North Carolina ................ } \\
\text { North Dakota .................. }\end{array}$ & $\begin{array}{l}6.33 \\
4.90 \\
7.24 \\
6.08 \\
4.84\end{array}$ & $\begin{array}{l}6.34 \\
4.80 \\
7.30 \\
6.58 \\
4.82\end{array}$ & $\begin{array}{l}6.49 \\
4.50 \\
7.67 \\
6.76 \\
4.71\end{array}$ & $\begin{array}{l}6.94 \\
4.75 \\
7.58 \\
6.60 \\
5.00\end{array}$ & $\begin{array}{l}6.80 \\
4.58 \\
7.74 \\
6.66 \\
4.85\end{array}$ & $\begin{array}{l}6.99 \\
5.17 \\
8.68 \\
7.12 \\
4.98\end{array}$ & $\begin{array}{l}7.23 \\
6.44 \\
8.62 \\
7.72 \\
5.64\end{array}$ & $\begin{array}{r}8.50 \\
6.81 \\
9.92 \\
10.03 \\
6.26\end{array}$ \\
\hline 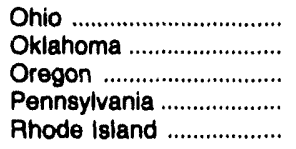 & $\begin{array}{l}5.31 \\
4.52 \\
5.99 \\
6.35 \\
7.67\end{array}$ & $\begin{array}{l}5.43 \\
4.58 \\
5.95 \\
6.34 \\
7.64\end{array}$ & $\begin{array}{l}5.33 \\
4.57 \\
5.90 \\
6.18 \\
7.69\end{array}$ & $\begin{array}{l}5.20 \\
4.96 \\
6.17 \\
6.60 \\
7.68\end{array}$ & $\begin{array}{l}5.38 \\
4.57 \\
5.92 \\
6.18 \\
7.62\end{array}$ & $\begin{array}{l}5.52 \\
4.82 \\
6.16 \\
6.36 \\
7.85\end{array}$ & $\begin{array}{l}5.76 \\
6.14 \\
6.66 \\
6.87 \\
8.28\end{array}$ & $\begin{array}{l}6.51 \\
6.71 \\
7.27 \\
8.34 \\
9.00\end{array}$ \\
\hline 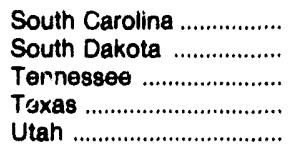 & $\begin{array}{l}6.78 \\
5.15 \\
5.20 \\
5.32 \\
5.15\end{array}$ & $\begin{array}{l}7.31 \\
5.12 \\
5.83 \\
5.52 \\
5.08\end{array}$ & $\begin{array}{l}7.69 \\
5.13 \\
5.45 \\
5.26 \\
5.14\end{array}$ & $\begin{array}{l}7.03 \\
5.15 \\
5.50 \\
5.78 \\
5.44\end{array}$ & $\begin{array}{l}6.86 \\
5.30 \\
5.34 \\
5.14 \\
5.21\end{array}$ & $\begin{array}{l}7.39 \\
5.37 \\
5.93 \\
6.02 \\
5.15\end{array}$ & $\begin{array}{l}7.17 \\
6.09 \\
6.29 \\
7.32 \\
5.35\end{array}$ & $\begin{array}{l}8.45 \\
6.36 \\
7.02 \\
7.48 \\
5.87\end{array}$ \\
\hline 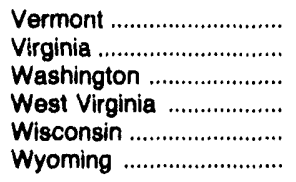 & $\begin{array}{l}5.94 \\
6.79 \\
4.80 \\
5.97 \\
5.96 \\
4.44\end{array}$ & $\begin{array}{l}5.96 \\
7.18 \\
4.76 \\
5.93 \\
5.94 \\
4.43\end{array}$ & $\begin{array}{l}5.59 \\
7.14 \\
4.59 \\
6.00 \\
5.91 \\
4.36\end{array}$ & $\begin{array}{l}6.70 \\
6.69 \\
5.00 \\
6.31 \\
5.87 \\
4.72\end{array}$ & $\begin{array}{l}6.36 \\
7.10 \\
4.60 \\
7.60 \\
5.98 \\
4.41\end{array}$ & $\begin{array}{l}6.73 \\
7.05 \\
4.94 \\
6.12 \\
6.27 \\
4.64\end{array}$ & $\begin{array}{l}7.14 \\
7.93 \\
5.57 \\
6.48 \\
6.68 \\
5.09\end{array}$ & $\begin{array}{l}8.02 \\
9.83 \\
6.35 \\
7.83 \\
6.89 \\
5.57\end{array}$ \\
\hline 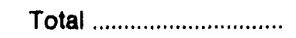 & 5.66 & 5.71 & 5.71 & 5.89 & 5.74 & 6.02 & 6.52 & 7.15 \\
\hline
\end{tabular}

See footnotes at end of table. 
Table 24. Average Price of Natural Gas Delivered to Residential Consumers by State, 1992-1993 (Continued) (Dollars per Thousand Cubic Feet)

\begin{tabular}{|c|c|c|c|c|c|c|c|c|}
\hline State & $\begin{array}{c}\text { August } \\
1992\end{array}$ & $\begin{array}{c}\text { July } \\
1992\end{array}$ & $\begin{array}{l}\text { June } \\
1992\end{array}$ & $\begin{array}{c}\text { May } \\
1992\end{array}$ & $\begin{array}{l}\text { April } \\
1992\end{array}$ & $\begin{array}{c}\text { March } \\
1992\end{array}$ & $\begin{array}{c}\text { February } \\
1992\end{array}$ & $\begin{array}{c}\text { January } \\
1992\end{array}$ \\
\hline 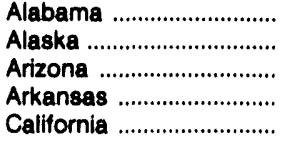 & $\begin{array}{l}9.06 \\
4.54 \\
9.87 \\
6.83 \\
6.21\end{array}$ & $\begin{array}{l}8.93 \\
4.20 \\
9.54 \\
6.46 \\
6.27\end{array}$ & $\begin{array}{l}8.00 \\
4.03 \\
9.02 \\
6.16 \\
6.28\end{array}$ & $\begin{array}{l}6.98 \\
3.83 \\
8.53 \\
5.53 \\
5.99\end{array}$ & $\begin{array}{l}6.24 \\
3.75 \\
7.43 \\
4.97 \\
5.52\end{array}$ & $\begin{array}{l}6.67 \\
3.69 \\
6.84 \\
4.87 \\
5.76\end{array}$ & $\begin{array}{l}6.01 \\
3.68 \\
6.48 \\
4.60 \\
6.03\end{array}$ & $\begin{array}{l}6.19 \\
3.69 \\
6.40 \\
4.64 \\
6.15\end{array}$ \\
\hline 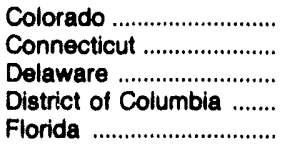 & $\begin{array}{r}6.06 \\
11.20 \\
8.08 \\
7.36 \\
11.26\end{array}$ & $\begin{array}{r}5.78 \\
10.67 \\
7.94 \\
6.77 \\
10.99\end{array}$ & $\begin{array}{r}5.25 \\
10.54 \\
7.39 \\
7.22 \\
10.69\end{array}$ & $\begin{array}{l}4.96 \\
9.84 \\
6.51 \\
7.95 \\
9.80\end{array}$ & $\begin{array}{l}4.52 \\
9.05 \\
5.98 \\
7.08 \\
8.66\end{array}$ & $\begin{array}{l}4.38 \\
8.89 \\
5.80 \\
7.23 \\
8.48\end{array}$ & $\begin{array}{l}4.30 \\
9.18 \\
5.52 \\
7.07 \\
7.54\end{array}$ & $\begin{array}{l}4.25 \\
9.14 \\
5.50 \\
7.31 \\
7.79\end{array}$ \\
\hline 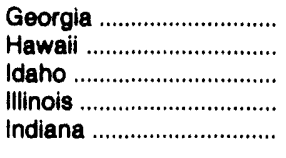 & $\begin{array}{r}6.38 \\
18.52 \\
6.61 \\
6.87 \\
6.97\end{array}$ & $\begin{array}{r}8.30 \\
18.19 \\
6.22 \\
6.91 \\
6.87\end{array}$ & $\begin{array}{r}7.88 \\
17.71 \\
6.17 \\
6.45 \\
6.42\end{array}$ & $\begin{array}{r}6.96 \\
18.04 \\
5.56 \\
5.52 \\
5.93\end{array}$ & $\begin{array}{r}6.29 \\
17.07 \\
5.42 \\
4.68 \\
5.30\end{array}$ & $\begin{array}{r}6.25 \\
17.48 \\
5.30 \\
4.33 \\
4.79\end{array}$ & $\begin{array}{r}6.51 \\
17.92 \\
5.05 \\
4.76 \\
5.40\end{array}$ & $\begin{array}{r}6.08 \\
18.12 \\
4.95 \\
4.66 \\
5.11\end{array}$ \\
\hline 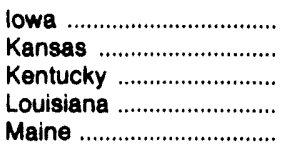 & $\begin{array}{l}7.89 \\
5.92 \\
6.42 \\
7.41 \\
7.65\end{array}$ & $\begin{array}{l}7.56 \\
5.95 \\
6.25 \\
7.06 \\
7.51\end{array}$ & $\begin{array}{l}6.71 \\
5.40 \\
5.79 \\
6.78 \\
6.76\end{array}$ & $\begin{array}{l}5.67 \\
5.11 \\
4.98 \\
6.27 \\
6.78\end{array}$ & $\begin{array}{l}4.68 \\
4.59 \\
4.69 \\
4.85 \\
7.08\end{array}$ & $\begin{array}{l}4.44 \\
4.57 \\
4.54 \\
5.09 \\
6.90\end{array}$ & $\begin{array}{l}4.31 \\
4.53 \\
4.46 \\
4.43 \\
6.79\end{array}$ & $\begin{array}{l}4.73 \\
4.33 \\
4.67 \\
4.80 \\
6.87\end{array}$ \\
\hline 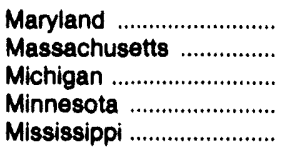 & $\begin{array}{l}8.51 \\
7.79 \\
6.71 \\
5.98 \\
5.84\end{array}$ & $\begin{array}{l}8.17 \\
7.61 \\
6.58 \\
5.56 \\
5.64\end{array}$ & $\begin{array}{l}7.58 \\
6.95 \\
6.02 \\
5.51 \\
5.51\end{array}$ & $\begin{array}{l}6.26 \\
6.12 \\
5.28 \\
4.85 \\
5.45\end{array}$ & $\begin{array}{l}5.40 \\
8.32 \\
4.89 \\
4.34 \\
4.62\end{array}$ & $\begin{array}{l}5.66 \\
8.05 \\
4.83 \\
4.17 \\
4.52\end{array}$ & $\begin{array}{l}5.89 \\
8.04 \\
4.82 \\
4.15 \\
4.46\end{array}$ & $\begin{array}{l}5.91 \\
8.11 \\
4.80 \\
4.58 \\
4.54\end{array}$ \\
\hline 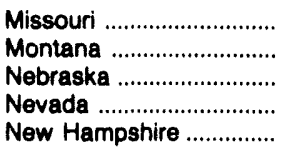 & $\begin{array}{l}7.40 \\
6.02 \\
6.24 \\
7.37 \\
9.16\end{array}$ & $\begin{array}{l}6.99 \\
5.78 \\
5.90 \\
6.89 \\
9.09\end{array}$ & $\begin{array}{l}6.24 \\
5.57 \\
5.47 \\
6.81 \\
8.58\end{array}$ & $\begin{array}{l}5.32 \\
5.06 \\
4.91 \\
6.41 \\
7.83\end{array}$ & $\begin{array}{l}4.60 \\
4.73 \\
4.41 \\
5.68 \\
6.94\end{array}$ & $\begin{array}{l}4.60 \\
4.66 \\
4.26 \\
5.29 \\
6.98\end{array}$ & $\begin{array}{l}4.61 \\
4.53 \\
4.28 \\
5.18 \\
6.87\end{array}$ & $\begin{array}{l}4.65 \\
4.47 \\
4.57 \\
5.10 \\
6.90\end{array}$ \\
\hline 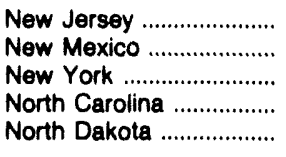 & $\begin{array}{r}8.77 \\
6.88 \\
10.28 \\
10.51 \\
6.75\end{array}$ & $\begin{array}{l}8.68 \\
6.63 \\
8.70 \\
8.89 \\
6.33\end{array}$ & $\begin{array}{l}8.41 \\
6.04 \\
8.70 \\
8.24 \\
6.04\end{array}$ & $\begin{array}{l}7.46 \\
5.35 \\
7.40 \\
6.74 \\
5.21\end{array}$ & $\begin{array}{l}6.71 \\
4.50 \\
6.79 \\
5.54 \\
4.78\end{array}$ & $\begin{array}{l}6.04 \\
4.27 \\
6.89 \\
6.03 \\
4.69\end{array}$ & $\begin{array}{l}6.75 \\
4.17 \\
6.83 \\
5.91 \\
4.62\end{array}$ & $\begin{array}{l}6.73 \\
4.10 \\
7.10 \\
6.30 \\
4.67\end{array}$ \\
\hline 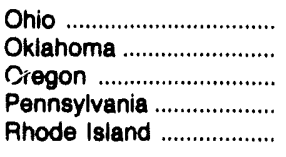 & $\begin{array}{l}6.88 \\
6.84 \\
7.64 \\
8.98 \\
9.53\end{array}$ & $\begin{array}{l}6.27 \\
6.59 \\
7.32 \\
8.94 \\
8.71\end{array}$ & $\begin{array}{l}5.92 \\
6.29 \\
6.96 \\
8.14 \\
8.45\end{array}$ & $\begin{array}{l}5.27 \\
5.87 \\
6.48 \\
7.20 \\
7.77\end{array}$ & $\begin{array}{l}4.83 \\
4.96 \\
6.21 \\
6.55 \\
7.45\end{array}$ & $\begin{array}{l}4.80 \\
4.79 \\
6.03 \\
6.30 \\
7.36\end{array}$ & $\begin{array}{l}4.66 \\
4.43 \\
5.92 \\
6.34 \\
7.33\end{array}$ & $\begin{array}{l}4.96 \\
4.42 \\
5.82 \\
6.14 \\
7.32\end{array}$ \\
\hline 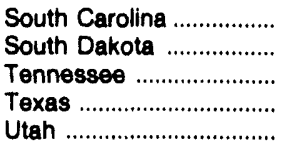 & $\begin{array}{l}8.61 \\
6.67 \\
7.14 \\
7.62 \\
5.85\end{array}$ & $\begin{array}{l}8.37 \\
6.31 \\
6.81 \\
7.42 \\
5.86\end{array}$ & $\begin{array}{l}7.67 \\
6.04 \\
6.38 \\
7.16 \\
5.80\end{array}$ & $\begin{array}{l}6.68 \\
4.64 \\
5.72 \\
6.92 \\
5.48\end{array}$ & $\begin{array}{l}6.27 \\
4.78 \\
5.19 \\
6.06 \\
5.50\end{array}$ & $\begin{array}{l}7.44 \\
4.60 \\
5.47 \\
5.52 \\
5.64\end{array}$ & $\begin{array}{l}6.88 \\
4.59 \\
5.22 \\
4.97 \\
5.43\end{array}$ & $\begin{array}{l}6.88 \\
4.94 \\
5.06 \\
4.97 \\
5.38\end{array}$ \\
\hline 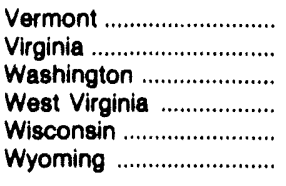 & $\begin{array}{l}8.42 \\
9.16 \\
7.05 \\
8.41 \\
6.80 \\
5.96\end{array}$ & $\begin{array}{l}8.22 \\
8.97 \\
7.17 \\
8.32 \\
6.50 \\
5.69\end{array}$ & $\begin{array}{l}7.78 \\
7.84 \\
6.24 \\
7.23 \\
6.40 \\
5.28\end{array}$ & $\begin{array}{l}7.05 \\
6.61 \\
5.46 \\
6.39 \\
5.67 \\
4.96\end{array}$ & $\begin{array}{l}6.68 \\
5.62 \\
5.00 \\
5.93 \\
5.44 \\
4.64\end{array}$ & $\begin{array}{l}6.59 \\
5.68 \\
4.81 \\
5.87 \\
5.46 \\
4.55\end{array}$ & $\begin{array}{l}6.54 \\
6.25 \\
4.67 \\
5.75 \\
5.43 \\
4.51\end{array}$ & $\begin{array}{l}6.22 \\
6.28 \\
4.52 \\
5.76 \\
5.75 \\
4.52\end{array}$ \\
\hline 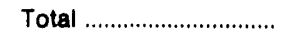 & 7.45 & 7.27 & 6.84 & 6.15 & 5.62 & 5.50 & 5.54 & 5.53 \\
\hline
\end{tabular}

A = Revised Data.

Notes: Data for 1992 are final. All other data are preliminary unless otherwise indicated. Geographic coverage is the 50 States and the District of Columbia. See Appendix A, Explanatory Note 5 for discussion of computations and revision policy. Source: Form ElA-857. 
Table 25. Average Price of Natural Gas Sold to Commercial Consumers by State, 1992-1993 (Dollars per Thousand Cubic Feet)

\begin{tabular}{|c|c|c|c|c|c|c|c|c|}
\hline State & $\begin{array}{l}\text { YTD } \\
1993\end{array}$ & $\begin{array}{l}\text { YTD } \\
1992\end{array}$ & $\begin{array}{l}\text { YTD } \\
1991\end{array}$ & $\begin{array}{c}\text { August } \\
1993\end{array}$ & $\begin{array}{l}\text { July } \\
1993\end{array}$ & $\begin{array}{l}\text { June } \\
1993\end{array}$ & $\begin{array}{l}\text { May } \\
1993\end{array}$ & $\begin{array}{l}\text { April } \\
1993\end{array}$ \\
\hline 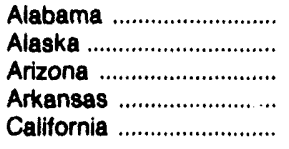 & $\begin{array}{l}6.03 \\
2.79 \\
4.99 \\
4.37 \\
5.76\end{array}$ & $\begin{array}{l}5.58 \\
2.64 \\
5.23 \\
4.35 \\
5.18\end{array}$ & $\begin{array}{l}5.82 \\
2.99 \\
5.03 \\
4.32 \\
5.66\end{array}$ & $\begin{array}{l}6.75 \\
2.54 \\
5.00 \\
4.68 \\
5.95\end{array}$ & $\begin{array}{l}6.86 \\
2.61 \\
4.86 \\
4.77 \\
4.83\end{array}$ & $\begin{array}{l}6.74 \\
2.67 \\
4.82 \\
4.96 \\
5.83\end{array}$ & $\begin{array}{l}6.33 \\
2.73 \\
5.03 \\
4.41 \\
5.24\end{array}$ & $\begin{array}{l}5.83 \\
2.83 \\
5.03 \\
4.35 \\
5.50\end{array}$ \\
\hline 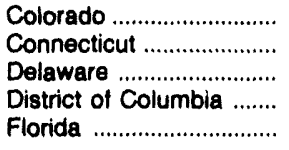 & $\begin{array}{l}3.84 \\
7.29 \\
5.31 \\
5.85 \\
5.78\end{array}$ & $\begin{array}{l}4.03 \\
7.38 \\
4.86 \\
5.24 \\
4.84\end{array}$ & $\begin{array}{l}4.03 \\
6.83 \\
4.85 \\
5.18 \\
4.87\end{array}$ & $\begin{array}{l}4.04 \\
7.58 \\
5.64 \\
5.56 \\
5.52\end{array}$ & $\begin{array}{l}4.08 \\
7.31 \\
5.64 \\
5.47 \\
5.82\end{array}$ & $\begin{array}{l}3.95 \\
6.97 \\
7.75 \\
5.59 \\
598\end{array}$ & $\begin{array}{l}3.87 \\
7.19 \\
5.46 \\
5.60 \\
6.01\end{array}$ & $\begin{array}{l}3.82 \\
7.36 \\
5.18 \\
5.72 \\
5.87\end{array}$ \\
\hline 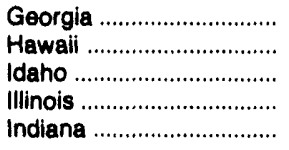 & $\begin{array}{r}5.74 \\
13.00 \\
4.46 \\
5.08 \\
4.98\end{array}$ & $\begin{array}{r}5.62 \\
13.17 \\
4.42 \\
4.48 \\
4.55\end{array}$ & $\begin{array}{r}5.75 \\
13.50 \\
4.39 \\
4.73 \\
4.82\end{array}$ & $\begin{array}{r}6.12 \\
13.08 \\
5.16 \\
6.55 \\
5.80\end{array}$ & $\begin{array}{r}5.78 \\
13.23 \\
4.93 \\
6.01 \\
6.33\end{array}$ & $\begin{array}{r}6.13 \\
12.92 \\
4.65 \\
6.80 \\
5.99\end{array}$ & $\begin{array}{r}6.14 \\
13.27 \\
4.65 \\
6.01 \\
5.29\end{array}$ & $\begin{array}{r}5.60 \\
13.18 \\
4.60 \\
5.26 \\
4.99\end{array}$ \\
\hline 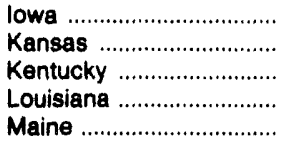 & $\begin{array}{l}4.74 \\
4.00 \\
4.70 \\
5.09 \\
6.58\end{array}$ & $\begin{array}{l}4.00 \\
3.53 \\
4.33 \\
4.53 \\
6.23\end{array}$ & $\begin{array}{l}3.88 \\
3.29 \\
4.50 \\
4.83 \\
5.98\end{array}$ & $\begin{array}{l}5.33 \\
3.44 \\
5.11 \\
5.66 \\
6.42\end{array}$ & $\begin{array}{l}5.66 \\
3.41 \\
5.27 \\
5.39 \\
6.96\end{array}$ & $\begin{array}{l}5.07 \\
4.14 \\
3.98 \\
5.46 \\
6.84\end{array}$ & $\begin{array}{l}5.48 \\
4.24 \\
5.00 \\
5.43 \\
6.81\end{array}$ & $\begin{array}{l}5.03 \\
4.12 \\
4.62 \\
5.05 \\
6.77\end{array}$ \\
\hline 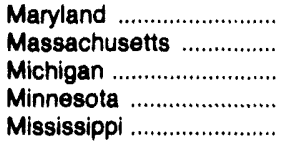 & $\begin{array}{l}5.79 \\
6.49 \\
4.44 \\
4.57 \\
4.07\end{array}$ & $\begin{array}{l}4.99 \\
5.85 \\
4.64 \\
3.79 \\
3.98\end{array}$ & $\begin{array}{l}5.10 \\
6.13 \\
4.72 \\
3.71 \\
4.38\end{array}$ & $\begin{array}{l}6.07 \\
4.45 \\
5.75 \\
5.20 \\
3.95\end{array}$ & $\begin{array}{l}6.13 \\
4.36 \\
5.57 \\
4.56 \\
3.94\end{array}$ & $\begin{array}{l}5.99 \\
4.58 \\
5.29 \\
4.71 \\
4.00\end{array}$ & $\begin{array}{l}5.91 \\
4.72 \\
4.77 \\
5.11 \\
4.54\end{array}$ & $\begin{array}{l}5.68 \\
7.02 \\
4.35 \\
4.70 \\
4.06\end{array}$ \\
\hline 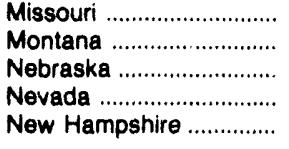 & $\begin{array}{l}4.62 \\
4.69 \\
4.27 \\
4.31 \\
6.62\end{array}$ & $\begin{array}{l}4.36 \\
4.45 \\
3.85 \\
4.33 \\
6.56\end{array}$ & $\begin{array}{l}4.54 \\
4.32 \\
3.83 \\
4.33 \\
6.35\end{array}$ & $\begin{array}{l}5.16 \\
5.14 \\
4.19 \\
4.42 \\
6.20\end{array}$ & $\begin{array}{l}5.18 \\
5.07 \\
4.55 \\
4.42 \\
6.21\end{array}$ & $\begin{array}{l}5.30 \\
5.12 \\
4.13 \\
4.41 \\
6.04\end{array}$ & $\begin{array}{l}4.73 \\
4.79 \\
4.58 \\
4.15 \\
5.78\end{array}$ & $\begin{array}{l}4.48 \\
4.65 \\
4.24 \\
4.30 \\
5.61\end{array}$ \\
\hline 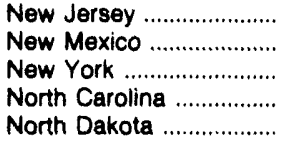 & $\begin{array}{l}5.39 \\
4.18 \\
6.09 \\
5.48 \\
4.72\end{array}$ & $\begin{array}{l}5.46 \\
3.34 \\
5.59 \\
4.59 \\
4.43\end{array}$ & $\begin{array}{l}5.38 \\
4.23 \\
5.48 \\
4.48 \\
4.24\end{array}$ & $\begin{array}{l}5.44 \\
4.34 \\
5.89 \\
5.68 \\
5.61\end{array}$ & $\begin{array}{l}4.95 \\
4.42 \\
6.11 \\
5.79 \\
5.45\end{array}$ & $\begin{array}{l}5.16 \\
4.79 \\
6.63 \\
5.77 \\
5.37\end{array}$ & $\begin{array}{l}5.29 \\
4.52 \\
7.12 \\
5.44 \\
5.04\end{array}$ & $\begin{array}{l}5.25 \\
4.38 \\
6.27 \\
5.15 \\
4.75\end{array}$ \\
\hline 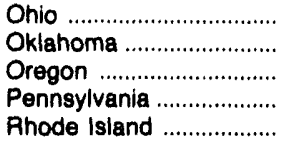 & $\begin{array}{l}5.15 \\
4.53 \\
4.90 \\
5.94 \\
7.07\end{array}$ & $\begin{array}{l}4.53 \\
4.19 \\
4.73 \\
5.96 \\
6.31\end{array}$ & $\begin{array}{l}4.83 \\
3.91 \\
4.75 \\
6.08 \\
6.06\end{array}$ & $\begin{array}{l}5.96 \\
4.48 \\
5.53 \\
6.88 \\
6.64\end{array}$ & $\begin{array}{r}5.65 \\
4.76 \\
5.43 \\
R \quad 6.89 \\
7.26\end{array}$ & $\begin{array}{l}5.89 \\
4.66 \\
5.29 \\
6.52 \\
7.55\end{array}$ & $\begin{array}{l}5.10 \\
4.44 \\
4.97 \\
6.43 \\
7.48\end{array}$ & $\begin{array}{l}5.13 \\
4.51 \\
4.77 \\
6.07 \\
7.01\end{array}$ \\
\hline $\begin{array}{l}\text { South Carolina } \\
\text { South Dakota } \\
\text { Tennessee } \ldots \ldots \ldots \ldots \ldots \ldots \ldots \ldots \\
\text { Texas } \\
\text { Utah }\end{array}$ & $\begin{array}{l}5.83 \\
4.49 \\
5.14 \\
4.25 \\
4.16\end{array}$ & $\begin{array}{l}5.57 \\
4.00 \\
4.96 \\
3.97 \\
4.48\end{array}$ & $\begin{array}{l}5.65 \\
3.98 \\
4.75 \\
3.93 \\
4.51\end{array}$ & $\begin{array}{l}5.43 \\
4.82 \\
5.30 \\
3.82 \\
4.32\end{array}$ & $\begin{array}{l}5.41 \\
4.72 \\
5.30 \\
3.79 \\
4.19\end{array}$ & $\begin{array}{l}5.46 \\
4.61 \\
5.50 \\
3.85 \\
4.18\end{array}$ & $\begin{array}{l}5.49 \\
5.21 \\
5.12 \\
4.07 \\
3.82\end{array}$ & $\begin{array}{l}5.67 \\
4.52 \\
4.95 \\
4.12 \\
3.96\end{array}$ \\
\hline 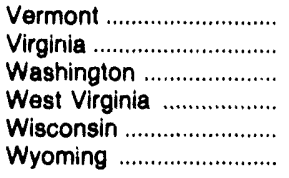 & $\begin{array}{l}5.35 \\
5.62 \\
4.43 \\
5.90 \\
4.88 \\
4.18\end{array}$ & $\begin{array}{l}5.72 \\
4.75 \\
4.37 \\
5.45 \\
4.62 \\
4.25\end{array}$ & $\begin{array}{l}5.28 \\
4.88 \\
4.03 \\
6.18 \\
4.66 \\
4.28\end{array}$ & $\begin{array}{l}5.68 \\
6.14 \\
5.00 \\
7.25 \\
5.34 \\
4.28\end{array}$ & $\begin{array}{l}5.75 \\
5.95 \\
4.80 \\
7.52 \\
5.20 \\
4.33\end{array}$ & $\begin{array}{l}5.73 \\
6.01 \\
4.66 \\
7.01 \\
5.20 \\
4.51\end{array}$ & $\begin{array}{l}5.54 \\
5.61 \\
4.52 \\
6.41 \\
5.53 \\
4.18\end{array}$ & $\begin{array}{l}5.32 \\
5.62 \\
4.34 \\
5.84 \\
4.93 \\
4.15\end{array}$ \\
\hline Total & 5.13 & 4.81 & 4.85 & 5.26 & 5.03 & 5.31 & 5.21 & 5.13 \\
\hline
\end{tabular}


Table 25. Average Price of Natural Gas Sold to Commercial Consumers by State, 1992-1993 (Continued) (Dollars per Thousand Cubic Feet)

\begin{tabular}{|c|c|c|c|c|c|c|c|c|}
\hline State & $\begin{array}{c}\text { March } \\
1993\end{array}$ & $\begin{array}{c}\text { Fobruary } \\
1993\end{array}$ & $\begin{array}{c}\text { January } \\
1993\end{array}$ & $\begin{array}{l}\text { Total } \\
1992\end{array}$ & $\begin{array}{c}\text { December } \\
1992\end{array}$ & $\begin{array}{c}\text { November } \\
1992\end{array}$ & $\begin{array}{l}\text { October } \\
1992\end{array}$ & $\begin{array}{c}\text { September } \\
1992\end{array}$ \\
\hline 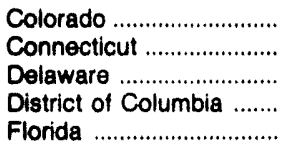 & $\begin{array}{l}3.82 \\
7.35 \\
5.11 \\
6.08 \\
5.94\end{array}$ & $\begin{array}{l}3.81 \\
7.22 \\
5.12 \\
5.99 \\
5.90\end{array}$ & $\begin{array}{l}3.75 \\
7.33 \\
5.17 \\
5.95 \\
5.20\end{array}$ & $\begin{array}{l}4.00 \\
7.20 \\
4.94 \\
5.36 \\
4.98\end{array}$ & $\begin{array}{l}3.84 \\
6.88 \\
5.18 \\
5.85 \\
5.36\end{array}$ & $\begin{array}{l}3.91 \\
6.80 \\
5.07 \\
5.90 \\
5.35\end{array}$ & $\begin{array}{l}4.19 \\
6.90 \\
5.07 \\
5.26 \\
5.30\end{array}$ & $\begin{array}{l}4.32 \\
6.45 \\
5.16 \\
4.99 \\
5.13\end{array}$ \\
\hline 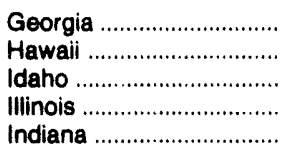 & $\begin{array}{r}5.21 \\
12.79 \\
4.36 \\
4.65 \\
4.62\end{array}$ & $\begin{array}{r}5.79 \\
12.40 \\
4.26 \\
4.83 \\
4.81\end{array}$ & $\begin{array}{r}5.92 \\
13.17 \\
4.33 \\
4.91 \\
4.95\end{array}$ & $\begin{array}{r}5.55 \\
13.34 \\
4.40 \\
4.65 \\
4.57\end{array}$ & $\begin{array}{r}5.62 \\
13.57 \\
4.22 \\
4.88 \\
4.85\end{array}$ & $\begin{array}{r}6.03 \\
13.71 \\
4.50 \\
4.93 \\
4.34\end{array}$ & $\begin{array}{r}5.98 \\
13.83 \\
4.53 \\
4.89 \\
4.21\end{array}$ & $\begin{array}{r}2.76 \\
13.67 \\
4.56 \\
5.28 \\
4.95\end{array}$ \\
\hline 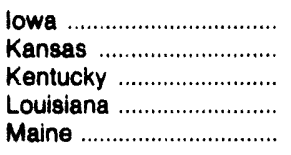 & $\begin{array}{l}4.64 \\
4.11 \\
4.60 \\
4.91 \\
6.77\end{array}$ & $\begin{array}{l}4.23 \\
4.09 \\
4.59 \\
4.55 \\
6.45\end{array}$ & $\begin{array}{l}4.73 \\
4.09 \\
4.91 \\
5.11 \\
6.30\end{array}$ & $\begin{array}{l}4.27 \\
3.53 \\
4.47 \\
4.79 \\
6.19\end{array}$ & $\begin{array}{l}4.58 \\
3.98 \\
4.78 \\
5.39 \\
6.46\end{array}$ & $\begin{array}{l}4.54 \\
3.68 \\
4.77 \\
5.54 \\
6.14\end{array}$ & $\begin{array}{l}5.31 \\
3.08 \\
4.52 \\
5.60 \\
5.38\end{array}$ & $\begin{array}{l}5.02 \\
2.69 \\
4.61 \\
4.91 \\
5.56\end{array}$ \\
\hline 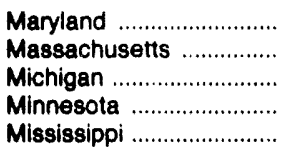 & $\begin{array}{l}5.57 \\
7.20 \\
4.27 \\
4.43 \\
3.93\end{array}$ & $\begin{array}{l}5.77 \\
7.38 \\
4.27 \\
4.32 \\
3.93\end{array}$ & $\begin{array}{l}5.85 \\
7.21 \\
4.32 \\
4.60 \\
4.25\end{array}$ & $\begin{array}{l}5.24 \\
5.86 \\
4.65 \\
4.10 \\
4.13\end{array}$ & $\begin{array}{l}5.89 \\
7.00 \\
4.49 \\
4.59 \\
4.53\end{array}$ & $\begin{array}{l}6.73 \\
6.40 \\
4.63 \\
4.61 \\
4.51\end{array}$ & $\begin{array}{l}5.57 \\
4.29 \\
4.89 \\
4.8 r \\
4.62\end{array}$ & $\begin{array}{l}5.54 \\
4.15 \\
5.40 \\
4.39 \\
4.02\end{array}$ \\
\hline 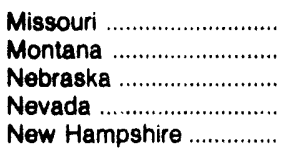 & $\begin{array}{l}4.47 \\
4.61 \\
4.16 \\
4.30 \\
7.14\end{array}$ & $\begin{array}{l}4.54 \\
4.66 \\
4.20 \\
4.31 \\
6.92\end{array}$ & $\begin{array}{l}4.60 \\
4.56 \\
4.34 \\
4.28 \\
6.99\end{array}$ & $\begin{array}{l}4.47 \\
4.46 \\
3.98 \\
4.33 \\
6.74\end{array}$ & $\begin{array}{l}4.68 \\
4.39 \\
4.31 \\
4.26 \\
7.18\end{array}$ & $\begin{array}{l}4.86 \\
4.46 \\
4.27 \\
4.31 \\
7.43\end{array}$ & $\begin{array}{l}4.62 \\
4.58 \\
4.21 \\
4.37 \\
6.75\end{array}$ & $\begin{array}{l}4.53 \\
4.76 \\
3.93 \\
4.42 \\
6.95\end{array}$ \\
\hline 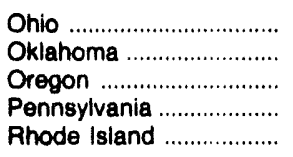 & $\begin{array}{l}4.97 \\
4.48 \\
4.75 \\
5.81 \\
6.94\end{array}$ & $\begin{array}{l}5.16 \\
4.52 \\
4.79 \\
5.79 \\
6.95\end{array}$ & $\begin{array}{l}5.03 \\
4.55 \\
4.78 \\
5.60 \\
7.21\end{array}$ & $\begin{array}{l}4.72 \\
4.23 \\
4.73 \\
5.87 \\
6.31\end{array}$ & $\begin{array}{l}5.15 \\
4.45 \\
4.72 \\
5.59 \\
6.95\end{array}$ & $\begin{array}{l}5.08 \\
4.22 \\
4.69 \\
5.67 \\
6.99\end{array}$ & $\begin{array}{l}5.00 \\
4.10 \\
4.73 \\
5.70 \\
5.411\end{array}$ & $\begin{array}{l}5.19 \\
4.11 \\
4.89 \\
6.23 \\
5.15\end{array}$ \\
\hline 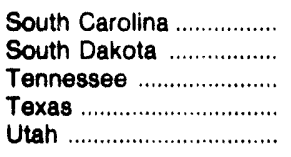 & $\begin{array}{l}5.85 \\
4.34 \\
4.36 \\
4.34 \\
4.23\end{array}$ & $\begin{array}{l}6.12 \\
4.31 \\
5.13 \\
4.74 \\
4.19\end{array}$ & $\begin{array}{l}6.22 \\
4.43 \\
5.31 \\
4.73 \\
4.26\end{array}$ & $\begin{array}{l}5.65 \\
4.19 \\
5.06 \\
4.09 \\
4.40\end{array}$ & $\begin{array}{l}6.02 \\
4.44 \\
5.25 \\
4.54 \\
4.27\end{array}$ & $\begin{array}{l}6.08 \\
4.45 \\
5.35 \\
4.45 \\
4.20\end{array}$ & $\begin{array}{l}5.31 \\
4.84 \\
5.25 \\
4.12 \\
4.04\end{array}$ & $\begin{array}{l}5.36 \\
4.62 \\
5.17 \\
4.00 \\
4.23\end{array}$ \\
\hline $\begin{array}{l}\text { Vermont } \\
\text { Virginia } \\
\text { Washington } \\
\text { West Virginia } \\
\text { Wisconsin } \\
\text { Wyoming }\end{array}$ & $\begin{array}{l}5.36 \\
5.26 \\
4.33 \\
5.67 \\
4.74 \\
4.15\end{array}$ & $\begin{array}{l}5.35 \\
5.61 \\
4.36 \\
5.67 \\
4.71 \\
4.16\end{array}$ & $\begin{array}{l}5.05 \\
5.66 \\
4.28 \\
5.69 \\
4.77 \\
4.12\end{array}$ & $\begin{array}{l}5.67 \\
4.97 \\
4.32 \\
5.48 \\
4.81 \\
4.26\end{array}$ & $\begin{array}{l}5.49 \\
5.66 \\
3.85 \\
5.30 \\
4.84 \\
4.21\end{array}$ & $\begin{array}{l}5.55 \\
5.27 \\
4.44 \\
5.45 \\
5.21 \\
4.28\end{array}$ & $\begin{array}{l}5.61 \\
5.42 \\
4.56 \\
5.75 \\
5.45 \\
4.36\end{array}$ & $\begin{array}{l}5.73 \\
5.06 \\
4.63 \\
6.83 \\
5.43 \\
4.36\end{array}$ \\
\hline Total ................................. & 5.06 & 5.08 & 5.18 & 4.88 & 5.11 & 5.12 & 4.90 & 4.69 \\
\hline
\end{tabular}

See footnotes at end of table 
Table 25. Average Price of Natural Gas Sold to Commerclal Consumers by State, 1992-1993 (Continued) (Dollars per Thousand Cubic Feet)

\begin{tabular}{|c|c|c|c|c|c|c|c|c|}
\hline State & $\begin{array}{c}\text { August } \\
1992\end{array}$ & $\begin{array}{c}\text { July } \\
1992\end{array}$ & $\begin{array}{l}\text { June } \\
1992\end{array}$ & $\begin{array}{l}\text { May } \\
1992\end{array}$ & $\begin{array}{l}\text { Aprll } \\
1992\end{array}$ & $\begin{array}{c}\text { March } \\
1992\end{array}$ & $\begin{array}{c}\text { February } \\
1992\end{array}$ & $\begin{array}{c}\text { January } \\
1892\end{array}$ \\
\hline 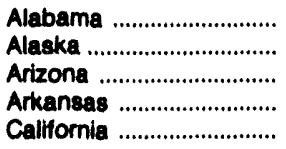 & $\begin{array}{l}6.14 \\
2.63 \\
5.28 \\
4.62 \\
4.40\end{array}$ & $\begin{array}{l}5.98 \\
2.48 \\
5.08 \\
4.36 \\
4.39\end{array}$ & $\begin{array}{l}5.70 \\
2.54 \\
5.37 \\
4.29 \\
3.91\end{array}$ & $\begin{array}{l}5.60 \\
2.61 \\
5.74 \\
4.25 \\
3.77\end{array}$ & $\begin{array}{l}5.38 \\
2.65 \\
5.29 \\
4.43 \\
5.83\end{array}$ & $\begin{array}{l}\mathbf{5 . 5 6} \\
2.70 \\
5.22 \\
\mathbf{4 . 3 3} \\
\mathbf{5 . 9 4}\end{array}$ & $\begin{array}{l}5.37 \\
2.70 \\
5.10 \\
4.28 \\
8.65\end{array}$ & $\begin{array}{l}5.65 \\
2.68 \\
5.09 \\
4.38 \\
4.80\end{array}$ \\
\hline 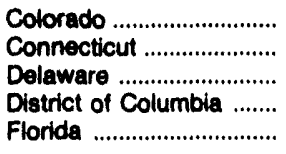 & $\begin{array}{l}4.38 \\
6.30 \\
5.14 \\
4.76 \\
4.95\end{array}$ & $\begin{array}{l}4.32 \\
6.37 \\
5.16 \\
4.85 \\
4.95\end{array}$ & $\begin{array}{l}4.23 \\
7.55 \\
5.14 \\
4.89 \\
4.97\end{array}$ & $\begin{array}{l}4.16 \\
7.21 \\
5.01 \\
5.18 \\
4.43\end{array}$ & $\begin{array}{l}4.01 \\
7.21 \\
4.89 \\
5.18 \\
4.84\end{array}$ & $\begin{array}{l}3.97 \\
7.21 \\
4.57 \\
5.24 \\
4.80\end{array}$ & $\begin{array}{l}3.94 \\
7.76 \\
4.99 \\
5.55 \\
4.84\end{array}$ & $\begin{array}{l}3.92 \\
7.77 \\
4.72 \\
5.37 \\
4.93\end{array}$ \\
\hline 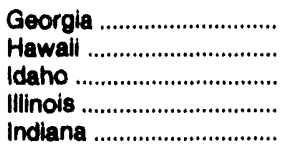 & $\begin{array}{r}5.53 \\
13.44 \\
4.60 \\
5.31 \\
4.64\end{array}$ & $\begin{array}{r}5.51 \\
13.24 \\
4.65 \\
5.48 \\
4.79\end{array}$ & $\begin{array}{r}5.53 \\
12.95 \\
4.64 \\
5.94 \\
4.75\end{array}$ & $\begin{array}{r}5.34 \\
13.34 \\
4.61 \\
4.80 \\
4.67\end{array}$ & $\begin{array}{r}5.28 \\
12.57 \\
4.60 \\
4.02 \\
4.52\end{array}$ & $\begin{array}{r}5.70 \\
12.87 \\
4.37 \\
4.11 \\
4.08\end{array}$ & $\begin{array}{r}5.93 \\
13.51 \\
4.31 \\
4.52 \\
4.82\end{array}$ & $\begin{array}{r}5.67 \\
13.46 \\
4.27 \\
4.44 \\
4.55\end{array}$ \\
\hline 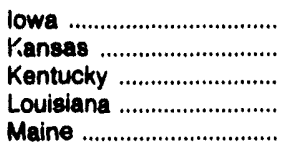 & $\begin{array}{l}4.99 \\
2.77 \\
4.75 \\
5.06 \\
5.95\end{array}$ & $\begin{array}{l}4.57 \\
2.88 \\
4.50 \\
4.71 \\
5.49\end{array}$ & $\begin{array}{l}4.58 \\
2.85 \\
4.46 \\
4.80 \\
5.47\end{array}$ & $\begin{array}{l}4.23 \\
3.15 \\
4.19 \\
4.69 \\
5.53\end{array}$ & $\begin{array}{l}3.85 \\
3.58 \\
4.27 \\
4.08 \\
6.37\end{array}$ & $\begin{array}{l}3.73 \\
4.01 \\
4.16 \\
4.43 \\
6.37\end{array}$ & $\begin{array}{l}3.71 \\
4.13 \\
4.27 \\
4.28 \\
6.74\end{array}$ & $\begin{array}{l}4.12 \\
3.97 \\
4.43 \\
4.67 \\
6.26\end{array}$ \\
\hline 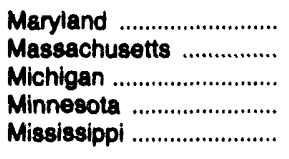 & $\begin{array}{l}5.37 \\
3.85 \\
5.47 \\
4.32 \\
3.90\end{array}$ & $\begin{array}{l}5.31 \\
3.76 \\
5.41 \\
3.96 \\
3.80\end{array}$ & $\begin{array}{l}5.01 \\
4.54 \\
5.11 \\
4.20 \\
3.89\end{array}$ & $\begin{array}{l}4.79 \\
4.49 \\
4.70 \\
3.99 \\
4.04\end{array}$ & $\begin{array}{l}4.63 \\
6.16 \\
4.52 \\
3.70 \\
3.80\end{array}$ & $\begin{array}{l}4.88 \\
6.44 \\
4.55 \\
3.51 \\
3.90\end{array}$ & $\begin{array}{l}5.06 \\
6.72 \\
4.58 \\
3.59 \\
4.00\end{array}$ & $\begin{array}{l}5.14 \\
6.75 \\
4.56 \\
4.00 \\
4.12\end{array}$ \\
\hline $\begin{array}{l}\text { Missouri ......................... } \\
\text { Montana .......................... } \\
\text { Nebraska ........................... } \\
\text { Nevada ............................. } \\
\text { New Hampshire .............. }\end{array}$ & $\begin{array}{l}4.71 \\
4.98 \\
3.78 \\
4.45 \\
6.85\end{array}$ & $\begin{array}{l}4.96 \\
4.93 \\
3.69 \\
4.37 \\
7.24\end{array}$ & $\begin{array}{l}4.35 \\
4.86 \\
3.98 \\
4.41 \\
6.50\end{array}$ & $\begin{array}{l}4.25 \\
4.58 \\
3.75 \\
4.40 \\
7.03\end{array}$ & $\begin{array}{l}4.11 \\
4.43 \\
3.69 \\
4.33 \\
6.46\end{array}$ & $\begin{array}{l}4.29 \\
4.36 \\
3.76 \\
4.26 \\
6.48\end{array}$ & $\begin{array}{l}4.39 \\
4.31 \\
3.87 \\
4.29 \\
6.45\end{array}$ & $\begin{array}{l}4.42 \\
4.32 \\
4.12 \\
4.30 \\
6.46\end{array}$ \\
\hline $\begin{array}{l}\text { New Jersey .................... } \\
\text { New Mexico .................... } \\
\text { New York ....................... } \\
\text { North Carolina .................. } \\
\text { North Dakota ................... }\end{array}$ & $\begin{array}{l}5.24 \\
2.99 \\
5.84 \\
4.82 \\
5.15\end{array}$ & $\begin{array}{l}4.99 \\
3.28 \\
5.76 \\
4.84 \\
5.00\end{array}$ & $\begin{array}{l}5.33 \\
3.82 \\
6.08 \\
4.78 \\
4.92\end{array}$ & $\begin{array}{l}5.36 \\
3.39 \\
5.77 \\
4.49 \\
4.55\end{array}$ & $\begin{array}{l}5.52 \\
3.23 \\
5.31 \\
4.16 \\
4.32\end{array}$ & $\begin{array}{l}5.05 \\
3.44 \\
5.25 \\
4.33 \\
4.26\end{array}$ & $\begin{array}{l}5.72 \\
3.47 \\
5.63 \\
4.55 \\
4.30\end{array}$ & $\begin{array}{l}5.79 \\
3.22 \\
5.76 \\
4.95 \\
4.40\end{array}$ \\
\hline 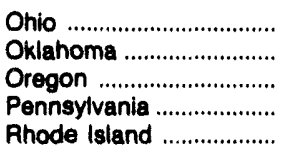 & $\begin{array}{l}5.28 \\
4.05 \\
5.02 \\
6.49 \\
5.07\end{array}$ & $\begin{array}{l}4.67 \\
4.06 \\
4.95 \\
6.73 \\
5.31\end{array}$ & $\begin{array}{l}4.84 \\
4.31 \\
4.82 \\
6.56 \\
5.67\end{array}$ & $\begin{array}{l}4.54 \\
4.28 \\
4.73 \\
6.25 \\
5.73\end{array}$ & $\begin{array}{l}4.42 \\
4.33 \\
4.71 \\
6.14 \\
6.01\end{array}$ & $\begin{array}{l}4.39 \\
4.34 \\
4.68 \\
5.77 \\
6.74\end{array}$ & $\begin{array}{l}4.39 \\
4.13 \\
4.68 \\
5.86 \\
7.05\end{array}$ & $\begin{array}{l}4.67 \\
4.09 \\
4.66 \\
5.67 \\
7.01\end{array}$ \\
\hline 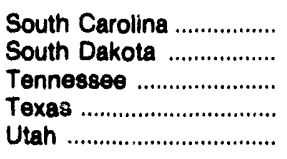 & $\begin{array}{l}5.32 \\
4.62 \\
5.25 \\
3.63 \\
4.33\end{array}$ & $\begin{array}{l}5.34 \\
4.40 \\
4.86 \\
3.53 \\
4.36\end{array}$ & $\begin{array}{l}5.32 \\
4.40 \\
4.91 \\
3.93 \\
4.38\end{array}$ & $\begin{array}{l}5.07 \\
4.19 \\
4.77 \\
3.81 \\
4.23\end{array}$ & $\begin{array}{l}5.36 \\
3.82 \\
4.77 \\
3.89 \\
4.40\end{array}$ & $\begin{array}{l}5.86 \\
3.70 \\
4.82 \\
4.08 \\
4.68\end{array}$ & $\begin{array}{l}5.82 \\
3.85 \\
4.98 \\
4.15 \\
4.50\end{array}$ & $\begin{array}{l}5.77 \\
4.15 \\
5.13 \\
4.30 \\
4.47\end{array}$ \\
\hline 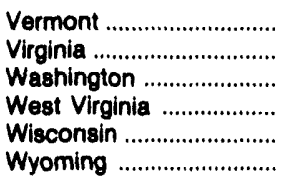 & $\begin{array}{l}6.01 \\
5.08 \\
4.69 \\
6.93 \\
5.42 \\
4.38\end{array}$ & $\begin{array}{l}6.15 \\
5.05 \\
4.64 \\
6.90 \\
4.91 \\
4.31\end{array}$ & $\begin{array}{l}6.20 \\
4.51 \\
4.63 \\
6.42 \\
5.11 \\
4.31\end{array}$ & $\begin{array}{l}5.96 \\
4.20 \\
4.49 \\
5.76 \\
4.44 \\
4.23\end{array}$ & $\begin{array}{l}5.66 \\
4.26 \\
4.39 \\
5.36 \\
4.46 \\
4.25\end{array}$ & $\begin{array}{l}5.62 \\
4.45 \\
4.29 \\
5.29 \\
4.49 \\
4.22\end{array}$ & $\begin{array}{l}5.69 \\
5.11 \\
4.28 \\
5.22 \\
4.46 \\
4.22\end{array}$ & $\begin{array}{l}5.53 \\
5.13 \\
4.24 \\
5.25 \\
4.80 \\
4.26\end{array}$ \\
\hline Total .............................. & 4.73 & 4.64 & 4.72 & 4.59 & 4.77 & 4.77 & 5.03 & 4.85 \\
\hline
\end{tabular}

R = Revised Data.

Notes: Data for 1992 are final. All other data are preliminary unless otherwise indicated. Gexgraphic coverage is the 50 States and the District of Columbia. Average prices for gas delivered to commercial consumers reflect onsystem sales prices only. See Appendix A, Explanatory Note 5 for discussion of computations and revision policy. See Table 28 for data on onsystem sales expressed as a percentage of both total commercial and total industrial deliveries.

Source: Form EIA-857. 
Table 26. Average Price of Natural Gas Sold to Industrial Consumers by State, 1992-1993 (Dollars per Thousand Cubic Feet)

\begin{tabular}{|c|c|c|c|c|c|c|c|c|}
\hline State & $\begin{array}{c}\text { YTD } \\
1993\end{array}$ & $\begin{array}{c}\text { YTD } \\
1992\end{array}$ & $\begin{array}{l}\text { YTD } \\
1991\end{array}$ & $\begin{array}{c}\text { August } \\
1993\end{array}$ & $\begin{array}{c}\text { July } \\
1993\end{array}$ & $\begin{array}{l}\text { June } \\
1993\end{array}$ & $\begin{array}{l}\text { May } \\
1993\end{array}$ & $\begin{array}{l}\text { April } \\
1993\end{array}$ \\
\hline 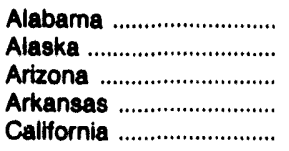 & $\begin{array}{l}3.42 \\
1.33 \\
4.03 \\
3.18 \\
3.62\end{array}$ & $\begin{array}{l}2.87 \\
1.18 \\
4.16 \\
3.08 \\
3.78\end{array}$ & $\begin{array}{l}2.96 \\
1.18 \\
3.39 \\
3.05 \\
3.97\end{array}$ & $\begin{array}{l}3.25 \\
1.24 \\
4.01 \\
3.15 \\
3.39\end{array}$ & $\begin{array}{l}3.16 \\
1.24 \\
3.98 \\
3.19 \\
2.84\end{array}$ & $\begin{array}{l}3.30 \\
1.35 \\
4.65 \\
3.07 \\
3.77\end{array}$ & $\begin{array}{l}3.78 \\
1.35 \\
4.03 \\
3.04 \\
3.52\end{array}$ & $\begin{array}{l}3.47 \\
1.35 \\
3.92 \\
3.15 \\
3.82\end{array}$ \\
\hline 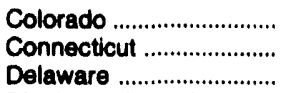 & $\begin{array}{l}2.28 \\
4.64 \\
3.32\end{array}$ & $\begin{array}{l}2.24 \\
5.04 \\
3.08\end{array}$ & $\begin{array}{l}2.27 \\
4.79 \\
3.06\end{array}$ & $\begin{array}{l}2.17 \\
4.01 \\
3.17\end{array}$ & $\begin{array}{l}2.10 \\
3.93 \\
3.11\end{array}$ & $\begin{array}{l}2.14 \\
3.90 \\
3.52\end{array}$ & $\begin{array}{l}2.48 \\
4.42 \\
3.69\end{array}$ & $\begin{array}{l}2.26 \\
4.77 \\
3.31\end{array}$ \\
\hline $\begin{array}{l}\text { District of Columbia } \\
\text { Florida }\end{array}$ & $3 . \overline{83}$ & $3 . \overline{07}$ & $3 . \overline{06}$ & $3 . \overline{85}$ & $4 . \overline{0}$ & $4 . \overline{01}$ & $4 . \overline{03}$ & $3 . \overline{86}$ \\
\hline 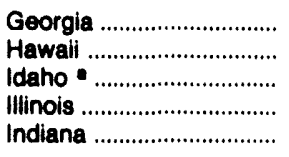 & $\begin{array}{r}4.01 \\
- \\
- \\
4.36 \\
3.54\end{array}$ & $\begin{array}{r}3.28 \\
- \\
3.80 \\
3.31\end{array}$ & $\begin{array}{r}3.30 \\
2 . \overline{9} \\
4.04 \\
3.69\end{array}$ & $\begin{array}{r}3.84 \\
-- \\
4 . \overline{77} \\
3.68\end{array}$ & $\begin{array}{r}3.61 \\
- \\
\overline{4.62} \\
3.97\end{array}$ & $\begin{array}{r}4.41 \\
- \\
4.62 \\
3.96\end{array}$ & $\begin{array}{r}4.49 \\
-- \\
4.64 \\
3.12\end{array}$ & $\begin{array}{r}4.11 \\
-- \\
-- \\
4.81 \\
3.36\end{array}$ \\
\hline 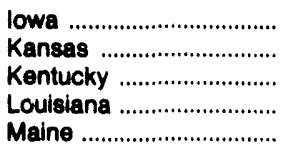 & $\begin{array}{l}3.95 \\
2.65 \\
3.73 \\
2.23 \\
4.83\end{array}$ & $\begin{array}{l}3.27 \\
2.46 \\
3.07 \\
1.70 \\
4.08\end{array}$ & $\begin{array}{l}2.45 \\
2.62 \\
3.50 \\
1.67 \\
4.29\end{array}$ & $\begin{array}{l}3.74 \\
2.56 \\
3.72 \\
2.22 \\
3.96\end{array}$ & $\begin{array}{l}4.04 \\
2.59 \\
3.66 \\
2.16 \\
4.03\end{array}$ & $\begin{array}{l}4.78 \\
2.55 \\
3.62 \\
2.43 \\
5.36\end{array}$ & $\begin{array}{l}3.75 \\
2.70 \\
3.65 \\
2.62 \\
5.31\end{array}$ & $\begin{array}{l}4.44 \\
2.60 \\
3.44 \\
2.27 \\
4.75\end{array}$ \\
\hline 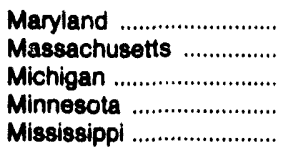 & $\begin{array}{l}3.71 \\
5.14 \\
4.08 \\
3.21 \\
2.87\end{array}$ & $\begin{array}{l}3.36 \\
4.12 \\
3.91 \\
2.83 \\
2.30\end{array}$ & $\begin{array}{l}3.54 \\
3.95 \\
4.02 \\
2.72 \\
2.30\end{array}$ & $\begin{array}{l}3.82 \\
3.71 \\
4.33 \\
3.47 \\
3.00\end{array}$ & $\begin{array}{l}3.67 \\
4.24 \\
4.37 \\
3.21 \\
2.62\end{array}$ & $\begin{array}{l}3.41 \\
3.68 \\
4.24 \\
2.70 \\
2.80\end{array}$ & $\begin{array}{l}4.33 \\
4.56 \\
4.16 \\
3.40 \\
3.34\end{array}$ & $\begin{array}{l}4.67 \\
5.80 \\
4.06 \\
3.34 \\
2.94\end{array}$ \\
\hline 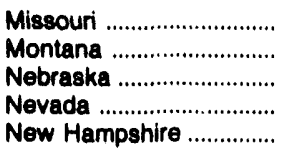 & $\begin{array}{l}4.20 \\
3.94 \\
3.11 \\
4.06 \\
4.40\end{array}$ & $\begin{array}{l}3.72 \\
3.99 \\
2.65 \\
4.11 \\
4.41\end{array}$ & $\begin{array}{l}4.17 \\
3.20 \\
2.68 \\
4.19 \\
4.33\end{array}$ & $\begin{array}{l}4.06 \\
4.23 \\
2.87 \\
4.33 \\
3.75\end{array}$ & $\begin{array}{l}4.19 \\
4.17 \\
2.91 \\
4.03 \\
3.60\end{array}$ & $\begin{array}{l}4.25 \\
4.09 \\
2.57 \\
4.36 \\
3.33\end{array}$ & $\begin{array}{l}4.10 \\
3.97 \\
3.94 \\
4.26 \\
3.00\end{array}$ & $\begin{array}{l}4.19 \\
3.82 \\
3.17 \\
4.12 \\
4.07\end{array}$ \\
\hline 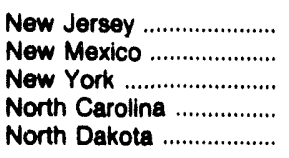 & $\begin{array}{l}3.62 \\
3.76 \\
5.73 \\
3.82 \\
3.39\end{array}$ & $\begin{array}{l}3.33 \\
6.65 \\
4.81 \\
3.04 \\
3.07\end{array}$ & $\begin{array}{l}3.82 \\
3.56 \\
4.76 \\
3.27 \\
3.14\end{array}$ & $\begin{array}{l}3.23 \\
3.50 \\
5.84 \\
3.44 \\
3.73\end{array}$ & $\begin{array}{l}3.32 \\
3.71 \\
5.44 \\
3.47 \\
3.43\end{array}$ & $\begin{array}{l}3.75 \\
4.44 \\
5.25 \\
3.55 \\
3.53\end{array}$ & $\begin{array}{l}3.61 \\
3.83 \\
6.28 \\
3.80 \\
3.49\end{array}$ & $\begin{array}{l}3.44 \\
3.92 \\
5.73 \\
3.62 \\
3.44\end{array}$ \\
\hline 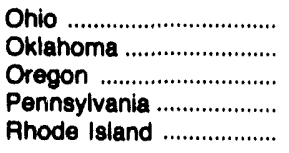 & $\begin{array}{l}4.64 \\
2.16 \\
3.49 \\
3.70 \\
5.27\end{array}$ & $\begin{array}{l}3.87 \\
1.91 \\
3.32 \\
3.85 \\
4.65\end{array}$ & $\begin{array}{l}4.18 \\
1.68 \\
3.41 \\
4.10 \\
5.48\end{array}$ & $\begin{array}{l}4.77 \\
2.26 \\
3.36 \\
3.35 \\
3.94\end{array}$ & $\begin{array}{r}4.56 \\
1.92 \\
3.40 \\
\text { R } 3.52 \\
4.46\end{array}$ & $\begin{array}{l}4.73 \\
2.51 \\
3.35 \\
3.58 \\
4.46\end{array}$ & $\begin{array}{l}4.57 \\
2.12 \\
3.62 \\
3.72 \\
4.99\end{array}$ & $\begin{array}{l}4.47 \\
2.07 \\
3.52 \\
3.66 \\
5.78\end{array}$ \\
\hline 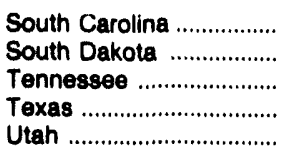 & $\begin{array}{l}3.29 \\
3.65 \\
3.64 \\
2.33 \\
3.07\end{array}$ & $\begin{array}{l}2.90 \\
3.52 \\
3.18 \\
1.89 \\
4.12\end{array}$ & $\begin{array}{l}2.96 \\
3.20 \\
3.15 \\
1.84 \\
3.72\end{array}$ & $\begin{array}{l}3.05 \\
3.99 \\
3.48 \\
2.42 \\
2.81\end{array}$ & $\begin{array}{l}3.03 \\
3.65 \\
3.59 \\
2.24 \\
3.00\end{array}$ & $\begin{array}{l}3.19 \\
3.79 \\
3.79 \\
2.16 \\
2.93\end{array}$ & $\begin{array}{l}3.53 \\
4.24 \\
3.78 \\
2.81 \\
2.91\end{array}$ & $\begin{array}{l}3.29 \\
3.84 \\
3.49 \\
2.47 \\
2.92\end{array}$ \\
\hline 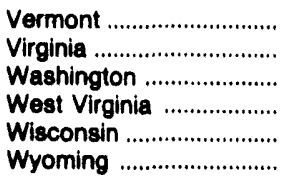 & $\begin{array}{l}3.64 \\
3.97 \\
3.11 \\
2.82 \\
3.92 \\
2.89\end{array}$ & $\begin{array}{l}3.07 \\
3.53 \\
2.85 \\
2.82 \\
3.15 \\
2.88\end{array}$ & $\begin{array}{l}3.07 \\
3.85 \\
2.80 \\
2.85 \\
3.10 \\
3.00\end{array}$ & $\begin{array}{l}3.48 \\
2.61 \\
3.31 \\
2.79 \\
3.69 \\
3.19\end{array}$ & $\begin{array}{l}3.56 \\
2.93 \\
3.14 \\
1.88 \\
3.77 \\
3.07\end{array}$ & $\begin{array}{l}3.74 \\
3.77 \\
3.15 \\
2.75 \\
3.87 \\
3.00\end{array}$ & $\begin{array}{l}3.65 \\
4.30 \\
3.45 \\
3.24 \\
4.33 \\
2.87\end{array}$ & $\begin{array}{l}3.59 \\
4.26 \\
3.19 \\
2.73 \\
3.99 \\
2.80\end{array}$ \\
\hline Total & 3.06 & 2.66 & 2.65 & 2.86 & 2.71 & 2.95 & 3.24 & 3.13 \\
\hline
\end{tabular}

See tootnotes at end of table. 
Table 26. Average Price of Natural Gas Sold to Industrial Consumers by State, 1992-1993 (Continued)

(Dollars per Thousand Cubic Feet)

\begin{tabular}{|c|c|c|c|c|c|c|c|c|}
\hline State & $\begin{array}{c}\text { March } \\
1993\end{array}$ & $\begin{array}{c}\text { February } \\
1993\end{array}$ & $\begin{array}{c}\text { January } \\
1893\end{array}$ & $\begin{array}{l}\text { Total } \\
1992\end{array}$ & $\begin{array}{c}\text { December } \\
1892\end{array}$ & $\begin{array}{c}\text { November } \\
1992\end{array}$ & $\begin{array}{c}\text { October } \\
1992\end{array}$ & $\begin{array}{c}\text { September } \\
1992\end{array}$ \\
\hline 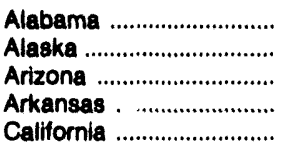 & $\begin{array}{l}3.47 \\
1.35 \\
3.97 \\
3.22 \\
3.31\end{array}$ & $\begin{array}{l}3.38 \\
1.35 \\
3.98 \\
3.31 \\
3.77\end{array}$ & $\begin{array}{l}3.58 \\
1.35 \\
3.97 \\
3.24 \\
4.85\end{array}$ & $\begin{array}{l}3.07 \\
1.18 \\
4.16 \\
3.13 \\
3.67\end{array}$ & $\begin{array}{l}3.59 \\
1.19 \\
4.09 \\
3.28 \\
3.82\end{array}$ & $\begin{array}{l}3.54 \\
1.17 \\
4.21 \\
3.28 \\
3.10\end{array}$ & $\begin{array}{l}3.61 \\
1.17 \\
4.11 \\
3.20 \\
3.44\end{array}$ & $\begin{array}{l}3.18 \\
1.17 \\
4.21 \\
3.03 \\
3.46\end{array}$ \\
\hline 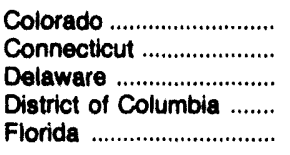 & $\begin{array}{r}2.27 \\
4.96 \\
3.26 \\
-\overline{3.82}\end{array}$ & $\begin{array}{r}2.27 \\
5.01 \\
3.22 \\
-\overline{3.64}\end{array}$ & $\begin{array}{r}2.49 \\
5.34 \\
3.32 \\
-\overline{3.48}\end{array}$ & $\begin{array}{r}2.20 \\
4.92 \\
3.25 \\
3 . \overline{2 .}\end{array}$ & $\begin{array}{r}2.32 \\
5.05 \\
3.59 \\
3 . \overline{51}\end{array}$ & $\begin{array}{r}2.29 \\
4.85 \\
3.47 \\
3 . \overline{60}\end{array}$ & $\begin{array}{r}2.34 \\
4.64 \\
3.69 \\
3 . \overline{-}\end{array}$ & $\begin{array}{r}1.63 \\
4.09 \\
3.22 \\
-\overline{3.28}\end{array}$ \\
\hline 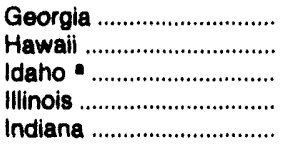 & $\begin{array}{r}3.97 \\
- \\
4 . \overline{23} \\
3.41\end{array}$ & $\begin{array}{r}3.81 \\
- \\
4.15 \\
3.48\end{array}$ & $\begin{array}{r}4.10 \\
- \\
4.12 \\
3.86\end{array}$ & $\begin{array}{r}3.50 \\
2 . \overline{2} \\
3.75 \\
3.39\end{array}$ & $\begin{array}{r}4.43 \\
- \\
4.14 \\
4.17\end{array}$ & $\begin{array}{r}4.28 \\
- \\
3.79 \\
3.51\end{array}$ & $\begin{array}{r}4.38 \\
- \\
- \\
3.21 \\
3.01\end{array}$ & $\begin{array}{r}2.81 \\
-- \\
-- \\
3.17 \\
3.36\end{array}$ \\
\hline 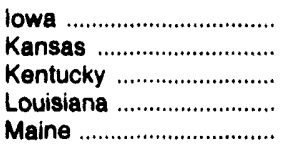 & $\begin{array}{l}3.85 \\
2.83 \\
3.76 \\
1.96 \\
5.49\end{array}$ & $\begin{array}{l}3.50 \\
2.66 \\
3.83 \\
1.96 \\
5.40\end{array}$ & $\begin{array}{l}4.11 \\
2.87 \\
4.23 \\
2.27 \\
4.76\end{array}$ & $\begin{array}{l}3.55 \\
2.61 \\
3.23 \\
1.93 \\
4.14\end{array}$ & $\begin{array}{l}3.86 \\
3.07 \\
4.14 \\
2.54 \\
4.97\end{array}$ & $\begin{array}{l}3.85 \\
2.86 \\
3.92 \\
2.70 \\
4.50\end{array}$ & $\begin{array}{l}4.29 \\
2.87 \\
3.11 \\
2.69 \\
3.88\end{array}$ & $\begin{array}{l}3.76 \\
2.70 \\
3.05 \\
2.19 \\
3.55\end{array}$ \\
\hline 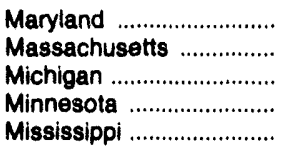 & $\begin{array}{l}3.96 \\
6.45 \\
4.02 \\
3.03 \\
2.76\end{array}$ & $\begin{array}{l}3.88 \\
6.55 \\
4.02 \\
3.33 \\
2.71\end{array}$ & $\begin{array}{l}3.25 \\
6.32 \\
4.04 \\
3.33 \\
2.80\end{array}$ & $\begin{array}{l}3.56 \\
4.14 \\
3.92 \\
3.05 \\
2.53\end{array}$ & $\begin{array}{l}4.13 \\
5.65 \\
3.86 \\
3.32 \\
3.02\end{array}$ & $\begin{array}{l}3.75 \\
5.05 \\
3.90 \\
3.51 \\
3.05\end{array}$ & $\begin{array}{l}3.65 \\
3.35 \\
3.97 \\
3.57 \\
3.34\end{array}$ & $\begin{array}{l}3.37 \\
3.06 \\
4.14 \\
3.21 \\
2.64\end{array}$ \\
\hline 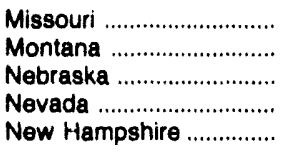 & $\begin{array}{l}4.15 \\
3.81 \\
2.97 \\
3.80 \\
6.06\end{array}$ & $\begin{array}{l}4.27 \\
3.81 \\
3.04 \\
4.09 \\
5.91\end{array}$ & $\begin{array}{l}4.26 \\
3.99 \\
3.24 \\
3.69 \\
5.66\end{array}$ & $\begin{array}{l}3.87 \\
4.19 \\
2.92 \\
4.07 \\
4.49\end{array}$ & $\begin{array}{l}4.56 \\
4.67 \\
3.36 \\
3.84 \\
5.58\end{array}$ & $\begin{array}{l}4.57 \\
4.81 \\
3.41 \\
4.11 \\
5.31\end{array}$ & $\begin{array}{l}3.49 \\
4.80 \\
3.62 \\
3.89 \\
4.20\end{array}$ & $\begin{array}{l}3.89 \\
4.66 \\
3.00 \\
4.25 \\
3.62\end{array}$ \\
\hline 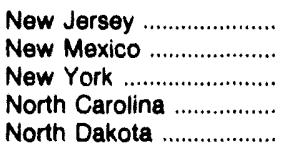 & $\begin{array}{l}3.83 \\
4.01 \\
5.57 \\
3.87 \\
3.28\end{array}$ & $\begin{array}{l}3.70 \\
3.78 \\
5.73 \\
4.14 \\
3.23\end{array}$ & $\begin{array}{l}3.91 \\
3.67 \\
5.94 \\
4.22 \\
3.37\end{array}$ & $\begin{array}{l}3.42 \\
6.86 \\
4.93 \\
3.34 \\
3.25\end{array}$ & $\begin{array}{l}4.38 \\
8.02 \\
5.51 \\
4.11 \\
3.47\end{array}$ & $\begin{array}{l}3.45 \\
7.00 \\
5.41 \\
4.02 \\
3.74\end{array}$ & $\begin{array}{l}3.29 \\
6.66 \\
4.75 \\
3.55 \\
3.71\end{array}$ & $\begin{array}{l}3.05 \\
5.85 \\
4.54 \\
3.38 \\
3.43\end{array}$ \\
\hline 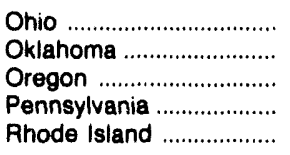 & $\begin{array}{l}4.52 \\
2.12 \\
3.54 \\
3.78 \\
6.08\end{array}$ & $\begin{array}{l}4.72 \\
2.10 \\
3.54 \\
3.85 \\
6.08\end{array}$ & $\begin{array}{l}4.85 \\
2.18 \\
3.59 \\
3.83 \\
6.27\end{array}$ & $\begin{array}{l}4.15 \\
2.02 \\
3.36 \\
3.75 \\
4.66\end{array}$ & $\begin{array}{l}4.68 \\
2.10 \\
3.56 \\
3.83 \\
5.85\end{array}$ & $\begin{array}{l}4.62 \\
2.17 \\
3.46 \\
3.54 \\
5.48\end{array}$ & $\begin{array}{l}4.13 \\
2.29 \\
3.42 \\
3.51 \\
3.91\end{array}$ & $\begin{array}{l}4.30 \\
2.03 \\
3.31 \\
3.54 \\
3.73\end{array}$ \\
\hline 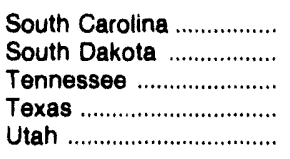 & $\begin{array}{l}3.25 \\
3.46 \\
3.58 \\
2.23 \\
3.27\end{array}$ & $\begin{array}{l}3.46 \\
3.57 \\
3.54 \\
2.06 \\
3.24\end{array}$ & $\begin{array}{l}3.52 \\
3.33 \\
3.84 \\
2.31 \\
3.31\end{array}$ & $\begin{array}{l}3.13 \\
3.63 \\
3.44 \\
2.12 \\
3.91\end{array}$ & $\begin{array}{l}3.68 \\
3.41 \\
4.18 \\
2.59 \\
3.60\end{array}$ & $\begin{array}{l}3.62 \\
3.62 \\
4.05 \\
2.56 \\
3.66\end{array}$ & $\begin{array}{l}3.60 \\
4.58 \\
3.92 \\
2.92 \\
3.37\end{array}$ & $\begin{array}{l}3.18 \\
4.03 \\
3.74 \\
2.28 \\
3.55\end{array}$ \\
\hline 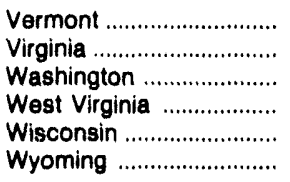 & $\begin{array}{l}3.54 \\
4.61 \\
2.89 \\
2.68 \\
3.85 \\
2.81\end{array}$ & $\begin{array}{l}3.67 \\
4.57 \\
2.92 \\
3.90 \\
3.83 \\
2.74\end{array}$ & $\begin{array}{l}3.81 \\
4.61 \\
2.89 \\
3.04 \\
3.97 \\
2.88\end{array}$ & $\begin{array}{l}3.28 \\
3.72 \\
2.91 \\
2.89 \\
3.38 \\
2.91\end{array}$ & $\begin{array}{l}3.81 \\
4.60 \\
2.69 \\
3.12 \\
3.68 \\
2.89\end{array}$ & $\begin{array}{l}3.75 \\
3.60 \\
3.13 \\
2.87 \\
3.87 \\
2.92\end{array}$ & $\begin{array}{l}3.56 \\
4.58 \\
3.37 \\
2.98 \\
4.04 \\
3.04\end{array}$ & $\begin{array}{l}3.56 \\
3.57 \\
2.96 \\
2.96 \\
3.47 \\
3.09\end{array}$ \\
\hline 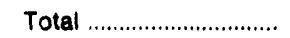 & 3.08 & 3.12 & 3.26 & 2.84 & 3.38 & 3.26 & 3.21 & 2.82 \\
\hline
\end{tabular}

See footnotes at end of table. 
Table 26. Average Price of Natural Gas Sold to Industrial Consumers by State, 1992-1993 (Continued) (Dollars per Thousand Cubic Feet)

\begin{tabular}{|c|c|c|c|c|c|c|c|c|}
\hline State & $\begin{array}{c}\text { August } \\
1992\end{array}$ & $\begin{array}{l}\text { July } \\
1992\end{array}$ & $\begin{array}{l}\text { June } \\
1892\end{array}$ & $\begin{array}{l}\text { May } \\
1992\end{array}$ & $\begin{array}{l}\text { April } \\
1992\end{array}$ & $\begin{array}{c}\text { March } \\
1992\end{array}$ & $\begin{array}{c}\text { February } \\
1992\end{array}$ & $\begin{array}{c}\text { January } \\
1992\end{array}$ \\
\hline 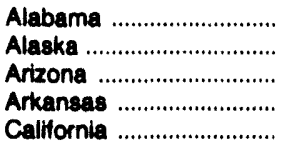 & $\begin{array}{l}3.13 \\
1.16 \\
4.14 \\
3.00 \\
3.29\end{array}$ & $\begin{array}{l}2.77 \\
1.17 \\
4.19 \\
2.97 \\
3.49\end{array}$ & $\begin{array}{l}2.76 \\
1.16 \\
4.23 \\
2.84 \\
3.42\end{array}$ & $\begin{array}{l}2.81 \\
1.16 \\
4.16 \\
2.90 \\
3.04\end{array}$ & $\begin{array}{l}2.81 \\
1.16 \\
4.11 \\
3.05 \\
3.98\end{array}$ & $\begin{array}{l}2.66 \\
1.19 \\
4.24 \\
3.22 \\
4.26\end{array}$ & $\begin{array}{l}2.83 \\
1.20 \\
4.09 \\
3.21 \\
4.43\end{array}$ & $\begin{array}{l}3.25 \\
1.20 \\
4.14 \\
3.39 \\
4.58\end{array}$ \\
\hline 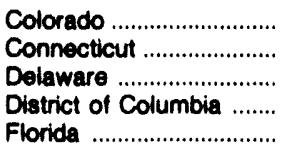 & $\begin{array}{r}2.30 \\
4.26 \\
3.17 \\
3 . \overline{16}\end{array}$ & $\begin{array}{l}1.88 \\
4.32 \\
2.97 \\
2 . \overline{97}\end{array}$ & $\begin{array}{r}2.18 \\
4.51 \\
3.15 \\
2 . \overline{81}\end{array}$ & $\begin{array}{l}2.31 \\
4.71 \\
2.94 \\
2 . \overline{97}\end{array}$ & $\begin{array}{r}2.29 \\
4.94 \\
3.03 \\
3 . \overline{0}\end{array}$ & $\begin{array}{r}2.29 \\
5.39 \\
3.02 \\
-\overline{3.22}\end{array}$ & $\begin{array}{r}2.30 \\
5.61 \\
3.09 \\
-\overline{3.17}\end{array}$ & $\begin{array}{r}2.44 \\
5.61 \\
3.25 \\
3 . \overline{19}\end{array}$ \\
\hline $\begin{array}{l}\text { Georgia } \\
\text { Hawaii } \\
\text { Idaho } \\
\text { Illinois } \\
\text { Indiana }\end{array}$ & $\begin{array}{r}3.35 \\
\overline{-} \\
3.84 \\
2.84\end{array}$ & $\begin{array}{r}3.20 \\
\overline{3} \\
3.68 \\
3.05\end{array}$ & $\begin{array}{r}3.19 \\
\overline{-} \\
4.06 \\
3.00\end{array}$ & $\begin{array}{r}3.01 \\
\bar{z} \\
3 . \overline{57} \\
2.90\end{array}$ & $\begin{array}{l}2.85 \\
- \\
-\overline{-} \\
3.37\end{array}$ & $\begin{array}{r}3.27 \\
- \\
3 . \overline{64} \\
3.39\end{array}$ & $\begin{array}{r}3.72 \\
\overline{-} \\
4.03 \\
3.85\end{array}$ & $\begin{array}{r}3.88 \\
\overline{-} \\
3 . \overline{83} \\
3.86\end{array}$ \\
\hline $\begin{array}{l}\text { lowa } \\
\text { Kansas } \\
\text { Kentucky } \\
\text { Louisiana } \\
\text { Maine }\end{array}$ & $\begin{array}{l}3.50 \\
2.58 \\
3.08 \\
1.98 \\
3.48\end{array}$ & $\begin{array}{l}3.23 \\
2.40 \\
2.75 \\
1.85 \\
3.36\end{array}$ & $\begin{array}{l}3.69 \\
2.39 \\
2.76 \\
1.84 \\
3.65\end{array}$ & $\begin{array}{l}2.97 \\
2.31 \\
2.79 \\
1.73 \\
3.43\end{array}$ & $\begin{array}{l}3.15 \\
2.32 \\
3.11 \\
1.54 \\
3.93\end{array}$ & $\begin{array}{l}3.15 \\
2.39 \\
3.22 \\
1.37 \\
3.88\end{array}$ & $\begin{array}{l}3.06 \\
2.60 \\
3.52 \\
1.49 \\
5.92\end{array}$ & $\begin{array}{l}3.45 \\
2.72 \\
3.53 \\
2.02 \\
5.41\end{array}$ \\
\hline 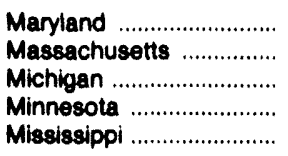 & $\begin{array}{l}3.80 \\
3.01 \\
3.99 \\
3.02 \\
2.56\end{array}$ & $\begin{array}{l}3.77 \\
3.38 \\
3.84 \\
2.87 \\
2.21\end{array}$ & $\begin{array}{l}3.77 \\
2.81 \\
3.91 \\
2.86 \\
2.42\end{array}$ & $\begin{array}{l}3.96 \\
3.40 \\
3.77 \\
2.70 \\
2.29\end{array}$ & $\begin{array}{l}3.00 \\
3.71 \\
3.88 \\
2.58 \\
2.20\end{array}$ & $\begin{array}{l}3.11 \\
5.16 \\
3.81 \\
2.51 \\
2.12\end{array}$ & $\begin{array}{l}3.41 \\
5.99 \\
4.03 \\
2.94 \\
2.14\end{array}$ & $\begin{array}{l}3.33 \\
5.83 \\
3.88 \\
3.08 \\
2.53\end{array}$ \\
\hline 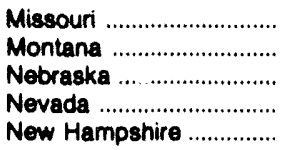 & $\begin{array}{l}3.55 \\
4.35 \\
2.96 \\
3.40 \\
3.47\end{array}$ & $\begin{array}{l}3.63 \\
4.21 \\
2.65 \\
4.32 \\
3.65\end{array}$ & $\begin{array}{l}3.57 \\
4.11 \\
2.60 \\
4.08 \\
3.67\end{array}$ & $\begin{array}{l}3.61 \\
4.00 \\
2.45 \\
4.15 \\
4.69\end{array}$ & $\begin{array}{l}3.38 \\
3.86 \\
2.39 \\
4.11 \\
4.08\end{array}$ & $\begin{array}{l}3.61 \\
3.87 \\
2.42 \\
4.13 \\
4.94\end{array}$ & $\begin{array}{l}3.89 \\
3.77 \\
2.61 \\
4.31 \\
5.43\end{array}$ & $\begin{array}{l}4.13 \\
4.05 \\
3.03 \\
4.32 \\
5.42\end{array}$ \\
\hline 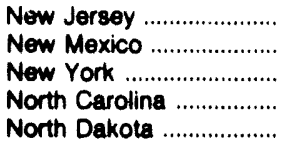 & $\begin{array}{l}2.85 \\
6.06 \\
5.04 \\
3.16 \\
3.27\end{array}$ & $\begin{array}{l}2.97 \\
6.71 \\
4.66 \\
3.00 \\
3.19\end{array}$ & $\begin{array}{l}2.96 \\
6.33 \\
4.47 \\
3.06 \\
3.13\end{array}$ & $\begin{array}{l}2.98 \\
5.83 \\
4.12 \\
2.86 \\
2.90\end{array}$ & $\begin{array}{l}3.42 \\
6.62 \\
4.91 \\
2.84 \\
2.86\end{array}$ & $\begin{array}{l}3.43 \\
7.14 \\
4.67 \\
2.82 \\
2.82\end{array}$ & $\begin{array}{l}4.04 \\
7.43 \\
5.17 \\
2.96 \\
3.08\end{array}$ & $\begin{array}{l}3.99 \\
7.61 \\
4.89 \\
3.56 \\
3.36\end{array}$ \\
\hline 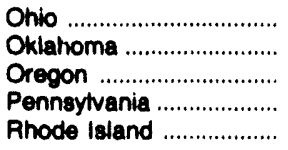 & $\begin{array}{l}4.13 \\
1.96 \\
3.30 \\
3.60 \\
2.75\end{array}$ & $\begin{array}{l}4.44 \\
2.00 \\
3.30 \\
3.80 \\
4.74\end{array}$ & $\begin{array}{l}3.82 \\
1.85 \\
3.32 \\
4.16 \\
3.84\end{array}$ & $\begin{array}{l}3.86 \\
1.81 \\
3.29 \\
4.01 \\
3.88\end{array}$ & $\begin{array}{l}3.80 \\
1.98 \\
3.28 \\
3.81 \\
4.14\end{array}$ & $\begin{array}{l}3.77 \\
1.91 \\
3.33 \\
3.83 \\
5.65\end{array}$ & $\begin{array}{l}3.87 \\
2.02 \\
3.31 \\
3.94 \\
6.46\end{array}$ & $\begin{array}{l}4.26 \\
1.83 \\
3.40 \\
3.78 \\
6.43\end{array}$ \\
\hline $\begin{array}{l}\text { South Carolina } \\
\text { South Dakota } \\
\text { Tonnesseo } \\
\text { Texas }\end{array}$ & $\begin{array}{l}3.06 \\
4.09 \\
3.37 \\
2.23 \\
3.58\end{array}$ & $\begin{array}{l}2.80 \\
3.79 \\
3.08 \\
1.91 \\
3.65\end{array}$ & $\begin{array}{l}2.84 \\
3.79 \\
3.30 \\
1.90 \\
3.74\end{array}$ & $\begin{array}{l}2.68 \\
3.45 \\
3.14 \\
1.84 \\
3.73\end{array}$ & $\begin{array}{l}2.64 \\
3.25 \\
3.01 \\
1.78 \\
3.66\end{array}$ & $\begin{array}{l}2.84 \\
3.22 \\
2.98 \\
1.67 \\
4.02\end{array}$ & $\begin{array}{l}2.98 \\
3.28 \\
3.02 \\
1.62 \\
6.10\end{array}$ & $\begin{array}{l}3.38 \\
3.65 \\
3.56 \\
2.07 \\
3.76\end{array}$ \\
\hline 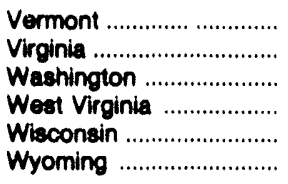 & $\begin{array}{l}3.57 \\
3.88 \\
2.71 \\
3.18 \\
3.24 \\
3.03\end{array}$ & $\begin{array}{l}3.32 \\
3.79 \\
2.72 \\
3.00 \\
3.10 \\
3.04\end{array}$ & $\begin{array}{l}3.22 \\
2.70 \\
2.82 \\
2.71 \\
3.07 \\
2.00\end{array}$ & $\begin{array}{l}3.09 \\
2.85 \\
2.85 \\
2.79 \\
2.98 \\
2.80\end{array}$ & $\begin{array}{l}2.97 \\
3.35 \\
2.81 \\
2.74 \\
2.97 \\
2.81\end{array}$ & $\begin{array}{l}2.76 \\
3.62 \\
2.84 \\
2.77 \\
3.09 \\
2.86\end{array}$ & $\begin{array}{l}2.88 \\
4.00 \\
2.81 \\
2.79 \\
3.10 \\
2.85\end{array}$ & $\begin{array}{l}3.09 \\
4.08 \\
3.11 \\
2.72 \\
3.46 \\
2.89\end{array}$ \\
\hline Total .......................... & 2.71 & 2.54 & 2.53 & 2.44 & 2.54 & 2.58 & 2.78 & 3.04 \\
\hline
\end{tabular}

- The price for small volumes of natural gas representing onsystem sales to industrial consumers in idaho is included as an annual figure but not distributed to monthly components.

= Revised Data.

$M=$ Not Available.

- $=$ Not Applicable.

Notes: Data for 1992 are final. All other data are preliminary unless otherwise indicated. Geographic coverage is the 50 States and the District of Columbia. Average prices for gas delivered to industrial consumers reflect onsystem sales prices only. See Appendix A, Explanatory Note 5 for discuseion of computations and revision policy. See Table 28 for data on onsystem sales expressed as a percentage of both total commercial and total industrial delverles.

Source: Form EIA-857. 
Table 27. Average Price of Natural Gas Delivered to Electric Utilitya Consumers by State, 1991-1993 (Dollars per Thousand Cubic Feet)

\begin{tabular}{|c|c|c|c|c|c|c|c|c|}
\hline State & $\begin{array}{l}\text { YTD } \\
1993\end{array}$ & $\begin{array}{c}\text { YTD } \\
1992\end{array}$ & $\begin{array}{c}\text { YTD } \\
1991\end{array}$ & $\begin{array}{c}\text { July } \\
1983\end{array}$ & $\begin{array}{l}\text { June } \\
1993\end{array}$ & $\begin{array}{c}\text { May } \\
1993\end{array}$ & $\begin{array}{l}\text { April } \\
1993\end{array}$ & $\begin{array}{c}\text { March } \\
1993\end{array}$ \\
\hline 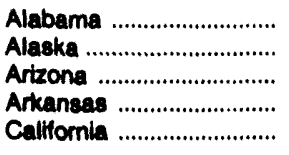 & $\begin{array}{r}2.51 \\
.63 \\
2.87 \\
2.07 \\
3.16\end{array}$ & $\begin{array}{r}1.86 \\
.58 \\
2.13 \\
1.48 \\
2.55\end{array}$ & $\begin{array}{r}1.86 \\
.53 \\
1.93 \\
1.41 \\
3.06\end{array}$ & $\begin{array}{r}2.42 \\
.63 \\
2.89 \\
2.23 \\
3.12\end{array}$ & $\begin{array}{r}2.50 \\
.61 \\
3.14 \\
2.14 \\
3.01\end{array}$ & $\begin{array}{r}3.18 \\
.63 \\
3.21 \\
1.71 \\
3.56\end{array}$ & $\begin{array}{r}2.69 \\
.63 \\
2.64 \\
1.61 \\
3.20\end{array}$ & $\begin{array}{r}2.42 \\
.64 \\
2.70 \\
1.50 \\
3.09\end{array}$ \\
\hline 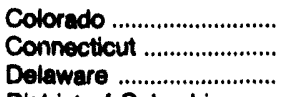 & $\begin{array}{l}2.32 \\
3.75 \\
3.29\end{array}$ & $\begin{array}{l}1.99 \\
2.56 \\
2.52\end{array}$ & $\begin{array}{l}2.20 \\
2.08 \\
2.40\end{array}$ & $\begin{array}{l}2.19 \\
2.53 \\
2.82\end{array}$ & $\begin{array}{l}2.14 \\
6.19 \\
3.05\end{array}$ & $\begin{array}{l}2.46 \\
9.99 \\
3.80\end{array}$ & $\begin{array}{l}2.22 \\
8.63 \\
5.31\end{array}$ & $\begin{array}{l}2.39 \\
6.07 \\
2.78\end{array}$ \\
\hline $\begin{array}{l}\text { Dletrict of Columbia ........ } \\
\text { Florida }\end{array}$ & $2 . \overline{43}$ & $2 . \overline{13}$ & $2 . \overline{13}$ & $2 . \overline{39}$ & $2 . \overline{37}$ & $2 . \overline{67}$ & $2 . \overline{59}$ & $2 . \overline{41}$ \\
\hline 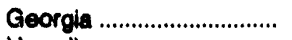 & 3.30 & 2.82 & 2.98 & 3.22 & 3.32 & 4.25 & 3.82 & 3.90 \\
\hline Hawail .................................. & - & - & - & - & - & - & - & - \\
\hline $\begin{array}{l}\text { Idaho } \\
\text { Illinols }\end{array}$ & $2 \overline{61}$ & $2 . \overline{11}$ & $2 . \overline{22}$ & $2 . \overline{43}$ & $2 . \overline{42}$ & $2 \overline{57}$ & $\overrightarrow{202}$ & $\overline{300}$ \\
\hline 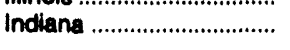 & 2.78 & 2.31 & $\begin{array}{l}2.22 \\
2.20\end{array}$ & $\begin{array}{l}2.43 \\
2.88\end{array}$ & $\begin{array}{l}2.42 \\
2.81\end{array}$ & $\begin{array}{l}2.57 \\
3.04\end{array}$ & $\begin{array}{l}2.62 \\
2.57\end{array}$ & $\begin{array}{l}3.00 \\
2.42\end{array}$ \\
\hline 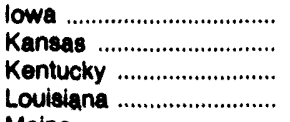 & $\begin{array}{l}3.08 \\
2.31 \\
3.10 \\
2.41\end{array}$ & $\begin{array}{l}2.78 \\
1.86 \\
2.62 \\
1.68\end{array}$ & $\begin{array}{l}2.47 \\
1.64 \\
2.77 \\
1.54\end{array}$ & $\begin{array}{l}2.84 \\
2.27 \\
2.82 \\
2.34\end{array}$ & $\begin{array}{l}3.30 \\
2.18 \\
3.06 \\
2.34\end{array}$ & $\begin{array}{l}3.65 \\
2.56 \\
3.87 \\
2.98\end{array}$ & $\begin{array}{l}3.33 \\
2.53 \\
3.22 \\
2.55\end{array}$ & $\begin{array}{l}3.30 \\
2.29 \\
2.76 \\
2.31\end{array}$ \\
\hline 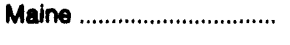 & - & - & - & - & - & - & - & - \\
\hline 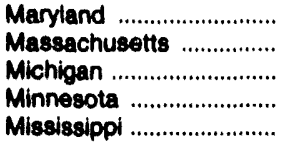 & $\begin{array}{r}2.87 \\
2.77 \\
.77 \\
2.52 \\
2.40\end{array}$ & $\begin{array}{r}2.41 \\
2.39 \\
.87 \\
1.70 \\
1.72\end{array}$ & $\begin{array}{r}2.33 \\
2.28 \\
.65 \\
1.62 \\
1.58\end{array}$ & $\begin{array}{l}2.70 \\
2.57 \\
1.36 \\
2.27 \\
2.42\end{array}$ & $\begin{array}{r}2.96 \\
2.47 \\
.87 \\
2.26 \\
2.44\end{array}$ & $\begin{array}{r}3.43 \\
3.01 \\
.30 \\
2.82 \\
2.85\end{array}$ & $\begin{array}{r}3.10 \\
2.88 \\
.38 \\
2.68 \\
2.30\end{array}$ & $\begin{array}{r}2.82 \\
2.92 \\
.45 \\
2.38 \\
2.23\end{array}$ \\
\hline 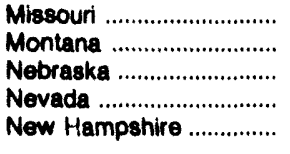 & $\begin{array}{l}2.38 \\
5.32 \\
2.49 \\
2.39 \\
2.21\end{array}$ & $\begin{array}{r}1.83 \\
3.20 \\
2.10 \\
1.71 \\
-\end{array}$ & $\begin{array}{r}1.46 \\
5.55 \\
1.74 \\
1.73 \\
-\end{array}$ & $\begin{array}{l}2.34 \\
4.81 \\
2.35 \\
2.17 \\
2.11\end{array}$ & $\begin{array}{l}2.26 \\
3.87 \\
2.26 \\
2.17 \\
2.21\end{array}$ & $\begin{array}{l}2.79 \\
3.34 \\
2.87 \\
2.45 \\
2.21\end{array}$ & $\begin{array}{l}2.56 \\
8.86 \\
2.45 \\
2.22 \\
2.22\end{array}$ & $\begin{array}{r}2.71 \\
10.41 \\
2.46 \\
2.42 \\
-\end{array}$ \\
\hline 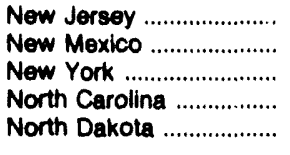 & $\begin{array}{l}2.33 \\
2.22 \\
2.75 \\
3.57 \\
2.20\end{array}$ & $\begin{array}{r}2.02 \\
1.73 \\
2.28 \\
2.74 \\
-\end{array}$ & $\begin{array}{l}2.00 \\
1.62 \\
2.24 \\
2.80 \\
3.38\end{array}$ & $\begin{array}{l}2.20 \\
2.08 \\
2.46 \\
3.26 \\
4.62\end{array}$ & $\begin{array}{r}2.44 \\
1.96 \\
2.64 \\
3.50 \\
-\end{array}$ & $\begin{array}{r}2.70 \\
2.61 \\
3.24 \\
3.90 \\
-\end{array}$ & $\begin{array}{l}2.72 \\
2.36 \\
2.87 \\
3.38 \\
4.27\end{array}$ & $\begin{array}{r}2.51 \\
2.21 \\
2.76 \\
3.71 \\
-\end{array}$ \\
\hline 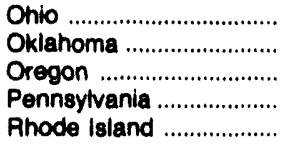 & $\begin{array}{l}2.98 \\
3.29 \\
2.23 \\
2.58 \\
2.65\end{array}$ & $\begin{array}{l}2.23 \\
3.03 \\
1.71 \\
2.66 \\
2.18\end{array}$ & $\begin{array}{l}2.16 \\
2.99 \\
1.34 \\
3.10 \\
1.96\end{array}$ & $\begin{array}{r}3.67 \\
2.94 \\
-\overline{54} \\
2.64\end{array}$ & $\begin{array}{r}3.32 \\
3.18 \\
-\overline{56} \\
2.66\end{array}$ & $\begin{array}{r}3.54 \\
3.68 \\
-\overline{3.21} \\
-\end{array}$ & $\begin{array}{r}3.72 \\
3.39 \\
-\overline{14} \\
-\end{array}$ & $\begin{array}{r}2.34 \\
3.57 \\
2.09 \\
2.58 \\
-\end{array}$ \\
\hline 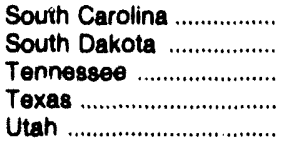 & $\begin{array}{r}2.85 \\
1.47 \\
2 . \overline{43} \\
2.11\end{array}$ & $\begin{array}{r}1.69 \\
.95 \\
-\overline{2.02} \\
1.79\end{array}$ & $\begin{array}{r}1.56 \\
1.71 \\
.85 \\
1.93 \\
1.74\end{array}$ & $\begin{array}{r}2.82 \\
- \\
2.32 \\
2.60\end{array}$ & $\begin{array}{r}3.08 \\
- \\
2 . \overline{31} \\
2.17\end{array}$ & $\begin{array}{r}3.37 \\
-- \\
-\overline{72} \\
2.73\end{array}$ & $\begin{array}{r}3.18 \\
- \\
-\overline{58} \\
1.87\end{array}$ & $\begin{array}{r}3.20 \\
2.62 \\
-\overline{40} \\
1.80\end{array}$ \\
\hline 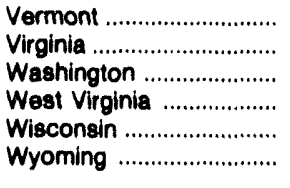 & $\begin{array}{l}2.02 \\
2.83 \\
4.28 \\
3.20 \\
2.66 \\
3.35\end{array}$ & $\begin{array}{l}1.91 \\
2.39 \\
4.05 \\
3.14 \\
2.11 \\
3.40\end{array}$ & $\begin{array}{l}1.59 \\
1.78 \\
4.02 \\
3.79 \\
2.71 \\
3.55\end{array}$ & $\begin{array}{l}1.86 \\
2.79 \\
3.44 \\
4.27 \\
2.46 \\
3.71\end{array}$ & $\begin{array}{l}1.83 \\
3.02 \\
5.80 \\
5.40 \\
2.62 \\
3.39\end{array}$ & $\begin{array}{l}2.28 \\
5.31 \\
7.68 \\
3.86 \\
3.02 \\
3.47\end{array}$ & $\begin{array}{l}2.28 \\
2.90 \\
6.52 \\
3.80 \\
2.66 \\
3.41\end{array}$ & $\begin{array}{l}3.33 \\
3.01 \\
4.05 \\
4.18 \\
2.33 \\
3.20\end{array}$ \\
\hline 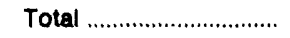 & 2.60 & 2.14 & 2.12 & 2.46 & 2.47 & 2.90 & 2.75 & 2.61 \\
\hline
\end{tabular}

See footnotes at end of table. 
Table 27. Average Price of Natural Gas Delivered to Electric Utilitya Consumers by State, 1991-1993 (Continued) (Dollars per Thousand Cubic Feet)

\begin{tabular}{|c|c|c|c|c|c|c|c|c|}
\hline State & $\begin{array}{c}\text { February } \\
1993\end{array}$ & $\begin{array}{c}\text { January } \\
1993\end{array}$ & $\begin{array}{l}\text { Total } \\
1992\end{array}$ & $\begin{array}{c}\text { December } \\
1992\end{array}$ & $\begin{array}{c}\text { November } \\
1992\end{array}$ & $\begin{array}{c}\text { October } \\
1992\end{array}$ & $\begin{array}{c}\text { September } \\
1992\end{array}$ & $\begin{array}{c}\text { August } \\
1992\end{array}$ \\
\hline 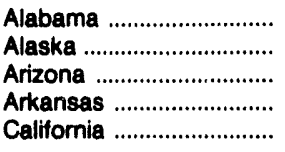 & $\begin{array}{r}2.29 \\
.64 \\
3.12 \\
1.38 \\
3.02\end{array}$ & $\begin{array}{r}2.59 \\
.64 \\
3.81 \\
1.64 \\
3.29\end{array}$ & $\begin{array}{r}2.28 \\
.57 \\
2.28 \\
1.57 \\
2.81\end{array}$ & $\begin{array}{r}2.88 \\
.56 \\
3.99 \\
1.35 \\
3.24\end{array}$ & $\begin{array}{r}2.79 \\
.56 \\
3.47 \\
1.15 \\
3.28\end{array}$ & $\begin{array}{r}3.20 \\
.56 \\
2.40 \\
2.18 \\
3.41\end{array}$ & $\begin{array}{r}2.43 \\
.57 \\
2.40 \\
1.85 \\
2.93\end{array}$ & $\begin{array}{r}2.36 \\
.54 \\
2.40 \\
1.85 \\
2.73\end{array}$ \\
\hline 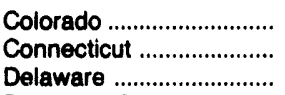 & $\begin{array}{l}2.33 \\
3.50 \\
4.38\end{array}$ & $\begin{array}{l}2.51 \\
6.86 \\
3.65\end{array}$ & $\begin{array}{l}2.14 \\
2.74 \\
2.70\end{array}$ & $\begin{array}{l}2.49 \\
6.11 \\
5.40\end{array}$ & $\begin{array}{l}2.45 \\
7.15 \\
4.89\end{array}$ & $\begin{array}{l}2.50 \\
6.29 \\
4.31\end{array}$ & $\begin{array}{l}1.94 \\
6.31 \\
3.32\end{array}$ & $\begin{array}{l}1.80 \\
2.82 \\
2.68\end{array}$ \\
\hline 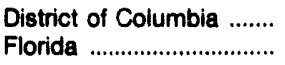 & $2 . \overline{23}$ & $2 . \overline{27}$ & $2 . \overline{30}$ & $2 . \overline{32}$ & $2 . \overline{65}$ & $2 . \overline{88}$ & $2 . \overline{59}$ & $2 . \overline{51}$ \\
\hline 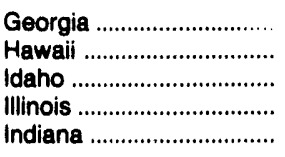 & $\begin{array}{r}3.51 \\
- \\
-\overline{0} \\
2.08\end{array}$ & $\begin{array}{r}3.90 \\
- \\
2 . \overline{9} \\
2.87\end{array}$ & $\begin{array}{r}2.89 \\
-- \\
2 . \overline{24} \\
2.48\end{array}$ & $\begin{array}{r}4.38 \\
-\overline{-} \\
3.11 \\
2.81\end{array}$ & $\begin{array}{r}4.05 \\
-- \\
3.05 \\
3.21\end{array}$ & $\begin{array}{r}4.32 \\
-- \\
-- \\
2.93 \\
3.14\end{array}$ & $\begin{array}{r}2.30 \\
- \\
2 . \overline{-} \\
3.00\end{array}$ & $\begin{array}{r}2.75 \\
-- \\
-\overline{2.43} \\
2.57\end{array}$ \\
\hline 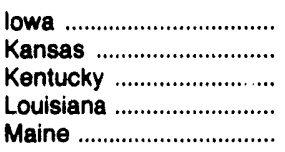 & $\begin{array}{r}3.34 \\
2.42 \\
2.74 \\
2.10 \\
--\end{array}$ & $\begin{array}{r}3.37 \\
2.40 \\
2.99 \\
2.35 \\
--\end{array}$ & $\begin{array}{r}3.08 \\
1.94 \\
2.77 \\
1.91 \\
--\end{array}$ & $\begin{array}{r}3.42 \\
2.42 \\
3.12 \\
2.35 \\
--\end{array}$ & $\begin{array}{r}3.37 \\
2.22 \\
3.24 \\
2.51 \\
--\end{array}$ & $\begin{array}{r}3.77 \\
2.14 \\
3.38 \\
2.73 \\
--\end{array}$ & $\begin{array}{r}3.51 \\
1.99 \\
2.67 \\
2.16 \\
--\end{array}$ & $\begin{array}{r}3.32 \\
1.98 \\
2.59 \\
2.04 \\
--\end{array}$ \\
\hline 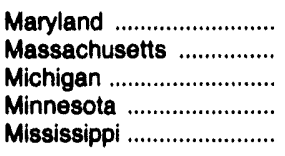 & $\begin{array}{r}2.86 \\
3.25 \\
.85 \\
2.52 \\
1.98\end{array}$ & $\begin{array}{r}3.14 \\
3.51 \\
.68 \\
2.81 \\
2.06\end{array}$ & $\begin{array}{r}2.66 \\
2.68 \\
.81 \\
1.85 \\
1.85\end{array}$ & $\begin{array}{r}4.07 \\
3.70 \\
.64 \\
2.74 \\
2.34\end{array}$ & $\begin{array}{r}3.79 \\
3.27 \\
.67 \\
2.52 \\
2.46\end{array}$ & $\begin{array}{r}3.78 \\
3.43 \\
.56 \\
3.35 \\
2.66\end{array}$ & $\begin{array}{l}2.82 \\
2.81 \\
1.28 \\
1.89 \\
2.22\end{array}$ & $\begin{array}{r}2.73 \\
2.67 \\
.51 \\
1.81 \\
2.17\end{array}$ \\
\hline 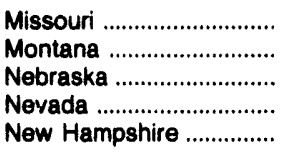 & $\begin{array}{l}2.42 \\
2.78 \\
2.61 \\
3.11 \\
--\end{array}$ & $\begin{array}{r}2.62 \\
7.62 \\
2.88 \\
3.17 \\
-\end{array}$ & $\begin{array}{l}1.89 \\
3.30 \\
2.28 \\
1.91 \\
2.20\end{array}$ & $\begin{array}{l}2.75 \\
2.44 \\
2.84 \\
3.12 \\
-\end{array}$ & $\begin{array}{r}2.61 \\
2.38 \\
2.32 \\
2.44 \\
--\end{array}$ & $\begin{array}{r}2.13 \\
9.41 \\
2.46 \\
2.55 \\
--\end{array}$ & $\begin{array}{l}2.31 \\
3.76 \\
2.27 \\
1.87 \\
2.20\end{array}$ & $\begin{array}{l}2.14 \\
2.93 \\
2.50 \\
2.25 \\
2.20\end{array}$ \\
\hline $\begin{array}{l}\text { New Jersey .................... } \\
\text { New Mexico ..................... } \\
\text { New York .......................... } \\
\text { North Carolina ................. } \\
\text { North Dakota ................... }\end{array}$ & $\begin{array}{r}2.35 \\
2.06 \\
2.66 \\
4.11 \\
--\end{array}$ & $\begin{array}{r}2.66 \\
2.35 \\
3.02 \\
4.16 \\
--\end{array}$ & $\begin{array}{l}2.18 \\
1.99 \\
2.48 \\
2.96 \\
4.18\end{array}$ & $\begin{array}{l}3.09 \\
2.40 \\
3.21 \\
4.15 \\
4.46\end{array}$ & $\begin{array}{r}2.98 \\
2.45 \\
3.12 \\
4.10 \\
--\end{array}$ & $\begin{array}{r}2.92 \\
2.67 \\
3.22 \\
3.22 \\
-\end{array}$ & $\begin{array}{l}2.42 \\
2.30 \\
2.55 \\
3.15 \\
4.05\end{array}$ & $\begin{array}{l}2.35 \\
2.11 \\
2.39 \\
2.83 \\
4.30\end{array}$ \\
\hline 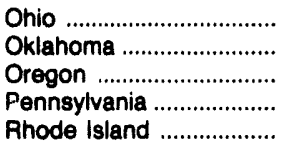 & $\begin{array}{r}2.64 \\
3.37 \\
1.99 \\
2.41 \\
-\end{array}$ & $\begin{array}{r}2.79 \\
3.31 \\
2.96 \\
2.47 \\
-\end{array}$ & $\begin{array}{l}2.31 \\
3.20 \\
1.97 \\
3.06 \\
2.20\end{array}$ & $\begin{array}{r}2.59 \\
3.25 \\
2.44 \\
3.51 \\
--\end{array}$ & $\begin{array}{r}4.17 \\
3.76 \\
2.15 \\
5.19 \\
--\end{array}$ & $\begin{array}{r}2.66 \\
4.11 \\
2.40 \\
4.07 \\
--\end{array}$ & $\begin{array}{l}1.97 \\
3.29 \\
2.05 \\
3.49 \\
2.84\end{array}$ & $\begin{array}{l}2.01 \\
3.19 \\
1.99 \\
3.20 \\
2.45\end{array}$ \\
\hline 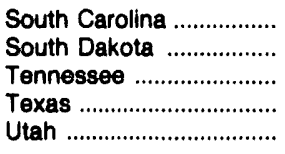 & $\begin{array}{r}3.61 \\
- \\
-\overline{32} \\
1.87\end{array}$ & $\begin{array}{r}3.78 \\
- \\
-\overline{46} \\
2.03\end{array}$ & $\begin{array}{r}1.73 \\
2.88 \\
-\overline{2.25} \\
1.87\end{array}$ & $\begin{array}{r}3.72 \\
2.95 \\
-\overline{-} \\
2.67 \\
2.27\end{array}$ & $\begin{array}{r}3.33 \\
2.92 \\
-- \\
2.69 \\
1.80\end{array}$ & $\begin{array}{r}3.30 \\
4.04 \\
-\overline{90} \\
2.90 \\
1.99\end{array}$ & $\begin{array}{r}2.74 \\
-\overline{-} \\
2.34 \\
1.98\end{array}$ & $\begin{array}{r}2.46 \\
2.61 \\
-\overline{2} \\
2.30 \\
1.92\end{array}$ \\
\hline 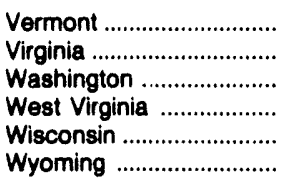 & $\begin{array}{r}3.60 \\
3.10 \\
3.73 \\
2 . \overline{68} \\
3.21\end{array}$ & $\begin{array}{l}3.30 \\
2.57 \\
3.70 \\
4.04 \\
3.22 \\
3.25\end{array}$ & $\begin{array}{l}2.00 \\
2.47 \\
3.31 \\
3.53 \\
2.42 \\
3.33\end{array}$ & $\begin{array}{l}3.14 \\
2.70 \\
4.54 \\
3.51 \\
3.41 \\
3.29\end{array}$ & $\begin{array}{r}3.02 \\
.48 \\
4.57 \\
3.27 \\
3.33 \\
3.22\end{array}$ & $\begin{array}{l}2.18 \\
5.05 \\
5.97 \\
3.34 \\
3.47 \\
3.21\end{array}$ & $\begin{array}{l}2.24 \\
2.20 \\
3.32 \\
4.27 \\
2.80 \\
3.19\end{array}$ & $\begin{array}{l}2.16 \\
3.10 \\
2.94 \\
3.40 \\
2.30 \\
3.22\end{array}$ \\
\hline 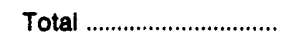 & 2.55 & 2.70 & 2.36 & 2.81 & 2.87 & 3.04 & 2.51 & 2.42 \\
\hline
\end{tabular}

See footnotes at end of table. 
Table 27. Average Price of Natural Gas Delivered to Electric Utilitya Consumers by State, 1991-1993 (Continued)

(Dollars per Thousand Cubic Feet)

\begin{tabular}{|c|c|c|c|c|c|c|c|c|}
\hline State & $\begin{array}{c}\text { July } \\
1992\end{array}$ & $\begin{array}{l}\text { June } \\
1992\end{array}$ & $\begin{array}{l}\text { May } \\
1992\end{array}$ & $\begin{array}{l}\text { April } \\
1992\end{array}$ & $\begin{array}{l}\text { March } \\
1992\end{array}$ & $\begin{array}{c}\text { February } \\
1992\end{array}$ & $\begin{array}{l}\text { January } \\
1992\end{array}$ & $\begin{array}{l}\text { Total } \\
1991\end{array}$ \\
\hline 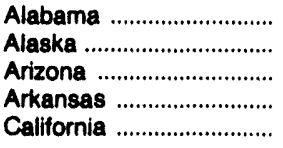 & $\begin{array}{r}1.98 \\
.55 \\
2.21 \\
1.64 \\
2.57\end{array}$ & $\begin{array}{r}2.15 \\
.57 \\
2.27 \\
1.50 \\
2.39\end{array}$ & $\begin{array}{r}2.05 \\
.55 \\
2.14 \\
1.52 \\
239\end{array}$ & $\begin{array}{r}1.86 \\
.56 \\
1.81 \\
1.44 \\
2.50\end{array}$ & $\begin{array}{r}1.70 \\
.59 \\
2.02 \\
1.36 \\
2.36\end{array}$ & $\begin{array}{r}1.62 \\
.60 \\
2.09 \\
1.33 \\
2.42\end{array}$ & $\begin{array}{r}2.28 \\
.62 \\
2.35 \\
1.33 \\
3.22\end{array}$ & $\begin{array}{r}1.91 \\
.53 \\
2.06 \\
1.44 \\
2.95\end{array}$ \\
\hline 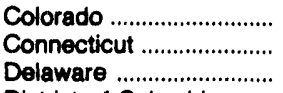 & $\begin{array}{l}1.98 \\
2.58 \\
2.22\end{array}$ & $\begin{array}{l}1.84 \\
2.62 \\
2.47\end{array}$ & $\begin{array}{l}1.98 \\
2.57 \\
2.86\end{array}$ & $\begin{array}{l}2.02 \\
2.25 \\
2.35\end{array}$ & $\begin{array}{l}1.81 \\
2.23 \\
2.68\end{array}$ & $\begin{array}{l}2.26 \\
6.29 \\
2.63\end{array}$ & $\begin{array}{l}2.20 \\
6.30 \\
2.80\end{array}$ & $\begin{array}{l}2.14 \\
2.16 \\
2.49\end{array}$ \\
\hline $\begin{array}{l}\text { District of Columbia } \\
\text { Florida }\end{array}$ & $2 . \overline{41}$ & $2 . \overline{41}$ & $2 . \overline{29}$ & 2.01 & $1 . \overline{82}$ & $1 . \overline{74}$ & $1 . \overline{93}$ & $2 . \overline{17}$ \\
\hline 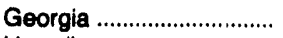 & 2.83 & 2.61 & 2.99 & 2.78 & 3.18 & 3.19 & 3.64 & 2.83 \\
\hline Hawaii ................................. & - & - & - & - & - & - & - & - \\
\hline 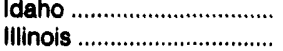 & $2 . \overline{37}$ & $2 . \overline{29}$ & $2 . \overline{07}$ & $2 . \overline{03}$ & $1 . \overline{69}$ & 2.10 & $2 \overline{64}$ & $2 . \overline{14}$ \\
\hline 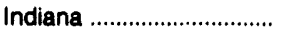 & 2.31 & 2.30 & 2.11 & 2.44 & 2.18 & 2.19 & 2.63 & 2.38 \\
\hline 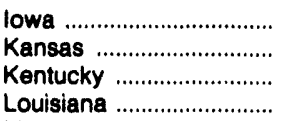 & $\begin{array}{l}2.80 \\
1.83 \\
2.46 \\
1.72\end{array}$ & $\begin{array}{l}3.01 \\
2.08 \\
2.60 \\
1.90\end{array}$ & $\begin{array}{l}2.93 \\
1.67 \\
2.71 \\
1.78\end{array}$ & $\begin{array}{l}2.76 \\
1.73 \\
2.67 \\
1.56\end{array}$ & $\begin{array}{l}2.56 \\
1.87 \\
2.57 \\
1.36\end{array}$ & $\begin{array}{l}2.35 \\
1.85 \\
2.32 \\
1.31\end{array}$ & $\begin{array}{l}3.23 \\
2.02 \\
2.80 \\
1.75\end{array}$ & $\begin{array}{l}2.70 \\
1.65 \\
2.65 \\
1.59\end{array}$ \\
\hline 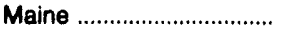 & -- & - & -- & -- & -- & - & $-\cdots$ & - \\
\hline 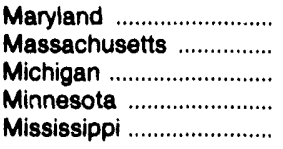 & $\begin{array}{r}2.33 \\
2.58 \\
.63 \\
1.76 \\
1.87\end{array}$ & $\begin{array}{r}2.45 \\
2.37 \\
.81 \\
1.64 \\
1.97\end{array}$ & $\begin{array}{l}2.15 \\
2.17 \\
1.34 \\
1.54 \\
1.72\end{array}$ & $\begin{array}{r}2.38 \\
2.19 \\
.93 \\
1.62 \\
1.65\end{array}$ & $\begin{array}{r}2.57 \\
2.39 \\
.71 \\
1.53 \\
1.49\end{array}$ & $\begin{array}{r}2.75 \\
3.98 \\
.94 \\
1.65 \\
1.33\end{array}$ & $\begin{array}{r}3.44 \\
4.48 \\
.66 \\
2.29 \\
2.26\end{array}$ & $\begin{array}{r}2.36 \\
2.27 \\
.76 \\
1.71 \\
1.61\end{array}$ \\
\hline 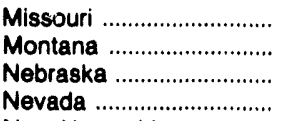 & $\begin{array}{l}1.49 \\
2.19 \\
2.12 \\
1.86\end{array}$ & $\begin{array}{l}1.94 \\
2.48 \\
2.10 \\
1.95\end{array}$ & $\begin{array}{l}2.12 \\
3.04 \\
2.31 \\
1.59\end{array}$ & $\begin{array}{l}1.60 \\
1.40 \\
1.54 \\
1.43\end{array}$ & $\begin{array}{l}2.89 \\
6.28 \\
2.02 \\
1.53\end{array}$ & $\begin{array}{l}1.72 \\
8.22 \\
2.58 \\
1.45\end{array}$ & $\begin{array}{l}2.89 \\
7.35 \\
2.80 \\
1.94\end{array}$ & $\begin{array}{l}1.51 \\
4.33 \\
1.85 \\
1.78\end{array}$ \\
\hline New Hampshire ............... & -- & -- & - & - & -- & - & - & - \\
\hline 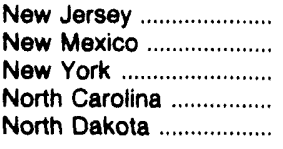 & $\begin{array}{l}2.02 \\
1.72 \\
2.06 \\
2.78 \\
4.03\end{array}$ & $\begin{array}{r}2.23 \\
1.77 \\
2.30 \\
2.83 \\
-\end{array}$ & $\begin{array}{r}2.26 \\
1.72 \\
2.28 \\
2.52 \\
--\end{array}$ & $\begin{array}{r}1.84 \\
1.61 \\
2.19 \\
2.53 \\
\ldots\end{array}$ & $\begin{array}{l}1.73 \\
1.64 \\
2.27 \\
2.69 \\
4.28\end{array}$ & $\begin{array}{r}2.22 \\
1.54 \\
2.37 \\
2.68 \\
-\end{array}$ & $\begin{array}{r}2.83 \\
2.09 \\
2.82 \\
2.86 \\
--\end{array}$ & $\begin{array}{l}2.02 \\
1.73 \\
2.30 \\
2.76 \\
4.36\end{array}$ \\
\hline 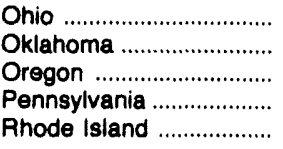 & $\begin{array}{l}2.11 \\
2.92 \\
1.72 \\
2.34 \\
2.45\end{array}$ & $\begin{array}{l}2.14 \\
3.08 \\
1.88 \\
2.73 \\
2.27\end{array}$ & $\begin{array}{r}2.06 \\
2.72 \\
-- \\
3.16 \\
2.13\end{array}$ & $\begin{array}{r}2.08 \\
2.74 \\
-- \\
2.90 \\
2.04\end{array}$ & $\begin{array}{r}2.49 \\
2.92 \\
-- \\
2.91 \\
1.86\end{array}$ & $\begin{array}{r}2.31 \\
3.35 \\
1.53 \\
3.07 \\
--\end{array}$ & $\begin{array}{r}2.46 \\
3.83 \\
1.81 \\
3.09 \\
--\end{array}$ & $\begin{array}{l}2.19 \\
2.98 \\
1.59 \\
3.05 \\
2.04\end{array}$ \\
\hline 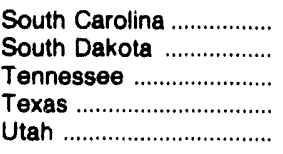 & $\begin{array}{r}1.91 \\
-- \\
1.99 \\
1.76\end{array}$ & $\begin{array}{r}1.71 \\
- \\
-\overline{0} \\
2.06 \\
1.74\end{array}$ & $\begin{array}{r}2.56 \\
-- \\
2.03 \\
1.67\end{array}$ & $\begin{array}{r}3.15 \\
-- \\
1.99 \\
1.82\end{array}$ & $\begin{array}{r}1.46 \\
-- \\
1.94 \\
1.83\end{array}$ & $\begin{array}{r}3.29 \\
- \\
1.92 \\
1.88\end{array}$ & $\begin{array}{r}3.45 \\
2.60 \\
-\overline{2.23} \\
1.82\end{array}$ & $\begin{array}{r}1.53 \\
1.77 \\
-\overline{2} \\
2.03 \\
1.72\end{array}$ \\
\hline 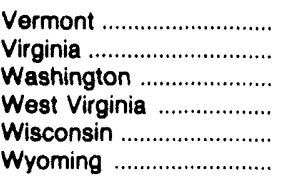 & $\begin{array}{l}2.09 \\
2.18 \\
2.95 \\
5.36 \\
2.21 \\
3.22\end{array}$ & $\begin{array}{l}1.94 \\
2.67 \\
3.31 \\
3.26 \\
2.06 \\
3.31\end{array}$ & $\begin{array}{l}1.77 \\
2.59 \\
3.57 \\
1.21 \\
2.01 \\
3.37\end{array}$ & $\begin{array}{r}1.74 \\
2.32 \\
7.71 \\
-\overline{1.94} \\
3.36\end{array}$ & $\begin{array}{l}1.63 \\
2.22 \\
4.18 \\
3.66 \\
1.88 \\
3.48\end{array}$ & $\begin{array}{l}2.57 \\
2.43 \\
3.13 \\
3.89 \\
2.72 \\
3.49\end{array}$ & $\begin{array}{l}2.85 \\
2.54 \\
3.01 \\
3.60 \\
2.89 \\
3.51\end{array}$ & $\begin{array}{l}1.72 \\
1.90 \\
4.02 \\
3.63 \\
2.72 \\
3.51\end{array}$ \\
\hline 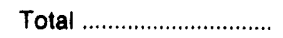 & 2.13 & 2.18 & 2.11 & R 2.07 & 1.99 & 2.03 & 2.49 & 2.18 \\
\hline
\end{tabular}

- Includes all steam electric utility generating plants with a combined capacity of 50 megawatts or greater.

- Average prices calculated from data reported on Form EIA-176.

R = Revised Data.

- $=$ Not Applicable.

Notes: Data for 1992 are final. All other data are preliminary unless otherwise indicated. Geographic coverage is the 50 States and the District of Columbia. See Appendix A, Explanatory Note 5 for discussion of computations and revision policy.

Sources: Form FERC-423 and Form EIA-176. 
Table 28. Percentage of Total Deliveries Represented by Onsystem Sales by State, 1991-1993

\begin{tabular}{|c|c|c|c|c|c|c|c|c|}
\hline \multirow{2}{*}{ State } & \multicolumn{2}{|c|}{$\begin{array}{c}\text { August } \\
1993\end{array}$} & \multicolumn{2}{|c|}{$\begin{array}{c}\text { July } \\
1993\end{array}$} & \multicolumn{2}{|c|}{$\begin{array}{l}\text { June } \\
1993\end{array}$} & \multicolumn{2}{|c|}{$\begin{array}{c}\text { May } \\
1993\end{array}$} \\
\hline & Commercial & Industrial & Commercial & Industrial & Commercial & Industrial & Commercial & Industrial \\
\hline 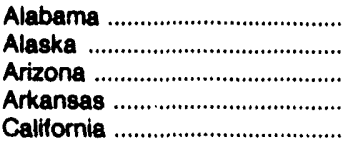 & $\begin{array}{r}68.8 \\
100.0 \\
88.2 \\
84.6 \\
68.9\end{array}$ & $\begin{array}{l}17.7 \\
61.3 \\
24.7 \\
14.5 \\
31.6\end{array}$ & $\begin{array}{r}70.9 \\
100.0 \\
87.9 \\
85.8 \\
75.1\end{array}$ & $\begin{array}{l}20.1 \\
73.3 \\
24.8 \\
13.1 \\
33.7\end{array}$ & $\begin{array}{r}71.9 \\
100.0 \\
90.3 \\
84.1 \\
70.0\end{array}$ & $\begin{array}{l}18.2 \\
79.7 \\
18.7 \\
14.3 \\
31.4\end{array}$ & $\begin{array}{r}75.3 \\
100.0 \\
90.9 \\
87.5 \\
75.6\end{array}$ & $\begin{array}{l}17.1 \\
70.9 \\
26.8 \\
14.3 \\
34.1\end{array}$ \\
\hline 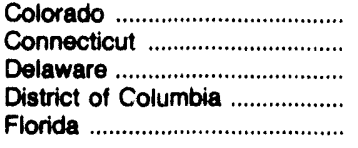 & $\begin{array}{r}92.7 \\
99.1 \\
100.0 \\
88.3 \\
96.9\end{array}$ & $\begin{array}{c}32.8 \\
93.0 \\
78.0 \\
\overline{17.2}\end{array}$ & $\begin{array}{r}92.6 \\
86.5 \\
100.0 \\
96.8 \\
97.0\end{array}$ & $\begin{array}{c}32.4 \\
68.1 \\
75.3 \\
\overline{17.5}\end{array}$ & $\begin{array}{r}95.2 \\
99.2 \\
100.0 \\
89.8 \\
97.6\end{array}$ & $\begin{array}{c}30.1 \\
71.9 \\
69.3 \\
- \\
35.4\end{array}$ & $\begin{array}{r}94.9 \\
99.3 \\
100.0 \\
100.0 \\
97.5\end{array}$ & $\begin{array}{c}30.1 \\
69.5 \\
75.0 \\
\overline{30.6}\end{array}$ \\
\hline 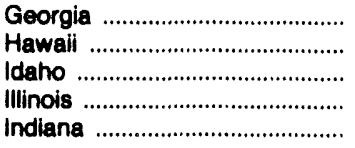 & $\begin{array}{r}83.9 \\
100.0 \\
81.4 \\
38.4 \\
90.5\end{array}$ & $\begin{array}{r}25.2 \\
-. \\
.0 \\
6.4 \\
15.6\end{array}$ & $\begin{array}{r}84.3 \\
100.0 \\
82.9 \\
43.0 \\
89.9\end{array}$ & $\begin{array}{r}30.3 \\
- \\
.0 \\
5.6 \\
13.0\end{array}$ & $\begin{array}{r}81.6 \\
100.0 \\
82.5 \\
43.5 \\
91.4\end{array}$ & $\begin{array}{r}23.1 \\
- \\
.0 \\
7.2 \\
14.2\end{array}$ & $\begin{array}{r}85.3 \\
100.0 \\
87.5 \\
51.6 \\
100.0\end{array}$ & $\begin{array}{r}23.4 \\
-- \\
.0 \\
8.5 \\
25.0\end{array}$ \\
\hline 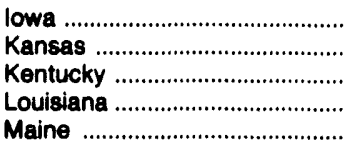 & $\begin{array}{r}88.2 \\
85.5 \\
85.2 \\
98.7 \\
100.0\end{array}$ & $\begin{array}{r}10.3 \\
19.9 \\
16.3 \\
31.6 \\
100.0\end{array}$ & $\begin{array}{r}89.7 \\
82.1 \\
89.2 \\
88.8 \\
100.0\end{array}$ & $\begin{array}{r}10.1 \\
16.9 \\
17.8 \\
31.0 \\
100.0\end{array}$ & $\begin{array}{r}90.3 \\
78.8 \\
93.0 \\
98.8 \\
100.0\end{array}$ & $\begin{array}{r}8.0 \\
14.4 \\
24.3 \\
31.8 \\
100.0\end{array}$ & $\begin{array}{r}92.3 \\
84.4 \\
91.9 \\
98.9 \\
100.0\end{array}$ & $\begin{array}{r}12.9 \\
12.3 \\
28.5 \\
34.6 \\
100.0\end{array}$ \\
\hline 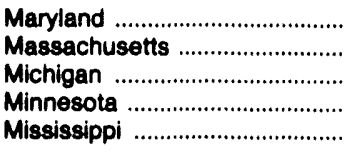 & $\begin{array}{l}93.3 \\
99.6 \\
52.9 \\
87.5 \\
99.1\end{array}$ & $\begin{array}{r}10.5 \\
54.0 \\
2.7 \\
30.2 \\
25.9\end{array}$ & $\begin{array}{l}94.9 \\
99.7 \\
48.9 \\
88.3 \\
88.4\end{array}$ & $\begin{array}{r}10.7 \\
52.3 \\
2.7 \\
32.8 \\
29.4\end{array}$ & $\begin{array}{l}96.1 \\
99.8 \\
50.0 \\
93.4 \\
91.6\end{array}$ & $\begin{array}{r}47.9 \\
53.0 \\
3.0 \\
48.4 \\
31.1\end{array}$ & $\begin{array}{l}94.6 \\
99.7 \\
62.2 \\
99.3 \\
95.6\end{array}$ & $\begin{array}{r}7.7 \\
58.6 \\
5.3 \\
50.1 \\
33.4\end{array}$ \\
\hline 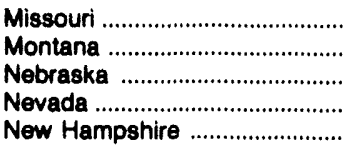 & $\begin{array}{r}68.2 \\
91.3 \\
66.2 \\
86.9 \\
100.0\end{array}$ & $\begin{array}{r}16.5 \\
8.3 \\
19.2 \\
4.2 \\
400.0\end{array}$ & $\begin{array}{r}71.2 \\
91.3 \\
87.5 \\
89.9 \\
100.0\end{array}$ & $\begin{array}{r}18.5 \\
7.7 \\
23.3 \\
2.3 \\
100.0\end{array}$ & $\begin{array}{r}73.8 \\
81.2 \\
87.7 \\
90.8 \\
100.0\end{array}$ & $\begin{array}{r}17.6 \\
9.9 \\
23.1 \\
3.8 \\
100.0\end{array}$ & $\begin{array}{r}80.2 \\
95.0 \\
97.8 \\
92.6 \\
100.0\end{array}$ & $\begin{array}{r}22.4 \\
13.8 \\
29.5 \\
3.4 \\
100.0\end{array}$ \\
\hline 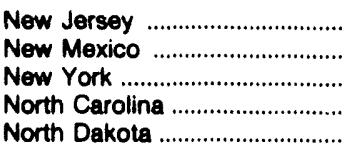 & $\begin{array}{l}80.3 \\
50.2 \\
73.3 \\
89.6 \\
54.0\end{array}$ & $\begin{array}{r}53.2 \\
7.1 \\
14.1 \\
54.0 \\
14.9\end{array}$ & $\begin{array}{l}87.5 \\
51.9 \\
70.3 \\
80.0 \\
58.1\end{array}$ & $\begin{array}{r}53.0 \\
4.9 \\
13.5 \\
53.0 \\
16.6\end{array}$ & $\begin{array}{l}86.8 \\
54.3 \\
67.5 \\
80.1 \\
62.6\end{array}$ & $\begin{array}{r}53.5 \\
2.1 \\
16.0 \\
54.8 \\
15.7\end{array}$ & $\begin{array}{r}88.8 \\
59.6 \\
72.5 \\
100.0 \\
73.3\end{array}$ & $\begin{array}{l}54.6 \\
10.4 \\
14.8 \\
94.3 \\
19.0\end{array}$ \\
\hline 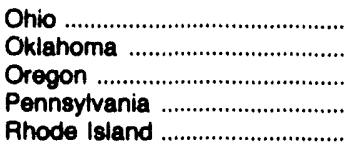 & $\begin{array}{r}69.1 \\
80.9 \\
97.5 \\
65.4 \\
100.0\end{array}$ & $\begin{array}{r}5.8 \\
31.9 \\
28.7 \\
19.3 \\
9.1\end{array}$ & $\begin{array}{r}74.3 \\
79.2 \\
97.6 \\
\text { ค } 72.5 \\
100.0\end{array}$ & $\begin{array}{r}8.1 \\
31.1 \\
25.7 \\
\text { ค } 17.1 \\
9.6\end{array}$ & $\begin{array}{r}73.6 \\
82.0 \\
97.8 \\
72.5 \\
100.0\end{array}$ & $\begin{array}{r}6.6 \\
28.3 \\
27.2 \\
17.6 \\
12.1\end{array}$ & $\begin{array}{r}75.8 \\
88.3 \\
97.6 \\
71.4 \\
100.0\end{array}$ & $\begin{array}{r}7.3 \\
27.3 \\
21.8 \\
23.6 \\
10.4\end{array}$ \\
\hline $\begin{array}{l}\text { South Carolina } \\
\text { South Dakota } \\
\text { Tennessee } \\
\text { Texas } \\
\text { Utah }\end{array}$ & $\begin{array}{r}93.5 \\
72.9 \\
89.4 \\
83.2 \\
100.0\end{array}$ & $\begin{array}{l}59.3 \\
46.4 \\
35.2 \\
31.5 \\
13.9\end{array}$ & $\begin{array}{r}94.0 \\
75.0 \\
89.9 \\
80.9 \\
100.0\end{array}$ & $\begin{array}{l}60.4 \\
55.2 \\
32.0 \\
29.7 \\
12.3\end{array}$ & $\begin{array}{r}100.0 \\
73.3 \\
89.6 \\
78.3 \\
100.0\end{array}$ & $\begin{array}{l}61.4 \\
38.4 \\
39.3 \\
27.5 \\
12.3\end{array}$ & $\begin{array}{r}100.0 \\
76.4 \\
97.3 \\
77.6 \\
100.0\end{array}$ & $\begin{array}{l}55.9 \\
43.7 \\
38.8 \\
29.9 \\
12.2\end{array}$ \\
\hline 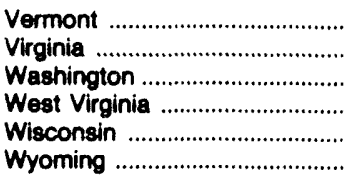 & $\begin{array}{r}100.0 \\
75.2 \\
90.5 \\
25.9 \\
87.0 \\
96.4\end{array}$ & $\begin{array}{r}100.0 \\
10.3 \\
33.3 \\
7.5 \\
37.3 \\
1.9\end{array}$ & $\begin{array}{r}100.0 \\
83.8 \\
90.6 \\
25.7 \\
87.6 \\
97.1\end{array}$ & $\begin{array}{r}100.0 \\
10.8 \\
34.6 \\
11.5 \\
38.1 \\
2.1\end{array}$ & $\begin{array}{r}100.0 \\
86.3 \\
88.8 \\
28.4 \\
88.3 \\
96.9\end{array}$ & $\begin{array}{r}100.0 \\
14.3 \\
38.8 \\
8.3 \\
36.6 \\
4.1\end{array}$ & $\begin{array}{r}100.0 \\
89.1 \\
90.9 \\
40.6 \\
90.1 \\
98.4\end{array}$ & $\begin{array}{r}100.0 \\
21.7 \\
42.1 \\
8.4 \\
40.7 \\
3.2\end{array}$ \\
\hline 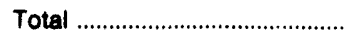 & 70.9 & 24.8 & 78.3 & 26.7 & 78.0 & 26.3 & 81.6 & 28.4 \\
\hline
\end{tabular}

See footnotes at end of table. 
Table 28. Percentage of Total Deliveries Represented by Onsystem Sales by State, 1991-1993 (Continued)

\begin{tabular}{|c|c|c|c|c|c|c|c|c|}
\hline \multirow{2}{*}{ State } & \multicolumn{2}{|c|}{$\begin{array}{l}\text { April } \\
1993\end{array}$} & \multicolumn{2}{|c|}{$\begin{array}{c}\text { March } \\
1993\end{array}$} & \multicolumn{2}{|c|}{$\begin{array}{c}\text { February } \\
1993\end{array}$} & \multicolumn{2}{|c|}{$\begin{array}{c}\text { January } \\
1993\end{array}$} \\
\hline & Commercial & Industrial & Commercial & Industrial & Commercial & Industrial & Commercial & Industrial \\
\hline $\begin{array}{l}\text { Alabama } \\
\text { Alaska } \\
\text { Artzona } \\
\text { Arkanses } \\
\text { Calitornia }\end{array}$ & $\begin{array}{r}80.3 \\
100.0 \\
89.7 \\
91.8 \\
79.6\end{array}$ & $\begin{array}{l}19.8 \\
73.4 \\
24.0 \\
16.0 \\
35.9\end{array}$ & $\begin{array}{r}82.4 \\
100.0 \\
92.2 \\
92.7 \\
76.5\end{array}$ & $\begin{array}{l}20.0 \\
70.1 \\
28.0 \\
16.1 \\
36.1\end{array}$ & $\begin{array}{r}81.8 \\
100.0 \\
93.1 \\
93.0 \\
81.3\end{array}$ & $\begin{array}{l}22.1 \\
73.4 \\
25.7 \\
16.6 \\
44.7\end{array}$ & $\begin{array}{r}80.4 \\
100.0 \\
93.1 \\
92.5 \\
78.8\end{array}$ & $\begin{array}{l}20.4 \\
76.0 \\
29.3 \\
16.7 \\
31.7\end{array}$ \\
\hline $\begin{array}{l}\text { Colorado } \\
\text { Connocticut } \\
\text { Delaware } \\
\text { District of Columbia } \\
\text { Florida }\end{array}$ & $\begin{array}{r}95.5 \\
98.0 \\
100.0 \\
100.0 \\
97.3\end{array}$ & $\begin{array}{l}34.2 \\
70.0 \\
72.5 \\
\overline{28.6}\end{array}$ & $\begin{array}{r}96.3 \\
97.1 \\
100.0 \\
100.0 \\
96.6\end{array}$ & $\begin{array}{l}32.7 \\
73.5 \\
73.7 \\
\overline{28.7}\end{array}$ & $\begin{array}{r}96.2 \\
97.6 \\
100.0 \\
100.0 \\
96.6\end{array}$ & $\begin{array}{l}34.9 \\
73.5 \\
75.5 \\
\overline{31.0}\end{array}$ & $\begin{array}{r}96.3 \\
97.1 \\
100.0 \\
100.0 \\
96.5\end{array}$ & $\begin{array}{l}35.4 \\
71.6 \\
71.8 \\
-- \\
32.6\end{array}$ \\
\hline $\begin{array}{l}\text { Georgia } \\
\text { Hawail } \\
\text { Idaho } \\
\text { Illinois } \\
\text { Indiana }\end{array}$ & $\begin{array}{r}90.1 \\
100.0 \\
88.0 \\
56.5 \\
99.4\end{array}$ & $\begin{array}{l}26.5 \\
- \\
.0 \\
16.1 \\
26.0\end{array}$ & $\begin{array}{r}94.1 \\
100.0 \\
91.1 \\
58.5 \\
99.6\end{array}$ & $\begin{array}{r}35.2 \\
- \\
.0 \\
15.4 \\
27.4\end{array}$ & $\begin{array}{r}92.3 \\
100.0 \\
90.0 \\
58.5 \\
93.7\end{array}$ & $\begin{array}{r}37.3 \\
-. \\
.0 \\
16.9 \\
26.9\end{array}$ & $\begin{array}{r}90.8 \\
100.0 \\
89.5 \\
61.2 \\
94.3\end{array}$ & $\begin{array}{r}32.0 \\
-. \\
.0 \\
21.2 \\
23.9\end{array}$ \\
\hline 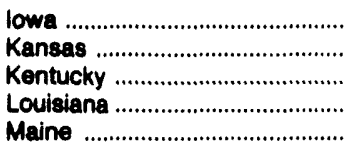 & $\begin{array}{r}96.0 \\
83.5 \\
93.1 \\
98.5 \\
100.0\end{array}$ & $\begin{array}{r}12.0 \\
12.6 \\
37.7 \\
35.3 \\
100.0\end{array}$ & $\begin{array}{r}95.4 \\
85.2 \\
95.0 \\
97.9 \\
100.0\end{array}$ & $\begin{array}{r}15.0 \\
10.6 \\
29.0 \\
31.8 \\
100.0\end{array}$ & $\begin{array}{r}96.3 \\
82.1 \\
95.1 \\
97.8 \\
100.0\end{array}$ & $\begin{array}{r}18.2 \\
8.8 \\
21.4 \\
34.7 \\
100.0\end{array}$ & $\begin{array}{r}96.3 \\
83.4 \\
94.9 \\
97.3 \\
100.0\end{array}$ & $\begin{array}{r}16.3 \\
10.2 \\
20.0 \\
34.7 \\
100.0\end{array}$ \\
\hline $\begin{array}{l}\text { Maryland } \\
\text { Massachusetts } \\
\text { Michigan } \\
\text { Minnesota } \\
\text { Mississippl }\end{array}$ & $\begin{array}{l}96.2 \\
99.7 \\
66.6 \\
99.2 \\
96.7\end{array}$ & $\begin{array}{r}9.1 \\
52.5 \\
8.6 \\
45.9 \\
32.3\end{array}$ & $\begin{array}{l}96.5 \\
99.8 \\
71.3 \\
96.5 \\
92.7\end{array}$ & $\begin{array}{l}22.1 \\
51.7 \\
10.3 \\
48.5 \\
35.9\end{array}$ & $\begin{array}{l}97.4 \\
99.8 \\
71.1 \\
96.9 \\
84.5\end{array}$ & $\begin{array}{l}15.0 \\
55.3 \\
10.8 \\
48.8 \\
34.6\end{array}$ & $\begin{array}{l}97.3 \\
99.8 \\
73.2 \\
98.8 \\
91.7\end{array}$ & $\begin{array}{l}18.6 \\
56.2 \\
11.6 \\
50.5 \\
34.9\end{array}$ \\
\hline $\begin{array}{l}\text { Missouri } \\
\text { Montana } \\
\text { Nebraska } \\
\text { Nevada } \\
\text { Now Hampshire }\end{array}$ & $\begin{array}{r}86.8 \\
94.0 \\
93.0 \\
91.1 \\
100.0\end{array}$ & $\begin{array}{r}24.3 \\
14.1 \\
24.2 \\
3.7 \\
100.0\end{array}$ & $\begin{array}{r}88.8 \\
95.0 \\
94.1 \\
94.3 \\
100.0\end{array}$ & $\begin{array}{r}27.3 \\
15.0 \\
42.4 \\
7.6 \\
100.0\end{array}$ & $\begin{array}{r}86.4 \\
94.1 \\
94.4 \\
96.0 \\
100.0\end{array}$ & $\begin{array}{r}28.7 \\
16.1 \\
45.4 \\
6.9 \\
100.0\end{array}$ & $\begin{array}{r}87.9 \\
95.5 \\
93.7 \\
97.1 \\
100.0\end{array}$ & $\begin{array}{r}29.4 \\
16.5 \\
45.0 \\
6.1 \\
100.0\end{array}$ \\
\hline 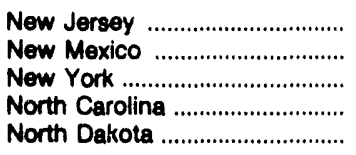 & $\begin{array}{r}91.5 \\
61.3 \\
78.8 \\
100.0 \\
76.9\end{array}$ & $\begin{array}{r}55.5 \\
1.7 \\
22.0 \\
95.7 \\
26.2\end{array}$ & $\begin{array}{r}93.9 \\
66.2 \\
83.2 \\
100.0 \\
77.4\end{array}$ & $\begin{array}{r}58.6 \\
1.6 \\
25.6 \\
92.9 \\
29.5\end{array}$ & $\begin{array}{r}94.2 \\
62.6 \\
82.4 \\
100.0 \\
73.9\end{array}$ & $\begin{array}{r}60.5 \\
2.9 \\
28.4 \\
94.0 \\
31.5\end{array}$ & $\begin{array}{r}93.8 \\
67.8 \\
82.3 \\
100.0 \\
77.1\end{array}$ & $\begin{array}{r}63.4 \\
4.8 \\
25.4 \\
97.1 \\
97.1 \\
34.3\end{array}$ \\
\hline 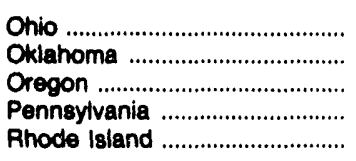 & $\begin{array}{r}83.6 \\
90.2 \\
97.9 \\
77.7 \\
100.0\end{array}$ & $\begin{array}{l}10.7 \\
31.1 \\
22.1 \\
27.0 \\
10.7\end{array}$ & $\begin{array}{r}86.7 \\
91.1 \\
98.5 \\
79.0 \\
100.0\end{array}$ & $\begin{array}{l}13.6 \\
27.2 \\
23.9 \\
25.3 \\
11.7\end{array}$ & $\begin{array}{r}86.8 \\
91.4 \\
99.0 \\
79.2 \\
100.0\end{array}$ & $\begin{array}{l}12.6 \\
37.5 \\
26.0 \\
26.7 \\
11.8\end{array}$ & $\begin{array}{r}89.3 \\
91.2 \\
98.6 \\
80.0 \\
100.0\end{array}$ & $\begin{array}{l}11.2 \\
41.3 \\
25.4 \\
29.3 \\
10.8\end{array}$ \\
\hline $\begin{array}{l}\text { South Carolina } \\
\text { South Dakota } \\
\text { Tennesseo } \\
\text { Texas }\end{array}$ & $\begin{array}{r}100.0 \\
81.2 \\
95.8 \\
83.2 \\
100.0\end{array}$ & $\begin{array}{l}58.3 \\
55.1 \\
45.9 \\
28.0 \\
12.5\end{array}$ & $\begin{array}{r}100.0 \\
84.4 \\
95.9 \\
83.8 \\
100.0\end{array}$ & $\begin{array}{l}67.0 \\
54.4 \\
46.5 \\
30.2 \\
12.0\end{array}$ & $\begin{array}{r}100.0 \\
83.9 \\
96.3 \\
85.2 \\
100.0\end{array}$ & $\begin{array}{l}70.0 \\
61.4 \\
44.9 \\
29.1 \\
12.3\end{array}$ & $\begin{array}{r}100.0 \\
86.5 \\
96.0 \\
83.9 \\
100.0\end{array}$ & $\begin{array}{r}72.2 \\
68.3 \\
39.2 \\
30.0 \\
9.9\end{array}$ \\
\hline 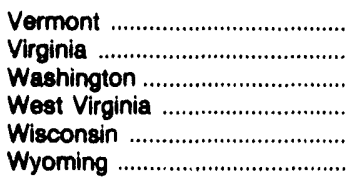 & $\begin{array}{r}100.0 \\
86.3 \\
93.7 \\
53.3 \\
91.8 \\
98.2\end{array}$ & $\begin{array}{r}100.0 \\
16.2 \\
45.2 \\
7.7 \\
42.7 \\
3.1\end{array}$ & $\begin{array}{r}100.0 \\
88.7 \\
93.9 \\
54.9 \\
94.2 \\
98.5\end{array}$ & $\begin{array}{r}100.0 \\
13.2 \\
44.4 \\
8.1 \\
49.0 \\
5.0\end{array}$ & $\begin{array}{r}100.0 \\
90.9 \\
93.3 \\
54.9 \\
95.1 \\
98.3\end{array}$ & $\begin{array}{r}100.0 \\
15.3 \\
46.0 \\
6.7 \\
48.6 \\
4.3\end{array}$ & $\begin{array}{r}100.0 \\
89.1 \\
93.8 \\
54.3 \\
94.1 \\
98.6\end{array}$ & $\begin{array}{r}100.0 \\
14.5 \\
48.0 \\
8.8 \\
46.6 \\
4.8\end{array}$ \\
\hline Total & 84.3 & 29.3 & 85.8 & 30.1 & 85.8 & 31.2 & 86.2 & 30.8 \\
\hline
\end{tabular}

See footnotes at end of table. 
Table 28. Percentage of Total Deliveries Represented by Onsystem Sales by State, 1991-1993 (Continued)

\begin{tabular}{|c|c|c|c|c|c|c|c|c|}
\hline \multirow{2}{*}{ State } & \multicolumn{2}{|c|}{$\begin{array}{l}\text { Total } \\
1992\end{array}$} & \multicolumn{2}{|c|}{$\begin{array}{c}\text { December } \\
1992\end{array}$} & \multicolumn{2}{|c|}{$\begin{array}{c}\text { November } \\
1992\end{array}$} & \multicolumn{2}{|c|}{$\begin{array}{l}\text { October } \\
1992\end{array}$} \\
\hline & Commercial & Industrial & Commercial & Industrial & Commercial & Industrial & Commercial & Industrial \\
\hline 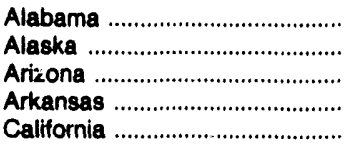 & $\begin{array}{r}80.7 \\
100.0 \\
92.6 \\
90.7 \\
74.5\end{array}$ & $\begin{array}{l}29.8 \\
69.8 \\
29.6 \\
13.8 \\
27.5\end{array}$ & $\begin{array}{r}80.6 \\
100.0 \\
92.9 \\
90.8 \\
81.4\end{array}$ & $\begin{array}{l}31.1 \\
73.9 \\
31.6 \\
20.1 \\
30.0\end{array}$ & $\begin{array}{r}74.2 \\
100.0 \\
89.1 \\
88.7 \\
76.7\end{array}$ & $\begin{array}{l}30.4 \\
72.3 \\
28.8 \\
15.3 \\
27.7\end{array}$ & $\begin{array}{r}73.2 \\
100.0 \\
91.2 \\
87.6 \\
79.0\end{array}$ & $\begin{array}{l}28.7 \\
71.2 \\
30.1 \\
14.4 \\
31.6\end{array}$ \\
\hline 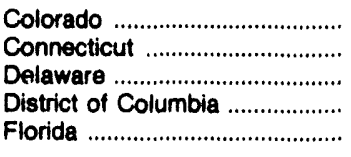 & $\begin{array}{r}95.5 \\
96.5 \\
100.0 \\
99.0 \\
97.7\end{array}$ & $\begin{array}{c}32.2 \\
65.6 \\
65.0 \\
-\overline{32.9}\end{array}$ & $\begin{array}{r}96.2 \\
98.4 \\
100.0 \\
100.0 \\
97.0\end{array}$ & $\begin{array}{c}37.0 \\
75.3 \\
74.0 \\
\overline{35.1}\end{array}$ & $\begin{array}{r}93.7 \\
98.2 \\
100.0 \\
100.0 \\
97.4\end{array}$ & $\begin{array}{l}32.4 \\
78.9 \\
72.3 \\
-- \\
31.8\end{array}$ & $\begin{array}{r}91.2 \\
97.8 \\
100.0 \\
100.0 \\
98.2\end{array}$ & $\begin{array}{c}37.3 \\
69.7 \\
68.8 \\
-\overline{28.9}\end{array}$ \\
\hline 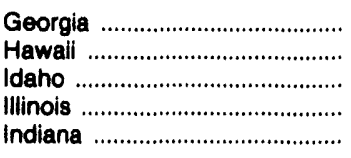 & $\begin{array}{r}88.1 \\
100.0 \\
85.7 \\
57.7 \\
96.8\end{array}$ & $\begin{array}{r}35.0 \\
- \\
.3 \\
15.0 \\
23.2\end{array}$ & $\begin{array}{r}92.9 \\
100.0 \\
88.3 \\
59.9 \\
98.0\end{array}$ & $\begin{array}{r}40.3 \\
-. \\
.0 \\
20.7 \\
22.5\end{array}$ & $\begin{array}{r}87.9 \\
100.0 \\
82.2 \\
60.5 \\
99.9\end{array}$ & $\begin{array}{r}29.4 \\
- \\
.0 \\
22.1 \\
29.8\end{array}$ & $\begin{array}{r}84.0 \\
100.0 \\
77.9 \\
55.5 \\
99.8\end{array}$ & $\begin{array}{r}30.0 \\
-- \\
19.5 \\
28.3\end{array}$ \\
\hline 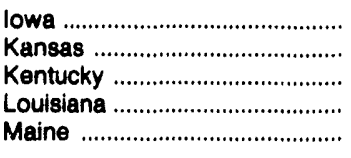 & $\begin{array}{r}95.7 \\
84.4 \\
93.1 \\
87.5 \\
100.0\end{array}$ & $\begin{array}{r}17.6 \\
10.2 \\
35.1 \\
37.6 \\
100.0\end{array}$ & $\begin{array}{r}96.7 \\
82.7 \\
94.9 \\
86.4 \\
100.0\end{array}$ & $\begin{array}{r}23.9 \\
8.6 \\
27.0 \\
34.0 \\
100.0\end{array}$ & $\begin{array}{r}96.1 \\
78.0 \\
94.6 \\
81.4 \\
100.0\end{array}$ & $\begin{array}{r}26.7 \\
10.4 \\
35.4 \\
32.8 \\
100.0\end{array}$ & $\begin{array}{r}91.0 \\
81.1 \\
93.0 \\
90.6 \\
100.0\end{array}$ & $\begin{array}{r}19.2 \\
9.8 \\
57.3 \\
33.5 \\
100.0\end{array}$ \\
\hline 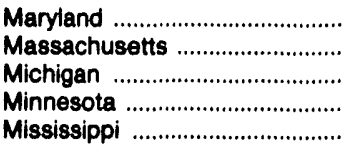 & $\begin{array}{l}96.0 \\
99.8 \\
68.8 \\
96.2 \\
96.4\end{array}$ & $\begin{array}{r}20.1 \\
45.4 \\
9.1 \\
42.3 \\
45.5\end{array}$ & $\begin{array}{l}97.4 \\
99.8 \\
73.1 \\
97.6 \\
97.4\end{array}$ & $\begin{array}{l}30.5 \\
33.9 \\
12.8 \\
54.4 \\
44.8\end{array}$ & $\begin{array}{l}97.0 \\
99.7 \\
68.6 \\
96.5 \\
95.9\end{array}$ & $\begin{array}{r}41.0 \\
36.6 \\
8.6 \\
48.9 \\
45.4\end{array}$ & $\begin{array}{l}95.0 \\
99.6 \\
61.1 \\
99.4 \\
95.6\end{array}$ & $\begin{array}{r}18.6 \\
43.6 \\
5.7 \\
45.0 \\
43.3\end{array}$ \\
\hline 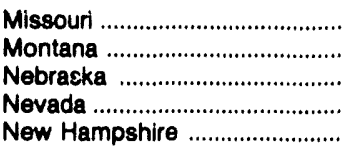 & $\begin{array}{r}85.5 \\
95.4 \\
88.2 \\
88.3 \\
100.0\end{array}$ & $\begin{array}{r}24.5 \\
17.5 \\
41.2 \\
7.5 \\
100.0\end{array}$ & $\begin{array}{r}87.2 \\
94.6 \\
90.9 \\
95.4 \\
100.0\end{array}$ & $\begin{array}{r}27.0 \\
13.2 \\
44.6 \\
14.1 \\
100.0\end{array}$ & $\begin{array}{r}81.0 \\
92.8 \\
87.1 \\
88.5 \\
100.0\end{array}$ & $\begin{array}{r}21.7 \\
14.1 \\
45.8 \\
6.4 \\
100.0\end{array}$ & $\begin{array}{r}76.1 \\
92.0 \\
84.5 \\
88.9 \\
100.0\end{array}$ & $\begin{array}{r}28.8 \\
11.1 \\
40.0 \\
6.5 \\
100.0\end{array}$ \\
\hline 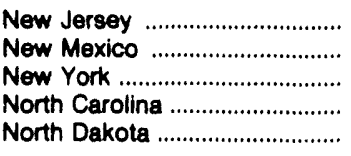 & $\begin{array}{l}92.4 \\
70.0 \\
77.7 \\
95.9 \\
68.9\end{array}$ & $\begin{array}{r}55.7 \\
4.3 \\
23.3 \\
81.0 \\
21.6\end{array}$ & $\begin{array}{r}96.9 \\
61.1 \\
81.5 \\
100.0 \\
68.9\end{array}$ & $\begin{array}{r}59.5 \\
9.8 \\
27.7 \\
98.9 \\
31.0\end{array}$ & $\begin{array}{r}94.8 \\
57.0 \\
82.6 \\
100.0 \\
67.3\end{array}$ & $\begin{array}{r}63.9 \\
8.2 \\
24.2 \\
97.5 \\
25.7\end{array}$ & $\begin{array}{l}86.4 \\
57.9 \\
76.0 \\
99.9 \\
56.8\end{array}$ & $\begin{array}{r}57.9 \\
3.2 \\
21.1 \\
96.7 \\
18.0\end{array}$ \\
\hline 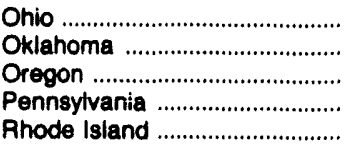 & $\begin{array}{r}85.6 \\
88.5 \\
97.8 \\
75.8 \\
100.0\end{array}$ & $\begin{array}{l}11.6 \\
23.7 \\
20.7 \\
22.9 \\
11.4\end{array}$ & $\begin{array}{r}88.5 \\
88.1 \\
98.4 \\
79.4 \\
100.0\end{array}$ & $\begin{array}{l}14.5 \\
41.5 \\
24.4 \\
34.3 \\
11.0\end{array}$ & $\begin{array}{r}85.7 \\
86.9 \\
97.4 \\
74.1 \\
100.0\end{array}$ & $\begin{array}{l}12.8 \\
31.8 \\
22.7 \\
30.4 \\
12.5\end{array}$ & $\begin{array}{r}84.2 \\
85.3 \\
94.6 \\
74.0 \\
100.0\end{array}$ & $\begin{array}{l}12.2 \\
28.3 \\
18.7 \\
29.4 \\
12.1\end{array}$ \\
\hline $\begin{array}{l}\text { South Carolina } \\
\text { South Dakota } \\
\text { Tennessee } \\
\text { Texas } \\
\text { Utah }\end{array}$ & $\begin{array}{r}98.6 \\
82.4 \\
96.4 \\
79.7 \\
100.0\end{array}$ & $\begin{array}{l}66.9 \\
52.6 \\
49.6 \\
31.3 \\
11.7\end{array}$ & $\begin{array}{r}100.0 \\
84.7 \\
97.4 \\
78.6 \\
100.0\end{array}$ & $\begin{array}{l}74.7 \\
68.6 \\
48.8 \\
32.0 \\
13.4\end{array}$ & $\begin{array}{r}100.0 \\
81.8 \\
97.5 \\
75.3 \\
100.0\end{array}$ & $\begin{array}{c}69.8 \\
64.4 \\
48.8 \\
28.0 \\
10.2\end{array}$ & $\begin{array}{r}99.7 \\
76.5 \\
97.3 \\
72.4 \\
100.0\end{array}$ & $\begin{array}{l}66.6 \\
59.2 \\
48.5 \\
33.3 \\
11.7\end{array}$ \\
\hline 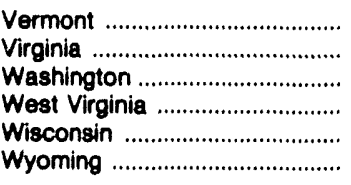 & $\begin{array}{r}100.0 \\
90.7 \\
87.3 \\
56.9 \\
91.3 \\
98.0\end{array}$ & $\begin{array}{r}100.0 \\
25.6 \\
37.4 \\
16.2 \\
41.5 \\
3.6\end{array}$ & $\begin{array}{r}100.0 \\
90.3 \\
92.3 \\
58.9 \\
95.1 \\
98.3\end{array}$ & $\begin{array}{r}100.0 \\
30.4 \\
44.8 \\
24.2 \\
49.0 \\
4.5\end{array}$ & $\begin{array}{r}100.0 \\
86.6 \\
86.8 \\
56.8 \\
93.4 \\
98.2\end{array}$ & $\begin{array}{r}100.0 \\
30.2 \\
36.6 \\
16.7 \\
46.2 \\
4.0\end{array}$ & $\begin{array}{r}100.0 \\
89.9 \\
83.4 \\
48.1 \\
90.7 \\
97.2\end{array}$ & $\begin{array}{r}100.0 \\
26.0 \\
37.1 \\
13.6 \\
38.4 \\
2.7\end{array}$ \\
\hline Total & 83.2 & 30.3 & 85.6 & 32.5 & 83.1 & 30.8 & 80.9 & 31.4 \\
\hline
\end{tabular}

See footnotes at end of table. 
Table 28. Percentage of Total Deliveries Represented by Onsystem Sales by State, 1991-1993 (Continued)

\begin{tabular}{|c|c|c|c|c|c|c|c|c|}
\hline \multirow{2}{*}{ State } & \multicolumn{2}{|c|}{$\begin{array}{c}\text { September } \\
1992\end{array}$} & \multicolumn{2}{|c|}{$\begin{array}{c}\text { August } \\
1992\end{array}$} & \multicolumn{2}{|c|}{$\begin{array}{c}\text { July } \\
1992\end{array}$} & \multicolumn{2}{|c|}{$\begin{array}{l}\text { June } \\
1992\end{array}$} \\
\hline & Commercial & Industrial & Commercial & Industrial & Commercial & Industrial & Commercial & Industrial \\
\hline 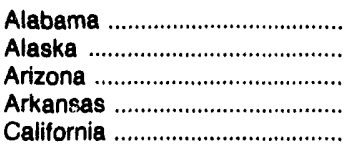 & $\begin{array}{r}74.4 \\
100.0 \\
90.5 \\
86.4 \\
79.7\end{array}$ & $\begin{array}{l}26.5 \\
81.3 \\
27.2 \\
12.2 \\
33.3\end{array}$ & $\begin{array}{r}74.7 \\
100.0 \\
86.0 \\
85.4 \\
64.6\end{array}$ & $\begin{array}{l}28.9 \\
60.1 \\
26.0 \\
12.2 \\
29.8\end{array}$ & $\begin{array}{r}75.3 \\
100.0 \\
86.3 \\
85.9 \\
60.9\end{array}$ & $\begin{array}{l}29.9 \\
66.7 \\
26.8 \\
12.4 \\
31.1\end{array}$ & $\begin{array}{r}77.6 \\
100.0 \\
88.9 \\
87.0 \\
74.5\end{array}$ & $\begin{array}{l}28.3 \\
69.9 \\
26.3 \\
13.1 \\
26.2\end{array}$ \\
\hline 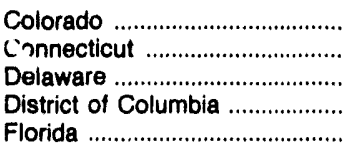 & $\begin{array}{r}93.6 \\
98.7 \\
100.0 \\
99.0 \\
98.3\end{array}$ & $\begin{array}{l}40.5 \\
63.9 \\
61.9 \\
\overline{32.3}\end{array}$ & $\begin{array}{r}93.3 \\
98.8 \\
100.0 \\
99.5 \\
98.1\end{array}$ & $\begin{array}{c}34.6 \\
61.8 \\
62.3 \\
-- \\
31.6\end{array}$ & $\begin{array}{r}94.2 \\
95.8 \\
100.0 \\
91.4 \\
98.1\end{array}$ & $\begin{array}{c}40.0 \\
54.2 \\
59.0 \\
-- \\
34.5\end{array}$ & $\begin{array}{r}96.0 \\
87.2 \\
100.0 \\
100.0 \\
98.2\end{array}$ & $\begin{array}{l}27.9 \\
52.1 \\
59.9 \\
-- \\
38.1\end{array}$ \\
\hline 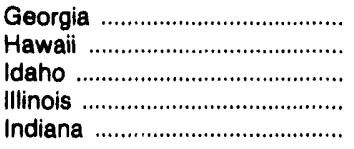 & $\begin{array}{r}83.4 \\
100.0 \\
80.2 \\
47.0 \\
91.9\end{array}$ & $\begin{array}{r}40.6 \\
- \\
.0 \\
12.8 \\
20.9\end{array}$ & $\begin{array}{r}83.3 \\
100.0 \\
82.4 \\
49.2 \\
99.6\end{array}$ & $\begin{array}{r}35.6 \\
-- \\
.0 \\
7.9 \\
25.6\end{array}$ & $\begin{array}{r}79.7 \\
100.0 \\
81.0 \\
44.4 \\
92.8\end{array}$ & $\begin{array}{r}31.4 \\
-- \\
.0 \\
7.4 \\
17.8\end{array}$ & $\begin{array}{r}82.7 \\
100.0 \\
80.2 \\
43.4 \\
99.9\end{array}$ & $\begin{array}{r}30.1 \\
-- \\
.0 \\
5.6 \\
22.8\end{array}$ \\
\hline 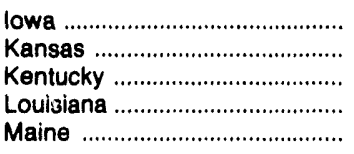 & $\begin{array}{r}88.7 \\
86.9 \\
87.9 \\
91.1 \\
100.0\end{array}$ & $\begin{array}{r}13.3 \\
10.7 \\
51.3 \\
36.4 \\
100.0\end{array}$ & $\begin{array}{r}90.6 \\
88.9 \\
87.4 \\
90.2 \\
100.0\end{array}$ & $\begin{array}{r}13.1 \\
13.6 \\
42.9 \\
30.2 \\
100.0\end{array}$ & $\begin{array}{r}91.3 \\
88.4 \\
86.7 \\
90.6 \\
100.0\end{array}$ & $\begin{array}{r}11.8 \\
12.2 \\
45.2 \\
30.6 \\
100.0\end{array}$ & $\begin{array}{r}93.2 \\
86.8 \\
86.9 \\
90.1 \\
100.0\end{array}$ & $\begin{array}{r}12.8 \\
9.8 \\
47.3 \\
28.7 \\
100.0\end{array}$ \\
\hline 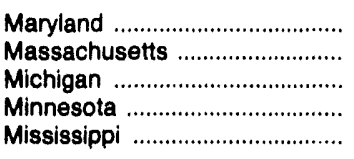 & $\begin{array}{l}94.1 \\
99.6 \\
49.9 \\
93.0 \\
95.6\end{array}$ & $\begin{array}{r}8.4 \\
44.7 \\
4.2 \\
39.9 \\
43.1\end{array}$ & $\begin{array}{l}92.7 \\
99.6 \\
51.6 \\
89.8 \\
94.8\end{array}$ & $\begin{array}{r}9.1 \\
44.9 \\
4.0 \\
36.3 \\
45.6\end{array}$ & $\begin{array}{l}93.1 \\
99.7 \\
48.5 \\
93.1 \\
95.5\end{array}$ & $\begin{array}{r}7.4 \\
47.7 \\
4.6 \\
32.7 \\
45.1\end{array}$ & $\begin{array}{l}92.8 \\
99.8 \\
55.5 \\
88.3 \\
95.4\end{array}$ & $\begin{array}{r}7.0 \\
44.2 \\
4.7 \\
37.1 \\
43.4\end{array}$ \\
\hline $\begin{array}{l}\text { Missouri } \\
\text { Montana } \\
\text { Nebraska } \\
\text { Nevada } \\
\text { New Hampshire }\end{array}$ & $\begin{array}{r}74.5 \\
90.6 \\
80.5 \\
82.7 \\
100.0\end{array}$ & $\begin{array}{r}18.5 \\
9.3 \\
33.4 \\
6.3 \\
100.0\end{array}$ & $\begin{array}{r}75.8 \\
96.8 \\
83.6 \\
81.4 \\
100.0\end{array}$ & $\begin{array}{r}21.5 \\
14.8 \\
36.9 \\
6.7 \\
100.0\end{array}$ & $\begin{array}{r}71.9 \\
95.4 \\
85.6 \\
84.8 \\
100.0\end{array}$ & $\begin{array}{r}18.2 \\
15.1 \\
32.1 \\
4.7 \\
100.0\end{array}$ & $\begin{array}{r}80.6 \\
94.9 \\
81.7 \\
84.0 \\
100.0\end{array}$ & $\begin{array}{r}18.6 \\
16.3 \\
33.0 \\
7.5 \\
100.0\end{array}$ \\
\hline 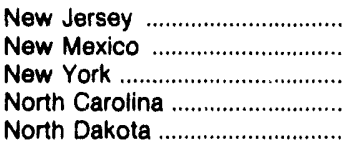 & $\begin{array}{l}80.7 \\
60.8 \\
69.3 \\
89.2 \\
57.2\end{array}$ & $\begin{array}{r}55.9 \\
9.1 \\
16.1 \\
75.1 \\
14.2\end{array}$ & $\begin{array}{l}80.7 \\
64.4 \\
69.3 \\
86.2 \\
58.8\end{array}$ & $\begin{array}{r}55.7 \\
2.1 \\
13.7 \\
61.1 \\
13.0\end{array}$ & $\begin{array}{l}88.6 \\
62.4 \\
72.9 \\
84.3 \\
61.6\end{array}$ & $\begin{array}{r}52.8 \\
1.9 \\
17.3 \\
46.9 \\
12.6\end{array}$ & $\begin{array}{l}91.3 \\
66.5 \\
70.9 \\
85.8 \\
63.8\end{array}$ & $\begin{array}{r}53.6 \\
2.4 \\
15.6 \\
61.1 \\
14.7\end{array}$ \\
\hline 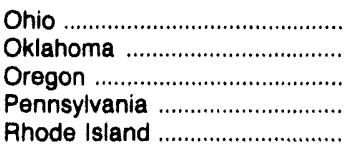 & $\begin{array}{r}73.1 \\
84.3 \\
97.8 \\
69.4 \\
100.0\end{array}$ & $\begin{array}{r}7.4 \\
25.4 \\
17.8 \\
20.5 \\
13.3\end{array}$ & $\begin{array}{r}73.5 \\
85.7 \\
96.7 \\
67.2 \\
100.0\end{array}$ & $\begin{array}{r}9.2 \\
21.8 \\
17.6 \\
17.9 \\
9.1\end{array}$ & $\begin{array}{r}72.0 \\
80.2 \\
97.9 \\
68.1 \\
100.0\end{array}$ & $\begin{array}{r}7.3 \\
16.2 \\
17.5 \\
14.1 \\
12.1\end{array}$ & $\begin{array}{r}73.9 \\
84.1 \\
97.8 \\
73.1 \\
100.0\end{array}$ & $\begin{array}{r}8.4 \\
17.0 \\
20.4 \\
12.3 \\
9.8\end{array}$ \\
\hline $\begin{array}{l}\text { South Carolina } \\
\text { South Dakota } \\
\text { Tennessee } \\
\text { Texas } \\
\text { Utah }\end{array}$ & $\begin{array}{r}97.0 \\
78.0 \\
91.6 \\
71.9 \\
100.0\end{array}$ & $\begin{array}{l}63.2 \\
54.7 \\
38.9 \\
29.4 \\
11.9\end{array}$ & $\begin{array}{r}95.8 \\
74.6 \\
90.4 \\
75.9 \\
100.0\end{array}$ & $\begin{array}{l}65.4 \\
50.3 \\
43.1 \\
32.8 \\
11.6\end{array}$ & $\begin{array}{r}95.5 \\
72.3 \\
97.3 \\
77.0 \\
100.0\end{array}$ & $\begin{array}{l}61.9 \\
32.7 \\
43.8 \\
31.4 \\
11.1\end{array}$ & $\begin{array}{r}95.8 \\
73.5 \\
95.8 \\
77.8 \\
100.0\end{array}$ & $\begin{array}{l}64.6 \\
39.2 \\
47.9 \\
35.0 \\
10.7\end{array}$ \\
\hline 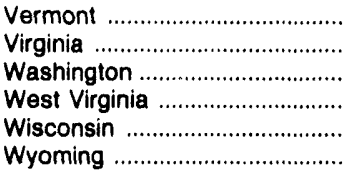 & $\begin{array}{r}100.0 \\
83.8 \\
81.6 \\
33.4 \\
85.2 \\
97.4\end{array}$ & $\begin{array}{r}100.0 \\
30.9 \\
39.3 \\
13.8 \\
34.8 \\
2.3\end{array}$ & $\begin{array}{r}100.0 \\
85.9 \\
80.3 \\
33.6 \\
84.1 \\
97.1\end{array}$ & $\begin{array}{r}100.0 \\
21.5 \\
34.6 \\
14.4 \\
33.2 \\
2.4\end{array}$ & $\begin{array}{r}100.0 \\
86.9 \\
78.5 \\
35.8 \\
83.3 \\
96.5\end{array}$ & $\begin{array}{r}100.0 \\
14.0 \\
36.1 \\
10.3 \\
32.0 \\
2.3\end{array}$ & $\begin{array}{r}100.0 \\
88.4 \\
80.7 \\
39.3 \\
82.3 \\
97.2\end{array}$ & $\begin{array}{r}100.0 \\
30.3 \\
36.1 \\
12.6 \\
31.9 \\
2.5\end{array}$ \\
\hline 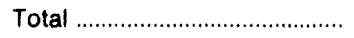 & 78.0 & 29.3 & 76.1 & 28.6 & 76.0 & 27.4 & 79.4 & 28.0 \\
\hline
\end{tabular}

See footnotes at end of table. 
Table 28. Percentage of Total Deliveries Represented by Onsystem Sales by State, 1991-1993 (Continued)

\begin{tabular}{|c|c|c|c|c|c|c|c|c|}
\hline \multirow{2}{*}{ State } & \multicolumn{2}{|c|}{$\begin{array}{c}\text { May } \\
1992\end{array}$} & \multicolumn{2}{|c|}{$\begin{array}{l}\text { April } \\
1992\end{array}$} & \multicolumn{2}{|c|}{$\begin{array}{c}\text { March } \\
1992\end{array}$} & \multicolumn{2}{|c|}{$\begin{array}{c}\text { February } \\
1982\end{array}$} \\
\hline & Commercial & Industrial & Commercial & Industrial & Commercial & Industrial & Commercial & Industrial \\
\hline 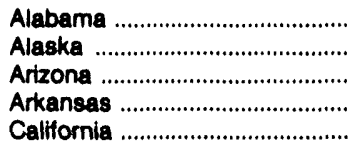 & $\begin{array}{r}79.0 \\
100.0 \\
89.9 \\
88.4 \\
63.5\end{array}$ & $\begin{array}{l}28.4 \\
67.7 \\
26.3 \\
12.6 \\
24.3\end{array}$ & $\begin{array}{r}83.2 \\
100.0 \\
92.7 \\
92.7 \\
79.0\end{array}$ & $\begin{array}{l}29.4 \\
68.8 \\
31.4 \\
14.4 \\
36.1\end{array}$ & $\begin{array}{r}84.0 \\
100.0 \\
96.3 \\
91.3 \\
75.7\end{array}$ & $\begin{array}{l}35.6 \\
69.1 \\
33.0 \\
10.3 \\
24.0\end{array}$ & $\begin{array}{r}86.8 \\
100.0 \\
97.0 \\
94.7 \\
65.6\end{array}$ & $\begin{array}{l}32.2 \\
68.1 \\
32.9 \\
16.4 \\
22.9\end{array}$ \\
\hline 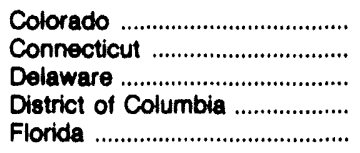 & $\begin{array}{r}95.1 \\
93.6 \\
100.0 \\
97.6 \\
98.3\end{array}$ & $\begin{array}{c}28.5 \\
54.1 \\
63.4 \\
- \\
38.5\end{array}$ & $\begin{array}{r}95.7 \\
94.4 \\
100.0 \\
97.4 \\
98.2\end{array}$ & $\begin{array}{c}25.8 \\
59.2 \\
61.0 \\
- \\
27.8\end{array}$ & $\begin{array}{r}95.9 \\
95.6 \\
100.0 \\
100.0 \\
97.4\end{array}$ & $\begin{array}{l}27.6 \\
62.7 \\
62.1 \\
- \\
29.0\end{array}$ & $\begin{array}{r}96.7 \\
97.2 \\
100.0 \\
100.0 \\
97.2\end{array}$ & $\begin{array}{c}25.0 \\
72.0 \\
63.7 \\
- \\
28.2\end{array}$ \\
\hline 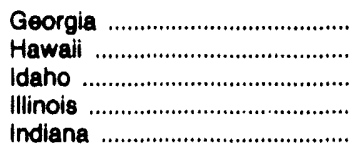 & $\begin{array}{r}85.0 \\
100.0 \\
83.1 \\
53.0 \\
99.7\end{array}$ & $\begin{array}{r}40.2 \\
-.0 \\
9.1 \\
24.7\end{array}$ & $\begin{array}{r}88.2 \\
100.0 \\
85.2 \\
57.5 \\
98.1\end{array}$ & $\begin{array}{c}46.0 \\
- \\
.0 \\
11.9 \\
22.4\end{array}$ & $\begin{array}{r}89.3 \\
100.0 \\
87.1 \\
59.5 \\
98.6\end{array}$ & $\begin{array}{c}32.6 \\
- \\
.0 \\
14.6 \\
20.2\end{array}$ & $\begin{array}{r}90.6 \\
100.0 \\
88.0 \\
58.2 \\
82.4\end{array}$ & $\begin{array}{r}28.8 \\
- \\
.0 \\
16.8 \\
18.8\end{array}$ \\
\hline 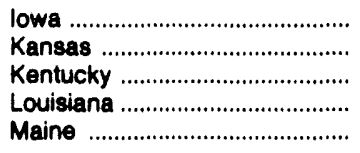 & $\begin{array}{r}94.3 \\
88.8 \\
87.8 \\
90.5 \\
100.0\end{array}$ & $\begin{array}{r}14.2 \\
13.5 \\
33.7 \\
46.3 \\
100.0\end{array}$ & $\begin{array}{r}96.2 \\
86.8 \\
91.8 \\
90.6 \\
100.0\end{array}$ & $\begin{array}{r}15.0 \\
10.5 \\
22.2 \\
42.4 \\
100.0\end{array}$ & $\begin{array}{r}96.5 \\
83.9 \\
83.6 \\
88.7 \\
100.0\end{array}$ & $\begin{array}{r}15.5 \\
7.4 \\
17.6 \\
43.1 \\
100.0\end{array}$ & $\begin{array}{r}97.4 \\
83.8 \\
94.2 \\
87.7 \\
100.0\end{array}$ & $\begin{array}{r}19.8 \\
9.4 \\
20.2 \\
44.8 \\
100.0\end{array}$ \\
\hline 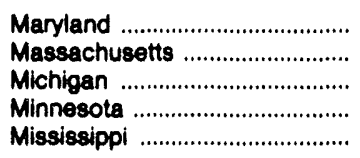 & $\begin{array}{l}94.1 \\
99.7 \\
66.6 \\
92.2 \\
95.2\end{array}$ & $\begin{array}{r}5.8 \\
48.9 \\
7.2 \\
35.6 \\
44.6\end{array}$ & $\begin{array}{l}95.6 \\
99.8 \\
70.5 \\
95.5 \\
86.6\end{array}$ & $\begin{array}{l}15.1 \\
53.1 \\
10.6 \\
39.8 \\
45.5\end{array}$ & $\begin{array}{l}96.3 \\
99.8 \\
71.4 \\
96.0 \\
96.4\end{array}$ & $\begin{array}{l}23.5 \\
42.4 \\
12.0 \\
42.4 \\
47.2\end{array}$ & $\begin{array}{l}97.2 \\
99.8 \\
74.5 \\
95.3 \\
97.3\end{array}$ & $\begin{array}{l}29.7 \\
47.4 \\
14.0 \\
40.9 \\
51.5\end{array}$ \\
\hline 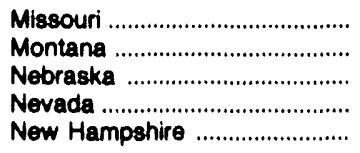 & $\begin{array}{r}82.7 \\
96.6 \\
88.7 \\
83.7 \\
100.0\end{array}$ & $\begin{array}{r}17.5 \\
17.3 \\
43.1 \\
6.2 \\
100.0\end{array}$ & $\begin{array}{r}88.1 \\
96.5 \\
90.9 \\
87.2 \\
100.0\end{array}$ & $\begin{array}{r}28.4 \\
23.4 \\
40.3 \\
6.9 \\
100.0\end{array}$ & $\begin{array}{r}89.1 \\
86.7 \\
89.9 \\
88.3 \\
100.0\end{array}$ & $\begin{array}{r}26.2 \\
27.3 \\
48.5 \\
6.6 \\
100.0\end{array}$ & $\begin{array}{r}90.1 \\
98.0 \\
91.6 \\
89.5 \\
100.0\end{array}$ & $\begin{array}{r}31.0 \\
28.6 \\
43.1 \\
9.1 \\
100.0\end{array}$ \\
\hline 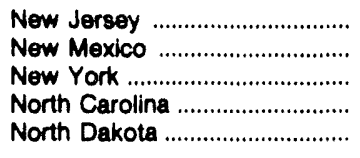 & $\begin{array}{l}94.1 \\
64.0 \\
72.4 \\
88.3 \\
73.6\end{array}$ & $\begin{array}{r}51.8 \\
7.8 \\
19.8 \\
61.8 \\
21.0\end{array}$ & $\begin{array}{l}87.7 \\
73.0 \\
77.3 \\
83.1 \\
71.6\end{array}$ & $\begin{array}{r}48.4 \\
.7 \\
24.6 \\
73.3 \\
24.9\end{array}$ & $\begin{array}{l}92.1 \\
75.3 \\
79.5 \\
99.2 \\
72.0\end{array}$ & $\begin{array}{r}53.9 \\
1.6 \\
27.4 \\
95.1 \\
21.3\end{array}$ & $\begin{array}{l}95.7 \\
84.9 \\
78.9 \\
99.2 \\
73.7\end{array}$ & $\begin{array}{r}58.6 \\
1.7 \\
31.0 \\
23.8 \\
28.9\end{array}$ \\
\hline 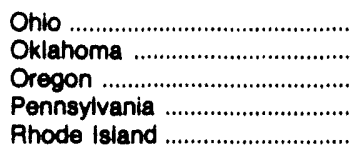 & $\begin{array}{r}78.9 \\
85.4 \\
97.7 \\
71.8 \\
100.0\end{array}$ & $\begin{array}{r}8.4 \\
16.8 \\
24.4 \\
14.7 \\
15.1\end{array}$ & $\begin{array}{r}85.7 \\
80.9 \\
97.7 \\
75.1 \\
100.0\end{array}$ & $\begin{array}{l}12.0 \\
16.4 \\
24.5 \\
20.4 \\
13.3\end{array}$ & $\begin{array}{r}88.4 \\
87.7 \\
97.4 \\
76.6 \\
100.0\end{array}$ & $\begin{array}{l}13.2 \\
18.6 \\
23.4 \\
21.5 \\
11.1\end{array}$ & $\begin{array}{r}88.0 \\
92.3 \\
88.4 \\
79.6 \\
100.0\end{array}$ & $\begin{array}{r}14.9 \\
18.3 \\
18.4 \\
23.4 \\
8.2\end{array}$ \\
\hline 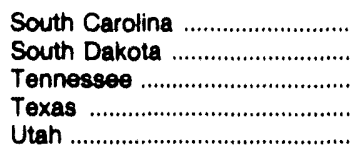 & $\begin{array}{r}98.5 \\
80.7 \\
83.7 \\
79.5 \\
100.0\end{array}$ & $\begin{array}{l}69.2 \\
40.9 \\
50.6 \\
31.0 \\
11.3\end{array}$ & $\begin{array}{r}88.6 \\
83.1 \\
87.2 \\
83.6 \\
100.0\end{array}$ & $\begin{array}{l}61.6 \\
44.4 \\
50.7 \\
32.4 \\
11.8\end{array}$ & $\begin{array}{r}88.8 \\
85.6 \\
86.8 \\
83.4 \\
100.0\end{array}$ & $\begin{array}{c}62.0 \\
46.5 \\
56.0 \\
28.3 \\
10.8\end{array}$ & $\begin{array}{r}99.1 \\
83.3 \\
97.1 \\
85.6 \\
100.0\end{array}$ & $\begin{array}{l}89.0 \\
53.4 \\
57.4 \\
32.5 \\
12.4\end{array}$ \\
\hline 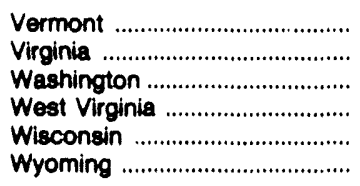 & $\begin{array}{r}100.0 \\
88.8 \\
83.5 \\
54.5 \\
85.8 \\
97.8\end{array}$ & $\begin{array}{r}100.0 \\
25.0 \\
34.8 \\
14.8 \\
36.8 \\
3.2\end{array}$ & $\begin{array}{r}100.0 \\
91.7 \\
85.5 \\
63.0 \\
90.0 \\
98.4\end{array}$ & $\begin{array}{r}100.0 \\
26.4 \\
35.0 \\
17.4 \\
42.3 \\
4.3\end{array}$ & $\begin{array}{r}100.0 \\
83.7 \\
88.7 \\
58.8 \\
81.6 \\
87.8\end{array}$ & $\begin{array}{r}100.0 \\
23.5 \\
37.5 \\
18.1 \\
44.5 \\
4.8\end{array}$ & $\begin{array}{r}100.0 \\
93.3 \\
89.0 \\
65.9 \\
92.1 \\
88.1\end{array}$ & $\begin{array}{r}100.0 \\
27.5 \\
38.1 \\
18.3 \\
46.6 \\
4.9\end{array}$ \\
\hline 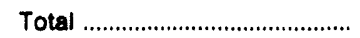 & 79.3 & 29.8 & 84.0 & 31.3 & 84.7 & 29.8 & 85.2 & 31.4 \\
\hline
\end{tabular}

See footnotes at end of table. 
Table 28. Percentage of Total Dellverles Represented by Onsystem Sales by State, 1991-1993 (Continued)

\begin{tabular}{|c|c|c|c|c|c|c|c|c|}
\hline \multirow[t]{2}{*}{ State } & \multicolumn{2}{|c|}{$\begin{array}{c}\text { January } \\
1992\end{array}$} & \multicolumn{2}{|c|}{$\begin{array}{l}\text { Total } \\
1891\end{array}$} & \multicolumn{2}{|c|}{$\begin{array}{c}\text { December } \\
1991\end{array}$} & \multicolumn{2}{|c|}{$\begin{array}{c}\text { November } \\
1991\end{array}$} \\
\hline & Commercial & Industrial & Commercial & Industrial & Commercial & Industrial & Commercial & Industrial \\
\hline 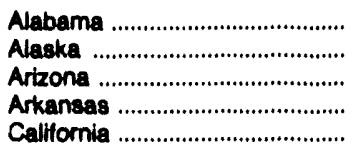 & $\begin{array}{r}83.7 \\
100.0 \\
97.2 \\
93.0 \\
86.6\end{array}$ & $\begin{array}{l}29.1 \\
68.7 \\
33.1 \\
11.5 \\
20.6\end{array}$ & $\begin{array}{r}82.7 \\
100.0 \\
93.2 \\
91.5 \\
77.8\end{array}$ & $\begin{array}{l}31.4 \\
69.1 \\
48.1 \\
19.4 \\
29.8\end{array}$ & $\begin{array}{r}81.7 \\
100.0 \\
97.7 \\
92.3 \\
80.3\end{array}$ & $\begin{array}{l}30.4 \\
70.8 \\
40.4 \\
19.0 \\
25.6\end{array}$ & $\begin{array}{r}75.5 \\
100.0 \\
92.8 \\
90.5 \\
69.8\end{array}$ & $\begin{array}{l}29.3 \\
68.4 \\
31.6 \\
19.1 \\
29.8\end{array}$ \\
\hline 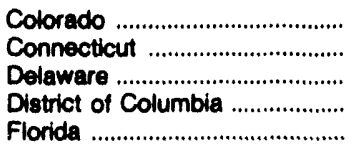 & $\begin{array}{r}96.7 \\
97.7 \\
100.0 \\
99.6 \\
96.8\end{array}$ & $\begin{array}{c}28.2 \\
75.8 \\
65.8 \\
- \\
38.2\end{array}$ & $\begin{array}{r}86.0 \\
83.0 \\
100.0 \\
97.3 \\
97.4\end{array}$ & $\begin{array}{c}34.7 \\
68.2 \\
65.9 \\
-\overline{40.7}\end{array}$ & $\begin{array}{r}96.3 \\
96.6 \\
100.0 \\
99.5 \\
96.8\end{array}$ & $\begin{array}{c}29.9 \\
73.5 \\
67.3 \\
-. \\
40.1\end{array}$ & $\begin{array}{r}94.7 \\
93.0 \\
100.0 \\
95.7 \\
96.8\end{array}$ & $\begin{array}{c}31.6 \\
67.2 \\
63.9 \\
- \\
37.4\end{array}$ \\
\hline 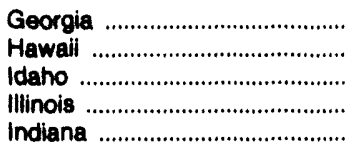 & $\begin{array}{r}90.8 \\
100.0 \\
89.4 \\
63.0 \\
93.6\end{array}$ & $\begin{array}{r}34.4 \\
- \\
.0 \\
21.2 \\
23.2\end{array}$ & $\begin{array}{r}87.5 \\
100.0 \\
87.6 \\
58.0 \\
94.2\end{array}$ & $\begin{array}{c}36.8 \\
- \\
.4 \\
15.4 \\
21.4\end{array}$ & $\begin{array}{r}92.5 \\
100.0 \\
87.5 \\
60.3 \\
95.4\end{array}$ & $\begin{array}{r}35.7 \\
- \\
.4 \\
21.1 \\
25.9\end{array}$ & $\begin{array}{r}90.7 \\
100.0 \\
85.1 \\
60.4 \\
97.9\end{array}$ & $\begin{array}{r}37.2 \\
-. \\
.5 \\
21.1 \\
28.5\end{array}$ \\
\hline 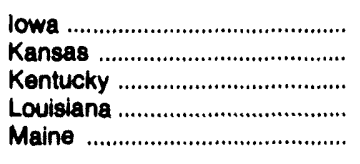 & $\begin{array}{r}97.6 \\
82.9 \\
95.4 \\
82.3 \\
100.0\end{array}$ & $\begin{array}{r}22.1 \\
8.5 \\
31.8 \\
43.6 \\
100.0\end{array}$ & $\begin{array}{r}97.7 \\
88.2 \\
84.0 \\
99.1 \\
100.0\end{array}$ & $\begin{array}{l}28.1 \\
15.1 \\
26.8 \\
45.3 \\
95.9\end{array}$ & $\begin{array}{r}98.0 \\
65.3 \\
95.5 \\
97.6 \\
100.0\end{array}$ & $\begin{array}{r}26.1 \\
11.5 \\
37.4 \\
44.5 \\
100.0\end{array}$ & $\begin{array}{r}98.5 \\
87.7 \\
95.7 \\
97.4 \\
100.0\end{array}$ & $\begin{array}{r}26.0 \\
12.2 \\
38.1 \\
45.0 \\
100.0\end{array}$ \\
\hline 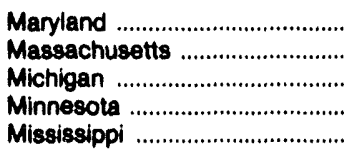 & $\begin{array}{l}97.2 \\
99.9 \\
73.7 \\
99.6 \\
96.9\end{array}$ & $\begin{array}{l}32.2 \\
60.0 \\
15.5 \\
46.4 \\
46.2\end{array}$ & $\begin{array}{l}96.6 \\
99.8 \\
68.3 \\
95.2 \\
95.9\end{array}$ & $\begin{array}{l}15.8 \\
71.0 \\
10.8 \\
41.8 \\
48.0\end{array}$ & $\begin{array}{l}97.4 \\
99.9 \\
70.0 \\
98.0 \\
96.4\end{array}$ & $\begin{array}{l}24.2 \\
68.7 \\
12.9 \\
55.5 \\
47.6\end{array}$ & $\begin{array}{l}96.5 \\
99.9 \\
87.2 \\
97.0 \\
95.5\end{array}$ & $\begin{array}{l}16.2 \\
64.6 \\
12.5 \\
53.2 \\
49.3\end{array}$ \\
\hline 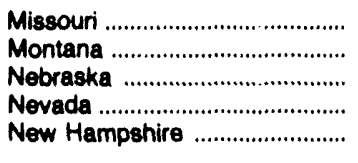 & $\begin{array}{r}89.7 \\
97.1 \\
91.7 \\
90.6 \\
100.0\end{array}$ & $\begin{array}{r}29.5 \\
24.7 \\
50.2 \\
8.6 \\
100.0\end{array}$ & $\begin{array}{r}85.8 \\
97.5 \\
91.8 \\
80.8 \\
100.0\end{array}$ & $\begin{array}{l}25.3 \\
54.5 \\
46.4 \\
14.1 \\
94.1\end{array}$ & $\begin{array}{r}88.5 \\
97.2 \\
91.4 \\
89.0 \\
100.0\end{array}$ & $\begin{array}{r}30.4 \\
24.4 \\
60.6 \\
12.5 \\
100.0\end{array}$ & $\begin{array}{r}85.9 \\
96.5 \\
80.5 \\
82.2 \\
100.0\end{array}$ & $\begin{array}{r}28.2 \\
22.6 \\
64.3 \\
14.7 \\
100.0\end{array}$ \\
\hline 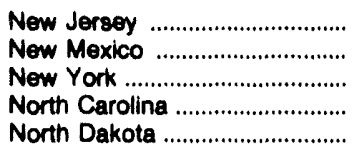 & $\begin{array}{l}96.8 \\
84.7 \\
81.0 \\
99.3 \\
71.7\end{array}$ & $\begin{array}{r}55.8 \\
3.8 \\
33.5 \\
98.4 \\
25.7\end{array}$ & $\begin{array}{l}93.9 \\
77.7 \\
80.7 \\
95.0 \\
70.0\end{array}$ & $\begin{array}{l}55.9 \\
10.5 \\
29.4 \\
72.5 \\
23.9\end{array}$ & $\begin{array}{l}97.0 \\
72.9 \\
81.1 \\
99.3 \\
70.1\end{array}$ & $\begin{array}{r}67.3 \\
6.3 \\
32.4 \\
97.8 \\
27.6\end{array}$ & $\begin{array}{l}93.2 \\
75.5 \\
79.5 \\
98.0 \\
67.6\end{array}$ & $\begin{array}{r}60.5 \\
8.3 \\
29.5 \\
94.8 \\
25.4\end{array}$ \\
\hline 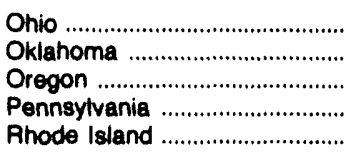 & $\begin{array}{r}89.1 \\
91.6 \\
98.6 \\
77.1 \\
100.0\end{array}$ & $\begin{array}{r}14.5 \\
30.2 \\
19.9 \\
29.3 \\
9.2\end{array}$ & $\begin{array}{r}86.7 \\
81.3 \\
97.8 \\
77.3 \\
100.0\end{array}$ & $\begin{array}{l}12.0 \\
42.0 \\
18.1 \\
20.9 \\
14.3\end{array}$ & $\begin{array}{r}89.8 \\
90.6 \\
98.0 \\
76.7 \\
100.0\end{array}$ & $\begin{array}{r}15.0 \\
30.0 \\
21.6 \\
27.4 \\
9.7\end{array}$ & $\begin{array}{r}87.8 \\
87.6 \\
97.6 \\
76.4 \\
100.0\end{array}$ & $\begin{array}{r}13.2 \\
23.2 \\
18.7 \\
23.6 \\
9.7\end{array}$ \\
\hline $\begin{array}{l}\text { South Carolina } \\
\text { South Dakota } \\
\text { Tennessee } \\
\text { Texas } \\
\text { Utah }\end{array}$ & $\begin{array}{r}99.1 \\
87.0 \\
86.6 \\
86.8 \\
100.0\end{array}$ & $\begin{array}{l}77.9 \\
67.2 \\
57.3 \\
30.1 \\
12.8\end{array}$ & $\begin{array}{r}88.2 \\
81.8 \\
95.7 \\
89.3 \\
100.0\end{array}$ & $\begin{array}{l}70.4 \\
55.1 \\
55.6 \\
30.7 \\
15.3\end{array}$ & $\begin{array}{r}98.8 \\
85.2 \\
97.8 \\
87.6 \\
100.0\end{array}$ & $\begin{array}{l}71.1 \\
62.8 \\
59.4 \\
27.4 \\
10.4\end{array}$ & $\begin{array}{r}98.9 \\
83.4 \\
97.1 \\
84.8 \\
100.0\end{array}$ & $\begin{array}{l}70.2 \\
59.2 \\
59.6 \\
28.7 \\
11.8\end{array}$ \\
\hline 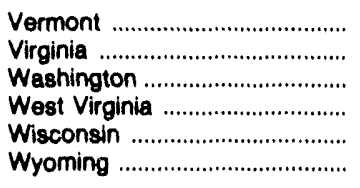 & $\begin{array}{r}100.0 \\
94.6 \\
92.1 \\
65.0 \\
92.7 \\
88.6\end{array}$ & $\begin{array}{r}100.0 \\
25.4 \\
38.0 \\
16.4 \\
46.3 \\
5.3\end{array}$ & $\begin{array}{r}100.0 \\
93.6 \\
92.2 \\
54.9 \\
91.0 \\
99.0\end{array}$ & $\begin{array}{r}100.0 \\
28.8 \\
44.2 \\
21.4 \\
42.7 \\
6.3\end{array}$ & $\begin{array}{r}100.0 \\
93.5 \\
92.1 \\
58.0 \\
92.1 \\
98.5\end{array}$ & $\begin{array}{r}100.0 \\
38.2 \\
40.2 \\
18.8 \\
48.7 \\
6.3\end{array}$ & $\begin{array}{r}100.0 \\
90.9 \\
88.7 \\
55.6 \\
91.5 \\
98.6\end{array}$ & $\begin{array}{r}100.0 \\
40.0 \\
40.6 \\
15.9 \\
47.3 \\
5.6\end{array}$ \\
\hline Total & 87.1 & 31.8 & 85.1 & 32.7 & 86.1 & 32.3 & 85.1 & 32.8 \\
\hline
\end{tabular}

$R_{M}=$ Revised Data
$=$ Not Available.

- $=$ Not Applicable

Notes: Volumes of natural gas reported for the commercial and industrial sectors in this publication include data for both sales and deliveries for the account of others. This table shows the percent of the total State volume that represents natural gas sales to the commercial and industrial sectors. This information may be helptul in evaluating commercial and industrial price data which are based on sales data only. See Appendix C, Statistical Considerations, for a discussion of the computation of natural gas prices.

Source: Form ElA-857. 


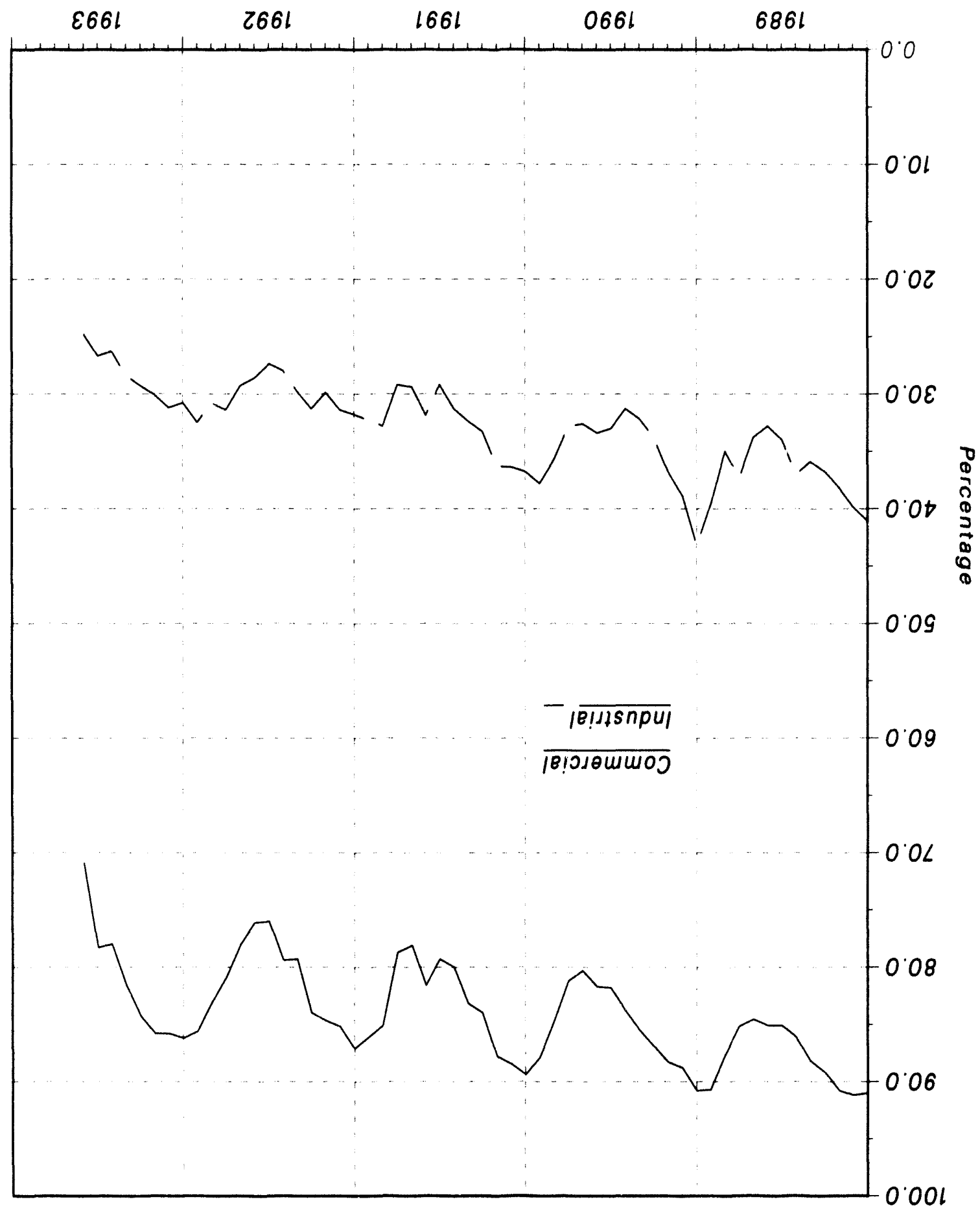


Appendix A

Explanatory Notes 


\section{Explanatory Notes}

The Energy Information Administration (EIA) publishes monthly data for the supply and disposition of natural gas in the United States in the Natural Gas Monthly. These data are preliminary when initially published. Some of these monthly data are estimates developed by EIA staff. Others are taken or estimated from submitted reports. The table below lists the methodologies for deriving the monthly data to be published initially for the components of supply and disposition.

Tablo A1. Mothodology for Roporting Initial Monthly Natural Gas Supply and Disposition Data

\begin{tabular}{|c|c|}
\hline Components & Reporting Methodology \\
\hline \multicolumn{2}{|l|}{ Supply and Disposition } \\
\hline Marketed Production & Estimated from Historical Data \\
\hline Extraction Loss & Derived from Marketed Production \\
\hline Dry Production & Marketed Production minus Extraction Loss \\
\hline Withdrawals from Storage & Reported on Form EIA-191 \\
\hline Supplemental Gaseous Fuels & Derived from Supply Estimates and Coal Gasification Information \\
\hline Imports & $\begin{array}{l}\text { Estimated from National Energy Board of Canada Information and Lique- } \\
\text { fied Natural Gas Information }\end{array}$ \\
\hline Additions to Storage & Reported on Form EIA-191 \\
\hline Exports & Estimated from Industry Trends and Liquefied Natural Gas Information \\
\hline Current-Month Consumption & Estimated from Historical Month-to-Month Percent Changes \\
\hline \multicolumn{2}{|l|}{ Prior-Month Consumption } \\
\hline Lease and Plant Fuel & Derived from Marketed Production \\
\hline Pipeline Fuel & $\begin{array}{l}\text { Derived from Estimates for Lease and Plant Fuel and Deliveries to } \\
\text { Consumers }\end{array}$ \\
\hline \multicolumn{2}{|l|}{ Deliveries to Consumers } \\
\hline Residential & Estimated from Reports to the Sample Survey Form EIA-857 \\
\hline Commercial & Estimated from Reports to the Sample Survey Form EIA-857 \\
\hline Industrial & Estimated from Reports to the Sample Survey Form EIA-857 \\
\hline Electric Utilities & Reported on Form EIA-759 \\
\hline
\end{tabular}


Note 1. Nonhydrocarbon Gases Removed

\section{Annual Data}

Data on nonhydrocarbon gases removed from marketed production--carbon dioxide, helium, hydrogen sulfide, and nitrogen--are reported by State agencies on the voluntary Form EIA-627. For 1992, of the 33 producing States, 23 reported data on nonhydrocarbon gases removed. The 23 States accounted for 61 percent of total 1992 gross withdrawals. Of the 23 States reporting nonhydrocarbon gases removed, 13 reported zero values: Alaska, Arizona, Arkansas, Colorado, Illinois, Indiana, Maryland, Missouri, Nevada, New York, Oregon, South Dakota, and Virginia. The eight States reporting volumes greater than zero are Alabama, California, Florida, Mississippi, New Mexico, North Dakota, Texas, and Wyoming. Two States (Kentucky and Nebraska) reported quantities unknown but considered insignificant. In addition, Kansas, Louisiana, Montana, and Oklahoma, which together accounted for 35 percent of gross withdrawals, did not report nonhydrocarbon gases removed separately. However, their gross withdrawal data excluded all or most of the nonhydrocarbon gases removed on leases. No estimates are made for States not reporting nonhydrocarbon gases removed.

\section{Preliminary Monthly Data}

All monthly data are considered preliminary until after publication of the Natural Gas Annual for the year in which the report month falls. Three States report monthly data on nonhydrocarbon gases removed: Alabama, Texas, and Mississippi. Monthly data for California, Colorado, Florida, New Mexico, North Dakota, and Wyoming are estimated based on annual data reported on Form EIA-627. Nonhydrocarbon gases as an annual percentage of gross withdrawals reported by each of the six States is applied to each State's monthly gross withdrawal data to produce an estimate of nonhydrocarbon gases removed.

\section{Final Monthly Data}

Beginning with report year 1990, States filing the Form EIA-627, "Annual Quantity and Value of Natural Gas Report," were asked to supply monthly breakdowns of all data previously reported on an annual basis. The sums of the reported figures were used to calculate monthly volumes.

For States not supplying monthly data on the EIA-627, final monthly data are calculated by proportionally allocating the differences between total annual data reported on the Form EIA-627 and the sum of monthly data (January-December).

\section{Note 2. Supplemental Gaseous Fuels}

\section{Annual Data}

Annual data are published from Form EIA-176.

\section{Preliminary Monthly Data}

All monthly data are considered preliminary until after the publication of the Natural Gas Annual for the year in which the report month falls. Monthly estimates are based on the annual ratio of supplemental gaseous fuels to the sum of dry gas production, net imports, and net withdrawals from storage. This ratio is applied to the monthly sum of these three elements to compute a monthly supplemental gaseous fuels figure.

\section{Final Monthly Data}

Monthly data are revised after publication of the Natural Gas Annual. Final monthly data are estimated based on the revised annual ratio of supplemental gaseous fuels to the sum of dry gas production, net imports, and net withdrawals from storage. This ratio is applied to the revised monthly sum of these three elements to compute final monthly data.

\section{Note 3. Production}

\section{Annual Data}

Natural gas production data are collected from 33 gasproducing States on Form EIA-627 which includes gross withdrawals, vented and flared, repressuring, nonhydrocarbon gases removed, fuel used on leases, marketed production (wet), and extraction loss. The U.S. Minerals Management Service (MMS) also supplies data on the quantity and value of natural gas production on the Gulf of Mexico and Outer Continental Shelf. No adjustments are made to the data.

\section{Estimated Monthly Data}

State marketed production data for a particular month are estimated if data are unavailable at the time of publication. The data are estimated based on final monthly data reported on the Form EIA-627 for the previous year.

Estimates for total U.S. marketed production are based on final monthly data reported on the Form EIA-627 
for the previous year. State estimates for nonhydrocarbon gas removed, gas used for repressuring, and gas vented and flared are based on the ratio of the that item to gross withdrawals as reported on the ElA-627. These ratios are applied to the month's estimates for gross withdrawals to calculate figures for nonhydrocarbon gases removed, gas used for repressuring, and gas vented and flared. Estimates for gross withdrawal data are calculated from final monthly data filed on Form EIA-627 for the previous year.

\section{Prellminary Monthly Data}

All monthly data are considered preliminary until after publication of the Natural Gas Annual for the year in which the report month falls. Preliminary monthly data are published from reports from the Interstate Oil and Gas Compact Commission (IOGCC) and the MMS. Volumetric data are converted, as necessary, to a standard 14.73 psia pressure base. Data are revised as Table 7 monthly data are updated.

\section{Final Monthly Data}

Final monthly data for 1991 and 1992 are the sums of monthly data reported on the annual Form EIA-627, "Annual Quantity and Value of Natural Gas Report." For prior years, the differences between each State's annual production data reported on the EIA-627 and the sum of its monthly IOGCC reports for the year were allocated proportionally to the monthly IOGCC data.

\section{Note 4. Imports and Exports}

\section{Annual Data and Final Monthly Data}

Annual and final monthly data are published from the annual Form FPC-14, which requires data to be reported by month for the calendar year.

\section{Preliminary Monthly Data - Imports}

Preliminary monthly import data are based on data from the National Energy Board of Canada and responses to informal industry contacts and EIA estimates. Preliminary data are revised after the publication of the article "U.S. Imports and Exports of Natural Gas" for the calendar year.

\section{Prellminary Monthly Data - Exports}

Preliminary monthly export data are based on historical data from the Form FPC-14, informal industry contacts, and information gathered from natural gas industry trade publications. Preliminary monthly data are revised after publication of "U.S. Imports and Exports of Natural Gas" for the calendar year in which the report month falls.

\section{Note 5. Consumption}

\section{All Annual Data}

All consumption data except electric utility data are from the Form EIA-857 and Form EIA-176. No adjustments are made to the data. Electric utility data are reported on Form EIA-759.

\section{Monthly Data}

All monthly data are considered preliminary until after publication of the Natural Gas Annual.

\section{Total Consumption}

\section{Preliminary Monthly Data}

The most current month estimate is calculated based on the arithmetic average change from the previous month for the previous 3 years. The following month this estimate is revised by summing the components (pipeline fuel, lease and plant fuel, and deliveries to consumers).

\section{Final Monthly Data}

Monthly data are revised after publication of the Natural Gas Annual. Final monthly total consumption is obtained by summing its components.

\section{Residential, Commercial, and Industrial Sector Consumption}

\author{
Preliminary Monthly Data \\ Preliminary monthly residential, commercial, and in- \\ dustrial data are from Form EIA-857. See Appendix \\ C, "Statistical Considerations," for a detailed explana- \\ tion of sample selection and estimation procedures.
}


Price data are representative of prices for gas sold and delivered to residential, commercial, and industrial consumers. These prices do not reflect average prices of natural gas transported to consumers for the account of third parties or "spot-market" prices.

\section{Final Monthly Data}

Monthly data are revised after the publication of the Natural Gas Annual. Final monthly data are estimated by allocating annual consumption data from the Form EIA-176 to each month in proportion to monthly volumes reported in Form EIA-857.

\section{Electric Utility Sector Consumption}

\author{
All Monthly Data \\ Monthly data published are from Form EIA-759.
}

\section{Pipeline Fuel Consumption}

\section{Preliminary Monthly Data}

Preliminary data are estimated based on the pipeline fuel consumption as an annual percentage of total consumption from the previous year's Form EIA-176. This percentage is applied to each month's total consumption figure to compute the monthly estimate.

\section{Final Monthly Data}

Monthly data are revised after the publication of the Natural Gas Annual. Final monthly data are based on the revised annual ratio of pipeline fuel consumption to total consumption from the Form EIA-176. This ratio is applied to each month's revised total consumption figure to compute final monthly pipeline fuel consumption estimates.

\section{Lease and Plant Fuel Consumption}

\section{Preliminary Monthly Data}

Preliminary monthly data are estimated based on lease and plant fuel consumption as an annual percentage of marketed production. This percentage is applied to each month's marketed production figure to compute estimated lease and plant fuel consumption.
Final Monthly Data

Monthly data are revised after publication of the Natural Gas Annual. Final monthly data are based on a revised annual ratio of lease and plant fuel consumption to marketed production from Form EIA-176. This ratio is applied to each month's revised marketed production figure to compute final monthly lease and plant fuel consumption estimates.

\section{Note 6. Extraction Loss}

\section{Annual Data}

Extraction loss data are calculated from filings of Form EIA-64A, "Annual Report of the Origin of Natural Gas Liquids Production." For a fuller discussion, see the Natural Gas Annual.

\section{Preliminary Monthly Data}

Preliminary data are estimated based on extraction loss as an annual percentage of marketed production. This percentage is applied to each month's marketed production to estimate monthly extraction loss.

\section{Final Monthly Data}

Monthly data are revised after the publication of the Natural Gas Annual. Final monthly data are estimated by allocating annual extraction loss data to each month based on its total natural gas marketed production.

\section{Note 7. Natural Gas Storage}

\section{Underground Natural Gas Storage}

All monthly data concerning underground storage are published from the EIA-191. A new EIA-191 became effective in January 1991. Injection and withdrawal data from the EIA-191 survey are adjusted to correspond to data from Form EIA-176 following publication of the Natural Gas Annual.

\section{Underground and Liquefied Natural Gas Storage}

The final monthly and annual storage and withdrawal data for 1987 through 1992 shown in Table 2 include both underground and liquefied natural gas (LNG) storage. Underground storage data are obtained from 
the EIA-191 and EIA-176 surveys in the manner described earlier. Annual data on LNG additions and withdrawals are taken from Form EIA-176. Monthly data are estimated by computing the ratio of each month's underground storage additions and withdrawals to annual underground storage additions and withdrawals and applying it to annual LNG data.

\section{Note 8. Average Wellhead Value}

\section{Annual Data}

Form EIA-627 requests State agencies to report the quan .cty and value of marketed production. When complete data are unavailable, the form instructs the State agency to report the available value and the quantity of marketed production a sociated with this value. A number of States reported volumes of production and associated values for other than marketed production. In addition, information for several States which were unable to provide data was obtained from Form EIA-176. It should be noted that Form EIA-176 reports a fraction of State production. The imputed value of marketed production in each State is calculated by dividing the State's reported value by its associated production. This unit price is then applied to the quantity of the State's marketed production to derive the imputed value of marketed production.

\section{Initial Monthly Data}

An initial estimate is calculated based on the statistical relationship between U.S. monthly wellhead gas prices and the monthly composite spot wellhead prices published in the Natural Gas Week. The estimate is prepared using the same methodology that generates monthly gas price estimates for EIA's Short-Term Energy Outlook. The initial estimate is the latest monthly estimate presented.

\section{Preliminary Monthly Data}

A preliminary estimate of the U.S. gas price is made each month based on the change in the productionweighted gas price from four States: Mississippi, New Mexico, Oklahoma, and Texas. Gas prices for these four States are used because both their gas production and value represent a substantial sample of the U.S. gas production and value (roughly 50 percent), and their prices are readily available and provide a consistent series. The latest preliminary U.S. gas price estimate is calculated by multiplying the preliminary U.S. gas price estimate for the prior month by the ratio of the four States' gas price for the latest month to that of the prior month. This estimate replaces the initial gas price estimate.

\section{Final Monthly Data}

Preliminary monthly gas price data for Mississippi, New Mexico, Oklahoma, and Texas are replaced by final monthly data that are adjusted to match the annual prices published in the Natural Gas Annual for each State. A revised set of the monthly U.S. gas price estimates are derived based on the monthly change in the production-weighted prices for these four States and adjusted to match the U.S. gas price published in the Natural Gas Annual.

\section{Note 9. Financial Data of Major Interstate Pipeline Companies}

The prices in Table 4 for imports and purchases from producers by major interstate pipeline companies, and all data in Tables 8 through 12 are derived from Form FERC-11. Form FERC-11 is filed monthly by the approximately 51 major interstate natural gas pipeline companies. A major pipeline company is defined as one "whose combined sales for resale, and gas transported interstate or stored for a fee exceeded 50 billion cubic feet in the previous calendar year."

Data reported by the major interstate pipeline companies on Form FERC-11 generally reflect the timing of data entry, revision, and/or reclassification of accounts in the companies' accounting records in accordance with the FERC regulations and regulatory filings. Certain data may also be estimated. Consequently, the data reported and shown in Tables 8 through 12 for any given month may include or reflect out-of-period dollar or volume adjustments, restatements or revisions, or account reclassifications. The dollar amounts reported as paid or received and volumes reported as delivered or received may also include amounts paid, delivered, or received under contractual provisions such as prepayment, take-or-pay, minimum take, or minimum bill provisions. Unless otherwise footnoted, the individual data items, computed averages, and aggregated totals shown include the effect of any and all such adjustments, revisions, estimates, reclassifications, and/or contractual provisions. A verage prices are not reported on the FERC-11. The averages shown are computed by dividing the total dollars reported for the particular item by the total volume reported for the same item.

\section{Final Monthly Data}

Final revisions for the prior year's data are made upon receipt of the current data which will indicate any revisions. Revisions are made on a month by month basis. 


\section{Note 10. Balancing Item}

The "balancing item" category represents the difference between the sum of the components of natural gas supply and the sum of the components of natural gas disposition. These differences may be due to quantities lost or to the effects of data reporting problems. Reporting problems include differences due to the net result of conversions of flow data metered at varying temperatures and pressure bases and converted to a standard temperature and pressure base; the effect of variations in company accounting and billing practices; differences between billing cycles and calendar periods; and imbalances resulting from the merger of data reporting systems, which vary in scope, format, definitions, and type of respondents.

\section{Annual Data}

Annual data are from the Natural Gas Annual. For an explanation of the methodology involved in calculating annual "balancing item" data, see the Natural Gas Annual.

\section{Preliminary Monthly Data}

Preliminary monthly data in the "balancing item" category are calculated by subtracting dry gas production, withdrawals from storage, supplemental gaseous fuels, and imports from total supply/disposition. 
Appendix B

Data Sources 


\section{Data Sources}

The data in this publication are taken from survey reports authorized by the U.S. Department of Energy (DOE), Energy Information Administration (EIA) and by the Federal Energy Regulatory Commission (FERC). The EIA is the independent statistical and analytical agency within the DOE. The FERC is an independent regulatory commission within the DOE which has jurisdiction primarily in the regulation of electric utilities and the interstate natural gas industry. The EIA conducts and processes some of the surveys authorized by the FERC.

Data are collected from two annual surveys and five monthly surveys. Filings with the FERC also provide sources of data for this publication.

The annual reports are the Form EIA-176, a mandatory survey of all companies that deliver natural gas to consumers or that transport gas across State lines, and the Form EIA-627, a voluntary survey completed by energy or conservation agencies in the gas-producing States.

The monthly reports include three surveys of the natural gas industry and two surveys of the electric utility industry. The natural gas industry survey is the Form EIA-191 filed by companies that operate underground storage facilities, the Form FERC-11 filed by major interstate natural gas pipeline companies, and the Form EIA-857 filed by a sample of companies that deliver natural gas to consumers. The electric utility industry surveys are the Form EIA-759 filed by all generating electric utilities and the Form FERC-423 filed by fossil fueled plants. Responses to these five monthly surveys are mandatory.

A description of the survey respondents, reporting requirements, and processing and editing of the data is given on the following pages for each of the surveys. Also shown are copies of the EIA and FERC survey forms that are sources of data for this publication.

\section{Form EIA-176, "Annual Report of Natural and Supplemental Gas Supply and Disposition"}

\section{Survey Design}

The original version of Form EIA-176 was approved in 1980 with a mandatory response requirement. Prior to 1980 , published data were based on voluntary responses to Bureau of Mines, U.S. Department of the Interior predecessor Forms BOM-6-1340-A and BOM-6-1341-A of the same title. Beginning with 1990 data, the responses are not considered proprietary.

In 1982, the scope of the revised EIA-176 survey was expanded to collect the number of electric utility consumers in each State, volumes of gas transported to industrial and electric utility consumers, detailed information on volumes transported across State borders by the respondent for others and for the responding company, and detailed information on other disposition. These changes were incorporated to provide more complete survey information with a minimal change in respondent burden.

In 1988, the Form EIA-176 was revised to include data collection for deliveries of natural gas to commercial consumers for the account of others. The revised form was approved for use during report years 1987 through 1989. Response to the form was mandatory.

A short version of Form EIA-176 was also approved in 1988. Companies engaged in purchase and delivery activities, but not in transportation and storage activities, may file the short form. Usually, these companies are municipals handling small volumes of gas.

In 1990 , revisions to the Form were approved that permitted collection of data on deliveries of natural gas for use as a vehicle fuel and detailed underground storage information. Data filed on the EIA-176 are no longer held confidential. 


\section{Survey Universe and Response Statistics}

The Form EIA-176 is mailed to all identified interstate and intrastate natural gas pipeline companies, investor and municipally owned natural gas distributors, underground natural gas storage operators, synthetic natural gas plant operators, and field, well, or processing plant operators that deliver natural gas directly to consumers (including their own industrial facilities) and/or that transport gas to, across, or from a State border through field or gathering facilities.

Each company and its parent company or subsidiaries were required to file if they met the survey specifications. The ori,ginal 1993 mailing for report year 1992 totaled 2,104 questionnaire packages. To this original mailing, 6 names were added and 46 were deleted as a result of the survey processing. Additions were the result of comparisons of the mailing list to other survey mailing lists. Deletions resulted from post office returns and determinations that companies were out of business, sold, or not within the scope of the survey. after all updates, the survey universe was 2,064 responses from approximately 1,800 companies.

Following the original mailing, second request mailing, and nonrespondents followup, 2,056 responses were entered into the data base, and there were eight nonrespondents.

\section{Summary of Form EIA-176 Data Reporting Requirements}

The EIA-176 is a multiline schedule for reporting all supplies of natural gas and supplemental gaseous fuels and their disposition within the State indicated. Respondents file completed forms with EIA in Washington, DC. Data for the report year are due by April 1 of the following year. Extensions of the filing deadline for up to 45 days are granted to any respondent on request.
All natural gas and supplemental gaseous fuels volumes are reported on a physical custody basis in thousand cubic feet (Mcf), and dollar values are reported to the nearest whole dollar. All volumes are reported at 14.73 pounds per square inch absolute pressure (psia) and 60 degrees Fahrenheit.

\section{Routine Form EIA-176 Edit Checks}

A series of manual and computerized edit checks are used to screen the Form EIA-176. The edits performed include validity, arithmetic, and analytical checks.

The incoming forms are reviewed prior to keying. This prescan determines if the respondent identification (ID) number and the company name and address are correct, if the data on the form appear complete and reasonable, and if the certifying information is complete.

Manual checks on the data are also made. Each form is prescanned to determine that data were reported on the correct lines. The flow of gas through interstate pipelines is checked at the company level to ensure that each delivery from a State is matched with a corresponding receipt in an adjoining State.

After the data are keyed, computer edit procedures are performed. Edit programs verify the report year, State code, and arithmetic totals. Further tests are made to ensure that all necessary data elements are present and that the data are reasonable and internally consistent. The computerized edit system produces error listings with messages for each failed edit test. When problems occur, respondents are contacted by telephone and required to file amended forms with corrected data.

\section{Other EIA Publications Referencing Form EIA-176}

Data from Form EIA-176 are also published in the Natural Gas Annual. 
Figure B1. Form EIA-176

ElA.176 (Rovised 1991)

U.S. DEPARTMENT OF ENERGY

Form Approved

OMB No. 19050175

ENERGY INFORMATION ADMINISTAATION

Expires: $12 / 31 / 93$

ANNUAL REPORT OF NATURAL AND SUPPLEMENTAL GAS SUPPLY AND DISPOSITION, 19

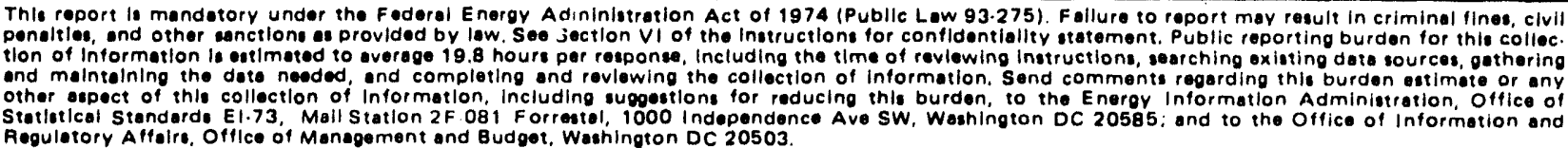

\section{EIA USE}

EIA COPY. Tear out, complete, and return to

Control (ID) No

Affix mailing label or enter mail address

Name:

Operations in (State)

Street or Post Otfice Box

City, State, Zip Code

Attention

Washington, D C 20585

Altn: Form EIA.176

Atrontion:

PART I: IDENTIFICATION

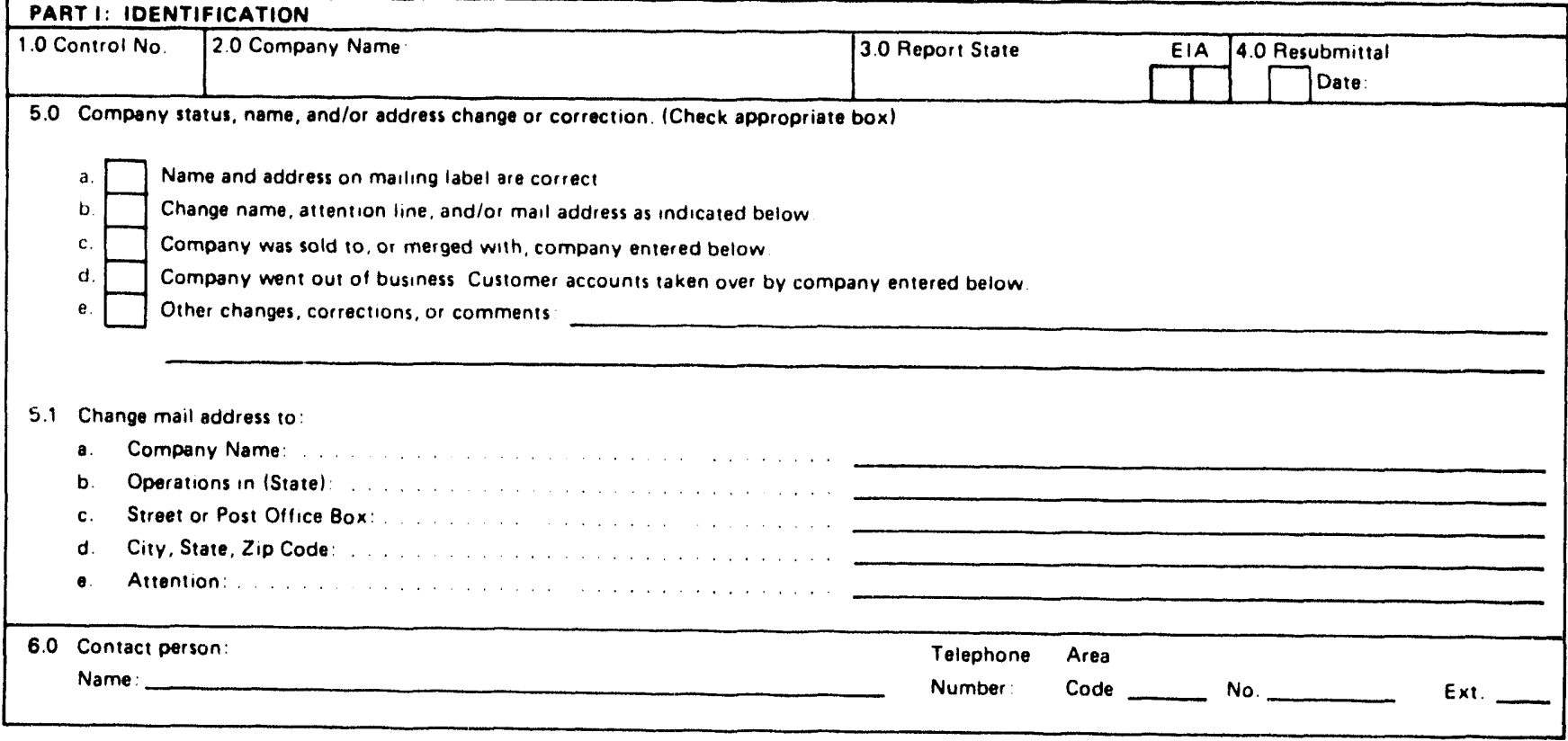

PART II: CERTIFICATION AND DISCLOSURE STATEMENT

1.0 ( certity that (Check appropriate box):

a. The information provided herein and appended hereto is true and accurate or, where indicated on the form, reasonable estimates to the best of my knowledge.

b. My company does not meet any of the criteria set forth in Section II, "Who must submit," of the instructions and is therefore not required to complete and submit a Form ElA.176 for the report State.

\begin{tabular}{|l|l|}
\hline 2.0 Name & 3.0 Title \\
\hline 4.0 Signature & 5.0 Date \\
\hline $\begin{array}{l}\text { Title 18, USC 1001, makes it a crime for any person knowingly and willingly to make to any agency or department of the United States any false, fictitious } \\
\text { or fraudulent statements as to any matter within hs jurisdiction. }\end{array}$ \\
\hline
\end{tabular}


EIA.176, ANNUAL REPORT OF NATURAL AND SUPPLEMENTAL GAS SUPPLY AND DISPOSITION, 19

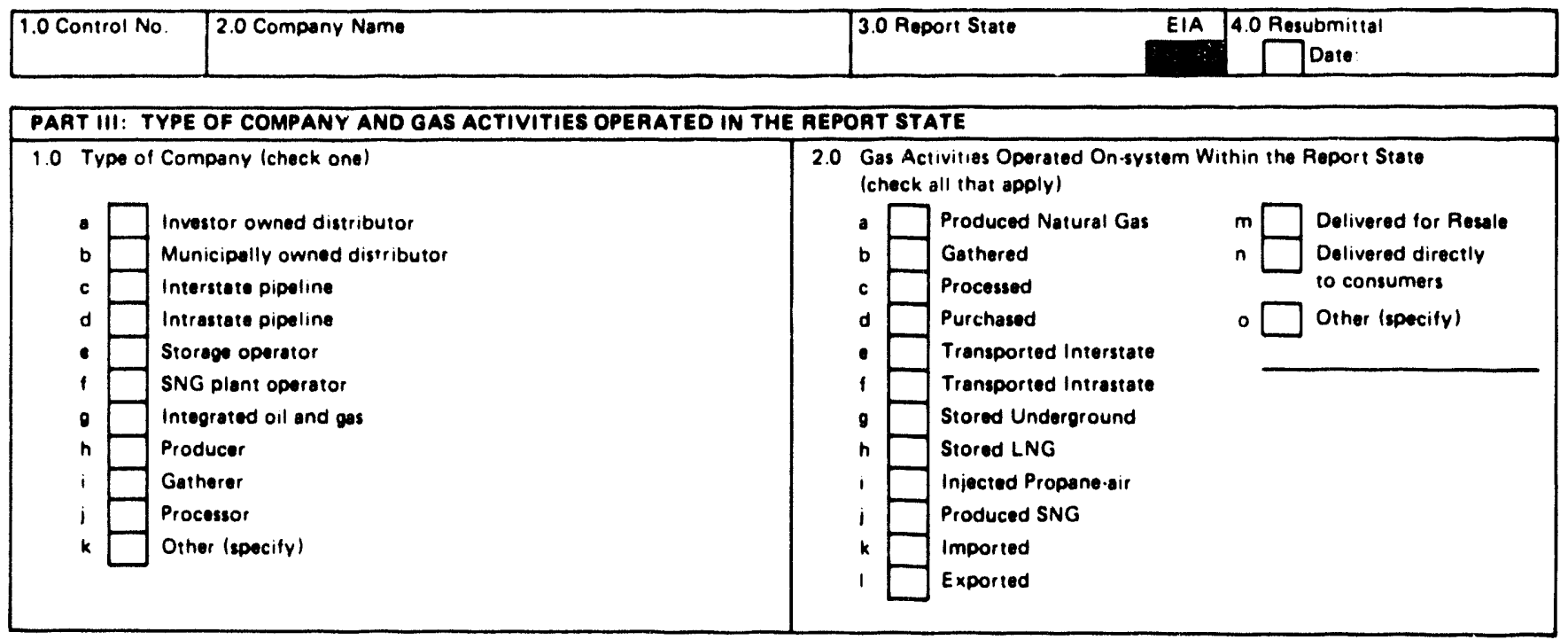

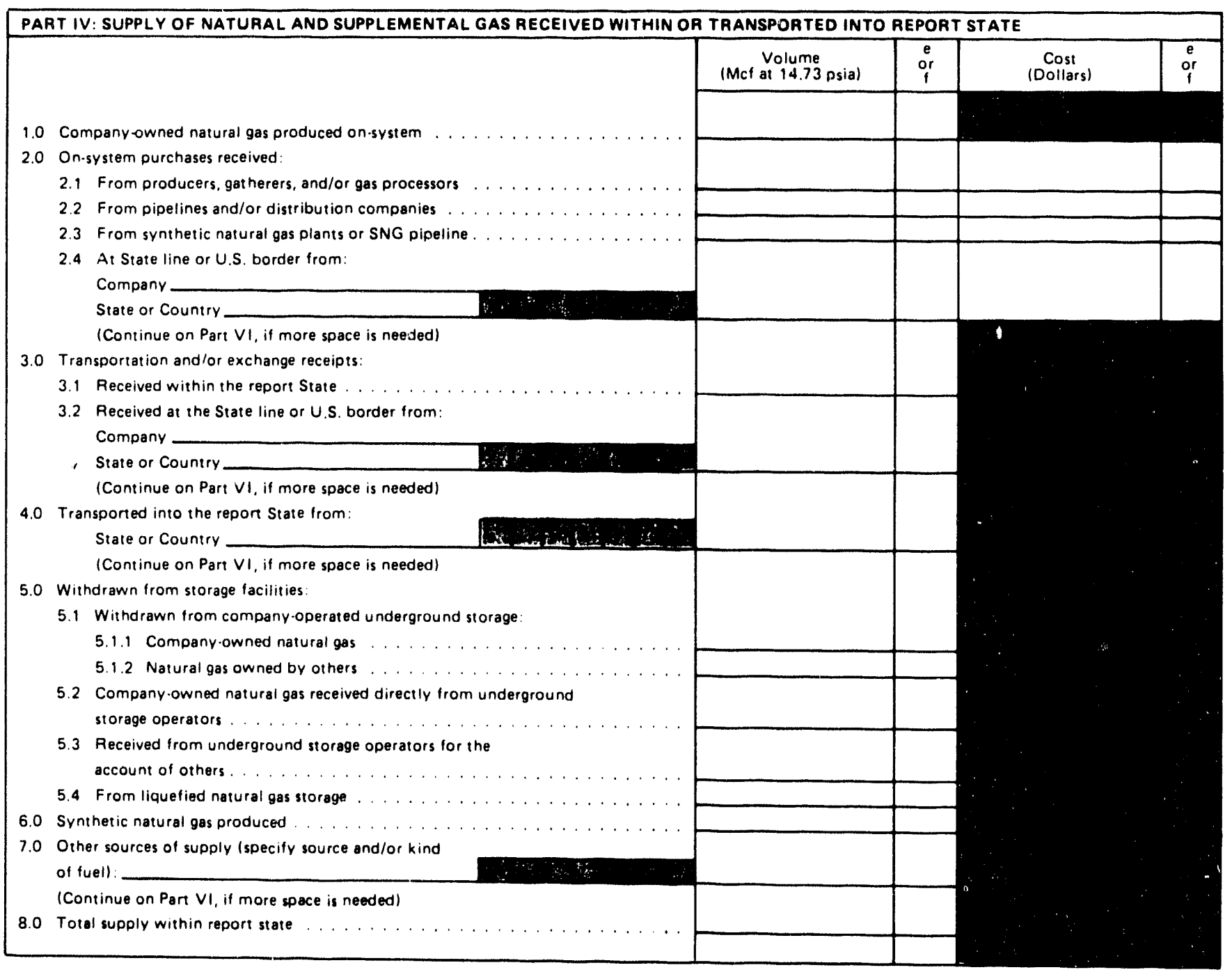


EIA.176, ANNUAL REPORT OF NATURAL AND SUPPLEMENTAL GAS SUPPLY AND DISPOSITION, 19

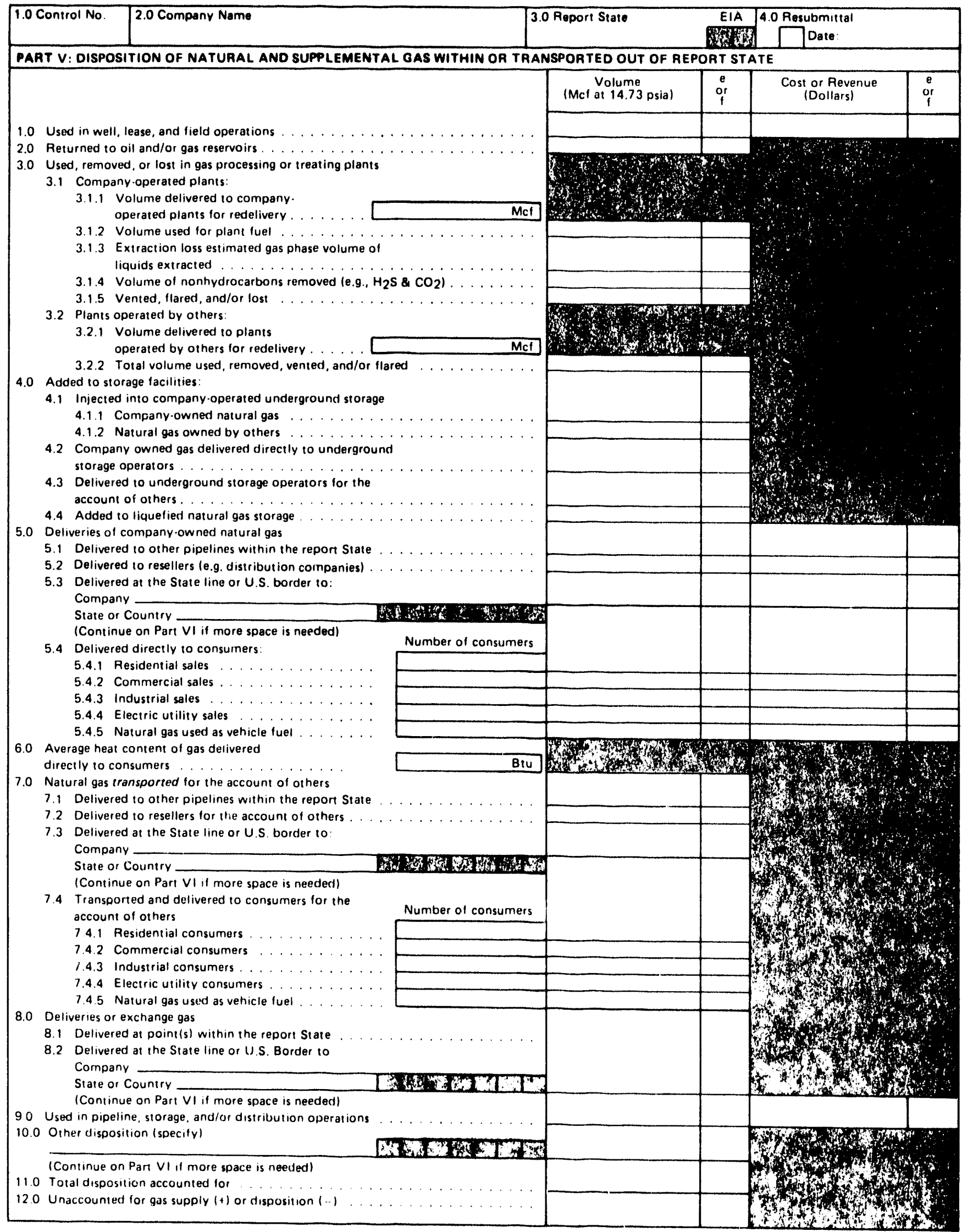


EIA.176, ANNUAL REPORT OF NATURAL AND SUPPLEMENTAL GAS ȘUPLIY AND DISPOSITION, 19 [D

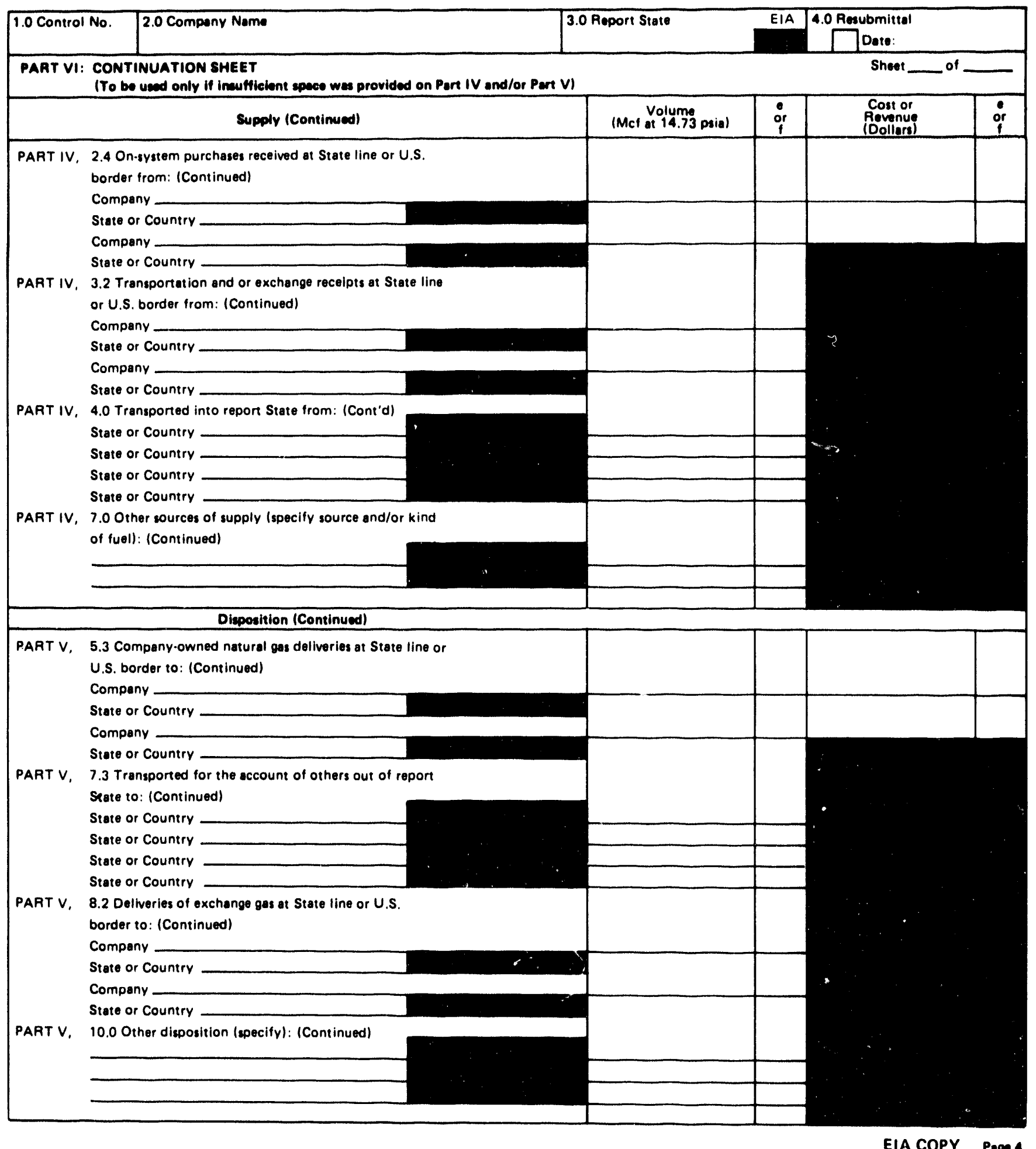


EIA.176, ANNUAL REPORT OF NATURAL AND SUPPLEMENTAL SAS SUPPLY AND DISPOSITION, $19 \square$

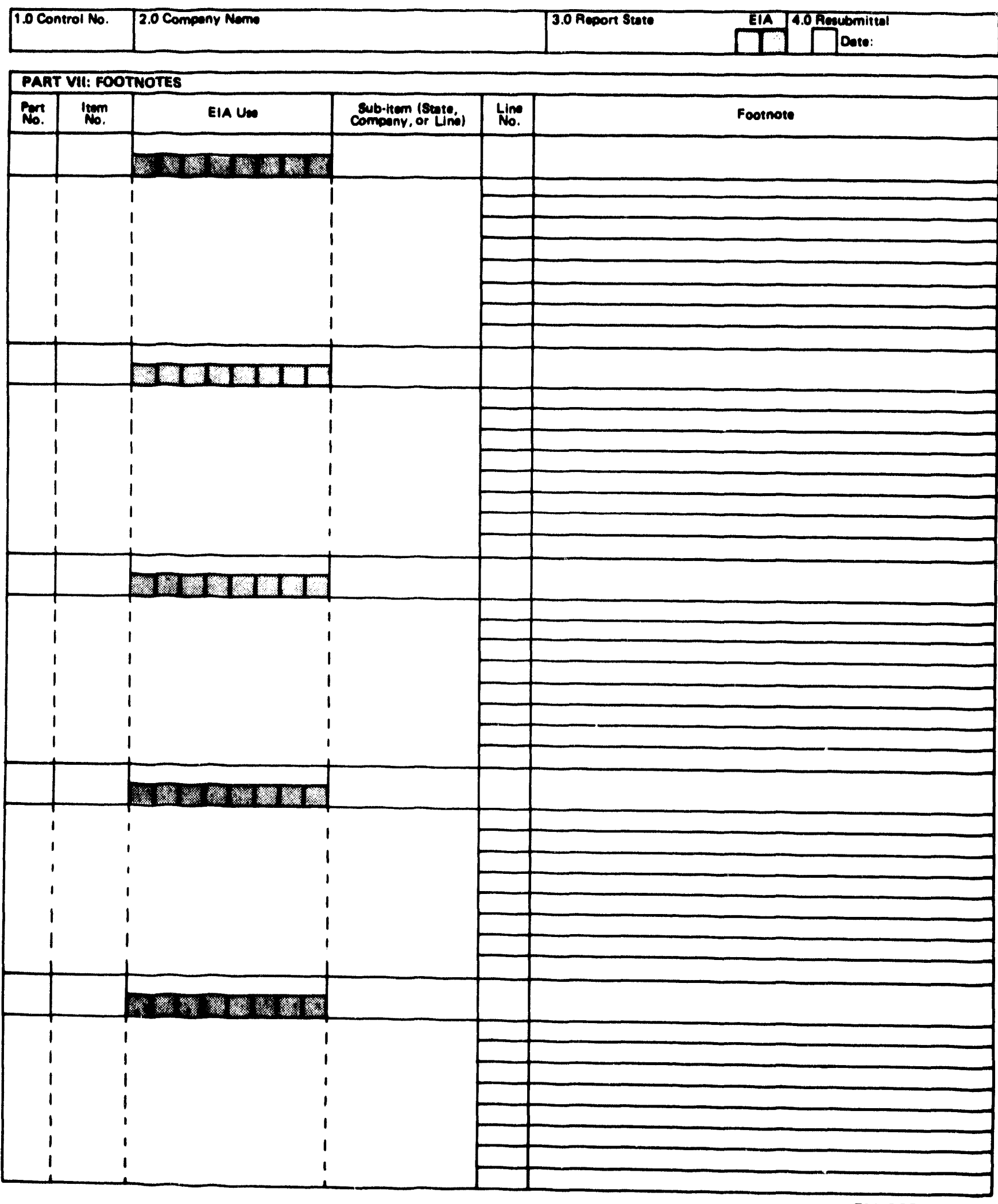




\title{
ANNUAL REPORT OF NATURAL AND SUPPLEMENTAL GAS SUPPLY AND DISPOSITION, $19 \square$
}

\begin{abstract}
Thle report le mendetory under the Federel Energy Administration Act of 1974 (Public Law 93.275). Fallure to report may result in criminal fines, civil penaltes, and other wnctions as provided bv lew. See Section $V I$ of the inetructions for conflidentiality stotement. Public reporting burden for this collec tion of information lo estimated to overage 2 hours per responso, including the time of reviewing Instructions, searching existing date sources, gethering ond maintelning the dete needed, end comploting end reviewing the collection of information. Send commente regarding thie burden estimate or any other espect of this collection of information, Inciuding eugeestions for reducing this burden. to the Energv informetion Administretion. Oifice of stetieticel Stenderde El.73. Mell Stetlon 2F.081 Forrestal, 1000 Independence Ave SW, Weehington DC 20885 , end to the Office of Information end Reguletory Affalrs, Office of Menagement and Budget. Weahington DC 20503
\end{abstract}

\section{EIA USE}

EIA COPY. Tear out, complete, and return to:

Erergy Information Administration

Mail Station: BG.094 FORSTL

U.S. Department of Energy

Washington, D.C. 20585

Attn: Form ElA.176

Affix mailing label or enter mail address

Control (ID) No.

Name:

Operations in (State)

Street or Post Otfice Box:

City, State, Zip Code:

Attention:

PART I: IDENTIFICATION

\begin{tabular}{|l|l|l|l|l|l|l|}
\hline 1.0 Control No. & 2.0 Company Name: & 3.0 Report State Resubmittal \\
\hline
\end{tabular}

5.0 Company status, name, and/or address change or correction. (Check appropriote box.)

a. $\square$ Name and address on mailing label are correct.

b. Change name, attention line, and/or mail oddress as ir dicated below.

c. Company was sold to, or merged with, company entered below.

d. Company went out of business. Customer accounts taken over by company entered below.

e. Other changes, corrections, or comments:

5.1 Change mail address to:

a. Company Name:

b. Operations in (State):

c. Street or Post Office Box

d. City, State, Zip Code

e. Attention:

6.0 Contact person:

Name:

Telephone Ares

Number: Code N_ No.

Ext.

PART II: CERTIFICATION AND DISCLOSURE STATEMENT

1.0 I certity that (Chock appropriate box):

a. The information provided herein and appended hereto is true and accurate or, where indicatec on the form, reasonable satimates to the best of my knowledpe

b. My company does not meet any of the criteria set forth in Section II, "Who must eubmit," of the instructions and is therefore not required to complete and submit a Form EIA.176 for the repon State.

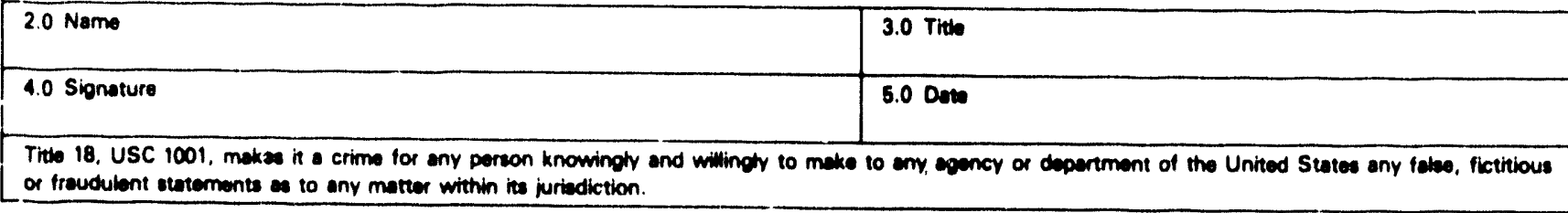


EIA-176, ANNUAL REPORT OF NATURAL AND SUPPLEMENTAL GAS SUPPLY AND DISPOSITION, 19

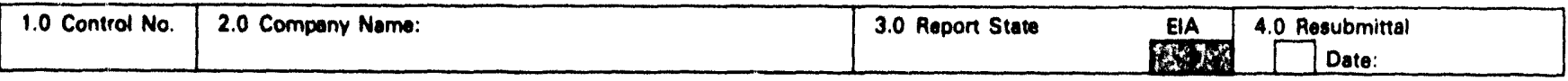

\begin{tabular}{|c|c|c|c|c|c|}
\hline \multicolumn{6}{|c|}{ PART III: TYPE OF COMPANY AND GAS ACTIVITIES OPERATED IN THE REPORT STATE } \\
\hline \multicolumn{2}{|c|}{ 1.0 Typo of Compeny (check onal } & \multicolumn{4}{|c|}{$\begin{array}{l}2.0 \text { Gas Activitie Operated On-svstem Within the Report State } \\
\text { (check all that apply) }\end{array}$} \\
\hline a. & Investor owned diatributor & \multirow{2}{*}{ a. } & \multirow{2}{*}{\multicolumn{2}{|c|}{$\begin{array}{ll}\text { Produced Natural Gas } & \mathrm{m} . \\
\text { Gathered } & \mathrm{n} .\end{array}$}} & \multirow{4}{*}{$\begin{array}{l}\text { Delivered for Resale } \\
\text { Delivered directly } \\
\text { to consumers } \\
\text { Other (specity) }\end{array}$} \\
\hline b. & Municipathy owned diatributor & & & & \\
\hline c. & Interstete pipeline & \multirow{2}{*}{ c. } & \multicolumn{2}{|l|}{ Procesered } & \\
\hline d. & Intruatute plpeline & & \multirow{2}{*}{$\begin{array}{l}\text { Purchased } \\
\text { Transported Interstate }\end{array}$} & \multirow[t]{2}{*}{0.} & \\
\hline •. & Storage operator & \multirow{2}{*}{-. } & & & \\
\hline f. & SNG plant operator & & Traneported Intrastate & & \\
\hline o. & Integrated oll and ges & f. & \multirow{2}{*}{$\begin{array}{l}\text { Stored Underground } \\
\text { Stored LNG }\end{array}$} & & \\
\hline h. & Producer & h. & & & \\
\hline i. & Gatherer & \multirow{2}{*}{ i. } & Injected Propane-air & & \\
\hline i. & Proceseror & & \multirow{3}{*}{$\begin{array}{l}\text { Produced SNG } \\
\text { Imported } \\
\text { Exported }\end{array}$} & & \\
\hline k. & Other (epecify) & k. & & & \\
\hline & & I. & & & \\
\hline
\end{tabular}

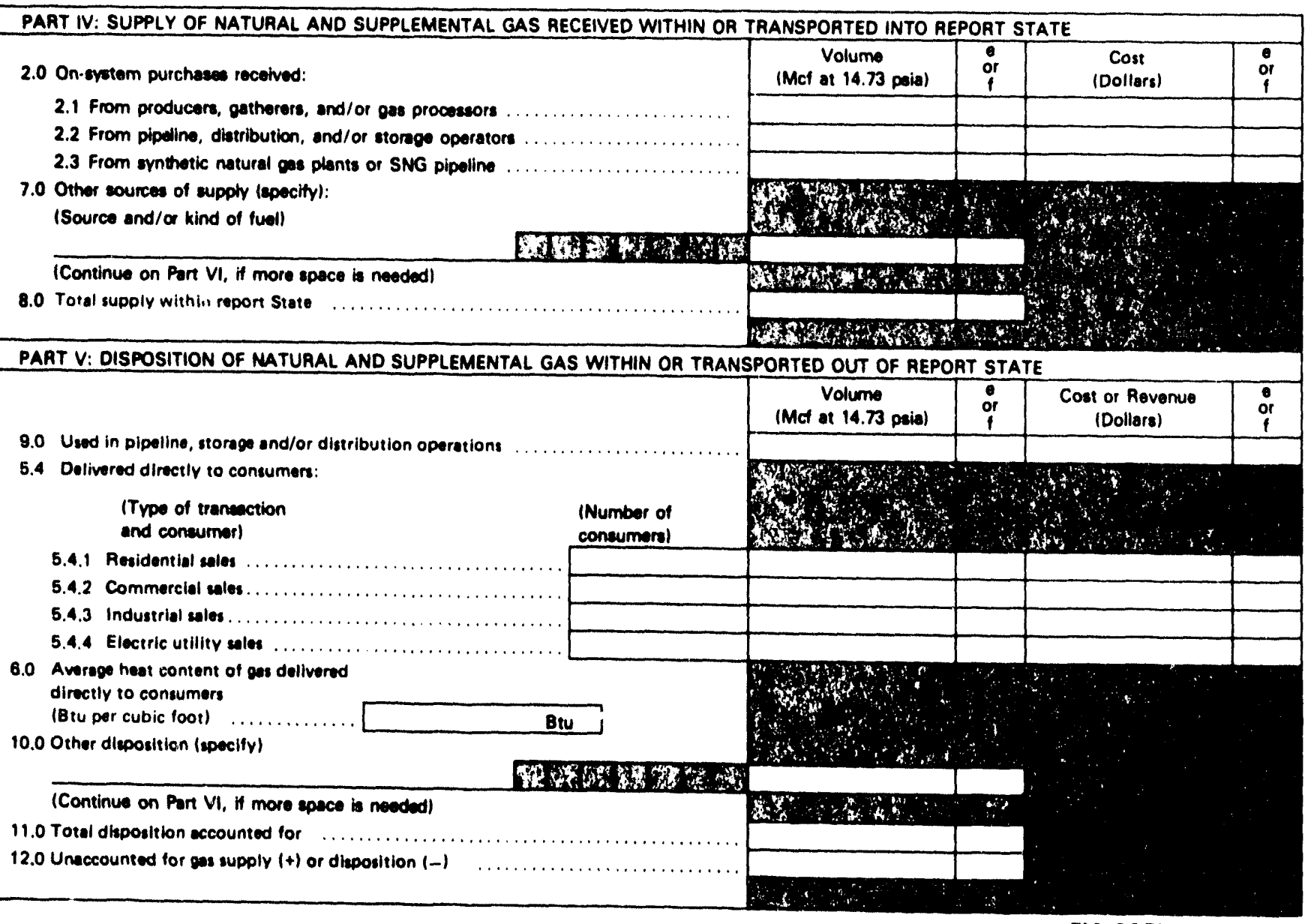


EIA.176, ANNUAL REPORT OF NATURAL AND SUPPLEMENTAL GAS SUPPLY AND DISPOSITION, $19 \square$

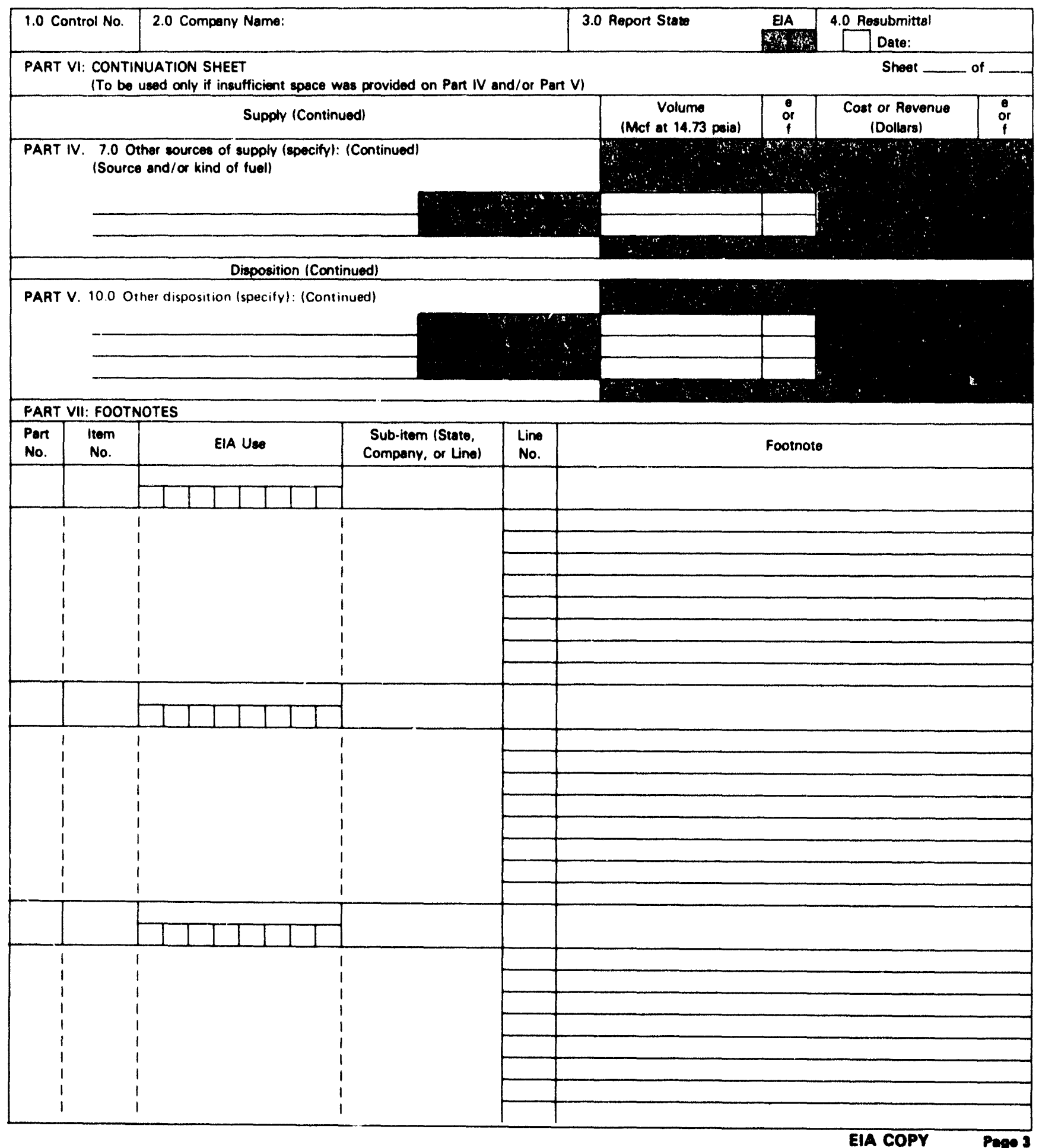




\section{Form EIA-627, "Annual Quantity and Value of Natural Gas Report"}

\section{Survey Design}

Beginning with 1980, natural gas production data previously obtained on an informal basis from State conservation agencies were collected on Form EIA-627. This form was designed by EIA to collect annual natural gas production data from the appropriate State agencies under a standard data reporting system within the limits imposed by the diversity of data collection systems of the various producing States. The form was redesigned in 1990 to collect monthly breakdowns of all annual data elements. Data are not considered proprietary. It was also designed to avoid duplication. of effort in collecting production and value data by producing States and to avoid an unnecessary respondent burden on gas and oil well operators.

\section{Survey Universe and Response Statistics}

Form EIA-627 is mailed to energy or conservation agencies in all 33 natural gas producing States. All producing States participate voluntarily in the EIA-627 survey by filing the completed form or by responding to telephone contacts. For 1992, data on the quantities of nonhydrocarbon gases removed were reported by the appropriate agencies of 23 of the 33 States. These 23 States accounted for 61 percent of total 1992 gross withdrawals. In addition, gross withdrawal data from Kansas, Oklahoma, Louisiana, and Montana, which together accounted for 35 percent of total production, excluded all or most of the nonhydrocarbon gases removed on leases.

\section{Summary of Form EIA-627 Data Reporting Requirements}

Form EIA-627 is a multipart annual form that collects data on the monthly and annual production volume of natural gas (including gross withdrawals from both gas and oil wells); volumes returned to formation for repressuring, pressure maintenance, and cycling; quantities vented and flared; quantities of nonhydrocarbon gases removed; quantities of fuel used on leases; marketed production; the value of marketed production; and the number of producing gas wells.

Respondents are asked to report all volumes in million cubic feet at the State's standard pressure base and at 60 degrees Fahrenheit. All dollar values are reported in thousands.

\section{Routine Form ElA-627 Edit Checks}

Each filing of Form EIA-627 is manually checked for reasonableness and mathematical accuracy. Information on the forms is compared to totals of monthly data reported to the Interstate Oil and Gas Compact Commission (see Appendix B, "Data Sources"). Volumes are converted, as necessary, to a standard 14.73 psia pressure base. Reasonableness of data is assessed by comparing reported data to the previous year's data. State agencies are contacted by telephone to correct errors. Amended filings or resubmissions are not a requirement, since participation in the survey is voluntary.

\section{Other EIA Publications Referencing Form EIA-627}

Data from Form EIA-627 are also published in the EIA publication, Natural Gas Annual.

\section{Interstate Oil and Gas Compact Commission Form "Monthly Report of Natural Gas Production"}

\section{Survey Design}

The Interstate Oil Gas Compact Commission (IOGCC) is an organization comprised of 32 gas and oil producing States; the Governor of each State sits on the board of the IOGCC. The IOGCC form, "Monthly Report of Natural Gas Production," (Figure B4) is a voluntary report filed to the IOGCC by most of the producing States. The IOGCC forwards copies of these forms to the EIA. The purpose of the form is to standardize, to the extent possible, the reporting of natural gas data by the States. Data are not considered proprietary.

\section{Survey Universe and Response Statistics}

Most of the 32 States report data to the IOGCC. Two exceptions are Florida, which submits its own form, and California, whose data are taken from the Conservation Committee of California Oil Producers publication. Reports on State production are forwarded to the EIA by the IOGCC approximately 80 days after the end of the report month. 
ANNUAL QUANTITY AND VALUE OF NATURAL GAS REPORT

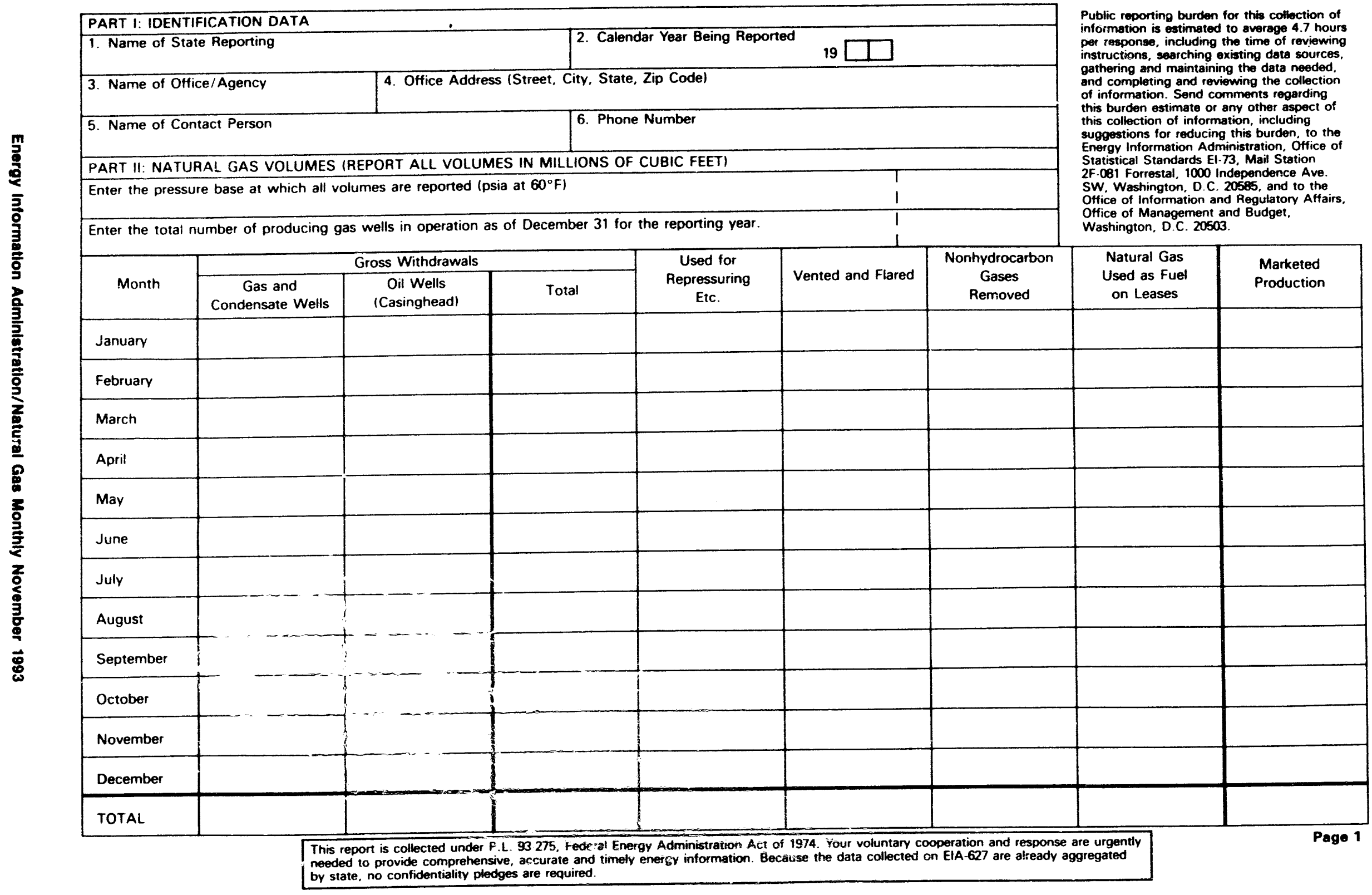




\section{EIA-627. ANNUAL QUANTITY AND VALUE OF NATURAL GAS REPORT}

PART III: Value of Marketed Production (Wellhead Sales Prices)

Enter the available value of marketed production

REPORT IN THOUSANDS OF DOLLARS

Enter the quantity of marketed production
REPORT IN MILLIONS OF CUBIC FEET

NOTE: The value reported should represent welliniad sales prices including charges for natural gas plant liquids subsequently removed

from the gas and for gathering and compression, in addition to state production, severance, and/or similar taxes.

Does your computation of wellhead value agree with the above conditions?

Yes

No

Complete the table below only if your method of reporting is inconsistent with the above note. For each item in column (a) of the table, eriter an " $X$ " in column (b) or (c) to indicate whether the item is included or excluded from the amount reported.

\begin{tabular}{|l|l|l|}
\hline \multicolumn{1}{|c|}{$\begin{array}{c}\text { Item } \\
\text { (a) }\end{array}$} & $\begin{array}{c}\text { Included } \\
\text { (b) }\end{array}$ & $\begin{array}{c}\text { Excluded } \\
\text { (c) }\end{array}$ \\
\hline Natural gas plant liquids & & \\
\hline Lease condensate & & \\
\hline Gathering and compression charges & & \\
\hline State production, severance, and/or similar taxes & & \\
\hline
\end{tabular}

\section{PART IV: COMMENTS}

Enter any additional comments including identification and explanation of any data elements submitted based on definitions differing from those provided for the previous year.

If more space is needed, please attach separate sheet(s). 


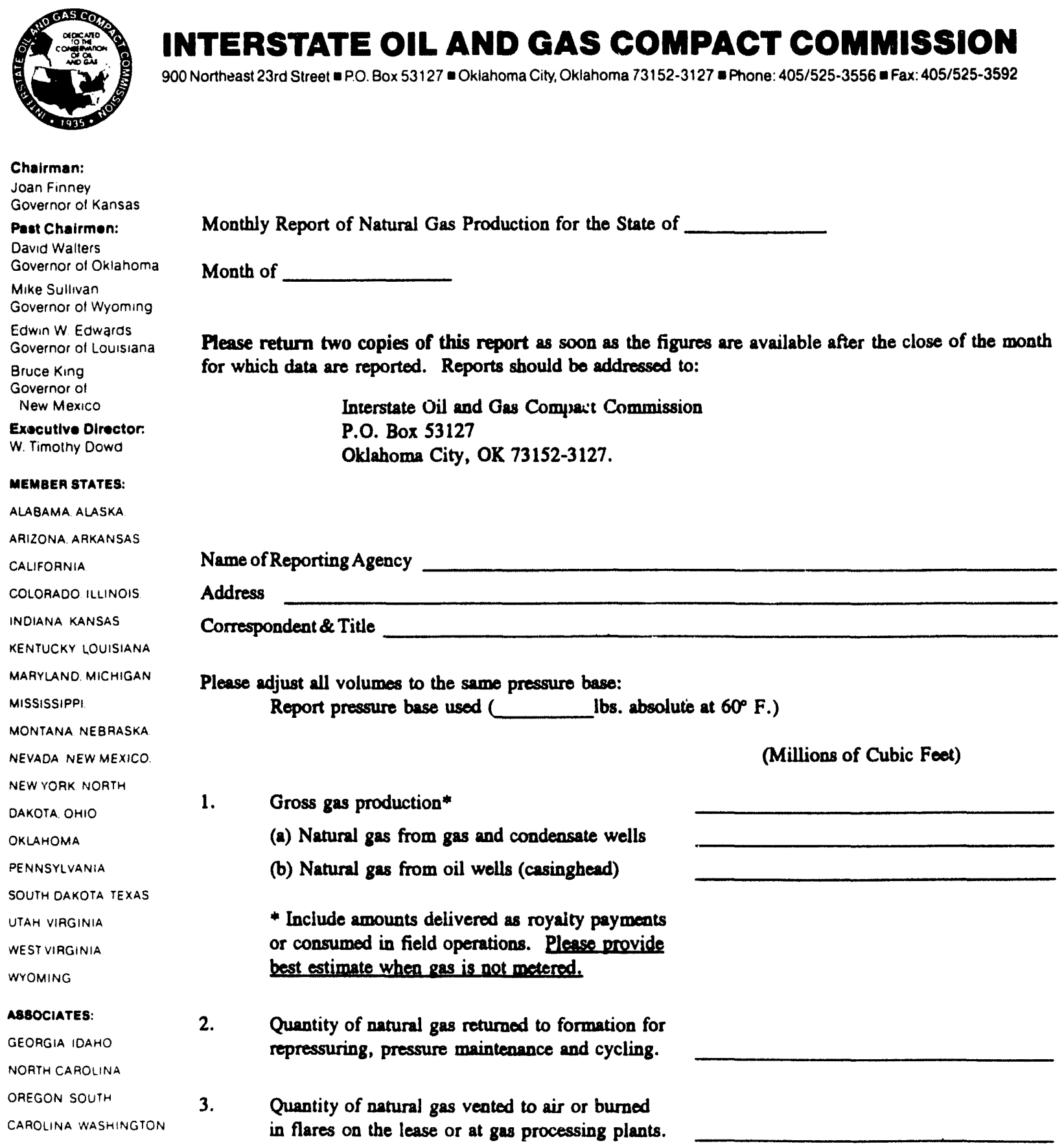




\section{Summary of Data Requirements}

The IOGCC form consists of three questions on one page, and requires volumetric information on gross production, quantities of gas vented or flared, and gas used for repressuring.

\section{Routine Edit Checks}

State data are checked for reasonableness and, in the event of problems, the appropriate State agency is called.

\section{EIA-191 Survey, "Underground Natural Gas Storage Report"}

\section{Survey Design}

Beginning in January 1991, the EIA-191, "Underground Natural Gas Storage Report," was revised. All prior data on the storage of natural gas was collected on a survey jointly implemented in 1975 by the Federal Power Commission (FPC), the Federal Energy Administration (FEA), and the Bureau of Mines (BOM) as the FPC-8/ FEA-G-318 system. The data received on both the FPC-8 and FEA-G-318 were computerized and aggregated by FPC. The new form will collect storage data by State, field, and reservoir of which there are approximately 400 operating reservoirs in the United States. Data are considered proprietary.

At the beginning of 1979, the EIA assumed responsibility for the collection, processing, and publication of the data gathered in the survey. Form FEA-G-318 was renewed on July 1, 1979, as Form EIA-191 and the survey was retitled the FPC-8/EIA-191 Survey (Figure D4 shows the EIA-191). Form FPC-8 was renewed in December 1985 and the survey retitled FERC-8/ EIA-191 Survey. The forms were not merged because of FERC's stated desire to maintain the separate identity of the FERC-8 for administrative reasons. FERC jurisdictional firms will continue to file the FERC-8 in addition to the new EIA-191.

\section{Survey Universe and Response Statistics}

The same group of 92 companies that operate underground facilities will file the new Form EIA-191. Of these companies, 40 are subject to the jurisdiction of
FERC and are required to report data on Form FERC-8.

The response rate as of the filing deadline is approximately 20 percent. Data from the remaining 80 percent of respondents are received in writing and/or by telephone within 3 to 4 days after the filing deadline. All data supplied by telephone are subsequently filed in writing, generally within 15 days of the filing deadline. The final response rate is 100 percent.

\section{Summary of EIA-191 Data Reporting Requirements}

The EIA-191 is a multipart form that reports the quantities of gas in storage, injections and withdrawals, and the location (including State and county, field, reservoirs) and capacity of underground storage reservoirs along with peak day send out during the reporting period. Information on co-owners of storage reservoirs is also required.

Collection of the survey is on a custody basis, although some respondent ownership data are required. Information requested must be provided within 10 days after the first day of each month. Twelve reports are required per calendar year. Respondents are required to indicate whether the data reported are actual or estimated. In the case of most estimated filings, the necessary revisions are reflected in subsequent scheduled filings or in a revised submission that provides actual data. Actual data on natural gas injections and withdrawals from underground storage are based on metered quantities. Data on quantities of gas in storage and on storage capacity represent, in part, reservoir engineering evaluations. All volumes are reported at 14.73 psia and 60 degrees Fahrenheit.

\section{Routine Form EIA-191 Edit Checks}

Data received on Form EIA-191 are entered into the survey processing system. The survey's five principal data elements (total, base, and working gas in storage, injections, and withdrawals) receive a preliminary visual edit to eliminate and correct obvious errors or omissions. Respondents are required to refile reports containing any inconsistencies or errors.

\section{Other EIA Publications Referensing Form EIA-191}

The EIA publication Monthly Energy Review contains data from the EIA-191 survey. 
UNDERGROUND GAS STORAGE REPORT

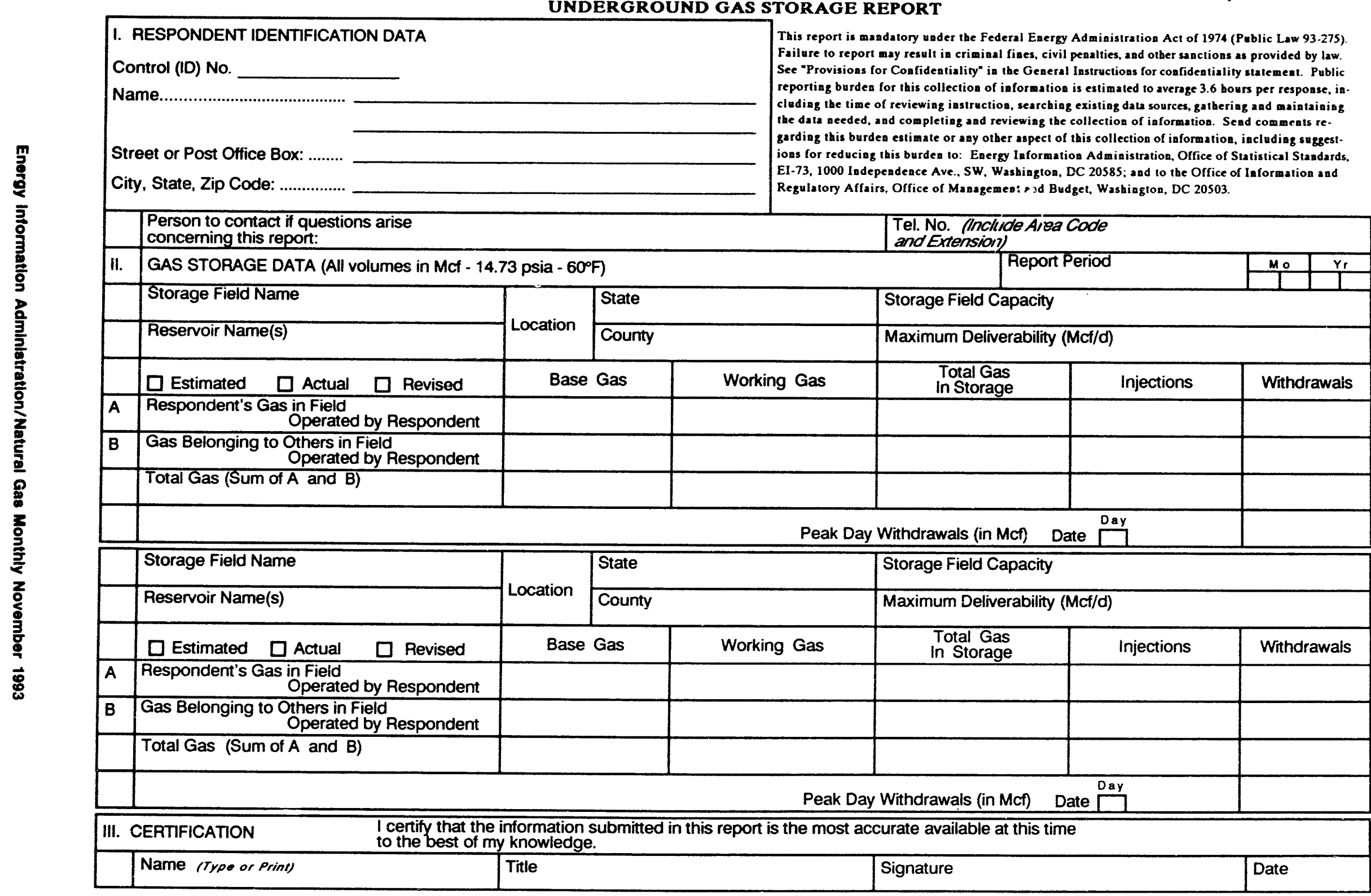

Person to contact if questions arise

I. GAS STORAGE DATA (All volumes in Mcf - $14.73 \mathrm{psia}-60^{\circ} \mathrm{F}$ )

\begin{tabular}{|l|l|l|l|}
\hline & Storage Field Name &
\end{tabular}

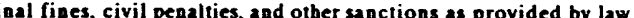
Instructions for confidentiality statement. Public 


\section{Form FERC-11, "Natural Gas Pipeline Company Monthly Statement"}

\section{Survey Design}

The collection of monthly data from major pipeline companies was begun in December 1964 by the Federal Power Commission (FPC). On October 1, 1977, FPC ceased to exist, and its functions and regulatory responsibilities were transferred to the Secretary of Energy and to the Federal Energy Regulatory Commission (FERC), an independent commission within the Department of Energy.

Information collected on Form FERC-11 (Figure B6) is used by FERC in carrying out its regulatory authority. Form FERC-11 is a monthly regulatory reporting form rather than one filed for statistical purposes. Data are not considered proprietary.

\section{Survey Universe and Response Statistics}

Form FERC-11 is filed by major interstate natural gas pipeline companies whose combined sales for resale and gas transported interstate or stored for a fee exceeded 50 billion cubic feet in the previous calendar year. Approximately 50 pipeline companies report data on Form FERC-11. Natural gas pipeline companies are monitored annually to determine whether each has met the requirements for classification as a major pipeline.

Information is collected monthly by mail. Historically, the response rate has been 100 percent.

\section{Summary of Form FERC-11 Data Requirements}

Form FERC-11 requires information on revenues, expenses, and sales data, as well as volumetric data on purchases and production.

Submission of Form FERC-11 is required no later than 40 days after the close of the report month. The form requires reporting of both preliminary data for the report month and final data for the same month in the previous year. All data are reported on an equity basis.

\section{Routine Form FERC-11 Edit Checks}

The completed Form FERC-11 is sent on disk along with two facsimiles of the form to FERC. FERC loads these disks on a electronic data file. This file is transmitted to EIA for further processing and editing. Edit reports are produced of the current file and are reviewed manually. This review is to ensure consistency in reporting within and among utilities in the presentation of current and 12-month financial sales data.

\section{Other EIA Publications Referencing Form FERC-11}

The Energy Information Administration publication Monthly Energy Review contains data from Form FERC-11.

\section{Form FPC-14, "Annual Report for Importers and Exporters of Natural Gas"}

\section{Survey Design}

The collection of data covering natural gas imports and exports was begun in 1973 by the Federal Power Commission (FPC). On October 1977, FPC ceased to exist and its data collection functions were transferred to the Federal Energy Regulatory Commission (FERC) within the Department of Energy (DOE). Since 1979, the Energy Information Administration (EIA) has had the responsibility for collecting Form FPC-14. Data are not considered proprietary.

\section{Survey Universe and Response Statistics}

The Form FPC-14 is filed annually by each organization or individual having authorization to import and export natural gas regardless of whether any imports or exports took place during the reporting year. The authorization to import and export was originally granted by the FPC. In 1977, it was tranferred to the Economic Regulatory Administration (ERA) and it now resides with the Office of Fuels Programs in the Office of Fossil Energy. In 1990, 228 companies met the reporting criteria, only 102 reported imports or exports of natural gas.

The respondent list for the Form FPC-14 is updated at the beginning of each year. All new respondents with authorization to import or export natural gas are added to the list and respondents whose licenses have expired are deleted. Five copies of Form FPC-14 are mailed in February to all companies authorized to importor export natural gas. The completed osiginal and three copies are to be filed with the EIA on or before March 31 of each year, for the preceding calendar year. Companies that have not filed by March 31 are contacted. 


\begin{tabular}{|c|c|c|c|c|c|c|}
\hline \multirow{3}{*}{\multicolumn{5}{|c|}{ 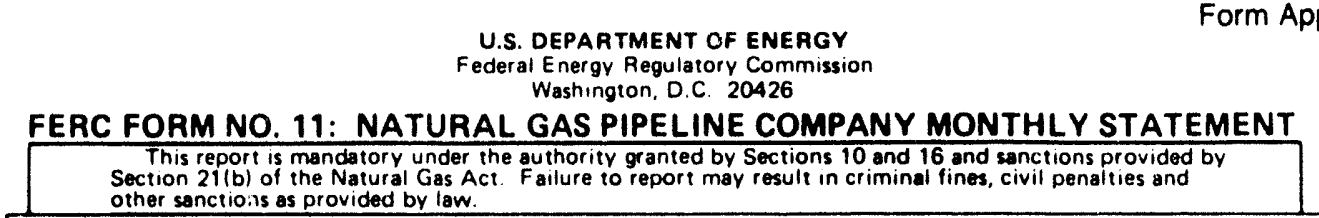 }} & \multicolumn{2}{|c|}{$\begin{array}{l}\text { oroved, OMB No. 1902-0032 } \\
\text { (Expires 6-30-90) }\end{array}$} \\
\hline & & & & & \\
\hline & & & & & \multicolumn{2}{|c|}{$\begin{array}{r}\text { Month Being Reported } \\
19 \\
\end{array}$} \\
\hline \multicolumn{7}{|c|}{ PART I: IDENTIFICATION } \\
\hline \multicolumn{7}{|c|}{ 1. Name of Company } \\
\hline \multicolumn{7}{|c|}{$\begin{array}{l}\text { 2. Address of Company } \\
\text { (1) Number and Street }\end{array}$} \\
\hline \multicolumn{7}{|c|}{$\begin{array}{l}\text { 3. Person Authorized To Sign This Report } \\
\begin{array}{ll}\text { (1) Signature } & \text { (2) Phone Number (Including area code) }\end{array}\end{array}$} \\
\hline \multicolumn{7}{|c|}{ PART II: REVENUE DATA } \\
\hline \multirow{2}{*}{$\begin{array}{l}\text { Line } \\
\text { No. } \\
\text { (a) }\end{array}$} & \multirow{2}{*}{$\begin{array}{l}\text { Item } \\
\text { (b) }\end{array}$} & \multicolumn{2}{|c|}{$\begin{array}{l}\text { Revenues } \\
\text { (In thousands of dollars) }\end{array}$} & \multicolumn{2}{|c|}{$\begin{array}{c}\text { Soles } \\
\text { (In millions of cubic feet) }\end{array}$} & \multirow{2}{*}{$\begin{array}{c}\text { Foot- } \\
\text { note } \\
(\mathrm{g})\end{array}$} \\
\hline & & $\begin{array}{l}\text { Current Yoar } \\
\text { (c) }\end{array}$ & $\begin{array}{l}\text { Previous Year } \\
\text { (d) }\end{array}$ & $\begin{array}{l}\begin{array}{c}\text { Current Year } \\
(e)\end{array} \\
\end{array}$ & $\begin{array}{l}\text { Previous Year } \\
(f)\end{array}$ & \\
\hline 4 & Sales of Natural Gas & & $\therefore$ & & & \\
\hline 5 & Firm Industrial & & & & & \\
\hline 6 & Off-peak Industrial & & & & & \\
\hline 7 & Interruptible Industrial & & & & & \\
\hline 8 & $\begin{array}{l}\text { TOTAL INDUSTRIAL } \\
\text { (Enter total of lines } 5,6 \text { and } 7 \text { ) }\end{array}$ & & & & & \\
\hline 9 & Other Ultimate Consumers & & & & & \\
\hline 10 & $\begin{array}{l}\text { TOTAL SALES TO ULTIMATE CONSUMERS } \\
\text { (Enter total of lines } 8 \text { and } 9 \text { ) }\end{array}$ & & & & & \\
\hline & Sales For Resale (483) & & & & & \\
\hline 11 & Total Major Gas Pipelines & & & & & \\
\hline 12 & Total to All Other Pipelines and Gas Utilities & & & & & \\
\hline 13 & $\begin{array}{l}\text { TOTAL SALES FOR RESALE } \\
\text { (Enter total of lines } 11 \text { and } 12)\end{array}$ & & & & & \\
\hline 14 & $\begin{array}{l}\text { TOTAL SALES OF NATURAL GAS } \\
\text { (Enter total of lines } 10 \text { and } 13)\end{array}$ & & & & & \\
\hline 15 & Intracompany Transfers (485) & & & & & \\
\hline 16 & Revenues From Transportation of Gas of Others (489) & & & (Enter volume c & jes transponted:) & \\
\hline 17 & Provision for Rate Refunds (496) & & & & & \\
\hline
\end{tabular}




\begin{tabular}{|c|c|c|c|c|}
\hline \multicolumn{2}{|c|}{ Company Code Number } & \multicolumn{3}{|c|}{ Month and Year Being Reported } \\
\hline \multicolumn{5}{|c|}{ PART III: INCOME DATA } \\
\hline $\begin{array}{l}\text { Line } \\
\text { No. } \\
\text { (a) }\end{array}$ & $\begin{array}{l}\text { Item } \\
\text { (b) }\end{array}$ & $\begin{array}{l}\text { Current Year } \\
\text { (In thousands of } \\
\text { dollars) } \\
\text { (c) }\end{array}$ & $\begin{array}{l}\text { Previous Year } \\
\text { (In thousands of } \\
\text { dollars) } \\
\text { (d) }\end{array}$ & $\mid \begin{array}{c}\text { Foot- } \\
\text { note } \\
\text { (e) }\end{array}$ \\
\hline 18 & Gas Operating Revenues (400) & & & \\
\hline 19 & Operation and Maintenance Expense $(401,402)$ & & & \\
\hline 20 & $\begin{array}{l}\text { Depreciation, Depletion and Amortization Expense } \\
\text { (403-407) }\end{array}$ & & & \\
\hline 21 & $\begin{array}{l}\text { Taxes Other Than Income Taxes, Utility Operating Income } \\
\text { (408.1) }\end{array}$ & & & \\
\hline 22 & $\begin{array}{l}\text { Total Gas Operating Expenses }(401,402,403-407,408.1,409.1,410.1 \text {, } \\
411.1,411.4)\end{array}$ & & & \\
\hline 23 & $\begin{array}{l}\text { Net Gas Operating Revenues (Enter the result of lines } 18 \text { minus } 22 \text { ) } \\
\text { (including Plent Leased to Others) }\end{array}$ & & & \\
\hline 24 & Total Gas Utility Operating Income (Refer to specific instruction for line 24.) & & & \\
\hline 25 & $\begin{array}{l}\text { Allowance For All Funds Used During Construction - Credit } \\
(419.1,432)\end{array}$ & & & \\
\hline 26 & $\begin{array}{l}\text { Total Income Before Interest Charges }(427.432) \text { and Extraordinary Items } \\
(409.3,434,435)\end{array}$ & & & \\
\hline 27 & $\begin{array}{l}\text { Net Income (433) Before Extraordinary l tems }(434,435) \text {, Income Taxes } \\
(409.1,409.2,409.3,410.1,410.2,411,1,411.2) \text {, and Investment Tax } \\
\text { Credits }(411.4,411.5,420)\end{array}$ & & & \\
\hline 28 & Net Income (Monthiv Amount Related to 433.) & & & \\
\hline \multicolumn{5}{|c|}{ PART IV: OTHER SELECTED DATA } \\
\hline $\begin{array}{l}\text { Line } \\
\text { No. } \\
\text { (a) }\end{array}$ & $\begin{array}{l}\text { Item } \\
\text { (b) }\end{array}$ & $\begin{array}{l}\text { Current Year } \\
\text { (In thousands of } \\
\text { dollars) } \\
\text { (c) }\end{array}$ & $\begin{array}{l}\text { Previous Year } \\
\text { (In thousands of } \\
\text { dollars) } \\
\text { (d) }\end{array}$ & $\begin{array}{c}\text { Foot- } \\
\text { note } \\
(e)\end{array}$ \\
\hline 29 & Gas Utility Plant in Service 11011 & & & \\
\hline 30 & $\begin{array}{l}\text { Accumulated Provision For Depreciation, Depletion, and } \\
\text { Amortization of Gas Utility Plant } 1108,111)\end{array}$ & & & \\
\hline 31 & Gas Plant Construction Work In Progress (107) & & & \\
\hline 32 & $\begin{array}{l}\text { Gross Additions To Construction Work in Progress (107) } \\
\text { For This Month Being Reported }\end{array}$ & & & \\
\hline 33 & $\begin{array}{l}\text { Amount Collected Which is Subject To Refund During This } \\
\text { Month Being Reported }\end{array}$ & & & \\
\hline 34 & $\begin{array}{l}\text { Cumplative Amount Collected Since January } 1 \text { This Year Subject To } \\
\text { Refund, At End of This Month Being Reported }\end{array}$ & & & \\
\hline 35 & $\begin{array}{l}\text { Monthly Amount Subject To Refund Actually Refunded During } \\
\text { This Month Being Reported }\end{array}$ & & & \\
\hline 36 & $\begin{array}{l}\text { Cumulative Amount Subject To Refund Refunded Since January } 1 \text { This } \\
\text { Year. To The End of This Month Being Reported }\end{array}$ & & & \\
\hline
\end{tabular}

FERC FORM NO. 11 (REVISED 12.84) 


\begin{tabular}{|c|c|c|c|c|c|c|}
\hline \multicolumn{2}{|c|}{ Company Code Number } & \multicolumn{5}{|c|}{ Month and Year Being Reported } \\
\hline \multicolumn{7}{|c|}{ PART $V:$ OPERATION AND MAINTENANCE EXPENSE DATA } \\
\hline \multirow{2}{*}{$\begin{array}{l}\text { Line } \\
\text { No. } \\
\text { (a) }\end{array}$} & \multirow{2}{*}{$\begin{array}{l}\text { Item } \\
\text { (b) } \\
\end{array}$} & \multicolumn{2}{|c|}{$\begin{array}{l}\text { Amount } \\
\text { (In thoussends of dollers) }\end{array}$} & \multicolumn{2}{|c|}{$\begin{array}{l}\text { Gas Volume } \\
\text { (In millions of cubic fest) }\end{array}$} & \multirow{2}{*}{$\begin{array}{c}\text { Foot- } \\
\text { note } \\
(g)\end{array}$} \\
\hline & & $\begin{array}{c}\text { Current Year } \\
\text { (c) }\end{array}$ & $\begin{array}{c}\text { Previous Year } \\
\text { (d) }\end{array}$ & $\begin{array}{c}\text { Current Year } \\
(e)\end{array}$ & Previous Yoer & \\
\hline 38 & Liquefied Petroleum Gas & & & & & \\
\hline 39 & Other Manufactured Gas & & & & & \\
\hline 40 & TOTAL (Enter total of lines 38 and 39 ) & & & & & \\
\hline 41 & Natural Gas Production & & & & & \\
\hline 42 & Production and Gathering (750- 769$)$ & & & & & \\
\hline 43 & Products Extraction $(770-791)$ & & & (Enter thou & of agllons) 7 & \\
\hline 44 & Purchased Natural Gas From & & & & & \\
\hline 45 & Producers $(800,801.803)$ & & & & & \\
\hline 46 & Intracompany Transfers 1800.11 & & & & & \\
\hline 47 & Tmports & & & & & \\
\hline 48 & Major Gas Pipellnes $(800,801.803)$ & & & & & \\
\hline 49 & Other Piplines $(800,801.803)$ & & & & & \\
\hline 50 & Other Gas Purchases $(804,805,805.1$ minus line 68.71$)$ & & & & & \\
\hline 51 & TOTAL (Enter total of lines 45 to 50 ) & & & & & \\
\hline 52 & Natural Gas Produced & & & & & \\
\hline 53 & Exchange Gas - In (806) & & & & & \\
\hline 54 & Exchange Gas - Out (806) & & & & & \\
\hline 55 & Purchased Gas Expenses (807.1-807.5) & & & & & \\
\hline 56 & $\begin{array}{l}\text { Gas Withdrawn From Underground Storage - Debit } \\
(808.1,809.1)\end{array}$ & & & & & \\
\hline 58 & Gas Used For Compressor Station Fuel - Credit (810) & & & & & \\
\hline 59 & Gas Used For Products Extraction - Credit (811) & & & & & \\
\hline 60 & Gas Used For Other Utility Operations - Credit (812) & & & & & \\
\hline 61 & Other Gas Supply Expenses (813) & & & & & \\
\hline 62 & $\begin{array}{l}\text { TOTAL GAS PRODUCTION (Enter total of } \\
\text { lines } 40,42,43,44,51,53 \text { to } 611\end{array}$ & & & & & \\
\hline 63 & Stor age Expenses (814-843.9) & & & & & \\
\hline 64 & $\begin{array}{l}\text { LNG Terminating and Processing Expenses } \\
(844.1-847.8)\end{array}$ & & & & & \\
\hline 65 & Transmission Expenses (850-867) & & & & & \\
\hline 66 & Distribution Expenses $(870-894)$ & & & & & \\
\hline 67 & $\begin{array}{l}\text { Other Gas Purchased and Produced (Entries hereshould } \\
\text { not be included innier any other item in this pars!) }\end{array}$ & & & & & \\
\hline 68 & Liquefied Natural Gas (804.1) & & & & & \\
\hline 69 & Gasified Coal & & & & & \\
\hline 70 & $\begin{array}{l}\text { Synthetic Gas (Reformed (gasified) lıquid } \\
\text { hydrocarbons. I }\end{array}$ & & & & & \\
\hline 71 & TOTAL (Enter total of lines 68 to 70 ) & & & & & \\
\hline 72 & $\begin{array}{l}\text { All Other Operating and Maintenance Expenses } \\
(901.905,907.916,920.931,935)\end{array}$ & & & & & \\
\hline 73 & $\begin{array}{l}\text { TOTAL OPERATING AND MAINTENANCE EXPENSES } \\
\text { (Enter total of lines } 62,63,64,65,66,71,72)\end{array}$ & & & & & \\
\hline
\end{tabular}




\section{Routine Form FPC-14 Edit Checks}

Respondents are required to certify the accuracy of all data reported. The survey forms are checked at the EIA for reasonableness and accuracy. If errors are found the companies are required to file corrected data. The data are processed at the EIA and published as reported. All natural gas volumes in this report are expressed at a pressure base of 14.73 pounds per square inch absolute and temperature of 60 degrees Fahrenheit, except as noted. All prices are in U.S. dollars and import/export prices are those paid at the U.S. border (except exports of LNG are those prices paid at the point of sale and delivery in Yokohama, Japan).

\section{"Quarterly Natural Gas Import and Export Sales and Price Report"}

This report is prepared quarterly by the Office of Fuels Programs in the Office of Fossil Energy based on information submitted by all firms having authorization to import or export natural gas. All data on this report are considered preliminary until the annual data on the Form FPC-14 are final, usually in September of the following year.

\section{Form EIA-857, "Monthly Report of Natural Gas Purchases and Deliveries to Consumers"}

\section{Survey Design}

The original Form EIA-857 was approved for use in December 1984. Response to the Form EIA-857 is mandatory on a monthly basis. Data collected on the Form EIA-857 cover the 50 States and the District of Columbia and include both price and volume data. Data are considered proprietary.

\section{Survey Universe and Response Statistics}

A sample of 391 natural gas companies including interstate pipelines, intrastate pipelines, and local distribution companies report on the survey. The sample was selected independently for each of the 50 States and the District of Columbia from a frame consisting of all respondents to Form EIA-176 who reported deliveries of natural gas to consumers in the residential, commercial, or industrial sectors. Each selected company is required to complete and file the Form EIA-857 on a monthly basis. Initial response statistics are as follows: responses received by due date, approximately 50 percent, and responses received after follow-up, 100 percent. Virtually all are received in time for incorporation in the current month's processing cycle. When a response is extremely late, and the company represents less than 25 percent of the naturai gas volumes delivered by all sampled companies in the State, values are imputed as described in Appendix C. When the company's submission is eventually received, the submitted data are used for future processing and revisions.

The Form EIA-857 is a monthly sample survey of firms delivering natural gas to consumers. It provides data that are used to estimate monthly sales of natural gas (volume and price) by State and monthly deliveries of natural gas on behalf of others (volume) by State to three consumer sectors - residential, commercial, and industrial. (Monthly deliveries and prices of natural gas to electric utilities are reported on the Form FERC-423, "Monthly Report of Cost and Quality of Fuels for Electric Plants," and the Form EIA-759, "Monthly Power Plant Report.")

See Appendix $\mathbf{C}$ for a discussion of the sample design and estimation procedures.

\section{Summary of Form EIA-857 Data Reporting Requirements}

Data collected monthly on the Form EIA-857 on a State level include the volume and cost of purchased gas, the volume and cost of natural gas consumed by sector (residential, commercial, and industrial), and the average heat content of all gas consumed. Respondents file completed forms with EIA in Washington, DC on or before the 30th day after the end of the report month.

All natural gas volumes are reported in thousand cubic feet at 14.73 psia at 60 degrees Fahrenheit and dollar values are reported to the nearest whole dollar.

\section{Routine Form EIA-857 Edit Checks}

A series of manual and computerized edit checks are used to screen the Form EIA-857. The edits performed include validity and analytical checks. 
FEDERAL ENEAGY AEGULATOAY COMMIESION Weanington, D.C. 20426

ANNUAL REPORT FOR
IMPORTERS AND EXPORTERS OF NATURAL GAS

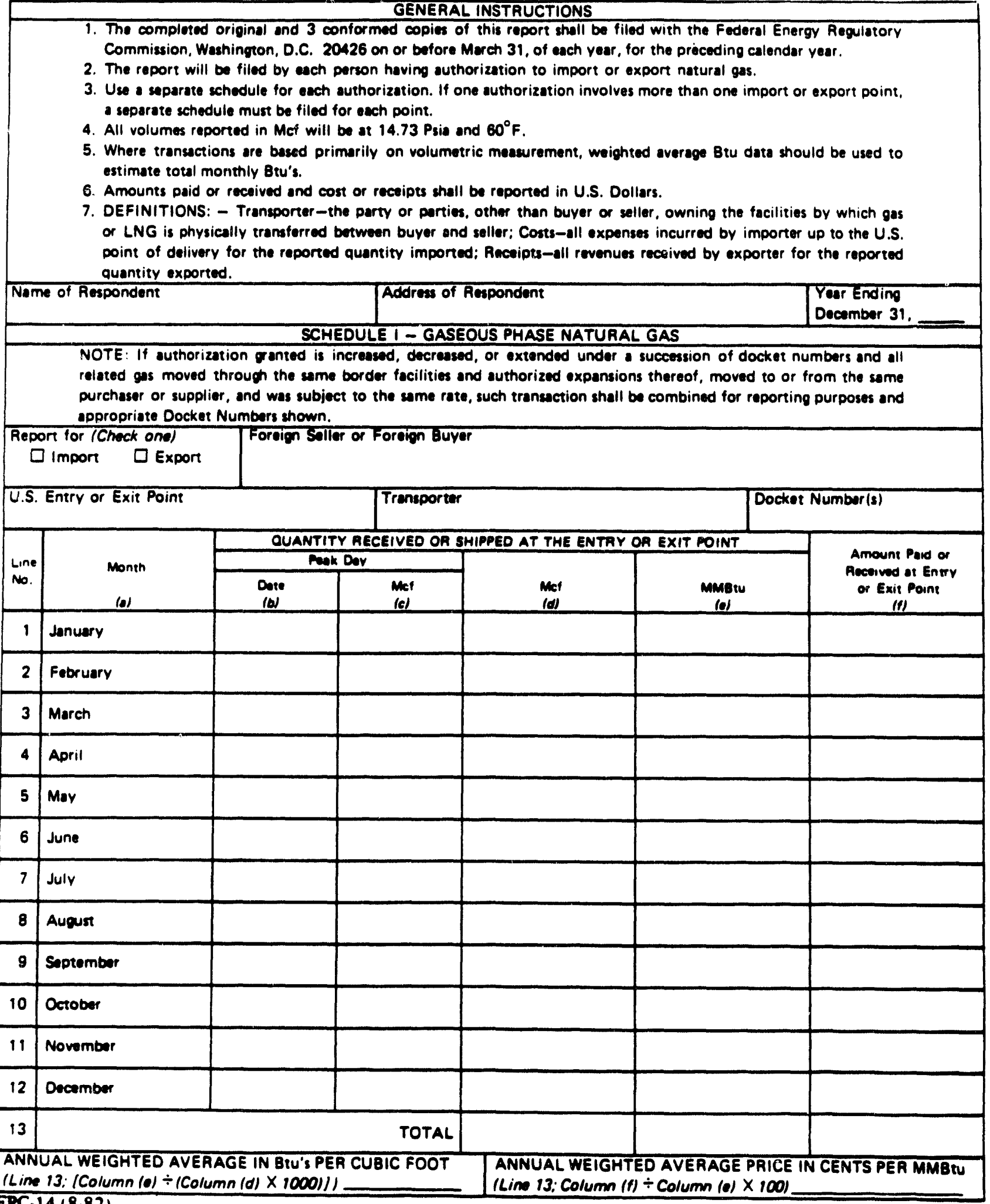
FPC. $14(8-82)$ 


\title{
MONTHLY REPORT OF NATURAL GAS PURCHASES AND DELIVERIES TO CONSUMERS
}

\begin{abstract}
This report is mandatory under the Federal Energy Administration Act of 1974 (Public Law 93-275). Failure to repon may result in criminal fines, civil penalties, and other sanctions as provided by law. See section VI of the instructions for the confidentiality statement. Public reponing burden for this collection of information is estimated to average 4 hours per response. including time of reviewing instructions, searching existing data sources, gathering and maintaining the data needed, and

completing and reviewing the collection of information. Send comments regarding this burden or any other aspect of this collection of information, including suggestions

for reducing this burden, to the Energy Information Administration, Office of Statistical Standards EI-73. Mail Station: 2F-081 Forrestal, 1000 Independence Ave. SW,

Washington. DC 20585: and to the Office of Information and Regulatory Affairs, Office of Management and Budget. Washington. DC 20503.
\end{abstract}

Affix Identification Label or Enter:
01 Control No.
02 Company Name:_
State of Operation:

SECTION I - Natural Gas Purchases and Deliveries to Consumers

Purchased Gas Received in Distribution Service Area

Delivered to Residential Consumers (Onsystem Sales).......

Delivered to Commercial Consumers (Onsystem Sales)

Delivered to Industrial Consumers (Onsystem Sales)

Delivered to Electric Utilities (Onsystem Sales)

Transponed to Residential Consumers

Transported to Commercial Consumers

Transported to Industrial Consumers

Transported to Electric Utilities

Total Delivered and Transported to Consumers

Average Btu Content of Gas Delivered to Consumers

\section{Return completed forms to:}

Energy Information Administration

Mail Station: BG-094

U.S. Department of Energy

Washington, D.C. 20585

Attn. Form EIA-857

SECTION II - Footnotes

SECTION III - CONTACT PERSON AND CERTIFICATION

\begin{tabular}{|l|l|}
\hline $\begin{array}{l}\text { Contact Person: } \\
16 \text { Name: }\end{array}$ & \multicolumn{1}{|c|}{17 Telephone: } \\
\hline $\begin{array}{l}\text { Certification: I certify that the information provided hereon is true and accurate or, where indicated on the form, reasonable estimates to the best } \\
\text { of my knowledge. }\end{array}$ & 19 Title: \\
\hline 18 Name: & Date: \\
\hline Signature: & \\
\hline
\end{tabular}


Appendix C

Statistical

Considerations 


\section{Statistical Considerations}

The monthly sales (volume and price) and monthly deliveries (volume) of natural gas to residential, commercial and industrial consumers presented in this report by State are estimated from data reported on the Form EIA-857, "Monthly Report of Natural Gas Purchases and Deliveries to Consumers." (See Appendix B for a description of this Form.) These estimations must be made from the reported data since the Form EIA-857 is a sample survey. A description of the sample design and the estimation procedures is given below.

\section{Sample Design}

The Form EIA-857 is a monthly sample survey of companies delivering natural gas to consumers. It includes interstate pipeline companies, intrastate pipeline companies, producers, and local distribution companies. The survey provides data that are used each month to estimate the volume and price for onsystem sales of natural gas by State and the volume of natural gas delivered for the account of others, by State, to three consumer sectors--residential, commercial, and industrial. Monthly deliveries and prices of natural gas to electric utilities are reported on the Form EIA-759, "Monthly Power Plant Report," and the Form FERC-423, "Monthly Report of Costs and Quality of Fucls for Electric Plants."

Sample Universe. The sample currently in use was selected from a universe of 1,705 companies. These companies were respondents to the Form EIA-176, "Annual Report of Natural and Supplemental Gas Supply and Disposition," for reporting year 1990 who reported sales or deliveries to consumers in the residential, commercial or industrial sectors. (See Appendix B for a description of the Form EIA-176.)

Sampling Plan. The goal was a sample that would provide estimates of monthly natural gas consumption by the three consuming sectors within each State and the District of Columbia. A stratified sample using a single stage and systematic selection with probability proportional to size was designed. The measure of size was the volume of natural gas physically delivered in the
State to the three consuming sectors by the company in 1990. As a supplement, two companies with no deliveries to end users during 1990 but having subsequently started delivering very large volumes were sarnpled with certainty. There were two strata--companies selected with certainty and companies selected under the systematic probability proportional to size design.

Initial calculations showed that a 25 percent sample of companies would yield reasonably accurate estimates. The sample was selected independently in each State, resulting in a national total of 391 respondent companies. While some mergers and acquisitions were uncovered as a result of the initial mail-out, all resulted in a substitution of respondent companies rather than a reduction in the number of respondents. The sample for the 1992 survey year contains a total of 391 respondent companies.

Certainty Stratum. Since estimates were needed for each of the 50 States and the District of Columbia, the strata were established independently within each State. In 16 States and the District of Columbia where sampling was not feasible due to small numbers of companies and/or small volumes of gas deliveries, all companies were selected. The 16 States were: Alaska, Connecticut, Delaware, Hawaii, Idaho, Maine, North Dakota, New Hampshire, New Jersey, Nevada, Oregon, Rhode Island, South Dakota, Utah, Vermont, and Washington.

For each of the remaining States, the total volumes of industrial sales and deliveries and of the combined residential/commercial sales and deliveries were determined. Cumpanies with natural gas deliveries to the inclustrial sector or to the combined residential/ commercial sector above a certain level were selected with certainty. Since a few large companies often account for most of the natural gas delivered within a State, this ensures those companies' inclusion in the sample. The formula for determining certainty was applied independently in the two consumer sectors--the industrial and the combined residential/commercial. These selected companies, together with the companies in the jurisdictions discussed where sampling was not feasible, formed the certainty stratum. 
All companies with natural gas deliveries in sector $j$ greater than the cut-off value $\left(C_{. j}\right)$ were included in the certainty stratum. The formula for $C_{. j}$ was:

$$
C_{. j}=\frac{X_{. j}}{2 n}
$$

where:

$C_{. j}=$ cutoff value for consumer sector $\mathrm{j}$,

$n=$ target sample size to be selected for the State, 25 percent of the companies in the State,

$X_{i j}=$ the annual volume of natural gas deliveries by company $i$ to customers in consumer sector $j$,

$X_{i .}=$ the sum within State of annual gas volumes for company i,

$X_{. j}=$ the sum within State of annual gas volumes in consumer sector $\mathrm{j}$,

$X_{. .}=$the sum within State of annual gas volumes in all consumer sectors.

Noncertainty Stratum. All other companies formed the noncertainty stratum. They were systematically sampled with probability proportional to size. The measure of size for each company was the total volume of gas sales to all consumer sectors $\left(X_{i}\right)$. The number of companies to be selected from the noncertainty stratum was calculated for each State, with a minimum of 2.

The formula for selecting the number of noncertainty stratum companies was:

$$
m=n \frac{X 2}{X}
$$

where:

$m=$ the sample size for the noncertainty stratum within a State,

$X 2=$ the sum within State of the $X_{i}$. for all companies in the noncertainty stratum.

Companies were listed in ascending order according to their measure of size and then a cumulative measure of size in the stratum was calculated for each company. The cimulative measure of size was the sum of the measures of size for that company and all preceding companies on the list. An interval of width I for selecting the companies systematically was calculated using $\left(I=\frac{X 2}{m}\right)$. A uniform random number $\mathrm{R}$ was selected between zero and $\mathrm{I}$. The first sampled company was the first company on the list to have a cumulative measure of size greater than $R$. The second company selected was the first company on the list to have a cu- mulative measure of size greater than $R+I . R+I$ was increased again by $I$ to determine the third company to be selected. This procedure was repeated until the entire sample was drawn.

Subgroups. In eight States, the noncertainty stratum was divided into subgroups to ensure that gas in each consumer sector could be estimated. The systematic sample with probability proportional to size design described above was applied independently in each subgroup. The methods for determining the subgroup sample size and calculating the subgroup interval for sample selection were the same as the methods described above for the noncertainty stratum, except that X2 was the sum within State of the $X_{i}$, for only those companies in the subgroup.

These subgroups were defined only for the purpose of sample selection. They are:

California: companies handling only industrial gas and all other companies.

Louisiana: companies handling only industrial gas and all other companies, with the latter being further subdivided according to size. The larger group is comprised of all companies with total deliveries of at least 200 million cubic feet while the smaller group consists of companies with less than that volume of delivered gas (three subgroups).

Texas: companies handling only residential/ cornmercial gas, companies handling only industrial gas, and all other companies (three subgroups).

West Virginia: companies delivering only residential or commercial gas and all other companies.

Arkansas, Colorado, Georgia, Pennsylvania, and Tennessee: companies delivering 1 billion cubic feet or more of gas and companies delivering less than 1 billion cubic feet of gas.

Oklahoma: Companies delivering less than 500 million cubic feet of gas and those delivering more than that volume.

New York: companies delivering 8 billion cubic feet or more of gas and companies delivering less than 8 billion cubic feet of gas.

\section{Estimation Procedures}

Estimates of Volumes. A ratio estimator is applied to the volumes reported in each State by the sampled companies to estimate the total gas sales and deliveries for the State. Ratio estimators are calculated for each consumer sector--residential, commercial, and industrial--in each State where companies are sampled. 
The following annual data are taken from the most recent 1990 submissions of Form EIA-176:

The formula for calculating the ratio estimator $\left(E_{v j}\right)$ for the volume of gas in consumer sector $j$ is:

$$
E_{v j}=\frac{Y_{. j}}{Y_{. j}^{\prime}}
$$

where:

$Y_{. j}=$ the sum within State of annual gas volumes in consumer sector $\mathrm{j}$ for all companies,

$Y_{. j}^{\prime}=$ the sum within State of annual gas volumes in consumer sector $\mathrm{j}$ for those companies in the sample.

The ratio estimator is applied as follows:

$$
V_{j}=y_{. j} \times E_{v j}
$$

where:

$V_{j}=$ the State estimate of monthly gas volumes in consumer sector $\mathrm{j}$,

$y_{. j}=$ the sum within State of reported monthly gas volumes in consumer sector $\mathrm{j}$.

Computation of Natural Gas Prices. The natural gas volumes that are included in the computation of prices represent only those volumes associated with natural gas sales.

The price of natural gas for a State within a sector is calculated as follows:

$P_{j=\frac{R_{j}}{V_{j}}}$

where:

$P_{j}=$ the average price for gas sales within the State in consumer sector $\mathrm{j}$,

$V_{j}=$ the reported revenue from natural gas sales within the State in consumer sector $j$.
$V_{j}=$ the reported volume of natural gas sales within the State in consumer sector $j$.

All average prices are weighted by their corresponding sales volume estimates when national average prices are computed.

The monthly average prices of natural gas are based on sales data only. Volumes of gas delivered for the account of others to these consumer sectors are not included in the State or national average prices.

Table 33 shows the percent of the total State volume that represents volumes from natural gas sales to the commercial and industrial sectors. This table may be helpful in evaluating commercial and industrial price data. Virtually all natural gas deliveries to the residential sector represent onsystem sales volumes only.

Estimation for Nonrespondents. A volume for each consumer category is imputed for companies that fail to respond. The imputation is based on the previous month's value reported by the non-responding company and the change from the previous month to the current month in volumes reported by other companies in the State. The imputed volumes are included in the State totals. To estimate prices for non-respondents, the unit price (dollars per thousand cubic feet) reported by the company in the previous month is used.

The formula for imputing volumes of gas sales for nonrespondents was:

$$
F_{t}=F_{t-1} \times \frac{y_{. j t}}{y_{. j} t-1}
$$

where:

$F_{t}=$ imputed gas volume for current month $\mathrm{t}$,

$F_{t-1}=$ gas volume for the company for the previous month,

$y_{. j t}=$ gas volume reported by companies in the State stratum for report month $t$,

$y_{. j} t-1=$ gas volume in the previous month for companies in the State stratum that reported in month $t$. 


\section{Final Revisions}

Adjusting Monthly Data to Annual Data. After the annual data reported on the Form EIA-176 have been submitted, edited, and prepared for publicat on in the Natural Gas Annual, revisions are ma e to monthly data. The revisions are made to the volumes and prices of natural gas delivered to consumers that have appeared in the Natural Gas Monthly to match them to the annual values appearing in the Natural Gas Annual. The revised monthly estimates allocate the difference between the sum of monthly estimates and the annual reports according to the distribution of the estimated values across the months.

Before the final revisions are made, changes or additions to submitted data received after publication of the monthly estimate and not sufficiently large to require a revision to be published in the Natural Gas Monthly, are used to derive an updated estimate of monthly consumption and revenues for each State's residential, commercial, or industrial natural gas consumption.

For each State, two numbers are revised, the estimated consumption and the estimated price per thousand cubic feet.

The formula for revising the estimated consumption is:

$$
V_{j m}^{*}=V_{j m}+\left[\left(V_{j a}-V_{j m}^{\prime}\right)\left(\frac{V_{j m}}{V_{j m}^{\prime}}\right)\right]
$$

where:

$V_{j m}^{*}=$ the final volume estimate for month $\mathrm{m}$ in consumer sector $\mathrm{j}$,

$V_{j m}=$ the estimated volume for month $\mathrm{m}$ in consumer sector $\mathrm{j}$,

$V_{j a}=$ the volume for the year reported on Form EIA-176,

$V_{j m}^{\prime}=$ The annual sum of estimated monthly volumes.

The price is calculated as described above in the Estimation Procedures section, using the final revised consumption estimate and a revised revenue estimate.

The formula for revising the estimated revenue is:

$$
R_{j m}^{*}=R_{j m}+\left[\left(R_{j a}-R_{j m}^{\prime}\right)\left(\frac{R_{j m}}{R_{j m}^{\prime}}\right)\right]
$$

where:

$R_{j m}^{*}=$ the final revenue estimate for month $\mathrm{m}$ in consumer sector $\mathrm{j}$.

$\boldsymbol{R}_{j m}=$ the estimated revenue for month $\mathrm{m}$ in consumer sector j,

$R_{j a}=$ the revenue for the year reported on Form EIA-176,

$R_{j m}^{\prime}=$ The annual sum of estimated monthly revenues.

Revision of Volumes and Prices for Deliveries to Electric Utilities. Revisions to monthly electric utilities data are published throughout the year as they become available.

\section{Reliability of Monthly Data}

The monthly data published in this report are subject to two sources of error - nonsampling error and sampling error. Nonsampling errors occur in the collection and processing of the data. See the discussion of the Form EIA-857 in Appendix B for a description of nonsampling errors for monthly data.

Sampling error may be defined as the difference between the results obtained from a sample and the results that a complete enumeration would provide. The standard error statistic is a measurement of sampling error.

Standard Errors. A standard error of an estimate is a statistical measure that indicates how the estimate from the sample compares to the result from a complete enumeration. Standard errors are calculated based on statistical theory that refers to all possible samples of the same size and design.

The standard errors for monthly natural gas volume estimates by State are given in Table C1. Ninety-five percent of the time, the volume that would have been obtained from a complete enumeration will lie in the range between the estimated volume minus two standard errors and the estimated volume plus two standard errors. 
Table C-1. Standard Error for Natural Gas Deliverles and Price to Consumers by State, August 1993

\begin{tabular}{|c|c|c|c|c|c|c|c|}
\hline \multirow[t]{2}{*}{ Stato } & \multicolumn{4}{|c|}{$\begin{array}{c}\text { Volume } \\
\text { Mumion Cubic Feet }\end{array}$} & \multicolumn{3}{|c|}{$\begin{array}{c}\text { Price } \\
\text { Dollare per Thousand Cuble Feet }\end{array}$} \\
\hline & Residential & Commercial & Industrial & Total & Residential & Commercial & Inchustrial \\
\hline $\begin{array}{l}\text { Alabama } \\
\text { Alaska } \\
\text { Arizona } \\
\text { Arkansas } \\
\text { California }\end{array}$ & $\begin{array}{r}78 \\
0 \\
15 \\
41 \\
287\end{array}$ & $\begin{array}{r}324 \\
0 \\
14 \\
61 \\
137\end{array}$ & $\begin{array}{r}1,982 \\
0 \\
0 \\
242 \\
3,319\end{array}$ & $\begin{array}{r}2,010 \\
0 \\
20 \\
253 \\
3,332\end{array}$ & $\begin{array}{l}0.26 \\
-.09 \\
.42 \\
.05\end{array}$ & $\begin{array}{l}0.18 \\
.01 \\
.10 \\
.01\end{array}$ & $\begin{array}{l}0.42 \\
- \\
.02 \\
.25\end{array}$ \\
\hline 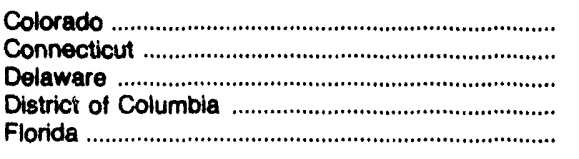 & $\begin{array}{r}6 \\
0 \\
0 \\
0 \\
19\end{array}$ & $\begin{array}{r}23 \\
0 \\
0 \\
0 \\
24\end{array}$ & $\begin{array}{r}0 \\
0 \\
0 \\
0 \\
1,984\end{array}$ & $\begin{array}{r}23 \\
0 \\
0 \\
0 \\
1,984\end{array}$ & $\bar{z}-\overline{-}$ & $\frac{.01}{-}$ & $\overline{-}$ \\
\hline $\begin{array}{l}\text { Georgia } \\
\text { Hawail } \\
\text { Idaho } \\
\text { Illinois } \\
\text { Indiana }\end{array}$ & $\begin{array}{r}539 \\
0 \\
0 \\
270 \\
54\end{array}$ & $\begin{array}{r}759 \\
0 \\
0 \\
569 \\
114\end{array}$ & $\begin{array}{r}501 \\
0 \\
0 \\
731 \\
1,245\end{array}$ & $\begin{array}{r}1,057 \\
0 \\
0 \\
865 \\
1,251\end{array}$ & $\begin{array}{l}.28 \\
- \\
.31 \\
1.13\end{array}$ & $\overline{-16}$ & $\frac{.69}{-}$ \\
\hline 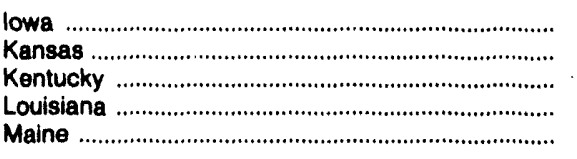 & $\begin{array}{r}28 \\
88 \\
65 \\
42 \\
0\end{array}$ & $\begin{array}{r}474 \\
6,353 \\
79 \\
78 \\
0\end{array}$ & $\begin{array}{r}368 \\
9,874 \\
751 \\
924 \\
0\end{array}$ & $\begin{array}{r}601 \\
11,742 \\
758 \\
928 \\
0\end{array}$ & $\begin{array}{l}.33 \\
.42 \\
.33 \\
.19 \\
-\end{array}$ & $\begin{array}{r}1.07 \\
.26 \\
.26 \\
.26 \\
-\end{array}$ & $\begin{array}{r}.24 \\
5.08 \\
.51 \\
.01 \\
-\end{array}$ \\
\hline $\begin{array}{l}\text { Maryland } \\
\text { Massachusetts } \\
\text { Michigan } \\
\text { Minnesota } \\
\text { Mississippi }\end{array}$ & $\begin{array}{r}5 \\
61 \\
150 \\
14 \\
185\end{array}$ & $\begin{array}{r}6 \\
52 \\
154 \\
57 \\
19\end{array}$ & $\begin{array}{r}68 \\
393 \\
1.349 \\
405 \\
573\end{array}$ & $\begin{array}{r}67 \\
401 \\
1,366 \\
409 \\
603\end{array}$ & $\begin{array}{l}.01 \\
.23 \\
.31 \\
.30 \\
.70\end{array}$ & $\begin{array}{l}.01 \\
.22 \\
.24 \\
.48 \\
.40\end{array}$ & $\begin{array}{l}.05 \\
.09 \\
.44 \\
.61 \\
.21\end{array}$ \\
\hline 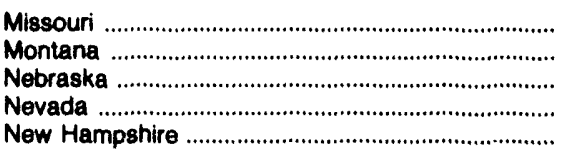 & $\begin{array}{r}210 \\
3 \\
17 \\
0 \\
0\end{array}$ & $\begin{array}{r}133 \\
2 \\
73 \\
0 \\
0\end{array}$ & $\begin{array}{r}712 \\
0 \\
383 \\
0 \\
0\end{array}$ & $\begin{array}{r}755 \\
3 \\
380 \\
0 \\
0\end{array}$ & $\begin{array}{l}.16 \\
.01 \\
.30 \\
- \\
-\end{array}$ & $\begin{array}{l}.15 \\
.02 \\
.02 \\
- \\
-\end{array}$ & $\begin{array}{l}.69 \\
- \\
-\end{array}$ \\
\hline $\begin{array}{l}\text { Ohio } \\
\text { Oklahoma } \\
\text { Oregon } \\
\text { Pennsytvania } \\
\text { Rhode Island }\end{array}$ & $\begin{array}{r}233 \\
7 \\
0 \\
26 \\
0\end{array}$ & $\begin{array}{r}368 \\
189 \\
0 \\
77 \\
0\end{array}$ & $\begin{array}{r}8,538 \\
27 \\
0 \\
2,111 \\
0\end{array}$ & $\begin{array}{r}8,549 \\
181 \\
0 \\
2,113 \\
0\end{array}$ & $\begin{array}{l}.09 \\
.01 \\
- \\
.11\end{array}$ & $\begin{array}{l}.13 \\
.40 \\
. \\
.10\end{array}$ & $\begin{array}{l}.13 \\
.01 \\
. \\
.57 \\
-\end{array}$ \\
\hline $\begin{array}{l}\text { South Carolina } \\
\text { South Dakota } \\
\text { Tennessee } \\
\text { Texas } \\
\text { Utah }\end{array}$ & $\begin{array}{r}8 \\
0 \\
545 \\
394 \\
0\end{array}$ & $\begin{array}{r}18 \\
0 \\
132 \\
15,780 \\
0\end{array}$ & $\begin{array}{r}1,202 \\
0 \\
766 \\
26,617 \\
0\end{array}$ & $\begin{array}{r}1,203 \\
0 \\
950 \\
30,951 \\
0\end{array}$ & $\begin{array}{l}.18 \\
2.11 \\
.41\end{array}$ & $\begin{array}{l}.28 \\
-23 \\
1.69 \\
-\end{array}$ & $\begin{array}{l}.36 \\
.07 \\
.12 \\
-\end{array}$ \\
\hline $\begin{array}{l}\text { Vermont } \\
\text { Virginia } \\
\text { Washington . } \\
\text { West Virginia } \\
\text { Wisconsin } \\
\text { Wyoming }\end{array}$ & $\begin{array}{r}0 \\
86 \\
0 \\
78 \\
197 \\
9\end{array}$ & $\begin{array}{r}0 \\
261 \\
0 \\
130 \\
1,058 \\
47\end{array}$ & $\begin{array}{r}0 \\
2,011 \\
0 \\
278 \\
429 \\
384\end{array}$ & $\begin{array}{r}0 \\
2,030 \\
0 \\
317 \\
1,157 \\
387\end{array}$ & $\begin{array}{l}-.65 \\
-. \\
.86 \\
.25 \\
.14\end{array}$ & $\begin{array}{l}-59 \\
\overline{2.84} \\
1.48 \\
.17\end{array}$ & $\begin{array}{l}\overline{3.00} \\
\overline{.31} \\
.76 \\
.12\end{array}$ \\
\hline U.S. Totais & 1,205 & 17,144 & 30,280 & 34,818 & .10 & .48 & .29 \\
\hline
\end{tabular}

- $=$ Not Applicable.

Source: Energy Information Administration, Form ElA-857 ."Monthly Report of Natural Gas Purchases and Detheries to Coneumers." 
The standard error of the natural gas volume estimate is the square root of the variance of the estimate. The formula for calculating the variance of the volume estimate is:

$$
\hat{V(Y)}=\sum_{h=1}^{\psi}\left[N_{h}^{2} \frac{\left(1-\frac{n_{h}}{N_{h}}\right)}{n_{h}\left(n_{h}-1\right)}\left(\sum_{i=1}\left(y_{i}-T x_{i}\right)^{2}\right)\right]
$$

where:
$H=$ the total number of strata

$N_{h}=$ the total number of companies in stratum $h$

$n_{h}=$ the sample size in stratum $\mathrm{h}$

$y_{i}=$ the reported monthly volume for company $\mathrm{i}$

$x_{i}=$ the reported annual volume for company $\mathrm{i}$

$T=$ the ratio of the sum of the reported monthly volumes for sample companies to the sum of the reported annual volumes for the sample companies. 
Appendix D

\author{
Natural Gas \\ Reports and \\ Feature Articles
}




\section{Natural Gas Reports and Feature Articles}

\section{Reports Dealling Principally with Natural Gas and/or Natural Gas Liquids}

- Natural Gas Annual 1991, DOE/EIA-0131(91), October 1992.

- Natural Gas Annual 1991 Supplement: Company Profiles DOE/EIA-0131(91/S), January 1993.

- Statistics of Interstate Natural Gas Pipeline Companies 1991, DOE/EIA-0145(91), December 1992.

- Gas Supplies of Interstate Natural Gas Pipeline Companies 1991, DOE/EIA-0167(91), December 1992.

\section{Other Reports Covering Natural Gas, Natural Gas Liquids, and Other Energy Sources}

- U.S. Crude Oil, Natural Gas, and Natural Gas Liquids Reserves - 1992 Annual Report, DOE/EIA-0216(92), October 1993.

- Monthly Energy Review, DOE/EIA-0035. Published monthly. Provides national aggregate data for natural gas, natural gas liquids, and other energy sources.

- Annual Report to Congress 1992, DOE/ EIA-0173(92), March 1993. Published annually.

- Annual Energy Outlook 1993, DOE/ EIA-0383(93), January 1993. Published annually.

- Annual Energy Review 1992, DOE/ EIA-0384(92), June 1993. Published annually.

- Short-Term Energy Outlook, DOE/EIA-0202. Published quarterly. Provides forecasts for next six quarters for natural gas and other energy sources.

\section{Selected One-Time Natural Gas and Related Reports}

- U.S. Production of Natural Gas from Tight Reservoirs, DOE/EIA-TR-0574, October 1993

- Energy Policy Act Transportation Rate Study, DOE/EIA-0571, October 1993

- Natural Gas 1992: Issues and Trends, DOE/EIA-0560(20), March 1993.

- Natural Gas Productive Capacity for the Lower 48 States, 1982 through 1993,

DOE/EIA-0542(93), March 1993.

- Geologic Distributions of U.S. Oil and Gas, DOE/ EIA-0557, July 1992.

- Capacity and Service on the Interstate Natural Gas Pipeline System 1990, DOE/EIA-0556, June 1992.

- Effects of Interruptible Natural Gas Service: Winter 1989-1990, SR/OG-91-01, June 1991.

- The Outlook for Natural Gas Imports: Supporting Analysis for the National Energy Strategy, SR/ NES/90-06, January 1991.

\section{Selected and Recurring Natural Gas and Related Data Reference Reports}

- Directory of Energy Data Collection Forms, DOE/ EIA-0249(92), January 1993.

- Oil and Gas Field Code Master List, 1991, EIA-0370(91), January 1992. 
January 1991

\section{Take-or-Pay Settlements}

(Presents information on the resolutions of the takeor-pay issue.)

\section{February 1991}

Recent Trends and Regional Variability in Underground Storage Activities

(Discusses underground storage in the expansion of end-use markets.)

\section{March 1991}

Natural Gas Transportation Services: An Update

(Reassesses transportation market activities by examining data through 1989 for major interstate pipeline companies.)

\section{April 1991}

An Overview of Recent Developments in the Underground Storage Market

(Reviews the changing market for underground natural gas storage services in the United States.)

\section{May 1991}

Analysis of Long-Term Contracts for Imports of Canadian Natural Gas

(Focuses on the contractual provisions that will govern imports of natural gas from Canada through the 1990's and beyond.)
Natural Gas Productive Capacity for the Lower 48 States, 1980 through 1991

(Summarizes the full report and contrasts the findings for two major producing regions: Texas and the Gulf of Mexico Outer Continental Shelf.)

\section{July 1991}

The Outlook for Natural Gas 1991

(Focuses upon the natural gas supply and market aspects of the forecasts contained in the Annual Outlook for Oil and Gas 1991.)

\section{August 1991}

\section{U.S. Natural Gas Imports and Exports - 1990}

(Contains final 1990 data on all U.S. imports and exports of natural gas.)

\section{September 1991}

\section{Background on the Natural Gas Industry}

(Focuses on changes in the industry that pertain to natural gas.)

Domestic Natural Gas Reserves and Production Dedicated to Interstate Pipeline Companies, 1990

(Provides preliminary data on natural gas reserves and production dedicated to the interstate market.)

\section{March 1992}

\section{Revisions to Monthly Natural Gas Data}

(Discusses the revision errors for natural gas data.) 
U.S. Natural Gas Imports and Exports - 1991

(Contains final 1991 data on all U.S. imports and exports of natural gas.)

\section{November 1992}

\section{Natural Gas Futures Contract Market - The First 2}

Years

(Reviews the financial and economic significance of trading in natural gas futures markets.)

\section{December 1992}

Three-Dimensional Seismology -- A New Perspective

(Describes the impact 3D seismology will have on future U.S. reserves and production.)

\section{Imports of Canadian Gas Under Long-Term Contracts}

(Addresses how regulatory changes have altered the contractual revisions of long-term agreements.)

\section{March 1993}

\section{Natural Gas 1992: Issues and Trends}

(Provides an overview of the natural gas industry in 1991 and 1992, focusing on trends in production, consumption, and pricing of natural gas.)
(Analyzes monthly natural gas wellhead productive capacity and projects this capacity for 1992 and 1993.)

\section{Aprll 1993}

Revisions to Monthly Natural Gas Data

(Discusses the revision errors for natural gas data.)

\section{August 1993}

U.S. Natural Gas Imports and Exports - 1992

(Contains final 1992 data on all U.S. imports and exports of natural gas.)

\section{November 1993}

\section{U.S. Production of Natural Gas from Tight Reservoirs}

(Discusses the economic incentives offered to induce operators to explore for and develop gas reservoirs from unconventional sources.)

\section{The Expanding Role of Underground Storage}

(Discusses the expanded role of underground natural gas storage in the restructured natural gas industry.) 
Appendix E

Technical

Contacts 


\section{Technical Contacts}

\section{Section}

\begin{abstract}
Summary Statistics:
Natural Gas Production

and Consumption
\end{abstract}

Supplemental Gaseous Fuels

Imports and Exports

Price:

City Gate, Residential,

Commercial, and Industrial

Imports and Purchases

from Producers

Wellhead

Electric Utility

Summary of Natural Gas Imports and Exports

Producer Related Activities:

Natural Gas Production

Interstate Pipeline Activities:

Underground Storage:

Distribution and Consumption:

Deliveries to:

Residential,

Commercial,

Industrial,

Electric Utility,

All Consumers

Average Price to:
Tables

\section{$1,2,3$}

Monthly:
Annual:

Principal Data Sources

Technical Contact

Interstate Oil and Gas Compact

Commission (IOGCC)

EIA-627, "Annual Quantity and

Donna Guerrina

(202) $586-6135$
Value of Natural Gas Report"

Monthly: Form EIA-857, "Monthly Report of Natiral Gas Purchases and Deliveries to $C$ insumers"

1 Monthly:

Annual:

Monthly:

Annual:

2

Monthly:

Annual:

4

4

Monthly:

$8,9,10$,

11,12

$13,14,15$, 16,17

Monthly:

Monthly:

EIf computations

Form EIA-816, "Monthly Natural

Gas Liquids Report" and

Form EIA-64A, "Annual Report of the Origin of Natural Gas Liquids

Production"

EIA computations

Form EIA-176, "Annual Report of

Natural and Supplemental Gas Supply and Disposition"

EIA computations

Form FPC-14, "Annual Reports for Importers and Exporters of Natural Gas"

Monthly:

Monthly:

Monthly:

Annual:

Monthly:

Monthly:

Form EIA-857, "Monthly Report of Natural Gas Purchases and Deliveries to Consumers"

Form FERC-11, "Natural Gas Pipeline Company Monthly Statement"

EIA computations

Form EIA-627, "Annual Quantity and Value of Natural Gas Report"

Form FPC-423, "Cost and Quality of Fuels for Electric Power Plants"

Form FPC-14, "Annual Report for Importers and Exporters of Natural Gas"

Interstate Oil and Gas Compact Commission (IOGCC)

Form FERC-11, "Natural Gas Pipeline Company Monthly Statement"

Forms FERC-8 and EIA-191, "Underground Gas Storage Report"

Monthly:

Form EIA-857, "Monthly Report of Natural Gas Purchases and Deliveries to Consumers"

Form FERC-423, "Cost and Quality of Fuels for Electric Power Plants"
Roy Kass

(202) $586-4790$

Margo Natof

(202) 586-6303

Donna Guerrina (202) 586-6135

Margo Natof

(202) 586-6303

Norman Crabtree

(202) 586-6181

Roy Kass

(202) $586-4790$

James Keeling (202) 586-6107

Donna Guerrina

(202) 586-6135

Roy Kass

(202) $586-4790$

Norman Crabtree

(202) 586-6181

Donna Guerrina (202) 586-6135

James Keeling (202) 586-6107

Rosemary Jameson (202) 586-6229

Roy Kass

(202) $586-4790$ 
City Gate

Residential,

Commercial,

Industrial,

Electric Utility

Onsystem Sales

Heating Degree Days

29

Highlights

Industry Overview
Monthly: Form ElA-857, "Monthly Report of Natural Gas Purchases and Deliveries to Consumers"

Form FERC-423, "Cost and Quality of Fuels for Electric Power Plants"

Monthly:

Form EIA-857, "Monthly Report of Natural Gas Purchases and Deliveries to Consumers"

Seasonal: National Oceanic and Atmospheric Administration
Roy Kass

(202) $586-4790$

Roy Kass

(202) $586-4790$

Rosemary Jameson (202) 586-6229

Audrey Corley (202) 586-4804

Eva Fleming (202) 586-6113 


\section{Glossary}

Balancing Item: Represents the difference between the sum of the components of natural gas supply and the sum of the components of natural gas disposition. These differences may be due to quantities lost or to the effects of data reporting problems. Reporting problems include differences due to the net result of conversions of flow data metered at varying temperature and pressure bases and converted to a standard temperature and pressure base; the effect of variations in company accounting and billing practices; differences between billing cycle and calendar period time frames; and imbalances resulting from the merger of data reporting systems which vary in scope, format, definitions, and type of respondents.

Base (Cushion) Gas: The volume of gas needed as a permanent inventory to maintain adequate underground storage reservoir pressures and deliverability rates throughout the withdrawal season. All native gas is included in the base gas volume.

British Thermal Unit (Btu): The heat required to raise the termperature of one pound of water by one degree Fahrenheit at or near 39.2 degrees Fahrenheit.

City-gate: A point or measuring station at which a gas distribution company receives gas from a pipeline company or transmission system.

Commercial Consumption: Gas used by nonmanufacturing organizations such as hotels, restaurants, retail stores, laundries, and other service enterprises, and gas used by local, State, and Federal agencies engaged in nonmanufacturing activities.

Depletion: The loss in service value incurred in connection with the exhaustion of the natural gas reserves in the course of service.

Depreciation: The loss in service value not restored by current maintenance, incurred in connection with the consumption or respective retirement of a gas plant in the course of service from causes that are known to be in current operation and against which the utility is not protected by insurance; for example, wear and tear, decay, obsolescence, changes in demand and requirements of public authorities, and the exhaustion of natural resources.

Dry Natural Gas Production: Marketed production less extraction loss.

Electric Utility Consumption: Gas used as fuel in electric utility plants.

Exports: Natural gas deliveries out of the continental United States and Alaska to foreign countries.

Extraction Loss: The reduction in volume of natural gas resulting from the removal of natural gas liquid constituents at natural gas processing plants.

Flared: The volume of gas burned in flares on the base site or at gas processing plants.

Gross Withdrawals: Full well stream volume, including all natural gas plant liquid and nonhydrocarbon gases, but excluding lease condensate. Also includes amounts delivered as royalty payments or consumed in field operations.

Hinshaw Pipeline: A pipeline or local distribution company that has received exemption, (by Section 1 (c) of the Natural Gas Act), from regulations pursuant to the Natural Gas Act. These companies transport interstate natural gas not subject to regulations under NGA.

Imports: Natural gas received in the Continental United States (including Alaska) from a foreign country. 
Independent Producers: Any person who is engaged in the production or gathering of natural gas and who sells natural gas in interstate commerce for resale but who is not engaged in the transportation of natural gas (other than gathering) by pipeline in interstate commerce.

Industrial Consumption: Natural gas used by manufacturing and mining establishments for heat, power, and chemical feedstock.

Interstate Companies: Natural gas pipeline companies subject to FERC jurisdiction.

Intransit Deliveries: Redeliveries to a foreign country of foreign gas received for transportation across U.S. territory and deliveries of U.S. gas to a foreign country for transportation across its territory and redelivery to the United States.

Intransit Receipts: Receipts of foreign gas for transportation across U.S. territory and redelivery to a foreign country and redeliveries to the United States of U.S. gas transported across foreign territory.

Intrastate Companies: Companies not subject to FERC jurisdiction.

Lease and Plant Fuel: Natural gas used in well, field, lease operations and as fuel in natural gas processing plants.

Liquefied Natural Gas (LNG): Natural gas that has been liquefied by reducing its temperature to minus 260 degrees Fahrenheit at atmospheric pressure.

Major Interstate Pipeline Company: A company whose combined sales for resale, and gas transported interstate or stored for a fee, exceeded 50 million thousand cubic feet in the previous year.

Marketed Production: Gross withdrawals less gas used for repressuring, quantities vented and flared, and nonhydrocarbon gases removed in treating or processing operations. Includes all quantities of gas used in field and processing operations. See Explanatory Note 1 for discussion of coverage of data concerning nonhydrocarbon gases removed.
Native Gas: Gas in place at the time that a reservoir was converted to use as an underground storage reservoir as in contrast to injected gas volumes.

Natural Gas: A mixture of hydrocarbon compounds and small quantities of various nonhydrocarbons existing in the gaseous phase or solution with oil in natural underground reservoirs at reservoir conditions.

Natural Gas Policy Act of 1978 (NGPA): Signed into law on November 9, 1978, the NGPA is a framework for the regulation of most facets of the natural gas industry. See Explanatory Note 10 for a full discussion.

Nonhydrocarbon Gases: Typical nonhydrocarbon gases that may be present in reservoir natural gas are carbon dioxide, helium, hydrogen sulfide, and nitrogen.

Onsystem Sales: Sales to customers where the delivery point is a point on, or directly interconnected with, a transportation, storage, and/or distribution system operated by the reporting company.

Pipeline Fuel: Gas consumed in the operation of pipelines, primarily in compressors.

Repressuring: The injection of gas into oil or gas formations to effect greater ultimate recovery.

Residential Consumption: Gas used in private dwellings, including apartments, for heating, cooking, water heating, and other household uses.

Storage Additions: The volume of gas injected or otherwise added to underground natural gas or liquefied natural gas storage during the applicable reporting period.

Storage Withdrawals: Total volume of gas withdrawn from underground storage or liquefied natural gas storage during the applicable reporting period.

Supplemental Gaseous Fuels Supplies: Synthetic natural gas, propane-air, refinery gas, biomass gas, air injected for stabilization of heating content, and manufactured gas commingled and distributed with natural gas. 
Synthetic Natural Gas (SNG): A manufactured product chemically similar in most respects to natural gas, that results from the conversion or reforming of petroleum hydrocarbons and may easily be substituted for or interchanged with pipeline quality natural gas.

Therm: One-hundred thousand British thermal units.

Underground Gas Storage Reservoir Capacity: Interstate company reservoir capacities are those certificated by FERC. Independent producer and intrastate company reservoir capacities are reported as developed capacity.
Vented Gas: Gas released into the air on the base site or at processing plants.

Wellhead Price: Represents the wellhead sales price, including charges for natural gas plant liquids subsequently removed from the gas, gathering and compression charges, and State production, severance, and/or similar charges.

Working (Top Storage) Gas: The volume of gas in an underground storage reservoir above the designed level of the base. It may or may not be completely withdrawn during any particular withdrawal season. Conditions permitting, the total working capacity could be used more than once during any season. 
Feature Article:

Feature Article: 
Statement of Ownership,

Management and

Circulation

(Required by 39 U.S.C. 3685)

1A. Title of Publication

NATURAL GAS MONTHLY

3. Frequency of lasue

MONTHLY

\begin{tabular}{|l|l|l|l|l|l|l|}
\hline \multicolumn{1}{|c|}{ 18. PUELICATION NO. } \\
\hline 7 & 3 & 7 & - & 1 & 7 & 1 \\
\hline
\end{tabular}

34. No. of lasues Publiohod Annually

12
2. Date of Filing

$09|28| 93$

36. Annual Subscription price

Domestic $\$ 85.00$

Forelgn $\$ 106.25$

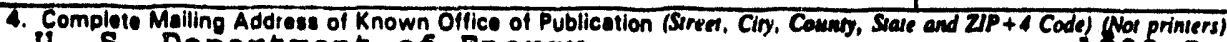

U. S. Department of Energy

Energy Information Administration

1000 Independence Avenue, SW

Washington, DC 20585

6. Comploto Moiling Address of the Haedquartors of Genaral Businass Offices of the Publisher (Na printer)

U. S. Department of Energy

Energy Information Administration

1000 Independence Avenue, sw

Washington, DC 20585

6. Full Nemes and Complote Mailing Addiass of Publisher, Editor, and Managing Editor (This term MUST NOT be Meak)

Publishor (Name and Complere Mailing Address)

U. S. Department of Energy

Energy Information Administration

1000 Independence Avenue, sw washington, DC 20585

Ediror (Name and Complece Mailing Address)

Doris Wells

U. S. Department

1000 Independence Avenue, SW Washington, DC 20585

Managing Edhor (Name and Compleie Malling Address)

Nancy Nicolett 1

U. S. Department of Energy

1000 Independence Avenue, SW

Washington, DC 20585

7. Owner tf owned by a corporation. its name and address must be stated and also immediately thereunder the nomes and addresses of suactholders ouning or holding I percent or more of tolal amount of stack. If not owned by a corporation, the names and addresses of the individual owners must be given. $V$ owned by a partinership or other unincorporated firm, its name and address. as well as that of each individual must be siven. If the publication is published by a nonproft organization. its name and address must be staled.) (hem must be complered.)

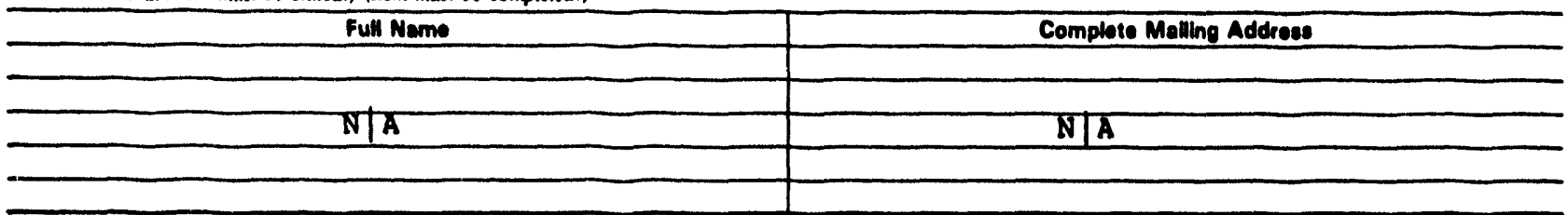

8. Known Bondholdors. Mortgegees, and Other Security Holders Owning or Holding I Percent or More of Total Amount of Bonds, Mortgages or Other Securitios (ff there are none. so stase)

\begin{tabular}{c|c|c|c|}
\hline Full Name & Complete Malling Addrens \\
\hline & & \\
\hline & N/A & N & A \\
\hline & & \\
\hline
\end{tabular}

9. For Complotion by Nonproflt Organizations Authorized To Mall et Special Retes (DMM Section 124.12 only)

The purpose, function, and nonprofit status of this orgenization and the exemps status for faderal income tax purposes (Check one) (1) (2)

X Has Not Changed During $\square$ Hes Changed During

(I) changed, publisher must submil explanation of change with this stasement.)

\begin{tabular}{|c|c|c|c|}
\hline 10. & $\begin{array}{l}\text { Extent end Nature of Cliculation } \\
\text { (Ste instructions on reverse side) }\end{array}$ & $\begin{array}{l}\text { Average No. Coples Eech lseue Ouring } \\
\text { Preceding } 12 \text { Months }\end{array}$ & $\begin{array}{l}\text { Actual No. Copios of Single lesue } \\
\text { Published Nearest to Flling Date }\end{array}$ \\
\hline \multicolumn{2}{|c|}{ A. Total No. Coplos (Net Press Run) } & 1475 & 1515 \\
\hline \multicolumn{2}{|r|}{$\begin{array}{l}\text { 8. Paid endlor Requested Clrculation } \\
\text { 1. Salos through dealors and carriers, street vendors and countor calos }\end{array}$} & 778 & 780 \\
\hline \multicolumn{2}{|r|}{$\begin{array}{l}\text { 2. Mail Subscription } \\
\text { (Pald and/or requested) }\end{array}$} & 556 & 596 \\
\hline \multicolumn{2}{|r|}{$\begin{array}{l}\text { C. Total Paid and/or Requested Circulotion } \\
\text { (Swm of } 1081 \text { and } 1082)\end{array}$} & 1334 & 1376 \\
\hline \multicolumn{2}{|r|}{$\begin{array}{l}\text { D. Free Diatribution by Mail, Cerriber or Other Means } \\
\text { Samples, Complimontery, and Other Free Copies }\end{array}$} & 74 & 77 \\
\hline \multicolumn{2}{|r|}{ E. Total Diatribution (Sum of C and D) } & 1408 & 1453 \\
\hline \multicolumn{2}{|r|}{$\begin{array}{l}\text { F. Copies Not Distributed } \\
\text { 1. Office use. loft ovar, unaccounted, spolled after printing }\end{array}$} & 67 & 62 \\
\hline \multicolumn{2}{|r|}{ 2. Return from Nows Agente } & 0 & 0 \\
\hline \multicolumn{2}{|r|}{ a. TOTAL (Sum of E, FI and 2-should equal net press nun shown in A) } & 1475 & 1515 \\
\hline \multicolumn{4}{|c|}{$\begin{array}{l}\text { I1. I certify that the statements made by } \\
\text { me above are correct and complete }\end{array}$} \\
\hline
\end{tabular}




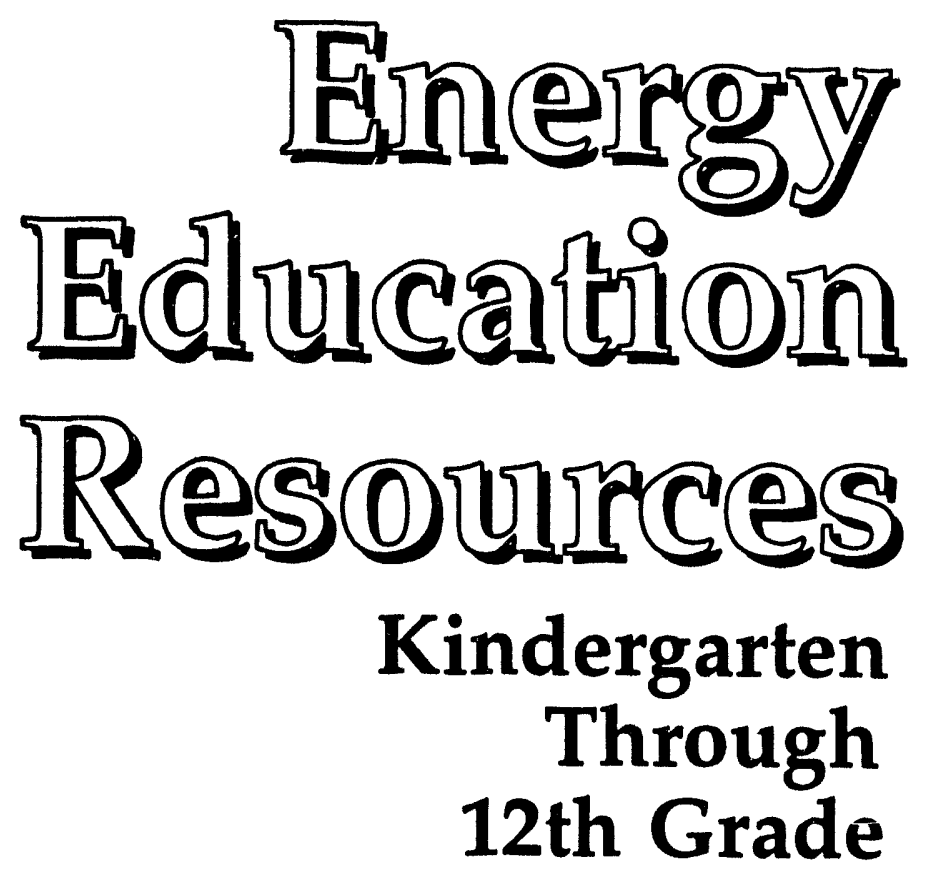

This brochure contains a listing of free or low-cost energy-related educational materials for educators and primary and secondary students.

Call the National Energy Information Center on (202)586-8800 to order your free copy. 

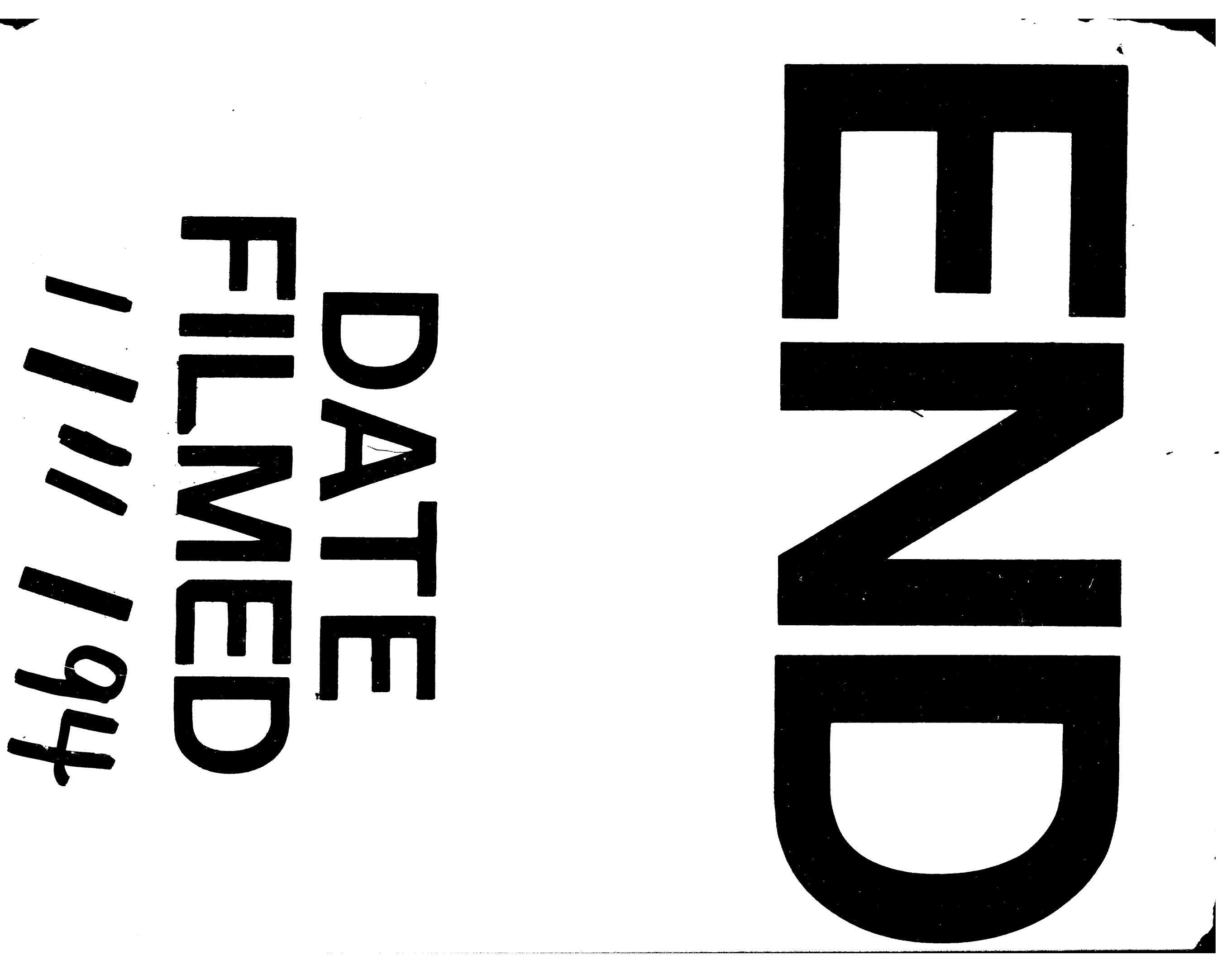